\title{
Savannah River Site Environmental Data for 1995
}

by

\author{
M. W. Arnett
}

Westinghouse Savannah River Company

Savannah River Site

Aiken, South Carolina 29808

A. Mamatey

DOE Contract No. DE-AC09-89SR18035

This paper was prepared in connection with work done under the above contract number with the U.S.

Department of Energy. By acceptance of this paper, the publisher and/or recipient acknowledges the U.S.

Government's right to retain a nonexclusive, royalty-free license in and to any copyright covering this paper, along with the right to reproduce and to authorize others to reproduce all or part of the copyrighted paper.

\section{DISTRIBUTKON OF THS DOCUNENT IS URLARTED}

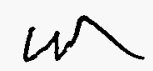




\section{DISCLAMMER}

This report was prepared as an account of work sponsored by an agency of the United States Government. Neither the United States Government nor any agency thereof, nor any of their employees, makes any warranty, express or implied, or assumes any legal liability or responsibility for the accuracy, completeness, or usefulness of any information, apparatus, product, or process disclosed, or represents that its use would not infringe privately owned rights. Reference herein to any specific commercial product, process, or service by trade name, trademark, manufacturer, or otherwise does not necessarily constitute or imply its endorsement, recommendation, or favoring by the United States Government or any agency thereof. The views and opinions of authors expressed herein do not necessarily state or reflect those of the United States Government or any agency thereof.

This report has been reproduced directly from the best available copy.

Available to DOE and DOE contractors from the Office of Scientific and Technical Information, P.O. Box 62, Oak Ridge, TN 37831; prices available from (615) 576-8401.

Available to the public from the National Technical Information Service, U.S. Department of Commerce, 5285 Port Royal Road, Springfield, VA 22161. 


\section{DISCLAIMER}

This report was prepared as an account of work sponsored by an agency of the United States Government. Neither the United States Government nor any agency thereof, nor any of their employees, makes any warranty, express or implied, or assumes any legal liability or responsibility for the accuracy, completeness, or usefulness of any information, apparatus, product, or process disclosed, or represents that its use would not infringe privately owned rights. Reference herein to any specific commercial product, process, or service by trade name, trademark, manufacturer, or otherwise does not necessarily constitute or imply its endorsement, recommendation, or favoring by the United States Government or any agency thereof. The views and opinions of authors expressed herein do not necessarily state or reflect those of the United States Government or any agency thereof. 


\section{DISCLAIMER}

Portions of this document may be illegible in electronic image products. Images are produced from the best available original document. 


\section{Savannah River Site Environmental Data for 1995}

Editor

Margaret W. Arnett

Prepared for the U.S. Department of Energy Under Contract No. DE-ACO9-89SR18035

Westinghouse Savannah River Company Savannah River Site, Aiken, SC 29808 


\section{Acknowledgments}

The editor acknowledges with appreciation the efforts of the following individuals and groups:

Environmental Monitoring Section/Environmental Protection Department (for technical expertise, review, clerical support, and oversight during the preparation of this book)

Chris Arenz
Sandra Boynton
Brian Crandall
Mary Dodgen
Larry Eldridge
Dave Filler
Pete Fledderman
Lynne Geary
Manley Grove
June Hall
Jim Heffner

Chris Arenz

Sandra Boynton

Mary Dodgen

Larry Eldridge

Pete Fledderman

Lynne Geary

June Hall

Jim Heffner

\author{
Bob Henderson \\ Tracey Humphrey \\ Moheb Khalil \\ Bill Littrell \\ Bob Lorenz \\ Al Mamatey \\ Phil Miller \\ Priscilla Patterson \\ Stuart Stinson \\ Neil Suttles \\ Robin Young
}

\section{Westinghouse Savannah River Company}

Timothy Jannik and Bill Carlton (for dose estimates)

John Ellinger and Karl Bergmann (for computer and software support)

Larry Koffman and Jim Bollinger (for help with graphic and text transfer)

WSRC Management Services (for illustrating, classification, and printing support-Julie Bean; Dawn Beam; Eleanor Justice; Dennis Hendrix; Carol Attaway; Bonita Bell; Rette Spencer; Jeanne Sellers; Trish Baughman; Pat Dominey; Julie Hearn; Sharon Lybrand; Lee Prim; Stephanie Doetsch; Ann Scott)

\section{Department of Energy-Savannah River}

Mary Langford, coordinator; Chuck Borup; and Ron Peterson (for DOE-SR review and approval)

Mina Perrin, Ann Thompson (for DOE-SR classification)

\section{Environmental Advisory Committee (for independent technical review)}

Dr. Edgar Berkey, National Environmental Technology Applications Corporation, University of Pittsburgh

Dr. Keros Cartwright, Illinois State Geological Survey

Dr. Bernd Kahn, Environmental Research Center, Georgia Institute of Technology

Dr. Ruth Patrick, Francis Boyer Chair of Limnology, Academy of Natural Sciences

Dr. Milton Russell, Energy, Environment and Research Center, University of Tennessee

Dr. Gordon Wolman, Department of Geography and Environmental Engineering, Johns Hopkins University 
This document presents data from Savannah River Site routine environmental monitoring and surveillance programs. An attempt also has been made to include all available data from environmental research programs.

The first section of the book is a collection of maps of radiological and nonradiological sampling locations. Next are a list of the media sampled, along with sample sizes and representative aliquots; minimum detectable concentrations for gamma analysis of water and air samples; minimum detectable concentrations for gamma analysis of soil, food, fish and wildlife, and vegetation samples; minimum detectable concentrations for Environmental Monitoring Section radiological analyses; minimum detectable activities for radionuclides in settleable solids; and nonradiological environmental surveillance detection/report limits.

Following the first sections are tables with radiological and nonradiological effluent monitoring results, radiological and nonradiological environmental surveillance results, dose estimates, and quality assurance data. Because of space requirements of data table columns, many abbreviations are used. To assist the reader, lists of radionuclide and chemical nomenclature and sampling location abbreviations are included following this introduction.

Data tables sometimes present fewer results than would be expected according to the frequency described in the sampling and analysis schedules. There are several reasons for this. Sample collection problems, such as loss of power to the sampling site or inaccessibility to the sampling site (locked gates, flooding, etc.) may have arisen. Results for collected samples can be rejected for such reasons as insufficient sample volume, low chemical yield, or equipment failure. The "number of samples" columns in the tables refer to the number of results used to determine maximum, minimum, and average concentrations.

The following should aid the reader in interpreting the data:

- The uncertainty term generally is reported with up to three significant figures. In most cases, the last significant figure is determined by the quantification of the uncertainty term.

- The reported uncertainty reflects only the counting error-not other components of random and systematic error in the measurement process. For this reason, some results may imply a greater confidence than the determination would suggest.

- Uncertainties quoted with means represent the deviation of measurements about the mean value. This number is calculated from the results themselves and does not account for the uncertainties of the individual results.

- Averages are calculated using both positive and negative results, except for gamma-emitting radionuclides, whose less-than-detectable results are not considered in the averages.

- In tables containing arithmetic mean, maximum, and minimum columns, when only one sample was collected, sometimes only the single result is reported in the arithmetic mean column.

An errata page follows the tables.

Information in this book is summarized in the Savannah River Site Environmental Report for 1995 (WSRCTR-96-0075). Information about the environmental monitoring program, including a complete description of the Environmental Protection Department/Environmental Monitoring Section sampling and analytical procedures, can be found in sections 1101-1111 (SRS EM Program) of the Savannah River Site Environmental Monitoring Section Plans and Procedures, WSRC-3Q1-2, Volume 1. Copies of environmental reports and documents may be obtained by contacting

\author{
Bob Lorenz \\ Manager, Environmental Sampling and Reporting \\ Westinghouse Savannah River Company \\ Building 735-16A \\ Aiken, SC 29808 \\ Telephone: 803-725-3556 \\ e-mail address: robert.lorenz@srs.gov
}




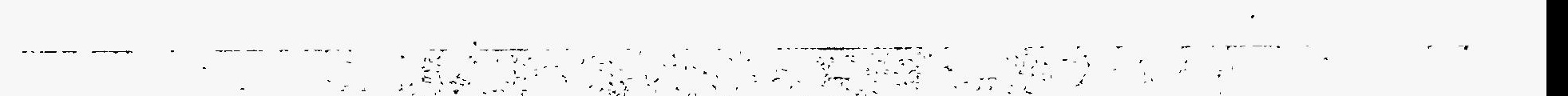




\section{Radionuclide and Chemical Nomenclature}

\begin{tabular}{|c|c|c|}
\hline \multicolumn{3}{|c|}{ Nomenclature and Half-Life for Radionuclides } \\
\hline Radionuclide & Symbol & Half-life ${ }^{a}, b$ \\
\hline Americium-241 & Am-241 & $432.7 y$ \\
\hline Americium-243 & Am-243 & 7.37E3 y \\
\hline Antimony-125 & $\mathrm{Sb}-125$ & $2.7 y$ \\
\hline Argon-41 & Ar-41 & $1.83 \mathrm{~h}$ \\
\hline Berylium-7 & $\mathrm{Be}-7$ & $53 d$ \\
\hline Californium-252 & Cf-252 & $2.638 y$ \\
\hline Carbon-14 & C-14 & $5,730 y$ \\
\hline Cerium-141 & $\mathrm{Ce}-141$ & $33 d$ \\
\hline Cerium-144 & Ce-144 & $284 d$ \\
\hline Cesium-134 & Cs-134 & $2.05 y$ \\
\hline Cesium-137 & Cs-137 & $30 y$ \\
\hline Cobalt-58 & Co-58 & $71.3 d$ \\
\hline Cobalt-60 & Co-60 & $5.26 \mathrm{y}$ \\
\hline Curium-242 & $\mathrm{Cm}-242$ & $163 d$ \\
\hline Curium-244 & $\mathrm{Cm}-244$ & $17.6 y$ \\
\hline lodine-129 & $1-129$ & $1.7 E 7 y$ \\
\hline lodine-131 & $|-13|$ & $8.05 d$ \\
\hline Krypton-85 & $\mathrm{Kr}-85$ & $10.76 y$ \\
\hline Krypton-88 & $\mathrm{Kr}-88$ & $2.8 \mathrm{~h}$ \\
\hline Manganese-54 & $\mathrm{Mn}-54$ & $312 \mathrm{~d}$ \\
\hline Niobium-95 & $\mathrm{Nb}-95$ & $35 d$ \\
\hline Osmium-185 & Os-185 & $94 d$ \\
\hline Phosphorus-32 & P-32 & $14.3 \mathrm{~d}$ \\
\hline Polonium-210 & Po-210 & $138.4 \mathrm{~d}$ \\
\hline Plutonium-238 & Pu-238 & $87.4 y$ \\
\hline Plutonium-239 & Pu-239 & $2.4 E 4 y$ \\
\hline Potassium-40 & $K-40$ & $1.26 E 9 y$ \\
\hline Promethium-147 & Pm-147 & $2.62 y$ \\
\hline Ruthenium-103 & Ru-103. & $39.6 \mathrm{~d}$ \\
\hline Ruthenium-106 & Ru-106 & $367 d$ \\
\hline Selenium-75 & Se-75 & $120.4 d$ \\
\hline Strontium-89 & Sr-89 & $52 d$ \\
\hline Strontium-90 & Sr-90 & $28.1 y$ \\
\hline Tritium & $H-3$ & $12.3 y$ \\
\hline Uranium-235 & U-235 & $7.1 \mathrm{E} 8 \mathrm{y}$ \\
\hline Uranium-238 & U-238 & 4.5E9 y \\
\hline Xenon-133 & $\mathrm{Xe}-133$ & $5.27 d$ \\
\hline Xenon-135 & $\mathrm{Xe}-135$ & $9.16 h$ \\
\hline Yttrium-90 & $Y-90$ & $64 \mathrm{~h}$ \\
\hline Zirconium-95 & $Z r-95$ & $65 d$ \\
\hline
\end{tabular}

a $h=$ hour; $d=$ day; $y=$ year

b Reference: Chart of the Nuclides, 14th edition, revised to April 1988, General Electric Company 
Nomenclature for Elements and Chemical Constituent Analyses

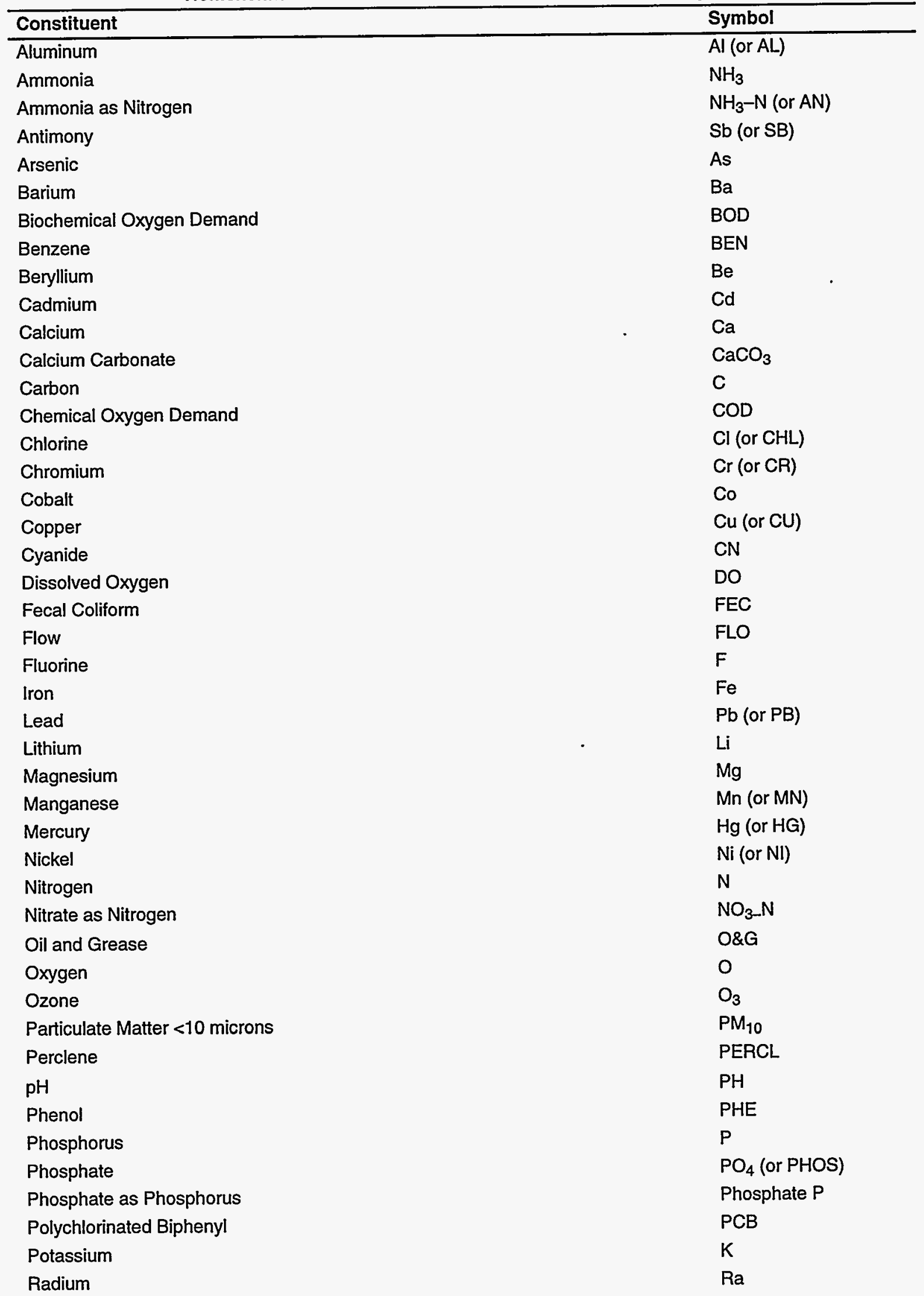


Nomenclature for Elements and Chemical Constituent Analyses

\begin{tabular}{ll}
\hline Constituent & Symbol \\
\hline Rhenium & $\mathrm{Re}$ \\
Selenium & $\mathrm{Se}$ (or SE) \\
Silver & $\mathrm{Ag}$ \\
Sodium & $\mathrm{Na}$ \\
Sulfate & $\mathrm{SO}_{4}$ \\
Sulfur Dioxide & $\mathrm{SO}_{2}$ \\
Temperature & $\mathrm{TMP}$ \\
Tetrachloroethylene (Perchloroethylene) & $\mathrm{PERCL}$ \\
Trichloroethylene & $\mathrm{TRICL}$ \\
1,1,1-Trichloroethane & $\mathrm{TCE}$ \\
Thallium & $\mathrm{TI}$ \\
Tin & $\mathrm{SN}$ \\
Total Dissolved Solids & $\mathrm{TDS}$ \\
Total Kjeldahl Nitrogen & $\mathrm{TKN}$ \\
Total Organic Carbon & $\mathrm{TOC}$ \\
Total Organic Halogens & $\mathrm{TOH}$ \\
Total Phosphates & $\mathrm{TPO}$ \\
Total Residual Chlorine & $\mathrm{TRC}$ \\
Total Solids & $\mathrm{TS}$ \\
Total Suspended Solids & $\mathrm{TSS}$ \\
Uranium & $\mathrm{U}$ \\
Uranium (tested as a heavy metal) & $\mathrm{U} \mathrm{O}_{8}$ \\
Vanadium & $\mathrm{V}$ \\
Volatile Organic Compound & $\mathrm{VOC}$ \\
Zinc & $\mathrm{Zn}$ \\
\hline
\end{tabular}





\section{Sampling-Location and Other Abbreviations}

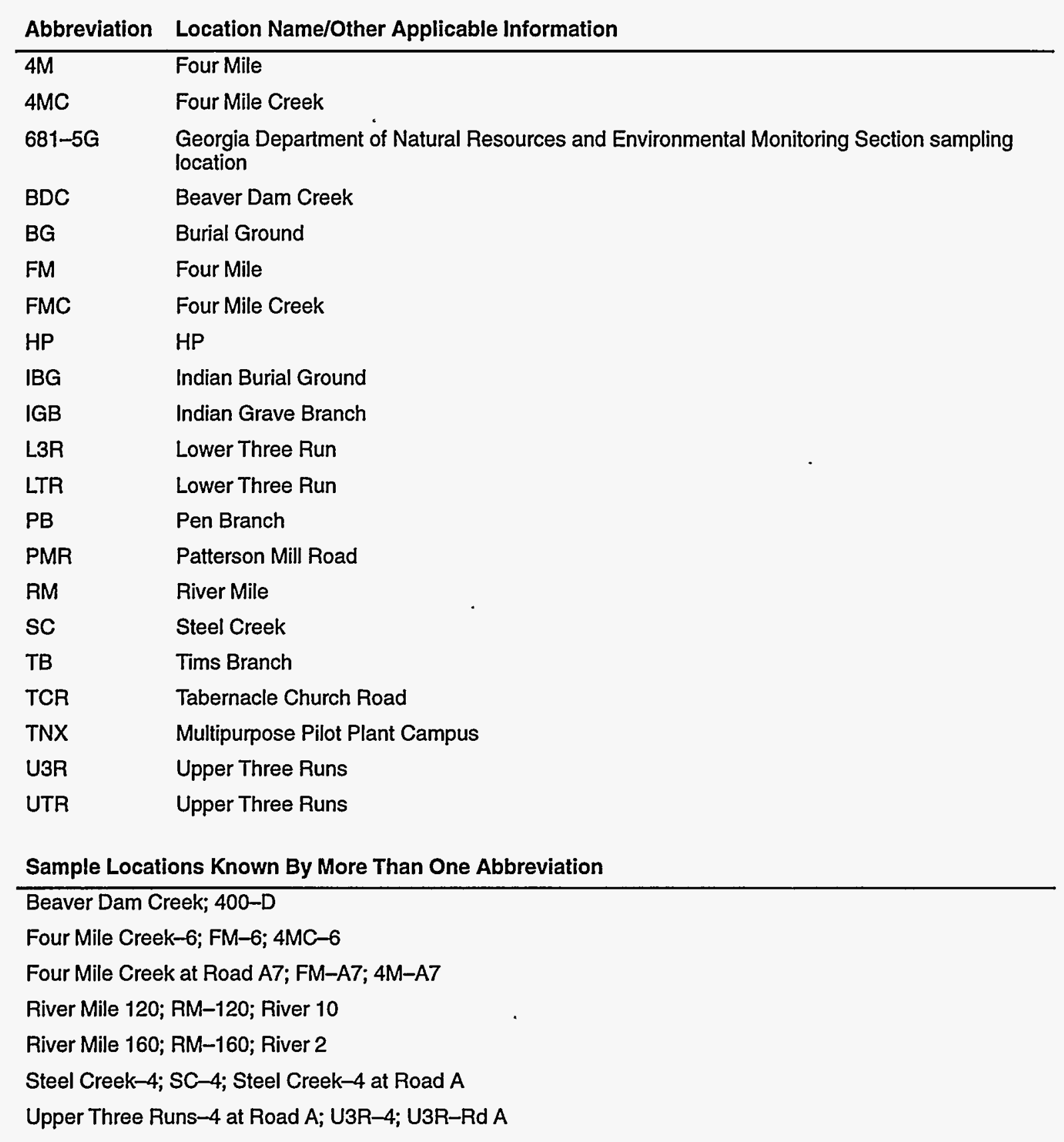


Other Abbreviations Used in This Book

\begin{tabular}{ll}
\hline AMAD & Activity median aerodynamic diameter \\
cfs & cubic feet per second \\
DCG & Derived Concentration Guide \\
Dup, DUP & Duplicate \\
ETF & Effluent Treatment Facility \\
GP & Georgia Power \\
MDL & Minimum Detectable Limit \\
NF & No Flow \\
NRC & Nuclear Regulatory Commission \\
ORA & Operations Recreation Association \\
PAR & "P and R" Pond \\
RBOF & Receiving Basin for Offsite Fuel \\
Rep, REP & Replicate \\
SRTC & Savannah River Technology Center \\
SWDF & Solid Waste Disposal Facility \\
TLD & Thermoluminescent Dosimeter \\
h & hour \\
d & day \\
y & year
\end{tabular}


Figure $1 \quad$ Radiological Sampling Locations - Air Surveillance $\ldots \ldots \ldots \ldots \ldots \ldots \ldots \ldots \ldots \ldots$

Figure 2 Radiological Sampling Locations - Offsite TLD Surveillance $\ldots \ldots \ldots \ldots \ldots \ldots \ldots \ldots 2$

Figure 3 Radiological Sampling Locations - Surface Water $\ldots \ldots \ldots \ldots \ldots \ldots \ldots \ldots \ldots \ldots \ldots$

Figure 4 Radiological Sampling Locations - Offsite Drinking Water $\ldots \ldots \ldots \ldots \ldots \ldots \ldots \ldots \ldots$

Figure $5 \quad$ Radiological and Nonradiological Sampling Locations - Fish $\ldots \ldots \ldots \ldots \ldots \ldots \ldots \ldots$

Figure $6 \quad$ Radiological Sampling Locations - Soil $\ldots \ldots \ldots \ldots \ldots \ldots \ldots \ldots \ldots \ldots \ldots \ldots \ldots \ldots \ldots \ldots \ldots$

Figure $7 \quad$ Radiological Sampling Locations - Sediment $\ldots \ldots \ldots \ldots \ldots \ldots \ldots \ldots \ldots \ldots \ldots \ldots$

Figure 8 Radiological Sampling Locations — Vegetation (Except Inside SWDF) $\ldots \ldots \ldots \ldots \ldots \ldots \ldots$

Figure 9 Radiological Sampling Locations - Vegetation (Inside SWDF) $\ldots \ldots \ldots \ldots \ldots \ldots \ldots \ldots$

Figure 10 Nonradiological Sampling Locations — SRS Streams and Savannah River Water $\ldots \ldots \ldots \ldots 10$

Figure 11 Nonradiological Sampling Locations — SRS Stream and Savannah River Sediment ........ 11 



\section{Sampling}

Table 1

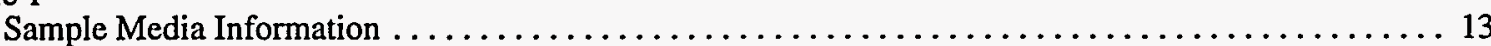

Table 2 Representative Minimum Detectable Concentrations for Gamma Analysis

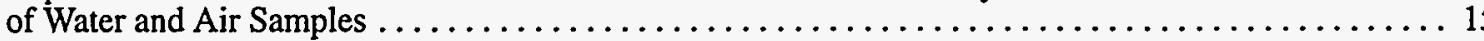

Table 3 Representative Minimum Detectable Concentrations for Gamma Analysis

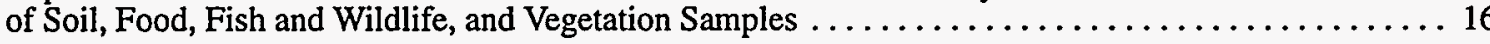

Table 4

Representative Minimum Detectable Concentrations for Radiological Analysis

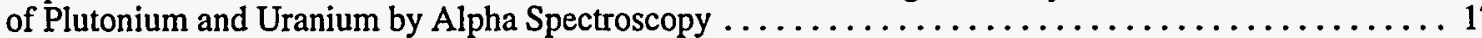

Table 5

Representative Minimum Detectable Concentrations for Radiological Analysis

by Gas-Flow Proportional Counters and by Liquid Scintillation $\ldots \ldots \ldots \ldots \ldots \ldots \ldots \ldots \ldots$

Table 6

Minimum Detectable Activities for Radionuclides in Settleable Solids . . . . . . . . . . . . . . . 19

Table 7

Nonradiological Environmental Surveillance Detection/Report Limits $\ldots \ldots \ldots \ldots \ldots \ldots \ldots \ldots$

\section{Radiological Effluent Monitoring}

Table 8

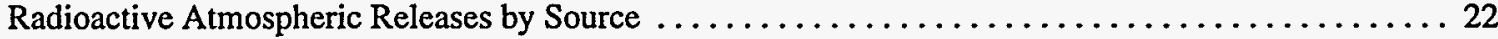

Table 9

Radioactive Atmospheric Releases by Stack/Facility and Comparison

of Annual Average Concentrations to DOE Derived Concentration Guides ................... 24

Table 10

Radioactive Liquid Releases by Source

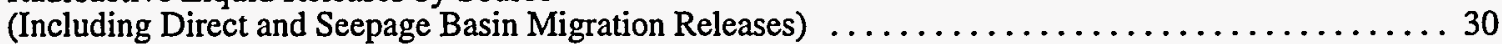

Table 11

Liquid Radioactive Releases by Outfall/Facility and Comparison of Annual

Average Radionuclide Concentrations to DOE Derived Concentration Guides $\ldots \ldots \ldots \ldots \ldots \ldots \ldots . \ldots \ldots$

Table 12

Calculated Migration of Radioactivity from Seepage Basins $\ldots \ldots \ldots \ldots \ldots \ldots \ldots \ldots \ldots \ldots \ldots$

Table 13

Estimated Tritium Releases in SRS Streams and the Savannah River $\ldots \ldots \ldots \ldots \ldots \ldots \ldots \ldots \ldots$

Table 14

Settleable Solids Results for the SRS Radionuclides in Settleable Solids Program ........... 41

\section{Radiological Environmental Surveillance}

Table 15

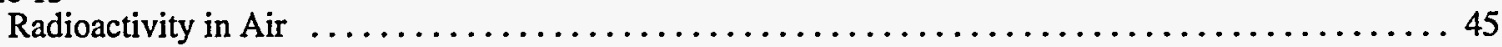

Table 16

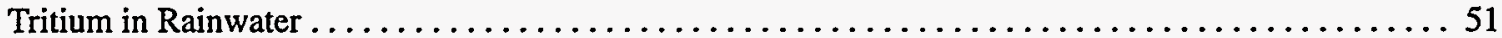

Table 17

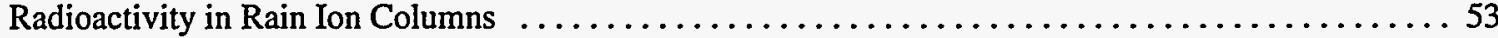


Table 18

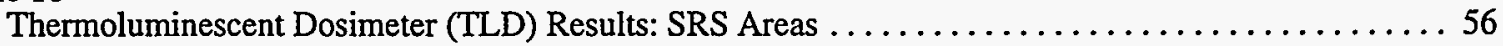

Table 19

Thermoluminescent Dosimeter (TLD) Results: Site Perimeter Stations $\ldots \ldots \ldots \ldots \ldots \ldots \ldots \ldots, 60$

Table 20

Thermoluminescent Dosimeter (TLD) Results:

Environmental Surveillance (Air Monitoring) Stations

Table 21

Thermoluminescent Dosimeter (TLD) Results: Population Centers $\ldots \ldots \ldots \ldots \ldots \ldots \ldots \ldots \ldots 69$

Table 22

Thermoluminescent Dosimeter (TLD) Results: Vogtle Electric Generating Plant Vicinity . . . . . . 72

Table 23

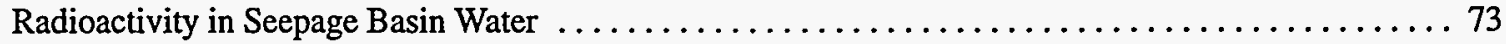

Table 24

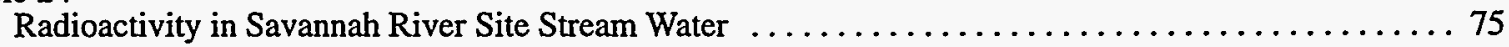

Table 25

Radioactivity in Transport at Sample Points on Four Mile Creek $\ldots \ldots \ldots \ldots \ldots \ldots \ldots \ldots \ldots \ldots \ldots \ldots \ldots$

Table 26

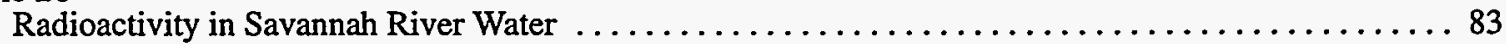

Table 27

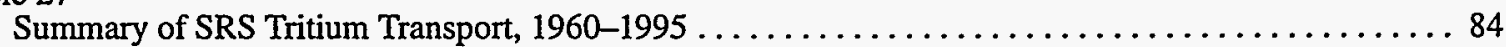

Table 28

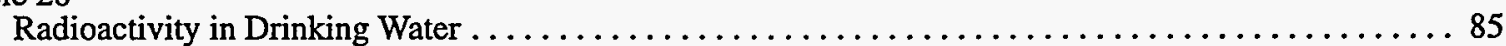

Table 29

Radioactivity in Terrestrial Food Products — Greens, Grain, Fruit, and Beef $\ldots \ldots \ldots \ldots \ldots \ldots$

Table 30

Radioactivity in Terrestrial Food Products - Milk $\ldots \ldots \ldots \ldots \ldots \ldots \ldots \ldots \ldots \ldots \ldots$

Table 31

Radioactivity in Aquatic Food Products - Freshwater Fish . . . . . . . . . . . . . . . 99

Table 32

Radioactivity in Aquatic Food Products - Marine (Saltwater) Fish . . . . . . . . . . . . . . 109

Table 33

Radioactivity in Aquatic Food Products - Marine Invertebrates (Shellfish) $\ldots \ldots \ldots \ldots \ldots \ldots \ldots \ldots$

Table 34

Comparison of Field and Laboratory Cesium-137 Measurements in Deer and Hog Muscle $\ldots \ldots \ldots 111$

Table 35

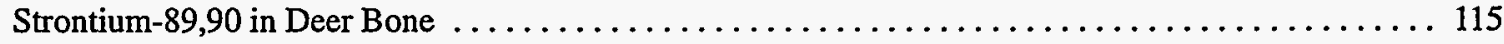

Table 36

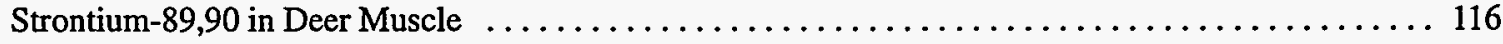

Table 37

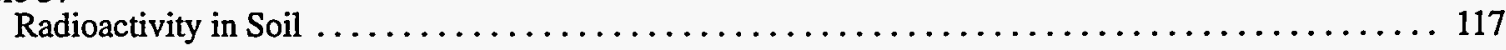

Table 38

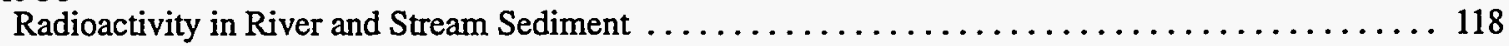

Table 39

Radioactivity in Terrestrial Vegetation (Quarterly Samples) $\ldots \ldots \ldots \ldots \ldots \ldots \ldots \ldots \ldots \ldots \ldots \ldots \ldots$

Table 40

Radioactivity in Terrestrial Vegetation (Outside Solid Waste Disposal Facility Fences) ......... 127

Table 41

Radioactivity in Terrestrial Vegetation (Inside Solid Waste Disposal Facility Fences) $\ldots \ldots \ldots \ldots \ldots 128$

Table 42

Radioactivity in Terrestrial Vegetation (Composites from Seepage and Retention Basins) . . ..... 130 


\section{Dose}

Table 43

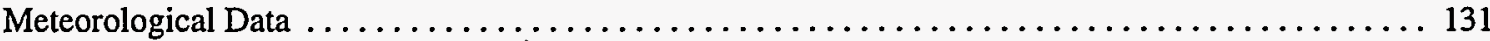

Table 44

80-km-Radius (50-Mile) Population Distribution Around SRS (1990 Census) . . . . . . . . . . . 138

Table 45

80-km-Radius (50-Mile) Milk, Meat, and Vegetation Production Around SRS . . . . . . . . . 139

Table 46

Release Locations for Maximally Exposed Individual Dose $\ldots \ldots \ldots \ldots \ldots \ldots \ldots \ldots \ldots \ldots$

Table 47

Parameters Used for Adult Consumption Rates and for Atmospheric Dose Calculations . . . . . . . . 142

Table 48

Site-Specific Parameters Used with CAP88 Code Used for NESHAPS Calculations ........... 143

Table 49

Parameters Used for Adult Consumption Rates and for Liquid Dose Calculations . . . . . . . . . 144

Table 50

Site-Specific Parameters Used in Liquid Dose Calculations $\ldots \ldots \ldots \ldots \ldots \ldots \ldots \ldots \ldots \ldots \ldots \ldots$

Table 51

Committed Dose to the Maximally Exposed Individual from Atmospheric Releases

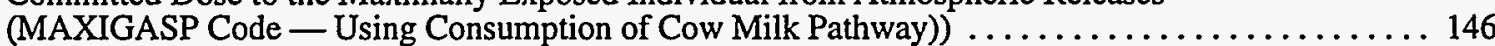

Table 52

Committed Dose to the Maximally Exposed Individual from Atmospheric Releases

(MAXIGASP Code - Using Consumption of Goat Milk Pathway) $\ldots \ldots \ldots \ldots \ldots \ldots \ldots \ldots \ldots \ldots$

Table 53

80-km (50-Mile) Collective Dose from Atmospheric Releases (POPGASP Code) $\ldots \ldots \ldots \ldots \ldots$

Table 54

Total Site Releases and Maximally Exposed Individual Effective Dose Equivalent by Radionuclide

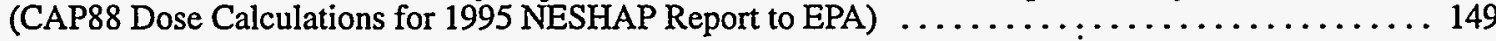

Table 55

NESHAP Report Data: CAP88 Compared With MAXIGASP $\ldots \ldots \ldots \ldots \ldots \ldots \ldots \ldots \ldots \ldots \ldots$

Table 56

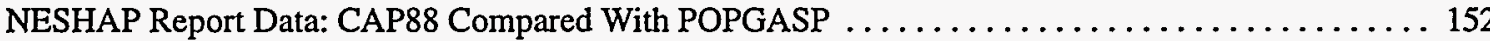

Table 57

Committed Dose to Maximally Exposed Individual from Liquid Releases $\ldots \ldots \ldots \ldots \ldots \ldots \ldots \ldots$

Table 58

Committed Dose to Maximally Exposed Individual from Public Water Supplies

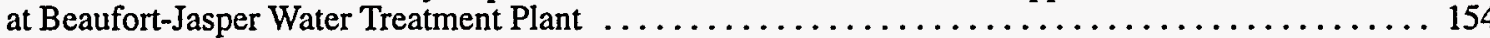

Table 59

Committed Dose to Maximally Exposed Individual from Public Water Supplies

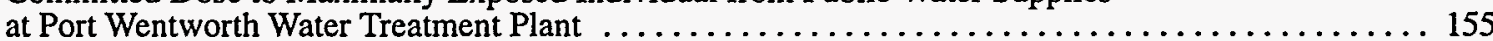

Table 60

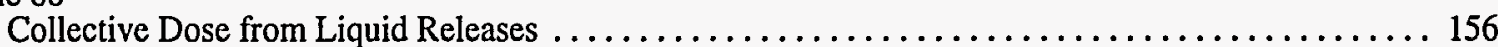

Table 61

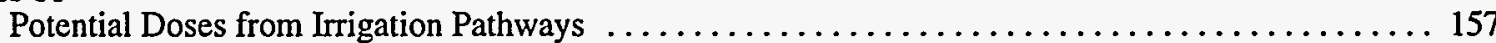

Table 62

Dose from Consumption of Fish from SRS Creek Mouths and River Mile $\ldots \ldots \ldots \ldots \ldots \ldots \ldots \ldots$

Table 63

Calculated Doses to Aquatic Biota from SRS Releases ........................ 160 


\section{Nonradiological Effluent Monitoring}

Table 64

Toxic/Hazardous Air Pollutant Emissions $(1994) \ldots \ldots \ldots \ldots \ldots \ldots \ldots \ldots \ldots \ldots \ldots \ldots \ldots \ldots \ldots \ldots \ldots \ldots$

Table 65

National Pollutant Discharge Elimination System Monitoring Data $\ldots \ldots \ldots \ldots \ldots \ldots \ldots \ldots$

Table 66

National Pollutant Discharge Elimination System Stormwater Monitoring Data $\ldots \ldots \ldots \ldots \ldots \ldots 7$

\section{Nonradiological Environmental Surveillance}

Table 67

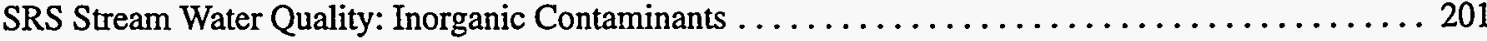

Table 68

SRS Stream Water Quality: Pesticides, Herbicides, and Volatile Organic Compounds .......... 221

Table 69

SRS Stream and Savannah River Water Quality: Pesticides and Herbicides ............. 222

Table 70

Savannah River Water Quality: Inorganic Contaminants ....................... 224

Table 71

Savannah River Water Quality:

Georgia Department of Natural Resources and EMS Sampling Location $\ldots \ldots \ldots \ldots \ldots \ldots \ldots$

Table 72

SRS Streams and Savannah River Water Quality: Fecal Coliform Bacteria Exceedances $\ldots \ldots \ldots \ldots 232$

Table 73

Sediment Surveillance: Pesticides and Herbicides .............................. 234

Table 74

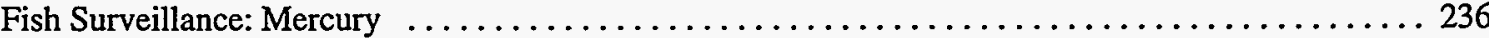

\section{Quality Assurance}

Table 75

Blind Sample Results for $\mathrm{pH}$ Field Measurements $\ldots \ldots \ldots \ldots \ldots \ldots \ldots \ldots \ldots \ldots \ldots \ldots \ldots \ldots \ldots \ldots \ldots$

Table 76

Blind Sample Results for Conductivity Field Measurements ...................... 239

Table 77

EMS Blind Sample Results for Tritium ................................. 240

Table 78

EMS Blind Sample Results for Gross Alpha and Beta and Gamma-Emitting Radionuclides ....... 241

Table 79

NPDES Blind Sample Results

Table 80

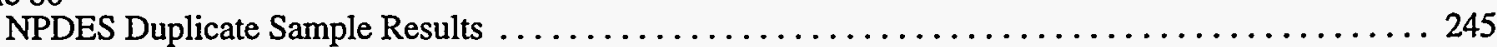

Table 81

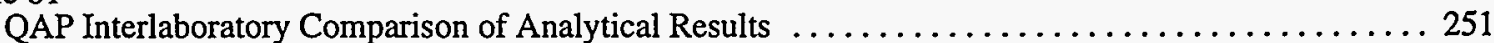

Table 82

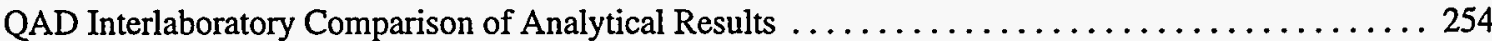

Table 83

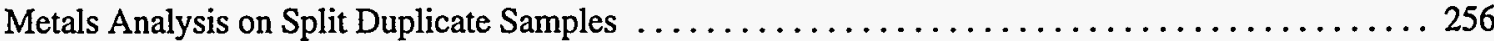

Table 84

Metals Analysis on Split Blind Quarterly Composites

258 


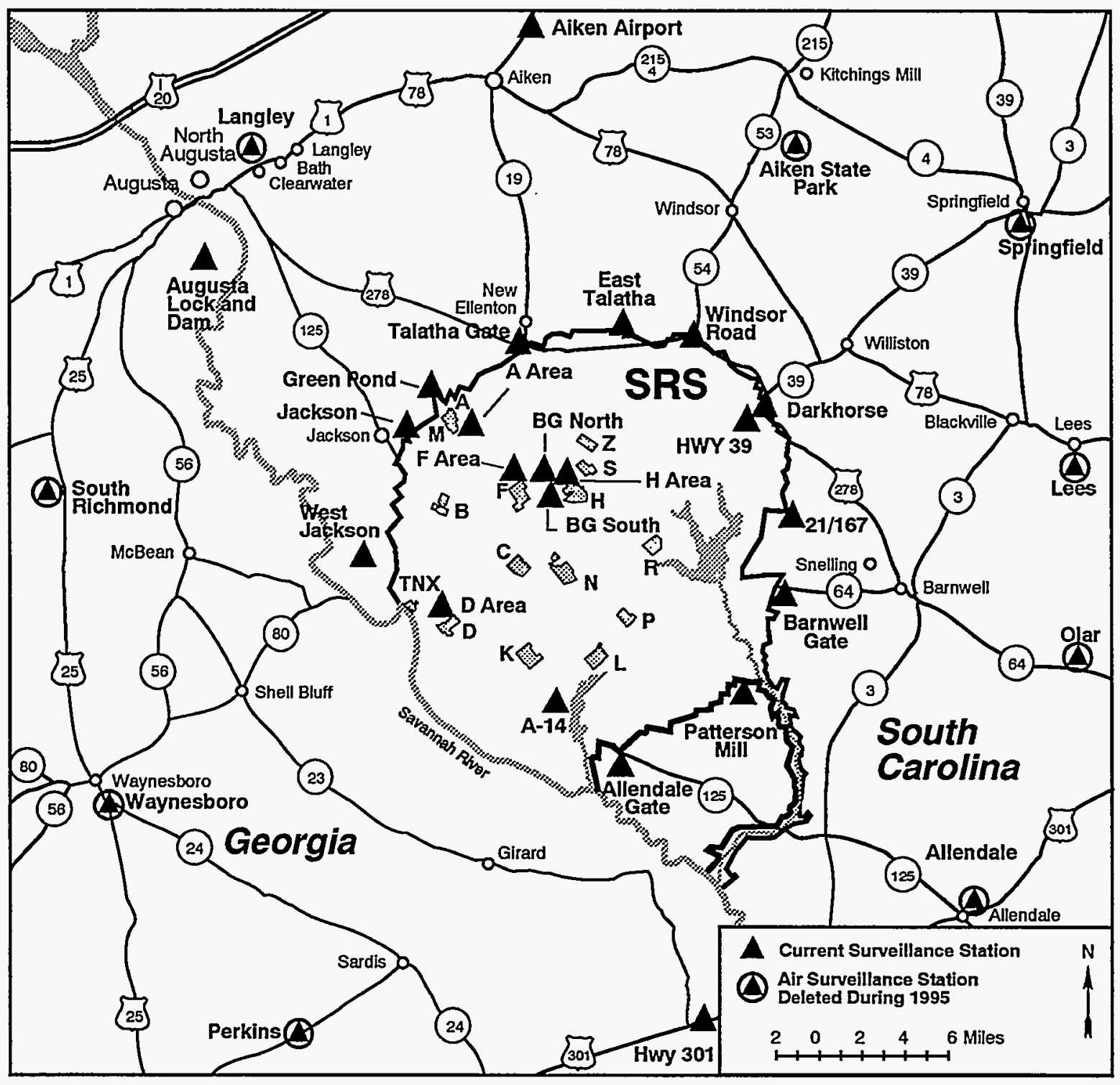

$96 \times 01678.02$

Figure 1 Radiological Sampling Locations - Air Surveillance

The SRS air surveillance program consists of 31 stations located within 25 miles of the site and four stations (not shown) approximately 100 miles from the site. 


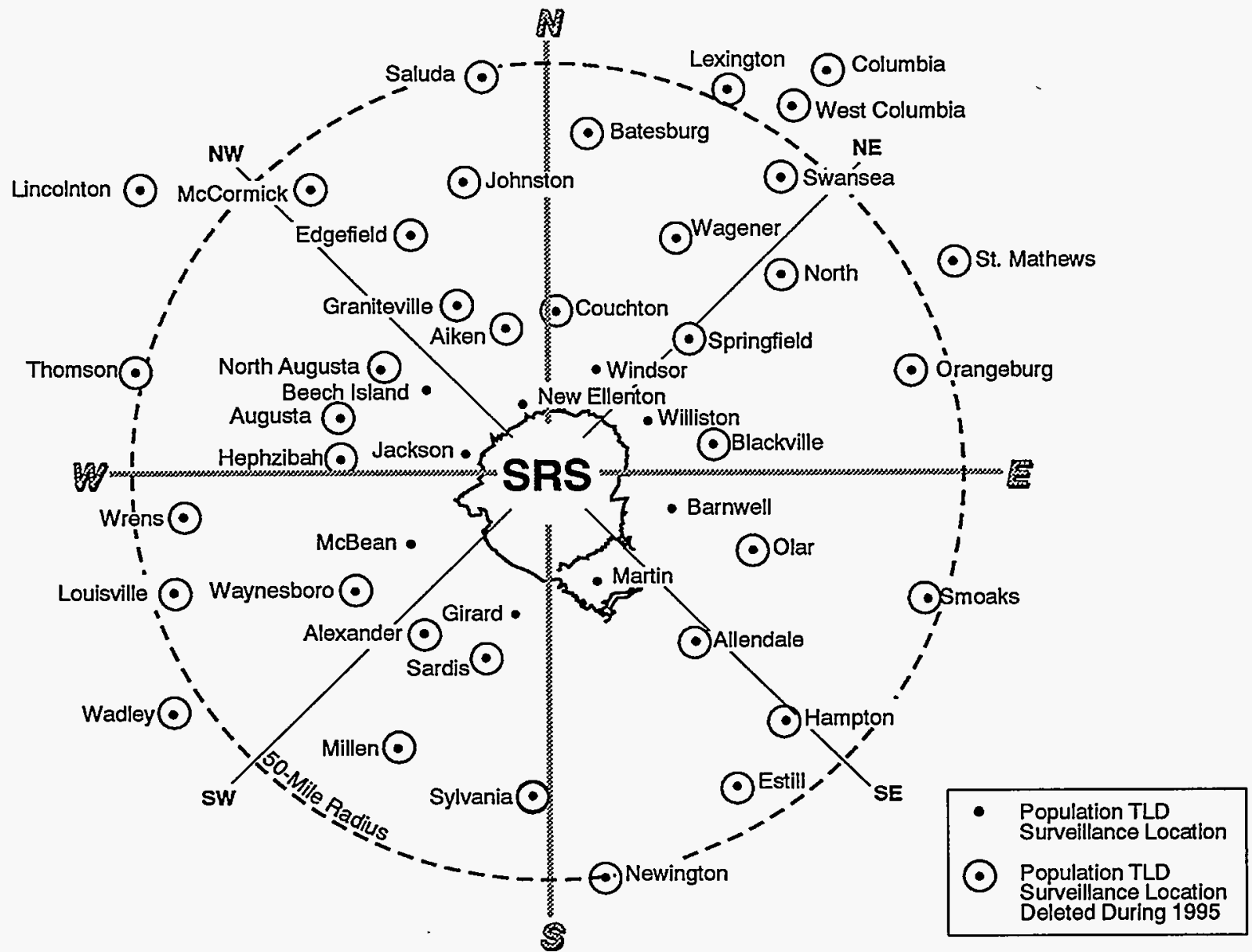

96X02355.AIL

Figure 2 Radiological Sampling Locations - Offsite TLD Surveillance

Ambient gamma exposure rates are monitored in cities and towns within a 50-mile radius of SRS. 


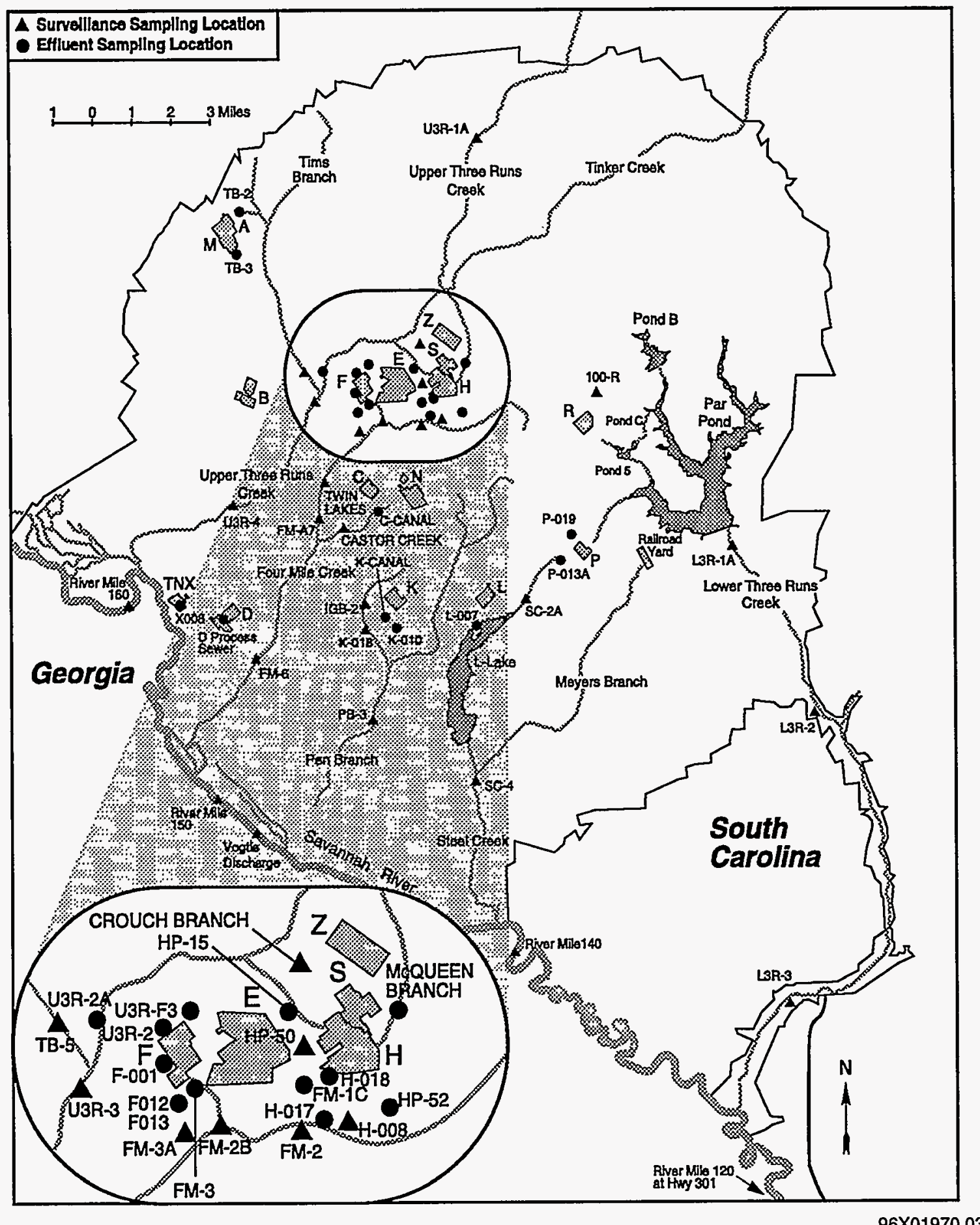

Figure 3 Radiological Sampling Locations - Surface Water

Surveillance and effluent sampling points are located on SRS seepage basins and streams and on the Savannah River. 


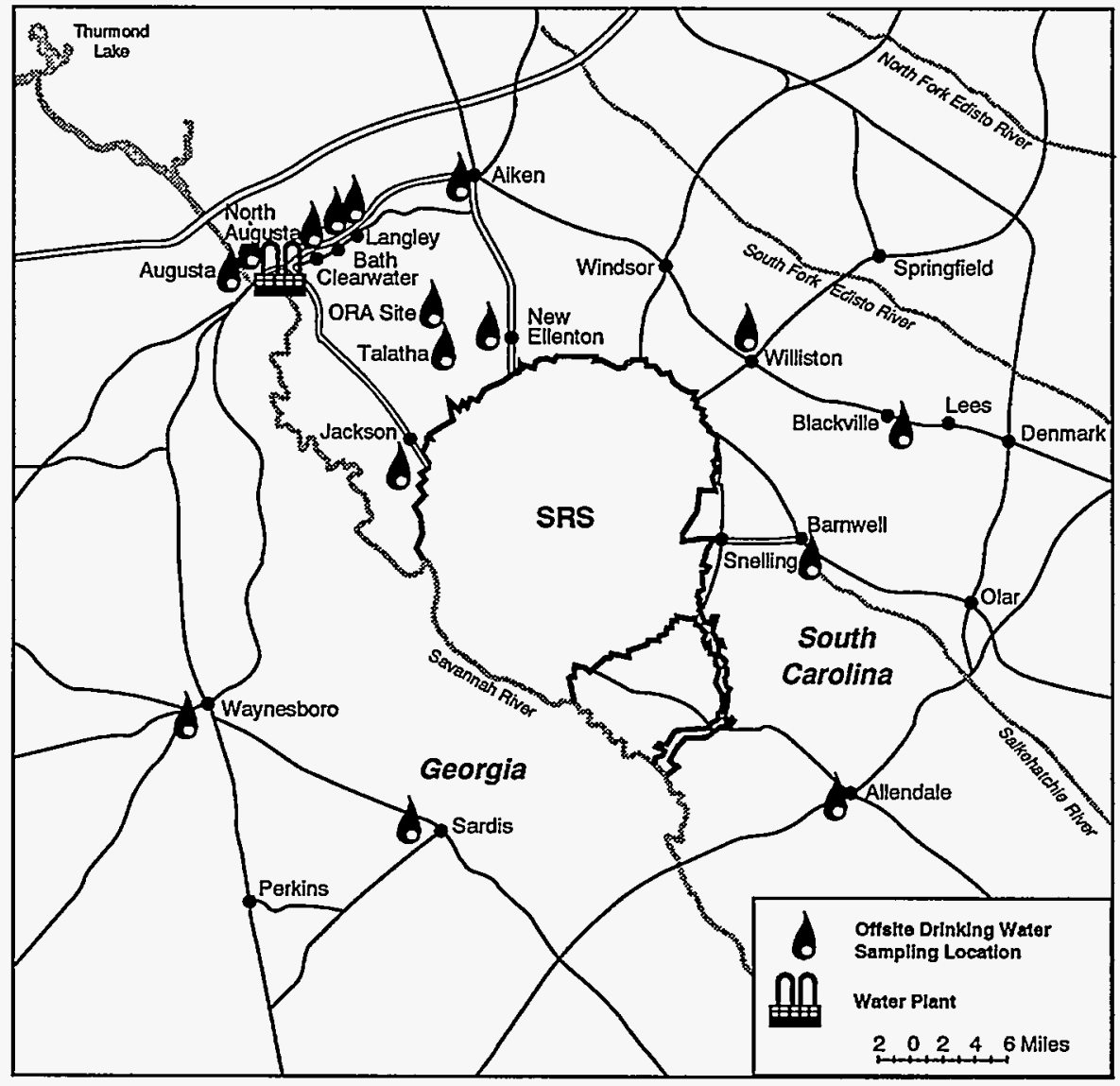

94X06608.15.AIL

Figure 4 Radiological Sampling Locations - Offsite Drinking Water

SRS collected drinking water samples during 1995 from 16 offsite locations within a 30-mile radius and from three water treatment plants, one of which is shown on the map. The 16 offsite locations were discontinued midway through 1995. 


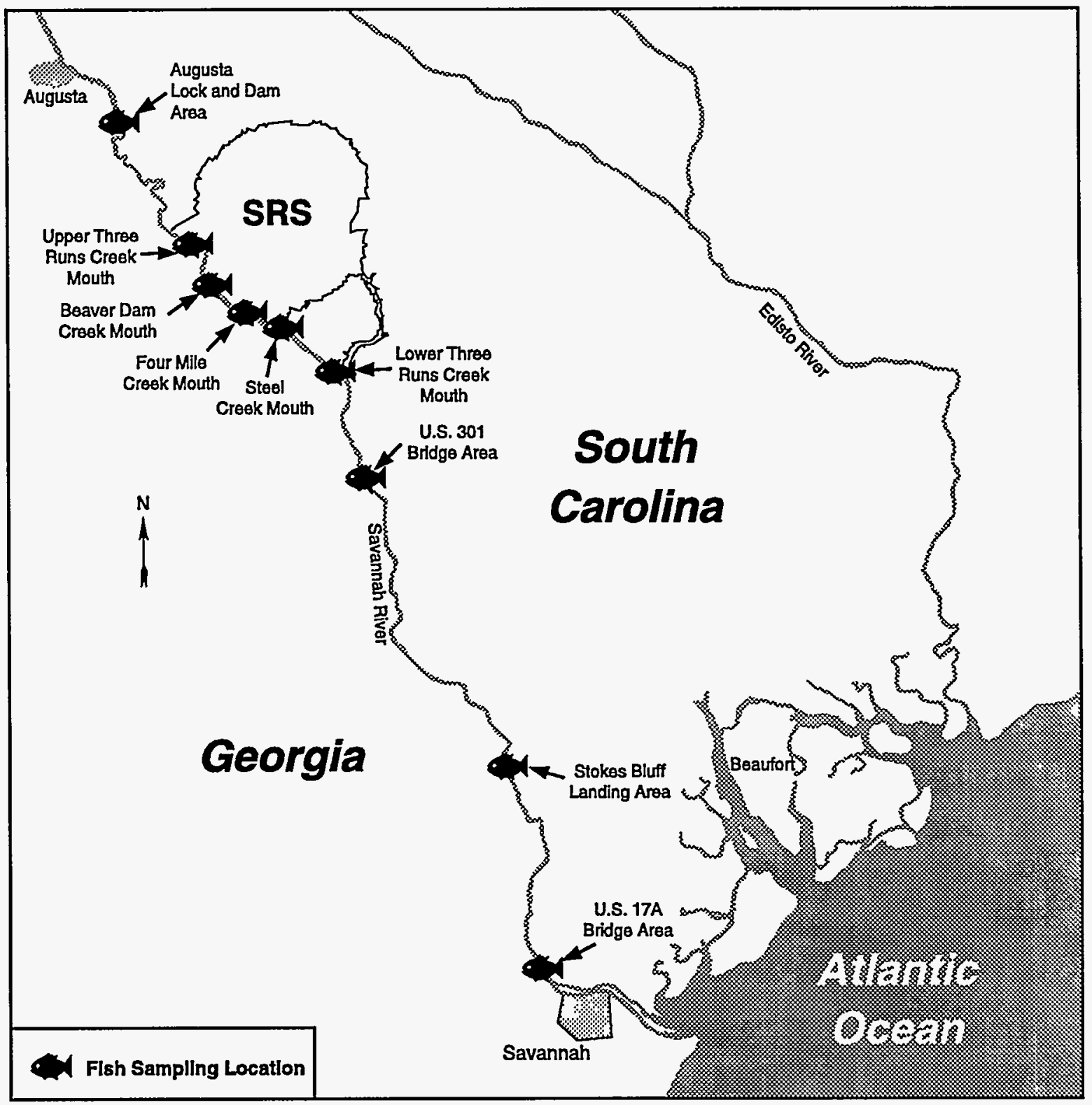

96X01678.08.AIL

Figure 5 Radiological and Nonradiological Sampling Locations - Fish

SRS collects fish from the Savannah River above, adjacent to, and below the site, as well as at Stokes Bluff Landing and near Savannah. 


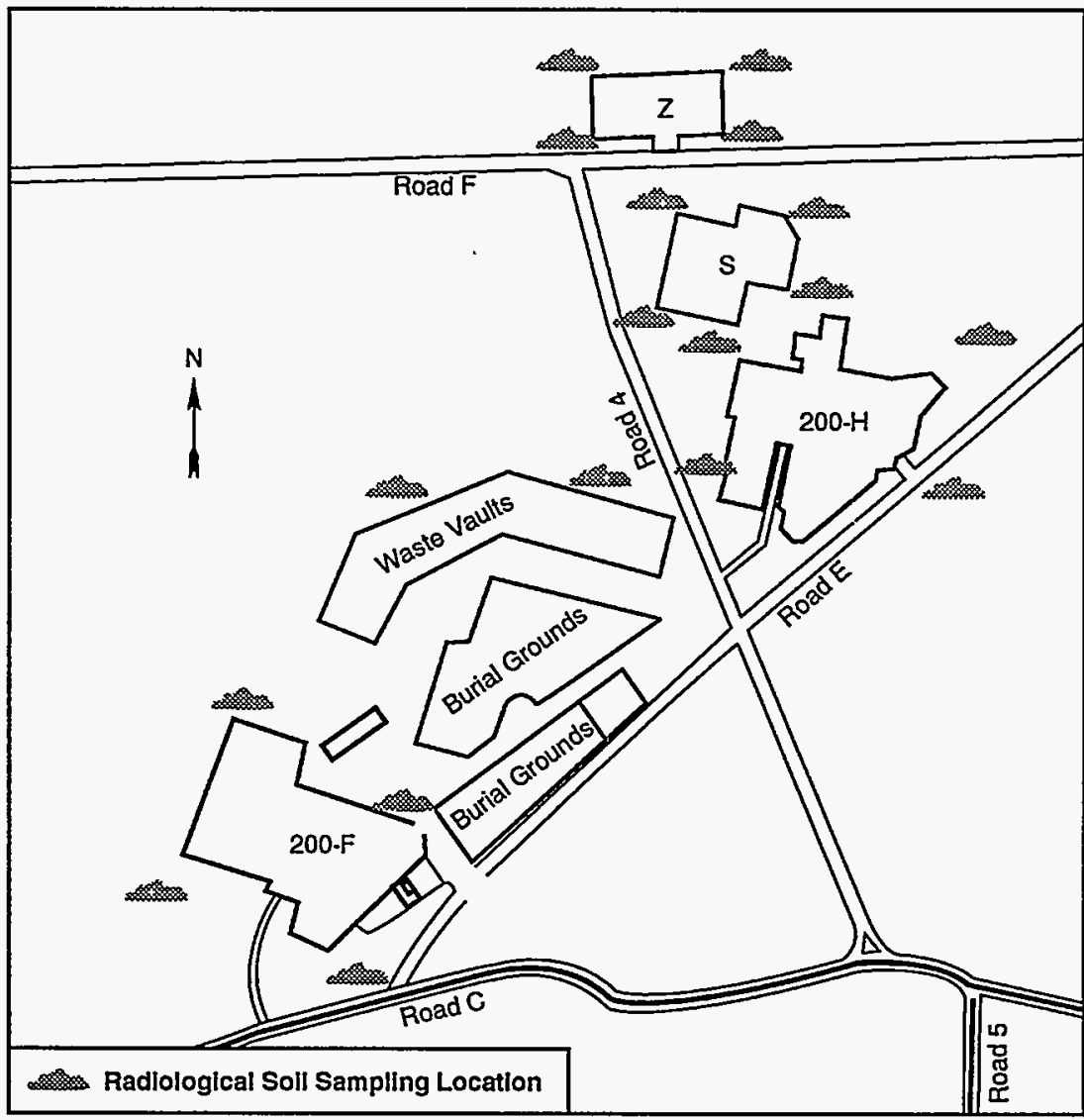

94X06608.19.AlL

Figure 6 Radiological Sampling Locations - Soil

SRS collected soil samples from 22 onsite locations (four perimeter locations not shown) and two offsite locations in 1995. 


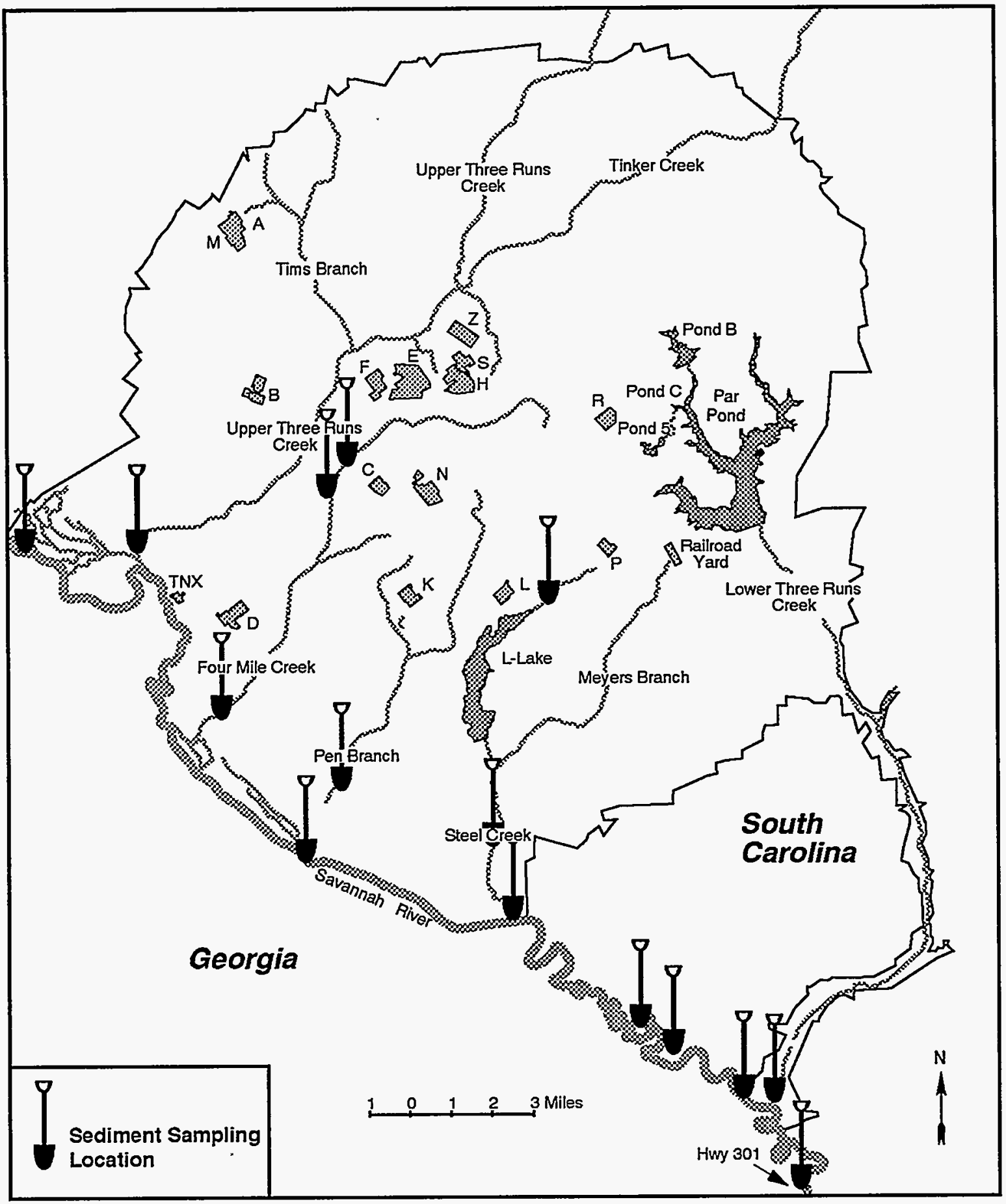

M94X06608.50.AlL

Figure 7 Radiological Sampling Locations - Sediment

Sediment samples were collected in 1995 at six Savannah River locations-upriver from, adjacent to, and downriver from the site-and at nine site stream locations. 


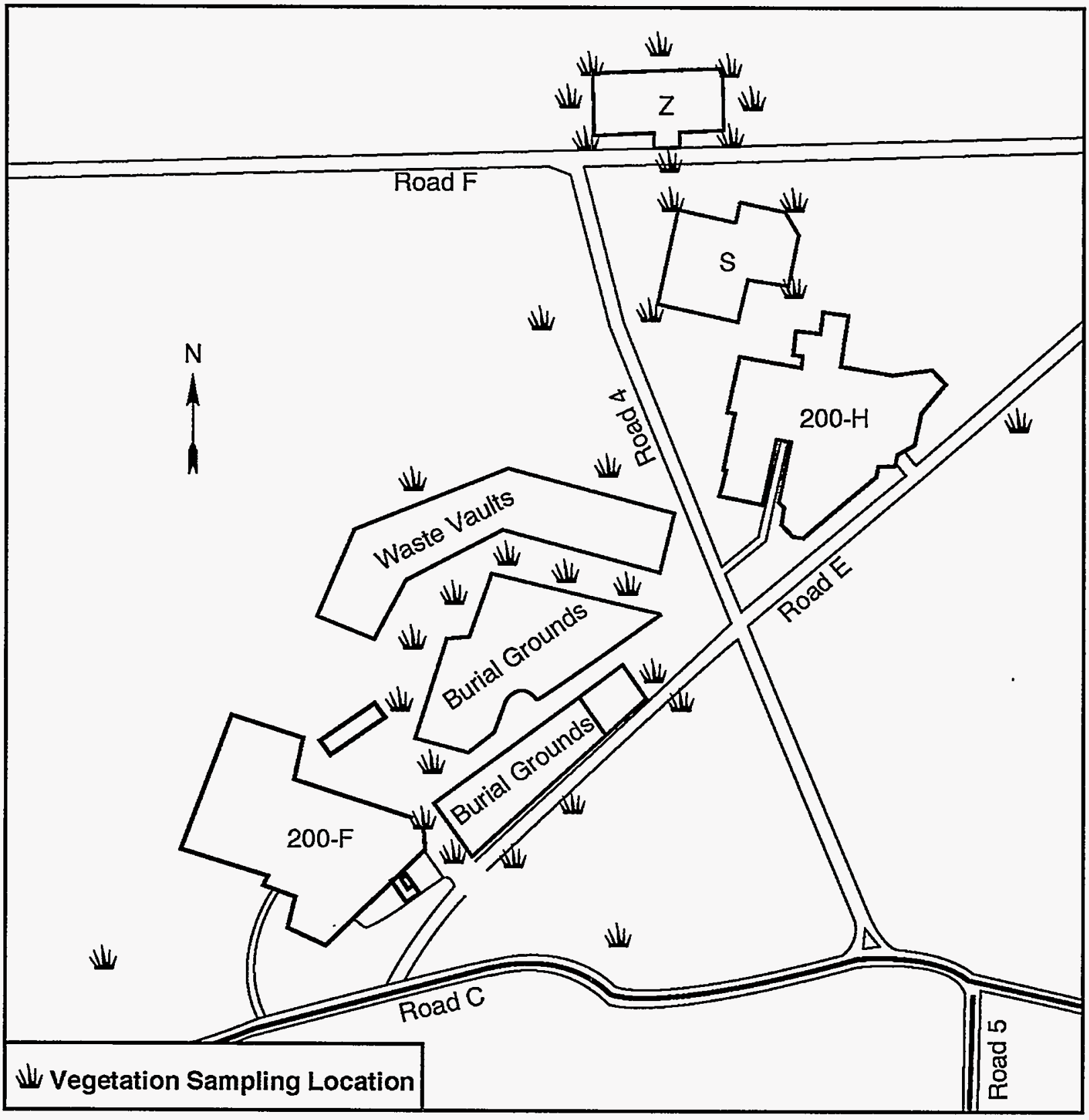

94X06608.21.AlL

Figure 8 Radiological Sampling Locations - Vegetation (Except Inside SWDF)

Samples are collected for radiological analysis from 18 onsite-quarterly and 13-outside SWDF locations. 


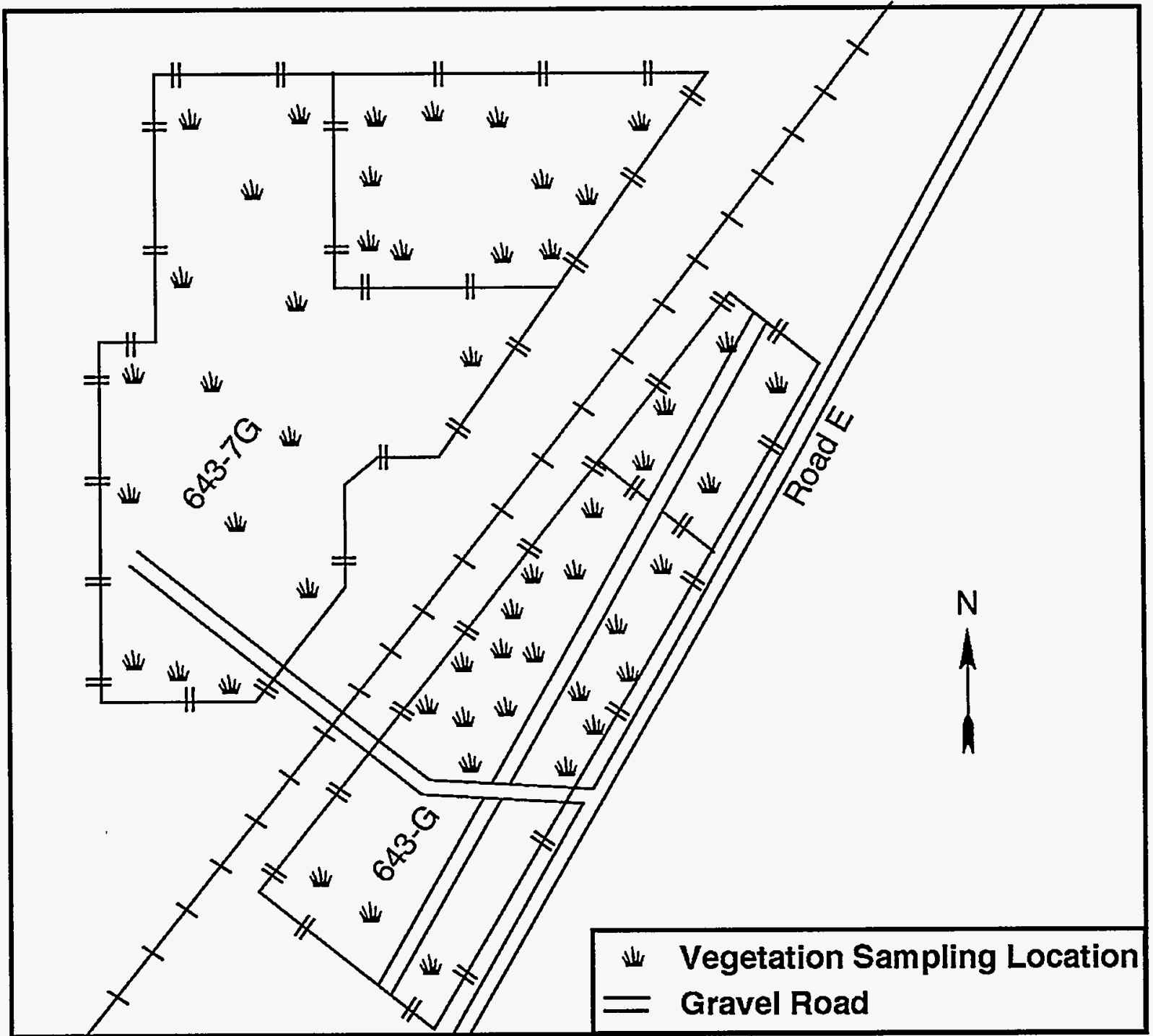

94X01185.21.AlL

Figure 9 Radiological Sampling Locations - Vegetation (Inside SWDF)

Vegetation samples are collected inside the SWDF to determine whether uptake of radioactivity by vegetation has occurred. 


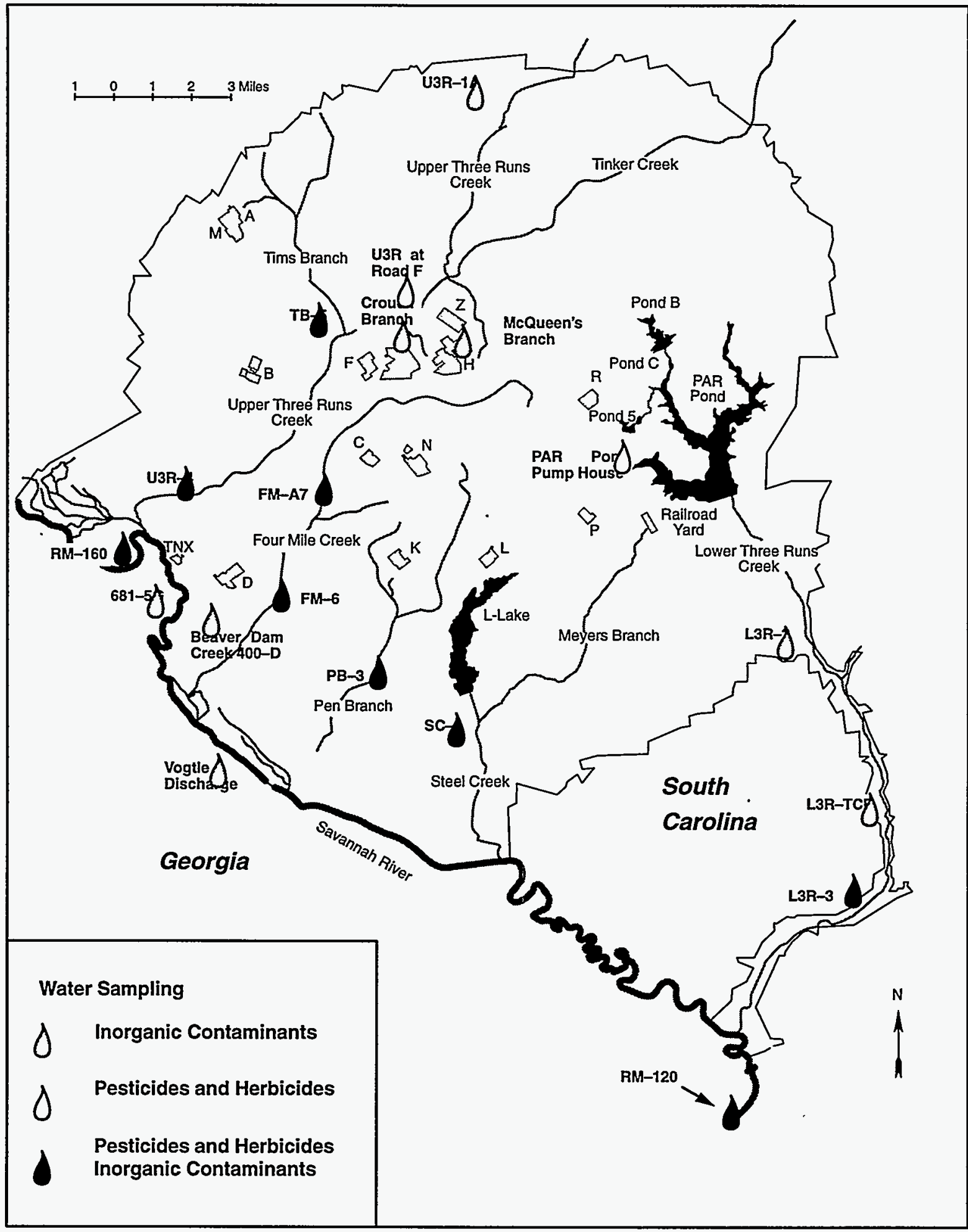

M94X06608.02 (Modified)

Figure 10 Nonradiological Sampling Locations - SRS Streams and Savannah River Water Surface water samples are collected from four Savannah River and 15 SRS stream locations for analysis of nonradiological contaminants, including pesticides, herbicides, and/or inorganic contaminants. Steel Creek-4 (SC-4), Tims Branch-5 (TB-5), Four Mile Creek at Road A7 (FM-A7), and Upper Three Runs-4 (U3R-4) are both EMS and SCDHEC sampling locations, from which samples are also analyzed for volatile organic compounds. 


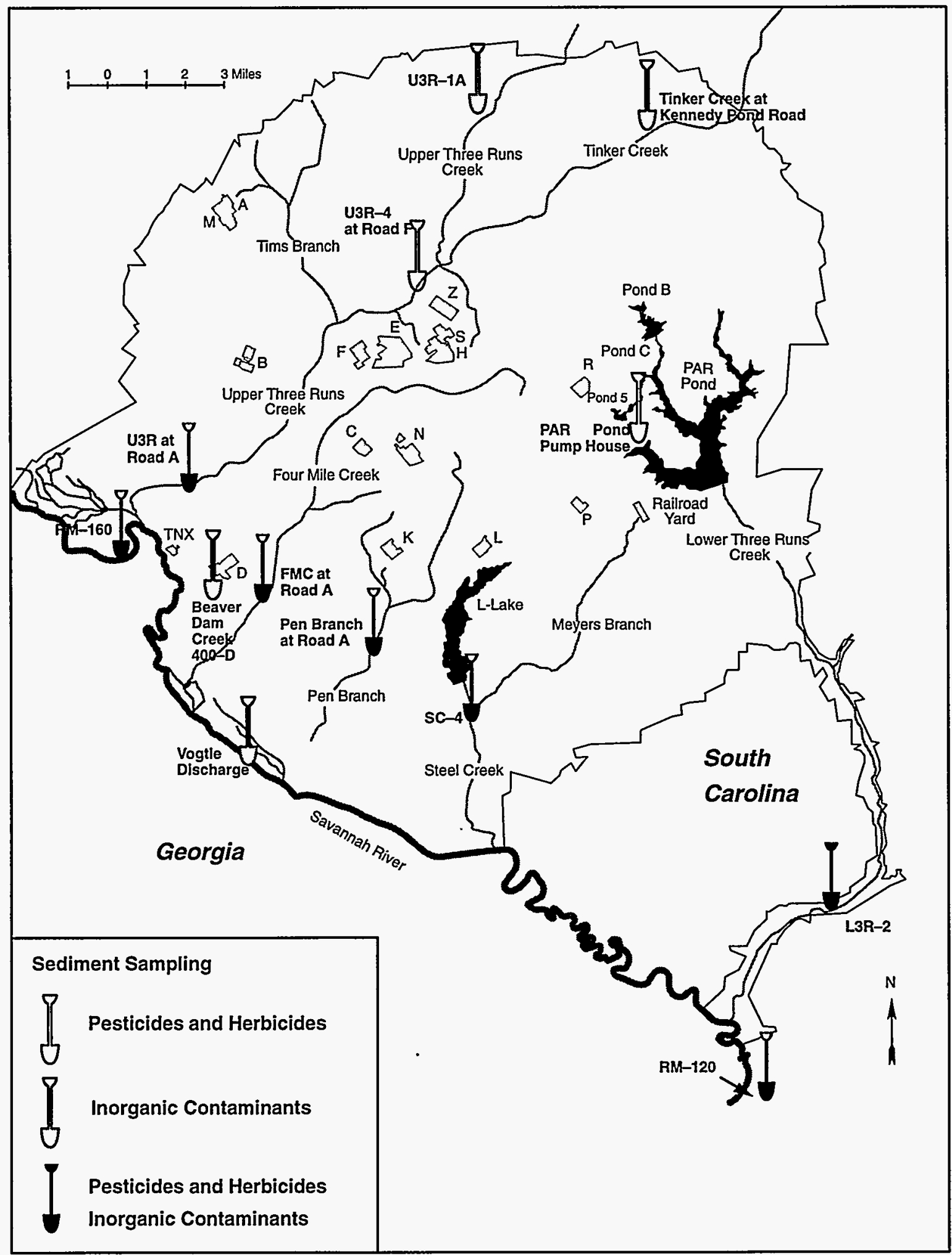

96X00447.01.AlL

Figure 11 Nonradiological Sampling Locations - SRS Stream and Savannah River Sediment

Sediment is sampled at three Savannah River and 10 SRS stream locations for nonradiological contaminants, including pesticides, herbicides, and inorganic contaminants. In 1995, however, sampling of the inorganic contaminants was accidentally omitted. 



\section{Table 1}

\section{Sample Media Information}

Page 1 of 2

\begin{tabular}{|c|c|c|}
\hline Sample Matrix or Media & Sample Size & Representative Aliquot \\
\hline \multicolumn{3}{|l|}{ Gross alpha } \\
\hline Water & $1 \mathrm{~L}$ & $1 \mathrm{~L}$ \\
\hline Vegetation & $1-2 \mathrm{~kg}$ & $0.5 \mathrm{~g}$ \\
\hline Rain (collection pan) & $0.37 \mathrm{~m}^{2}$ & $0.093 \mathrm{~m}^{2}$ (1/4 total sample) \\
\hline Air & whole filter & $800 \mathrm{~m}^{3}$ \\
\hline \multicolumn{3}{|l|}{ Nonvolatile beta } \\
\hline Water & $1 \mathrm{~L}$ & $1 \mathrm{~L}$ \\
\hline Vegetation & $1-2 \mathrm{~kg}$ & $0.5 \mathrm{~g}$ \\
\hline Rain (collection pan) & $0.37 \mathrm{~m}^{2}$ & $0.093 \mathrm{~m}^{2}$ (1/4 total sample) \\
\hline Air & whole filter & $800 \mathrm{~m}^{3}$ \\
\hline \multicolumn{3}{|l|}{ Strontium-89,90 } \\
\hline Vegetation & $5 \mathrm{~g}$ & $5 \mathrm{~g}$ \\
\hline Food & $20 \mathrm{~g}$ & $20 \mathrm{~g}$ \\
\hline River water & $1 \mathrm{~L}$ & $1 \mathrm{~L}$ \\
\hline Rain & $0.37 \mathrm{~m}^{2}$ & $0.031 \mathrm{~m}^{2}(1 / 12$ total sample $)$ \\
\hline Streams & $1-3 L$ & $1-3 L$ \\
\hline Soil & $20 \mathrm{~g}$ & $20 \mathrm{~g}$ \\
\hline \multicolumn{3}{|l|}{ Air composites } \\
\hline Site perimeter & $20,000 \mathrm{~m}^{3}$ & $4,200 \mathrm{~m}^{3}$ \\
\hline 25-mile radius & $18,000 \mathrm{~m}^{3}$ & $3,600 \mathrm{~m}^{3}$ \\
\hline 100-mile radius & $6,000 \mathrm{~m}^{3}$ & $1,200 \mathrm{~m}^{3}$ \\
\hline \multicolumn{3}{|l|}{ Strontium-90 } \\
\hline Milk & $0.5 \mathrm{~L}$ & $0.5 \mathrm{~L}$ \\
\hline \multicolumn{3}{|c|}{ Plutonium-238, Plutonium-239 } \\
\hline River water & $1 \mathrm{~L}$ & $1 \mathrm{~L}$ \\
\hline Vegetation & $5 \mathrm{~g}$ & $5 \mathrm{~g}$ \\
\hline Sediment & $10 \mathrm{~g}$ & $10 \mathrm{~g}$ \\
\hline Soil & $10 \mathrm{~g}$ & $10 \mathrm{~g}$ \\
\hline Food & $100 \mathrm{~g}$ & $100 \mathrm{~g}$ \\
\hline
\end{tabular}


Table 1

Sample Media Information

Page 2 of 2

\begin{tabular}{lll}
\hline Sample Matrix or Media & Sample Size & Representative Aliquot \\
\hline Tritium & & \\
River water & $60 \mathrm{~mL}$ & $5 \mathrm{~mL}$ \\
Vegetation & $60 \mathrm{~g}$ & $5 \mathrm{~mL}$ \\
Sediment & $5 \mathrm{~mL}$ & $5 \mathrm{~mL}$ \\
Soil (special samples) & $5 \mathrm{~mL}$ & $5 \mathrm{~mL}$ \\
Food & $25 \mathrm{~g}$ & $5 \mathrm{~mL}$
\end{tabular}




\section{Table 2}

\section{Representative Minimum Detectable Concentrations for Gamma Analysis of Water and Air Samples ${ }^{a}$}

Page 1 of 1

\begin{tabular}{|c|c|c|c|c|}
\hline \multirow[b]{2}{*}{ Nuclide } & \multicolumn{2}{|c|}{ pCi/L } & $\mathrm{pCi} / \mathrm{m}^{2}$ & \multirow{2}{*}{$\frac{\mathrm{pCi} / \mathrm{m}^{3}}{\text { Air Filter }}$} \\
\hline & River Water & Stream Water & Rainwater & \\
\hline Ce-141 & 0.8 & 8 & 36 & 12 \\
\hline Ce-144 & 2.9 & 29 & 125 & 33 \\
\hline Co-58 & 0.6 & 7 & 29 & 10 \\
\hline Co-60 & 1.0 & 9 & 32 & 11 \\
\hline Cr-51 & 5.8 & 56 & 246 & 93 \\
\hline Cs-134 & 0.7 & 6 & 27 & 8 \\
\hline Cs-137 & 0.7 & 7 & 26 & 8 \\
\hline $\mid-131$ & 1.2 & 16 & 72 & 16 \\
\hline$M n-54$ & 0.7 & 7 & 32 & 11 \\
\hline $\mathrm{Nb}-95$ & 0.8 & 10 & 36 & 14 \\
\hline$R u-103$ & 0.6 & 7 & 28 & 10 \\
\hline Ru-106 & 5.5 & 59 & 278 & 88 \\
\hline$S b-125$ & 1.8 & 15 & 63 & 19 \\
\hline $\mathrm{Zn}-65$ & 1.4 & 17 & 64 & 22 \\
\hline Zr-95 & 1.4 & 12 & 55 & 19 \\
\hline
\end{tabular}

a The minimum detectable concentrations (MDCs) are calculated at the $95 \%$ confidence level with Canberra Industries Inc.'s VAXNMS gamma spectroscopy software. The values are based on a background measurement using a $32 \%$ relative efficiency high purity germanium detector and typical decay times and counting intervals. Chemical recoveries are assumed to be $100 \%$. Air filter values are for a single $47 \mathrm{~mm}$ particulate filter with a flow rate of approximately $2.5 \mathrm{CFM}$ (cubic feet per minute) for 7 days (I-131 value is obtained from a charcoal cartridge). Rainwater values are for a collection area of $0.031 \mathrm{~m}^{2}$. The sample size for stream water is $1 \mathrm{~L}$ and for river water is $10 \mathrm{~L}$. The MDCs for actual samples may be different because of variations in the sample preparation, size, and content, and because of variations in the chemical recoveries, counting efficiencies, decay times, and instrument backgrounds. 
Table 3

Representative Minimum Detectable Concentrations for Gamma Analysis of Soil, Food, Fish and Wildlife, and Vegetation Samples ${ }^{a}$

Page 1 of 1

\begin{tabular}{|c|c|c|c|c|c|}
\hline \multirow[b]{2}{*}{ Nuclide } & \multicolumn{3}{|c|}{$\mathrm{fCi} / \mathrm{g}$} & \multirow{2}{*}{$\frac{\mathrm{pCi} / \mathrm{g}}{\text { Vegetation }}$} & \multirow{2}{*}{$\frac{\text { pCi/L }}{\text { Milk }}$} \\
\hline & Soil & Foods & $\begin{array}{l}\text { Fish and } \\
\text { Wildlife }\end{array}$ & & \\
\hline Ce-141 & 97 & 98 & 158 & 0.4 & 3 \\
\hline Ce-144 & 139 & 40 & 227 & 0.5 & 12 \\
\hline Co-58 & 42 & 21 & 74 & 0.2 & 2 \\
\hline Co-60 & 29 & 7 & 52 & 0.1 & 3 \\
\hline $\mathrm{Cr}-51$ & 771 & 979 & 1,322 & 2.9 & 19 \\
\hline Cs-134 & 26 & 7 & 45 & 0.1 & 2 \\
\hline Cs-137 & 24 & 7 & 42 & 0.1 & 3 \\
\hline $\mid-131$ & 3,939 & Decayed & 6,790 & 15.0 & 3 \\
\hline$M n-54$ & 32 & 8 & 57 & 0.1 & 2 \\
\hline $\mathrm{Nb}-95$ & 84 & 74 & 147 & 0.3 & 3 \\
\hline Ru-103 & 60 & 49 & 104 & 0.2 & 2 \\
\hline Ru-106 & 279 & 72 & 486 & 1.1 & 23 \\
\hline$S b-125$ & 61 & 18 & 106 & 0.2 & 6 \\
\hline Zn-65 & 66 & 21 & 117 & 0.3 & 5 \\
\hline Zr-95 & 83 & 39 & 145 & 0.3 & 4 \\
\hline
\end{tabular}

a The minimum detectable concentrations (MDCs) are calculated at the $95 \%$ confidence level with Canberra Industries Inc.'s VAXNMS gamma spectroscopy software. The values are based on a background measurement using a $32 \%$ relative efficiency high purity germanium detector and typical decay times and counting intervals. Chemical recoveries are assumed to be $100 \%$. Sample sizes are $700 \mathrm{~g}$ for soil, $1,000 \mathrm{~g}$ for foods, $200 \mathrm{~g}$ for fish and wildlife, $100 \mathrm{~g}$ for vegetation, and $1 \mathrm{~L}$ for milk. The MDCs for actual samples may be different because of variations in the sample preparation, size, and content, and because of variations in the chemical recoveries, counting efficiencies, decay times, and instrument backgrounds. 
Table 4

Representative Minimum Detectable Concentrations for Radiological Analysis of Plutonium and Uranium by Alpha Spectroscopya

Page 1 of 1

\begin{tabular}{lllllll}
\hline Sample Type & Units & Pu-239 & Pu-238 & U-238 & U-234 & U-235 \\
\hline Air filter & $\mu \mathrm{Ci} / \mathrm{m}^{3}$ & $2.4 \mathrm{E}-11$ & $4.3 \mathrm{E}-11$ & $2.4 \mathrm{E}-11$ & $2.8 \mathrm{E}-11$ & $2.9 \mathrm{E}-11$ \\
Rainwater & $\mu \mathrm{Ci} / \mathrm{m}^{2}$ & $3.3 \mathrm{E}-7$ & $5.8 \mathrm{E}-7$ & $3.1 \mathrm{E}-7$ & $3.5 \mathrm{E}-7$ & $3.6 \mathrm{E}-7$ \\
Stream water & $\mu \mathrm{Ci} / \mathrm{mL}$ & $1.0 \mathrm{E}-11$ & $1.8 \mathrm{E}-11$ & $9.5 \mathrm{E}-12$ & $1.1 \mathrm{E}-11$ & $1.1 \mathrm{E}-11$ \\
River water & $\mu \mathrm{Ci} / \mathrm{mL}$ & $1.0 \mathrm{E}-12$ & $1.8 \mathrm{E}-12$ & $9.5 \mathrm{E}-13$ & $1.1 \mathrm{E}-12$ & $1.1 \mathrm{E}-12$ \\
Soil and sediment & $\mu \mathrm{Ci} / \mathrm{g}$ & $5.9 \mathrm{E}-10$ & $7.3 \mathrm{E}-10$ & & & \\
Vegetation & $\mu \mathrm{Ci} / \mathrm{g}$ & $7.3 \mathrm{E}-11$ & $1.1 \mathrm{E}-10$ & & &
\end{tabular}

a The minimum detectable concentrations (MDCs) are calculated at the 95\% confidence level with Canberra Industries Inc.'s VAXNMS alpha management spectroscopy sotware. The values are based on the average regent blank activity, detector efficiency, and chemical recovery for each sample matrix. The counting time is 1,000 minutes. Air filter values are for one-half of a single $47 \mathrm{~mm}$ particulate filter with a flow rate of approximately 2.5 CFM (cubic feet per minute) for 7 days. The rainwater values are for a collection area of $0.031 \mathrm{~m}^{2}$. The other sample sizes are $1 \mathrm{~L}$ for stream water, $10 \mathrm{~L}$ for river water, $10 \mathrm{~g}$ for soil and sediment, and $100 \mathrm{~g}$ for vegetation. The MDCs for actual samples may be different because of variations in the sample preparation, size, and content, and because of variations in the chemical recoveries, counting efficiencies, batch reagent blanks, and instrument backgrounds. 
Table 5

Representative Minimum Detectable Concentrations for Radiological Analysis by Gas-Flow Proportional Counters ${ }^{a}$ and by Liquid Scintillation ${ }^{b}$

Page 1 of 1

Analysis of Gross Alpha, Nonvolatile Beta, Strontium-89,90, and Strontium-90 by Gas-Flow Proportional Counters

Nuclide

Gross alpha

Nonvolatile beta

Strontium-89, 90

Strontium-90
Typical Minimum Detectable Concentration ( $\mu$ Ci/sample)

$$
\begin{array}{r}
6.8 \mathrm{E}-7 \\
1.52 \mathrm{E}-6 \\
2.38 \mathrm{E}-6 \\
1.89 \mathrm{E}-6
\end{array}
$$

Analysis for Weak Beta Emitters by Liquid Scintillation

$\begin{array}{lc}\text { Sample } & \text { Typical Minimum Detectable Concentration }(\mu \mathrm{Ci} / \mathrm{mL}) \\ \text { Tritium }^{\mathrm{c}} & 1.3 \mathrm{E}-6 \\ \text { Tritium }^{\mathrm{d}} & 4.7 \mathrm{E}-7 \\ \text { Promethium-147 }^{-147} & 8.6 \mathrm{E}-9\end{array}$

a The instrument minimum detectable concentration (MDC) values for the gas-flow proportional counter were calculated at the 95\% confidence level using the formula given in the section, "Lower Limits of Detection," in chapter 4 of the Savannah River Site Environmental Report for 1991 . The counting efficiencies were $28 \%$ for alpha and $40 \%$ for beta, strontium-90, and strontium-89,90. The MDC for the actual sample is variable because of the effects of sample aliquot size, sample preparation, chemical recovery, counting efficiency, and radioactive decay. The sample counting time was 20 minutes.

b Instrumental minimum detectable concentration (MDC) values for the liquid scintillation counter were calculated at the 95\% confidence level using the formula given in the section, "Lower Limits of Detection," in chapter 4 of the Savannah River Site Environmental Report for 1991 . The average counting efficiencies were $37 \%$ for tritium and $88 \%$ for promethium-147. The MDC for the actual sample is variable because of the effects of sample preparation, sample aliquot size, chemical recovery, counting efficiency, counting time, and radioactive decay.

c Routine environmental samples (e.g. stream samples and silica gels) are analyzed for tritium using a 20-minute count.

d Environmental samples such as drinking water, foodstuffs, and rainwater are analyzed using a 150-minute count. 


\section{Table 6}

Minimum Detectable Activities for Radionuclides in Settleable Solids

Page 1 of 1

\begin{tabular}{|c|c|c|c|}
\hline Nuclide & MDA (pci/sample) & Nuclide & MDA (pCi/sample) \\
\hline $\mathrm{Be}-7$ & $3.32 E+01$ & $\mathrm{Na}-24$ & $1.21 E+01$ \\
\hline$K-40$ & $1.09 E+02$ & Cr-51 & $4.02 E+01$ \\
\hline$M n-54$ & $7.83 E+00$ & Mn-56 & $8.04 \mathrm{E}+00$ \\
\hline Co-57 & $3.31 E+00$ & Co-58 & $6.43 E+00$ \\
\hline $\mathrm{Fe}-59$ & $1.19 \mathrm{E}+01$ & Co-60 & $8.22 E+00$ \\
\hline$Z n-65$ & $1.59 \mathrm{E}+01$ & $Y-88$ & $1.12 \mathrm{E}+01$ \\
\hline $\mathrm{Nb}-95$ & $6.88 E+00$ & $\mathrm{Nb}-95 \mathrm{M}$ & $1.34 \mathrm{E}+01$ \\
\hline$Z r-95$ & $1.18 \mathrm{E}+01$ & Mo-99 & $4.40 E+01$ \\
\hline$R u-103$ & $5.29 E+00$ & Ru-106 & $6.63 E+01$ \\
\hline$C d-109$ & $6.74 E+01$ & Sn-113 & $5.69 \mathrm{E}+00$ \\
\hline$S b-124$ & $6.41 E+00$ & Sb-125 & $1.48 E+01$ \\
\hline Sb-127 & $1.82 E+01$ & $|-13|$ & $5.03 E+00$ \\
\hline $1-132$ & $9.26 E+00$ & Te-132 & $3.65 E+00$ \\
\hline $\mathrm{Ba}-133$ & $7.69 \mathrm{E}+00$ & $1-133$ & $6.82 E+00$ \\
\hline Cs-134 & $6.54 E+00$ & $1-135$ & $2.49 E+01$ \\
\hline $\mathrm{Xe-135}$ & $4.66 E+00$ & Cs-136 & $6.6 E+00$ \\
\hline Cs-137 & $6.47 E+00$ & Ce-139 & $3.50 \mathrm{E}+00$ \\
\hline $\mathrm{Ba}-140$ & $2.30 E+01$ & La-140 & $9.98 E+00$ \\
\hline Ce-141 & $5.68 E+00$ & Ce-143 & $5.59 E+00$ \\
\hline Ce-144 & $2.57 E+01$ & Nd-147 & $1.19 \mathrm{E}+01$ \\
\hline Eu-154 & $7.01 E+00$ & TI-208 & $9.15 E+00$ \\
\hline TI-210 & $6.15 E+00$ & $\mathrm{~Pb}-211$ & $1.34 \mathrm{E}+02$ \\
\hline $\mathrm{Bi}-212$ & $6.17 E+01$ & $\mathrm{~Pb}-212$ & $1.25 \mathrm{E}+01$ \\
\hline $\mathrm{Bi}-214$ & $1.69 E+01$ & $\mathrm{~Pb}-214$ & $1.29 E+01$ \\
\hline $\mathrm{Rn}-219$ & $3.78 E+01$ & Ra-223 & $1.35 \mathrm{E}+01$ \\
\hline $\mathrm{Ra}-224$ & $1.16 \mathrm{E}+02$ & Ra-226 & $1.27 \mathrm{E}+02$ \\
\hline Th-227 & $3.00 \mathrm{E}+01$ & Ac-228 & $3.22 E+01$ \\
\hline Th-228 & $2.89 E+02$ & Th-231 & $5.41 E+01$ \\
\hline Th-234 & $6.87 E+01$ & U-235 & $7.65 \mathrm{E}+00$ \\
\hline \multirow[t]{2}{*}{ Am-241 } & $1.25 E+01$ & Alpha & $6.80 \mathrm{E}-01$ \\
\hline & & Beta & $1.53 \mathrm{E}-00$ \\
\hline
\end{tabular}


Table 7

Nonradiological Environmental Surveillance Detection/Report Limits

Page 1 of 2

\begin{tabular}{|c|c|c|c|c|}
\hline \multirow[b]{2}{*}{ Parameter } & \multirow[b]{2}{*}{ Units } & \multicolumn{2}{|c|}{ Quantitation Limit } & \multirow[b]{2}{*}{$\begin{array}{c}\text { Report Value } \\
\text { Round to Nearest }\end{array}$} \\
\hline & & $\begin{array}{c}\text { First and Second } \\
\text { Quarters }\end{array}$ & $\begin{array}{l}\text { Third and Fourth } \\
\text { Quarters }^{a}\end{array}$ & \\
\hline
\end{tabular}

Note: "NA" denotes "not applicable."

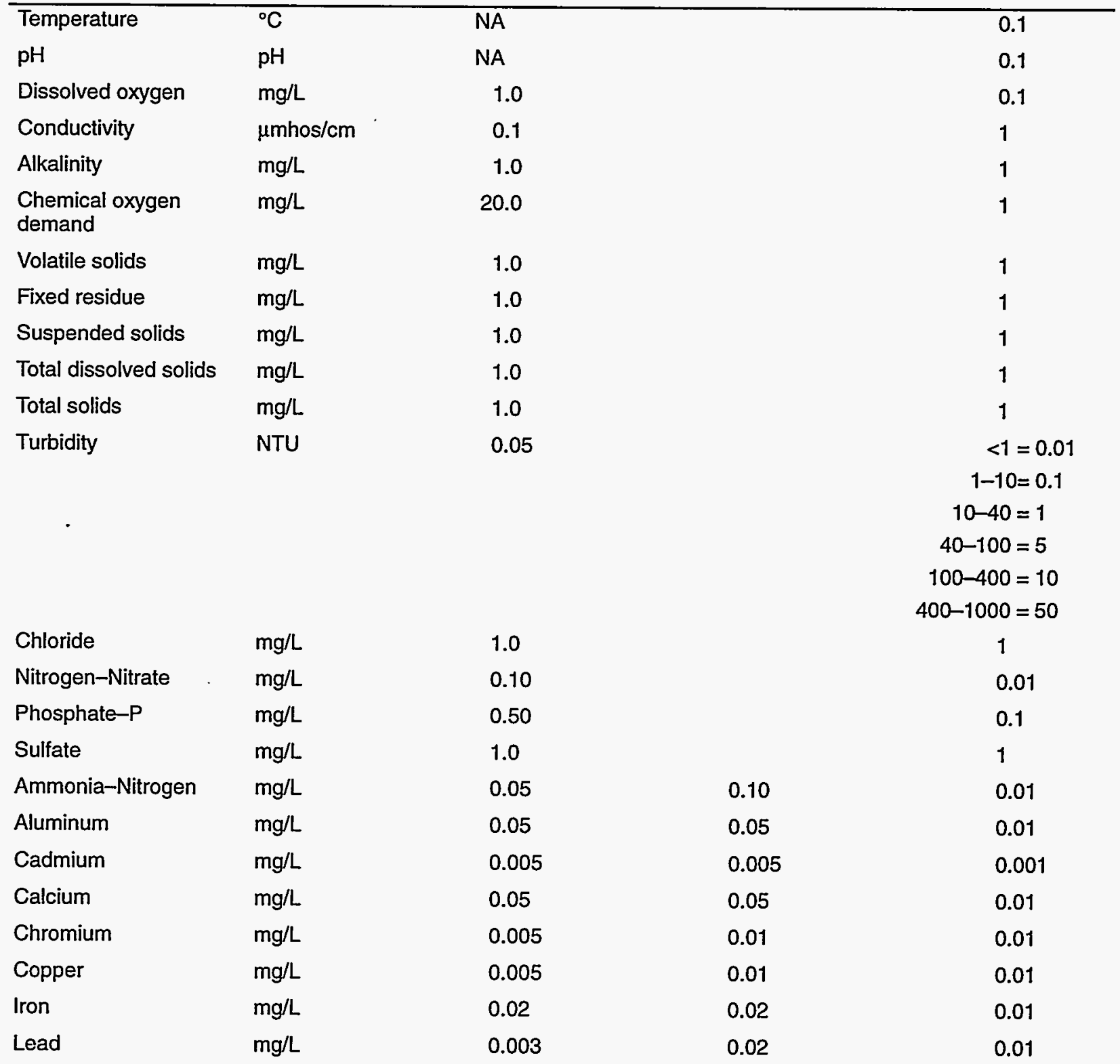

a Contract vendor changed. 
Table 7

Nonradiological Environmental Surveillance Detection/Report Limits

Page 2 of 2

Quantitation Limit

\begin{tabular}{llccc}
\hline Parameters & Units & $\begin{array}{c}\text { First and Second } \\
\text { Quarters }\end{array}$ & $\begin{array}{c}\text { Third and Fourth } \\
\text { Quarters }\end{array}$ & $\begin{array}{c}\text { Report Value Round } \\
\text { to Nearest: }\end{array}$ \\
\hline Magnesium & $\mathrm{mg} / \mathrm{L}$ & 0.05 & 0.05 & 0.01 \\
Manganese & $\mathrm{mg} / \mathrm{L}$ & 0.005 & 0.01 & 0.01 \\
Mercury & $\mathrm{mg} / \mathrm{L}$ & 0.0001 & 0.0001 & 0.0001 \\
Nickel & $\mathrm{mg} / \mathrm{L}$ & 0.005 & 0.01 & 0.01 \\
Sodium & $\mathrm{mg} / \mathrm{L}$ & 0.05 & 0.10 & 0.01 \\
Zinc & $\mathrm{mg} / \mathrm{L}$ & 0.005 & 0.005 & 0.001 \\
Total organic carbon & $\mathrm{mg} / \mathrm{L}$ & 1.0 & 1.0 & 0.1 \\
Total kjeldahl nitrogen & $\mathrm{mg} / \mathrm{L}$ & 0.20 & 0.10 & 0.01
\end{tabular}

a Contract vendor changed. 
Table 8

Radioactive Atmospheric Releases by Source

Page 1 of 2

\begin{tabular}{|c|c|c|c|c|c|c|c|c|}
\hline \multirow[b]{2}{*}{$\begin{array}{l}\text { Radio- } \\
\text { nuclide }\end{array}$} & \multirow[b]{2}{*}{ Half-life } & \multicolumn{7}{|c|}{ Curies $^{a}$} \\
\hline & & Reactors & $\begin{array}{l}\text { Separa- } \\
\text { tions }{ }^{b}\end{array}$ & $\begin{array}{l}\text { Reactor } \\
\text { Materials }\end{array}$ & $\begin{array}{l}\text { Heavy } \\
\text { Water }\end{array}$ & SRTCC & $\begin{array}{c}\text { Diffuse } \\
\text { and } \\
\text { Fugitived }^{\text {d }}\end{array}$ & Total \\
\hline \multicolumn{9}{|c|}{ Blank space indicates no quantifiable activity. } \\
\hline \multicolumn{9}{|c|}{ GASES AND VAPORS } \\
\hline $\mathrm{H}-3$ (oxide) & $12.3 y$ & $1.26 \mathrm{E}+04$ & $4.20 \mathrm{E}+04$ & & $3.28 E+02$ & & $3.32 E+01$ & $5.50 E+04$ \\
\hline $\mathrm{H}-3$ (elem) & $12.3 y$ & & $4.17 E+04$ & & & & & 4.17E+04 \\
\hline H-3 Total & $12.3 y$ & $1.26 \mathrm{E}+04$ & $8.37 E+04$ & & $3.28 \mathrm{E}+02$ & & $3.32 E+01$ & $9.67 \mathrm{E}+04$ \\
\hline C-14 & $5.73 E 3 y$ & & & & & & $9.80 E-15$ & $9.90 \mathrm{E}-15$ \\
\hline $1-129$ & 1.57E7 y & & $4.70 E-03$ & & & & & $4.70 \mathrm{E}-03$ \\
\hline $1-131$ & $8.040 \mathrm{~d}$ & & $1.29 \mathrm{E}-05$ & & & 4.07E-05 & $2.05 E-02$ & $2.06 \mathrm{E}-02$ \\
\hline $1-133$ & $20.8 \mathrm{~h}$ & & & & & $1.72 E-03$ & & $1.72 E-03$ \\
\hline $1-135$ & $6.57 \mathrm{~h}$ & & & & & $7.19 \mathrm{E}-02$ & & $7.19 \mathrm{E}-02$ \\
\hline \multicolumn{9}{|c|}{ PARTICULATES } \\
\hline $\mathrm{Al}-26$ & $7.3 \mathrm{E} 5 \mathrm{y}$ & & & & & & $1.50 E-14$ & $1.50 \mathrm{E}-14$ \\
\hline S-35 & $87.2 d$ & & & & & & $5.26 \mathrm{E}-12$ & $5.26 \mathrm{E}-12$ \\
\hline Cr-51 & $27.70 \mathrm{~d}$ & & & & & & $1.00 \mathrm{E}-16$ & $1.00 E-16$ \\
\hline Co-57 & $271.8 d$ & & & & & & $2.50 E-14$ & $2.50 \mathrm{E}-14$ \\
\hline Co-58 & $77.88 \mathrm{~d}$ & & & & & & $2.60 E-05$ & $2.60 E-05$ \\
\hline Co-60 & $5.271 \mathrm{y}$ & 2.78E-05 & $2.84 \mathrm{E}-07$ & & & $2.46 \mathrm{E}-06$ & $2.71 E-05$ & $5.76 \mathrm{E}-05^{\circ}$ \\
\hline $\mathrm{Ni}-63$ & $100 y$ & & & & & & $2.00 E-13$ & $2.00 E-13$ \\
\hline $\mathrm{Zn}-65$ & $243.8 d$ & & & & & & $6.24 \mathrm{E}-05$ & $6.24 \mathrm{E}-05$ \\
\hline $\mathrm{Rb}-86$ & $18.65 d$ & & & & & & $2.00 E-15$ & $2.00 E-15$ \\
\hline Sr-85 & $64.84 \mathrm{~d}$ & & & & & & $5.20 \mathrm{E}-16$ & $5.20 \mathrm{E}-16$ \\
\hline $\mathrm{Sr}-89,90^{e}$ & $29.1 y$ & $3.29 \mathrm{E}-03$ & $1.59 \mathrm{E}-03$ & $1.69 \mathrm{E}-04$ & $1.57 \mathrm{E}-04$ & $7.31 \mathrm{E}-06$ & 3.03E-04 & $5.52 E-03$ \\
\hline Y-88 & $106.6 \mathrm{~d}$ & & & & & & $9.10 \mathrm{E}-16$ & $9.10 E-16$ \\
\hline Zr-95 & $6402 d$ & & & & & & $4.51 E-05$ & $4.51 \mathrm{E}-05$ \\
\hline $\mathrm{Nb}-95$ & $34.97 \mathrm{~d}$ & & & & & & 2.67E-05 & 2.67E-05 \\
\hline$R u-103$ & $39.27 \mathrm{~d}$ & & & & & & $3.72 E-05$ & $3.72 E-05$ \\
\hline
\end{tabular}

One curie equals $3.7 \mathrm{E}+10$ Becquerels.

Includes separations, waste management, and tritium facilities

Savannah River Technology Center

Estimated releases from minor unmonitored diffuse and fugitive sources

Includes unidentified beta-gamma emissions 


\section{Table 8}

\section{Radioactive Atmospheric Releases by Source}

Page 2 of 2

\begin{tabular}{|c|c|c|c|c|c|c|c|c|}
\hline \multirow[b]{2}{*}{$\begin{array}{l}\text { Radio- } \\
\text { nuclide }\end{array}$} & \multirow[b]{2}{*}{ Half-life } & \multicolumn{7}{|c|}{ Curies ${ }^{a}$} \\
\hline & & Reactors & $\begin{array}{c}\text { Separa- } \\
\text { tions }\end{array}$ & $\begin{array}{l}\text { Reactor } \\
\text { Materials }\end{array}$ & $\begin{array}{l}\text { Heavy } \\
\text { Water }\end{array}$ & SRTCC & $\begin{array}{l}\text { Diffuse } \\
\text { and } \\
\text { Fugitive }^{d}\end{array}$ & Total \\
\hline Ru-106 & $1.020 y$ & & $6.46 E-07$ & & & & $1.80 \mathrm{E}-04$ & $1.81 \mathrm{E}-04$ \\
\hline Cd-109 & $462.0 \mathrm{~d}$ & & & & & & $5.21 \mathrm{E}-14$ & $5.21 E-14$ \\
\hline$S n-113$ & $115.1 d$ & & & & & & $3.80 E-16$ & $3.80 E-16$ \\
\hline Sb-124 & $60.20 d$ & & $1.81 E-07$ & & & & & $1.81 E-07$ \\
\hline$S b-125$ & $2.758 y$ & & $9.45 \mathrm{E}-07$ & & & & $1.19 \mathrm{E}-04$ & $1.20 E-04$ \\
\hline Cs-134 & $2.065 \mathrm{y}$ & & $3.22 E-07$ & & & & $2.98 E-05$ & $3.01 E-05$ \\
\hline Cs-137 & $30.17 y$ & $4.68 \mathrm{E}-04$ & $5.25 \mathrm{E}-04$ & $3.01 E-06$ & $2.58 E-06$ & $2.94 \mathrm{E}-07$ & $1.40 \mathrm{E}-02$ & $1.50 E-02$ \\
\hline $\mathrm{Ce}-139$ & $137.6 \mathrm{~d}$ & & & & & & $1.00 \mathrm{E}-16$ & $1.00 \mathrm{E}-16$ \\
\hline Ce-141 & $32.50 \mathrm{~d}$ & & & & & & $5.30 \mathrm{E}-05$ & $5.30 \mathrm{E}-05$ \\
\hline Ce-144 & $284.6 \mathrm{~d}$ & & $2.22 \mathrm{E}-07$ & & & & $2.32 E-04$ & $2.32 E-04$ \\
\hline Pm-147 & $2.623 \mathrm{y}$ & & & & & & $7.92 E-07$ & $7.92 \mathrm{E}-07$ \\
\hline Eu-154 & $8.59 \mathrm{y}$ & & $3.02 E-07$ & & & & & $3.02 E-07$ \\
\hline Eu-155 & $4.71 y$ & & $7.50 \mathrm{E}-07$ & & & & & $7.50 E-07$ \\
\hline $\mathrm{Hg}-203$ & $46.61 d$ & & & & & & $1.00 E-12$ & $1.00 \mathrm{E}-12$ \\
\hline$U-234$ & $2.46 E 5 y$ & & $1.27 \mathrm{E}-04$ & $1.73 E-06$ & & & & $1.29 \mathrm{E}-04$ \\
\hline$U-235$ & 7.04E8 y & & $8.32 E-04$ & $2.66 \mathrm{E}-05$ & & & $1.44 \mathrm{E}-15$ & $8.59 E-04$ \\
\hline$U-238$ & 4.47E9 $y$ & & $5.78 \mathrm{E}-04$ & $1.20 \mathrm{E}-06$ & & & $2.87 E-09$ & $5.79 E-04$ \\
\hline Pu-238 & $87.7 y$ & & $5.85 \mathrm{E}-04$ & $4.40 E-09$ & & & $6.61 E-06$ & $5.92 E-04$ \\
\hline Pu-239 & $2.410 \mathrm{E} 4$ & $2.78 E-04$ & $4.04 \mathrm{E}-04$ & $1.62 E-05$ & $2.39 E-05$ & $1.75 E-06$ & $2.21 E-06$ & $7.26 \mathrm{E}-04$ \\
\hline Am-241 & $432.7 \mathrm{y}$ & & $3.04 \mathrm{E}-05$ & $3.61 E-08$ & & & $1.81 E-16$ & 3.04E-05 \\
\hline$A m-243$ & 7.37E3 y & & & & & & $2.30 \mathrm{E}-17$ & $2.30 \mathrm{E}-17$ \\
\hline $\mathrm{Cm}-242$ & $162.8 d$ & & & & & & $2.03 E-16$ & $2.03 E-16$ \\
\hline $\mathrm{Cm}-243$ & $29.1 y$ & & & & & & $4.90 E-14$ & $4.90 E-14$ \\
\hline $\mathrm{Cm}-244$ & $18.1 \mathrm{y}$ & & $3.39 E-06$ & $9.02 E-09$ & & & & $3.40 E-06$ \\
\hline $\mathrm{Cm}-248$ & $3.48 E 5 y$ & & & & & & $9.20 E-18$ & $9.20 E-18$ \\
\hline
\end{tabular}

a One curie equals $3.7 E+10$ Becquerels.

Includes separations, waste management and tritium facilities

Savannah River Technology Center

Estimated releases from minor unmonitored diffuse and fugitive sources

Includes unidentified alpha emissions 
Table 9

Radioactive Atmospheric Releases by Stack/Facility and Comparison of Annual Average Concentrations to DOE Derived Concentration Guides

Page 1 of 6

\begin{tabular}{|c|c|c|c|c|c|}
\hline Stack/Facility & Radionuclide & $\begin{array}{c}\text { Quantity } \\
\text { Released During } \\
1995(\mu \mathrm{Ci})\end{array}$ & $\begin{array}{l}\text { Atmospheric } \\
\text { Release } \\
\text { Volume }(\mathrm{mL})\end{array}$ & $\begin{array}{c}\text { Average Effluent } \\
\text { Concentration } \\
\text { During } 1995 \\
(\mu \mathrm{Ci} / \mathrm{mL})\end{array}$ & $\begin{array}{c}\text { DOE DCGs }{ }^{\mathrm{a}} \\
(\mu \mathrm{Ci} / \mathrm{mL})\end{array}$ \\
\hline \multicolumn{6}{|c|}{ Note: Blank space indicates no quantifiable activity. } \\
\hline \multicolumn{6}{|c|}{ A-Area (Savannah River Technology Center) } \\
\hline \multicolumn{6}{|l|}{$735-A$} \\
\hline $773-A B$ & Cs-137 & $2.94 E-01$ & $9.84 E+14$ & $2.99 \mathrm{E}-16$ & $4.0 E-10$ \\
\hline \multicolumn{6}{|l|}{ 773-A C } \\
\hline \multirow[t]{5}{*}{ 773-A Sandfilter } & Co-60 & $2.46 \mathrm{E}+00$ & $9.27 \mathrm{E}+14$ & $2.65 E-15$ & $8.0 E-11$ \\
\hline & $1-131$ & $4.05 \mathrm{E}+01$ & $9.27 \mathrm{E}+14$ & 4.37E-14 & 4.0E-10 \\
\hline & $1-133$ & $1.71 E+03$ & $9.27 E+14$ & $1.84 \mathrm{E}-12$ & $2.0 E-09$ \\
\hline & $\mathrm{l}-135$ & $7.19 \mathrm{E}+04$ & $9.27 \mathrm{E}+14$ & $7.78 \mathrm{E}-11$ & $1.0 \mathrm{E}-08$ \\
\hline & $\mathrm{Xe}-135$ & $1.49 E+04$ & $9.27 \mathrm{E}+14$ & $1.61 E-11$ & none listed \\
\hline \multirow[t]{2}{*}{ 776-A } & $\mid-131$ & $1.99 E-01$ & $1.30 E+14$ & $1.53 E-15$ & $4.0 \mathrm{E}-10$ \\
\hline & l-133 & $7.43 E+00$ & $1.30 E+14$ & $5.72 \mathrm{E}-14$ & $2.0 \mathrm{E}-09$ \\
\hline \multicolumn{6}{|c|}{ C-Area (C-Reactor) } \\
\hline \multicolumn{6}{|l|}{ 717-C Hot Shop } \\
\hline \multicolumn{6}{|l|}{$728-N$} \\
\hline \multicolumn{6}{|l|}{ C Decon } \\
\hline \multicolumn{6}{|l|}{ C Disassembly } \\
\hline C Main (148') & H-3 (oxide) & $1.28 \mathrm{E}+09$ & $1.98 E+15$ & $8.27 E-08$ & $1.0 E-07$ \\
\hline \multicolumn{6}{|c|}{ D-Area (Heavy Water Rework) } \\
\hline $420-D$ & H-3 (oxide) & $2.58 \mathrm{E}+08$ & $2.33 E+14$ & $1.11 E-06$ & $1.0 \mathrm{E}-07$ \\
\hline \multirow[t]{2}{*}{$421-2 D$} & H-3 (oxide) & $1.94 \mathrm{E}+07$ & $1.59 \mathrm{E}+14$ & $1.22 \mathrm{E}-07$ & $1.0 E-07$ \\
\hline & Cs-137 & $2.58 \mathrm{E}+00$ & $1.59 \mathrm{E}+14$ & $1.62 \mathrm{E}-14$ & $40 \mathrm{E}-10$ \\
\hline $772-D$ & H-3 (oxide) & $5.08 \mathrm{E}+07$ & $2.65 E+14$ & $1.92 E-07$ & $1.0 E-07$ \\
\hline \multicolumn{6}{|c|}{ F-Area (Separations and Waste Management) } \\
\hline \multirow[t]{4}{*}{ 235-F Sandfilter } & U-234 & $3.78 \mathrm{E}-01$ & $5.64 \mathrm{E}+14$ & $6.67 \mathrm{E}-16$ & $9.0 \mathrm{E}-14$ \\
\hline & U-235 & 3.33E-02 & $5.64 \mathrm{E}+14$ & $5.90 E-17$ & $1.0 E-13$ \\
\hline & U-238 & $7.26 \mathrm{E}-01$ & $5.64 \mathrm{E}+14$ & $1.29 \mathrm{E}-15$ & $1.0 \mathrm{E}-13$ \\
\hline & Pu-238 & $6.20 \mathrm{E}-03$ & $5.64 \mathrm{E}+14$ & $1.10 \mathrm{E}-17$ & $3.0 E-14$ \\
\hline
\end{tabular}

a Source: DOE Order 5400.5. In cases where different chemical forms have different DCGs, the lowest DCG for the radionuclide is given. These DCGs are defined as the air concentration of that radionuclide that will give a 50 -year committed effective dose equivalent of 100 mrem under conditions of continuous exposure for one year. DCGs are reference values only and are not considered release limits or standards. 
Table 9

Radioactive Atmospheric Releases by Stack/Facility and Comparison of Annual Average Concentrations to DOE Derived Concentration Guides

Page 2 of 6

\begin{tabular}{|c|c|c|c|c|c|}
\hline Stack/Facility & Radionuclide & $\begin{array}{c}\text { Quantity } \\
\text { Released During } \\
1995(\mu \mathrm{Ci})\end{array}$ & $\begin{array}{l}\text { Atmospheric } \\
\text { Release } \\
\text { Volume }(\mathrm{mL})\end{array}$ & $\begin{array}{c}\text { Average Effluent } \\
\text { Concentration } \\
\text { During } 1995 \\
(\mu \mathrm{Ci} / \mathrm{mL})\end{array}$ & $\begin{array}{c}\text { DOE DCGs }{ }^{a} \\
(\mu \mathrm{Ci} / \mathrm{mL})\end{array}$ \\
\hline & Pu-239 & $8.28 \mathrm{E}-02$ & $5.64 \mathrm{E}+14$ & $1.47 \mathrm{E}-16$ & $2.0 \mathrm{E}-14$ \\
\hline & $A M-241$ & $5.70 E-02$ & $5.64 E+14$ & $1.01 E-16$ & $2.0 E-14$ \\
\hline & $\mathrm{CM}-244$ & $1.62 \mathrm{E}-02$ & $5.64 E+14$ & $2.87 E-17$ & $4.0 E-14$ \\
\hline 241-F Misc & Cs-137 & $1.65 E+01$ & $6.16 E+14$ & $2.68 E-14$ & $4.0 E-10$ \\
\hline 241-F Purge & Cs-137 & $3.91 E+01$ & $9.43 E+14$ & 4.15E-14 & $4.0 E-10$ \\
\hline 241-F Annulus & Cs-137 & $7.47 E+01$ & $6.74 E+13$ & $1.11 \mathrm{E}-12$ & $4.0 E-10$ \\
\hline \multirow[t]{7}{*}{ 247-F Main } & $U-234$ & $9.01 E+00$ & $1.06 \mathrm{E}+15$ & $8.50 E-15$ & $9.0 E-14$ \\
\hline & $U-235$ & $2.16 E+01$ & $1.06 E+15$ & $2.04 E-14$ & $1.0 \mathrm{E}-13$ \\
\hline & $U-238$ & $1.41 \mathrm{E}-01$ & $1.06 E+15$ & $1.33 E-16$ & $1.0 \mathrm{E}-13$ \\
\hline & $\mathrm{Pu}-238$ & $2.83 E+00$ & $1.06 E+15$ & 2.67E-14 & $3.0 E-14$ \\
\hline & Pu-239 & $7.06 \mathrm{E}+02$ & $1.06 \mathrm{E}+15$ & $6.66 E-13$ & $2.0 E-14$ \\
\hline & $A m-241$ & $1.60 \mathrm{E}-01$ & $1.06 E+15$ & $1.51 E-16$ & $2.0 E-14$ \\
\hline & $\mathrm{Cm}-244$ & $5.55 E-02$ & $1.06 \mathrm{E}+15$ & $5.24 \mathrm{E}-17$ & $4.0 \mathrm{E}-14$ \\
\hline \multirow[t]{12}{*}{ 291-F Isokinetic } & $\mathrm{Sr}-89,90$ & $2.61 E-01$ & $3.35 \mathrm{E}+15$ & $7.79 E-14$ & $9.0 \mathrm{E}-12$ \\
\hline & $1-129$ & $3.00 \mathrm{E}+03$ & $3.35 E+15$ & $8.96 \mathrm{E}-13$ & $7.0 \mathrm{E}-11$ \\
\hline & $\mid-131$ & $4.99 E+00$ & $3.35 E+15$ & $1.49 \mathrm{E}-14$ & $4.0 E-10$ \\
\hline & $X e-135$ & $1.87 E+04$ & $3.35 E+15$ & $5.58 \mathrm{E}-12$ & $8.0 E-08$ \\
\hline & Cs-137 & $1.55 E+01$ & $3.35 E+15$ & 4.63E-14 & $4.0 E-10$ \\
\hline & $U-234$ & 1.17E+02 & $3.35 E+15$ & $3.49 E-14$ & $9.0 E-14$ \\
\hline & $U-235$ & $7.93 E+02$ & $3.35 E+15$ & 2.37E-13 & $1.0 E-13$ \\
\hline & $U-238$ & $5.75 E+02$ & $3.35 E+15$ & $1.72 E-13$ & $1.0 E-13$ \\
\hline & $\mathrm{Pu}-238$ & $1.16 E+02$ & $3.35 E+15$ & $3.46 E-14$ & $3.0 E-14$ \\
\hline & Pu-239 & $2.47 \mathrm{E}+02$ & $3.35 E+15$ & 7.37E-14 & $2.0 E-14$ \\
\hline & $A m-241$ & $2.68 E+01$ & $3.35 E+15$ & 8.00E-14 & $2.0 E-14$ \\
\hline & $\mathrm{Cm}-244$ & $2.46 E+00$ & $3.35 E+15$ & $7.34 \mathrm{E}-16$ & $4.0 E-14$ \\
\hline \multirow[t]{3}{*}{ 6.1 D Dissolver } & Co-60 & $2.84 \mathrm{E}-01$ & $9.59 \mathrm{E}+11$ & $2.96 E-13$ & $8.0 E-11$ \\
\hline & $R u-106$ & $6.46 \mathrm{E}-01$ & $9.59 E+11$ & $6.74 E-13$ & $3.0 \mathrm{E}-11$ \\
\hline & $\mathrm{Sb}-124$ & $1.81 E-01$ & $9.59 E+11$ & $1.89 \mathrm{E}-13$ & $6.0 \mathrm{E}-10$ \\
\hline
\end{tabular}

a Source: DOE Order 5400.5. In cases where different chemical forms have different DCGs, the lowest DCG for the radionuclide is given. These DCGs are defined as the air concentration of that radionuclide that will give a 50-year committed effective dose equivalent of 100 mrem under conditions of continuous exposure for one year. DCGs are reference values only and are not considered release limits or standards. 


\section{Table 9}

\section{Radioactive Atmospheric Releases by Stack/Facility and Comparison of Annual Average Concentrations to DOE Derived Concentration Guides}

Page 3 of 6

\begin{tabular}{|c|c|c|c|c|c|}
\hline Stack/Facility & Radionuclide & $\begin{array}{c}\text { Quantity } \\
\text { Released During } \\
1995(\mu \mathrm{Ci})\end{array}$ & $\begin{array}{c}\text { Atmospheric } \\
\text { Release } \\
\text { Volume (mL) }\end{array}$ & $\begin{array}{c}\text { Average Effluent } \\
\text { Concentration } \\
\text { During } 1995 \\
(\mu \mathrm{Ci} / \mathrm{mL})\end{array}$ & $\begin{array}{c}\text { DOE DCGsa } \\
(\mu \mathrm{Ci} / \mathrm{mL})\end{array}$ \\
\hline & $\mathrm{Sb}-125$ & $8.44 \mathrm{E}-01$ & $9.59 E+11$ & $8.80 E-13$ & $1.0 E-09$ \\
\hline & $\mathrm{Cs}-134$ & $1.32 \mathrm{E}-01$ & $9.59 E+11$ & $1.38 E-13$ & $2.0 E-10$ \\
\hline & $\mathrm{Cs}-137$ & $7.85 \mathrm{E}+01$ & $9.59 E+11$ & $8.19 E-11$ & $4.0 E-10$ \\
\hline & $\mathrm{Ce}-144$ & $2.22 E-01$ & $9.59 \mathrm{E}+11$ & $2.31 E-13$ & $3.0 \mathrm{E}-11$ \\
\hline & Eu-154 & $3.02 E-01$ & $9.59 E+11$ & $3.15 E-13$ & $5.0 E-11$ \\
\hline & Eu-155 & $7.50 \mathrm{E}-01$ & $9.59 E+11$ & $7.82 E-13$ & $3.0 \mathrm{E}-10$ \\
\hline & $A m-241$ & $2.61 \mathrm{E}+00$ & $9.59 \mathrm{E}+11$ & $2.72 E-12$ & $2.0 E-14$ \\
\hline & $\mathrm{Sb}-125$ & $1.01 E-01$ & $8.36 E+11$ & $1.21 \mathrm{E}-13$ & $1.0 E-09$ \\
\hline & Cs-137 & $2.21 E+00$ & $8.36 E+1.1$ & $2.64 \mathrm{E}-12$ & $4.0 E-10$ \\
\hline \multicolumn{6}{|l|}{$723-F$} \\
\hline \multicolumn{6}{|l|}{$772-1 F$} \\
\hline \multirow[t]{8}{*}{$772-4 F$} & $\mathrm{Sr}-89,90$ & $2.10 E+00$ & $2.20 E+15$ & $9.55 E-16$ & $9.0 E-12$ \\
\hline & Cs-137 & $3.86 \mathrm{E}-01$ & $2.20 E+15$ & $1.75 E-16$ & $4.0 E-10$ \\
\hline & $U-234$ & $1.36 \mathrm{E}-02$ & $2.20 E+15$ & $6.18 E-18$ & $9.0 E-14$ \\
\hline & $U-235$ & 3.47E-03 & $2.20 E+15$ & $1.58 \mathrm{E}-18$ & $1.0 E-13$ \\
\hline & $\mathrm{U}-238$ & $1.98 E-02$ & $2.20 \mathrm{E}+15$ & $9.00 \mathrm{E}-18$ & $1.0 E-13$ \\
\hline & $P u-239$ & $1.64 \mathrm{E}-02$ & $2.20 E+15$ & $7.45 E-18$ & $2.0 \mathrm{E}-14$ \\
\hline & Am-241 & $3.11 \mathrm{E}-02$ & $2.20 E+15$ & $1.41 \mathrm{E}-17$ & $2.0 \mathrm{E}-14$ \\
\hline & $\mathrm{Cm}-244$ & $1.19 \mathrm{E}-02$ & $2.20 E+15$ & $5.41 E-18$ & $4.0 E-14$ \\
\hline 800 Cell $(211-F)$ & $\mathrm{Sr}-89,90$ & $1.35 \mathrm{E}+01$ & $2.51 E+12$ & $5.38 \mathrm{E}-12$ & $9.0 \mathrm{E}-12$ \\
\hline \multirow[t]{7}{*}{ A-Line $(221-1 F)$} & $U-234$ & $3.42 E-01$ & $3.54 \mathrm{E}+13$ & $9.66 E-15$ & $9.0 E-14$ \\
\hline & $\mathrm{U}-235$ & $2.68 \mathrm{E}+00$ & $3.54 E+13$ & $7.57 E-14$ & $1.0 E-13$ \\
\hline & $U-238$ & $6.87 E-01$ & $3.54 E+13$ & $1.94 E-14$ & $1.0 E-13$ \\
\hline & $\mathrm{Pu}-238$ & $3.22 \mathrm{E}-01$ & $3.54 E+13$ & $9.10 E-15$ & $3.0 E-14$ \\
\hline & $P u-239$ & $2.71 \mathrm{E}-01$ & $3.54 E+13$ & $7.66 \mathrm{E}-15$ & 2.0E-14 \\
\hline & $A m-241$ & $2.83 E-02$ & $3.54 E+13$ & $7.99 E-16$ & $2.0 E-14$ \\
\hline & $\mathrm{Cm}-244$ & 3.07E-02 & $3.54 \mathrm{E}+13$ & 8.67E-16 & $4.0 E-14$ \\
\hline
\end{tabular}

a Source: DOE Order 5400.5. In cases where different chemical forms have different DCGs, the lowest DCG for the radionuclide is given. These DCGs are defined as the air concentration of that radionuclide that will give a 50-year committed effective dose equivalent of $100 \mathrm{mrem}$ under conditions of continuous exposure for one year. DCGs are reference values only and are not considered release limits or standards. 
Table 9

Radioactive Atmospheric Releases by Stack/Facility and Comparison of Annual Average Concentrations to DOE Derived Concentration Guides

Page 4 of 6

\begin{tabular}{|c|c|c|c|c|c|}
\hline Stack/Facility & Radionuclide & $\begin{array}{c}\text { Quantity } \\
\text { Released During } \\
1995(\mu \mathrm{Ci})\end{array}$ & $\begin{array}{l}\text { Atmospheric } \\
\text { Release } \\
\text { Volume }(\mathrm{mL})\end{array}$ & $\begin{array}{c}\text { Average Effluent } \\
\text { Concentration } \\
\text { During } 1995 \\
(\mu \mathrm{Ci} / \mathrm{mL})\end{array}$ & $\begin{array}{c}\text { DOE DCGsa } \\
(\mu \mathrm{Ci} / \mathrm{mL})\end{array}$ \\
\hline \multicolumn{6}{|l|}{ F Main (195') } \\
\hline \multicolumn{6}{|c|}{ H-Area (Separations and Waste Management) } \\
\hline \multicolumn{6}{|l|}{$230-H$ Lag } \\
\hline \multicolumn{6}{|l|}{ 230-H Process } \\
\hline $\begin{array}{l}241-84 H \text { (ETF } \\
\text { Lab) }\end{array}$ & Cs-137 & $9.88 E-02$ & $4.51 E+13$ & $2.19 \mathrm{E}-15$ & 4.0E-10 \\
\hline \multicolumn{6}{|l|}{$\begin{array}{l}\text { 241-84H (ETF } \\
\text { Process) }\end{array}$} \\
\hline 241-H Misc & Cs-137 & $8.11 \mathrm{E}+00$ & $5.52 E+15$ & $1.47 \mathrm{E}-15$ & $4.0 E-10$ \\
\hline \multirow[t]{2}{*}{ 241-H Purge } & Cs-134 & $1.90 E-01$ & $1.46 \mathrm{E}+15$ & $1.30 E-16$ & $2.0 E-10$ \\
\hline & Cs-137 & $1.06 \mathrm{E}+02$ & $1.46 E+15$ & $7.26 \mathrm{E}-14$ & $4.0 E-10$ \\
\hline 241-H Annulus & Cs-137 & $1.34 E+02$ & $5.94 \mathrm{E}+15$ & $2.26 \mathrm{E}-14$ & $4.0 \mathrm{E}-10$ \\
\hline 244-H RBOF & Cs137 & $2.42 E+01$ & $3.60 E+14$ & $6.72 E-14$ & $4.0 E-10$ \\
\hline $\begin{array}{l}\text { 244-HRBOF } \\
\text { Vessel Vent }\end{array}$ & Cs137 & $5.62 E-01$ & $3.70 E+12$ & $1.52 \mathrm{E}-13$ & $4.0 E-10$ \\
\hline \multicolumn{6}{|l|}{$\begin{array}{l}\text { 253-H Waste } \\
\text { Compactor }\end{array}$} \\
\hline \multirow[t]{11}{*}{ 291-H Isokinetic } & $\mathrm{Sr}-89,90$ & $1.20 E+01$ & $4.05 E+15$ & $2.96 \mathrm{E}-15$ & $9.0 E-12$ \\
\hline & $1-129$ & $1.70 E+03$ & $4.05 E+15$ & $4.20 \mathrm{E}-13$ & $7.0 E-12$ \\
\hline & $1-131$ & $7.98 \mathrm{E}+00$ & $4.05 E+15$ & $1.95 \mathrm{E}-15$ & $4.0 E-10$ \\
\hline & Cs-137 & $2.50 E+01$ & $4.05 E+15$ & $6.17 E-15$ & $4.0 E-10$ \\
\hline & $U-234$ & $2.26 \mathrm{E}-01$ & $4.05 E+15$ & $5.58 \mathrm{E}-17$ & $9.02 E-14$ \\
\hline & $U-235$ & $1.43 E+01$ & $4.05 E+15$ & $3.53 E-15$ & $1.0 \mathrm{E}-13$ \\
\hline & $U-238$ & $1.00 \mathrm{E}+00$ & $4.05 E+15$ & $2.47 E-16$ & $1.0 \mathrm{E}-13$ \\
\hline & $\mathrm{Pu}-238$ & $4.66 E+02$ & $4.05 E+15$ & $1.15 E-13$ & $3.0 \mathrm{E}-14$ \\
\hline & $\mathrm{Pu}-239$ & $8.70 E+00$ & $4.05 E+15$ & 2.15E-15 & $2.0 E-14$ \\
\hline & Am-241 & $6.26 \mathrm{E}-01$ & $4.05 E+15$ & 1.55E-16 & $2.0 \mathrm{E}-14$ \\
\hline & $\mathrm{Cm}-244$ & $8.21 E-01$ & $4.05 E+15$ & $2.03 E-16$ & $4.0 \mathrm{E}-14$ \\
\hline
\end{tabular}

a Source: DOE Order 5400.5. In cases where different chemical forms have different DCGs, the lowest DCG for the radionuclide is given. These DCGs are defined as the air concentration of that radionuclide that will give a 50-year committed effective dose equivalent of 100 mrem under conditions of continuous exposure for one year. DCGs are reference values only and are not considered release limits or standards. 


\section{Table 9}

\section{Radioactive Atmospheric Releases by Stack/Facility and Comparison of Annual Average Concentrations to DOE Derived Concentration Guides}

Page 5 of 6

\begin{tabular}{|c|c|c|c|c|c|}
\hline Stack/Facility & Radionuclide & $\begin{array}{c}\text { Quantity } \\
\text { Released During } \\
1995(\mu \mathrm{Ci})\end{array}$ & $\begin{array}{c}\text { Atmospheric } \\
\text { Release } \\
\text { Volume (mL) }\end{array}$ & $\begin{array}{c}\text { Average Effluent } \\
\text { Concentration } \\
\text { During } 1995 \\
(\mu \mathrm{Ci} / \mathrm{mL})\end{array}$ & $\begin{array}{c}\text { DOE DCGsa } \\
(\mu \mathrm{Ci} / \mathrm{mL})\end{array}$ \\
\hline $\begin{array}{l}\text { 299-H (Bldg + } \\
\text { HP Hood) }\end{array}$ & Cs -137 & $9.95 E-03$ & $2.76 E+14$ & $3.61 \mathrm{E}-17$ & $4.0 E-10$ \\
\hline \multicolumn{6}{|l|}{ H Main (195') } \\
\hline \multirow[t]{2}{*}{ Tritium } & $\mathrm{H}-3$ (oxide) & $4.20 E+10$ & $1.32 E+15$ & $3.18 E-05$ & $1.0 \mathrm{E}-07$ \\
\hline & H-3 (elem.) & $4.17 E+10$ & $1.32 E+15$ & $3.16 \mathrm{E}-05$ & $2.0 \mathrm{E}-02$ \\
\hline \multicolumn{6}{|c|}{ K-Area (K-Reactor) } \\
\hline K-Main (148') & $\mathrm{H}-3$ (oxide) & 2.17E+09 & $2.27 E+15$ & $9.56 \mathrm{E}-07$ & $1.0 \mathrm{E}-07$ \\
\hline \multirow[t]{3}{*}{ K Disassembly } & $\mathrm{H}-3$ (oxide) & $5.46 E+08$ & $3.12 E+15$ & $1.75 E-07$ & $1.0 \mathrm{E}-07$ \\
\hline & Co-60 & $2.78 E+01$ & $3.12 E+15$ & $8.91 E-15$ & $8.0 E-11$ \\
\hline & Cs-137 & $4.20 \mathrm{E}+02$ & $3.12 E+15$ & $1.34 \mathrm{E}-13$ & $4.0 \mathrm{E}-10$ \\
\hline \multicolumn{6}{|c|}{ L-Area (L-Reactor) } \\
\hline L Main (148') & $\mathrm{H}-3$ (oxide) & $1.58 \mathrm{E}+09$ & $1.43 E+15$ & $1.10 \mathrm{E}-06$ & $1.0 \mathrm{E}-07$ \\
\hline \multirow{2}{*}{ L Disassembly } & H-3 (oxide) & $3.14 E+08$ & $1.43 E+15$ & $2.20 \mathrm{E}-07$ & $1.0 \mathrm{E}-07$ \\
\hline & Cs-137 & 4.27E+01 & $1.43 E=15$ & $2.99 \mathrm{E}-14$ & $4.0 E-10$ \\
\hline \multicolumn{6}{|c|}{ M-Area (Reactor Materials) } \\
\hline \multirow[t]{6}{*}{$313-M$} & U-234 & $3.56 \mathrm{E}-03$ & $5.22 \mathrm{E}-13$ & $6.82 E-13$ & $9.0 E-14$ \\
\hline & $U-235$ & $1.97 E-03$ & $5.22 E+13$ & $3.77 E-15$ & $1.0 E-13$ \\
\hline & $U-238$ & $2.81 E-03$ & $5.22 E+13$ & $5.38 \mathrm{E}-15$ & $1.0 E-13$ \\
\hline & $\mathrm{Pu}-238$ & $3.24 E-04$ & $5.22 \mathrm{E}+13$ & $6.21 E-16$ & $3.0 E-14$ \\
\hline & $\mathrm{Pu}-239$ & $9.64 E-04$ & $5.22 E+13$ & $1.85 \mathrm{E}-17$ & $2.0 E-14$ \\
\hline & $A m-241$ & $1.07 \mathrm{E}-03$ & $5.22 E+13$ & $2.05 E-15$ & $2.0 E-14$ \\
\hline $320-M$ & $\mathrm{U}-235$ & $3.48 E-01$ & $9.62 E+12$ & $3.62 E-14$ & $1.0 E-13$ \\
\hline \multirow[t]{5}{*}{$321-M$} & $U-234$ & $8.41 \mathrm{E}-01$ & $5.06 \mathrm{E}+14$ & $1.66 \mathrm{E}-15$ & $9.0 E-14$ \\
\hline & U-235 & $6.65 E+00$ & $5.06 E+14$ & $1.31 \mathrm{E}-14$ & $1.0 E-13$ \\
\hline & $U-238$ & $1.89 E-01$ & $5.06 E+14$ & $3.74 E-16$ & $1.0 \mathrm{E}-13^{\circ}$ \\
\hline & Pur-238 & $3.06 E-03$ & $5.06 \mathrm{E}+14$ & $6.05 \mathrm{E}-18$ & $3.0 E-14$ \\
\hline & $\mathrm{Pu}-239$ & $7.01 E-03$ & $5.06 E+14$ & $1.39 E-17$ & $2.0 \mathrm{E}-14$ \\
\hline
\end{tabular}

a Source: DOE Order 5400.5. In cases where different chemical forms have different DCGs, the lowest DCG for the radionuclide is given. These DCGs are defined as the air concentration of that radionuclide that will give a 50 -year committed effective dose equivalent of 100 mrem under conditions of continuous exposure for one year. DCGs are reference values only and are not considered release limits or standards. 


\section{Table 9}

\section{Radioactive Atmospheric Releases by Stack/Facility and Comparison of Annual Average Concentrations to DOE Derived Concentration Guides}

Page 6 of 6

\begin{tabular}{|c|c|c|c|c|c|}
\hline Stack/Facility & Radionuclide & $\begin{array}{c}\text { Quantity } \\
\text { Released During } \\
1995(\mu \mathrm{Ci})\end{array}$ & $\begin{array}{l}\text { Atmospheric } \\
\text { Release } \\
\text { Volume }(\mathrm{mL})\end{array}$ & $\begin{array}{c}\text { Average Effluent } \\
\text { Concentration } \\
\text { During } 1995 \\
(\mu \mathrm{Ci} / \mathrm{mL})\end{array}$ & $\begin{array}{c}\text { DOE DCGs } \\
(\mu \mathrm{Ci} / \mathrm{mL})\end{array}$ \\
\hline & $A m-241$ & $2.05 E-02$ & $5.06 E+14$ & $4.05 E-17$ & $2.0 E-14$ \\
\hline & $\mathrm{Cm}-244$ & $3.86 E-03$ & $5.06 E+14$ & $7.63 E-18$ & $4.0 E-14$ \\
\hline \multirow{7}{*}{$\begin{array}{l}\text { 321-M Mach Rm } \\
\text { Isokinetic }\end{array}$} & $U-234$ & $8.65 E-02$ & $5.33 E+14$ & $1.62 E-16$ & $9.0 E-13$ \\
\hline & $U-235$ & $1.74 E-01$ & $5.33 E+14$ & $3.26 \mathrm{E}-16$ & $1.0 E-13$ \\
\hline & $U-238$ & $3.84 \mathrm{E}-03$ & $5.33 E+14$ & $7.20 E-18$ & $1.0 E-13$ \\
\hline & $P u-238$ & $1.02 E-03$ & $5.33 E+14$ & $1.91 E-18$ & $3.0 E-14$ \\
\hline & $P u-239$ & $3.42 E-05$ & $5.33 E+14$ & $6.42 E-20$ & $2.0 E-14$ \\
\hline & $A m-241$ & $7.68 \mathrm{E}-04$ & $5.33 E+14$ & $1.44 E-18$ & $2.0 E-14$ \\
\hline & $\mathrm{Cm}-244$ & $2.26 \mathrm{E}-03$ & $5.33 E+14$ & $4.24 E-18$ & $4.0 E-14$ \\
\hline \multirow[t]{4}{*}{ 321-M Mach Rm } & $U-234$ & $1.36 \mathrm{E}-01$ & $7.43 E+13$ & $1.83 E-15$ & $9.0 \mathrm{E}-13$ \\
\hline & $U-235$ & $1.71 \mathrm{E}+01$ & $7.43 E+13$ & $2.30 E-13$ & $1.0 E-13$ \\
\hline & $U-238$ & $7.48 \mathrm{E}-02$ & $7.43 E+13$ & $1.01 E-15$ & $1.0 E-13$ \\
\hline & $P u-239$ & $1.07 E-02$ & $7.43 E+13$ & $1.44 \mathrm{E}-16$ & $2.0 E-14$ \\
\hline \multirow[t]{7}{*}{$322-M$} & Cs-137 & $3.01 E+00$ & $5.43 E+14$ & $5.54 \mathrm{E}-15$ & $4.0 E-10$ \\
\hline & $U-234$ & $6.66 \mathrm{E}-01$ & $5.43 E+14$ & $1.23 E-15$ & $9.0 E-13$ \\
\hline & $U-235$ & $2.35 E+00$ & $5.43 E+14$ & 4.33E-15 & $1.0 E-13$ \\
\hline & $U-238$ & $9.29 E-01$ & $5.43 E+14$ & $1.71 E-15$ & $1.0 \mathrm{E}-13$ \\
\hline & Pu-239 & $1.16 \mathrm{E}-02$ & $5.43 E+14$ & $2.14 \mathrm{E}-15$ & $2.0 E-14$ \\
\hline & $A m-241$ & $1.38 \mathrm{E}-02$ & $5.43 E+14$ & $2.54 \mathrm{E}-15$ & $2.0 E-14$ \\
\hline & $\mathrm{Cm}-244$ & $2.90 E-03$ & $5.43 E+14$ & $5.34 \mathrm{E}-18$ & $4.0 E-14$ \\
\hline \multicolumn{6}{|c|}{ P-Area (P-Reactor) } \\
\hline P Main (148') & H-3 (oxide) & $6.25 E+09$ & 2.67E+15 & 2.34E-06 & $1.0 \mathrm{E}-07$ \\
\hline \multirow[t]{2}{*}{ P Disassembly } & $\mathrm{H}-3$ (oxide) & $4.68 \mathrm{E}+08$ & $2.73 E+14$ & $1.71 \mathrm{E}-06$ & $1.0 \mathrm{E}-07$ \\
\hline & $\mathrm{Cs}-137$ & $5.24 \mathrm{E}+00$ & $2.73 E+14$ & $1.92 E-14$ & $4.0 E-10$ \\
\hline
\end{tabular}

a Source: DOE Order 5400.5. In cases where different chemical forms have different DCGs, the lowest DCG for the radionuclide is given. These DCGs are defined as the air concentration of that radionuclide that will give a 50-year committed effective dose equivalent of $100 \mathrm{mrem}$ under conditions of continuous exposure for one year. DCGs are reference values only and are not considered release limits or standards. 
Table 10

Radioactive Liquid Releases by Source

(Including Direct and Seepage Basin Migration Releases)

Page 1 of 1

\begin{tabular}{|c|c|c|c|c|c|c|c|}
\hline \multirow[b]{2}{*}{$\begin{array}{l}\text { Radio- } \\
\text { nuclide }\end{array}$} & \multirow[b]{2}{*}{ Half-life } & \multicolumn{6}{|c|}{ Curies $^{a}$} \\
\hline & & Reactors & $\begin{array}{c}\text { Separa- } \\
\text { tions }^{b}\end{array}$ & $\begin{array}{l}\text { Reactor } \\
\text { Materials }\end{array}$ & $\begin{array}{l}\text { Heavy } \\
\text { Water }\end{array}$ & $\begin{array}{c}\text { Savannah } \\
\text { River } \\
\text { Technology } \\
\text { Center/TNX }\end{array}$ & Total \\
\hline \multicolumn{8}{|l|}{ Note: } \\
\hline $\mathrm{H}-3$ (oxide) & $12.3 y$ & 2.97E+03 & $7.83 E+03$ & & $6.28 E+02$ & $8.84 E-01$ & $1.14 E+04$ \\
\hline Co-60 & $5.271 y$ & & & & $2.28 E-03$ & & $2.28 \mathrm{E}-03$ \\
\hline Sr-89,90c & $29.1 \mathrm{y}$ & 1.97E-01 & $1.88 \mathrm{E}-01$ & $1.04 \mathrm{E}-03$ & $1.15 E-02$ & $1.28 E-03$ & $3.98 E-01$ \\
\hline $\mid-129$ & 1.6E7 y & & $9.49 E-03$ & & & & $9.49 E-03$ \\
\hline Cs-137 & $30.2 y$ & $1.76 \mathrm{E}-04$ & $6.55 E-02$ & & & & $6.57 E-02^{d}$ \\
\hline Pm-147 & $2.6 y$ & & $2.63 E-03$ & & & & $2.63 E-03$ \\
\hline U-234 & $2.46 E 5 y$ & & $1.03 E-05$ & $1.17 \mathrm{E}-05$ & $1.63 \mathrm{E}-06$ & $1.24 \mathrm{E}-04$ & $1.48 E-04$ \\
\hline U-235 & 7.04E8 y & & $3.46 E-06$ & $9.37 \mathrm{E}-07$ & $1.19 \mathrm{E}-07$ & $7.29 E-06$ & $1.18 \mathrm{E}-05$ \\
\hline U-238 & 4.47E9 y & & $1.22 E-05$ & $1.98 \mathrm{E}-05$ & $3.88 \mathrm{E}-06$ & $1.33 E-04$ & $1.69 E-04$ \\
\hline Pu-238 & $87.7 y$ & & 2.48E-.06 & $2.86 \mathrm{E}-05$ & $1.63 E-06$ & $7.80 \mathrm{E}-06$ & $4.05 E-05$ \\
\hline Pu-239e & $2.410 E 4 y$ & $4.95 E-03$ & $9.57 E-03$ & $1.05 E-05$ & $4.98 E-04$ & $6.01 E-04$ & $1.56 E-02$ \\
\hline Am-241 & $432.7 y$ & & $8.60 E-07$ & $1.14 \mathrm{E}-06$ & & & $2.00 E-06$ \\
\hline $\mathrm{Cm}-244$ & $18.1 y$ & & $1.11 E-07$ & $3.52 E-06$ & & & $3.63 E-06$ \\
\hline
\end{tabular}

a One curie equals 3.7E+10 Becquerels.

b Includes separations, waste management, and tritium facilities

c Includes unidentified beta-gamma

d For conservatism, the higher release number (2.01E-01 Ci), calculated from River Mile 120 fish concentrations, was used for dose calculations.

e Includes unidentified alpha 
Table 11

\section{Liquid Radioactive Releases by Outfall/Facility and Comparison of Annual Average Radionuclide Concentrations to DOE Derived Concentration Guides}

Page 1 of 5

\begin{tabular}{|c|c|c|c|c|c|}
\hline $\begin{array}{l}\text { Outfall or } \\
\text { Facility }\end{array}$ & Radionuclide & $\begin{array}{c}\text { Quantity of } \\
\text { Radionuclides } \\
\text { Released During } \\
1995(\mathrm{Ci})\end{array}$ & $\begin{array}{c}\text { Average Effluent } \\
\text { Concentration } \\
\text { During 1995 } \\
(\mu \mathrm{Ci} / \mathrm{mL})\end{array}$ & $\begin{array}{l}\text { DOE DCGsa } \\
(\mu \mathrm{Ci} / \mathrm{mL})\end{array}$ & $\begin{array}{c}\text { Fraction of DOE } \\
\text { DCG }\end{array}$ \\
\hline Note: $\quad$ "MDL"C & enotes "minimum & detectable level." & & & \\
\hline \multicolumn{6}{|c|}{ A-Area (Savannah River Technology Center) } \\
\hline \multirow[t]{8}{*}{$T B-2 A-1$} & H-3 (oxide) & $8.84 \mathrm{E}-01$ & $9.32 \mathrm{E}-07$ & $2.00 \mathrm{E}-03$ & 4.66E-04 \\
\hline & U-234 & $1.24 \mathrm{E}-04$ & $1.65 \mathrm{E}-10$ & $6.00 \mathrm{E}-07$ & $2.75 E-04$ \\
\hline & U-235 & $7.29 \mathrm{E}-06$ & $8.29 E-12$ & $6.00 \mathrm{E}-07$ & $1.38 E-05$ \\
\hline & $U-238$ & $1.33 E-04$ & $1.83 E-10$ & $6.00 E-07$ & $3.05 E-04$ \\
\hline & Pu-238 & $7.80 \mathrm{E}-06$ & $1.41 \mathrm{E}-11$ & $4.00 E-08$ & $3.51 E-04$ \\
\hline & Pu-239 & $1.17 \mathrm{E}-06$ & $3.28 \mathrm{E}-13$ & $3.00 \mathrm{E}-08$ & $1.09 E-05$ \\
\hline & $\mathrm{U} / \mathrm{Pu}$ (Pu-239) & $6.65 \mathrm{E}-05$ & 4.06E-10 & $3.00 \mathrm{E}-08$ & $1.35 \mathrm{E}-02$ \\
\hline & & & & Sum of Fractions & $1.49 E-02$ \\
\hline \multicolumn{6}{|c|}{ C-Area (C-Reactor) } \\
\hline \multirow[t]{3}{*}{ C-Canal } & H-3 (oxide) & $2.84 \mathrm{E}+00$ & 4.64E-07 & $2.00 E-03$ & $2.32 E-04$ \\
\hline & Sr-89,90 & Below MDL & 3.95E-10 & $1.00 \mathrm{E}-06$ & 3.95E-04 \\
\hline & & & & Sum of Fractions & $6.27 E-04$ \\
\hline \multicolumn{6}{|c|}{ D-Area (Heavy Water Rework) } \\
\hline \multirow{3}{*}{$\begin{array}{l}\text { 400-D Effluent } \\
\text { Discharge }\end{array}$} & H-3 (oxide) & $6.28 \mathrm{E}+02$ & $5.68 E-05$ & $2.00 E-03$ & $2.84 E-02$ \\
\hline & Co-60 & $2.28 E-03$ & $1.03 E-08$ & $5.00 E-02$ & $2.06 \mathrm{E}-07$ \\
\hline & & & & Sum of Fractions & $2.84 E-02$ \\
\hline \multicolumn{6}{|c|}{ F-Area (Separations and Waste Management) } \\
\hline \multirow[t]{3}{*}{$F-001$} & $H-3$ (oxide) & $9.26 \mathrm{E}-02$ & $3.60 E-07$ & $2.00 E-03$ & $1.80 \mathrm{E}-04$ \\
\hline & Sr-89,90 & Below MDL & $5.45 \mathrm{E}-10$ & $1.00 E-06$ & $5.45 E-04$ \\
\hline & & & & Sum of Fractions & $7.25 \mathrm{E}-04$ \\
\hline \multirow{4}{*}{$\begin{array}{l}F-012(281-8 F \\
\text { Retention Basin) }\end{array}$} & $H-3$ (oxide) & $5.76 \mathrm{E}-01$ & $1.10 E-05$ & $2.00 E-03$ & $5.50 \mathrm{E}-03$ \\
\hline & Sr-89,90 & $7.59 E-04$ & $7.98 \mathrm{E}-0.9$ & $1.00 E-06$ & $7.98 \mathrm{E}-03$ \\
\hline & Cs-137 & $2.01 E-03$ & $2.10 E-08$ & $3.00 E-06$ & $6.99 \mathrm{E}-03$ \\
\hline & & & & Sum of Fractions & $2.05 E-02$ \\
\hline
\end{tabular}

a Source: DOE Order 5400.5. In cases where different chemical forms have different DCGs, the lowest DCG for the radionuclide is given. DCGs are defined as the concentration of that radionuclide that will give a 50 -year committed effective dose equivalent of 100 mrem under conditions of continuous exposure for one year. DCGs are reference values only and are not considered release limits or standards. 
Table 11

Liquid Radioactive Releases by Outfall/Facility and Comparison of Annual Average Radionuclide Concentrations to DOE Derived Concentration Guides

Page 2 of 5

\begin{tabular}{|c|c|c|c|c|c|}
\hline $\begin{array}{l}\text { Outfall or } \\
\text { Facility }\end{array}$ & Radionuclide & $\begin{array}{c}\text { Quantity of } \\
\text { Radionuclides } \\
\text { Released During } \\
1995(\mathrm{Ci})\end{array}$ & $\begin{array}{c}\text { Average Effluent } \\
\text { Concentration } \\
\text { During } 1995 \\
(\mu \mathrm{Ci} / \mathrm{mL})\end{array}$ & $\begin{array}{c}\text { DOE DCGs } \\
(\mu \mathrm{Ci} / \mathrm{mL})\end{array}$ & $\begin{array}{c}\text { Fraction of DOE } \\
\text { DCG }\end{array}$ \\
\hline \multirow{4}{*}{$\begin{array}{l}\text { F-013 (200-F } \\
\text { Cooling Basin) }\end{array}$} & $H-3$ (oxide) & $5.42 E-02$ & $3.62 \mathrm{E}-06$ & $2.00 E-03$ & $1.81 \mathrm{E}-03$ \\
\hline & Sr-89,90 & $6.71 E-03$ & $1.96 \mathrm{E}-07$ & $1.00 \mathrm{E}-06$ & $1.96 \mathrm{E}-01$ \\
\hline & Cs-137 & $1.37 \mathrm{E}-02$ & 3.67E-07 & $3.00 E-06$ & $1.22 E-01$ \\
\hline & & & & Sum of Fractions & $3.20 E-01$ \\
\hline \multirow{5}{*}{$\begin{array}{l}\text { FM-3 (F-Area } \\
\text { Effluent) }\end{array}$} & H-3 (oxide) & $1.77 E+00$ & $1.00 E-06$ & $2.00 E-03$ & $5.01 E-04$ \\
\hline & Sr-89,90 & $2.31 \mathrm{E}-03$ & $2.43 E-09$ & $1.00 \mathrm{E}-06$ & $2.43 \mathrm{E}-03$ \\
\hline & Cs-137 & $2.81 \mathrm{E}-03$ & $2.10 \mathrm{E}-08$ & $3.00 \mathrm{E}-06$ & $7.00 E-03$ \\
\hline & Pm-147 & $2.25 \mathrm{E}-03$ & $2.05 E-10$ & $1.00 E-04$ & $2.05 \mathrm{E}-06$ \\
\hline & & & & Sum of Fractions & $9.94 E-03$ \\
\hline \multirow{12}{*}{$\begin{array}{l}\text { U3R-2 (F Storm } \\
\text { Sewer) }\end{array}$} & H-3 (oxide) & $4.88 E-02$ & $9.50 E-07$ & $2.00 E-03$ & $4.75 E-04$ \\
\hline & Sr-89,90 & $6.00 \mathrm{E}-06$ & $5.04 \mathrm{E}-10$ & $1.00 E-06$ & $5.04 \mathrm{E}-04$ \\
\hline & Pm-147 & $1.85 \mathrm{E}-04$ & $8.23 E-10$ & $1.00 E-04$ & $8.23 E-06$ \\
\hline & $U-234$ & 2.87E-06 & $1.15 E-10$ & $6.00 \mathrm{E}-07$ & $1.91 \mathrm{E}-04$ \\
\hline & U-235 & $2.28 \mathrm{E}-07$ & $1.43 E-11$ & $6.00 E-07$ & $2.38 \mathrm{E}-05$ \\
\hline & U-238 & $9.45 E-06$ & $4.28 \mathrm{E}-10$ & $6.00 \mathrm{E}-07$ & $7.13 E-04$ \\
\hline & Pu-238 & $2.14 \mathrm{E}-06$ & $5.55 \mathrm{E}-11$ & $4.00 E-08$ & $1.39 E-03$ \\
\hline & Pu-239 & $2.97 E-07$ & $2.79 E-11$ & $3.00 E-08$ & $9.30 \mathrm{E}-04$ \\
\hline & $A m-241$ & $9.19 E-08$ & $1.38 \mathrm{E}-12$ & $3.00 E-08$ & $4.61 E-05$ \\
\hline & $\mathrm{Cm}-244$ & $1.11 E-07$ & $1.47 \mathrm{E}-11$ & $6.00 \mathrm{E}-08$ & $2.45 \mathrm{E}-04$ \\
\hline & $\mathrm{U} / \mathrm{Pu}(\mathrm{Pu}-239)$ & $9.06 \mathrm{E}-06$ & $8.48 E-10$ & $3.00 E-08$ & $2.83 \mathrm{E}-02$ \\
\hline & & & & Sum of Fractions & $3.28 \mathrm{E}-02$ \\
\hline \multirow{4}{*}{$\begin{array}{l}\text { U3R-F3 (Naval } \\
\text { Fuels Effluent) }\end{array}$} & $H-3$ (oxide) & $3.81 E-01$ & $3.01 E-06$ & $2.00 E-03$ & $1.50 E-03$ \\
\hline & Sr-89,90 & Below MDL & $2.49 E-10$ & $1.00 E-06$ & $2.49 E-04$ \\
\hline & Pm-147 & Below MDL & $-1.11 E-09$ & $1.00 \mathrm{E}-04$ & $0.00 E+00$ \\
\hline & U-234 & $7.44 E-06$ & $1.30 \mathrm{E}-10$ & $6.00 E-07$ & 2.17E-04 \\
\hline
\end{tabular}

a Source: DOE Order 5400.5. In cases where different chemical forms have different DCGs, the lowest DCG for the radionuclide is given. DCGs are defined as the concentration of that radionuclide that will give a 50-year committed effective dose equivalent of 100 mrem under conditions of continuous exposure for one year. DCGs are reference values only and are not considered release limits or standards. 
Table 11

Liquid Radioactive Releases by Outfall/Facility and Comparison of Annual Average Radionuclide Concentrations to DOE Derived Concentration Guides

Page 3 of 5

\begin{tabular}{|c|c|c|c|c|c|}
\hline $\begin{array}{l}\text { Outfall or } \\
\text { Facility }\end{array}$ & Radionuclide & $\begin{array}{c}\text { Quantity of } \\
\text { Radionuclides } \\
\text { Released During } \\
1995 \text { (Ci) }\end{array}$ & $\begin{array}{l}\text { Average Effluent } \\
\text { Concentration } \\
\text { During } 1995 \\
(\mu \mathrm{Ci} / \mathrm{mL})\end{array}$ & $\begin{array}{l}\text { DOE DCGs } \\
(\mu \mathrm{Ci} / \mathrm{mL})\end{array}$ & $\begin{array}{c}\text { Fraction of DOE } \\
\text { DCG }\end{array}$ \\
\hline & U-235 & $4.21 E-07$ & $5.56 \mathrm{E}-12$ & $6.00 \mathrm{E}-07$ & $9.27 \mathrm{E}-06$ \\
\hline & $U-238$ & $2.76 \mathrm{E}-06$ & $4.86 E-11$ & $6.00 E-07$ & $8.10 E-05$ \\
\hline & Pu-238 & $3.44 \mathrm{E}-07$ & $1.01 E-11$ & $4.00 E-08$ & $2.52 E-04$ \\
\hline & Pu-239 & $7.72 \mathrm{E}-07$ & $9.02 E-12$ & $3.00 E-08$ & $3.01 E-04$ \\
\hline & Am-241 & $7.68 \mathrm{E}-07$ & $-5.14 E-10$ & $3.00 E-08$ & $0.00 E+00$ \\
\hline & $\mathrm{Cm}-244$ & Below MDL & $-1.12 E-10$ & $6.00 \mathrm{E}-08$ & $0.00 E+00$ \\
\hline & $U-235,238$ & $2.81 E-06$ & $2.68 \mathrm{E}-10$ & $6.00 \mathrm{E}-07$ & 4.47E-04 \\
\hline & & & & Sum of Fractions & $3.06 \mathrm{E}-03$ \\
\hline \multicolumn{6}{|c|}{ H-Area (Separations and Waste Management) } \\
\hline \multirow{5}{*}{$\begin{array}{l}\text { FM-1C (H-Area } \\
\text { Effluent) }\end{array}$} & $\mathrm{H}-3$ (oxide) & $5.97 \mathrm{E}+00$ & $1.09 \mathrm{E}-05$ & $2.00 \mathrm{E}-03$ & $5.44 \mathrm{E}-03$ \\
\hline & Sr-89,90 & $5.58 \mathrm{E}-04$ & $1.73 E-09$ & $1.00 \mathrm{E}-06$ & $1.73 E-03$ \\
\hline & Cs-137 & $2.11 E-03$ & $1.08 E-08$ & $3.00 \mathrm{E}-06$ & $3.60 \mathrm{E}-03$ \\
\hline & Pm-147 & $1.16 \mathrm{E}-04$ & $-4.63 E-10$ & $1.00 \mathrm{E}-04$ & $0.00 \mathrm{E}+00$ \\
\hline & & & & Sum of Fractions & $1.08 E-02$ \\
\hline \multirow{4}{*}{$\begin{array}{l}\mathrm{H}-017 \text { (281-8H } \\
\text { Retention Basin) }\end{array}$} & H-3 (oxide) & $1.39 \mathrm{E}+00$ & $1.86 \mathrm{E}-05$ & $2.00 \mathrm{E}-03$ & $9.28 \mathrm{E}-03$ \\
\hline & Sr-89,90 & $6.81 E-04$ & $9.31 E-09$ & $1.00 \mathrm{E}-06$ & $9.31 E-03$ \\
\hline & Cs-137 & $1.76 E-02$ & $1.51 E-07$ & $3.00 E-06$ & $5.03 E-02$ \\
\hline & & & & Sum of Fractions & $6.89 E-02$ \\
\hline \multirow{4}{*}{$\begin{array}{l}\mathrm{H}-018(200-\mathrm{H} \\
\text { Cooling Basin) }\end{array}$} & $\mathrm{H}-3$ (oxide) & $1.99 E-01$ & $2.67 E-05$ & $2.00 E-03$ & $1.33 E-02$ \\
\hline & Sr-89,90 & $6.00 E-04$ & $8.03 E-08$ & $1.00 E-06$ & $8.03 E-02$ \\
\hline & Cs-137 & $1.79 E-03$ & $2.41 E-07$ & $3.00 \mathrm{E}-06$ & $8.03 E-02$ \\
\hline & & & & Sum of Fractions & $1.74 \mathrm{E}-01$ \\
\hline \multirow[t]{2}{*}{ HP-15 (Tritium) } & $H-3$ (oxide) & $3.54 E+00$ & $1.45 \mathrm{E}-05$ & $2.00 E-03$ & $7.25 \mathrm{E}-03$ \\
\hline & & & & Sum of Fractions & $7.25 \mathrm{E}-03$ \\
\hline
\end{tabular}

a Source: DOE Order 5400.5. In cases where different chemical forms have different DCGs, the lowest DCG for the radionuclide is given. DCGs are defined as the concentration of that radionuclide that will give a 50-year committed effective dose equivalent of $100 \mathrm{mrem}$ under conditions of continuous exposure for one year. DCGs are reference values only and are not considered release limits or standards. 


\section{Table 11}

\section{Liquid Radioactive Releases by Outfall/Facility and Comparison of Annual Average Radionuclide Concentrations to DOE Derived Concentration Guides}

Page 4 of 5

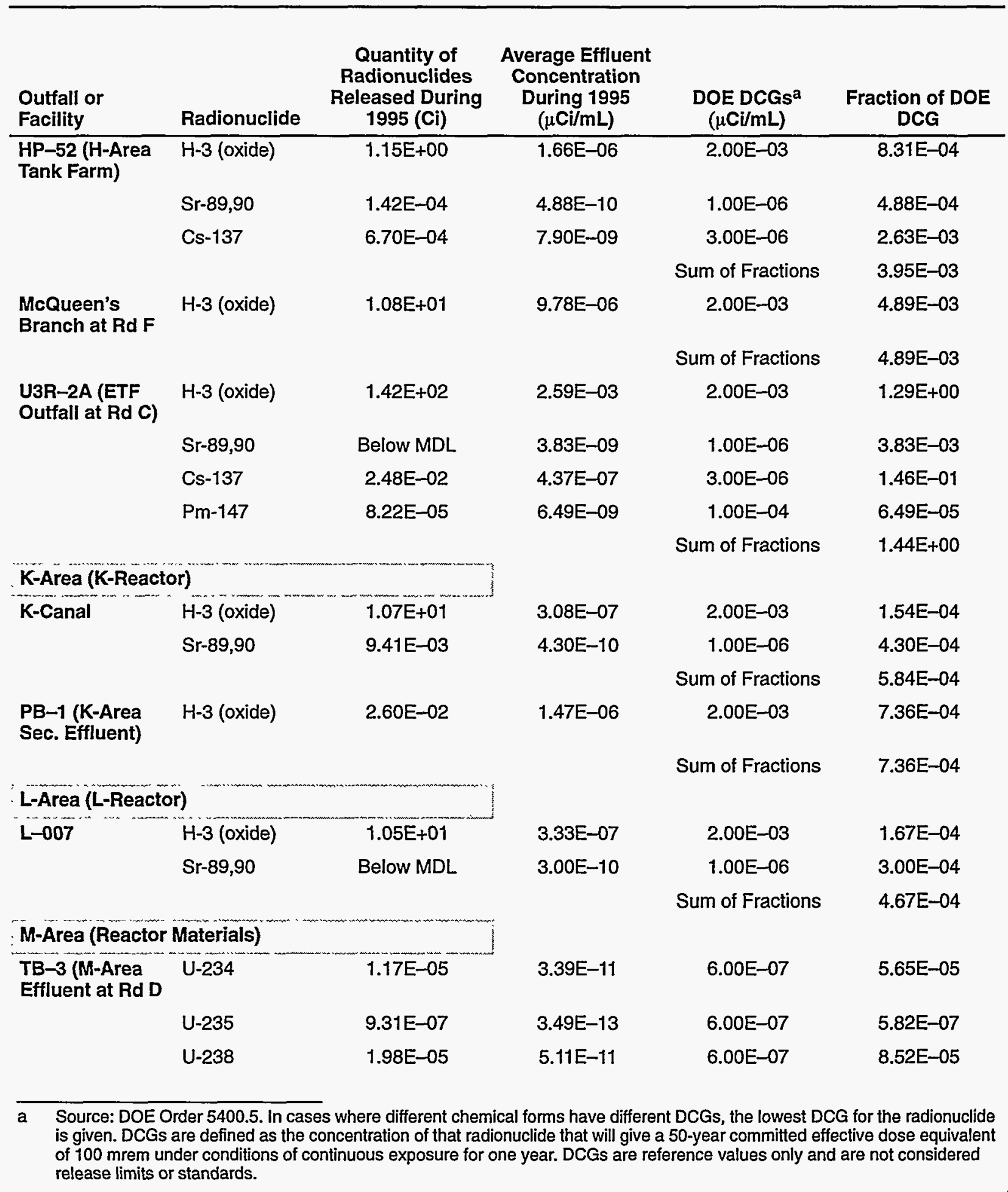


Table 11

Liquid Radioactive Releases by Outfall/Facility and Comparison of Annual Average Radionuclide Concentrations to DOE Derived Concentration Guides

Page 5 of 5

\begin{tabular}{|c|c|c|c|c|c|}
\hline $\begin{array}{l}\text { Outfall or } \\
\text { Facility }\end{array}$ & Radionuclide & $\begin{array}{c}\text { Quantity of } \\
\text { Radionuclides } \\
\text { Released During } \\
1995 \text { (Ci) }\end{array}$ & $\begin{array}{l}\text { Average Effluent } \\
\text { Concentration } \\
\text { During } 1995 \\
(\mu \mathrm{Ci} / \mathrm{mL})\end{array}$ & $\begin{array}{c}\text { DOE DCGsa } \\
(\mu \mathrm{Ci} / \mathrm{mL})\end{array}$ & $\begin{array}{c}\text { Fraction of DOE } \\
\text { DCG }\end{array}$ \\
\hline & Pu-238 & $2.86 \mathrm{E}-05$ & $3.66 \mathrm{E}-11$ & $4.00 E-08$ & $9.15 \mathrm{E}-04$ \\
\hline & Pu-239 & $1.04 \mathrm{E}-05$ & $3.55 \mathrm{E}-11$ & $3.00 \mathrm{E}-08$ & $1.18 E-03$ \\
\hline & Am-241 & $1.14 \mathrm{E}-06$ & $-8.57 E-11$ & $3.00 E-08$ & $0.00 \mathrm{E}+00$ \\
\hline & $\mathrm{Cm}-244$ & $3.52 E-06$ & $-1.23 E-11$ & $6.00 \mathrm{E}-08$ & $0.00 \mathrm{E}+00$ \\
\hline & $U-235,238$ & Below MDL & $1.75 \mathrm{E}-10$ & $6.00 \mathrm{E}-07$ & $2.92 E-04$ \\
\hline & & & & Sum of Fractions & $2.53 E-03$ \\
\hline \multicolumn{6}{|c|}{ P-Area (P-Reactor) } \\
\hline \multirow[t]{4}{*}{ 105-R Sumps } & $\mathrm{H}-3$ (oxide) & $7.24 \mathrm{E}-01$ & $3.14 \mathrm{E}-04$ & $2.00 E-03$ & $1.57 \mathrm{E}-01$ \\
\hline & Sr-89,90 & $1.54 E-04$ & $6.50 E-09$ & $1.00 E-06$ & $6.50 E-03$ \\
\hline & Cs-137 & $1.76 \mathrm{E}-04$ & $9.57 E-08$ & $3.00 E-06$ & $3.19 \mathrm{E}-02$ \\
\hline & & & & Sum of Fractions & $1.95 \mathrm{E}-01$ \\
\hline \multirow[t]{3}{*}{$P-013 A$} & $H-3$ (oxide) & 4.13E+00 & $8.23 \mathrm{E}-07$ & $2.00 \mathrm{E}-03$ & $4.12 E-04$ \\
\hline & Sr-89,90 & $4.02 E-03$ & $2.58 E-10$ & $1.00 \mathrm{E}-06$ & $2.58 E-04$ \\
\hline & & & & Sum of Fractions & $6.69 E-04$ \\
\hline \multirow{3}{*}{$\begin{array}{l}\text { P-019 (P-Area } \\
\text { Canal PAR Pond) }\end{array}$} & $H-3$ (oxide) & $3.89 E+00$ & $6.92 \mathrm{E}-07$ & $2.00 \mathrm{E}-03$ & $3.46 E-04$ \\
\hline & Sr-89,90 & Below MDL & $3.12 \mathrm{E}-10$ & $1.00 \mathrm{E}-06$ & $3.12 E-04$ \\
\hline & & & & Sum of Fractions & $6.58 E-04$ \\
\hline \multicolumn{6}{|l|}{ TNX Area } \\
\hline \multirow[t]{8}{*}{ TNX-008 } & $\mathrm{H}-3$ (oxide) & $2.21 \mathrm{E}-02$ & $7.18 \mathrm{E}-08$ & $2.00 E-03$ & $3.59 E-05$ \\
\hline & U-234 & $1.63 \mathrm{E}-06$ & $1.04 \mathrm{E}-11$ & $6.00 \mathrm{E}-07$ & $1.74 \mathrm{E}-05$ \\
\hline & U-235 & $1.19 \mathrm{E}-07$ & $1.88 \mathrm{E}-12$ & $6.00 \mathrm{E}-07$ & $3.14 \mathrm{E}-06$ \\
\hline & U-238 & $3.88 \mathrm{E}-06$ & $2.46 \mathrm{E}-11$ & $6.00 \mathrm{E}-07$ & $4.10 E-05$ \\
\hline & Pu-238 & $1.63 \mathrm{E}-06$ & $1.35 E-11$ & $4.00 E-08$ & $3.38 E-04$ \\
\hline & Pu-239 & Below MDL & $-8.18 E-13$ & $3.00 E-08$ & $0.00 E+00$ \\
\hline & U/Pu (Pu-239) & Below MDL & 1.29E-10 & $3.00 \mathrm{E}-08$ & $4.29 \mathrm{E}-03$ \\
\hline & & & & Sum of Fractions & 4.73E- -03 \\
\hline
\end{tabular}

a Source: DOE Order 5400.5. In cases where different chemical forms have different DCGs, the lowest DCG for the radionuclide is given. DCGs are defined as the concentration of that radionuclide that will give a 50-year committed effective dose equivalent of 100 mrem under conditions of continuous exposure for one year. DCGs are reference values only and are not considered release limits or standards. 


\section{Table 12}

\section{Calculated Migration of Radioactivity from Seepage Basins}

Page 1 of 2

\begin{tabular}{lllllll}
\hline & & \multicolumn{5}{c}{ Tritium (Curies) } \\
\cline { 4 - 7 } Location & \multicolumn{1}{c}{ Source Description } & 1991 & 1992 & 1993 & 1994 & 1995 \\
\hline FMA7-(FM3A+FM2B) & $\begin{array}{l}\text { 200-F seepage basins to } \\
\text { Four Mile Creek }\end{array}$ & 5,750 & 4,260 & 2,180 & 2880 & 2,370 \\
$\begin{array}{l}\text { FM2B-(FM1C+H017+ } \\
\text { H018+HP52) }\end{array}$ & $\begin{array}{l}\text { 200-H seepage basins to } \\
\text { Four Mile Creek }\end{array}$ & 1,810 & 1,470 & 1,020 & 739 & 528 \\
$\begin{array}{l}\text { FM3A-(FM3+F012+ } \\
\text { F013) }\end{array}$ & $\begin{array}{l}\text { 200-H seepage basin 4 } \\
\text { and Solid Waste Disposal } \\
\text { Facility to Four Mile Creek }\end{array}$ & 6,420 & 4,090 & 5,330 & 3090 & 4,010 \\
K018-K Canal & $\begin{array}{l}\text { K-Area retention basin to } \\
\text { Indian Grave Branch } \\
\text { 100-P seepage basin to } \\
\text { Steel Greek }\end{array}$ & 2,160 & 1,530 & 1,100 & 878 & 1,650 \\
SC2A & 364 & 232 & 382 & 386 & 355
\end{tabular}

Total Strontium (Millicuries)

\begin{tabular}{llrrrrr}
\cline { 3 - 6 } Location & Source Description & 1991 & 1992 & 1993 & 1994 & 1995 \\
\hline FMA7-(FM3A+FM2B) & $\begin{array}{l}\text { 200-F seepage basins to } \\
\text { Four Mile Creek }\end{array}$ & 460 & 194 & 150 & 78 & $\mathrm{~b}$ \\
FM2B-FM1C & $\begin{array}{l}\text { 200-H seepage basins to } \\
\text { Four Mile Creek }\end{array}$ & 72 & 78 & 65 & 35 & $\mathrm{~b}$
\end{tabular}

Cesium-137 (Millicuries)

\begin{tabular}{llrrrrr}
\cline { 2 - 6 } Location & Source Description & 1991 & 1992 & 1993 & 1994 & 1995 \\
\hline $\begin{array}{l}\text { FMA7-(direct } \\
\text { discharges to Four Mile }\end{array}$ & $\begin{array}{l}\text { 200-F seepage basins to } \\
\text { Four Mile Creek }\end{array}$ & $\mathrm{b}$ & $\mathrm{b}$ & $\mathrm{b}$ & 3 & $\mathrm{~b}$
\end{tabular}
Creek)

200-H seepage basins to Four Mile Creek

200-H seepage basin 4 and Solid Waste Disposal Facility to Four Mile Creek

lodine-129 (Millicuries)

\begin{tabular}{llrrrrr}
\cline { 3 - 7 } Location & Source Description & 1991 & 1992 & 1993 & 1994 & 1995 \\
\hline $\begin{array}{l}\text { FMA7-(direct dis- } \\
\text { charges to Four Mile }\end{array}$ & $\begin{array}{l}\text { 200-F seepage basins to } \\
\text { Four Mile Creek }\end{array}$ & 22 & 22 & 22 & 74 & 10
\end{tabular}

200-H seepage basins to Four Mile Creek

200-H seepage basin 4 and Solid Waste Disposal Facility to Four Mile Creek

a Includes some secondary effluent releases from P-Area when water is diverted from PAR Pond.

b Not detected 


\section{Table 12}

Calculated Migration of Radioactivity from Seepage Basins

Page 2 of 2

\begin{tabular}{|c|c|c|c|c|c|c|}
\hline \multirow[b]{2}{*}{ Location } & \multirow[b]{2}{*}{ Source Description } & \multicolumn{5}{|c|}{ Technicium-99 (Millicuries) } \\
\hline & & 1991 & 1992 & 1993 & 1994 & 1995 \\
\hline \multirow{3}{*}{$\begin{array}{l}\text { FMA7-(direct dis- } \\
\text { charges to Four Mile } \\
\text { Creek) }\end{array}$} & $\begin{array}{l}\text { 200-F seepage basins to } \\
\text { Four Mile Creek }\end{array}$ & $\overline{\mathbf{a}}$ & $\bar{a}$ & $\bar{a}$ & $\overline{9}$ & $\bar{b}$ \\
\hline & $\begin{array}{l}200-H \text { seepage basins to } \\
\text { Four Mile Creek }\end{array}$ & & & & & \\
\hline & $\begin{array}{l}200-H \text { seepage basin } 4 \\
\text { and Solid Waste Disposal } \\
\text { Facility to Four Mile Creek }\end{array}$ & & & & & \\
\hline
\end{tabular}

a Not measured

b Not detected 
Table 13

Estimated Tritium Releases in SRS Streams and the Savannah River

Page 1 of 3

\begin{tabular}{|c|c|c|c|c|c|c|}
\hline \multirow[b]{2}{*}{ Area } & \multirow[b]{2}{*}{ Release Point } & \multicolumn{5}{|c|}{ Direct Releases (Curies) } \\
\hline & & 1991 & 1992 & 1993 & 1994 & 1995 \\
\hline \multicolumn{7}{|c|}{ Reactor } \\
\hline \multirow[t]{4}{*}{$100-P$} & $\begin{array}{l}\text { PAR Pond overflow to Lower } \\
\text { Three Runs Creek (L3R2) }\end{array}$ & 221 & 100 & 64 & 59 & 56 \\
\hline & ${ }^{*}$ Process sewer to PAR Pond & $(43)^{a}$ & & & & \\
\hline & $\begin{array}{l}\text { *Reactor heat exchanger cool- } \\
\text { ing water to PAR Pond }\end{array}$ & $(67)^{a}$ & & & & \\
\hline & ${ }^{\star}$ Combined in 1992 (P019) & & $(8)^{a}$ & $(3)^{a}$ & $(2)^{a}$ & $(5)^{a}$ \\
\hline \multirow[t]{4}{*}{$100-L$} & $\begin{array}{l}\text { L-Lake overflow to Steel Creek } \\
\text { (SC4) }\end{array}$ & 723 & 515 & 650 & 473 & 472 \\
\hline & ${ }^{*}$ Process sewer to L Lake & $(11)^{a}$ & & & & \\
\hline & $\begin{array}{l}\text { *Reactor heat exchanger cool- } \\
\text { ing water to L Lake }\end{array}$ & $(112)^{a}$ & & & & \\
\hline & ${ }^{*}$ Combined in 1992 (L007) & & $(58)^{a}$ & $(9)^{a}$ & $(19)^{a}$ & $(11)^{\mathrm{a}}$ \\
\hline \multirow[t]{5}{*}{$100-K$} & *Process sewer to Pen Branch & 74 & & & & \\
\hline & $\begin{array}{l}{ }^{*} \text { Reactor heat exchanger cool- } \\
\text { ing water to Pen Branch }\end{array}$ & $6,470^{\mathrm{b}}$ & & & & \\
\hline & $\begin{array}{l}\text { *Combined in } 1992 \\
\text { (K-Canal) }\end{array}$ & & 126 & 16 & 15 & 11 \\
\hline & (K008) & & 3 & & & \\
\hline & K-Area secondary effluent (PB1) & 6 & 3 & & & \\
\hline \multirow[t]{2}{*}{$100-C$} & $\begin{array}{l}\text { Process Sewer to Four Mile } \\
\text { Creek (C-Canal) }\end{array}$ & 13 & 28 & 12 & 1 & 3 \\
\hline & Subtotal & 7,510 & 775 & 742 & 548 & 542 \\
\hline \multicolumn{7}{|c|}{ Separations } \\
\hline \multirow[t]{2}{*}{$200-F$} & $\begin{array}{l}\text { Effluent to Four Mile Creek } \\
\text { (FM3) }\end{array}$ & 6 & 5 & 4 & 13 & 2 \\
\hline & Effluent to Upper Three Runs & 0 & 1 & 1 & 1 & 1 \\
\hline \multirow[t]{4}{*}{ 200-H } & Effluent to Four Mile Creek & 14 & 13 & 12 & 10 & 9 \\
\hline & $\begin{array}{l}\text { Effluent to Upper Three Runs } \\
\text { (HP15+MQB) }\end{array}$ & 5 & 15 & 17 & 13 & 14 \\
\hline & $\begin{array}{l}\text { Effiluent Treatment Facility } \\
\text { (U3R2A) }\end{array}$ & 3,070 & 1,010 & 395 & 389 & 142 \\
\hline & Subtotal & 3,090 & 1,040 & 426 & 426 & 168 \\
\hline
\end{tabular}

a Not used in totals because release was counted elsewhere

b Includes heat exchanger leak of December 22-25, 1991 
Table 13

Estimated Tritium Releases in SRS Streams and the Savannah River

Page 2 of 3

\begin{tabular}{llrrrrr}
\hline & \multicolumn{6}{c}{ Direct Releases (Curies) } \\
\cline { 3 - 7 } Area & \multicolumn{1}{c}{ Release Point } & 1991 & 1992 & 1993 & 1994 & 1995 \\
\hline $400-D$ & $\begin{array}{l}\text { Process sewer to Beaver Dam } \\
\text { Creek }\end{array}$ & 681 & 576 & 499 & 235 & 628 \\
& Subtotal & 681 & 576 & 499 & 235 & 628 \\
& Total Direct Releases & & & & & \\
& & 11,300 & 2,390 & 1,670 & 1,210 & 1,338
\end{tabular}

\begin{tabular}{|c|c|c|c|c|c|c|}
\hline \multirow[b]{2}{*}{ Area } & \multirow[b]{2}{*}{ Release Point } & \multicolumn{5}{|c|}{ Migration (Curies) } \\
\hline & & 1991 & 1992 & 1993 & 1994 & 1995 \\
\hline \multirow[t]{3}{*}{$\begin{array}{l}200- \\
F \& H\end{array}$} & $\begin{array}{l}\text { Solid Waste Disposal Facility } \\
\text { (FM3A-FM3) and H-Area seep- } \\
\text { age basin to Four Mile Creek }\end{array}$ & 6,420 & 4,090 & 5,330 & 3090 & 4,010 \\
\hline & $\begin{array}{l}200-F \text { seepage basin to Four } \\
\text { Mile Creek }\end{array}$ & 5,750 & 4,260 & 2,180 & 2880 & 2,370 \\
\hline & $\begin{array}{l}200-H \text { seepage basin to Four } \\
\text { Mile Creek }\end{array}$ & 1,810 & 1,470 & 1,020 & 739 & 528 \\
\hline $100-C$ & $\begin{array}{l}\text { Seepage basin to Four Mile } \\
\text { Creek }\end{array}$ & & & & & \\
\hline $100-K$ & $\begin{array}{l}904-88 G \text { to Indian Grave } \\
\text { Branch }\end{array}$ & 2,160 & 1,530 & 1,100 & 878 & $1,650^{\mathrm{b}}$ \\
\hline \multirow[t]{3}{*}{$100-P$} & Seepage basin to Steel Creek & $(364)^{c}$ & $(232)^{c}$ & $(382)^{c}$ & $(386)^{c}$ & $(355)^{c}$ \\
\hline & Subtotal & 16,100 & 11,400 & 9,630 & 7,500 & 8,558 \\
\hline & $\begin{array}{l}\text { Total Direct Releases } \\
\text { and Migration }\end{array}$ & 27,400 & 13,800 & 11,300 & 8,800 & 9,896 \\
\hline
\end{tabular}

Stream Transport (Curies)

\begin{tabular}{llrrrrr} 
Area & \multicolumn{1}{c}{ Release Point } & 1991 & 1992 & 1993 & 1994 & 1995 \\
\hline $400-D$ & Beaver Dam Creek at swamp & 801 & 576 & 499 & 235 & 628 \\
$20-$ & Four Mile Creek at Road A13 & 13,300 & 8,710 & 9,000 & 6,980 & 7,350 \\
F\&H & (FM6) & & & & & \\
$100-K$ & Pen Branch at Road A (PB3) & 7,100 & 1,850 & 1,580 & 1,890 & 2,440 \\
$100-$ L & Steel Creek at Road A (SC4) & 723 & 515 & 650 & 473 & 472 \\
$100-P$ & Lower Three Runs at Road B & 221 & 100 & 64 & 59 & 56 \\
& (L3R2 & & & & & \\
ETF & Upper Three Runs at Road A & 4,410 & 1,300 & 879 & 747 & 483 \\
& (U3R4) & 26,600 & 13,100 & 12,700 & 10,400 & 11,429
\end{tabular}

a Because of rounding, sums of individual columns might not equal totals.

b In 1995, this value was determined from K018-K Canal.

c Not used in totals because release was counted elsewhere 
Table 13

Estimated Tritium Releases in SRS Streams and the Savannah River

Page 3 of 3

\begin{tabular}{|c|c|c|c|c|c|}
\hline & \multicolumn{5}{|c|}{ River Transport (Curies) } \\
\hline & 1991 & 1992 & 1993 & 1994 & 1995 \\
\hline $\begin{array}{l}\text { Tritium measured in the Savannah River } \\
\text { below SRS (RM 120) }\end{array}$ & 28,700 & 16,000 & 12,600 & 12,100 & 12,600 \\
\hline $\begin{array}{l}\text { Tritium measured in the Savannah River } \\
\text { above SRS (RM 160) }\end{array}$ & 2,420 & 2,210 & 433 & 1,170 & 1,940 \\
\hline $\begin{array}{l}\text { Tritium measured in the Savannah River } \\
\text { below SRS (downriver minus upriver)a }\end{array}$ & 26,300 & 13,800 & 12,200 & 10,900 & 10,660 \\
\hline
\end{tabular}

a Because of rounding, differences in individual columns might not equal totals. 


\section{Table 14}

Settleable Solids Results

for the SRS Radionuclides in Settleable Solids Program

Page 1 of 4

\begin{tabular}{|c|c|c|c|c|}
\hline \multirow[b]{2}{*}{ Location } & \multirow[b]{2}{*}{ Date } & \multicolumn{3}{|c|}{ First Quarter } \\
\hline & & $\begin{array}{l}\text { Total Suspended } \\
\text { Solids (mg/L) }\end{array}$ & $\begin{array}{l}\text { Nonsettleable } \\
\text { Solids (mg/L) }\end{array}$ & $\begin{array}{c}\text { Settleable } \\
\text { Solids (mg/L) }\end{array}$ \\
\hline BDC & $2 / 8 / 95$ & 14 & 8 & 6 \\
\hline BDC Rep & $2 / 8 / 95$ & 15 & 8 & 7 \\
\hline C Canal & $2 / 8 / 95$ & 1 & $<1$ & 1 \\
\hline$F-001$ & 2/9/95 & 1 & $<1$ & 1 \\
\hline$F-012$ & $2 / 21 / 95$ & 10 & 9 & 1 \\
\hline F-012 Dup & $2 / 21 / 95$ & 10 & 8 & 2 \\
\hline FMC-1C & $2 / 9 / 95$ & 2 & 1 & 1 \\
\hline FMC-1C Rep & $2 / 9 / 95$ & 2 & 1 & 1 \\
\hline FMC-3 & $2 / 9 / 95$ & 2 & 1 & 1 \\
\hline $\mathrm{H}-017$ & $2 / 21 / 95$ & 4 & $<1$ & 4 \\
\hline H-017 Dup & $2 / 21 / 95$ & 9 & 8 & 1 \\
\hline H-017 Rep & $2 / 21 / 95$ & 10 & 6 & 4 \\
\hline $\mathrm{H}-018$ & $3 / 18 / 95$ & 4 & 3 & 1 \\
\hline H-018 Dup & $3 / 18 / 95$ & 4 & 3 & 1 \\
\hline$H P-15$ & $2 / 9 / 95$ & 2 & $<1$ & 2 \\
\hline HP-52 & 2/9/95 & 3 & 3 & 0 \\
\hline K Canal & 2/8/95 & 3 & 1 & 2 \\
\hline$L-007$ & $2 / 8 / 95$ & 1 & 1 & 0 \\
\hline McQueen's Branch & $2 / 9 / 95$ & 8 & 1 & 7 \\
\hline P-019 & $2 / 8 / 95$ & 1 & 1 & 0 \\
\hline PB-1 & 2/8/95 & 1 & $<1$ & 1 \\
\hline TB-2 & 2/8/95 & $<1$ & $<1$ & $<1$ \\
\hline TB-3 & 2/8/95 & 1 & $<1$ & 1 \\
\hline$T N X-1$ & $2 / 8 / 95$ & 5 & $<1$ & 5 \\
\hline U3R-1A & 2/8/95 & 1 & $<1$ & 1 \\
\hline U3R-2A & $2 / 9 / 95$ & 1 & 1 & 0 \\
\hline U3R-2F & $2 / 9 / 95$ & 1 & $<1$ & 1 \\
\hline U3R-F3 & 2/9/95 & 3 & 1 & 2 \\
\hline
\end{tabular}


Table 14

Settleable Solids Results

for the SRS Radionuclides in Settleable Solids Program

Page 2 of 4

\begin{tabular}{|c|c|c|c|c|}
\hline \multirow[b]{2}{*}{ Location } & \multirow[b]{2}{*}{ Date } & \multicolumn{3}{|c|}{ Second Quarter } \\
\hline & & $\begin{array}{l}\text { Total Suspended } \\
\text { Solids (mg/L) }\end{array}$ & $\begin{array}{l}\text { Nonsettlable } \\
\text { Solids (mg/L) }\end{array}$ & $\begin{array}{c}\text { Settleable } \\
\text { Solids (mg/L) }\end{array}$ \\
\hline $\mathrm{BDC}$ & $6 / 13 / 95$ & 8 & $<1$ & 8 \\
\hline C Canal & $6 / 13 / 95$ & $<1$ & $<1$ & $<1$ \\
\hline$F-001$ & $6 / 13 / 95$ & 2 & 1 & 1 \\
\hline F-001 Rep & $6 / 13 / 95$ & 2 & 1 & 1 \\
\hline$F-012$ & $6 / 13 / 95$ & 12 & 9 & 3 \\
\hline$F-013$ & $6 / 28 / 95$ & 22 & 11 & 11 \\
\hline F-013 Rep & $6 / 28 / 95$ & 23 & 9 & 14 \\
\hline FMC-1C & $6 / 13 / 95$ & 1 & $<1$ & 1 \\
\hline FMC-1C Rep & $6 / 13 / 95$ & 1 & $<1$ & 1 \\
\hline FMC-3 & $6 / 13 / 95$ & $<1$ & $<1$ & $<1$ \\
\hline $\mathrm{H}-017$ & $6 / 26 / 95$ & 12 & 10 & 2 \\
\hline H-017 Rep & $6 / 26 / 95$ & 11 & 10 & 1 \\
\hline $\mathrm{H}-018$ & $6 / 10 / 95$ & $<1$ & $<1$ & $<1$ \\
\hline HP-15 & $6 / 13 / 95$ & $<1$ & $<1$ & $<1$ \\
\hline $\mathrm{HP}-52$ & $6 / 13 / 95$ & 1 & $<1$ & 1 \\
\hline HP-52 Dup & $6 / 13 / 95$ & 1 & $<1$ & 1 \\
\hline K Canal & $6 / 13 / 95$ & 4 & $<1$ & 4 \\
\hline$L-007$ & $6 / 13 / 95$ & 2 & $<1$ & 2 \\
\hline$L-008$ & $6 / 13 / 95$ & $<1$ & $<1$ & $<1$ \\
\hline McQueen's Branch & $6 / 13 / 95$ & 6 & 1 & 5 \\
\hline P-019 & $6 / 13 / 95$ & $<1$ & $<1$ & $<1$ \\
\hline TB-2 & $6 / 13 / 95$ & $<1$ & $<1$ & $<1$ \\
\hline TB-3 & $6 / 13 / 95$ & $<1$ & $<1$ & $<1$ \\
\hline TB-3 Dup & $6 / 13 / 95$ & $<1$ & $<1$ & $<1$ \\
\hline TNX-1 & $6 / 13 / 95$ & 3 & $<1$ & 3 \\
\hline U3R-1A & $6 / 13 / 95$ & 5 & 2 & 3 \\
\hline U3R-2A & $6 / 19 / 95$ & 8 & $<1$ & 8 \\
\hline U3R-2F & $6 / 13 / 95$ & 2 & $<1$ & 2 \\
\hline U3R-2F Rep & $6 / 13 / 95$ & 2 & $<1$ & 2 \\
\hline U3R-F3 & $6 / 13 / 95$ & 6 & 1 & 5 \\
\hline
\end{tabular}




\section{Table 14}

Settleable Solids Results

for the SRS Radionuclides in Settleable Solids Program

Page 3 of 4

\begin{tabular}{|c|c|c|c|c|}
\hline & & & Third Quarter & \\
\hline Location & Date & $\begin{array}{l}\text { Total Suspended } \\
\text { Solids (mg/L) }\end{array}$ & $\begin{array}{l}\text { Nonsettleable } \\
\text { Solids (mg/L) }\end{array}$ & $\begin{array}{c}\text { Settleable } \\
\text { Solids (mg/L) }\end{array}$ \\
\hline $\mathrm{BDC}$ & $8 / 18 / 95$ & 9 & $<1$ & 9 \\
\hline C Canal & $8 / 18 / 95$ & $<1$ & $<1$ & $<1$ \\
\hline$F-001$ & $8 / 22 / 95$ & $<1$ & $<1$ & $<1$ \\
\hline F-001 Rep & $8 / 22 / 95$ & $<1$ & $<1$ & $<1$ \\
\hline$F-012$ & $8 / 18 / 95$ & 1 & 1 & 0 \\
\hline F-012 Rep & $8 / 18 / 95$ & 1 & 1 & 0 \\
\hline FMC $-1 C$ & $8 / 17 / 95$ & $<1$ & $<1$ & $<1$ \\
\hline FMC-3 & $8 / 17 / 95$ & 3 & 1 & 2 \\
\hline $\mathrm{H}-017$ & $7 / 13 / 95$ & 5 & 4 & 1 \\
\hline H-017 Rep & $7 / 13 / 95$ & 5 & 4 & 1 \\
\hline $\mathrm{H}-017$ & $8 / 28 / 95$ & 8 & 7 & 1 \\
\hline H-017 Rep & $8 / 28 / 95$ & 8 & 7 & 1 \\
\hline $\mathrm{H}-017$ & $9 / 25 / 95$ & 5 & $<1$ & 5 \\
\hline $\mathrm{H}-017$ Rep & $9 / 25 / 95$ & 6 & $<1$ & 6 \\
\hline HP-15 & $8 / 17 / 95$ & 1 & $<1$ & 1 \\
\hline HP-52 & $8 / 17 / 95$ & 1 & 1 & 0 \\
\hline HP-52 Rep & $8 / 17 / 95$ & 1 & 1 & 0 \\
\hline K Canal & $8 / 18 / 95$ & 5 & 1 & 4 \\
\hline$K-008$ & $8 / 18 / 95$ & 2 & 1 & 1 \\
\hline$L-007$ & $8 / 22 / 95$ & 1 & $<1$ & 1 \\
\hline L-007 Rep & $8 / 22 / 95$ & 1 & $<1$ & 1 \\
\hline McQueen's Branch & $8 / 17 / 95$ & 3 & 2 & 1 \\
\hline$P-019$ & $8 / 18 / 95$ & 1 & $<1$ & 1 \\
\hline P-019 Rep & $8 / 18 / 95$ & 1 & $<1$ & 1 \\
\hline PB-1 & $8 / 18 / 95$ & $<1$ & $<1$ & $<1$ \\
\hline TB-2 & $8 / 17 / 95$ & $<1$ & $<1$ & $<1$ \\
\hline TB-3 & $8 / 22 / 95$ & $<1$ & $<1$ & $<1$ \\
\hline TB-3 Rep & $8 / 22 / 95$ & $<1$ & $<1$ & $<1$ \\
\hline TNX-1 & $8 / 18 / 95$ & $<1$ & $<1$ & $<1$ \\
\hline U3R-1A & $8 / 17 / 95$ & 3 & 1 & 2 \\
\hline U3R-2A & $8 / 17 / 95$ & 6 & 1 & 5 \\
\hline U3R-2F & $8 / 17 / 95$ & $<1$ & $<1$ & $<1$ \\
\hline U3R-F3 & $8 / 17 / 95$ & $<1$ & $<1$ & $<1$ \\
\hline
\end{tabular}


Table 14

Settleable Solids Results

for the SRS Radionuclides in Settleable Solids Program

Page 4 of 4

\begin{tabular}{|c|c|c|c|c|}
\hline \multirow[b]{2}{*}{ Location } & \multirow[b]{2}{*}{ Date } & \multicolumn{3}{|c|}{ Fourth Quarter } \\
\hline & & $\begin{array}{l}\text { Total Suspended } \\
\text { Solids (mg/L) }\end{array}$ & $\begin{array}{l}\text { Nonsettleable } \\
\text { Solids (mg/L) }\end{array}$ & $\begin{array}{c}\text { Settleable } \\
\text { Solids (mg/L) }\end{array}$ \\
\hline $\mathrm{BDC}$ & $11 / 14 / 95$ & 13 & $<1$ & 13 \\
\hline C Canal & $11 / 14 / 95$ & $<1$ & $<1$ & $<1$ \\
\hline$F-001$ & $11 / 14 / 95$ & $<1$ & $<1$ & $<1$ \\
\hline $\mathrm{F}-012$ & $12 / 12 / 95$ & 3 & 3 & 0 \\
\hline F-012 Rep & $12 / 12 / 95$ & 3 & 3 & 0 \\
\hline$F-013$ & $12 / 9 / 95$ & 2 & 2 & 0 \\
\hline F-013 Rep & $12 / 9 / 95$ & 2 & 2 & 0 \\
\hline FMC-1C & $11 / 14 / 95$ & $<1$ & $<1$ & $<1$ \\
\hline FMC-3 & $11 / 14 / 95$ & 1 & $<1$ & 1 \\
\hline $\mathrm{H}-017$ & $11 / 10 / 95$ & 11 & 9 & 2 \\
\hline $\mathrm{H}-017$ & $12 / 4 / 95$ & 4 & 3 & 1 \\
\hline $\mathrm{H}-017$ & $12 / 8 / 95$ & 8 & 3 & 5 \\
\hline $\mathrm{H}-017$ & $12 / 20 / 95$ & 10 & 8 & 2 \\
\hline $\mathrm{H}-017$ & $11 / 1 / 95$ & 8 & 6 & 2 \\
\hline H-017 Rep & $11 / 10 / 95$ & 10 & 10 & 0 \\
\hline H-017 Rep & $12 / 4 / 95$ & 5 & 3 & 2 \\
\hline H-017 Rep & $12 / 8 / 95$ & 8 & 3 & 5 \\
\hline H-017 Rep & $12 / 20 / 95$ & 10 & 8 & 2 \\
\hline H-017 Rep & $11 / 1 / 95$ & 7 & 6 & 1 \\
\hline $\mathrm{H}-018$ & $11 / 29 / 95$ & 3 & 2 & 1 \\
\hline H-018 Rep & 11/29/95 & 3 & 3 & 0 \\
\hline$H P-15$ & $11 / 14 / 95$ & $<1$ & $<1$ & $<1$ \\
\hline HP-15 Rep & $11 / 14 / 95$ & $<1$ & $<1$ & $<1$ \\
\hline$H P-52$ & $11 / 14 / 95$ & $<1$ & $<1$ & $<1$ \\
\hline K Canal & $11 / 14 / 95$ & 3 & $<1$ & 3 \\
\hline $\mathrm{K}-008$ & $11 / 14 / 95$ & $<1$ & $<1$ & $<1$ \\
\hline K-008 Rep & $11 / 14 / 95$ & $<1$ & $<1$ & $<1$ \\
\hline$L-007$ & $11 / 14 / 95$ & 4 & $<1$ & 4 \\
\hline McQueen's Branch & $11 / 9 / 95$ & 5 & $<1$ & 5 \\
\hline$P-019$ & $11 / 14 / 95$ & $<1$ & $<1$ & $<1$ \\
\hline PB-1 & $11 / 16 / 95$ & $<1$ & $<1$ & $<1$ \\
\hline TB-2 & $11 / 14 / 95$ & $<1$ & $<1$ & $<1$ \\
\hline TB-3 & $11 / 14 / 95$ & $<1$ & $<1$ & $<1$ \\
\hline TNX-1 & $11 / 14 / 95$ & 1 & $<1$ & 1 \\
\hline U3R-1A & $11 / 9 / 95$ & 1 & $<1$ & 1 \\
\hline U3R-2A & $11 / 21 / 95$ & $<1$ & $<1$ & $<1$ \\
\hline
\end{tabular}


Table 15

Radioactivity in Air

Page 1 of 6

\begin{tabular}{|c|c|c|c|c|}
\hline Location & $\begin{array}{l}\text { No. of } \\
\text { Samples }\end{array}$ & Arithmetic Mean $\pm \sigma$ & Maximum $\pm \sigma$ & Minimum $\pm \sigma$ \\
\hline \multicolumn{5}{|l|}{ H-3, pCi/cu m } \\
\hline \multicolumn{5}{|l|}{ On Site } \\
\hline A-Area & 26 & $(2.11 \pm 1.22) E+01$ & $(5.17 \pm 0.96) E+01$ & $(6.67 \pm 2.22) E+00$ \\
\hline Burial Ground North & 26 & $(2.37 \pm 1.84) E+02$ & $(6.56 \pm 0.25) E+02$ & $(5.23 \pm 0.30) E+01$ \\
\hline Burial Ground South & 25 & $(2.57 \pm 1.57) E+02$ & $(5.89 \pm 0.12) E+02$ & $(2.08 \pm 0.08) E-01$ \\
\hline F-Area & 26 & $(6.17 \pm 3.34) E+01$ & $(1.28 \pm 0.09) \mathrm{E}+02$ & $(7.03 \pm 0.68) E-02$ \\
\hline H-Area & 25 & $(0.65 \pm 1.05) E+03$ & $(4.33 \pm 0.06) E+03$ & $(9.74 \pm 0.56) E-02$ \\
\hline \multicolumn{5}{|l|}{ Site Perimeter } \\
\hline Allendale Gate & 24 & $(1.20 \pm 0.66) E+01$ & $(2.52 \pm 1.00) E+01$ & $(0.04 \pm 2.66) E+00$ \\
\hline Barnwell Gate & 26 & $(1.28 \pm 0.53) E+01$ & $(2.27 \pm 0.44) E+01$ & $(2.99 \pm 5.62) E+00$ \\
\hline D-Area & 26 & $(3.18 \pm 1.90) E+01$ & $(9.56 \pm 0.73) E+01$ & $(8.59 \pm 2.09) E+00$ \\
\hline $\begin{array}{l}\text { Darkhorse @ Williston } \\
\text { Gate }\end{array}$ & 25 & $(1.46 \pm 0.78) E+01$ & $(3.48 \pm 0.92) E+01$ & $(3.07 \pm 1.97) \mathrm{E}+00$ \\
\hline East Talatha & 26 & $(1.49 \pm 0.83) E+01$ & $(3.10 \pm 0.70) E+01$ & $(2.27 \pm 4.90) E+00$ \\
\hline Green Pond & 26 & $(1.73 \pm 1.03) E+01$ & $(5.25 \pm 1.07) E+01$ & $(3.82 \pm 1.72) E+00$ \\
\hline $\begin{array}{l}\text { Highway } 125 @ \\
\text { Road A-14 }\end{array}$ & 25 & $(1.64 \pm 0.83) E+01$ & $(4.32 \pm 0.78) E+01$ & $(7.02 \pm 2.74) E+00$ \\
\hline Highway 21/167 & 26 & $(1.55 \pm 1.73) E+01$ & $(9.10 \pm 2.13) E+01$ & $(1.51 \pm 2.44) E+00$ \\
\hline $\begin{array}{l}\text { Highway-39 @ Williston } \\
\text { Gate }\end{array}$ & 26 & $(1.22 \pm 0.66) E+01$ & $(2.62 \pm 0.27) E+01$ & $(-0.50 \pm 4.54) E+00$ \\
\hline Jackson & 26 & $(1.99 \pm 1.53) E+01$ & $(6.83 \pm 0.67) E+01$ & $(5.37 \pm 1.46) E+00$ \\
\hline Patterson Mill Road & 26 & $(1.18 \pm 0.47) E+01$ & $(2.27 \pm 0.92) E+01$ & $(3.09 \pm 5.10) E+00$ \\
\hline Talatha Gate & 25 & $(1.61 \pm 0.89) E+01$ & $(3.79 \pm 0.70) E+01$ & $(1.88 \pm 4.93) E+00$ \\
\hline West Jackson & 26 & $(1.59 \pm 0.90) E+01$ & $(3.82 \pm 0.94) E+01$ & $(0.39 \pm 1.03) E+01$ \\
\hline Windsor Road & 26 & $(1.75 \pm 1.10) E+01$ & $(5.00 \pm 0.43) E+01$ & $(2.13 \pm 2.33) E+00$ \\
\hline \multicolumn{5}{|l|}{ 25-Mile Radius } \\
\hline Aiken Airport & 9 & $(1.14 \pm 0.60) E+01$ & $(1.79 \pm 0.27) E+01$ & $(3.39 \pm 1.87) E+00$ \\
\hline Aiken State Park & 15 & $(1.01 \pm 0.63) E+01$ & $(2.03 \pm 0.66) E+01$ & $(0.17 \pm 6.16) E+00$ \\
\hline Allendale, S.C. & 9 & $(7.74 \pm 4.05) E+00$ & $(1.29 \pm 0.50) E+01$ & $(1.22 \pm 2.12) E+00$ \\
\hline $\begin{array}{l}\text { Augusta Lock and } \\
\text { Dam } 614\end{array}$ & 25 & $(7.75 \pm 8.73) E+00$ & $(4.24 \pm 0.20) E+01$ & $(-2.24 \pm 2.36) E+00$ \\
\hline Highway $301 @$ state line & 23 & $(6.60 \pm 5.55) E+00$ & $(1.65 \pm 0.49) E+01$ & $(-2.80 \pm 3.97) E+00$ \\
\hline Langley, S.C. & 15 & $(1.16 \pm 0.70) E+01$ & $(3.00 \pm 0.84) E+01$ & $(4.25 \pm 1.97) \mathrm{E}+00$ \\
\hline Lees, S.C.' & 15 & $(1.07 \pm 0.78) E+01$ & $(2.83 \pm 0.21) E+01$ & $(-0.52 \pm 1.67) E+00$ \\
\hline Olar, S.C. & 15 & $(9.15 \pm 6.17) E+00$ & $(2.24 \pm 0.18) E+01$ & $(-0.84 \pm 4.39) E+00$ \\
\hline Perkins, Ga. & 15 & $(9.94 \pm 5.60) E+00$ & $(2.03 \pm 0.96) E+01$ & $(2.18 \pm 4.98) E+00$ \\
\hline
\end{tabular}


Table 15

Radioactivity in Air

Page 2 of 6

\begin{tabular}{lcccr} 
Location & $\begin{array}{c}\text { No. of } \\
\text { Samples }\end{array}$ & Arithmetic Mean $\pm \sigma$ & Maximum $\pm \sigma$ & \multicolumn{1}{c}{ Minimum $\pm \sigma$} \\
\hline $\begin{array}{l}\text { South Richmond, Ga. } \\
\text { Springfield, S.C. }\end{array}$ & 15 & $(1.16 \pm 1.09) \mathrm{E}+01$ & $(3.63 \pm 1.74) \mathrm{E}+01$ & $(-2.12 \pm 6.50) \mathrm{E}+00$ \\
$\begin{array}{l}\text { Waynesboro, Ga. } \\
\text { 100-Mile Radius }\end{array}$ & 15 & $(1.20 \pm 0.75) \mathrm{E}+01$ & $(2.61 \pm 0.74) \mathrm{E}+01$ & $(2.27 \pm 7.30) \mathrm{E}+00$ \\
$\quad \begin{array}{l}\text { Columbia, S.C. } \\
\text { Greenville, S.C. }\end{array}$ & 15 & $(1.18 \pm 0.61) \mathrm{E}+01$ & $(2.31 \pm 0.64) \mathrm{E}+01$ & $(3.21 \pm 2.66) \mathrm{E}+00$ \\
$\begin{array}{l}\text { Macon, Ga. } \\
\text { Savannah, Ga. }\end{array}$ & 1 & $(1.34 \pm 0.75) \mathrm{E}+01$ & & \\
\hline Cs-137, pCi/cu m & 2 & $(9.61 \pm 1.42) \mathrm{E}+00$ & $(1.06 \pm 0.66) \mathrm{E}+01$ & $(8.61 \pm 3.18) \mathrm{E}+00$ \\
\hline $\begin{array}{l}\text { On Site } \\
\text { Burial Ground North }\end{array}$ & 9 & $(4.13 \pm 7.19) \mathrm{E}+00$ & $(1.86 \pm 0.43) \mathrm{E}+01$ & $(-3.64 \pm 3.97) \mathrm{E}+00$ \\
$\begin{array}{l}\text { Site Perimeter } \\
\text { Barnwell Gate }\end{array}$ & 1 & $(2.72 \pm 0.95) \mathrm{E}-03$ & & \\
$\quad$ Site perimeter & 1 & $(6.68 \pm 2.13) \mathrm{E}-03$ & & \\
\hline
\end{tabular}

Pu-238, pCi/cu m

On Site

A-Area

Burial Ground North

$(0.22 \pm 1.06) E-06$

$(2.40 \pm 1.05) E-06$

$(-1.64 \pm 1.01) E-06$

Burial Ground South

$(3.72 \pm 5.75) \mathrm{E}-06$

$(2.00 \pm 0.27) \mathrm{E}-05$

$(-1.38 \pm 1.03) \mathrm{E}-06$

F-Area

(3.49 \pm 2.70$) E-06$

$(9.63 \pm 1.84) \mathrm{E}-06$

$(-5.49 \pm 5.49) \mathrm{E}-07$

$\mathrm{H}$-Area

(7.54 \pm 4.55$) \mathrm{E}-06$

$(1.79 \pm 0.14) \mathrm{E}-05$

(2.22 \pm 1.33$) \mathrm{E}-06$

11

$(5.95 \pm 4.25) \mathrm{E}-06$

$(1.43 \pm 0.26) E-05$

$(1.26 \pm 0.79) E-06$

Site Perimeter

Site perimeter

48

$(0.35 \pm 1.27) E-06$

$(7.91 \pm 0.97) E-06$

$(-1.07 \pm 0.47) E-06$

25-Mile Radius

25-mile radius

51

$(0.16 \pm 2.30) E-06$

$(1.20 \pm 0.88) E-05$

$(-5.37 \pm 3.17) E-06$

100-Mile Radius

100-mile radius

52

$(0.18 \pm 2.82) E-06$

$(6.43 \pm 5.26) E-06$

$(-8.73 \pm 4.54) E-06$

Pu-239, pCi/cu m

On Site

A-Area

Burial Ground North

Burial Ground South

F-Area

H-Area
10

11

11

11

11
$(2.45 \pm 6.74) \mathrm{E}-07$

(1.22 \pm 2.12$) E-06$

$(1.74 \pm 3.15) E-06$

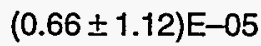

(1.02 \pm 2.92$) E-05$
$(1.53 \pm 1.40) E-06$

$(4.83 \pm 1.34) \mathrm{E}-06$

$(9.92 \pm 3.92) E-06$

(3.98 \pm 0.22$) E-05$

$(9.81 \pm 0.55) E-05$
$(-6.22 \pm 5.49) E-07$

$(-1.33 \pm 0.90) E-06$

$(-3.31 \pm 1.18) E-06$

$(4.20 \pm 5.15) E-07$

$(-0.27 \pm 1.04) E-06$ 


\section{Table 15}

\section{Radioactivity in Air}

Page 3 of 6

\begin{tabular}{|c|c|c|c|c|}
\hline Location & $\begin{array}{c}\text { No. of } \\
\text { Samples }\end{array}$ & Arithmetic Mean $\pm \sigma$ & Maximum $\pm \sigma$ & Minimum $\pm \sigma$ \\
\hline \multicolumn{5}{|l|}{ Site Perimeter } \\
\hline Site perimeter & 48 & $(0.88 \pm 5.05) E-07$ & $(2.12 \pm 1.47) E-06$ & $(-9.40 \pm 9.42) E-07$ \\
\hline \multicolumn{5}{|l|}{ 25-Mile Radius } \\
\hline 25 -mile radius & 51 & $(-0.24 \pm 1.44) E-06$ & $(2.87 \pm 1.73) E-06$ & $(-4.38 \pm 2.20) E-06$ \\
\hline \multicolumn{5}{|l|}{ 100-Mile Radius } \\
\hline 100 -mile radius & 52 & $(-0.96 \pm 4.08) E-06$ & $(1.20 \pm 0.43) E-05$ & $(-1.09 \pm 0.57) E-05$ \\
\hline \multicolumn{5}{|l|}{ Sr-89,90, pCi/cu m } \\
\hline \multicolumn{5}{|l|}{ On Site } \\
\hline A-Area & 12 & $(2.87 \pm 3.11) \mathrm{E}-04$ & $(1.18 \pm 0.25) E-03$ & $(-0.35 \pm 1.96) E-04$ \\
\hline Burial Ground North & 12 & $(0.58 \pm 3.24) E-04$ & $(9.45 \pm 2.39) \mathrm{E}-04$ & $(-3.75 \pm 2.34) E-04$ \\
\hline Burial Ground South & 12 & $(3.27 \pm 5.36) \mathrm{E}-04$ & $(1.83 \pm 0.33) E-03$ & $(-2.20 \pm 3.58) E-04$ \\
\hline F-Area & 12 & $(2.09 \pm 2.89) E-04$ & $(9.74 \pm 2.54) \mathrm{E}-04$ & $(-0.57 \pm 2.38) E-04$ \\
\hline H-Area & 12 & $(3.07 \pm 3.36) E-04$ & $(1.07 \pm 0.24) E-03$ & $(-0.09 \pm 2.06) E-04$ \\
\hline \multicolumn{5}{|l|}{ Site Perimeter } \\
\hline Site perimeter & 52 & $(1.08 \pm 1.57) E-04$ & $(5.78 \pm 1.06) \mathrm{E}-04$ & $(-1.03 \pm 0.91) E-04$ \\
\hline \multicolumn{5}{|l|}{ 25-Mile Radius } \\
\hline 25 -mile radius & 53 & $(0.76 \pm 3.35) E-04$ & $(7.14 \pm 5.47) E-04$ & $(-1.65 \pm 1.14) E-03$ \\
\hline \multicolumn{5}{|l|}{ 100-Mile Radius } \\
\hline 100-mile radius & 53 & $(4.65 \pm 6.07) E-04$ & $(1.79 \pm 0.90) E-03$ & $(-1.15 \pm 0.89) E-03$ \\
\hline \multicolumn{5}{|l|}{ Gross Beta, pCi/cu m } \\
\hline \multicolumn{5}{|l|}{ On Site } \\
\hline A-Area & 53 & $(1.67 \pm 0.45) E-02$ & $(2.87 \pm 0.14) E-02$ & $(7.86 \pm 0.92) E-03$ \\
\hline Burial Ground North & 52 & $(1.89 \pm 1.39) E-02$ & $(1.14 \pm 0.07) E-01$ & $(9.85 \pm 0.59) E-03$ \\
\hline Burial Ground South & 53 & $(1.84 \pm 0.42) E-02$ & $(2.74 \pm 0.14) E-02$ & $(9.55 \pm 0.59) \mathrm{E}-03$ \\
\hline F-Area & 53 & $(1.71 \pm 0.45) E-02$ & $(2.58 \pm 0.13) E-02$ & $(6.86 \pm 0.53) E-03$ \\
\hline H-Area & 52 & $(1.78 \pm 0.45) E-02$ & $(2.90 \pm 0.14) E-02$ & $(5.31 \pm 0.79) E-03$ \\
\hline \multicolumn{5}{|l|}{ Site Perimeter } \\
\hline Allendale Gate & 46 & $(1.97 \pm 0.54) E-02$ & $(3.28 \pm 0.19) E-02$ & $(9.55 \pm 0.59) E-03$ \\
\hline Barnwell Gate & 52 & $(1.87 \pm 0.56) E-02$ & $(2.92 \pm 0.15) E-02$ & $(2.76 \pm 0.19) E-05$ \\
\hline D-Area & 51 & $(1.62 \pm 0.45) E-02$ & $(2.55 \pm 0.14) E-02$ & $(5.52 \pm 0.66) E-03$ \\
\hline $\begin{array}{l}\text { Darkhorse @ Williston } \\
\text { Gate }\end{array}$ & 48 & $(1.97 \pm 0.45) E-02$ & $(2.79 \pm 0.14) E-02$ & $(8.96 \pm 0.57) E-03$ \\
\hline East Talatha & 52 & $(1.92 \pm 0.48) E-02$ & $(3.50 \pm 0.16) E-02$ & $(9.98 \pm 0.60) E-03$ \\
\hline Green Pond & 52 & $(1.80 \pm 0.43) E-02$ & $(2.64 \pm 0.14) E-02$ & $(8.57 \pm 0.95) E-03$ \\
\hline
\end{tabular}




\section{Table 15}

\section{Radioactivity in Air}

Page 4 of 6

\begin{tabular}{|c|c|c|c|c|}
\hline Location & $\begin{array}{l}\text { No. of } \\
\text { Samples }\end{array}$ & Arithmetic Mean $\pm \sigma$ & Maximum $\pm \sigma$ & Minimum $\pm \sigma$ \\
\hline $\begin{array}{l}\text { Highway } 125 @ \\
\text { Road A-14 }\end{array}$ & 52 & $(1.80 \pm 0.48) E-02$ & $(2.94 \pm 0.15) \mathrm{E}-02$ & $(9.07 \pm 0.95) E-03$ \\
\hline Highway $21 / 167$ & 53 & $(2.05 \pm 0.49) E-02$ & $(3.21 \pm 0.30) E-02$ & $(8.76 \pm 0.94) E-03$ \\
\hline $\begin{array}{l}\text { Highway } 39 \text { @ Williston } \\
\text { Gate }\end{array}$ & 52 & $(1.85 \pm 0.45) E-02$ & $(2.88 \pm 0.14) E-02$ & $(9.08 \pm 0.66) E-03$ \\
\hline Jackson & 52 & $(1.45 \pm 0.41) E-02$ & $(2.52 \pm 0.14) \mathrm{E}-02$ & $(5.27 \pm 0.46) E-03$ \\
\hline Patterson Mill Road & 52 & $(1.84 \pm 0.45) E-02$ & $(2.79 \pm 0.14) \mathrm{E}-02$ & $(1.02 \pm 0.10) E-02$ \\
\hline Talatha Gate & 51 & $(1.61 \pm 0.41) E-02$ & $(2.42 \pm 0.14) E-02$ & $(7.84 \pm 0.53) E-03$ \\
\hline West Jackson & 52 & $(1.81 \pm 0.44) \mathrm{E}-02$ & $(2.68 \pm 0.14) \mathrm{E}-02$ & $(8.18 \pm 0.55) E-03$ \\
\hline Windsor Road & 52 & $(1.90 \pm 0.49) E-02$ & $(2.90 \pm 0.15) E-02$ & $(8.29 \pm 0.87) E-03$ \\
\hline \multicolumn{5}{|l|}{ 25-Mile Radius } \\
\hline Aiken Airport & 16 & $(1.67 \pm 0.39) E-02$ & $(2.25 \pm 0.17) E-02$ & $(1.11 \pm 0.10) E-02$ \\
\hline Aiken State Park & 31 & $(1.94 \pm 0.46) E-02$ & $(2.95 \pm 0.15) \mathrm{E}-02$ & $(9.07 \pm 0.95) E-03$ \\
\hline Allendale, S.C. & 19 & $(1.72 \pm 0.45) E-02$ & $(2.77 \pm 0.10) E-02$ & $(9.38 \pm 0.84) E-03$ \\
\hline $\begin{array}{l}\text { Augusta Lock and } \\
\text { Dam } 614\end{array}$ & 52 & $(1.64 \pm 0.51) E-02$ & $(2.70 \pm 0.14) E-02$ & $(4.46 \pm 0.23) E-05$ \\
\hline Highway $301 @$ state line & 45 & $(1.73 \pm 0.49) E-02$ & $(2.64 \pm 0.14) E-02$ & $(4.58 \pm 0.24) E-05$ \\
\hline Langley, S.C. & 31 & $(1.89 \pm 0.46) E-02$ & $(3.29 \pm 0.15) E-02$ & $(9.74 \pm 0.97) E-03$ \\
\hline Lees, S.C. & 31 & $(1.89 \pm 0.49) E-02$ & $(2.95 \pm 0.15) \mathrm{E}-02$ & $(9.43 \pm 0.96) E-03$ \\
\hline Olar, S.C. & 31 & $(1.81 \pm 0.41) E-02$ & $(2.41 \pm 0.14) E-02$ & $(1.06 \pm 0.10) E-02$ \\
\hline Perkins, Ga. & 30 & $(1.74 \pm 0.41) E-02$ & $(2.82 \pm 0.23) \mathrm{E}-02$ & $(1.05 \pm 0.10) E-02$ \\
\hline South Richmond, Ga. & 30 & $(1.91 \pm 0.48) E-02$ & $(3.23 \pm 0.15) E-02$ & $(1.15 \pm 0.10) E-02$ \\
\hline Springfield, S.C. & 31 & $(1.88 \pm 0.46) E-02$ & $(3.14 \pm 0.15) E-02$ & $(1.12 \pm 0.10) E-02$ \\
\hline Waynesboro, Ga. & 31 & $(1.63 \pm 0.34) E-02$ & $(2.50 \pm 0.13) \mathrm{E}-02$ & $(9.74 \pm 0.96) E-03$ \\
\hline \multicolumn{5}{|l|}{ 100-Mile Radius } \\
\hline Columbia, S.C. & 31 & $(1.82 \pm 0.43) E-02$ & $(2.65 \pm 0.14) \mathrm{E}-02$ & $(8.47 \pm 0.94) E-03$ \\
\hline Greenville, S.C. & 20 & $(1.87 \pm 0.53) E-02$ & $(3.22 \pm 0.16) \mathrm{E}-02$ & $(3.55 \pm 0.66) \mathrm{E}-03$ \\
\hline Macon, Ga. & 27 & $(1.87 \pm 0.42) E-02$ & $(2.97 \pm 0.16) E-02$ & $(1.17 \pm 0.11) E-02$ \\
\hline Savannah, Ga. & 50 & $(1.75 \pm 0.55) E-02$ & $(3.06 \pm 0.16) E-02$ & $(8.39 \pm 0.55) E-03$ \\
\hline \multicolumn{5}{|l|}{ Gross Alpha, pCi/cu m } \\
\hline \multicolumn{5}{|l|}{ On Site } \\
\hline A-Area & 53 & $(1.34 \pm 0.58) E-03$ & $(2.47 \pm 0.63) E-03$ & $(0.43 \pm 2.36) E-04$ \\
\hline Burial Ground North & 52 & $(1.73 \pm 1.31) \mathrm{E}-03$ & $(9.95 \pm 2.87) E-03$ & $(4.35 \pm 3.26) E-04$ \\
\hline Burial Ground South & 53 & $(1.43 \pm 0.59) E-03$ & $(2.48 \pm 0.63) E-03$ & $(0.46 \pm 2.34) E-04$ \\
\hline
\end{tabular}


Table 15

Radioactivity in Air

Page 5 of 6

\begin{tabular}{|c|c|c|c|c|}
\hline Location & $\begin{array}{l}\text { No. of } \\
\text { Samples }\end{array}$ & Arithmetic Mean $\pm \sigma$ & Maximum $\pm \sigma$ & Minimum $\pm \sigma$ \\
\hline F-Area & 53 & $(1.58 \pm 0.68) E-03$ & $(3.11 \pm 0.84) \mathrm{E}-03$ & $(4.33 \pm 3.25) \mathrm{E}-04$ \\
\hline H-Area & 52 & $(1.47 \pm 0.60) E-03$ & $(3.41 \pm 0.72) E-03$ & $(1.81 \pm 1.99) E-04$ \\
\hline \multicolumn{5}{|l|}{ Site Perimeter } \\
\hline Allendale Gate & 46 & $(1.52 \pm 0.78) E-03$ & $(4.32 \pm 1.26) E-03$ & $(4.54 \pm 3.31) E-04$ \\
\hline Barnwell Gate & 52 & $(1.38 \pm 0.71) E-03$ & $(3.17 \pm 0.75) E-03$ & $(2.20 \pm 0.82) E-06$ \\
\hline D-Area & 51 & $(1.17 \pm 0.52) E-03$ & $(2.32 \pm 0.61) E-03$ & $(1.61 \pm 2.73) E-04$ \\
\hline $\begin{array}{l}\text { Darkhorse @ Williston } \\
\text { Gate }\end{array}$ & 48 & $(1.58 \pm 0.62) E-03$ & $(2.91 \pm 1.12) E-03$ & $(3.76 \pm 2.35) E-04$ \\
\hline East Talatha & 52 & $(1.53 \pm 0.72) E-03$ & $(4.33 \pm 0.78) E-03$ & $(0.38 \pm 2.27) E-04$ \\
\hline Green Pond & 52 & $(1.55 \pm 0.69) E-03$ & $(3.01 \pm 0.68) E-03$ & $(0.66 \pm 3.07) E-04$ \\
\hline $\begin{array}{l}\text { Highway } 125 @ \\
\text { Road A-14 }\end{array}$ & 52 & $(1.26 \pm 0.57) E-03$ & $(2.72 \pm 0.64) E-03$ & $(1.72 \pm 2.68) E-04$ \\
\hline Highway $21 / 167$ & 53 & $(1.49 \pm 0.54) E-03$ & $(2.55 \pm 0.86) E-03$ & $(0.55 \pm 2.57) E-04$ \\
\hline $\begin{array}{l}\text { Highway } 39 \text { @ Williston } \\
\text { Gate }\end{array}$ & 52 & $(1.27 \pm 0.59) E-03$ & $(3.08 \pm 0.68) E-03$ & $(3.27 \pm 3.22) E-04$ \\
\hline Jackson & 52 & $(1.07 \pm 0.59) E-03$ & $(3.43 \pm 0.70) E-03$ & $(-0.79 \pm 2.16) E-04$ \\
\hline Patterson Mill Road & 52 & $(1.45 \pm 0.62) E-03$ & $(3.06 \pm 0.69) E-03$ & $(1.78 \pm 2.71) E-04$ \\
\hline Talatha Gate & 51 & $(1.19 \pm 0.49) E-03$ & $(2.47 \pm 0.61) E-03$ & $(0.54 \pm 2.54) E-04$ \\
\hline West Jackson & 52 & $(1.48 \pm 0.60) E-03$ & $(2.90 \pm 0.63) E-03$ & $(3.23 \pm 3.18) E-04$ \\
\hline Windsor Road & 52 & $(1.54 \pm 0.63) E-03$ & $(2.77 \pm 0.66) E-03$ & $(1.91 \pm 1.98) E-04$ \\
\hline \multicolumn{5}{|l|}{ 25-Mile Radius } \\
\hline Aiken Airport & 16 & $(1.29 \pm 0.57) E-03$ & $(2.49 \pm 0.58) E-03$ & $(3.28 \pm 3.23) E-04$ \\
\hline Aiken State Park & 31 & $(1.35 \pm 0.65) E-03$ & $(2.74 \pm 0.64) E-03$ & $(1.73 \pm 2.75) E-04$ \\
\hline Allendale, S.C. & 19 & $(1.18 \pm 0.62) E-03$ & $(2.49 \pm 0.43) E-03$ & $(1.88 \pm 2.86) E-04$ \\
\hline $\begin{array}{l}\text { Augusta Lock and } \\
\text { Dam } 614\end{array}$ & 52 & $(1.27 \pm 0.63) E-03$ & $(2.70 \pm 0.63) E-03$ & $(1.89 \pm 0.72) E-06$ \\
\hline Highway $301 @$ state line & 45 & $(1.31 \pm 0.67) E-03$ & $(2.52 \pm 0.59) E-03$ & $(-0.89 \pm 1.95) E-04$ \\
\hline Langley, S.C. & 31 & $(1.39 \pm 0.57) E-03$ & $(2.73 \pm 0.67) E-03$ & $(4.69 \pm 3.42) E-04$ \\
\hline Lees, S.C. & 31 & $(1.39 \pm 0.53) E-03$ & $(2.72 \pm 0.66) E-03$ & $(2.99 \pm 2.97) E-04$ \\
\hline Olar, S.C. & 31 & $(1.30 \pm 0.46) E-03$ & $(2.00 \pm 0.53) E-03$ & $(3.25 \pm 3.20) E-04$ \\
\hline Perkins, Ga. & 30 & $(1.27 \pm 0.66) E-03$ & $(2.97 \pm 0.66) E-03$ & $(1.89 \pm 2.88) E-04$ \\
\hline South Richmond, Ga. & 30 & $(1.39 \pm 0.65) E-03$ & $(2.70 \pm 0.63) E-03$ & $(0.54 \pm 2.52) E-04$ \\
\hline Springfield, S.C. & 31 & $(1.50 \pm 0.80) E-03$ & $(3.63 \pm 0.74) E-03$ & $(0.35 \pm 2.36) \mathrm{E}-04$ \\
\hline Waynesboro, Ga. & 31 & $(1.50 \pm 0.57) E-03$ & $(2.88 \pm 0.67) E-03$ & $(5.77 \pm 3.61) E-04$ \\
\hline
\end{tabular}




\section{Table 15}

Radioactivity in Air

Page 6 of 6

\begin{tabular}{lcccc}
\hline Location & $\begin{array}{c}\text { No. of } \\
\text { Samples }\end{array}$ & Arithmetic Mean $\pm \sigma$ & Maximum $\pm \sigma$ & Minimum $\pm \sigma$ \\
\hline $\begin{array}{c}\text { 100-Mile Radius } \\
\text { Columbia, S.C. }\end{array}$ & 31 & $(1.79 \pm 0.93) \mathrm{E}-03$ & $(4.13 \pm 0.75) \mathrm{E}-03$ & $(3.28 \pm 3.23) \mathrm{E}-04$ \\
Greenville, S.C. & 20 & $(1.52 \pm 0.69) \mathrm{E}-03$ & $(2.64 \pm 0.64) \mathrm{E}-03$ & $(3.23 \pm 2.33) \mathrm{E}-04$ \\
Macon, Ga. & 27 & $(1.55 \pm 0.56) \mathrm{E}-03$ & $(2.94 \pm 0.65) \mathrm{E}-03$ & $(4.58 \pm 3.34) \mathrm{E}-04$ \\
Savannah, Ga. & 50 & $(1.51 \pm 0.60) \mathrm{E}-03$ & $(3.37 \pm 0.74) \mathrm{E}-03$ & $(3.99 \pm 3.42) \mathrm{E}-04$
\end{tabular}




\section{Table 16}

\section{Tritium in Rainwater}

Page 1 of 2

\begin{tabular}{|c|c|c|c|c|}
\hline Location & $\begin{array}{l}\text { No. of } \\
\text { Samples }\end{array}$ & Arithmetic Mean $\pm \sigma$ & Maximum $\pm \sigma$ & Minimum $\pm \sigma$ \\
\hline \multicolumn{5}{|l|}{$\mathrm{H}-3, \mu \mathrm{Ci} / \mathrm{mL}$} \\
\hline \multicolumn{5}{|l|}{ On Site } \\
\hline A-Area & 25 & $(3.61 \pm 5.34) \mathrm{E}-07$ & $(1.70 \pm 0.26) E-06$ & $(-4.45 \pm 3.46) E-07$ \\
\hline Burial Ground North & 25 & $(4.52 \pm 4.95) \mathrm{E}-06$ & $(1.82 \pm 0.06) \mathrm{E}-05$ & $(2.14 \pm 1.29) \mathrm{E}-07$ \\
\hline Burial Ground South & 25 & $(0.84 \pm 1.72) E-05$ & $(8.70 \pm 0.07) E-05$ & $(-1.20 \pm 3.86) E-07$ \\
\hline F-Area & 24 & $(2.55 \pm 2.51) \mathrm{E}-06$ & $(7.33 \pm 0.45) E-06$ & $(-1.61 \pm 3.91) \mathrm{E}-07$ \\
\hline H-Area & 25 & $(3.22 \pm 3.57) E-05$ & $(1.59 \pm 0.02) E-04$ & $(2.61 \pm 0.39) E-06$ \\
\hline \multicolumn{5}{|l|}{ Site Perimeter } \\
\hline Allendale Gate & 25 & $(1.29 \pm 3.35) E-07$ & $(8.42 \pm 3.56) E-07$ & $(-5.47 \pm 3.52) E-07$ \\
\hline Barnwell Gate & 25 & $(4.34 \pm 6.91) \mathrm{E}-07$ & $(2.68 \pm 0.42) E-06$ & $(-4.21 \pm 3.38) E-07$ \\
\hline D-Area & 24 & $(1.39 \pm 1.75) \mathrm{E}-06$ & $(6.12 \pm 0.39) \mathrm{E}-06$ & $(-1.17 \pm 1.83) E-07$ \\
\hline $\begin{array}{l}\text { Darkhorse @ Williston } \\
\text { Gate }\end{array}$ & 24 & $(2.07 \pm 2.63) \mathrm{E}-07$ & $(1.07 \pm 0.35) E-06$ & $(-1.63 \pm 3.40) \mathrm{E}-07$ \\
\hline East Talatha & 25 & $(4.74 \pm 6.49) \mathrm{E}-07$ & $(2.42 \pm 0.19) \mathrm{E}-06$ & $(-2.53 \pm 3.88) E-07$ \\
\hline Green Pond & 25 & $(3.54 \pm 4.15) \mathrm{E}-07$ & $(1.42 \pm 0.36) \mathrm{E}-06$ & $(-2.31 \pm 3.53) \mathrm{E}-07$ \\
\hline Highway $125 @$ Road A-14 & 25 & $(5.55 \pm 6.69) \mathrm{E}-07$ & $(2.13 \pm 0.37) \mathrm{E}-06$ & $(-3.76 \pm 3.71) \mathrm{E}-07$ \\
\hline Highway $21 / 167$ & 25 & $(2.44 \pm 3.34) \mathrm{E}-07$ & $(9.60 \pm 4.09) \mathrm{E}-07$ & $(-3.13 \pm 2.87) E-07$ \\
\hline $\begin{array}{l}\text { Highway } 39 @ \text { Williston } \\
\text { Gate }\end{array}$ & 25 & $(2.35 \pm 3.92) \mathrm{E}-07$ & $(1.07 \pm 0.39) E-06$ & $(-3.86 \pm 3.60) E-07$ \\
\hline Jackson & 25 & $(4.24 \pm 5.52) E-07$ & $(1.82 \pm 0.13) E-06$ & $(-4.06 \pm 3.44) E-07$ \\
\hline Patterson Mill Road & 24 & $(1.87 \pm 3.55) \mathrm{E}-07$ & $(9.59 \pm 3.58)$ E- 07 & $(-4.39 \pm 3.34) \mathrm{E}-07$ \\
\hline Talatha Gate & 25 & $(3.37 \pm 4.75) \mathrm{E}-07$ & $(1.50 \pm 0.39) E-06$ & $(-2.72 \pm 3.31) \mathrm{E}-07$ \\
\hline West Jackson & 25 & $(3.96 \pm 4.53) \mathrm{E}-07$ & $(1.66 \pm 0.38) E-06$ & $(-2.65 \pm 3.44) E-07$ \\
\hline Windsor Road & 25 & $(4.29 \pm 5.61) \mathrm{E}-07$ & $(2.28 \pm 0.40) E-06$ & $(-4.56 \pm 2.84) E-07$ \\
\hline \multicolumn{5}{|l|}{ 25-Mile Radius } \\
\hline Aiken Airport & 8 & $(3.56 \pm 6.26) \mathrm{E}-07$ & $(1.66 \pm 0.32) E-06$ & $(-4.28 \pm 2.72) E-07$ \\
\hline Aiken State Park & 15 & $(1.74 \pm 3.58) E-07$ & $(9.80 \pm 3.92) E-07$ & $(-3.03 \pm 3.90) E-07$ \\
\hline Allendale, S.C. & 8 & $(3.99 \pm 4.37) \mathrm{E}-07$ & $(1.03 \pm 0.39) E-06$ & $(-1.43 \pm 2.81) E-07$ \\
\hline $\begin{array}{l}\text { Augusta Lock and Dam } \\
614\end{array}$ & 23 & $(2.11 \pm 5.84) \mathrm{E}-07$ & $(2.49 \pm 0.39) E-06$ & $(-4.51 \pm 3.66) \mathrm{E}-07$ \\
\hline Highway $301 @$ state line & 21 & $(0.90 \pm 2.65) \mathrm{E}-07$ & $(5.04 \pm 3.47)$ E- -07 & $(-5.23 \pm 3.52) E-07$ \\
\hline Langley, S.C. & 14 & $(1.14 \pm 3.85) \mathrm{E}-07$ & $(7.23 \pm 3.80) E-07$ & $(-8.30 \pm 3.76) E-07$ \\
\hline Lees, S.C. & 13 & $(2.59 \pm 4.54) \mathrm{E}-07$ & $(1.16 \pm 0.36) E-06$ & $(-5.66 \pm 3.82) E-07$ \\
\hline Olar, S.C. & 14 & $(1.42 \pm 3.49) \mathrm{E}-07$ & $(7.14 \pm 3.82)$ E- 07 & $(-4.14 \pm 3.53) \mathrm{E}-07$ \\
\hline Perkins, Ga. & 15 & $(1.16 \pm 4.67) E-07$ & $(1.02 \pm 0.37) E-06$ & $(-6.90 \pm 3.55) E-07$ \\
\hline
\end{tabular}


Table 16

Tritium in Rainwater

Page 2 of 2

\begin{tabular}{lcccc}
\hline & $\begin{array}{c}\text { No. of } \\
\text { Samples }\end{array}$ & Arithmetic Mean $\pm \sigma$ & Maximum $\pm \sigma$ & Minimum $\pm \sigma$ \\
\hline Socation & 15 & $(0.54 \pm 3.99) E-07$ & $(1.06 \pm 0.39) E-06$ & $(-6.29 \pm 3.78) E-07$ \\
Springfield, S.C. & 13 & $(2.40 \pm 3.32) E-07$ & $(8.80 \pm 3.55) E-07$ & $(-2.83 \pm 3.56) E-07$ \\
Waynesboro, Ga. & 15 & $(4.76 \pm 6.36) E-07$ & $(2.11 \pm 0.40) E-06$ & $(-2.68 \pm 3.37) E-07$ \\
100-Mile Radius & 2 & $(1.89 \pm 7.04) E-07$ & $(6.87 \pm 2.99) E-07$ & $(-3.09 \pm 3.57) E-07$ \\
Columbia, S.C. & 1 & $(1.20 \pm 3.75) E-07$ & & \\
Greenville, S.C. & 2 & $(4.68 \pm 0.18) E-07$ & $(4.81 \pm 3.81) E-07$ & $(4.56 \pm 2.96) E-07$ \\
Macon, Ga. & 9 & $(1.29 \pm 3.43) E-07$ & $(7.40 \pm 3.75) E-07$ & $(-3.79 \pm 3.70) E-07-$
\end{tabular}




\section{Table 17}

\section{Radioactivity in Rain lon Columns}

Page 1 of 3

\begin{tabular}{|c|c|c|c|c|}
\hline Location & $\begin{array}{l}\text { No. of } \\
\text { Samples }\end{array}$ & Arithmetic Mean $\pm \sigma$ & Maximum $\pm \sigma$ & Minimum $\pm \sigma$ \\
\hline \multicolumn{5}{|l|}{ Cs-137, pCi/sq m } \\
\hline \multicolumn{5}{|l|}{ Site Perimeter } \\
\hline Barnwell Gate & 1 & $(9.83 \pm 8.14) E+00$ & & \\
\hline \multicolumn{5}{|l|}{ Pu-238, pCi/sq m } \\
\hline \multicolumn{5}{|l|}{ On Site } \\
\hline H-Area & 4 & $(2.64 \pm 4.04) \mathrm{E}-02$ & $(6.78 \pm 1.81) \mathrm{E}-02$ & $(-2.82 \pm 2.22) E-02$ \\
\hline \multicolumn{5}{|l|}{ Site Perimeter } \\
\hline Barnwell Gate & 4 & $(-0.23 \pm 2.02) E-02$ & $(2.40 \pm 3.56) \mathrm{E}-02$ & $(-2.42 \pm 0.99) \mathrm{E}-02$ \\
\hline $\begin{array}{l}\text { Darkhorse @ Williston } \\
\text { Gate }\end{array}$ & 4 & $(0.25 \pm 2.03) E-02$ & $(3.28 \pm 1.41) \mathrm{E}-02$ & $(-0.96 \pm 2.71) E-02$ \\
\hline \multicolumn{5}{|l|}{ 25-Mile Radius } \\
\hline Olar, S.C. & 3 & $(1.02 \pm 1.15) E-02$ & $(2.26 \pm 2.26) \mathrm{E}-02$ & $(00.00 \pm 1.29) E-02$ \\
\hline \multicolumn{5}{|l|}{ 100-Mile Radius } \\
\hline Columbia, S.C & 3 & $(1.37 \pm 0.72) \mathrm{E}-02$ & $(1.78 \pm 5.38) \mathrm{E}-02$ & $(0.53 \pm 1.41) \mathrm{E}-02$ \\
\hline Greenville, S.C. & 1 & $(1.44 \pm 1.44) \mathrm{E}-02$ & & \\
\hline Macon, Ga. & 2 & $(7.99 \pm 5.63) \mathrm{E}-08$ & $(0.00 \pm 1.01) \mathrm{E}-02$ & $(0.00 \pm 8.49) E-03$ \\
\hline Savannah, Ga. & 3 & $(-0.78 \pm 1.36) E-02$ & $(0.00 \pm 2.02) E-02$ & $(-2.35 \pm 1.41) \mathrm{E}-02$ \\
\hline \multicolumn{5}{|l|}{ Pu-239, pCi/sq m } \\
\hline \multicolumn{5}{|l|}{ On Site } \\
\hline H-Area & 4 & $(1.53 \pm 2.10) \mathrm{E}-02$. & $(3.80 \pm 1.64)-02$ & $(-0.50 \pm 3.92) E-02$ \\
\hline \multicolumn{5}{|l|}{ Site Perimeter } \\
\hline Barnwell Gate & 4 & $(-0.52 \pm 2.28) E-02$ & $(1.35 \pm 1.49) \mathrm{E}-02$ & $(-3.83 \pm 1.38) E-02$ \\
\hline $\begin{array}{l}\text { Darkhorse @ Williston } \\
\text { Gate }\end{array}$ & 4 & $(-0.52 \pm 1.47) \mathrm{E}-02$ & $(0.82 \pm 2.18) \mathrm{E}-02$ & $(-2.40 \pm 1.08) E-02$ \\
\hline \multicolumn{5}{|l|}{ 25-Mile Radius } \\
\hline Olar; S.C. & 3 & $(-1.57 \pm 1.38) E-02$ & $(-0.75 \pm 1.68) E-02$ & $(-3.16 \pm 1.83) \mathrm{E}-02$ \\
\hline \multicolumn{5}{|l|}{ 100-Mile Radius } \\
\hline Columbia, S.C. & 3 & $(-0.30 \pm 2.56) E-02$ & $(2.65 \pm 1.40) \mathrm{E}-02$ & $(-1.78 \pm 6.92) E-02$ \\
\hline Greenville, S.C. & 1 & $(1.01 \pm 0.38) E-01$ & & \\
\hline Macon, Ga. & 2 & $(-3.56 \pm 5.04) E-03$ & $(0.00 \pm 4.30) E-01$ & $(-0.71 \pm 1.24) E-02$ \\
\hline Savannah, Ga. & 3 & $(-0.64 \pm 1.36) E-02$ & $(0.94 \pm 1.33) \mathrm{E}-02$ & $(-1.42 \pm 2.01) \mathrm{E}-02$ \\
\hline \multicolumn{5}{|l|}{ Sr-89,90, pci/sq m } \\
\hline \multicolumn{5}{|l|}{ On Site } \\
\hline H-Area & 4 & $(0.98 \pm 1.00) E+01$ & $(2.18 \pm 0.33) E+01$ & $(-0.58 \pm 3.78) E+00$ \\
\hline
\end{tabular}


Table 17

Radioactivity in Rain lon Columns

Page 2 of 3

\begin{tabular}{|c|c|c|c|c|}
\hline Location & $\begin{array}{l}\text { No. of } \\
\text { Samples }\end{array}$ & Arithmetic Mean $\pm \sigma$ & Maximum $\pm \sigma$ & Minimum $\pm \sigma$ \\
\hline \multicolumn{5}{|l|}{ Site Perimeter } \\
\hline Barnwell Gate & 4 & $(4.16 \pm 4.54) E+00$ & $(1.06 \pm 0.32) E+01$ & $(0.70 \pm 4.53) E+00$ \\
\hline Dakhorse @ Williston Gate & 4 & $(5.53 \pm 4.40) E+00$ & $(1.19 \pm 0.40) E+01$ & $(1.77 \pm 4.13) E+00$ \\
\hline \multicolumn{5}{|l|}{ 25-Mile Radius } \\
\hline Olar, S.C. & 3 & $(5.51 \pm 7.22) E+00$ & $(1.21 \pm 0.41) \mathrm{E}+01$ & $(-2.22 \pm 4.35) E+00$ \\
\hline \multicolumn{5}{|l|}{ 100-Mile Radius } \\
\hline Columbia, S.C. & 3 & $(1.29 \pm 0.00) E+01$ & $(1.29 \pm 0.79) E+01$ & $(1.29 \pm 0.38) E+01$ \\
\hline Greenville, S.C. & 1 & $(1.17 \pm 0.33) E+01$ & & \\
\hline Macon, Ga. & 2 & $(1.06 \pm 0.45) E+01$ & $(1.38 \pm 0.39) E+01$ & $(7.46 \pm 5.27) E+00$ \\
\hline Savannah, Ga. & 3 & $(1.01 \pm 1.20) E+01$ & $(1.70 \pm 0.42) E+01$ & $(-3.70 \pm 3.80) E+00$ \\
\hline Gross Beta & & $\mathrm{pCi} / \mathrm{sq} \mathrm{m}$ per yeara & $\mathrm{pCi} / \mathrm{sq} \mathrm{m}$ & $\mathrm{pCi} / \mathrm{sq} \mathrm{m}$ \\
\hline \multicolumn{5}{|l|}{ On Site } \\
\hline H-Area & 13 & $(6.27 \pm 7.04) E+01$ & $(2.28 \pm 0.17) E+02$ & $(-2.27 \pm 7.79) E+00$ \\
\hline \multicolumn{5}{|l|}{ Site Perimeter } \\
\hline Barnwell Gate & 11 & $(2.60 \pm 2.29) E+01$ & $(6.43 \pm 0.74) E+01$ & $(3.69 \pm 5.30) E+00$ \\
\hline $\begin{array}{l}\text { Darkhorse @ Williston } \\
\text { Gate }\end{array}$ & 11 & $(5.93 \pm 5.64) E+01$ & $(1.62 \pm 0.12) E+02$ & $(0.94 \pm 3.75) E+00$ \\
\hline \multicolumn{5}{|l|}{ 25-Mile Radius } \\
\hline Olar, S.C. & 8 & $(5.61 \pm 3.76) E+01$ & $(1.26 \pm 0.10) E+02$ & $(2.03 \pm 0.57) E+01$ \\
\hline \multicolumn{5}{|l|}{ 100-Mile Radius } \\
\hline Columbia, S.C. & 3 & $(2.56 \pm 0.56) E+02$ & $(3.21 \pm 0.15) E+02$ & $(2.24 \pm 0.13) E+02$ \\
\hline Greenville, S.C. & 1 & $(2.08 \pm 0.14) E+02$ & & \\
\hline Macon, Ga. & 2 & $(1.43 \pm 1.11) E+02$ & $(2.22 \pm 0.17) E+02$ & $(6.50 \pm 1.25) E+01$ \\
\hline Savannah,Ga. & 7 & $(1.12 \pm 1.13) E+02$ & $(3.29 \pm 0.14) E+02$ & $(3.29 \pm 3.83) E+00$ \\
\hline Gross Alpha & & pCi/sq m per yeara & pCi/sq m & pCi/sq m \\
\hline \multicolumn{5}{|l|}{ On Site } \\
\hline H-Area & 13 & $(1.22 \pm 2.60) E+00$ & $(6.57 \pm 5.34) E+00$ & $(-2.25 \pm 1.77) E+00$ \\
\hline \multicolumn{5}{|l|}{ Site Perimeter } \\
\hline Barnwell Gate & 11 & $(-3.81 \pm 6.00) E-01$ & $(0.81 \pm 1.85) E+00$ & $(-0.96 \pm 1.66) E+00$ \\
\hline $\begin{array}{l}\text { Darkhorse @ Williston } \\
\text { Gate }\end{array}$ & 11 & $(1.17 \pm 2.74) E+00$ & $(6.43 \pm 4.92) E+00$ & $(-1.92 \pm 1.36) E+00$ \\
\hline 25-Mile Radius & & • & & \\
\hline Olar, S.C. & 8 & $(2.13 \pm 3.25) \mathrm{E}+00$ & $(7.48 \pm 3.16) E+00$ & $(-2.28 \pm 1.81) E+00$ \\
\hline
\end{tabular}

a The mean concentrations for gross beta and gross alpha have been normalized to $\mathrm{pCi} / \mathrm{sq} \mathrm{m}$ per year to allow comparison between concentrations collected at different intervals. 


\section{Table 17}

Radioactivity in Rain lon Columns

Page 3 of 3

\begin{tabular}{lcccr}
\hline Location & $\begin{array}{c}\text { No. of } \\
\text { Samples }\end{array}$ & Arithmetic Mean $\pm \sigma$ & Maximum $\pm \sigma$ & Minimum $\pm \sigma$ \\
\hline 100-Mile Radius & 3 & $(1.13 \pm 0.90) \mathrm{E}+01$ & $(2.17 \pm 0.78) \mathrm{E}+01$ & $(6.17 \pm 4.59) \mathrm{E}+00$ \\
Columbia, S.C. & 1 & $(3.58 \pm 4.56) \mathrm{E}+00$ & & \\
Greenville, S.C. & 2 & $(3.58 \pm 3.56) \mathrm{E}+00$ & $(6.09 \pm 4.43) \mathrm{E}+00$ & $(1.06 \pm 5.02) \mathrm{E}+00$ \\
Macon, Ga. & 7 & $(2.40 \pm 6.34) \mathrm{E}+00$ & $(1.64 \pm 0.78) \mathrm{E}+01$ & $(-2.02 \pm 1.43) \mathrm{E}+00$ \\
Savannah, Ga. & & & &
\end{tabular}


Table 18

Thermoluminescent Dosimeter (TLD) Results:

SRS Areas

Page 1 of 4

Quarterly Exposure in $\mathrm{mR} / \mathrm{Day}$

Yearly Exposure in mR/Yeara

Note: Blank spaces indicate missing TLD results.

\begin{tabular}{lllllr}
\hline 643-26E 1 & 0.29 & 0.3 & 0.34 & 0.33 & 114.33 \\
643-26E 2 & 0.23 & 0.22 & 0.25 & 0.25 & 86.33 \\
643-7G 1 & 0.27 & 0.25 & 0.29 & 0.29 & 100.19 \\
643-7G 2 & 0.28 & 0.25 & 0.29 & 0.29 & 102.06 \\
643-7G 3 & 0.32 & 0.32 & 0.36 & 0.36 & 123.87 \\
643-7G 4 & 0.65 & 0.63 & 0.71 & 0.69 & 243.64 \\
643-G 1 & 0.24 & 0.22 & 0.25 & 0.25 & 88.17 \\
643-G 2 & 0.29 & 0.29 & 0.32 & 0.31 & 110.45 \\
643-G 3 & 0.88 & 0.59 & 0.68 & 0.5 & 240.88 \\
643-G 4 & 0.3 & 0.28 & 0.32 & 0.33 & 112.15 \\
B 1 & 0.76 & 0.55 & 0.62 & & 233.15 \\
B 2 & 0.21 & 0.2 & 0.23 & 0.22 & 78.45 \\
B 3 & 0.22 & 0.2 & 0.23 & 0.24 & 81.13 \\
B 4 & 0.21 & 0.21 & 0.23 & 0.22 & 79.22 \\
C 1 & 0.22 & 0.2 & 0.21 & 0.22 & 78.61 \\
C 2 & 0.21 & 0.2 & 0.21 & 0.21 & 76.59 \\
C 3 & 0.22 & 0.21 & 0.21 & 0.23 & 80.22 \\
C 4 & 0.21 & 0.2 & 0.21 & 0.21 & 76.8 \\
CY 1 & 0.21 & 0.19 & 0.2 & 0.21 & 75.54 \\
CY 2 & 0.22 & 0.21 & 0.21 & 0.23 & 79.76 \\
CY 3 & 0.23 & 0.23 & 0.23 & 0.24 & 86.21 \\
CY 4 & 0.19 & 0.18 & 0.18 & 0.19 & 69.38 \\
D 1 & 0.22 & 0.2 & 0.21 & 0.21 & 77.9 \\
D 2 & 0.21 & 0.2 & 0.21 & 0.22 & 77.73 \\
D 3 & 0.23 & 0.22 & 0.23 & 0.23 & 85.07 \\
D 4 & 0.22 & 0.21 & 0.22 & 0.23 & 82.34
\end{tabular}

\footnotetext{
a $\mathrm{mR}=$ milliRoentgen, exposure unit for gamma radiation. One $\mathrm{mR}$ is approximately equal to $1 \mathrm{mrem}$. Although the TLD is an integrating device, the derived unit $\mathrm{mR} /$ day is used to facilitate data comparison between locations or through time at a single location.

b An $18 \%$ uncertainty is associated with each result.
} 


\section{Table 18}

\section{Thermoluminescent Dosimeter (TLD) Results: \\ SRS Areas}

Page 2 of 4

\begin{tabular}{|c|c|c|c|c|c|}
\hline \multicolumn{6}{|c|}{$\begin{array}{l}\text { Quarterly Exposure in mR/Day } \\
\text { Yearly Exposure in mR/Yeara }\end{array}$} \\
\hline Location & Quarter $1^{b}$ & Quarter $2^{b}$ & Quarter $3^{b}$ & Quarter $4^{b}$ & mR/Yearb \\
\hline$\overline{D 5}$ & 0.19 & 0.19 & 0.19 & 0.19 & 71.05 \\
\hline D 6 & 0.17 & 0.16 & 0.17 & 0.18 & 62.61 \\
\hline$F 1$ & 0.24 & 0.22 & 0.24 & 0.27 & 88.44 \\
\hline $\mathrm{F} 2$ & 0.25 & 0.25 & 0.28 & 0.28 & 95.96 \\
\hline F 3 & 0.26 & 0.25 & 0.28 & 0.28 & 96.72 \\
\hline $\mathrm{F} 4$ & 0.26 & 0.26 & 0.29 & 0.29 & 99.82 \\
\hline F 5 & 0.4 & 0.36 & 0.4 & 0.39 & 140.54 \\
\hline F 6 & 0.27 & 0.27 & 0.3 & 0.29 & 103.49 \\
\hline$H 1$ & 0.25 & 0.24 & 0.27 & 0.27 & 93.47 \\
\hline $\mathrm{H} 2$ & 0.26 & 0.25 & 0.28 & 0.27 & 97.18 \\
\hline H 3 & 0.26 & 0.25 & 0.28 & 0.29 & 99.17 \\
\hline $\mathrm{H} 4$ & 0.41 & 0.39 & 0.44 & 0.41 & 149.44 \\
\hline $\mathrm{H} 5$ & 0.39 & 0.37 & 0.42 & 0.44 & 147.03 \\
\hline H6 & 0.27 & 0.26 & 0.29 & 0.28 & 99.81 \\
\hline $\mathrm{H} 7$ & 0.26 & 0.24 & 0.28 & & 93.76 \\
\hline H 8 & 0.26 & 0.26 & 0.29 & 0.28 & 99.35 \\
\hline $\mathrm{K} 1$ & 0.21 & 0.19 & 0.2 & 0.21 & 75.85 \\
\hline $\mathrm{K} 2$ & 0.19 & 0.18 & 0.19 & 0.2 & 69.64 \\
\hline K3 & 0.26 & 0.24 & 0.26 & 0.24 & 93.02 \\
\hline K 4 & 0.21 & $0.2^{\prime}$ & 0.21 & 0.22 & 77.5 \\
\hline L 1 & 0.22 & 0.21 & 0.22 & 0.22 & 79.9 \\
\hline L2 & 0.21 & 0.2 & 0.2 & 0.21 & 76.29 \\
\hline L3 & 0.21 & 0.2 & 0.21 & 0.21 & 77.38 \\
\hline L 4 & 0.23 & 0.22 & 0.23 & 0.25 & 86.97 \\
\hline M1 & 0.28 & 0.27 & 0.3 & 0.3 & 104.7 \\
\hline$M 2$ & 0.21 & 0.2 & 0.22 & 0.22 & 76.74 \\
\hline M 3 & 0.3 & 0.29 & 0.33 & 0.31 & 112.29 \\
\hline M 4 & 0.23 & 0.22 & 0.25 & 0.24 & 85.14 \\
\hline M5 & 0.25 & 0.24 & 0.27 & 0.26 & 92.73 \\
\hline
\end{tabular}

a $m R=$ milliRoentgen, exposure unit for gamma radiation. One $m R$ is approximately equal to $1 \mathrm{mrem}$. Although the TLD is an integrating device, the derived unit $\mathrm{mR} /$ day is used to facilitate data comparison between locations or through time at a single location.

b An $18 \%$ uncertainty is associated with each result 


\section{Table 18}

Thermoluminescent Dosimeter (TLD) Results:

SRS Areas

Page 3 of 4

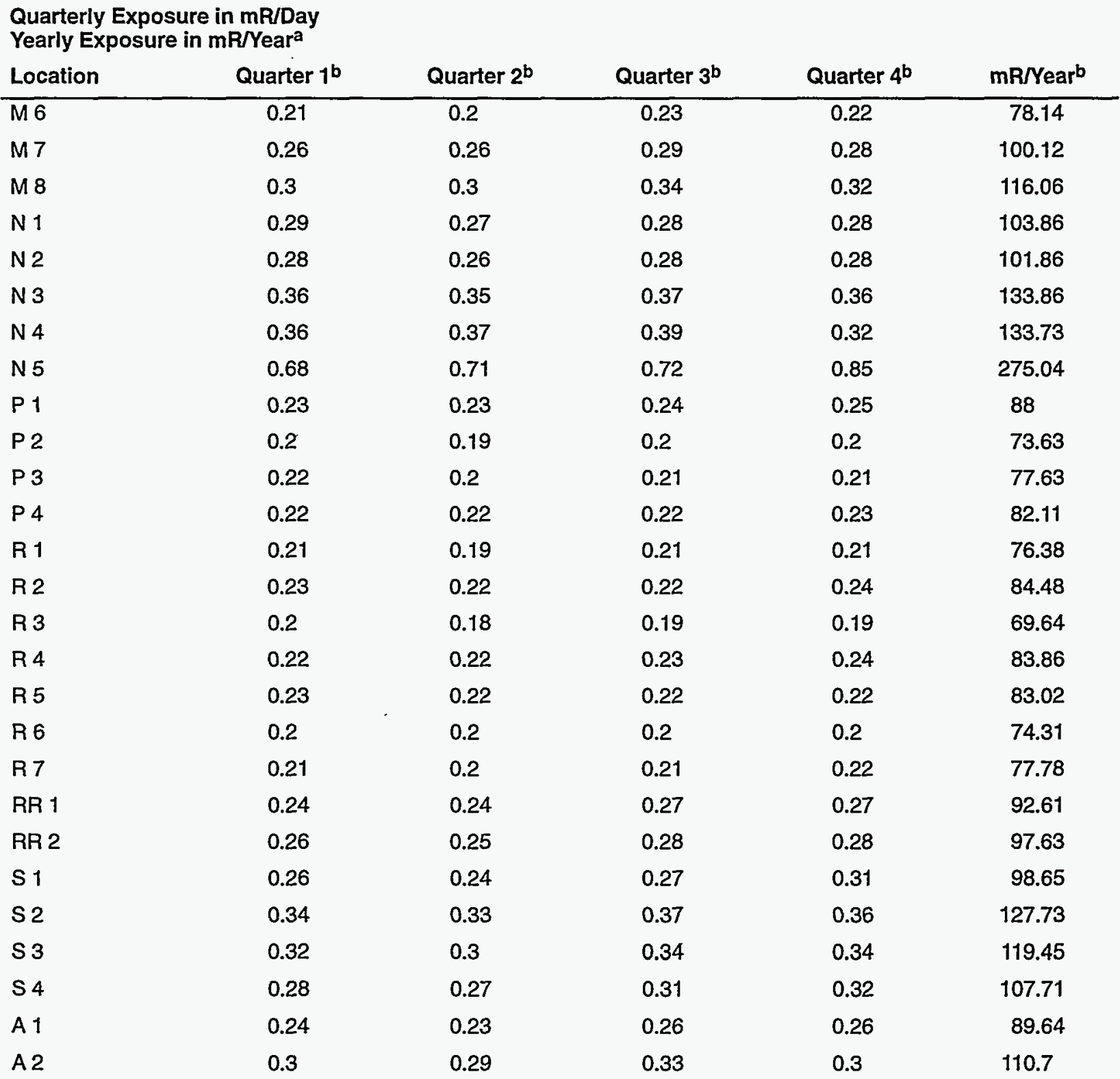

a $m R=$ milliRoentgen, exposure unit for gamma radiation. One $m R$ is approximately equal to $1 \mathrm{mrem}$. Although the TLD is an integrating device, the derived unit $\mathrm{mR} /$ day is used to facilitate data comparison between locations or through time at a single location.

b An $18 \%$ uncertainty is associated with each result. 


\section{Table 18}

\section{Thermoluminescent Dosimeter (TLD) Results:}

SRS Areas

Page 4 of 4

Quarterly Exposure in mR/Day

Yearly Exposure in mR/Yeara

\begin{tabular}{|c|c|c|c|c|c|}
\hline Location & Quarter $\mathbf{f}^{\mathbf{b}}$ & Quarter $2^{b}$ & Quarter $3^{\mathbf{b}}$ & Quarter $4^{b}$ & $\mathrm{mR} /$ Year $^{\mathrm{b}}$ \\
\hline A3 & 0.25 & 0.24 & 0.27 & & 93.22 \\
\hline A 4 & 0.27 & 0.25 & 0.29 & 0.29 & 100.4 \\
\hline A 5 & 0.23 & 0.23 & 0.26 & & 88.07 \\
\hline A 6 & 0.24 & 0.25 & 0.27 & & 92.27 \\
\hline A 7 & 0.28 & 0.26 & 0.3 & 0.27 & 101.2 \\
\hline A 8 & 0.23 & 0.2 & 0.27 & 0.22 & 83.6 \\
\hline$T 1$ & 0.19 & 0.18 & 0.19 & 0.2 & 71.18 \\
\hline T2 & 0.26 & 0.24 & 0.25 & 0.25 & 93.9 \\
\hline T 3 & 0.23 & 0.22 & 0.23 & 0.24 & 85.39 \\
\hline $\mathrm{T} 4$ & 0.29 & 0.25 & 0.24 & 0.29 & 99.47 \\
\hline $\mathrm{Z1}$ & 0.21 & 0.21 & 0.23 & 0.23 & 79.8 \\
\hline $\mathrm{Z2}$ & 0.21 & 0.2 & 0.22 & 0.22 & 78.01 \\
\hline Z3 & 0.2 & 0.2 & 0.22 & 0.22 & 75.78 \\
\hline$Z 4$ & 0.24 & 0.22 & 0.25 & 0.25 & 87.89 \\
\hline
\end{tabular}

a $m R=$ milliRoentgen, exposure unit for gamma radiation. One $m R$ is approximately equal to $1 \mathrm{mrem}$. Although the TLD is an integrating device, the derived unit $\mathrm{mR} /$ day is used to facilitate data comparison between locations or through time at a single location.

b An $18 \%$ uncertainty is associated with each result. 
Table 19

Thermoluminescent Dosimeter (TLD) Results:

Site Perimeter Stations

Page 1 of 7

Quarterly Exposure in mR/Day

Yearly Exposure in mR/Yeara

Location

Quarter $1^{b}$

Quarter 2b

Quarter $3^{b}$

Quarter 4b

mR/Yearb

Note: Blank spaces indicate missing TLD results.

\begin{tabular}{llllll}
\hline 1A & 0.18 & 0.16 & 0.17 & 0.19 & 66.64 \\
1B & 0.19 & 0.17 & 0.19 & 0.21 & 70.9 \\
1C & 0.19 & 0.17 & 0.19 & 0.21 & 72.33 \\
1D & 0.17 & 0.15 & 0.16 & 0.2 & 65.02 \\
2A & 0.26 & 0.22 & 0.25 & 0.27 & 94.58 \\
2B & 0.2 & & 0.2 & 0.23 & 79.33 \\
2C & 0.19 & 0.16 & 0.19 & 0.21 & 70.4 \\
2D & 0.17 & 0.15 & 0.17 & 0.2 & 65.49 \\
3A & 0.18 & 0.16 & 0.18 & 0.21 & 68.2 \\
3B & 0.17 & 0.16 & 0.18 & 0.2 & 66.21 \\
3C & 0.18 & 0.16 & 0.18 & 0.21 & 67.8 \\
3D & & 0.2 & 0.22 & 0.24 & 81.85 \\
4A & 0.21 & 0.19 & 0.21 & 0.23 & 79.16 \\
4B & 0.18 & 0.16 & 0.18 & 0.2 & 68.14 \\
4C & 0.19 & 0.17 & 0.18 & 0.2 & 69.92 \\
4D & 0.18 & 0.16 & 0.18 & 0.2 & 68.28 \\
5A & 0.22 & 0.2 & 0.22 & 0.24 & 82.45 \\
5B & 0.2 & 0.18 & 0.2 & 0.22 & 76.36 \\
5C & 0.22 & 0.19 & 0.22 & 0.24 & 82.74 \\
5D & 0.27 & 0.24 & 0.27 & 0.3 & 101.93 \\
6A & 0.26 & 0.24 & 0.27 & 0.29 & 100.04 \\
6B & 0.28 & 0.25 & 0.27 & 0.3 & 103.84 \\
6C & 0.23 & 0.2 & 0.22 & 0.24 & 84.29 \\
6D & 0.17 & 0.16 & 0.17 & 0.2 & 65.67 \\
7A & 0.22 & 0.19 & 0.22 & 0.24 & 82.69 \\
7B & 0.23 & 0.21 & 0.24 & 0.27 & 89.41
\end{tabular}

\footnotetext{
a $m R=$ milliRoentgen, exposure unit for gamma radiation. One $m R$ is approximately equal to $1 \mathrm{mrem}$. Although the TLD is an integrating device, the derived unit $\mathrm{mR} /$ day is used to facilitate data comparison between locations or through time at a single location.

b An $18 \%$ uncertainty is associated with each result.
} 


\section{Table 19}

Thermoluminescent Dosimeter (TLD) Results:

Site Perimeter Stations

Page 2 of 7

\begin{tabular}{|c|c|c|c|c|c|}
\hline \multicolumn{6}{|c|}{$\begin{array}{l}\text { Quarterly Exposure in mR/Day } \\
\text { Yearly Exposure in } \mathrm{mR} / \mathrm{Year}^{\mathrm{a}}\end{array}$} \\
\hline Location & Quarter $1^{b}$ & Quarter $2^{b}$ & Quarter $3^{b}$ & Quarter $\mathbf{4}^{b}$ & $\mathrm{mR} /$ Year $^{\mathrm{b}}$ \\
\hline $7 \mathrm{C}$ & 0.2 & 0.18 & 0.21 & 0.23 & 77 \\
\hline $7 \mathrm{D}$ & 0.21 & 0.18 & 0.2 & 0.23 & 77.91 \\
\hline $8 \mathrm{~A}$ & 0.19 & 0.17 & 0.19 & 0.22 & 73.87 \\
\hline $8 B$ & 0.22 & 0.19 & 0.22 & 0.25 & 82.38 \\
\hline $8 \mathrm{C}$ & 0.21 & 0.19 & 0.22 & 0.24 & 81.36 \\
\hline $8 D$ & 0.2 & 0.18 & 0.19 & 0.21 & 74.12 \\
\hline $9 \mathrm{~A}$ & 0.21 & 0.19 & 0.21 & 0.23 & 79.18 \\
\hline $9 \mathrm{~B}$ & 0.18 & 0.17 & 0.18 & 0.21 & 69.78 \\
\hline $9 \mathrm{C}$ & 0.18 & 0.16 & 0.18 & 0.2 & 68.31 \\
\hline $9 D$ & 0.15 & 0.14 & 0.15 & 0.16 & 56.84 \\
\hline 10 & 0.16 & 0.15 & 0.16 & 0.19 & 63.4 \\
\hline 11 & 0.18 & & & 0.2 & 70.92 \\
\hline 12 & 0.18 & & 0.18 & 0.21 & 72.95 \\
\hline 13 & 0.18 & 0.17 & 0.18 & 0.2 & 68.24 \\
\hline 14 & 0.2 & 0.18 & 0.2 & 0.22 & 75.36 \\
\hline 15 & 0.19 & 0.18 & 0.2 & 0.22 & 75.3 \\
\hline 16 & 0.22 & 0.21 & & 0.25 & 85.62 \\
\hline 17 & 0.25 & 0.21 & 0.24 & 0.25 & 90.07 \\
\hline 18 & 0.21 & 0.19 & 0.18 & 0.22 & 76.51 \\
\hline 19 & 0.19 & 0.17 & 0.19 & 0.23 & 73.96 \\
\hline 20 & 0.25 & 0.25 & 0.26 & 0.29 & 98.64 \\
\hline 21 & 0.16 & 0.16 & 0.18 & 0.19 & 64.63 \\
\hline 22 & 0.17 & 0.18 & 0.18 & 0.21 & 69.31 \\
\hline 23 & 0.19 & 0.19 & 0.2 & 0.23 & 75.34 \\
\hline 24 & 0.17 & 0.17 & 0.18 & 0.2 & 66.7 \\
\hline 25 & 0.16 & 0.16 & 0.17 & 0.2 & 63.73 \\
\hline 26 & 0.17 & 0.15 & 0.17 & 0.19 & 63.44 \\
\hline 27 & 0.18 & 0.18 & 0.19 & 0.2 & 70.67 \\
\hline
\end{tabular}

a $m R=$ milliRoentgen, exposure unit for gamma radiation. One $m R$ is approximately equal to $1 \mathrm{mrem}$. Although the TLD is an integrating device, the derived unit $\mathrm{mR} /$ day is used to facilitate data comparison between locations or through time at a single location.

b An $18 \%$ uncertainty is associated with each result. 
Table 19

Thermoluminescent Dosimeter (TLD) Results:

Site Perimeter Stations

Page 3 of 7

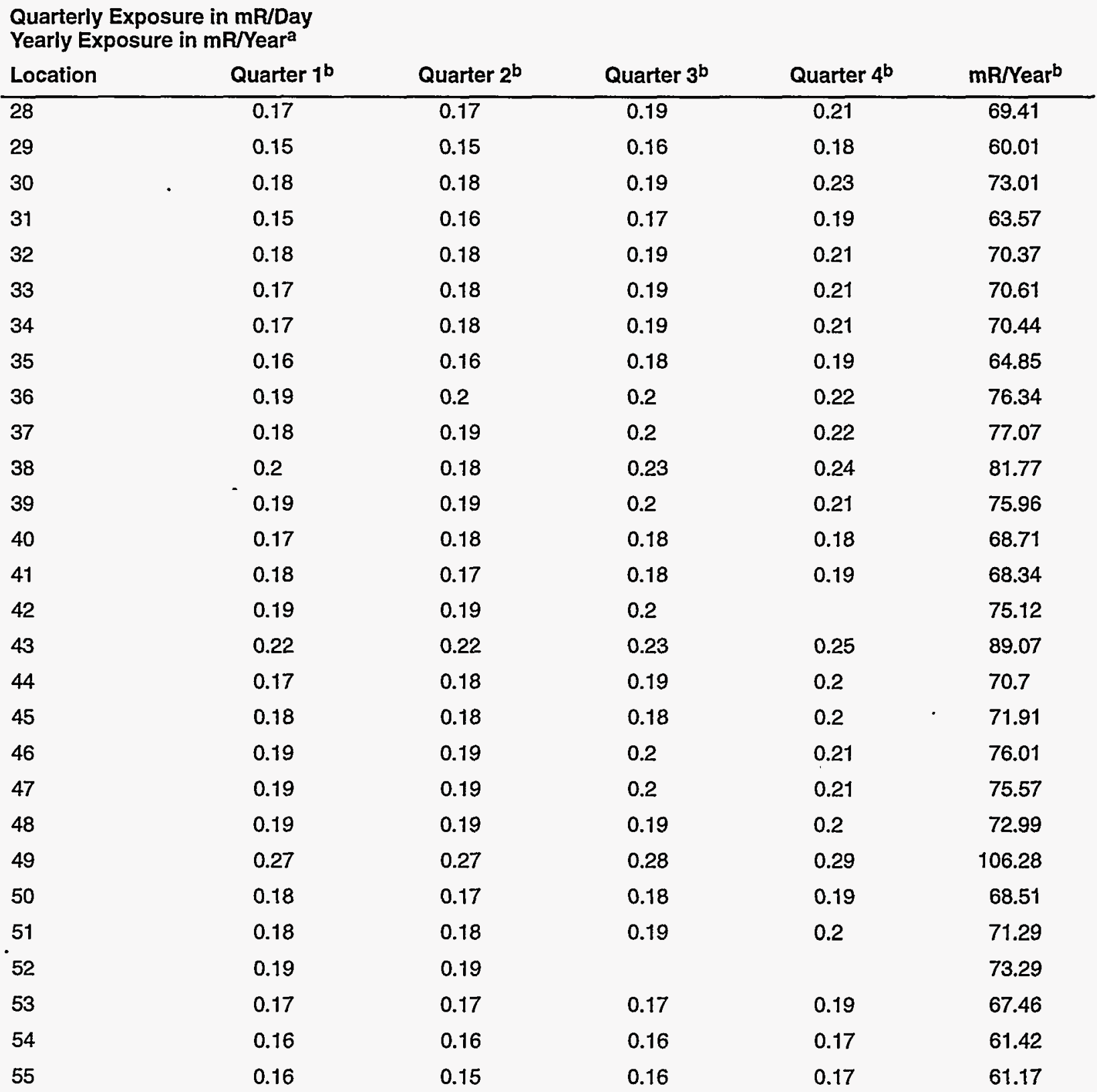

a $m R=$ milliRoentgen, exposure unit for gamma radiation. One $m R$ is approximately equal to $1 \mathrm{mrem}$. Although the TLD is an integrating device, the derived unit $\mathrm{mR} /$ day is used to facilitate data comparison between locations or through time at a single location.

b An $18 \%$ uncertainty is associated with each result. 


\section{Table 19}

\section{Thermoluminescent Dosimeter (TLD) Results:}

\section{Site Perimeter Stations}

Page 4 of 7

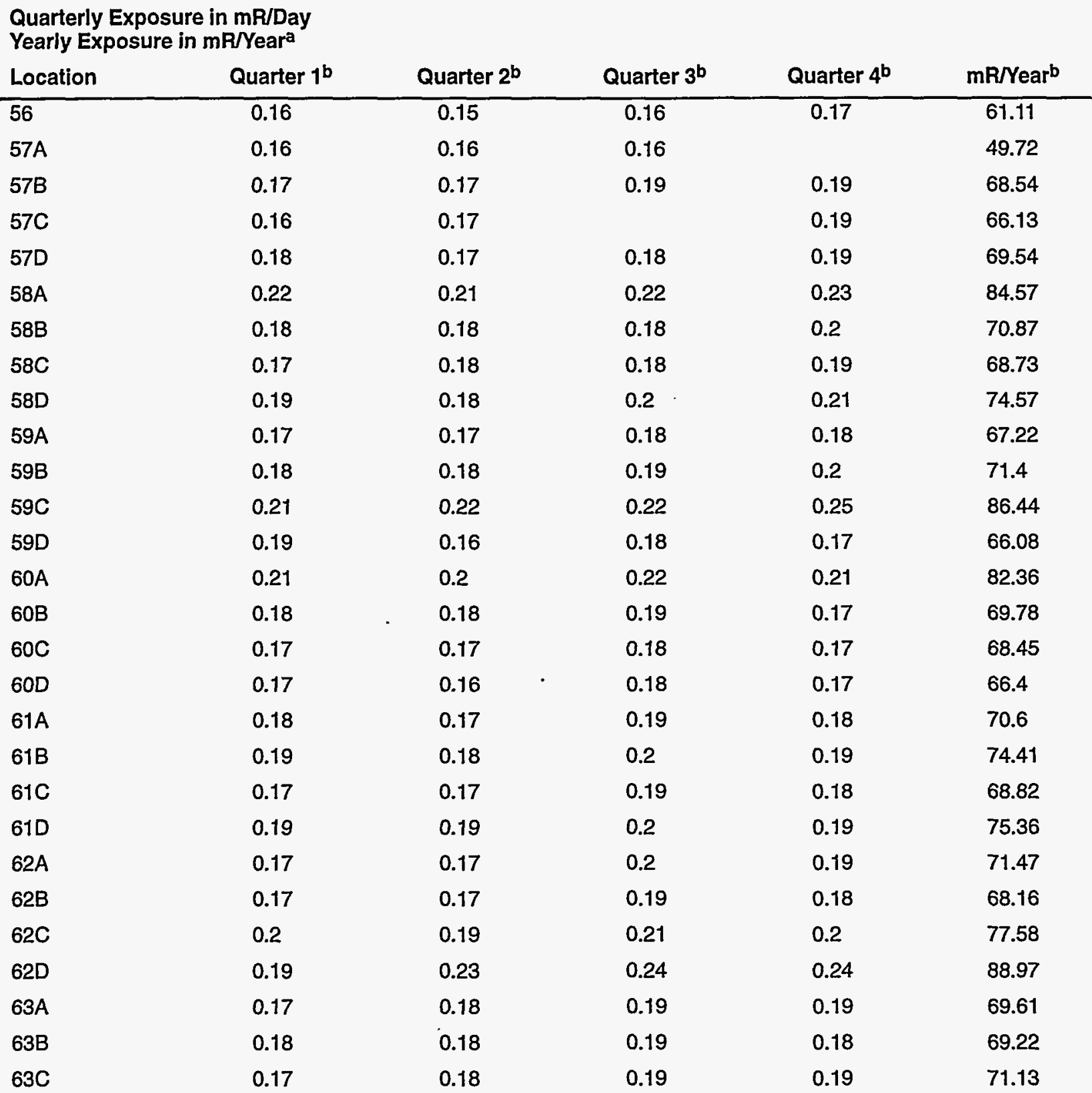

\footnotetext{
a $\quad m R=$ milliRoentgen, exposure unit for gamma radiation. One $\mathrm{mR}$ is approximately equal to $1 \mathrm{mrem}$. Although the TLD is an integrating device, the derived unit $\mathrm{mR} /$ day is used to facilitate data comparison between locations or through time at a single location.

b An $18 \%$ uncertainty is associated with each result.
} 
Table 19

Thermoluminescent Dosimeter (TLD) Results:

Site Perimeter Stations

Page 5 of 7

\begin{tabular}{|c|c|c|c|c|c|}
\hline \multicolumn{6}{|c|}{$\begin{array}{l}\text { Quarterly Exposure in } \mathrm{mR} / \mathrm{Day} \\
\text { Yearly Exposure in } \mathrm{mR} / \mathrm{Fear}^{\mathrm{a}}\end{array}$} \\
\hline Location & Quarter $1^{b}$ & Quarter $\mathbf{2}^{\mathbf{b}}$ & Quarter $3^{b}$ & Quarter $4^{b}$ & mR/Yearb \\
\hline $63 D$ & 0.16 & 0.17 & 0.18 & 0.17 & 64.82 \\
\hline $64 \mathrm{~A}$ & 0.16 & 0.18 & 0.18 & 0.17 & 67.64 \\
\hline $64 \mathrm{~B}$ & 0.16 & 0.16 & 0.17 & 0.16 & 63.21 \\
\hline $64 \mathrm{C}$ & 0.17 & 0.17 & 0.18 & 0.18 & 67.47 \\
\hline $64 \mathrm{D}$ & 0.16 & 0.17 & 0.18 & 0.17 & 64.67 \\
\hline $65 \mathrm{~A}$ & 0.18 & 0.19 & 0.2 & 0.2 & 74.6 \\
\hline $65 B$ & 0.21 & 0.23 & 0.23 & 0.23 & 86.88 \\
\hline $65 \mathrm{C}$ & 0.25 & 0.26 & 0.26 & 0.26 & 98.79 \\
\hline 65D & 0.2 & 0.22 & 0.24 & 0.22 & 84.85 \\
\hline $66 \mathrm{~A}$ & 0.24 & 0.25 & 0.26 & 0.26 & 96.92 \\
\hline $66 \mathrm{~B}$ & 0.24 & 0.25 & 0.28 & 0.27 & 100.28 \\
\hline $66 \mathrm{C}$ & 0.19 & 0.2 & 0.2 & 0.2 & 76.83 \\
\hline $66 \mathrm{D}$ & 0.21 & 0.22 & 0.23 & 0.22 & 83.82 \\
\hline $67 \mathrm{~A}$ & 0.18 & 0.19 & 0.21 & 0.19 & 73.65 \\
\hline $67 B$ & 0.2 & 0.21 & 0.22 & 0.21 & 80.93 \\
\hline $67 \mathrm{C}$ & 0.2 & 0.2 & 0.22 & 0.21 & 79.15 \\
\hline 67D & 0.16 & 0.17 & 0.18 & 0.17 & 65.17 \\
\hline $68 \mathrm{~A}$ & 0.16 & 0.17 & 0.18 & 0.17 & 65.72 \\
\hline $68 B$ & 0.16 & 0.16 & 0.18 & 0.17 & 65.38 \\
\hline $68 \mathrm{C}$ & 0.19 & 0.2 & 0.2 & 0.21 & 76.5 \\
\hline $68 D$ & 0.21 & 0.21 & 0.23 & 0.23 & 84.16 \\
\hline $69 \mathrm{~A}$ & 0.22 & 0.23 & 0.24 & 0.25 & 90.55 \\
\hline 698 & 0.16 & 0.17 & 0.18 & 0.17 & 65.23 \\
\hline $69 \mathrm{C}$ & 0.17 & 0.18 & 0.19 & 0.18 & 68.68 \\
\hline $69 D$ & 0.2 & 0.2 & 0.21 & 0.2 & 77.46 \\
\hline $70 \mathrm{~A}$ & 0.19 & 0.19 & 0.21 & 0.2 & 75.37 \\
\hline $70 \mathrm{~B}$ & 0.16 & 0.16 & 0.18 & 0.16 & 63.25 \\
\hline $70 \mathrm{C}$ & 0.17 & 0.18 & 0.19 & 0.18 & 70.48 \\
\hline
\end{tabular}

a $m R=$ milliRoentgen, exposure unit for gamma radiation. One $m R$ is approximately equal to 1 mrem. Although the TLD is an integrating device, the derived unit $\mathrm{mR} /$ day is used to facilitate data comparison between locations or through time at a single location.

b An $18 \%$ uncertainty is associated with each result. 
Table 19

Thermoluminescent Dosimeter (TLD) Results:

Site Perimeter Stations

Page 6 of 7

Quarterly Exposure in $\mathrm{mR} /$ Day

Yearly Exposure in $\mathrm{mR} / \mathrm{Year}^{\mathrm{a}}$

Location

Quarter $1^{\text {b }}$

$70 \mathrm{D}$

0.19

0.18

0.2

0.19

0.2

0.19

0.18

0.18

0.18

0.16

0.17

0.17

0.17

0.16

0.15

0.15

0.16

0.16

0.17

0.15

0.17

0.17

0.15

0.16

0.16

0.17

0.18

0.18

0.18

Quarter 2b

Quarter 3

Quarter 4

0.22

0.21

0.18

79.36

0.18

0.21

0.21

71.98

0.21

0.22

0.2

80.55

0.2

0.21

0.21

75.85

0.21

0.22

80.48

0.19

0.21

0.2

76.88

0.18

0.21

0.19

72.81

0.19

0.19

0.18

72.07

0.18

0.19

0.18

70.72

0.16

0.18

0.17

65.49

0.18

0.19

0.19

70.04

0.19

0.19

0.18

70.04

0.17

0.19

0.17

67.7

0.18

0.18

0.17

66.15

0.16

0.17

0.16

61.36

0.16

0.16

0.15

61.08

0.17

0.19

0.17

65.99

0.16

0.17

0.16

62.28

0.17

0.19

0.18

67.82

0.15

0.17

59.58

0.16

0.18

0.15

63.52

0.18

0.18

0.17

67.82

0.18

0.19

0.18

67.19

0.18

0.18

0.17

66.67

0.17

0.18

0.17

66.81

0.18

0.19

0.18

70.6

78

0.18

0.2

0.19

71.78

$79 \mathrm{~A}$

0.2

0.21

0.2

75.5

$79 B$

0.19

0.21

0.19

73.91

a $\quad \mathrm{mR}=$ milliRoentgen, exposure unit for gamma radiation. One $m R$ is approximately equal to 1 mrem. Although the TLD is an integrating device, the derived unit $\mathrm{mR} /$ day is used to facilitate data comparison between locations or through time at a single location.

b An $18 \%$ uncertainty is associated with each result. 
Table 19

Thermoluminescent Dosimeter (TLD) Results:

Site Perimeter Stations

Page 7 of 7

Quarterly Exposure in mR/Day

Yearly Exposure in mR/Yeara

\begin{tabular}{lccccc} 
Location & Quarter $1^{b}$ & Quarter 2 & Quarter 3b & Quarter $4^{b}$ & mR/Yearb $^{b}$ \\
\hline 79C & 0.2 & 0.21 & 0.22 & 0.21 & 81.19 \\
79D & 0.16 & 0.16 & 0.17 & 0.17 & 63.25 \\
AG 1 & 0.17 & 0.19 & & & 70.16 \\
AG 2 & 0.15 & 0.15 & 0.16 & 0.17 & 60.42 \\
AG 3 & 0.16 & 0.17 & 0.17 & 0.17 & 66.54 \\
AG 4 & 0.14 & 0.15 & 0.15 & 0.17 & 59.44 \\
PH RD 1 & 0.2 & 0.22 & 0.24 & 0.23 & 85.54 \\
PH RD 2 & 0.2 & 0.21 & 0.23 & 0.23 & 83.57 \\
PH RD 3 & 0.16 & 0.16 & 0.17 & 0.16 & 62.1 \\
PH RD 4 & 0.16 & 0.17 & 0.18 & 0.17 & 65.05 \\
PHRD 5 & 0.24 & 0.24 & 0.27 & 0.26 & 96.98
\end{tabular}

a $m R=$ milliRoentgen, exposure unit for gamma radiation. One $m R$ is approximately equal to $1 \mathrm{mrem}$. Although the TLD is an integrating device, the derived unit $\mathrm{mR} /$ day is used to facilitate data comparison between locations or through time at a single location.

b An $18 \%$ uncertainty is associated with each result. 


\section{Table 20}

\section{Thermoluminescent Dosimeter (TLD) Results: Environmental Surveillance (Air Monitoring) Stations}

Page 1 of 2

Quarterly Exposure in mR/Day

Yearly Exposure in mR/Yeara

\begin{tabular}{|c|c|c|c|c|c|c|}
\hline \multicolumn{2}{|c|}{ Location } & 2uarter $1^{b}$ & Quarter $2^{b}$ & Quarter $3^{b}$ & Quarter $4^{b}$ & $\mathrm{mR} / \mathrm{Year}^{\mathrm{b}}$ \\
\hline Note: & \multicolumn{6}{|c|}{$\begin{array}{l}\text { Blank spaces indicate missing TLD results. Program modifications implemented in mid-1995 resulted in } \\
\text { the elimination of } 65 \text { locations. Fourth quarter and annual exposures are not presented for these locations. }\end{array}$} \\
\hline \multicolumn{2}{|c|}{ Columbia 1} & 0.26 & 0.26 & & & \\
\hline \multicolumn{2}{|c|}{ Columbia 2} & 0.22 & 0.2 & & & \\
\hline \multicolumn{2}{|c|}{ Hwy 301} & 0.21 & 0.19 & 0.22 & 0.28 & 83.22 \\
\hline \multicolumn{2}{|c|}{ Augusta } & 0.23 & & 0.14 & 0.25 & 77.01 \\
\hline \multicolumn{2}{|c|}{ Langley } & 0.25 & 0.26 & 0.24 & & \\
\hline \multicolumn{2}{|c|}{ Perkins } & 0.19 & 0.19 & 0.16 & . & \\
\hline \multicolumn{2}{|c|}{ S. Richmond } & 0.26 & 0.27 & 0.24 & & \\
\hline \multicolumn{2}{|c|}{ Waynesboro } & 0.24 & 0.21 & 0.17 & & \\
\hline \multicolumn{2}{|c|}{ Greenville 1} & 0.31 & 0.3 & & & \\
\hline \multicolumn{2}{|c|}{ Greenville 2} & 0.24 & 0.27 & & & \\
\hline \multicolumn{2}{|c|}{ Macon 1} & 0.26 & 0.29 & & & \\
\hline \multicolumn{2}{|c|}{ Macon 2} & 0.21 & 0.19 & & & \\
\hline \multicolumn{2}{|c|}{ Hwy 39} & 0.21 & 0.21 & 0.21 & 0.2 & 76.49 \\
\hline \multicolumn{2}{|c|}{ A-Area } & 0.25 & 0.25 & 0.26 & 0.25 & 93.22 \\
\hline \multicolumn{2}{|c|}{ BG North } & 0.35 & 0.33 & 0.34 & 0.34 & 124.75 \\
\hline \multicolumn{2}{|c|}{ BG South } & 0.24 & 0.24 & 0.24 & 0.28 & 93.99 \\
\hline \multicolumn{2}{|c|}{ Dark Horse } & 0.2 & 0.2 & 0.21 & 0.21 & 76.28 \\
\hline \multicolumn{2}{|c|}{ East Talatha } & 0.19 & 0.2 & 0.19 & 0.19 & 70.19 \\
\hline \multicolumn{2}{|c|}{ F-Area } & 0.3 & 0.3 & 0.29 & 0.34 & 113.88 \\
\hline \multicolumn{2}{|c|}{ H-Area } & 0.3 & 0.31 & 0.26 & 0.32 & 110.33 \\
\hline \multicolumn{2}{|c|}{ Aiken Gate } & 0.23 & 0.23 & 0.23 & 0.23 & 84.79 \\
\hline \multicolumn{2}{|c|}{ Windsor Rd. } & 0.18 & 0.16 & 0.16 & 0.19 & 64.21 \\
\hline \multicolumn{2}{|c|}{ Hwy 21/167 } & 0.25 & 0.27 & 0.27 & 0.26 & 98.81 \\
\hline \multicolumn{2}{|c|}{ Rd A-14 } & 0.19 & 0.2 & 0.19 & 0.19 & 71.51 \\
\hline \multicolumn{2}{|c|}{ Allendale Gate } & 0.16 & 0.16 & 0.16 & 0.17 & 59.68 \\
\hline \multicolumn{2}{|c|}{ Barnwell Gate } & 0.21 & 0.21 & 0.21 & 0.22 & 78.41 \\
\hline
\end{tabular}

a $m R=$ milliRoentgen, exposure unit for gamma radiation. One $m R$ is approximately equal to $1 \mathrm{mrem}$. Although the TLD is an integrating device, the derived unit $\mathrm{mR} /$ day is used to facilitate data comparison between locations or through time at a single location.

b An $18 \%$ uncertainty is associated with each result. 
Table 20

Thermoluminescent Dosimeter (TLD) Results:

Environmental Surveillance (Air Monitoring) Stations

Page 2 of 2

\begin{tabular}{|c|c|c|c|c|c|}
\hline \multicolumn{6}{|c|}{$\begin{array}{l}\text { Quarterly Exposure in mR/Day } \\
\text { Yearly Exposure in } \mathrm{mR} / \mathrm{Year}^{\mathrm{a}}\end{array}$} \\
\hline Location & Quarter $1^{b}$ & Quarter $2^{b}$ & Quarter $3^{\mathbf{b}}$ & Quarter $4^{b}$ & $\mathrm{mR} /$ Year $^{\mathrm{b}}$ \\
\hline D-Area & 0.2 & 0.2 & 0.2 & 0.21 & 75.77 \\
\hline Green Pond & 0.19 & 0.19 & 0.2 & 0.18 & 70.76 \\
\hline Jackson & 0.21 & 0.22 & 0.22 & 0.22 & 81.23 \\
\hline Patterson Mill Road & 0.18 & 0.18 & 0.18 & 0.19 & 67.76 \\
\hline West Jackson & 0.24 & 0.25 & 0.25 & 0.25 & 92.64 \\
\hline Savannah 1 & 0.17 & 0.16 & 0.16 & 0.16 & 61.56 \\
\hline Savannah 2 & 0.15 & 0.15 & 0.14 & 0.17 & 57.54 \\
\hline Aiken Airport & 0.21 & 0.21 & & & 77.13 \\
\hline \multicolumn{6}{|l|}{ Allendale } \\
\hline Aiken State Park & 0.17 & 0.16 & 0.14 & & \\
\hline Lees & 0.18 & 0.17 & 0.15 & & \\
\hline Olar & 0.18 & 0.15 & 0.16 & & \\
\hline Springfield & & & 0.21 & & \\
\hline
\end{tabular}

a $m R=$ milliRoentgen, exposure unit for gamma radiation. One $m R$ is approximately equal to 1 mrem. Although the TLD is an integrating device, the derived unit $\mathrm{mR} /$ day is used to facilitate data comparison between locations or through time at a single location.

b An $18 \%$ uncertainty is associated with each result. 
Table 21

Thermoluminescent Dosimeter (TLD) Results: Population Centers

Page 1 of 3

Quarterly Exposure in mR/Day

Yearly Exposure in mR/Yeara

$\begin{array}{llllll}\text { Location } & \text { Quarter } 1^{b} & \text { Quarter } 2^{b} & \text { Quarter } 3^{b} & \text { Quarter } 4^{b} & m R / Y e a r^{b}\end{array}$

Note: Blank spaces indicate missing TLD results. Program modifications implemented in mid-1995 resulted in the elimination of 65 locations. Fourth quarter and annual exposures are not presented for these locations.

\begin{tabular}{llllll}
\hline Barnwell & 0.26 & 0.26 & 0.26 & 0.27 & 90.45 \\
Beech Island & 0.27 & 0.26 & 0.26 & 0.25 & 90.58 \\
Graniteville & & 0.21 & 0.21 & & \\
Jackson & 0.22 & 0.21 & 0.21 & 0.21 & 73.75 \\
Martin & 0.17 & 0.15 & 0.15 & 0.15 & 53.82 \\
North Augusta & 0.33 & 0.3 & 0.31 & & \\
Williston & 0.29 & 0.27 & 0.27 & 0.24 & 52.92 \\
Windsor & 0.16 & 0.15 & 0.14 & 0.17 & \\
Aiken 1 & 0.24 & 0.22 & 0.22 & & \\
Aiken 2 & 0.26 & 0.23 & 0.25 & \\
Allendale & & & & \\
Blackville & & & 0.24 & & \\
Couchton & 0.23 & & 0.25 & & \\
New Ellenton & 0.26 & 0.23 & 0.24 & 0.27 \\
Olar & 0.29 & 0.27 & 0.27 & & \\
Springfield & & 0.23 & 0.25 & \\
Wagener & 0.2 & 0.17 & 0.22 & \\
Alexander & 0.18 & & 0.18 & \\
Augusta 1 & 0.27 & 0.26 & 0.27 & \\
Augusta 2 & 0.28 & 0.29 & 0.29 & \\
Augusta 3 & 0.3 & 0.29 & 0.31 & \\
Augusta 4 & 0.22 & 0.22 & 0.22 & \\
Augusta 5 & 0.3 & 0.3 & 0.3 & \\
Augusta 7 & 0.23 & 0.22 & 0.24 & \\
Augusta 8 & 0.27 & 0.26 & 0.27 & \\
Girard & 0.28 & 0.26 & 0.28 & \\
\end{tabular}

a $\quad \mathrm{mR}=$ milliRoentgen, exposure unit for gamma radiation. One $\mathrm{mR}$ is approximately equal to $1 \mathrm{mrem}$. Although the TLD is an integrating device, the derived unit $\mathrm{mR} /$ day is used to facilitate data comparison between locations or through time at a single location.

b An $18 \%$ uncertainty is associated with each result. 
Table 21

Thermoluminescent Dosimeter (TLD) Results:

Population Centers

Page 2 of 3

\begin{tabular}{|c|c|c|c|c|c|}
\hline \multicolumn{6}{|c|}{$\begin{array}{l}\text { Quarterly Exposure in } \mathrm{mR} / \text { Day } \\
\text { Yearly Exposure in } \mathrm{mR} / \mathrm{Year}^{\mathrm{a}}\end{array}$} \\
\hline Location & Quarter $1^{b}$ & Quarter 2b & Quarter $3^{b}$ & Quarter $4^{b}$ & mR/Yearb \\
\hline Hephzibah & 0.19 & 0.18 & 0.19 & & \\
\hline McBean & 0.28 & 0.26 & 0.28 & & 99.45 \\
\hline Sardis & 0.22 & & 0.23 & & \\
\hline Waynesboro & 0.18 & 0.17 & 0.19 & & \\
\hline Batesburg & & 0.34 & 0.31 & & \\
\hline Edgefield & 0.26 & 0.3 & 0.26 & & \\
\hline Johnston & 0.3 & 0.34 & 0.31 & & \\
\hline Lincolnton & 0.28 & & 0.27 & & \\
\hline McCormick & 0.24 & 0.28 & 0.25 & & \\
\hline Saluda & & 0.41 & 0.41 & & \\
\hline Thompson & 0.29 & 0.3 & 0.33 & & \\
\hline Estill & 0.28 & 0.31 & 0.26 & & \\
\hline Hampton & 0.3 & & 0.26 & & \\
\hline North & 0.29 & 0.3 & 0.28 & & \\
\hline Orangeburg & 0.33 & 0.35 & 0.29 & & \\
\hline Smoaks & 0.23 & 0.26 & 0.2 & & \\
\hline St. Matthews & 0.32 & 0.32 & 0.3 & & \\
\hline Swansea & 0.26 & 0.27 & 0.33 & & \\
\hline Louisville & 0.33 & 0.35 & 0.32 & & \\
\hline Millen & 0.24 & 0.27 & 0.23 & & \\
\hline Newington & 0.28 & 0.31 & 0.26 & & \\
\hline Statesboro & & 0.28 & 0.22 & & \\
\hline Sylvania & 0.28 & 0.26 & 0.23 & & \\
\hline Wadley & 0.3 & 0.24 & 0.3 & & \\
\hline Wrens & 0.21 & & & & \\
\hline Columbia 1 & 0.34 & 0.29 & 0.27 & & \\
\hline Columbia 2 & 0.34 & 0.31 & 0.29 & & \\
\hline Columbia 3 & 0.37 & 0.34 & 0.3 & & \\
\hline Columbia 4 & 0.32 & 0.27 & 0.26 & & \\
\hline
\end{tabular}

a $\quad \mathrm{mR}=$ milliRoentgen, exposure unit for gamma radiation. One $\mathrm{mR}$ is approximately equal to $1 \mathrm{mrem}$. Although the TLD is an integrating device, the derived unit $\mathrm{mR} /$ day is used to facilitate data comparison between locations or through time at a single location.

b An $18 \%$ uncertainty is associated with each result 


\section{Table 21}

Thermoluminescent Dosimeter (TLD) Results:

Population Centers

Page 3 of 3

Quarterly Exposure in $\mathrm{mR} / \mathrm{Day}$

Yearly Exposure in $\mathrm{mR} / \mathrm{Year}^{\mathrm{a}}$

\begin{tabular}{|c|c|c|c|c|c|}
\hline Location & Quarter $1^{b}$ & Quarter $2^{b}$ & Quarter $\mathbf{3}^{\mathrm{b}}$ & Quarter $4^{b}$ & mR/Yearb \\
\hline Columbia 5 & 0.3 & 0.27 & 0.26 & & \\
\hline Columbia 6 & 0.35 & 0.31 & 0.29 & & \\
\hline Columbia 7 & 0.35 & 0.31 & 0.31 & & \\
\hline Columbia 8 & 0.34 & 0.31 & 0.3 & & \\
\hline Columbia 9 & 0.31 & 0.27 & 0.26 & & \\
\hline Lexington & 0.27 & 0.23 & 0.21 & & \\
\hline West Columbia & 0.32 & 0.31 & 0.29 & & \\
\hline
\end{tabular}

a $m R=$ milliRoentgen, exposure unit for gamma radiation. One $m R$ is approximately equal to 1 mrem. Although the TLD is an integrating device, the derived unit $\mathrm{mR} / \mathrm{day}$ is used to facilitate data comparison between locations or through time at a single location.

b An $18 \%$ uncertainty is associated with each result. 
Table 22

Thermoluminescent Dosimeter (TLD) Results:

Vogtle Electric Generating Plant Vicinity

Page 1 of 1

\begin{tabular}{|c|c|c|c|c|c|}
\hline Location & Quarter $1^{b}$ & Quarter $2^{b}$ & Quarter $3^{b}$ & Quarter $4^{b}$ & mR/Yearb \\
\hline NRC 1 & 0.21 & 0.2 & 0.2 & & 74.9 \\
\hline NRC 2 & 0.16 & 0.16 & 0.13 & 0.19 & 60.2 \\
\hline NRC 3 & 0.16 & 0.16 & 0.14 & 0.19 & 60.82 \\
\hline NRC 4 & 0.17 & 0.18 & 0.15 & 0.21 & 66.58 \\
\hline NRC 5 & 0.23 & 0.24 & 0.22 & 0.28 & 89.76 \\
\hline NRC 6 & 0.19 & 0.19 & 0.16 & 0.22 & 69.82 \\
\hline NRC 7 & 0.17 & 0.17 & 0.15 & 0.2 & 64.63 \\
\hline NRC 8 & 0.18 & 0.15 & 0.13 & 0.19 & 61.05 \\
\hline GP $1 \mathrm{H}$ & 0.17 & 0.17 & 0.15 & 0.16 & 60.58 \\
\hline GP $1 \mathrm{~L}$ & 0.18 & 0.17 & 0.15 & 0.15 & 60.37 \\
\hline GP $2 \mathrm{H}$ & 0.18 & 0.19 & 0.17 & 0.17 & 66.56 \\
\hline GP $2 L$ & 0.19 & 0.2 & 0.18 & 0.16 & 67.16 \\
\hline GP $3 \mathrm{H}$ & 0.2 & 0.2 & 0.18 & 0.17 & 69.43 \\
\hline GP $3 \mathrm{~L}$ & 0.19 & 0.19 & 0.17 & 0.17 & 66.48 \\
\hline GP $4 \mathrm{H}$ & 0.18 & 0.18 & 0.17 & 0.16 & 64.04 \\
\hline GP $4 \mathrm{~L}$ & 0.18 & 0.18 & 0.17 & 0.16 & 63.83 \\
\hline GP $5 \mathrm{H}$ & 0.18 & 0.19 & 0.16 & 0.15 & 62.97 \\
\hline GP $5 \mathrm{~L}$ & 0.18 & 0.15 & 0.13 & 0.14 & 55.78 \\
\hline
\end{tabular}

a $m R=$ milliRoentgen, exposure unit for gamma radiation. One $m R$ is approximately equal to 1 mrem. Although the TLD is an integrating device, the derived unit $\mathrm{mR} /$ day is used to facilitate data comparison between locations or through time at a single location.

b An $18 \%$ uncertainty is associated with each result. 


\section{Table 23}

\section{Radioactivity in Seepage Basin Water}

Page 1 of 2

\begin{tabular}{|c|c|c|c|c|}
\hline Location & $\begin{array}{l}\text { No. of } \\
\text { Samples }\end{array}$ & Arithmetic Mean $\pm \sigma$ & Maximum $\pm \sigma$ & Minimum $\pm \sigma$ \\
\hline \multicolumn{5}{|l|}{$\mathrm{H}-3, \mu \mathrm{Ci} / \mathrm{mL}$} \\
\hline $\begin{array}{l}\text { CSB-1 C-Area seepage } \\
\text { basin }\end{array}$ & 3 & $(3.76 \pm 4.62) E-06$ & $(8.51 \pm 3.02) E-06$ & $(-0.72 \pm 1.71) E-06$ \\
\hline EAV basin north (E-004) & 12 & $(1.06 \pm 1.29) E-05$ & $(5.01 \pm 0.05) E-05$ & $(2.38 \pm 0.33) E-06$ \\
\hline EAV basin south $(E-003)$ & 1 & $(5.63 \pm 0.48) E-06$ & & \\
\hline $\begin{array}{l}\text { LSB-1 L-Area seepage ba- } \\
\sin \end{array}$ & 4 & $(-0.28 \pm 3.45) E-06$ & $(3.64 \pm 3.91) \mathrm{E}-06$ & $(-4.71 \pm 2.79) E-06$ \\
\hline $\begin{array}{l}\text { PSB-1 P-Area seepage } \\
\text { basin }\end{array}$ & 2 & $(-0.25 \pm 1.63) E-05$ & $(0.90 \pm 5.98) E-05$ & $(-1.40 \pm 6.88) E-05$ \\
\hline SWDF basin south $(E-001)$ & 12 & $(2.63 \pm 1.94) E-05$ & $(5.65 \pm 0.11) E-05$ & $(7.82 \pm 0.30) E-06$ \\
\hline TNX-SB seepage basin & 1 & $(1.81 \pm 0.39) E-06$ & & \\
\hline \multicolumn{5}{|l|}{$\mathrm{Co}-60, \mu \mathrm{Ci} / \mathrm{mL}$} \\
\hline $\begin{array}{l}\text { CSB-1 C-Area seepage } \\
\text { basin }\end{array}$ & 1 & $(1.42 \pm 0.16) \mathrm{E}-08$ & & \\
\hline \multicolumn{5}{|l|}{$\mathrm{Cs}-137, \mu \mathrm{Ci} / \mathrm{mL}$} \\
\hline $\begin{array}{l}\text { CSB-1 C-Area seepage } \\
\text { basin }\end{array}$ & 3 & $(2.03 \pm 0.82) E-08$ & $(2.93 \pm 0.30) E-08$ & $(1.32 \pm 0.35) E-08$ \\
\hline $\begin{array}{l}\text { PSB-1 P-Area seepage } \\
\text { basin }\end{array}$ & 2 & $(4.13 \pm 2.39) E-08$ & $(5.82 \pm 0.35) \mathrm{E}-08$ & $(2.44 \pm 0.27) E-08$ \\
\hline \multicolumn{5}{|l|}{$\mathrm{U}-234, \mu \mathrm{Ci} / \mathrm{mL}$} \\
\hline TNX-SB seepage basin & 1 & $(8.21 \pm 1.85) E-10$ & & \\
\hline \multicolumn{5}{|l|}{$\mathrm{U}-235, \mu \mathrm{Ci} / \mathrm{mL}$} \\
\hline TNX-SB seepage basin & 1 & $(5.03 \pm 5.07) \mathrm{E}-11$ & & \\
\hline \multicolumn{5}{|l|}{$\mathrm{U}-238, \mu \mathrm{Ci} / \mathrm{mL}$} \\
\hline TNX-SB seepage basin & 1 & $(8.61 \pm 1.88) \mathrm{E}-10$ & & \\
\hline \multicolumn{5}{|l|}{$\overline{P u-238, \mu \mathrm{Ci} / \mathrm{mL}}$} \\
\hline TNX-SB seepage basin & $\overline{1}$ & $(4.05 \pm 1.78) E-10$ & & \\
\hline \multicolumn{5}{|l|}{$\mathrm{Sr}-89,90, \mu \mathrm{Ci} / \mathrm{mL}$} \\
\hline $\begin{array}{l}\text { LSB-1 L-Area seepage ba- } \\
\text { sin }\end{array}$ & 4 & $(2.61 \pm 1.19) E-08$ & $(3.55 \pm 1.90) \mathrm{E}-08$ & $(1.05 \pm 1.86) E-08$ \\
\hline \multicolumn{5}{|l|}{ Gross Beta, $\mu \mathrm{Ci} / \mathrm{mL}$} \\
\hline $\begin{array}{l}\text { CSB-1 C-Area seepage } \\
\text { basin }\end{array}$ & 3 & $(4.97 \pm 4.18) E-08$ & $(9.80 \pm 0.85) \mathrm{E}-08$ & $(2.50 \pm 0.46) E-08$ \\
\hline EAV basin north $(E-004)$ & 12 & $(4.91 \pm 1.94) E-09$ & $(9.65 \pm 1.20) \mathrm{E}-09$ & $(2.59 \pm 0.74) E-09$ \\
\hline EAV basin south $(E-003)$ & 1 & $(4.22 \pm 0.51) E-09$ & & \\
\hline $\begin{array}{l}\text { LSB-1 L-Area seepage ba- } \\
\text { sin }\end{array}$ & 4 & $(7.98 \pm 3.68) E-09$ & $(1.10 \pm 0.48) \mathrm{E}-08$ & $(2.68 \pm 3.49) E-09$ \\
\hline
\end{tabular}


Table 23

Radioactivity in Seepage Basin Water

Page 2 of 2

\begin{tabular}{|c|c|c|c|c|}
\hline Location & $\begin{array}{l}\text { No. of } \\
\text { Samples }\end{array}$ & Arithmetic Mean $\pm \sigma$ & Maximum $\pm \sigma$ & Minimum $\pm \sigma$ \\
\hline $\begin{array}{l}\text { PSB-1 P-Area seepage } \\
\text { basin }\end{array}$ & 2 & $(2.98 \pm 0.82) E-08$ & $(3.56 \pm 0.60) \mathrm{E}-08$ & $(2.40 \pm 0.45) E-08$ \\
\hline SWDF basin south (E-001) & 12 & $(6.43 \pm 2.06) E-09$ & $(9.01 \pm 1.17) \mathrm{E}-09$ & $(3.33 \pm 0.79) E-09$ \\
\hline TNX-SB seepage basin & 1 & $(-3.74 \pm 7.16) \mathrm{E}-09$ & & \\
\hline \multicolumn{5}{|l|}{ Gross Alpha, $\mu \mathrm{Ci} / \mathrm{mL}$} \\
\hline $\begin{array}{l}\text { CSB-1 C-Area seepage } \\
\text { basin }\end{array}$ & 3 & $(-1.98 \pm 6.40) E-10$ & $(0.52 \pm 1.16) E-09$ & $(-0.71 \pm 1.65) E-09$ \\
\hline EAV basin north (E-004) & 12 & $(1.26 \pm 0.77) E-09$ & $(2.76 \pm 1.14) E-09$ & $(1.46 \pm 5.16) E-10$ \\
\hline EAV basin south ( $E-003)$ & 1 & $(5.98 \pm 3.24) \mathrm{E}-10$ & & \\
\hline $\begin{array}{l}\text { LSB-1 L-Area seepage ba- } \\
\text { sin }\end{array}$ & 4 & $(-3.83 \pm 8.92) E-10$ & $(0.56 \pm 1.14) E-09$ & $(-1.57 \pm 1.26) \mathrm{E}-09$ \\
\hline $\begin{array}{l}\text { PSB-1 P-Area seepage } \\
\text { basin }\end{array}$ & 2 & $(0.42 \pm 1.39) E-09$ & $(1.40 \pm 1.39) E-09$ & $(-0.56 \pm 1.65) E-09$ \\
\hline SWDF basin south (E-001) & 12 & $(1.73 \pm 1.17) E-09$ & $(4.28 \pm 1.18) E-09$ & $(1.22 \pm 4.68) E-10$ \\
\hline TNX-SB seepage basin & 1 & $(-0.99 \pm 3.53) E-09$ & & \\
\hline
\end{tabular}


Table 24

Radioactivity in Savannah River Site Stream Water

Page 1 of 7

\begin{tabular}{|c|c|c|c|c|}
\hline Location & $\begin{array}{l}\text { No. of } \\
\text { Samples }\end{array}$ & Arithmetic Mean $\pm \sigma$ & Maximum $\pm \sigma$ & Minimum $\pm \sigma$ \\
\hline \multicolumn{5}{|l|}{$\mathrm{H}-3, \mu \mathrm{Cl} / \mathrm{mL}$} \\
\hline \multicolumn{5}{|l|}{ Tims Branch } \\
\hline TB-5 near Road C & 26 & $(9.66 \pm 3.41) \mathrm{E}-07$ & $(1.70 \pm 0.40) E-06$ & $(3.77 \pm 4.04) E-07$ \\
\hline \multicolumn{5}{|l|}{ Upper Three Runs } \\
\hline Crouch Branch at Road 4 & 26 & $(1.83 \pm 0.53) E-05$ & $(3.13 \pm 0.08) E-05$ & $(1.11 \pm 0.03) E-05$ \\
\hline $\begin{array}{l}\text { U3R-1A Treadway Bridge } \\
\text { Road 8-1 }\end{array}$ & 52 & $(5.08 \pm 3.45) E-07$ & $(1.12 \pm 0.38) E-06$ & $(-1.66 \pm 3.99) E-07$ \\
\hline U3R-3 at Road C & 26 & $(2.24 \pm 2.38) E-06$ & $(1.34 \pm 0.06) E-05$ & $(6.92 \pm 3.68) E-07$ \\
\hline U3R-4 at Road A & 24 & $(2.21 \pm 1.57) E-06$ & $(8.74 \pm 0.36) \mathrm{E}-06$ & $(1.01 \pm 0.37) E-06$ \\
\hline \multicolumn{5}{|l|}{ Four Mile Creek } \\
\hline $\begin{array}{l}\text { Caster Creek - southeast } \\
\text { of C-Area }\end{array}$ & 52 & $(3.82 \pm 0.43) E-06$ & $(4.95 \pm 0.44) E-06$ & $(2.85 \pm 0.41) E-06$ \\
\hline FM-2 at Road 4 & 25 & $(2.26 \pm 1.06) E-05$ & $(4.46 \pm 0.10) E-05$ & $(6.97 \pm 0.49) E-06$ \\
\hline $\begin{array}{l}\text { FM-2B above F-Area } \\
\text { effluent }\end{array}$ & 26 & $(8.80 \pm 1.67) E-05$ & $(1.21 \pm 0.06) E-04$ & $(5.76 \pm 0.46) E-05$ \\
\hline $\begin{array}{l}\text { FM-3A below F-Area } \\
\text { effluent }\end{array}$ & 26 & $(1.85 \pm 0.37) E-03$ & $(2.63 \pm 0.02) E-03$ & $(1.11 \pm 0.02) E-03$ \\
\hline FM-6 at Road A-12.2 & 26 & $(2.28 \pm 0.33) E-04$ & $(2.98 \pm 0.03) \mathrm{E}-04$ & $(1.58 \pm 0.02) E-04$ \\
\hline FM-A7 at Road $A-7$ & 26 & $(4.55 \pm 1.38) E-04$ & $(6.95 \pm 0.12) E-04$ & $(5.66 \pm 0.10) E-05$ \\
\hline $\mathrm{H}-008$ Outfall & 53 & $(2.17 \pm 1.59) E-06$ & $(9.12 \pm 0.44) E-06$ & $(-1.21 \pm 0.21) E-08$ \\
\hline $\begin{array}{l}\text { HP-50 Tritium Facility } \\
\text { Outfall }\end{array}$ & 26 & $(1.73 \pm 2.02) E-05$ & $(9.00 \pm 0.14) \mathrm{E}-05$ & $(9.10 \pm 3.60) E-07$ \\
\hline $\begin{array}{l}\text { Twin Lakes - West of } \\
\text { C-Area }\end{array}$ & 53 & $(1.40 \pm 0.24) E-05$ & $(1.73 \pm 0.05) E-05$ & $(0.13 \pm 1.72) E-09$ \\
\hline
\end{tabular}

Pen Branch

IGB-21 800 feet south of Road 6-1

$(8.23 \pm 1.88) E-04$

$(1.21 \pm 0.02) \mathrm{E}-03$

$(4.23 \pm 0.08) E-04$

K-011 Outfall at Road B

54

$(5.07 \pm 0.98) E-05$

$(6.99 \pm 0.11) E-05$

$(5.50 \pm 0.25) E-06$

PB-3 at Road A-13.2

$(6.89 \pm 1.33) E-05$

$(8.53 \pm 0.07) E-05$

$(3.57 \pm 0.09) E-05$

Steel Creek

SC-2A 1 mile above

Road $B$

$(9.82 \pm 4.38) E-05$

$(1.97 \pm 0.02) E-04$

$(1.14 \pm 0.05) E-05$

Road A

26

$(6.97 \pm 0.93) E-06$

$(8.79 \pm 0.51) E-06$

$(5.05 \pm 0.42) E-06$

Lower Three Runs Creek

L3R-1A at Road B

26

$(8.84 \pm 3.73) E-07$

$(1.55 \pm 0.37) E-06$

(1.46 \pm 3.67$) \mathrm{E}-07$

L3R-2 Patterson Mill Road

25

$(9.88 \pm 3.47) \mathrm{E}-07$

$(1.83 \pm 0.40) E-06$

(3.10 \pm 3.57$) E-07$ 
Table 24

Radioactivity in Savannah River Site Stream Water

Page 2 of 7

\begin{tabular}{|c|c|c|c|c|}
\hline Location & $\begin{array}{l}\text { No. of } \\
\text { Samples }\end{array}$ & Arithmetic Mean $\pm \sigma$ & Maximum $\pm \sigma$ & Minimum $\pm \sigma$ \\
\hline L3R-3 at Highway 125 & 12 & $(6.53 \pm 4.11) \mathrm{E}-07$ & $(1.14 \pm 0.36) \mathrm{E}-06$ & $(-2.38 \pm 3.70) E-07$ \\
\hline R-Area downstream of R-1 & 54 & $(6.29 \pm 9.10) \mathrm{E}-06$ & $(4.37 \pm 0.09) E-05$ & $(1.17 \pm 0.36) E-06$ \\
\hline \multicolumn{5}{|l|}{$\mathrm{Co}-60, \mu \mathrm{Ci} / \mathrm{mL}$} \\
\hline \multicolumn{5}{|l|}{ Upper Three Runs } \\
\hline U3R-4 at Road A & 1 & $(0.78 \pm 2.57) E-09$ & & \\
\hline \multicolumn{5}{|l|}{ Pen Branch } \\
\hline K-011 Outfall at Road B & 1 & $(4.57 \pm 1.65) E-09$ & & \\
\hline \multicolumn{5}{|l|}{ Lower Three Runs Creek } \\
\hline L3R-2 Patterson Mill Road & 1 & $(2.00 \pm 1.88) E-09$ & & \\
\hline R-Area downstream of R-1 & 1 & $(1.31 \pm 1.92) E-09$ & & \\
\hline \multicolumn{5}{|l|}{ Cs-137, $\mu \mathrm{Ci} / \mathrm{mL}$} \\
\hline \multicolumn{5}{|l|}{ Four Mile Creek } \\
\hline FM-2 at Road 4 & 5 & $(1.32 \pm 0.83) E-08$ & $(2.79 \pm 0.28) \mathrm{E}-08$ & $(7.98 \pm 2.85) E-09$ \\
\hline $\begin{array}{l}\text { FM-2B above F-Area } \\
\text { effluent }\end{array}$ & 12 & $(1.08 \pm 0.29) E-08$ & $(1.43 \pm 0.23) E-08$ & $(5.46 \pm 1.35) E-09$ \\
\hline $\begin{array}{l}\text { FM-3A below F-Area } \\
\text { effluent }\end{array}$ & 6 & $(1.13 \pm 0.50) E-08$ & $(1.65 \pm 0.21) E-08$ & $(2.90 \pm 1.07) E-09$ \\
\hline FM-6 at Road A-12.2 & 2 & $(4.89 \pm 0.90) E-09$ & $(5.52 \pm 2.02) E-09$ & $(4.25 \pm 1.30) E-09$ \\
\hline FM-A7 at Road A-7 & 6 & $(1: 03 \pm 0.54) E-08$ & $(1.87 \pm 0.57) E-08$ & $(3.33 \pm 1.12) E-09$ \\
\hline $\mathrm{H}-008$ Outfall & 6 & $(7.63 \pm 1.99) E-09$ & $(1.03 \pm 0.22) E-08$ & $(4.90 \pm 1.25) E-09$ \\
\hline
\end{tabular}

Pen Branch

K-011 Outfall at Road B $\quad 1 \quad(0.54 \pm 1.49) E-09$

Steel Creek

Road B

7

$(9.32 \pm 2.29) \mathrm{E}-09$

$(1.42 \pm 0.42) \mathrm{E}-08$

$(7.02 \pm 2.45) \mathrm{E}-09$

Lower Three Runs Creek

L3R-2 Patterson Mill Road

$1 \quad(0.81 \pm 2.16) E-09$

R-Area downstream of $\mathrm{R}-1$

$18 \quad(1.17 \pm 0.52) E-08$

$(2.43 \pm 0.39) E-08$

$(5.28 \pm 1.94) \mathrm{E}-09$

$\mathrm{U}-234, \mu \mathrm{Ci} / \mathrm{mL}$

Upper Three Runs

U3R-4 at Road A

12

$(5.71 \pm 2.81) \mathrm{E}-11$

$(1.16 \pm 0.16) \mathrm{E}-10$

$(2.65 \pm 1.02) \mathrm{E}-11$

Four Mile Creek

FM-6 at Road A-12.2

14

$(3.75 \pm 2.23) E-11$

$(7.39 \pm 1.36) \mathrm{E}-11$

$(3.53 \pm 6.41) E-12$

Pen Branch

PB-3 at Road A-13.2

14

$(1.73 \pm 1.44) \mathrm{E}-11$

$(5.16 \pm 1.06) E-11$

$(-4.13 \pm 5.45) E-12$ 


\section{Table 24}

\section{Radioactivity in Savannah River Site Stream Water}

Page 3 of 7

\begin{tabular}{|c|c|c|c|c|}
\hline Location & $\begin{array}{c}\text { No. of } \\
\text { Samples }\end{array}$ & Arithmetic Mean $\pm \sigma$ & Maximum $\pm \sigma$ & Minimum $\pm \sigma$ \\
\hline \multicolumn{5}{|l|}{ Lower Three Runs Creek } \\
\hline L3R-2(weekly grab) & 26 & $(1.95 \pm 1.40) E-11$ & $(5.53 \pm 1.23) \mathrm{E}-11$ & $(-8.84 \pm 6.19) E-12$ \\
\hline L3R-2 Patterson Mill Road & 13 & $(1.75 \pm 1.38) \mathrm{E}-11$ & $(3.97 \pm 1.03) E-11$ & $(-7.29 \pm 5.43) E-12$ \\
\hline \multicolumn{5}{|l|}{$\mathrm{U}-235, \mu \mathrm{Ci} / \mathrm{mL}$} \\
\hline \multicolumn{5}{|l|}{ Upper Three Runs } \\
\hline U3R-4 at Road A & 12 & $(6.62 \pm 6.39) E-12$ & $(2.04 \pm 0.84) E-11$ & $(-3.04 \pm 4.50) E-12$ \\
\hline \multicolumn{5}{|l|}{ Four Mile Creek } \\
\hline FM-6 at Road A-12.2 & 14 & $(3.47 \pm 5.55) E-12$ & $(1.38 \pm 0.62) E-11$ & $(-6.18 \pm 2.81) E-12$ \\
\hline \multicolumn{5}{|l|}{ Pen Branch } \\
\hline PB-3 at Road A-13.2 & 14 & $(2.91 \pm 7.06) \mathrm{E}-12$ & $(1.24 \pm 0.80) E-11$ & $(-8.95 \pm 0.41) E-12$ \\
\hline \multicolumn{5}{|l|}{ Lower Three Runs Creek } \\
\hline L3R-2(weekly grab) & 26 & $(1.39 \pm 6.38) E-12$ & $(1.38 \pm 0.94) E-11$ & $(-1.12 \pm 0.04) E-11$ \\
\hline L3R-2 Patterson Mill Road & 13 & $(0.97 \pm 5.14) E-12$ & $(7.87 \pm 5.33) E-12$ & $(-9.79 \pm 2.44) E-12$ \\
\hline \multicolumn{5}{|l|}{$\mathrm{U}-238, \mu \mathrm{Ci} / \mathrm{mL}$} \\
\hline \multicolumn{5}{|l|}{ Upper Three Runs } \\
\hline U3R-4 at Road A & 12 & $(6.91 \pm 3.40) E-11$ & $(1.31 \pm 0.18) E-10$ & $(1.74 \pm 0.94) E-11$ \\
\hline \multicolumn{5}{|l|}{ Four Mile Creek } \\
\hline FM-6 at Road A-12.2 & 14 & $(4.51 \pm 1.85) E-11$ & $(7.87 \pm 1.64) E-11$ & $(0.43 \pm 1.04) E-11$ \\
\hline \multicolumn{5}{|l|}{ Pen Branch } \\
\hline PB-3 at Road A-13.2 & 14 & $(1.78 \pm 1.79) E-11$ & $(5.56 \pm 1.09) E-11$ & $(-8.40 \pm 8.04) E-12$ \\
\hline \multicolumn{5}{|l|}{ Lower Three Runs Creek } \\
\hline L3R-2(weekly grab) & 26 & $(1.90 \pm 1.34) E-11$ & $(4.18 \pm 1.07) E-11$ & $(-1.30 \pm 0.55) E-11$ \\
\hline L3R-2 Patterson Mill Road & 13 & $(1.76 \pm 1.35) \mathrm{E}-11$ & $(3.52 \pm 0.91) E-11$ & $(-1.68 \pm 0.48) E-11$ \\
\hline \multicolumn{5}{|l|}{$\mathrm{Pu}-238, \mu \mathrm{Ci} / \mathrm{mL}$} \\
\hline \multicolumn{5}{|l|}{ Upper Three Runs } \\
\hline U3R-4 at Road A & 10 & $(-0.64 \pm 2.13) E-09$ & $(1.47 \pm 0.20) E-10$ & $(-6.70 \pm 2.99) E-09$ \\
\hline \multicolumn{5}{|l|}{ Four Mile Creek } \\
\hline FM-6 at Road A-12.2 & 13 & $(1.95 \pm 2.48) E-11$ & $(8.52 \pm 1.33) E-11$ & $(-5.90 \pm 3.97) E-12$ \\
\hline \multicolumn{5}{|l|}{ Pen Branch } \\
\hline PB-3 at Road A-13.2 & 12 & $(0.47 \pm 5.11) E-11$ & $(6.00 \pm 1.29) E-11$ & $(-1.46 \pm 0.71) E-10$ \\
\hline \multicolumn{5}{|l|}{ Lower Three Runs Creek } \\
\hline L3R-2(weekly grab) & 24 & $(-0.69 \pm 3.45) E-10$ & $(5.73 \pm 1.03) \mathrm{E}-11$ & $(-1.63 \pm 0.82) E-09$ \\
\hline L3R-2 Patterson Mill Road & 11 & $(2.08 \pm 2.25) E-11$ & $(6.63 \pm 1.48) E-11$ & $(-7.84 \pm 2.64) E-12$ \\
\hline
\end{tabular}




\section{Table 24}

\section{Radioactivity in Savannah River Site Stream Water}

Page 4 of 7

\begin{tabular}{|c|c|c|c|c|}
\hline Location & $\begin{array}{l}\text { No. of } \\
\text { Samples }\end{array}$ & Arithmetic Mean $\pm \sigma$ & Maximum $\pm \sigma$ & Minimum $\pm \sigma$ \\
\hline \multicolumn{5}{|l|}{$\mathrm{Pu}-239, \mu \mathrm{Ci} / \mathrm{mL}$} \\
\hline \multicolumn{5}{|l|}{ Upper Three Runs } \\
\hline U3R-4 at Road A & 10 & $(1.95 \pm 7.62) E-11$ & $(2.31 \pm 3.89) E-10$ & $(-4.95 \pm 0.36) E-11$ \\
\hline Four Mile Creek & & & & ' \\
\hline FM-6 at Road A-12.2 & 13 & $(-0.10 \pm 1.62) E-11$ & $(3.08 \pm 0.87) E-11$ & $(-4.64 \pm 0.47) E-11$ \\
\hline \multicolumn{5}{|l|}{ Pen Branch } \\
\hline PB-3 at Road A-13.2 & 11 & $(-0.48 \pm 1.54) E-11$ & $(2.79 \pm 2.31) E-12$ & $(-5.08 \pm 0.27) E-11$ \\
\hline \multicolumn{5}{|l|}{ Lower Three Runs Creek } \\
\hline L3R-2(weekly grab) & 23 & $(-0.51 \pm 1.90) E-10$ & $(3.02 \pm 0.82) E-11$ & $(-8.14 \pm 5.76) E-10$ \\
\hline L3R-2 Patterson Mill Road & 10 & $(-0.41 \pm 1.60) E-11$ & $(5.24 \pm 4.68) \mathrm{E}-12$ & $(-4.93 \pm 0.36) E-11$ \\
\hline \multicolumn{5}{|l|}{ Sr-89,90, $\mu \mathrm{Ci} / \mathrm{mL}$} \\
\hline \multicolumn{5}{|l|}{ Upper Three Runs } \\
\hline Crouch Branch at Road 4 & 12 & $(6.69 \pm 9.47) E-10$ & $(2.32 \pm 0.37) E-09$ & $(-2.87 \pm 4.25) E-10$ \\
\hline U3R-3 at Road C & 12 & $(7.10 \pm 9.22) E-10$ & $(2.72 \pm 0.50) E-09$ & $(-1.82 \pm 2.89) E-10$ \\
\hline \multicolumn{5}{|l|}{ Four Mile Creek } \\
\hline FM-2 at Road 4 & 12 & $(2.57 \pm 1.18) E-09$ & $(5.89 \pm 0.58) E-09$ & $(1.61 \pm 0.34) E-09$ \\
\hline $\begin{array}{l}\text { FM-2B above F-Area } \\
\text { effluent }\end{array}$ & 12 & $(6.35 \pm 1.47) E-09$ & $(9.26 \pm 0.83) E-09$ & $(4.29 \pm 0.50) E-09$ \\
\hline $\begin{array}{l}\text { FM-3A below F-Area } \\
\text { effluent }\end{array}$ & 12 & $(2.34 \pm 2.77) E-09$ & $(9.75 \pm 0.95) \mathrm{E}-09$ & $(3.33 \pm 8.94) \mathrm{E}-10$ \\
\hline FM-6 at Road A-12.2 & 12 & $(4.23 \pm 0.81) E-09$ & $(5.52 \pm 0.63) E-09$ & $(2.12 \pm 0.24) E-09$ \\
\hline FM-A7 at Road A-7 & 12 & $(8.90 \pm 1.26) E-09$ & $(1.15 \pm 0.10) E-08$ & $(6.80 \pm 0.75) E-09$ \\
\hline $\mathrm{H}-008$ Outfall & 12 & $(5.56 \pm 6.04) \mathrm{E}-10$ & $(1.98 \pm 0.42) E-09$ & $(-0.59 \pm 4.11) E-10$ \\
\hline \multicolumn{5}{|l|}{ Pen Branch } \\
\hline $\begin{array}{l}\text { IGB-21 } 800 \text { feet south of } \\
\text { Road 6-1 }\end{array}$ & 4 & $(2.30 \pm 5.33) \mathrm{E}-10$ & $(9.60 \pm 2.95) \mathrm{E}-10$ & $(-2.73 \pm 3.99) E-10$ \\
\hline $\mathrm{K}-011$ Outfall at Road B & 12 & $(5.36 \pm 7.10) E-10$ & $(1.90 \pm 0.40) E-09$ & $(-2.45 \pm 3.72) E-10$ \\
\hline PB-3 at Road A-13.2 & 12 & $(2.27 \pm 2.31) E-10$ & $(6.32 \pm 1.10) \mathrm{E}-10$ & $(-0.89 \pm 1.40) E-10$ \\
\hline \multicolumn{5}{|l|}{ Steel Creek } \\
\hline Road B & 12 & $(5.00 \pm 6.18) E-10$ & $(1.37 \pm 0.34) \mathrm{E}-09$ & $(-1.69 \pm 1.71) E-10$ \\
\hline Road A & 12 & $(1.61 \pm 1.70) \mathrm{E}-10$ & $(4.39 \pm 1.14) E-10$ & $(-1.39 \pm 3.42) E-10$ \\
\hline \multicolumn{5}{|l|}{ Lower Three Runs Creek } \\
\hline L3R-1A at Road B & 12 & $(5.60 \pm 5.14) \mathrm{E}-10$ & $(1.67 \pm 0.36) E-09$ & $(-0.31 \pm 1.82) E-10$ \\
\hline L3R-2 Patterson Mill Road & 11 & $(2.12 \pm 1.17) E-10$ & $(3.51 \pm 3.70) E-10$ & $(-0.21 \pm 1.36) E-10$ \\
\hline L3R-3 at Highway 125 & 12 & $(6.58 \pm 4.47) E-10$ & $(1.53 \pm 0.37) \mathrm{E}-09$ & $(1.07 \pm 4.38) E-10$ \\
\hline
\end{tabular}


Table 24

Radioactivity in Savannah River Site Stream Water

Page 5 of 7

\begin{tabular}{|c|c|c|c|c|}
\hline Location & $\begin{array}{l}\text { No. of } \\
\text { Samples }\end{array}$ & Arithmetic Mean $\pm \sigma$ & Maximum $\pm \sigma$ & Minimum $\pm \sigma$ \\
\hline \multicolumn{5}{|l|}{ U/Pu, $\mu \mathrm{Ci} / \mathrm{mL}$} \\
\hline \multicolumn{5}{|l|}{ Upper Three Runs } \\
\hline U3R-4 at Road $A$ & 13 & $(4.51 \pm 3.98) E-10$ & $(1.36 \pm 0.56) \mathrm{E}-09$ & $(-1.04 \pm 2.79) E-10$ \\
\hline \multicolumn{5}{|l|}{ Four Mile Creek } \\
\hline FM-6 at Road A-12.2 & 13 & $(3.09 \pm 3.59) E-10$ & $(9.56 \pm 5.58) \mathrm{E}-10$ & $(-1.00 \pm 2.68) E-10$ \\
\hline \multicolumn{5}{|l|}{ Pen Branch } \\
\hline PB-3 at Road A-13.2 & 13 & $(1.47 \pm 2.60) E-10$ & $(6.86 \pm 4.91) \mathrm{E}-10$ & $(-2.66 \pm 2.10) E-10$ \\
\hline \multicolumn{5}{|l|}{ Lower Three Runs Creek } \\
\hline L3R-(weekly grab) & 28 & $(0.32 \pm 2.72) E-10$ & $(7.38 \pm 4.34) \mathrm{E}-10$ & $(-3.94 \pm 3.47) E-10$ \\
\hline L3R-2 Patterson Mill Road & 13 & $(0.82 \pm 4.59) E-10$ & $(1.49 \pm 0.67) E-09$ & $(-2.66 \pm 2.10) E-10$ \\
\hline \multicolumn{5}{|l|}{ Gross Beta, $\mu \mathrm{Ci} / \mathrm{mL}$} \\
\hline \multicolumn{5}{|l|}{ Tims Branch } \\
\hline TB-5 near Road C & 25 & $(2.39 \pm 0.99) E-09$ & $(5.23 \pm 0.39) \mathrm{E}-09$ & $(1.35 \pm 0.28) E-09$ \\
\hline \multicolumn{5}{|l|}{ Upper Three Runs } \\
\hline Crouch Branch at Road 4 & 25 & $(1.65 \pm 0.88) E-09$ & $(3.25 \pm 0.68) E-09$ & $(4.47 \pm 3.43) E-10$ \\
\hline $\begin{array}{l}\text { U3R-1A Treadway Bridge } \\
\text { Road 8-1 }\end{array}$ & 50 & $(1.59 \pm 0.72) E-09$ & $(3.22 \pm 0.51) E-09$ & $(-0.22 \pm 3.56) E-10$ \\
\hline U3R-3 at Road C & 25 & $(1.40 \pm 0.66) E-09$ & $(2.68 \pm 0.42) E-09$ & $(2.55 \pm 3.30) E-10$ \\
\hline U3R-4 at Road A & 24 & $(1.27 \pm 0.59) E-09$ & $(2.68 \pm 0.38) E-09$ & $(0.66 \pm 7.35) E-10$ \\
\hline \multicolumn{5}{|l|}{ Four Mile Creek } \\
\hline FM-2 at Road 4 & 25 & $(9.83 \pm 6.24) E-09$ & $(3.43 \pm 0.13) E-08$ & $(2.38 \pm 0.55) E-09$ \\
\hline $\begin{array}{l}\text { FM-2B above F-Area efflu- } \\
\text { ent }\end{array}$ & 26 & $(1.84 \pm 0.52) E-08$ & $(2.83 \pm 0.13) E-08$ & $(9.87 \pm 0.84) E-09$ \\
\hline $\begin{array}{l}\text { FM-3A below F-Area efflu- } \\
\text { ent }\end{array}$ & 26 & $(9.60 \pm 8.18) E-09$ & $(3.17 \pm 0.13) E-08$ & $(2.74 \pm 0.59) E-09$ \\
\hline FM-6 at Road A-12.2 & 26 & $(1.03 \pm 0.26) E-08$ & $(1.92 \pm 0.14) E-08$ & $(6.67 \pm 0.48) E-09$ \\
\hline FM-A7 at Road $A-7$ & 26 & $(2.38 \pm 0.50) E-08$ & $(3.41 \pm 0.14) E-08$ & $(1.47 \pm 0.08) E-08$ \\
\hline $\mathrm{H}-008$ Outfall & 51 & $(6.41 \pm 2.52) E-09$ & $(1.31 \pm 0.11) \mathrm{E}-08$ & $(-3.59 \pm 0.49) E-09$ \\
\hline $\begin{array}{l}\text { HP-50 Tritium Facility Out- } \\
\text { fall }\end{array}$ & 25 & $(3.53 \pm 1.62) E-09$ & $(8.58 \pm 1.13) E-09$ & $(1.34 \pm 0.50) E-09$ \\
\hline \multicolumn{5}{|l|}{ Pen Branch } \\
\hline $\begin{array}{l}\text { IGB-21 } 800 \text { feet south of } \\
\text { Road } 6-1\end{array}$ & 3 & $(1.24 \pm 0.86) E-09$ & $(2.22 \pm 0.43) \mathrm{E}-09$ & $(6.17 \pm 3.56) E-10$ \\
\hline $\mathrm{K}-011$ Outfall at Road B & 54 & $(2.21 \pm 0.66) \mathrm{E}-09$ & $(3.47 \pm 0.77) E-09$ & $(1.02 \pm 0.49) E-09$ \\
\hline PB-3 at Road A-13.2 & 26 & $(1.25 \pm 0.64) E-09$ & $(2.78 \pm 0.43) E-09$ & $(3.65 \pm 7.39) E-10$ \\
\hline
\end{tabular}




\section{Table 24}

\section{Radioactivity in Savannah River Site Stream Water}

Page 6 of 7

\begin{tabular}{|c|c|c|c|c|}
\hline Location & $\begin{array}{l}\text { No. of } \\
\text { Samples }\end{array}$ & Arithmetic Mean $\pm \sigma$ & Maximum $\pm \sigma$ & Minimum $\pm \sigma$ \\
\hline \multicolumn{5}{|l|}{ Steel Creek } \\
\hline $\begin{array}{l}\text { SC-2A } 1 \text { mile above } \\
\text { Road B }\end{array}$ & 26 & $(3.40 \pm 1.28) E-09$ & $(6.53 \pm 0.74) E-09$ & $(1.44 \pm 0.29) E-09$ \\
\hline $\begin{array}{l}\text { SC-4 Steel Creek at } \\
\text { Road A }\end{array}$ & 26 & $(1.62 \pm 0.78) E-09$ & $(3.65 \pm 0.82) E-09$ & $(5.75 \pm 3.56) \mathrm{E}-10$ \\
\hline \multicolumn{5}{|l|}{ Lower Three Runs Creek } \\
\hline L3R-1A at Road B & 26 & $(3.80 \pm 0.89) E-09$ & $(5.56 \pm 0.56) E-09$ & $(2.00 \pm 0.23) E-09$ \\
\hline L3R-2 Patterson Mill Road & 24 & $(2.24 \pm 0.79) E-09$ & $(3.67 \pm 0.60) E-09$ & $(8.78 \pm 3.80) E-10$ \\
\hline L3R-3 at Highway 125 & 12 & $(1.84 \pm 0.71) E-09$ & $(2.88 \pm 0.50) E-09$ & $(8.20 \pm 4.04) E-10$ \\
\hline R-Area downstream of $R-1$ & 53 & $(7.72 \pm 2.97) E-09$ & $(1.72 \pm 0.11) E-08$ & $(3.15 \pm 0.52) E-09$ \\
\hline \multicolumn{5}{|l|}{ Gross Alpha, $\mu \mathrm{Ci} / \mathrm{mL}$} \\
\hline \multicolumn{5}{|l|}{ Tims Branch } \\
\hline TB-5 near Road C & 25 & $(1.47 \pm 0.59) E-09$ & $(3.21 \pm 0.47) E-09$ & $(5.62 \pm 2.25) E-10$ \\
\hline \multicolumn{5}{|l|}{ Upper Three Runs } \\
\hline Crouch Branch at Road 4 & 25 & $(3.36 \pm 3.84) E-10$ & $(1.59 \pm 0.48) E-09$ & $(-0.92 \pm 1.56) E-10$ \\
\hline $\begin{array}{l}\text { U3R-1A Treadway Bridge } \\
\text { Road 8-1 }\end{array}$ & 50 & $(2.12 \pm 0.85) E-09$ & $(4.88 \pm 0.73) E-09$ & $(5.91 \pm 2.70) E-10$ \\
\hline U3R-3 at Road C & 25 & $(1.36 \pm 0.46) E-09$ & $(2.02 \pm 0.52) E-09$ & $(6.38 \pm 3.46) E-10$ \\
\hline U3R-4 at Road A & 24 & $(1.30 \pm 0.56) E-09$ & $(2.52 \pm 0.64) E-09$ & $(2.62 \pm 2.60) E-10$ \\
\hline \multicolumn{5}{|l|}{ Four Mile Creek } \\
\hline FM-2 at Road 4 & 25 & $(1.06 \pm 0.70) E-09$ & $(3.37 \pm 0.64) E-09$ & $(0.31 \pm 2.50) E-10$ \\
\hline $\begin{array}{l}\text { FM-2B above F-Area } \\
\text { effluent }\end{array}$ & 26 & $(7.58 \pm 7.13) \mathrm{E}-10$ & $(3.65 \pm 0.75) E-09$ & $(0.46 \pm 2.22) E-10$ \\
\hline $\begin{array}{l}\text { FM-3A below F-Area } \\
\text { effluent }\end{array}$ & 26 & $(1.61 \pm 0.76) E-09$ & $(3.59 \pm 0.68) E-09$ & $(5.05 \pm 5.05) \mathrm{E}-10$ \\
\hline FM-6 at Road A-12.2 & 26 & $(2.81 \pm 3.01) \mathrm{E}-10$ & $(6.90 \pm 3.15) E-10$ & $(-2.22 \pm 1.51) E-10$ \\
\hline FM-A7 at Road A-7 & 26 & $(8.52 \pm 4.82) \mathrm{E}-10$ & $(1.58 \pm 0.51) E-09$ & $(0.47 \pm 4.17) E-10$ \\
\hline $\mathrm{H}-008$ Outfall & 51 & $(3.15 \pm 1.94) \mathrm{E}-09$ & $(8.22 \pm 1.06) E-09$ & $(-2.11 \pm 0.62) E-09$ \\
\hline $\begin{array}{l}\text { HP-50 Tritium Facility } \\
\text { Outfall }\end{array}$ & 25 & $(2.34 \pm 1.37) E-09$ & $(6.01 \pm 1.64) E-09$ & $(5.66 \pm 4.38) \mathrm{E}-10$ \\
\hline \multicolumn{5}{|l|}{ Pen Branch } \\
\hline $\begin{array}{l}\text { IGB-21 } 800 \text { feet south of } \\
\text { Road 6-1 }\end{array}$ & 3 & $(1.16 \pm 4.28) E-10$ & $(6.06 \pm 2.77) E-10$ & $(-1.87 \pm 1.31) \mathrm{E}-10$ \\
\hline K-011 Outfall at Road B & 54 & $(4.23 \pm 5.05) E-10$ & $(2.30 \pm 0.60) E-09$ & $(-1.97 \pm 1.60) E-10$ \\
\hline PB-3 at Road A-13.2 & 26 & $(1.07 \pm 2.38) E-10$ & $(6.39 \pm 4.79) E-10$ & $(-2.67 \pm 2.10) E-10$ \\
\hline
\end{tabular}




\section{Table 24}

Radioactivity in Savannah River Site Stream Water

Page 7 of 7

\begin{tabular}{lcccc}
\hline & $\begin{array}{c}\text { No. of } \\
\text { Samples }\end{array}$ & Arithmetic Mean $\pm \sigma$ & Maximum $\pm \sigma$ & Minimum $\pm \sigma$ \\
\hline $\begin{array}{l}\text { Steel Creek } \\
\quad \text { Road B } \\
\quad \text { Road A }\end{array}$ & 26 & $(4.67 \pm 4.23) \mathrm{E}-10$ & $(1.51 \pm 0.47) \mathrm{E}-09$ & $(-2.07 \pm 1.63) \mathrm{E}-10$ \\
$\begin{array}{l}\text { Lower Three Runs Creek } \\
\text { L3R-1A at Road B }\end{array}$ & 26 & $(0.84 \pm 1.95) \mathrm{E}-10$ & $(3.96 \pm 2.33) \mathrm{E}-10$ & $(-2.00 \pm 1.41) \mathrm{E}-10$ \\
$\quad$ & 26 & $(1.03 \pm 1.79) \mathrm{E}-10$ & $(4.04 \pm 2.37) \mathrm{E}-10$ & $(-2.26 \pm 2.34) \mathrm{E}-10$ \\
L3R-2 Patterson Mill Road & 24 & $(3.25 \pm 3.08) \mathrm{E}-10$ & $(9.30 \pm 4.74) \mathrm{E}-10$ & $(-1.95 \pm 3.37) \mathrm{E}-10$ \\
$\quad$ L3R-3 at Highway 125 & 12 & $(1.61 \pm 3.22) \mathrm{E}-10$ & $(8.85 \pm 3.13) \mathrm{E}-10$ & $(-2.77 \pm 2.23) \mathrm{E}-10$ \\
R-Area downstream of R-1 & 53 & $(1.77 \pm 2.52) \mathrm{E}-10$ & $(8.92 \pm 3.52) \mathrm{E}-10$ & $(-1.73 \pm 1.37) \mathrm{E}-10 \mathrm{~B}$
\end{tabular}


Table 25

Radioactivity in Transport at Sample Points on Four Mile Creek

Page 1 of 1

\begin{tabular}{|c|c|c|c|c|}
\hline \multirow[b]{2}{*}{ Location } & \multicolumn{4}{|c|}{ Tritium (Curies) } \\
\hline & 1992 & 1993 & 1994 & 1995 \\
\hline FM-3 & 5 & 4 & 13 & 2 \\
\hline $\mathrm{FM}-1 \mathrm{C}$ & 5 & 6 & 10 & 6 \\
\hline $\mathrm{HP}-52$ & & & & 1 \\
\hline $\mathrm{H}-017$ & & 1 & 1 & 1 \\
\hline $\mathrm{H}-018$ & & & & 1 \\
\hline $\mathrm{H}-008$ & 4 & 583 & 7 & 5 \\
\hline$F M-2$ & 421 & 322 & 260 & 200 \\
\hline$F M-2 B$ & 1480 & 1040 & 757 & 537 \\
\hline FM-3A & 4100 & 5320 & 3100 & 4010 \\
\hline FM-A7 & 9840 & 8560 & 6740 & 6920 \\
\hline \multirow[t]{2}{*}{ FM-6 } & 8710 & 9000 & 6980 & 7350 \\
\hline & \multicolumn{4}{|c|}{ Total Strontium (Millicuries) } \\
\hline Location & 1992 & 1993 & 1994 & 1995 \\
\hline FM-3 & 1 & $a$ & $\bar{a}$ & 2 \\
\hline FM-1C & 1 & a & 1 & 1 \\
\hline$F-012$ & & & & 1 \\
\hline$F-013$ & & & & 7 \\
\hline $\mathrm{HP}-52$ & & & & 0.1 \\
\hline $\mathrm{H}-017$ & 7 & 1 & 1 & 1 \\
\hline $\mathrm{H}-018$ & & & & 1 \\
\hline FM-2 & 42 & 26 & 43 & 15 \\
\hline FM-2B & 86 & 66 & 45 & 43 \\
\hline$F M-3 A$ & 1 & a & 2 & 5 \\
\hline $\mathrm{FM}-\mathrm{A} 7$ & 280 & 216 & 107 & 159 \\
\hline \multirow[t]{4}{*}{ FM-6 } & 219 & 227 & 113 & 143 \\
\hline & \multicolumn{4}{|c|}{ Desorption of Cesium-137 from Four Mile Creek (Millicuries) } \\
\hline & & 1993 & 1994 & \\
\hline & & $160^{\mathrm{b}}$ & 3 & \\
\hline
\end{tabular}

a No activity quantified for year

b Amount of Cs-137 estimated in transport at FM-A7 minus direct releases. This value is included in dose calculations and reflects previous releases to Four Mile Creek that were sorbed in the creek bed. 
Table 26

Radioactivity in Savannah River Water

Page 1 of 1

\begin{tabular}{|c|c|c|c|c|}
\hline Location & $\begin{array}{l}\text { No. of } \\
\text { Samples }\end{array}$ & Arithmetic Mean $\pm \sigma$ & Maximum $\pm \sigma$ & Minimum $\pm \sigma$ \\
\hline \multicolumn{5}{|l|}{$\mathrm{H}-3, \mu \mathrm{Ci} / \mathrm{mL}$} \\
\hline \multicolumn{5}{|l|}{ Edisto River } \\
\hline $\begin{array}{l}\text { E-1 Edisto River, } \\
\text { Aiken State Park }\end{array}$ & 50 & $(2.22 \pm 1.65) E-07$ & $(5.91 \pm 1.39) \mathrm{E}-07$ & $(-0.98 \pm 1.06) E-07$ \\
\hline \multicolumn{5}{|l|}{ Savannah River } \\
\hline River Mile 120 & 52 & $(1.28 \pm 0.45) E-06$ & $(2.39 \pm 0.14) E-06$ & $(4.15 \pm 1.37) E-07$ \\
\hline River Mile 140 & 52 & $(1.54 \pm 0.63) E-06$ & $(3.11 \pm 0.07) E-06$ & $(1.87 \pm 1.08) E-07$ \\
\hline River Mile 150 & 52 & $(1.74 \pm 1.00) E-06$ & $(3.27 \pm 0.11) E-06$ & $(1.87 \pm 1.26) E-07$ \\
\hline River Mile 160 & 52 & $(2.09 \pm 1.86) E-07$ & $(1.00 \pm 0.14) E-06$ & $(-0.71 \pm 1.38) E-07$ \\
\hline $\begin{array}{l}\text { River Mile } 120 \text { (Monthly } \\
\text { Grab) }\end{array}$ & 5 & $(1.05 \pm 0.58) E-06$ & $(1.49 \pm 0.15) E-06$ & $(4.08 \pm 1.10) E-07$ \\
\hline Vogtle Discharge & 52 & $(0.79 \pm 1.01) E-06$ & $(6.88 \pm 0.17) \mathrm{E}-06$ & $(0.73 \pm 1.24) E-07$ \\
\hline \multicolumn{5}{|l|}{ Gross Beta, $\mu$ Ci/mL } \\
\hline \multicolumn{5}{|l|}{ Edisto River } \\
\hline $\begin{array}{l}\text { E-1 Edisto River, } \\
\text { Aiken State Park }\end{array}$ & 48 & $(1.58 \pm 0.67) E-09$ & $(3.82 \pm 0.49) E-09$ & $(-1.00 \pm 3.73) E-10$ \\
\hline \multicolumn{5}{|l|}{ Savannah River } \\
\hline River Mile 120 & 51 & $(1.98 \pm 0.59) E-09$ & $(3.35 \pm 0.34) E-09$ & $(5.12 \pm 1.70) E-10$ \\
\hline River Mile 140 & 51 & $(2.33 \pm 0.96) E-09$ & $(5.17 \pm 0.70) E-09$ & $(7.76 \pm 2.46) E-10$ \\
\hline River Mile 150 & 51 & $(1.98 \pm 0.62) E-09$ & $(3.43 \pm 0.50) E-09$ & $(6.46 \pm 2.46) E-10$ \\
\hline River Mile 160 & 51 & $(2.19 \pm 1.52) \mathrm{E}-09$ & $(1.18 \pm 0.15) E-08$ & $(5.50 \pm 3.71) \mathrm{E}-10$ \\
\hline $\begin{array}{l}\text { River Mile } 120 \text { (Monthly } \\
\text { Grab) }\end{array}$ & 4 & $(2.07 \pm 0.86) E-09$ & $(2.94 \pm 0.46) \mathrm{E}-09$ & $(1.23 \pm 0.51) E-09$ \\
\hline Vogtle Discharge & 51 & $(1.94 \pm 0.74) E-09$ & $(4.00 \pm 0.54) E-09$ & $(4.96 \pm 2.85) E-10$ \\
\hline \multicolumn{5}{|l|}{ Gross Alpha, $\mu \mathrm{Ci} / \mathrm{mL}$} \\
\hline \multicolumn{5}{|l|}{ Edisto River } \\
\hline $\begin{array}{l}\text { E-1 Edisto River, } \\
\text { Aiken State Park }\end{array}$ & 48 & $(7.67 \pm 4.29) E-10$ & $(2.37 \pm 0.58) E-09$ & $(-0.23 \pm 1.69) E-10$ \\
\hline \multicolumn{5}{|l|}{ Savannah River } \\
\hline River Mile 120 & 51 & $(0.82 \pm 2.40) E-10$ & $(8.76 \pm 2.47) \mathrm{E}-10$ & $(-2.23 \pm 1.81) E-10$ \\
\hline River Mile 140 & 51 & $(1.96 \pm 3.44) E-10$ & $(1.65 \pm 0.59) \mathrm{E}-09$ & $(-2.37 \pm 1.88) E-10$ \\
\hline River Mile 150 & 51 & $(1.42 \pm 3.17) E-10$ & $(1.20 \pm 0.46) E-09$ & $(-3.09 \pm 2.39) E-10$ \\
\hline River Mile 160 & 51 & $(0.83 \pm 2.71) \mathrm{E}-10$ & $(7.91 \pm 4.14) \mathrm{E}-10$ & $(-3.26 \pm 2.66) E-10$ \\
\hline $\begin{array}{l}\text { River Mile } 120 \text { (Monthly } \\
\text { Grab) }\end{array}$ & 4 & $(1.85 \pm 2.60) E-10$ & $(5.20 \pm 3.96) E-10$ & $(-0.96 \pm 1.37) E-10$ \\
\hline Vogtle Discharge & 51 & $(1.73 \pm 2.92) \mathrm{E}-10$ & $(1.13 \pm 0.47) E-09$ & $(-2.66 \pm 2.10) E-10$ \\
\hline
\end{tabular}


Table 27

Summary of SRS Tritium Transport, 1960-1995

Page 1 of 1

\begin{tabular}{|c|c|c|c|}
\hline \multirow[b]{2}{*}{ Year } & \multicolumn{3}{|c|}{ Estimated Tritium Transport (Ci) } \\
\hline & $\begin{array}{c}\text { Based on Point-of- } \\
\text { Release } \\
\text { Concentrations } \\
\text { and Flow Rates }\end{array}$ & $\begin{array}{l}\text { Based on Stream } \\
\text { Concentrations and } \\
\text { Flow Rates }\end{array}$ & $\begin{array}{c}\text { Based on Savannah River } \\
\text { Concentrations and } \\
\text { Flow Rates }\end{array}$ \\
\hline 1960 & $64,000^{b}$ & 69,600 & 73,700 \\
\hline 1961 & $69,000^{b}$ & 83,000 & 77,000 \\
\hline 1962 & $58,000^{b}$ & 64,000 & 63,000 \\
\hline 1963 & $97,000^{\mathrm{b}}$ & 96,900 & 122,800 \\
\hline 1964 & $111,000^{\mathrm{b}}$ & 131,600 & 143,000 \\
\hline 1965 & 108,400 & 109,200 & 100,200 \\
\hline 1966 & 84,900 & 97,800 & 78,300 \\
\hline 1967 & 70,600 & 77,000 & 68,500 \\
\hline 1968 & 63,800 & 67,200 & 61,800 \\
\hline 1969 & 64,600 & 64,000 & 58,100 \\
\hline 1970 & 36,900 & 43,200 & 31,800 \\
\hline 1971 & 38,200 & 44,700 & 39,100 \\
\hline 1972 & 46,800 & 47,300 & 45,300 \\
\hline 1973 & 71,100 & 62,800 & 61,100 \\
\hline 1974 & 59,900 & 54,600 & 46,000 \\
\hline 1975 & 55,600 & 50,000 & 49,500 \\
\hline 1976 & 59,600 & 47,400 & 51,100 \\
\hline 1977 & 43,800 & 39,700 & 42,500 \\
\hline 1978 & 37,600 & 35,300 & 36,600 \\
\hline 1979 & 29,400 & 27,100 & 30,600 \\
\hline 1980 & 24,900 & 28,800 & 30,700 \\
\hline 1981 & 23,900 & 22,100 & 25,100 \\
\hline 1982 & 32,200 & 31,300 & 30,600 \\
\hline 1983 & 34,200 & 33,000 & 33,000 \\
\hline 1984 & 32,800 & 32,600 & 33,200 \\
\hline 1985 & 25,000 & 22,300 & 24,100 \\
\hline 1986 & 27,800 & 22,300 & 22,100 \\
\hline 1987 & 22,700 & 20,500 & 26,200 \\
\hline 1988 & 19,300 & 18,300 & 14,600 \\
\hline 1989 & 17,300 & 17,800 & 15,600 \\
\hline 1990 & 16,100 & 15,600 & 14,500 \\
\hline 1991 & 27,400 & 26,600 & 26,300 \\
\hline 1992 & 13,800 & 13,100 & 13,800 \\
\hline 1993 & 11,300 & 12,700 & 12,200 \\
\hline 1994 & 8,800 & 10,400 & 10,900 \\
\hline 1995 & 9,900 & 11,400 & 10,700 \\
\hline
\end{tabular}

a Includes direct releases to streams and migration from F-Area, $\mathrm{H}$-Area, $\mathrm{K}$-Area, and P-Area seepage basins and the Solid Waste Disposal Facility to streams

b Includes heat exchanger cooling water released from P-Area (of PAR Pond origin) to Steel Creek 
Table 28

Radioactivity in Drinking Water

Page 1 of 10

\begin{tabular}{|c|c|c|c|c|}
\hline Location & $\begin{array}{l}\text { No. of } \\
\text { Samples }\end{array}$ & Arithmetic Mean $\pm \sigma$ & Maximum $\pm \sigma$ & Minimum $\pm \sigma$ \\
\hline \multicolumn{5}{|l|}{$\mathrm{H}-3, \mu \mathrm{Ci} / \mathrm{mL}$} \\
\hline \multicolumn{5}{|l|}{ On Site } \\
\hline $\begin{array}{l}105-C \text { C-Area domestic } \\
\text { water faucet }\end{array}$ & 8 & $(1.69 \pm 1.77) E-07$ & $(3.92 \pm 1.38) E-07$ & $(-0.51 \pm 1.36) E-07$ \\
\hline $\begin{array}{l}\text { 105-K K-Area domestic } \\
\text { water faucet }\end{array}$ & 12 & $(1.44 \pm 1.43) E-07$ & $(3.29 \pm 1.35) E-07$ & $(-0.82 \pm 1.36) E-07$ \\
\hline $\begin{array}{l}\text { 105-L L-Area domestic } \\
\text { water faucet }\end{array}$ & 12 & $(1.00 \pm 1.52) E-07$ & $(3.66 \pm 1.36) E-07$ & $(-1.37 \pm 1.29) E-07$ \\
\hline $\begin{array}{l}\text { 105-P P-Area domestic } \\
\text { water faucet }\end{array}$ & 12 & $(1.41 \pm 2.94) E-06$ & $(1.00 \pm 0.01) E-05$ & $(-1.71 \pm 1.43) E-07$ \\
\hline $\begin{array}{l}\text { 221-F F-Area domestic } \\
\text { water faucet }\end{array}$ & 12 & $(0.46 \pm 1.63) E-07$ & $(3.62 \pm 1.35) E-07$ & $(-1.75 \pm 1.35) E-07$ \\
\hline $\begin{array}{l}221-\mathrm{H} \text { H-Area domestic } \\
\text { water faucet }\end{array}$ & 12 & $(0.60 \pm 2.01) E-07$ & $(3.71 \pm 1.35) E-07$ & $(-2.51 \pm 1.50) E-07$ \\
\hline $\begin{array}{l}241-24 \mathrm{H} \mathrm{H}-\text { Area domestic } \\
\text { water faucet }\end{array}$ & 4 & $(1.93 \pm 1.36) E-07$ & $(3.38 \pm 0.79) E-07$ & $(0.52 \pm 1.26) E-07$ \\
\hline $\begin{array}{l}617-G \text { Wackenhut training } \\
\text { facility }\end{array}$ & 4 & $(7.13 \pm 3.50) E-07$ & $(1.20 \pm 0.15) E-06$ & $(3.71 \pm 1.27) E-07$ \\
\hline $\begin{array}{l}\text { 618-G class. yard } \\
\text { lunchroom }\end{array}$ & 4 & $(0.23 \pm 1.10) E-07$ & $(1.61 \pm 1.34) E-07$ & $(-0.94 \pm 1.42) E-07$ \\
\hline $\begin{array}{l}\text { 661-G firing range } \\
\text { (pumphouse) }\end{array}$ & 4 & $(1.16 \pm 0.29) E-06$ & $(1.55 \pm 0.12) E-06$ & $(9.03 \pm 1.30) E-07$ \\
\hline $\begin{array}{l}\text { 679-T TNX domestic water } \\
\text { faucet }\end{array}$ & 4 & $(1.16 \pm 2.30) E-07$ & $(3.24 \pm 1.45) E-07$ & $(-1.22 \pm 1.24) E-07$ \\
\hline $\begin{array}{l}681-3 G \text { domestic water } \\
\text { faucet }\end{array}$ & 4 & $(-0.29 \pm 1.16) E-07$ & $(0.74 \pm 1.45) E-07$ & $(-1.45 \pm 1.30) E-07$ \\
\hline 701-12G Patrol Gate 7 & 4 & $(7.23 \pm 3.82) E-07$ & $(1.15 \pm 0.15) E-06$ & $(2.34 \pm 1.27) E-07$ \\
\hline 701-13G Patrol Gate 6 & 4 & $(2.05 \pm 1.24) E-06$ & $(2.89 \pm 0.16) E-06$ & $(2.03 \pm 0.80) E-07$ \\
\hline $\begin{array}{l}701-1 F \text { F-Area domestic } \\
\text { water faucet }\end{array}$ & 4 & $(0.93 \pm 1.00) E-07$ & $(1.91 \pm 1.26) E-07$ & $(-0.13 \pm 1.24) E-07$ \\
\hline $\begin{array}{l}701-1 \mathrm{H} \mathrm{H} \text {-Area domestic } \\
\text { water faucet }\end{array}$ & 4 & $(1.16 \pm 1.10) E-07$ & $(2.59 \pm 1.33) E-07$ & $(-0.07 \pm 1.45) E-07$ \\
\hline 701-3G Barnwell Gate & 4 & $(0.50 \pm 1.76) E-07$ & $(3.07 \pm 1.08) E-07$ & $(-0.76 \pm 1.30) E-07$ \\
\hline 701-4G Williston Gate & 4 & $(1.97 \pm 1.38) E-07$ & $(3.61 \pm 1.07) E-07$ & $(0.26 \pm 1.30) E-07$ \\
\hline 701-5G Talatha Gate & 4 & $(5.57 \pm 0.47) E-07$ & $(6.13 \pm 0.97) E-07$ & $(4.97 \pm 1.28) E-07$ \\
\hline $701-6 G$ Jackson Gate & 4 & $(1.08 \pm 1.63) E-07$ & $(3.29 \pm 1.07) \mathrm{E}-07$ & $(-0.27 \pm 1.24) E-07$ \\
\hline $701-8 G$ Patrol Gate 8 & 4 & $(1.75 \pm 0.92) E-06$ & $(2.35 \pm 0.16) E-06$ & $(3.84 \pm 1.09) E-07$ \\
\hline 703-A EOC (kitchen) & 4 & $(1.27 \pm 2.01) E-07$ & $(3.93 \pm 1.36) E-07$ & $(-9.48 \pm 9.14) E-08$ \\
\hline 704-F F-Area domestic & 4 & $(0.04 \pm 2.26) E-07$ & $(2.97 \pm 1.34) E-07$ & $(-2.33 \pm 1.36) E-07$ \\
\hline
\end{tabular}




\section{Table 28}

\section{Radioactivity in Drinking Water}

Page 2 of 10

\begin{tabular}{|c|c|c|c|c|}
\hline Location & $\begin{array}{l}\text { No. of } \\
\text { Samples }\end{array}$ & Arithmetic Mean $\pm \sigma$ & Maximum $\pm \sigma$ & Minimum $\pm \sigma$ \\
\hline $\begin{array}{l}\text { 704-H H-Area domestic } \\
\text { water faucet }\end{array}$ & 4 & $(0.86 \pm 2.46) E-07$ & $(4.20 \pm 1.35) E-07$ & $(-1.63 \pm 1.31) E-07$ \\
\hline $\begin{array}{l}704-S \text { S-Area domestic } \\
\text { water faucet }\end{array}$ & 4 & $(-1.29 \pm 7.63) E-08$ & $(0.86 \pm 1.07) E-07$ & $(-0.89 \pm 1.44) E-07$ \\
\hline $\begin{array}{l}\text { 708-A A-Area cafeteria } \\
\text { (restroom) }\end{array}$ & 4 & $(0.17 \pm 2.05) E-07$ & $(1.89 \pm 1.46) E-07$ & $(-2.67 \pm 1.28) E-07$ \\
\hline 735-7G PAR Pond Lab & 4 & $(0.51 \pm 1.48) E-07$ & $(2.66 \pm 1.44) \mathrm{E}-07$ & $(-0.57 \pm 1.04) E-07$ \\
\hline $760-G$ Forestry Building & 4 & $(8.79 \pm 4.12) E-07$ & $(1.19 \pm 0.11) E-06$ & $(2.79 \pm 0.75) E-07$ \\
\hline $\begin{array}{l}\text { 772-F F-Area domestic } \\
\text { water faucet }\end{array}$ & 4 & $(1.42 \pm 2.22) E-07$ & $(4.17 \pm 1.38) \mathrm{E}-07$ & $(-0.52 \pm 1.51) E-07$ \\
\hline TC-1 B-Area cafeteria & 4 & $(-0.44 \pm 1.69) E-07$ & $(1.15 \pm 1.05) E-07$ & $(-2.56 \pm 1.23) E-07$ \\
\hline Z-Area Building 704-Z & 4 & $(0.90 \pm 1.09) E-07$ & $(2.22 \pm 1.07) \mathrm{E}-07$ & $(-0.45 \pm 1.46) E-07$ \\
\hline \multicolumn{5}{|l|}{ Surrounding Towns } \\
\hline Aiken, S.C., Post Office & 1 & $(1.67 \pm 9.91) E-08$ & & \\
\hline Allendale, S.C., Post Office & 1 & $(4.47 \pm 1.36) E-07$ & & \\
\hline $\begin{array}{l}\text { Augusta, Ga., Post Office } \\
\text { 8th St }\end{array}$ & 1 & $(2.88 \pm 1.35) E-07$ & & \\
\hline Barnwell, S.C., Post Office & 1 & $(0.07 \pm 1.38) E-07$ & & \\
\hline Bath, S.C., Post Office & 1 & $(3.12 \pm 1.39) \mathrm{E}-07$ & & \\
\hline Blackville, S.C., Post Office & 1 & $(-0.27 \pm 1.37) E-07$ & & \\
\hline $\begin{array}{l}\text { Clearwater, S.C., Post } \\
\text { Office }\end{array}$ & 1 & $(4.57 \pm 1.35) E-07$ & & \\
\hline Jackson, S.C., Post Office & 1 & $(8.56 \pm 1.42) E-07$ & & \\
\hline Langley, S.C., Post Office & 1 & $(-0.99 \pm 1.38) E-07$ & & \\
\hline $\begin{array}{l}\text { North Augusta, S.C., Post } \\
\text { Office }\end{array}$ & 1 & $(1.77 \pm 1.39) \mathrm{E}-07$ & & \\
\hline $\begin{array}{l}\text { New Ellenton, S.C., Post } \\
\text { Office }\end{array}$ & 1 & $(9.40 \pm 1.44) \mathrm{E}-07$ & & \\
\hline ORA Site, ORA Building & 1 & $(-7.63 \pm 9.77) E-08$ & & \\
\hline $\begin{array}{l}\text { Sardis, Ga., Post Office, } \\
\text { Farm St }\end{array}$ & 1 & $(3.39 \pm 1.35) \mathrm{E}-07$ & & \\
\hline $\begin{array}{l}\text { Talatha, S.C., tap near } \\
\text { water tank }\end{array}$ & 1 & $(3.57 \pm 1.40) E-07$ & & \\
\hline $\begin{array}{l}\text { Waynesboro, Ga., Post } \\
\text { Office }\end{array}$ & 1 & $(-0.05 \pm 1.37) E-07$ & & \\
\hline Williston, S.C., Post Office & 1 & $(4.10 \pm 1.36) E-07$ & & \\
\hline
\end{tabular}


Table 28

Radioactivity in Drinking Water

Page 3 of 10

\begin{tabular}{|c|c|c|c|c|}
\hline Location & $\begin{array}{l}\text { No. of } \\
\text { Samples }\end{array}$ & Arithmetic Mean $\pm \sigma$ & Maximum $\pm \sigma$ & Minimum $\pm \sigma$ \\
\hline \multicolumn{5}{|l|}{ Treatment Plants } \\
\hline $\begin{array}{l}\text { Beaufort Public Water } \\
\text { Works - raw }\end{array}$ & 12 & $(8.64 \pm 2.59) E-07$ & $(1.34 \pm 0.15) E-06$ & $(4.31 \pm 1.32) E-07$ \\
\hline $\begin{array}{l}\text { Beaufort Public Water } \\
\text { Works - finished }\end{array}$ & 11 & $(8.25 \pm 2.26) E-07$ & $(1.15 \pm 0.13) E+03$ & $(5.17 \pm 1.31) E+02$ \\
\hline $\begin{array}{l}\text { D-Area Powerhouse Lab - } \\
\text { raw }\end{array}$ & 12 & $(2.44 \pm 1.69) E-07$ & $(5.82 \pm 0.98) E-07$ & $(-0.70 \pm 1.43) E-07$ \\
\hline $\begin{array}{l}\text { D-Area Powerhouse Lab - } \\
\text { finished }\end{array}$ & 12 & $(0.06 \pm 1.35) E-07$ & $(2.31 \pm 0.96) E+02$ & $(-1.66 \pm 1.27) E+02$ \\
\hline $\begin{array}{l}\text { North Augusta Public } \\
\text { Water Works - raw }\end{array}$ & 12 & $(1.26 \pm 0.99) E-07$ & $(2.80 \pm 1.37) E-07$ & $(-0.55 \pm 1.27) E-07$ \\
\hline $\begin{array}{l}\text { North Augusta Public } \\
\text { Water Works - finished }\end{array}$ & 12 & $(1.57 \pm 1.48) E-07$ & $(4.42 \pm 1.36) E+02$ & $(-0.85 \pm 1.41) \mathrm{E}+02$ \\
\hline $\begin{array}{l}\text { City of Savannah Industrial } \\
\text { and Domestic Water } \\
\text { Supply Plant }{ }^{\mathrm{a}}-\text { raw }\end{array}$ & 12 & $(1.02 \pm 0.19) E-06$ & $(1.33 \pm 0.15) E-06$ & $(8.25 \pm 1.39) E-07$ \\
\hline $\begin{array}{l}\text { City of Savannah Industrial } \\
\text { and Domestic Water } \\
\text { Supply Plant }{ }^{\mathrm{a}}-\text { finished }\end{array}$ & 12 & $(9.82 \pm 3.43) E-07$ & $(1.50 \pm 0.13) E+03$ & $(4.66 \pm 1.31) E+02$ \\
\hline \multicolumn{5}{|l|}{ EMS Sampler } \\
\hline $\begin{array}{l}\text { Beaufort Public Water } \\
\text { Works - raw }\end{array}$ & 16 & $(7.78 \pm 2.46) E-07$ & $(1.22 \pm 0.15) E-06$ & $(4.19 \pm 0.90) E-07$ \\
\hline \multicolumn{5}{|l|}{$\mathrm{Sr}-90, \mu \mathrm{Ci} / \mathrm{mL}$} \\
\hline \multicolumn{5}{|l|}{ On Site } \\
\hline $\begin{array}{l}617-G \text { Wackenhut training } \\
\text { facility }\end{array}$ & 1 & $(2.44 \pm 3.73) E-10$ & & \\
\hline $\begin{array}{l}\text { 618-G class. yard } \\
\text { lunchroom }\end{array}$ & 1 & $(0.36 \pm 3.41) E-10$ & & \\
\hline $\begin{array}{l}661-G \text { firing range } \\
\text { (pumphouse) }\end{array}$ & 1 & $(3.48 \pm 4.25) \mathrm{E}-10$ & & \\
\hline $\begin{array}{l}679-T \text { TNX domestic water } \\
\text { faucet }\end{array}$ & 1 & $(-3.58 \pm 3.83) E-10$ & & \\
\hline 701-3G Barnwell Gate & 1 & $(1.73 \pm 3.34) E-10$ & & \\
\hline 701-4G Williston Gate & 1 & $(1.04 \pm 3.72) E-10$ & & \\
\hline 701-5G Talatha Gate & 1 & $(1.07 \pm 3.94) E-10$ & & \\
\hline $760-G$ Forestry Building & 1 & $(1.20 \pm 4.08) E-10$ & & \\
\hline
\end{tabular}

a This water treatment plant, formerly the Cherokee Hill Water Treatment Plant, supplies water primarily to industries in Port Wentworth, Ga. 
Table 28

Radioactivity in Drinking Water

Page 4 of 10

\begin{tabular}{|c|c|c|c|c|}
\hline Location & $\begin{array}{l}\text { No. of } \\
\text { Samples }\end{array}$ & Arithmetic Mean $\pm \sigma$ & Maximum $\pm \sigma$ & Minimum $\pm \sigma$ \\
\hline \multicolumn{5}{|l|}{ Surrounding Towns } \\
\hline Aiken, S.C., Post Office & 1 & $(-9.42 \pm 7.19) E-10$ & & \\
\hline Allendale, S.C., Post Office & 1 & $(-1.11 \pm 5.56) E-10$ & & \\
\hline $\begin{array}{l}\text { Augusta, Ga., Post Office, } \\
\text { 8th St }\end{array}$ & 1 & $(-1.34 \pm 0.76) E-09$ & & \\
\hline Barnwell, S.C., Post Office & 1 & $(-3.05 \pm 7.50) E-10$ & & \\
\hline Bath, S.C., Post Office & 1 & $(-2.49 \pm 5.94) E-10$ & & \\
\hline $\begin{array}{l}\text { Clearwater, S.C., Post } \\
\text { Office }\end{array}$ & 1 & $(-6.37 \pm 7.39) E-10$ & & \\
\hline Jackson, S.C., Post Office & 1 & $(-4.68 \pm 5.34) E-10$ & & \\
\hline Langley, S.C., Post Office & 1 & $(-2.97 \pm 6.61) E-10$ & & \\
\hline $\begin{array}{l}\text { North Augusta, S.C., Post } \\
\text { Office }\end{array}$ & 1 & $(5.58 \pm 6.27) E-10$ & & \\
\hline $\begin{array}{l}\text { Talatha, S.C., tap near } \\
\text { water tank }\end{array}$ & 1 & $(4.17 \pm 8.34) E-10$ & & \\
\hline \multicolumn{5}{|l|}{ Treatment Plants } \\
\hline $\begin{array}{l}\text { Beaufort Public Water } \\
\text { Works - raw }\end{array}$ & 1 & $(1.72 \pm 7.27) E-10$ & & \\
\hline $\begin{array}{l}\text { Beaufort Public Water } \\
\text { Works - finished }\end{array}$ & 1 & $(-1.32 \pm 4.99) \mathrm{E}-10$ & & \\
\hline $\begin{array}{l}\text { D-Area Powerhouse Lab - } \\
\text { finished }\end{array}$ & 1 & $(1.28 \pm 2.95) E-10$ & & \\
\hline \multicolumn{5}{|l|}{$\mathrm{U}-235, \mu \mathrm{Ci} / \mathrm{mL}$} \\
\hline \multicolumn{5}{|l|}{ On Site } \\
\hline $284-H$ & 1 & $(5.59 \pm 0.24) E-09$ & & \\
\hline \multicolumn{5}{|l|}{$\mathrm{Sr}-89,90, \mu \mathrm{Ci} / \mathrm{mL}$} \\
\hline \multicolumn{5}{|l|}{ On Site } \\
\hline $\begin{array}{l}\text { 681-3G domestic water } \\
\text { faucet }\end{array}$ & 1 & $(2.62 \pm 8.59) E-10$ & & \\
\hline 701-12G Patrol Gate 7 & 1 & $(8.87 \pm 7.56) \mathrm{E}-10$ & & \\
\hline 701-13G Patrol Gate 6 & 1 & $(-0.81 \pm 7.19) E-10$ & & \\
\hline $701-6$ G Jackson Gate & 1 & $(-5.36 \pm 6.49) \mathrm{E}-10$ & & \\
\hline 701-8G Patrol Gate 8 & 1 & $(1.21 \pm 7.01) \mathrm{E}-10$ & & \\
\hline TC-1 B-Area cafeteria & 1 & $(8.87 \pm 7.56) \mathrm{E}-10$ & & \\
\hline
\end{tabular}




\section{Table 28}

\section{Radioactivity in Drinking Water}

Page 5 of 10

\begin{tabular}{|c|c|c|c|c|}
\hline Location & $\begin{array}{l}\text { No. of } \\
\text { Samples }\end{array}$ & Arithmetic Mean $\pm \sigma$ & Maximum $\pm \sigma$ & Minimum $\pm \sigma$ \\
\hline \multicolumn{5}{|l|}{ Surrounding Towns } \\
\hline Blackville, S.C., Post Office & 1 & $(7.06 \pm 6.09) E-10$ & & \\
\hline $\begin{array}{l}\text { New Ellenton, S.C., Post } \\
\text { Office }\end{array}$ & 1 & $(-5.24 \pm 4.04) E-10$ & & \\
\hline ORA Site, ORA Building & 1 & $(1.67 \pm 5.37) E-10$ & & \\
\hline $\begin{array}{l}\text { Sardis, Ga., Post Office } \\
\text { Farm St }\end{array}$ & 1 & $(-2.10 \pm 8.72) E-10$ & & \\
\hline $\begin{array}{l}\text { Waynesboro, Ga., Post } \\
\text { Office }\end{array}$ & 1 & $(0.18 \pm 1.01) E-09$ & & \\
\hline Williston, S.C., Post Office & 1 & $(6.42 \pm 6.85) E-10$ & & \\
\hline \multicolumn{5}{|l|}{ Treatment Plants } \\
\hline $\begin{array}{l}\text { City of Savannah Industrial } \\
\text { and Domestic Water } \\
\text { Supply Plant }{ }^{\mathrm{a}} \text { - raw }\end{array}$ & 1 & $(-3.51 \pm 5.27) E-10$ & & \\
\hline $\begin{array}{l}\text { City of Savannah Industrial } \\
\text { and Domestic Water } \\
\text { Supply Plant }{ }^{\mathrm{a}} \text { - finished }\end{array}$ & 1 & $(2.12 \pm 4.58) E-10$ & & \\
\hline \multicolumn{5}{|l|}{ Gross Beta, $\mu \mathrm{Ci} / \mathrm{mL}$} \\
\hline \multicolumn{5}{|l|}{ On Site } \\
\hline $\begin{array}{l}\text { 105-C C-Area domestic } \\
\text { water faucet }\end{array}$ & 8 & $(1.58 \pm 0.81) E-09$ & $(2.95 \pm 0.72) E-09$ & $(1.81 \pm 3.52) \mathrm{E}-10$ \\
\hline $\begin{array}{l}\text { 105-K K-Area domestic } \\
\text { water faucet }\end{array}$ & 11 & $(1.83 \pm 0.72) E-09$ & $(3.08 \pm 0.49) E-09$ & $(7.10 \pm 3.90) E-10$ \\
\hline $\begin{array}{l}\text { 105-L L-Area domestic } \\
\text { water faucet }\end{array}$ & 11 & $(1.83 \pm 0.62) E-09$ & $(2.90 \pm 1.15) E-09$ & $(1.05 \pm 0.56) E-09$ \\
\hline $\begin{array}{l}\text { 105-P P-Area domestic } \\
\text { water faucet }\end{array}$ & 11 & $(2.82 \pm 2.79) E-09$ & $(1.08 \pm 0.15) E-08$ & $(9.71 \pm 4.07) E-10$ \\
\hline $\begin{array}{l}221-F \text { F-Area domestic } \\
\text { water faucet }\end{array}$ & 10 & $(5.03 \pm 1.99) E-09$ & $(9.01 \pm 1.30) E-09$ & $(1.34 \pm 0.45) E-09$ \\
\hline $\begin{array}{l}\text { 221-H H-Area domestic } \\
\text { water faucet }\end{array}$ & 10 & $(3.28 \pm 1.70) E-09$ & $(7.42 \pm 1.30) \mathrm{E}-09$ & $(1.72 \pm 0.58) E-09$ \\
\hline $\begin{array}{l}241-24 \mathrm{H} \text { H-Area domestic } \\
\text { water faucet }\end{array}$ & 4 & $(5.24 \pm 1.53) E-09$ & $(7.45 \pm 0.86) E-09$ & $(3.97 \pm 0.59) E-09$ \\
\hline $\begin{array}{l}617-G \text { Wackenhut training } \\
\text { facility }\end{array}$ & 3 & $(1.24 \pm 0.25) E-09$ & $(1.52 \pm 0.53) E-09$ & $(1.03 \pm 0.38) E-09$ \\
\hline $\begin{array}{l}\text { 618-G class. yard } \\
\text { lunchroom }\end{array}$ & 3 & $(1.59 \pm 0.46) E-09$ & $(2.02 \pm 0.54) E-09$ & $(1.10 \pm 0.42) E-09$ \\
\hline
\end{tabular}

a This water treatment plant, formerly the Cherokee Hill Water Treatment Plant, supplies water primarily to industries in Port Wentworth, Ga. 
Table 28

Radioactivity in Drinking Water

Page 6 of 10

\begin{tabular}{|c|c|c|c|c|}
\hline Location & $\begin{array}{l}\text { No. of } \\
\text { Samples }\end{array}$ & Arithmetic Mean $\pm \sigma$ & Maximum $\pm \sigma$ & Minimum $\pm \sigma$ \\
\hline $\begin{array}{l}\text { 661-G firing range } \\
\text { (pumphouse) }\end{array}$ & 3 & $(2.18 \pm 0.85) E-09$ & $(2.77 \pm 0.63) \mathrm{E}-09$ & $(1.21 \pm 0.44) E-09$ \\
\hline $\begin{array}{l}679-T \text { TNX domestic water } \\
\text { faucet }\end{array}$ & 2 & $(2.64 \pm 0.31) E-09$ & $(2.86 \pm 0.53) E-09$ & $(2.41 \pm 0.51) E-09$ \\
\hline $\begin{array}{l}681-3 G \text { domestic water } \\
\text { faucet }\end{array}$ & 3 & $(2.89 \pm 0.39) \mathrm{E}-09$ & $(3.17 \pm 0.58) E-09$ & $(2.45 \pm 0.52) E-09$ \\
\hline 701-12G Patrol Gate 7 & 3 & $(1.36 \pm 0.62) E-09$ & $(1.79 \pm 0.59) E-09$ & $(6.47 \pm 3.98) E-10$ \\
\hline 701-13G Patrol Gate 6 & 3 & $(8.43 \pm 4.40) E-10$ & $(1.20 \pm 0.54) \mathrm{E}-09$ & $(3.52 \pm 4.29) E-10$ \\
\hline $\begin{array}{l}701-1 F \text { F-Area domestic } \\
\text { water faucet }\end{array}$ & 4 & $(3.87 \pm 1.84) E-09$ & $(6.26 \pm 1.20) \mathrm{E}-09$ & $(1.84 \pm 0.47) E-09$ \\
\hline $\begin{array}{l}701-1 \mathrm{H} \mathrm{H} \text {-Area domestic } \\
\text { water faucet }\end{array}$ & 4 & $(6.73 \pm 2.80) E-09$ & $(1.03 \pm 0.06) \mathrm{E}-08$ & $(3.50 \pm 0.59) E-09$ \\
\hline 701-3G Barnwell Gate & 3 & $(4.59 \pm 2.90) E-10$ & $(6.92 \pm 4.47) E-10$ & $(1.34 \pm 3.42) E-10$ \\
\hline 701-4G Williston Gate & 4 & $(2.53 \pm 3.57) E-10$ & $(7.03 \pm 3.98) E-10$ & $(-1.63 \pm 3.21) E-10$ \\
\hline 701-5G Talatha Gate & 3 & $(2.38 \pm 1.31) E-09$ & $(3.15 \pm 0.52) E-09$ & $(8.72 \pm 4.08) \mathrm{E}-10$ \\
\hline 701-6G Jackson Gate & 3 & $(1.45 \pm 1.33) E-09$ & $(2.96 \pm 0.45) E-09$ & $(4.50 \pm 3.88) E-10$ \\
\hline 701-8G Patrol Gate 8 & 3 & $(7.36 \pm 9.63) E-10$ & $(1.76 \pm 0.56) E-09$ & $(-1.55 \pm 3.10) E-10$ \\
\hline 703-A EOC (kitchen) & 4 & $(7.55 \pm 0.88) E-10$ & $(8.36 \pm 4.74) E-10$ & $(6.76 \pm 4.75) E-10$ \\
\hline $\begin{array}{l}\text { 704-F F-Area domestic } \\
\text { water faucet }\end{array}$ & 2 & $(2.05 \pm 2.52) E-09$ & $(3.83 \pm 0.73) \mathrm{E}-09$ & $(2.75 \pm 7.29) \mathrm{E}-10$ \\
\hline $\begin{array}{l}\text { 704-H H-Area domestic } \\
\text { water faucet }\end{array}$ & 3 & $(7.05 \pm 1.71) E-09$ & $(8.96 \pm 1.38) E-09$ & $(5.67 \pm 0.74) \mathrm{E}-09$ \\
\hline $\begin{array}{l}\text { 704-S S-Area domestic } \\
\text { water faucet }\end{array}$ & 4 & $(2.13 \pm 0.46) E-09$ & $(2.74 \pm 0.58) E-09$ & $(1.65 \pm 0.45) E-09$ \\
\hline $\begin{array}{l}\text { 708-A A-Area } \\
\text { cafeteria(restroom) }\end{array}$ & 4 & $(1.00 \pm 0.38) E-09$ & $(1.50 \pm 0.45) E-09$ & $(6.26 \pm 4.68) E-10$ \\
\hline 735-7G PAR Pond Lab & 2 & $(2.83 \pm 0.48) E-09$ & $(3.17 \pm 0.61) E-09$ & $(2.49 \pm 0.51) E-09$ \\
\hline 760-G Forestry Building & 2 & $(1.04 \pm 0.05) E-09$ & $(1.08 \pm 0.42) E-09$ & $(1.01 \pm 0.54) E-09$ \\
\hline $\begin{array}{l}\text { 772-F F-Area domestic } \\
\text { water faucet }\end{array}$ & 4 & $(5.28 \pm 1.30) E-09$ & $(7.13 \pm 0.88) E-09$ & $(4.31 \pm 0.73) E-09$ \\
\hline TC-1 B-Area cafeteria & 2 & $(1.38 \pm 0.76) E-09$ & $(1.92 \pm 0.62) E-09$ & $(8.40 \pm 4.31) E-10$ \\
\hline Z-Area Building 704-Z & 4 & $(1.55 \pm 0.53) E-09$ & $(2.20 \pm 0.49) E-09$ & $(9.26 \pm 4.55) \mathrm{E}-10$ \\
\hline \multicolumn{5}{|l|}{ Surrounding Towns } \\
\hline Aiken, S.C., Post Office & 1 & $(2.31 \pm 0.42) E-09$ & & \\
\hline Allendale, S.C., Post Office & 1 & $(2.69 \pm 0.46) E-09$ & & \\
\hline $\begin{array}{l}\text { Augusta, Ga., Post Office } \\
\text { 8th St }\end{array}$ & 1 & $(2.04 \pm 0.39) E-09$ & & \\
\hline Barnwell, S.C., Post Office & 1 & $(1.17 \pm 0.32) E-09$ & & \\
\hline
\end{tabular}




\section{Table 28}

\section{Radioactivity in Drinking Water}

Page 7 of 10

\begin{tabular}{|c|c|c|c|c|}
\hline Location & $\begin{array}{l}\text { No. of } \\
\text { Samples }\end{array}$ & Arithmetic Mean $\pm \sigma$ & Maximum $\pm \sigma$ & Minimum $\pm \sigma$ \\
\hline Bath, S.C., Post Office & 1 & $(1.42 \pm 0.34) E-09$ & & \\
\hline Blackville, S.C., Post Office & 1 & $(3.27 \pm 0.49) E-09$ & & \\
\hline $\begin{array}{l}\text { Clearwater, S.C., Post } \\
\text { Office }\end{array}$ & 1 & $(1.33 \pm 0.35) E-09$ & & \\
\hline Jackson, S.C., Post Office & 1 & $(3.81 \pm 0.52) E-09$ & & \\
\hline Langley, S.C., Post Office & 1 & $(2.10 \pm 0.38) E-09$ & & \\
\hline $\begin{array}{l}\text { North Augusta, S.C., Post } \\
\text { Office }\end{array}$ & 1 & $(1.28 \pm 0.33) E-09$ & & \\
\hline $\begin{array}{l}\text { New Ellenton, S.C., Post } \\
\text { Office }\end{array}$ & 1 & $(2.44 \pm 0.44) E-09$ & & \\
\hline ORA Site, ORA Building & 1 & $(2.19 \pm 0.45) E-09$ & & \\
\hline $\begin{array}{l}\text { Sardis, Ga., Post Office } \\
\text { Farm St }\end{array}$ & 1 & $(3.55 \pm 0.95) E-09$ & & \\
\hline $\begin{array}{l}\text { Talatha, S.C., tap near } \\
\text { water tank }\end{array}$ & 1 & $(1.71 \pm 0.38) E-09$ & & \\
\hline $\begin{array}{l}\text { Waynesboro, Ga., Post } \\
\text { Office }\end{array}$ & 1 & $(2.56 \pm 0.48) E-09$ & & \\
\hline Williston, S.C., Post Office & 1 & $(1.82 \pm 0.39) E-09$ & & \\
\hline \multicolumn{5}{|l|}{ Treatment Plants } \\
\hline $\begin{array}{l}\text { Beaufort Public Water } \\
\text { Works - raw }\end{array}$ & 12 & $(2.04 \pm 0.52) E-09$ & $(2.75 \pm 0.48) E-09$ & $(9.45 \pm 4.91) E-10$ \\
\hline $\begin{array}{l}\text { Beaufort Public Water } \\
\text { Works - finished }\end{array}$ & 12 & $(1.80 \pm 0.66) E-09$ & $(3.09 \pm 0.52) E+00$ & $(9.33 \pm 5.24) E-01$ \\
\hline $\begin{array}{l}\text { D-Area Powerhouse Lab - } \\
\text { raw }\end{array}$ & 12 & $(1.77 \pm 0.71) E-09$ & $(3.09 \pm 0.51) E-09$ & $(8.08 \pm 2.58) E-10$ \\
\hline $\begin{array}{l}\text { D-Area Powerhouse Lab - } \\
\text { finished }\end{array}$ & 11 & $(3.34 \pm 1.06) E-09$ & $(4.74 \pm 0.60) E+00$ & $(1.53 \pm 0.73) E+00$ \\
\hline $\begin{array}{l}\text { North Augusta Public } \\
\text { Water Works - raw }\end{array}$ & 12 & $(1.54 \pm 0.55) E-09$ & $(2.64 \pm 0.32) E-09$ & $(7.71 \pm 2.92) E-10$ \\
\hline $\begin{array}{l}\text { North Augusta Public } \\
\text { Water Works - finished }\end{array}$ & 12 & $(1.74 \pm 0.61) E-09$ & $(2.73 \pm 0.41) E+00$ & $(9.27 \pm 4.59) E-01$ \\
\hline $\begin{array}{l}\text { City of Savannah Industrial } \\
\text { and Domestic Water } \\
\text { Supply Plant }{ }^{\mathrm{a}} \text { - raw }\end{array}$ & 12 & $(1.96 \pm 0.73) E-09$ & $(2.90 \pm 0.47) E-09$ & $(5.21 \pm 4.15) E-10$ \\
\hline $\begin{array}{l}\text { City of Savannah Industrial } \\
\text { and Domestic Water } \\
\text { Supply Plant }{ }^{\mathrm{a}} \text { - finished }\end{array}$ & 12 & $(1.97 \pm 0.76) E-09$ & $(3.71 \pm 0.57) E+00$ & $(1.15 \pm 0.52) E+00$ \\
\hline
\end{tabular}

a This water treatment plant, formerly the Cherokee Hill Water Treatment Plant, supplies water primarily to industries in Port Wentworth, Ga. 
Table 28

Radioactivity in Drinking Water

Page 8 of 10

\begin{tabular}{|c|c|c|c|c|}
\hline Location & $\begin{array}{l}\text { No. of } \\
\text { Samples }\end{array}$ & Arithmetic Mean $\pm \sigma$ & Maximum $\pm \sigma$ & Minimum $\pm \sigma$ \\
\hline \multicolumn{5}{|l|}{ EMS Sampler } \\
\hline $\begin{array}{l}\text { Beaufort Public Water } \\
\text { Works - raw }\end{array}$ & 16 & $(2.37 \pm 0.87) E-09$ & $(4.55 \pm 0.51) E-09$ & $(9.23 \pm 3.77) \mathrm{E}-10$ \\
\hline \multicolumn{5}{|l|}{ Gross Alpha, $\mu \mathrm{Ci} / \mathrm{mL}$} \\
\hline \multicolumn{5}{|l|}{ On Site } \\
\hline $\begin{array}{l}\text { 105-C C-Area domestic } \\
\text { water faucet }\end{array}$ & 8 & $(0.94 \pm 1.14) E-09$ & $(3.54 \pm 0.81) E-09$ & $(-0.74 \pm 1.97) E-10$ \\
\hline $\begin{array}{l}\text { 105-K K-Area domestic } \\
\text { water faucet }\end{array}$ & 11 & $(0.06 \pm 1.72) E-10$ & $(3.68 \pm 3.69) \mathrm{E}-10$ & $(-3.05 \pm 2.67) E-10$ \\
\hline $\begin{array}{l}\text { 105- } L \text { L-Area domestic } \\
\text { water faucet }\end{array}$ & 11 & $(2.41 \pm 2.46) E-10$ & $(6.16 \pm 4.35) E-10$ & $(-2.30 \pm 1.82) E-10$ \\
\hline $\begin{array}{l}\text { 105-P P-Area domestic } \\
\text { water faucet }\end{array}$ & 11 & $(4.13 \pm 7.13) E-10$ & $(1.91 \pm 0.63) E-09$ & $(-1.61 \pm 4.39) E-10$ \\
\hline $\begin{array}{l}221-F \text { F-Area domestic } \\
\text { water faucet }\end{array}$ & 10 & $(3.62 \pm 2.89) E-09$ & $(1.05 \pm 0.22) E-08$ & $(8.93 \pm 5.65) E-10$ \\
\hline $\begin{array}{l}221-\mathrm{H} \mathrm{H} \text {-Area domestic } \\
\text { water faucet }\end{array}$ & 10 & $(1.17 \pm 0.79) E-09$ & $(3.22 \pm 1.02) \mathrm{E}-09$ & $(4.00 \pm 5.97) E-10$ \\
\hline $\begin{array}{l}241-24 \mathrm{H} H \text {-Area domestic } \\
\text { water faucet }\end{array}$ & 4 & $(2.64 \pm 2.22) E-09$ & $(5.83 \pm 1.07) E-09$ & $(7.19 \pm 3.92) \mathrm{E}-10$ \\
\hline $\begin{array}{l}617-G \text { Wackenhut training } \\
\text { facility }\end{array}$ & 3 & $(7.76 \pm 3.34) E-10$ & $(1.06 \pm 0.34) E-09$ & $(4.07 \pm 4.11) \mathrm{E}-10$ \\
\hline $\begin{array}{l}\text { 618-G class. yard } \\
\text { lunchroom }\end{array}$ & 3 & $(9.74 \pm 2.62) E-10$ & $(1.23 \pm 0.44) E-09$ & $(7.08 \pm 3.52) E-10$ \\
\hline $\begin{array}{l}661-G \text { firing range } \\
\text { (pumphouse) }\end{array}$ & 3 & $(2.41 \pm 1.02) E-09$ & $(3.58 \pm 0.70) E-09$ & $(1.73 \pm 0.44) E-09$ \\
\hline $\begin{array}{l}679-T \text { TNX domestic water } \\
\text { faucet }\end{array}$ & 2 & $(1.03 \pm 0.16) E-09$ & $(1.14 \pm 0.59) E-09$ & $(9.18 \pm 4.77) \mathrm{E}-10$ \\
\hline $\begin{array}{l}681-3 G \text { domestic water } \\
\text { faucet }\end{array}$ & 3 & $(1.11 \pm 0.25) E-09$ & $(1.35 \pm 0.45) \mathrm{E}-09$ & $(8.43 \pm 7.94) E-10$ \\
\hline 701-12G Patrol Gate 7 & 3 & $(4.24 \pm 4.33) E-10$ & $(7.49 \pm 4.61) \mathrm{E}-10$ & $(-0.68 \pm 1.02) E-10$ \\
\hline 701-13G Patrol Gate 6 & 3 & $(4.60 \pm 5.46) E-10$ & $(8.14 \pm 3.85) E-10$ & $(-1.68 \pm 1.33) E-10$ \\
\hline $\begin{array}{l}701-1 F \text { F-Area domestic } \\
\text { water faucet }\end{array}$ & 4 & $(2.49 \pm 0.26) E-09$ & $(2.75 \pm 0.83) \mathrm{E}-09$ & $(2.13 \pm 0.62) E-09$ \\
\hline $\begin{array}{l}701-1 \mathrm{H} \mathrm{H} \text {-Area domestic } \\
\text { water faucet }\end{array}$ & 4 & $(2.62 \pm 1.47) E-09$ & $(4.65 \pm 0.61) E-09$ & $(1.20 \pm 0.49) E-09$ \\
\hline 701-3G Barnwell Gate & 3 & $(2.31 \pm 0.96) E-10$ & $(3.41 \pm 2.03) \mathrm{E}-10$ & $(1.70 \pm 2.60) E-10$ \\
\hline 701-4G Williston Gate & 4 & $(1.07 \pm 1.52) E-10$ & $(2.89 \pm 2.03) \mathrm{E}-10$ & $(-0.72 \pm 1.91) E-10$ \\
\hline 701-5G Talatha Gate & 3 & $(6.18 \pm 5.12) E-09$ & $(9.36 \pm 0.97) \mathrm{E}-09$ & $(2.76 \pm 1.97) \mathrm{E}-10$ \\
\hline $701-6 G$ Jackson Gate & 3 & $(1.46 \pm 1.61) E-09$ & $(3.27 \pm 0.48) E-09$ & $(1.72 \pm 1.66) \mathrm{E}-10$ \\
\hline 701-8G Patrol Gate 8 & 3 & $(6.27 \pm 4.63) E-10$ & $(1.07 \pm 0.42) E-09$ & $(1.50 \pm 1.46) E-10$ \\
\hline
\end{tabular}


Table 28

Radioactivity in Drinking Water

Page 9 of 10

\begin{tabular}{|c|c|c|c|c|}
\hline Location & $\begin{array}{l}\text { No. of } \\
\text { Samples }\end{array}$ & Arithmetic Mean $\pm \sigma$ & Maximum $\pm \sigma$ & Minimum $\pm \sigma$ \\
\hline 703-A EOC (kitchen) & 4 & $(4.85 \pm 3.63) E-10$ & $(8.27 \pm 4.85) \mathrm{E}-10$ & $(1.03 \pm 4.88) \mathrm{E}-10$ \\
\hline $\begin{array}{l}\text { 704-F F-Area domestic } \\
\text { water faucet }\end{array}$ & 2 & $(1.87 \pm 0.14) E-09$ & $(1.97 \pm 0.79) E-09$ & $(1.77 \pm 0.82) E-09$ \\
\hline $\begin{array}{l}\text { 704-H H-Area domestic } \\
\text { water faucet }\end{array}$ & 3 & $(3.09 \pm 1.22) E-09$ & $(4.48 \pm 0.88) E-09$ & $(2.17 \pm 0.63) E-09$ \\
\hline $\begin{array}{l}\text { 704-S S-Area domestic } \\
\text { water faucet }\end{array}$ & 4 & $(1.78 \pm 0.51) E-09$ & $(2.31 \pm 0.60) E-09$ & $(1.10 \pm 0.42) E-09$ \\
\hline $\begin{array}{l}\text { 708-A A-Area cafeteria } \\
\text { (restroom) }\end{array}$ & 4 & $(5.73 \pm 3.54) E-10$ & $(9.76 \pm 3.56) \mathrm{E}-10$ & $(1.13 \pm 2.32) E-10$ \\
\hline 735-7G PAR Pond Lab & 2 & $(-0.03 \pm 1.63) E-10$ & $(1.13 \pm 2.45) E-10$ & $(-1.18 \pm 3.16) E-10$ \\
\hline 760-G Forestry Building & 2 & $(5.83 \pm 4.65) E-10$ & $(9.12 \pm 4.83) \mathrm{E}-10$ & $(2.55 \pm 2.52) E-10$ \\
\hline $\begin{array}{l}772-F \text { F-Area domestic } \\
\text { water faucet }\end{array}$ & 4 & $(3.89 \pm 2.99) E-09$ & $(7.45 \pm 1.49) \mathrm{E}-09$ & $(1.12 \pm 0.61) E-09$ \\
\hline TC-1 B-Area cafeteria & 2 & $(2.20 \pm 0.44) E-09$ & $(2.51 \pm 0.82) \mathrm{E}-09$ & $(1.89 \pm 0.72) E-09$ \\
\hline Z-Area Building 704-Z & 4 & $(1.32 \pm 1.24) E-09$ & $(3.15 \pm 0.70) E-09$ & $(4.20 \pm 2.53) E-10$ \\
\hline \multicolumn{5}{|l|}{ Surrounding Towns } \\
\hline Aiken, S.C., Post Office & 1 & $(6.53 \pm 4.63) E-10$ & & \\
\hline Allendale, S.C., Post Office & 1 & $(3.81 \pm 5.24) E-10$ & & \\
\hline $\begin{array}{l}\text { Augusta, Ga., Post Office } \\
\text { 8th St }\end{array}$ & 1 & $(-0.63 \pm 2.09) E-10$ & & \\
\hline Barnwell, S.C., Post Office & 1 & $(-1.93 \pm 1.59) E-10$ & & \\
\hline Bath, S.C., Post Office & 1 & $(4.63 \pm 3.22) E-10$ & & \\
\hline Blackville, S.C., Post Office & 1 & $(9.53 \pm 6.78) E-10$ & & \\
\hline $\begin{array}{l}\text { Clearwater, S.C., Post } \\
\text { Office }\end{array}$ & 1 & $(-1.20 \pm 4.22) E-10$ & & \\
\hline Jackson, S.C., Post Office & 1 & $(4.08 \pm 0.81) E-09$ & & \\
\hline Langley, S.C., Post Office & 1 & $(3.81 \pm 2.62) E-10$ & & \\
\hline $\begin{array}{l}\text { North Augusta, S.C., Post } \\
\text { Office }\end{array}$ & 1 & $(-0.63 \pm 2.18) E-10$ & & \\
\hline $\begin{array}{l}\text { New Ellenton, S.C., Post } \\
\text { Office }\end{array}$ & 1 & $(6.80 \pm 3.52) E-10$ & & \\
\hline ORA Site, ORA Building & 1 & $(-0.96 \pm 3.24) E-10$ & & \\
\hline $\begin{array}{l}\text { Sardis, Ga., Post Office } \\
\text { Farm St }\end{array}$ & 1 & $(1.62 \pm 1.15) E-09$ & & \\
\hline $\begin{array}{l}\text { Talatha, S.C., tap near } \\
\text { water tank }\end{array}$ & 1 & $(2.61 \pm 3.55) E-10$ & & \\
\hline $\begin{array}{l}\text { Waynesboro, Ga., Post } \\
\text { Office }\end{array}$ & 1 & $(-3.71 \pm 3.03) E-10$ & & \\
\hline Williston, S.C., Post Office & 1 & $(1.22 \pm 0.73) E-09$ & & \\
\hline
\end{tabular}




\section{Table 28}

Radioactivity in Drinking Water

Page 10 of 10

\begin{tabular}{|c|c|c|c|c|}
\hline Location & $\begin{array}{l}\text { No. of } \\
\text { Samples }\end{array}$ & Arithmetic Mean $\pm \sigma$ & Maximum $\pm \sigma$ & Minimum $\pm \sigma$ \\
\hline \multicolumn{5}{|l|}{ Treatment Plants } \\
\hline $\begin{array}{l}\text { Beaufort Public Water } \\
\text { Works - raw }\end{array}$ & 12 & $(3.54 \pm 2.95) E-10$ & $(7.41 \pm 4.72) \mathrm{E}-10$ & $(-1.19 \pm 1.65) \mathrm{E}-10$ \\
\hline $\begin{array}{l}\text { Beaufort Public Water } \\
\text { Works - finished }\end{array}$ & 12 & $(0.36 \pm 3.26) E-10$ & $(6.91 \pm 4.09) E-01$ & $(-3.71 \pm 2.95) E-01$ \\
\hline $\begin{array}{l}\text { D-Area Powerhouse Lab - } \\
\text { raw }\end{array}$ & 12 & $(1.70 \pm 2.28) E-10$ & $(5.50 \pm 4.14) E-10$ & $(-2.21 \pm 1.77) E-10$ \\
\hline $\begin{array}{l}\text { D-Area Powerhouse Lab - } \\
\text { finished }\end{array}$ & 11 & $(0.18 \pm 2.47) E-10$ & $(5.87 \pm 5.51) \mathrm{E}-01$ & $(-2.80 \pm 2.25) E-01$ \\
\hline $\begin{array}{l}\text { North Augusta Public } \\
\text { Water Works - raw }\end{array}$ & 12 & $(1.18 \pm 2.05) E-10$ & $(4.50 \pm 3.25) E-10$ & $(-1.92 \pm 1.51) E-10$ \\
\hline $\begin{array}{l}\text { North Augusta Public } \\
\text { Water Works - finished }\end{array}$ & 12 & $(0.40 \pm 3.81) E-10$ & $(1.19 \pm 0.43) E+00$ & $(-2.47 \pm 1.98) E-01$ \\
\hline $\begin{array}{l}\text { City of Savannah Industrial } \\
\text { and Domestic Water } \\
\text { Supply Plant }{ }^{\mathrm{a}} \text { - raw }\end{array}$ & 12 & $(1.39 \pm 2.81) \mathrm{E}-10$ & $(6.22 \pm 3.62) E-10$ & $(-2.65 \pm 1.93) E-10$ \\
\hline $\begin{array}{l}\text { City of Savannah Industrial } \\
\text { and Domestic Water } \\
\text { Supply Plant }{ }^{\mathrm{a}}-\text { finished }\end{array}$ & 12 & $(0.20 \pm 2.34) E-10$ & $(5.00 \pm 2.71) E-01$ & $(-3.83 \pm 3.03) E-01$ \\
\hline \multicolumn{5}{|l|}{ EMS Sampler } \\
\hline $\begin{array}{l}\text { Beaufort Public Water } \\
\text { Works - raw }\end{array}$ & 16 & $(6.13 \pm 8.67) E-10$ & $(3.12 \pm 0.90) E-09$ & $(-1.11 \pm 2.93) E-10$ \\
\hline
\end{tabular}

a This water treatment plant, formerly the Cherokee Hill Water Treatment Plant, supplies water primarily to industries in Port Wentworth, Ga. 


\section{Table 29 \\ Radioactivity in Terrestrial Food Products ${ }^{a}$ - Greens, Grain, Fruit, and Beef}

Page 1 of 3

\begin{tabular}{|c|c|c|c|c|c|c|}
\hline Location & $H-3^{b}(p C i / g \pm \sigma)$ & $C s-137^{c}(\mathrm{pCi} / \mathrm{g} \pm \sigma)$ & $\mathrm{Pu}-238^{\mathrm{b}}(\mathrm{pCi} / \mathrm{g} \pm \sigma)$ & Pu-239b (pci/gto) & Sr-89,90b $(p C i / g \pm \sigma)$ & $\mathbf{U} / \mathbf{P u}^{\mathbf{b}}(\mathbf{p C i} / \mathbf{g} \pm \sigma)$ \\
\hline \multicolumn{7}{|l|}{ Greens } \\
\hline $\begin{array}{l}\text { Northeast quadrant } \\
0-10 \text { miles }\end{array}$ & $(5.43 \pm 2.36) E-02$ & & $(2.93 \pm 1.64) E-05$ & $(0.73 \pm 2.00) E-05$ & $(5.36 \pm 1.31) E-02$ & $(7.16 \pm 5.83) E-03$ \\
\hline $\begin{array}{l}\text { Northwest quadrant } \\
0-10 \text { miles }\end{array}$ & $(6.53 \pm 4.02) E-02$ & & $(1.59 \pm 2.03) E-05$ & $(0.40 \pm 1.73) E-05$ & $(1.79 \pm 1.27) E-02$ & $(-0.51 \pm 3.78) E-03$ \\
\hline $\begin{array}{l}\text { Southeast quadrant } \\
0-10 \text { miles }\end{array}$ & $(1.44 \pm 0.32) E-01$ & & $(6.44 \pm 2.00) E-05$ & $(1.21 \pm 1.21) E-05$ & $(2.52 \pm 2.05) E-02$ & $(2.09 \pm 4.69) \mathrm{E}-03$ \\
\hline $\begin{array}{l}\text { Southeast quadrant } \\
25 \text { miles }\end{array}$ & $(9.77 \pm 2.94) E-02$ & & $(4.17 \pm 2.66) E-05$ & $(0.38 \pm 2.36) E-05$ & $(-0.15 \pm 1.69) E-02$ & $(1.25 \pm 3.06) \mathrm{E}-03$ \\
\hline $\begin{array}{l}\text { Southwest quadrant } \\
0-10 \text { miles }\end{array}$ & $(6.20 \pm 3.21) E-02$ & & $(5.92 \pm 2.49) E-05$ & $(7.49 \pm 2.36) E-05$ & $(4.30 \pm 2.55) E-02$ & $(-0.50 \pm 3.73) E-03$ \\
\hline \multicolumn{7}{|l|}{ Grain } \\
\hline FS \#4 Talatha area & & & & & $(0.70 \pm 1.54) E-02$ & $(2.78 \pm 0.93) E-02$ \\
\hline FS \#13 Hilda area & & $(1.48 \pm 0.44) E-02$ & & & $(1.45 \pm 1.77) E-02$ & $(1.63 \pm 0.77) E-02$ \\
\hline FS \#14 Ulmers area & & & $(-1.21 \pm 2.29) E-05$ & $(-0.54 \pm 2.20) E-05$ & $(1.13 \pm 1.34) E-02$ & $(1.07 \pm 0.48) E-02$ \\
\hline FS \#8 Sardis area & & & $(-0.85 \pm 1.59) E-05$ & $(3.81 \pm 1.76) E-05$ & $(2.54 \pm 1.84) E-02$ & $(2.34 \pm 5.24) E-03$ \\
\hline $\begin{array}{l}\text { FS \#12 Springfield } \\
\text { area }\end{array}$ & & $(1.19 \pm 0.34) E-02$ & $(0.97 \pm 1.68) E-05$ & $(0.97 \pm 2.16) E-05$ & & $(8.15 \pm 2.37) E-02$ \\
\hline FS \#3 Jackson area & & & $(-3.84 \pm 3.95) E-05$ & $(2.55 \pm 3.25) E-05$ & $(-5.39 \pm 5.89) E-03$ & $(9.40 \pm 6.64) E-03$ \\
\hline $\begin{array}{l}\text { FS \#5 Darkhorse } \\
\text { area }\end{array}$ & & . & & & $(-4.55 \pm 4.34) E-02$ & $(2.11 \pm 0.84) E-02$ \\
\hline $\begin{array}{l}\text { FS \#11 Hwy } 19- \\
7 \text { mi. north of Aiken }\end{array}$ & & & & & $(-0.52 \pm 1.73) E-02$ & $(0.00 \pm 4.68) E-03$ \\
\hline
\end{tabular}

a All results except those for $\mathrm{H}-3$ are expressed in dry weight.

b Blank spaces indicate that no analyses were performed.

c Blank spaces indicate that activity was below the lower limit of detection. The typical lower limit of detection for Cs-137 in food products was $7.00 \mathrm{E}-03 \mathrm{pCi} / \mathrm{g}$. 
Table 29

Radioactivity in Terrestrial Food Products ${ }^{a}$ - Greens, Grain, Fruit, and Beef

Page 2 of 3

\begin{tabular}{|c|c|c|c|c|c|c|}
\hline Location & $H-3^{b}(p C i / g \pm \sigma)$ & Cs-137c (pCi/g $\pm \sigma)$ & $\mathrm{Pu}-238^{b}(\mathrm{pCi} / \mathrm{g} \pm \sigma)$ & $\mathrm{Pu}-239^{b}(\mathrm{pCi} / \mathrm{g} \pm \sigma)$ & Sr-89,90b $(p C i / g \pm \sigma)$ & $U / P u^{b}(p C i / g \pm \sigma)$ \\
\hline \multicolumn{7}{|l|}{ Fruit } \\
\hline $\begin{array}{l}\text { FS \#10 Gracewood } \\
\text { School area }\end{array}$ & $(4.72 \pm 3.50) E-02$ & & $(2.57 \pm 3.04) E-05$ & $(-2.04 \pm 2.40) E-05$ & $(-1.49 \pm 2.10) E-02$ & $(-2.34 \pm 4.06) E-03$ \\
\hline $\begin{array}{l}\text { FS \#11 Hwy } 19- \\
7 \text { mi. north of Aiken }\end{array}$ & $(5.45 \pm 2.40) E-02$ & & $(4.74 \pm 2.43) E-05$ & $(2.36 \pm 1.95) E-05$ & $(-1.78 \pm 2.07) E-02$ & $(0.00 \pm 7.84) E-03$ \\
\hline $\begin{array}{l}\text { FS \#12 Springfield } \\
\text { area }\end{array}$ & $(5.01 \pm 2.71) E-02$ & & & & $(-1.67 \pm 1.95) E-02$ & $(-2.47 \pm 4.28) E-03$ \\
\hline FS \#3 Jackson area & $(1.77 \pm 0.28) E-01$ & & $(2.70 \pm 2.63) E-05$ & $(-0.44 \pm 2.41) E-05$ & $(-1.63 \pm 1.89) E-02$ & $(4.99 \pm 6.11) E-03$ \\
\hline FS \#4 Talatha area & $(2.97 \pm 0.41) E-01$ & & $(7.39 \pm 2.25) E-05$ & $(3.17 \pm 1.50) E-05$ & $(0.37 \pm 1.94) E-02$ & $(-2.44 \pm 4.23) E-03$ \\
\hline $\begin{array}{l}\text { FS \#1 Below Barri- } \\
\text { cade \#5 }\end{array}$ & $(-2.23 \pm 0.13) E-01$ & & $(4.61 \pm 1.09) \mathrm{E}-04$ & $(5.92 \pm 5.93) E-05$ & $(0.83 \pm 1.80) E-02$ & $(0.00 \pm 4.94) E-03$ \\
\hline $\begin{array}{l}\text { FS \#9 Boyce Dairy } \\
\text { area }\end{array}$ & $(3.00 \pm 3.20) E-02$ & & $(-0.42 \pm 2.44) E-0.5$ & $(-0.42 \pm 1.93) E-05$ & $(-1.39 \pm 1.97) \mathrm{E}-02$ & $(-2.34 \pm 4.06) E-03$ \\
\hline $\begin{array}{l}\text { FS \#5 Darkhorse } \\
\text { area }\end{array}$ & $(4.18 \pm 3.39) E-02$ & & $(3.46 \pm 1.35) E-05$ & $(1.20 \pm 1.09) E-05$ & $(-0.87 \pm 1.96) E-02$ & $(2.47 \pm 5.52) E-03$ \\
\hline $\begin{array}{l}\text { FS \#7 Wade } \\
\text { Plantation vicinity }\end{array}$ & & & $(4.94 \pm 1.75) E-05$ & $(00.00 \pm 1.01) E-05$ & $(-0.32 \pm 1.87) \mathrm{E}-02$ & $(-3.80 \pm 6.59) E-02$ \\
\hline FS \#13 Hilda area & $(4.07 \pm 2.06) E-02$ & & $(-1.94 \pm 2.28) E-05$ & $(-0.96 \pm 1.67) E-05$ & $(1.61 \pm 1.97) \mathrm{E}-02$ & $(-2.49 \pm 4.32) E-03$ \\
\hline FS \#8 Sardis area & $(-1.45 \pm 0.15) E-01$ & & $(2.67 \pm 2.21) E-05$ & $(1.07 \pm 1.07) E-05$ & $(-1.38 \pm 1.36) \mathrm{E}-02$ & $(-3.33 \pm 2.88) E-03$ \\
\hline $\begin{array}{l}\text { FS \#2 near } \\
\text { Savannah River - } \\
\text { east of Sardis }\end{array}$ & $(6.47 \pm 2.60) E-02$ & & $(0.86 \pm 1.84) E-05$ & $(0.43 \pm 2.15) E-05$ & $(3.68 \pm 2.05) E-02$ & $(-2.49 \pm 4.32) E-03$ \\
\hline FS\#14 UImers area & & & $(1.23 \pm 0.88) E-05$ & $(-8.74 \pm 8.03) E-06$ & $(-1.05 \pm 1.88) E-02$ & $(-4.94 \pm 3.49) E-03$ \\
\hline
\end{tabular}

a All results except those for $\mathrm{H}-3$ are expressed in dry weight.

b Blank spaces indicate that no analyses were performed.

c Blank spaces indicate that activity was below the lower limit of detection. The typical lower limit of detection for Cs-137 in food products was $7.00 \mathrm{E}-03 \mathrm{pCi/g}$. 
Table 29

Radioactivity in Terrestrial Food Products ${ }^{\mathrm{a}}$ - Greens, Grain, Fruit, and Beef

Page 3 of 3

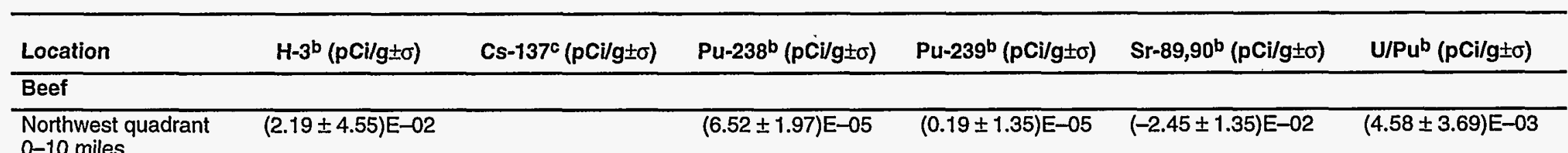

0-10 miles

All results except those for $\mathrm{H}-3$ are expressed in dry weight

Blank spaces indicate that no analyses were performed.

Blank spaces indicate that activity was below the lower limit of detection. The typical lower limit of detection for Cs-137 in food products was $7.00 \mathrm{E}-03 \mathrm{pCi} / \mathrm{g}$. 
Table 30

Radioactivity in Terrestrial Food Products - Milk

Page 1 of 1

\begin{tabular}{|c|c|c|c|c|}
\hline Location & $\begin{array}{c}\text { No. of } \\
\text { Samples }\end{array}$ & Arithmetic Mean $\pm \sigma$ & Maximum $\pm \sigma$ & Minimum $\pm \sigma$ \\
\hline \multicolumn{5}{|l|}{$\mathrm{H}-3, \mu \mathrm{Ci} / \mathrm{mL}$} \\
\hline Dairy (major distributor) & 12 & $(1.14 \pm 0.89) E-07$ & $(2.81 \pm 1.35) \mathrm{E}-07$ & $(-0.05 \pm 1.29) E-07$ \\
\hline Denmark, S.C. & 9 & $(0.96 \pm 1.30) E-07$ & $(3.05 \pm 1.35) E-07$ & $(-0.79 \pm 1.04) E-07$ \\
\hline Girard, Ga., dairy & 12 & $(1.97 \pm 1.55) E-07$ & $(5.45 \pm 1.31) E-07$ & $(0.05 \pm 1.29) E-07$ \\
\hline Gracewood, Ga., dairy & 10 & $(2.17 \pm 1.79) E-07$ & $(5.91 \pm 1.36) \mathrm{E}-07$ & $(-0.14 \pm 1.04) E-07$ \\
\hline Jackson, S.C., dairy & 11 & $(6.85 \pm 1.76) E-07$ & $(1.08 \pm 0.14) E-06$ & $(4.29 \pm 1.06) E-07$ \\
\hline NE S.C. 50 -mile radius & 7 & $(2.02 \pm 1.67) E-07$ & $(5.17 \pm 1.39) \mathrm{E}-07$ & $(0.73 \pm 1.36) E-07$ \\
\hline NW S.C. 50-mile radius & 6 & $(2.20 \pm 1.34) E-07$ & $(3.40 \pm 1.38) E-07$ & $(0.08 \pm 1.35) E-07$ \\
\hline SE S.C. 50 -mile radius & 6 & $(2.54 \pm 3.06) E-07$ & $(5.68 \pm 1.36) E-07$ & $(-0.72 \pm 1.36) E-07$ \\
\hline SW Ga. 50-mile radius & 3 & $(1.13 \pm 2.67) E-07$ & $(3.94 \pm 1.39) E-07$ & $(-1.36 \pm 1.34) E-07$ \\
\hline Waynesboro, Ga., dairy & 10 & $(5.50 \pm 8.24) E-08$ & $(1.58 \pm 1.33) \mathrm{E}-07$ & $(-0.66 \pm 1.35) E-07$ \\
\hline \multicolumn{5}{|l|}{ Sr-90, $\mu \mathrm{Ci} / \mathrm{mL}$} \\
\hline Girard, Ga., dairy & 1 & $(7.47 \pm 4.43) \mathrm{E}-09$ & & \\
\hline Gracewood, Ga., dairy & 1 & $(1.94 \pm 1.48) E-09$ & & \\
\hline Jackson, S.C., dairy & 1 & $(2.35 \pm 1.58) E-09$ & & \\
\hline Waynesboro, Ga., dairy & 1 & $(3.29 \pm 1.73) E-09$ & & \\
\hline \multicolumn{5}{|c|}{ Cs- $137^{\mathrm{a}}, \mu \mathrm{Ci} / \mathrm{mL}$ (The typical lower limit of detection for Cs-137 in milk was $3.00 \mathrm{E}-09 \mu \mathrm{Ci} / \mathrm{mL}$. ) } \\
\hline Dairy (major distributor) & 2 & $(3.32 \pm 1.23) \mathrm{E}-09$ & $(4.19 \pm 1.13) \mathrm{E}-09$ & $(2.45 \pm 0.92) \mathrm{E}-09$ \\
\hline Gracewood, Ga., dairy & 2 & $(3.64 \pm 0.32) E-09$ & $(3.87 \pm 1.16) E-09$ & $(3.41 \pm 1.26) E-09$ \\
\hline NE S.C. 50-mile radius & 5 & $(5.64 \pm 1.34) E-09$ & $(7.73 \pm 1.37) \mathrm{E}-09$ & $(4.19 \pm 0.95) E-09$ \\
\hline SE S.C. 50-mile radius & 1 & $(3.65 \pm 1.19) E-09$ & & \\
\hline SW Ga. 50-mile radius & 1 & $(3.16 \pm 0.95) E-09$ & & \\
\hline
\end{tabular}

a The gamma analysis package currently used by Environmental Monitoring does not force an activity determination if a threshold setting is not met. For these reported radionuclides, "No. of Samples" is displayed as "number with activities quantified/number of samples counted"; only the number quantified are used in the mean, maximum, and minimum generation. 
Table 31

Radioactivity in Aquatic Food Products - Freshwater Fish

Page 1 of 10

\begin{tabular}{|c|c|c|c|c|}
\hline Location & $\begin{array}{c}\text { No. of } \\
\text { Samples }\end{array}$ & Arithmetic Mean $\pm \sigma$ & Maximum $\pm \sigma$ & Minimum $\pm \sigma$ \\
\hline \multicolumn{5}{|l|}{$\mathrm{H}-3, \mathrm{pCi} / \mathrm{g}$} \\
\hline \multicolumn{5}{|c|}{ Offsite (Edible) } \\
\hline \multicolumn{5}{|c|}{ Augusta Lock and Dam } \\
\hline Bream & 2 & $(-0.07 \pm 1.50) E-01$ & $(9.91 \pm 4.15) E-02$ & $(-1.13 \pm 0.48) E-01$ \\
\hline Catfish & 3 & $(9.71 \pm 7.13) E-03$ & $(1.77 \pm 2.69) E-02$ & $(0.39 \pm 3.04) E-02$ \\
\hline Crappie & 1 & $(1.86 \pm 1.52) E-02$ & & \\
\hline \multicolumn{5}{|c|}{ Beaver Dam Creek River Mouth } \\
\hline Catfish & 3 & $(9.36 \pm 3.05) E-02$ & $(1.23 \pm 0.26) E-01$ & $(6.19 \pm 3.26) E-02$ \\
\hline \multicolumn{5}{|c|}{ Four Mile Creek River Mouth } \\
\hline Catfish & 1 & $(7.19 \pm 0.24) \mathrm{E}-01$ & & \\
\hline \multicolumn{5}{|c|}{ Hwy-301 Bridge Area } \\
\hline Bream & 3 & $(2.79 \pm 0.10) E-01$ & $(2.90 \pm 0.34) E-01$ & $(2.73 \pm 0.45) E-01$ \\
\hline Catfish & 2 & $(3.06 \pm 0.87) E-01$ & $(3.67 \pm 0.28) E-01$ & $(2.44 \pm 0.24) E-01$ \\
\hline \multicolumn{5}{|c|}{ L3R Creek River Mouth } \\
\hline Bream & 1 & $(2.66 \pm 0.43) E-01$ & & \\
\hline Catfish & 2 & $(1.71 \pm 0.60) E-01$ & $(2.14 \pm 0.24) E-01$ & $(1.28 \pm 0.21) E-01$ \\
\hline \multicolumn{5}{|c|}{ Steel Creek River Mouth } \\
\hline Bass & 1 & $(1.97 \pm 0.03) E+00$ & & \\
\hline Bream & 2 & $(2.08 \pm 0.88) E+00$ & $(2.70 \pm 0.05) E+00$ & $(1.45 \pm 0.03) E+00$ \\
\hline Catfish & 2 & $(1.59 \pm 1.40) E+00$ & $(2.58 \pm 0.05) E+00$ & $(6.06 \pm 0.35) E-01$ \\
\hline \multicolumn{5}{|c|}{ U3R Creek River Mouth } \\
\hline Catfish & 2 & $(1.89 \pm 0.23) E-01$ & $(2.06 \pm 0.31) E-01$ & $(1.73 \pm 0.29) E-01$ \\
\hline \multicolumn{5}{|c|}{ Cs-137, pCi/g } \\
\hline \multicolumn{5}{|c|}{ Offsite (Edible) } \\
\hline \multicolumn{5}{|c|}{ Augusta Lock and Dam } \\
\hline Catfish & 2 & $(4.29 \pm 0.06) E-02$ & $(4.33 \pm 1.39) E-02$ & $(4.25 \pm 1.51) E-02$ \\
\hline \multicolumn{5}{|c|}{ Beaver Dam Creek River Mouth } \\
\hline Catfish & 2 & $(9.00 \pm 2.12) E-02$ & $(1.05 \pm 0.21) E-01$ & $(7.51 \pm 1.95) E-02$ \\
\hline \multicolumn{5}{|c|}{ Hwy-301 Bridge Area } \\
\hline Bream & 3 & $(7.50 \pm 1.62) \mathrm{E}-02$ & $(8.93 \pm 1.43) E-02$ & $(5.74 \pm 1.22) E-02$ \\
\hline Catfish & 2 & $(5.69 \pm 1.08) E-02$ & $(6.45 \pm 0.54) E-02$ & $(4.92 \pm 0.67) E-02$ \\
\hline \multicolumn{5}{|c|}{ L3R Creek River Mouth } \\
\hline Bream & 1 & $(1.55 \pm 0.27) E-01$ & & \\
\hline Catfish & 2 & $(2.54 \pm 2.53) \mathrm{E}-01$ & $(4.33 \pm 0.33) E-01$ & $(7.54 \pm 2.09) E-02$ \\
\hline
\end{tabular}


Table 31

Radioactivity in Aquatic Food Products - Freshwater Fish

Page 2 of 10

\begin{tabular}{|c|c|c|c|c|}
\hline Location & $\begin{array}{c}\text { No. of } \\
\text { Samples }\end{array}$ & Arithmetic Mean $\pm \sigma$ & Maximum $\pm \sigma$ & Minimum $\pm \sigma$ \\
\hline \multicolumn{5}{|c|}{ Steel Creek River Mouth } \\
\hline Bass & 1 & $(1.21 \pm 0.04) \mathrm{E}+00$ & & \\
\hline Bream & 2 & $(1.71 \pm 0.38) \mathrm{E}-01$ & $(1.98 \pm 0.28) E-01$ & $(1.44 \pm 0.35) E-01$ \\
\hline Catfish & 2 & $(8.23 \pm 1.13) E-02$ & $(9.03 \pm 2.61) E-02$ & $(7.43 \pm 1.24) E-02$ \\
\hline \multicolumn{5}{|c|}{ U3R Creek River Mouth } \\
\hline Catfish & 2 & $(9.10 \pm 2.62) E-02$ & $(1.09 \pm 0.24) E-01$ & $(7.24 \pm 2.41) E-02$ \\
\hline \multicolumn{5}{|c|}{ Onsite (Edible) } \\
\hline \multicolumn{5}{|c|}{ Beaver Dam Creek } \\
\hline Bream & 1 & $(1.67 \pm 0.30) E-01$ & & \\
\hline \multicolumn{5}{|l|}{ L-Lake } \\
\hline Bass & 3 & $(8.33 \pm 0.46) E-01$ & $(8.73 \pm 0.30) E-01$ & $(7.83 \pm 0.55) E-01$ \\
\hline Bream & 3 & $(3.13 \pm 0.65) E-01$ & $(3.87 \pm 0.50) \mathrm{E}-01$ & $(2.68 \pm 0.57) E-01$ \\
\hline \multicolumn{5}{|l|}{ PAR Pond } \\
\hline Bass & 3 & $(1.42 \pm 0.19) E+01$ & $(1.58+0.01) \mathrm{E}$ & $(1.21 \pm 0.02) E+01$ \\
\hline Bluegill & 1 & $(6.96 \pm 0.16) E+00$ & & \\
\hline Bream & 1 & $(2.66 \pm 0.11) E+00$ & & \\
\hline Crappie & 1 & $(9.43 \pm 0.19) E+00$ & & \\
\hline \multicolumn{5}{|l|}{ Pond $B$} \\
\hline Bream & 1 & $(3.39 \pm 0.03) E+01$ & & \\
\hline \multicolumn{5}{|c|}{ SC-4 Steel Creek at Road A } \\
\hline Bass & 3 & $(1.21 \pm 0.39) E+00$ & $(1.65 \pm 0.10) E+00$ & $(9.13 \pm 0.14) E-01$ \\
\hline Bream & 3 & $(3.40 \pm 1.81) E-01$ & $(5.45 \pm 0.43) \mathrm{E}-01$ & $(2.03 \pm 0.35) E-01$ \\
\hline \multicolumn{5}{|c|}{ Offsite (Nonedible) } \\
\hline \multicolumn{5}{|c|}{ Beaver Dam Creek River Mouth } \\
\hline Catfish & 2 & $(6.68 \pm 2.54) E-02$ & $(8.48 \pm 2.78) E-02$ & $(4.88 \pm 1.80) E-02$ \\
\hline \multicolumn{5}{|c|}{ Hwy-301 Bridge Area } \\
\hline Bream & 2 & $(5.05 \pm 3.60) E-02$ & $(7.59 \pm 0.89) E-02$ & $(2.50 \pm 0.91) \mathrm{E}-02$ \\
\hline Catfish & 2 & $(2.72 \pm 1.08) E-02$ & $(3.49 \pm 0.57) E-02$ & $(1.95 \pm 0.57) E-02$ \\
\hline \multicolumn{5}{|c|}{ L3R Creek River Mouth } \\
\hline Cattish & 2 & $(1.92 \pm 1.60) E-01$ & $(3.05 \pm 0.31) E-01$ & $(7.88 \pm 1.68) \mathrm{E}-02$ \\
\hline \multicolumn{5}{|c|}{ Steel Creek River Mouth } \\
\hline Bass & 1 & $(7.30 \pm 0.35) \mathrm{E}-01$ & & \\
\hline Bream & 2 & $(5.74 \pm 5.51) E-02$ & $(9.64 \pm 2.74) E-02$ & $(1.85 \pm 0.43) E-02$ \\
\hline Catfish & 2 & $(4.44 \pm 1.02) \mathrm{E}-02$ & $(5.16 \pm 1.64) E-02$ & $(3.72 \pm 1.00) E-02$ \\
\hline
\end{tabular}




\section{Table 31}

Radioactivity in Aquatic Food Products - Freshwater Fish

Page 3 of 10

\begin{tabular}{|c|c|c|c|c|}
\hline Location & $\begin{array}{l}\text { No. of } \\
\text { Samples }\end{array}$ & Arithmetic Mean $\pm \sigma$ & Maximum $\pm \sigma$ & Minimum $\pm \sigma$ \\
\hline \multicolumn{5}{|c|}{ U3R Creek River Mouth } \\
\hline Cattish & 1 & $(4.73 \pm 1.45) E-02$ & & \\
\hline \multicolumn{5}{|c|}{$\mathrm{U}-235, \mathrm{pCi} / \mathrm{g}$} \\
\hline \multicolumn{5}{|c|}{ Offsite (Nonedible) } \\
\hline \multicolumn{5}{|c|}{ Hwy-301 Bridge Area } \\
\hline Bream & 1 & $(6.57 \pm 2.10) E-02$ & & \\
\hline \multicolumn{5}{|c|}{$\mathrm{Pu}-238, \mathrm{pCi} / \mathrm{g}$} \\
\hline \multicolumn{5}{|c|}{ Offsite (Edible) } \\
\hline \multicolumn{5}{|c|}{ Augusta Lock and Dam } \\
\hline Bream & 2 & $(0.84 \pm 1.26) E-04$ & $(1.73 \pm 0.43) E-04$ & $(-0.44 \pm 2.59) E-05$ \\
\hline Catfish & 3 & $(1.80 \pm 2.18) E-05$ & $(4.32 \pm 1.19) E-05$ & $(0.40 \pm 1.37) E-05$ \\
\hline Crappie & 1 & $(5.99 \pm 1.98) E-05$ & & \\
\hline \multicolumn{5}{|c|}{ Beaver Dam Creek River Mouth } \\
\hline Catfish & 3 & $(4.46 \pm 7.74) E-04$ & $(1.34 \pm 0.50) E-03$ & $(-0.85 \pm 1.24) E-05$ \\
\hline \multicolumn{5}{|c|}{ Four Mile Creek River Mouth } \\
\hline Catfish & 1 & $(1.12 \pm 0.15) E-04$ & & \\
\hline \multicolumn{5}{|c|}{ Hwy-301 Bridge Area } \\
\hline Bream & 3 & $(1.08 \pm 2.11) E-05$ & $(3.28 \pm 2.58) E-05$ & $(-0.92 \pm 1.96) E-05$ \\
\hline Catfish & 2 & $(-3.56 \pm 5.46) E-05$ & $(3.04 \pm 3.92) E-06$ & $(-7.42 \pm 5.25) E-05$ \\
\hline \multicolumn{5}{|c|}{ L3A Creek River Mouth } \\
\hline Bream & 1 & $(-1.23 \pm 2.04) E-05$ & & \\
\hline Cattish & 1 & $(4.23 \pm 2.13) \mathrm{E}-05$ & & \\
\hline \multicolumn{5}{|c|}{ Steel Creek River Mouth } \\
\hline Bass & 1 & $(7.34 \pm 7.35) \mathrm{E}-06$ & & \\
\hline Bream & 2 & $(3.10 \pm 2.18) E-06$ & $(0.46 \pm 1.39) E-05$ & $(1.56 \pm 7.74) E-06$ \\
\hline Cattish & 2 & $(2.42 \pm 0.06) \mathrm{E}-05$ & $(2.46 \pm 1.27) \mathrm{E}-05$ & $(2.38 \pm 1.93) E-05$ \\
\hline \multicolumn{5}{|c|}{ U3A Creek River Mouth } \\
\hline Catfish & 2 & $(0.73 \pm 1.04) E-05$ & $(1.47 \pm 0.50) E-05$ & $(0.00 \pm 1.11) E-05$ \\
\hline \multicolumn{5}{|c|}{ Onsite (Edible) } \\
\hline \multicolumn{5}{|l|}{ L-Lake } \\
\hline Bream & 3 & $(-2.09 \pm 3.15) E-05$ & $(1.43 \pm 1.85) E-05$ & $(-4.65 \pm 4.26) E-05$ \\
\hline \multicolumn{5}{|l|}{ Pond $B$} \\
\hline Bream & 1 & $(6.13 \pm 2.19) E-05$ & & \\
\hline
\end{tabular}




\section{Table 31}

Radioactivity in Aquatic Food Products - Freshwater Fish

Page 4 of 10

\begin{tabular}{|c|c|c|c|c|}
\hline Location & $\begin{array}{c}\text { No. of } \\
\text { Samples }\end{array}$ & Arithmetic Mean $\pm \sigma$ & Maximum $\pm \sigma$ & Minimum $\pm \sigma$ \\
\hline \multicolumn{5}{|c|}{ SC-4 Steel Creek at Road A } \\
\hline Bream & 2 & $(0.79 \pm 1.10) E-02$ & $(1.56 \pm 0.08) E-02$ & $(1.32 \pm 0.28) E-04$ \\
\hline \multicolumn{5}{|c|}{ Pu-239, pCi/g } \\
\hline \multicolumn{5}{|c|}{ Offsite (Edible) } \\
\hline \multicolumn{5}{|c|}{ Augusta Lock and Dam } \\
\hline Bream & 2 & $(-8.08 \pm 5.26) E-06$ & $(-0.44 \pm 2.34) E-05$ & $(-1.18 \pm 2.20) E-05$ \\
\hline Catfish & 3 & $(1.43 \pm 1.35) E-05$ & $(2.87 \pm 1.06) E-05$ & $(2.09 \pm 6.92) \mathrm{E}-06$ \\
\hline Crappie & 1 & $(2.72 \pm 1.22) E-05$ & & \\
\hline \multicolumn{5}{|c|}{ Beaver Dam Creek River Mouth } \\
\hline Catfish & 3 & $(-1.14 \pm 9.43) E-06$ & $(7.32 \pm 9.46) E-06$ & $(-1.13 \pm 1.06) E-05$ \\
\hline \multicolumn{5}{|c|}{ Four Mile Creek River Mouth } \\
\hline Catfish & 1 & $(6.07 \pm 5.35) E-06$ & & \\
\hline \multicolumn{5}{|c|}{ Hwy-301 Bridge Area } \\
\hline Bream & 3 & $(0.36 \pm 3.77) E-05$ & $(3.52 \pm 1.77) E-05$ & $(-3.82 \pm 1.97) E-05$ \\
\hline Catfish & 2 & $(-4.29 \pm 5.64) E-05$ & $(-3.03 \pm 3.64) E-06$ & $(-8.27 \pm 4.25) E-05$ \\
\hline \multicolumn{5}{|c|}{ L3R Creek River Mouth } \\
\hline Bream & 1 & $(1.63 \pm 0.82) E-05$ & & \\
\hline Catfish & 1 & $(-2.11 \pm 1.41) \mathrm{E}-05$ & & \\
\hline \multicolumn{5}{|c|}{ Steel Creek River Mouth } \\
\hline Bass & 1 & $(4.89 \pm 6.91) E-06$ & & \\
\hline Bream & 2 & $(2.69 \pm 2.75) E-06$ & $(0.46 \pm 1.39) E-05$ & $(0.74 \pm 5.50) E-06$ \\
\hline Catfish & 2 & $(2.74 \pm 3.87) E-06$ & $(5.48 \pm 6.73) E-06$ & $(0.00 \pm 2.75) E-04$ \\
\hline \multicolumn{5}{|c|}{ U3R Creek River Mouth } \\
\hline Catfish & 2 & $(2.09 \pm 8.84) E-06$ & $(8.34 \pm 3.92) E-06$ & $(-4.16 \pm 5.89) E-06$ \\
\hline \multicolumn{5}{|c|}{ Onsite (Edible) } \\
\hline \multicolumn{5}{|l|}{ L-Lake } \\
\hline Bream & 3 & $(-0.37 \pm 1.87) E-05$ & $(0.92 \pm 4.42) E-05$ & $(-2.52 \pm 2.08) E-05$ \\
\hline \multicolumn{5}{|l|}{ Pond $B$} \\
\hline Bream & 1 & $(4.07 \pm 2.70) E-05$ & & \\
\hline \multicolumn{5}{|c|}{ SC-4 Steel Creek at Road A } \\
\hline Bream & 2 & $(4.33 \pm 8.79) E-05$ & $(1.05 \pm 0.23) E-04$ & $(-1.89 \pm 1.79) E-05$ \\
\hline
\end{tabular}




\section{Table 31}

Radioactivity in Aquatic Food Products - Freshwater Fish

Page 5 of 10

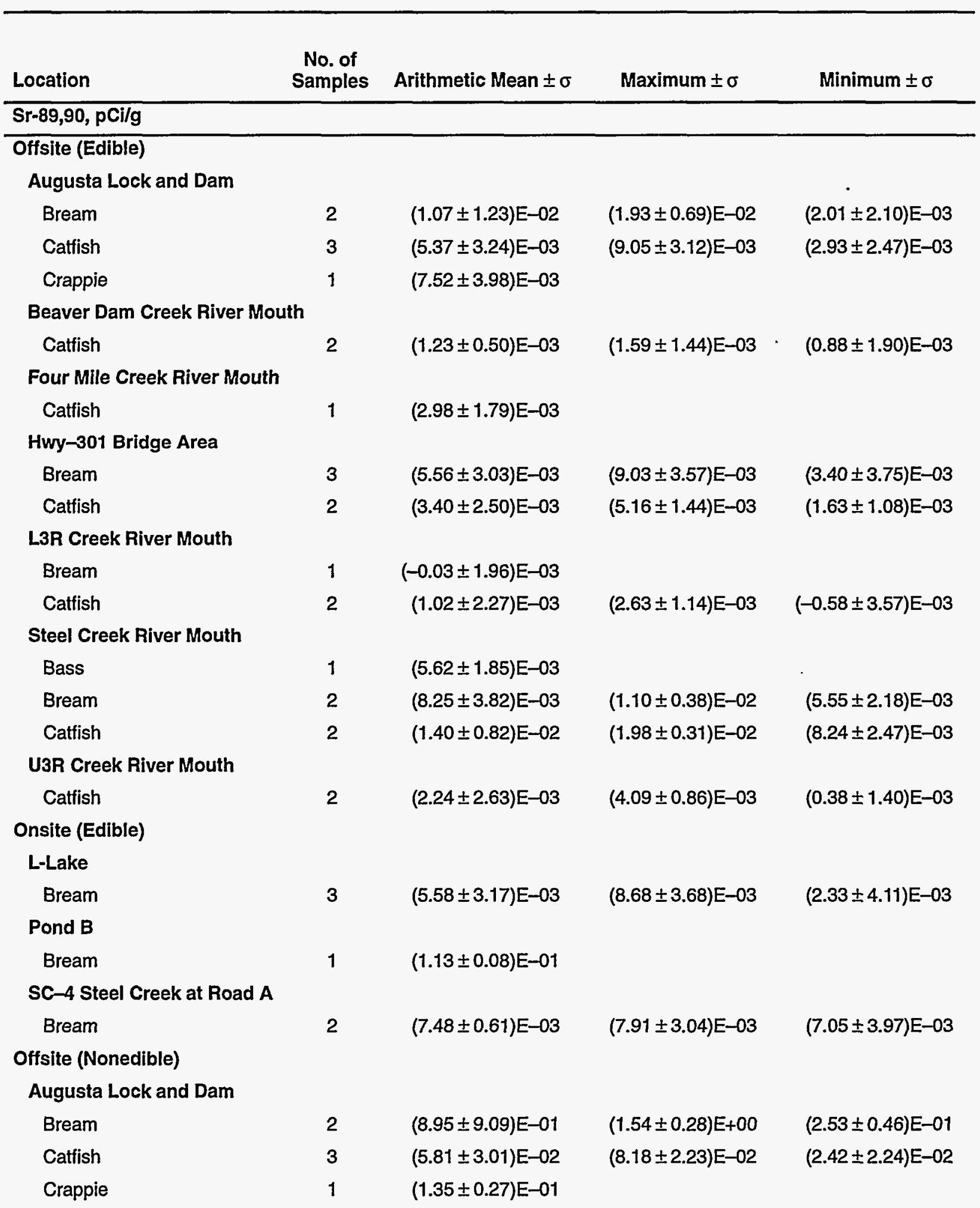




\section{Table 31}

\section{Radioactivity in Aquatic Food Products - Freshwater Fish}

Page 6 of 10

\begin{tabular}{|c|c|c|c|c|}
\hline Location & $\begin{array}{c}\text { No. of } \\
\text { Samples }\end{array}$ & Arithmetic Mean $\pm \sigma$ & Maximum $\pm \sigma$ & Minimum $\pm \sigma$ \\
\hline \multicolumn{5}{|c|}{ Beaver Dam Creek River Mouth } \\
\hline Catfish & 3 & $(4.16 \pm 4.46) \mathrm{E}-01$ & $(9.31 \pm 1.51) E-01$ & $(1.38 \pm 0.26) E-01$ \\
\hline \multicolumn{5}{|c|}{ Four Mile Creek River Mouth } \\
\hline Catfish & 1 & $(1.73 \pm 0.25) E-01$ & & \\
\hline \multicolumn{5}{|c|}{ Hwy-301 Bridge Area } \\
\hline Bream & 3 & $(2.50 \pm 1.62) E-01$ & $(4.36 \pm 1.39) \mathrm{E}-01$ & $(1.56 \pm 0.31) \mathrm{E}-01$ \\
\hline Catfish & 2 & $(2.96 \pm 3.28) E-01$ & $(5.28 \pm 1.56) E-01$ & $(6.34 \pm 2.15) E-02$ \\
\hline \multicolumn{5}{|c|}{ L3R Creek River Mouth } \\
\hline Bream & 1 & $(1.86 \pm 0.52) E-01$ & & \\
\hline Catfish & 2 & $(1.09 \pm 0.11) E-01$ & $(1.16 \pm 0.25) E-01$ & $(1.01 \pm 0.21) E-01$ \\
\hline \multicolumn{5}{|c|}{ Steel Creek River Mouth } \\
\hline Bass & 1 & $(5.68 \pm 4.57) 巨-02$ & & \\
\hline Bream & 2 & $(1.83 \pm 0.55) E-01$ & $(2.22 \pm 0.21) E-01$ & $(1.44 \pm 0.30) E-01$ \\
\hline Catfish & 2 & $(1.10 \pm 0.39) E-01$ & $(1.38 \pm 0.18) E-01$ & $(8.27 \pm 1.38) E-02$ \\
\hline \multicolumn{5}{|c|}{ U3R Creek River Mouth } \\
\hline Catfish & 2 & $(4.60 \pm 5.02) E-01$ & $(8.15 \pm 2.02) E-01$ & $(1.04 \pm 0.15) E-01$ \\
\hline \multicolumn{5}{|c|}{ Gross Beta, pCi/g } \\
\hline \multicolumn{5}{|c|}{ Offsite (Edible) } \\
\hline \multicolumn{5}{|c|}{ Augusta Lock and Dam } \\
\hline Bream & 2 & $(2.40 \pm 0.40) E+00$ & $(2.69 \pm 0.52) E+00$ & $(2.12 \pm 0.32) E+00$ \\
\hline Catfish & 3 & $(2.34 \pm 0.24) E+00$ & $(2.56 \pm 0.38) E+00$ & $(2.09 \pm 0.26) E+00$ \\
\hline Crappie & 1 & $(2.98 \pm 0.42) E+00$ & & \\
\hline \multicolumn{5}{|c|}{ Beaver Dam Creek River Mouth } \\
\hline Catfish & 3 & $(2.65 \pm 0.47) E+00$ & $(3.03 \pm 0.36) E+00$ & $(2.12 \pm 0.32) E+00$ \\
\hline \multicolumn{5}{|c|}{ Four Mile Creek River Mouth } \\
\hline Catfish & 1 & $(2.91 \pm 0.31) E+00$ & & \\
\hline \multicolumn{5}{|c|}{ Hwy-301 Bridge Area } \\
\hline Bream & 3 & $(2.06 \pm 0.48) E+00$ & $(2.42 \pm 0.40) E+00$ & $(1.51 \pm 0.30) E+00$ \\
\hline Catfish & 2 & $(2.42 \pm 0.43) E+00$ & $(2.73 \pm 0.35) E+00$ & $(2.12 \pm 0.32) E+00$ \\
\hline \multicolumn{5}{|c|}{ L3R Creek River Mouth } \\
\hline Bream & 1 & $(1.82 \pm 0.31) E+00$ & & \\
\hline Catfish & 2 & $(2.87 \pm 0.67) E+00$ & $(3.34 \pm 0.44) E+00$ & $(2.40 \pm 0.23) E+00$ \\
\hline
\end{tabular}


Table 31

Radioactivity in Aquatic Food Products - Freshwater Fish

Page 7 of 10

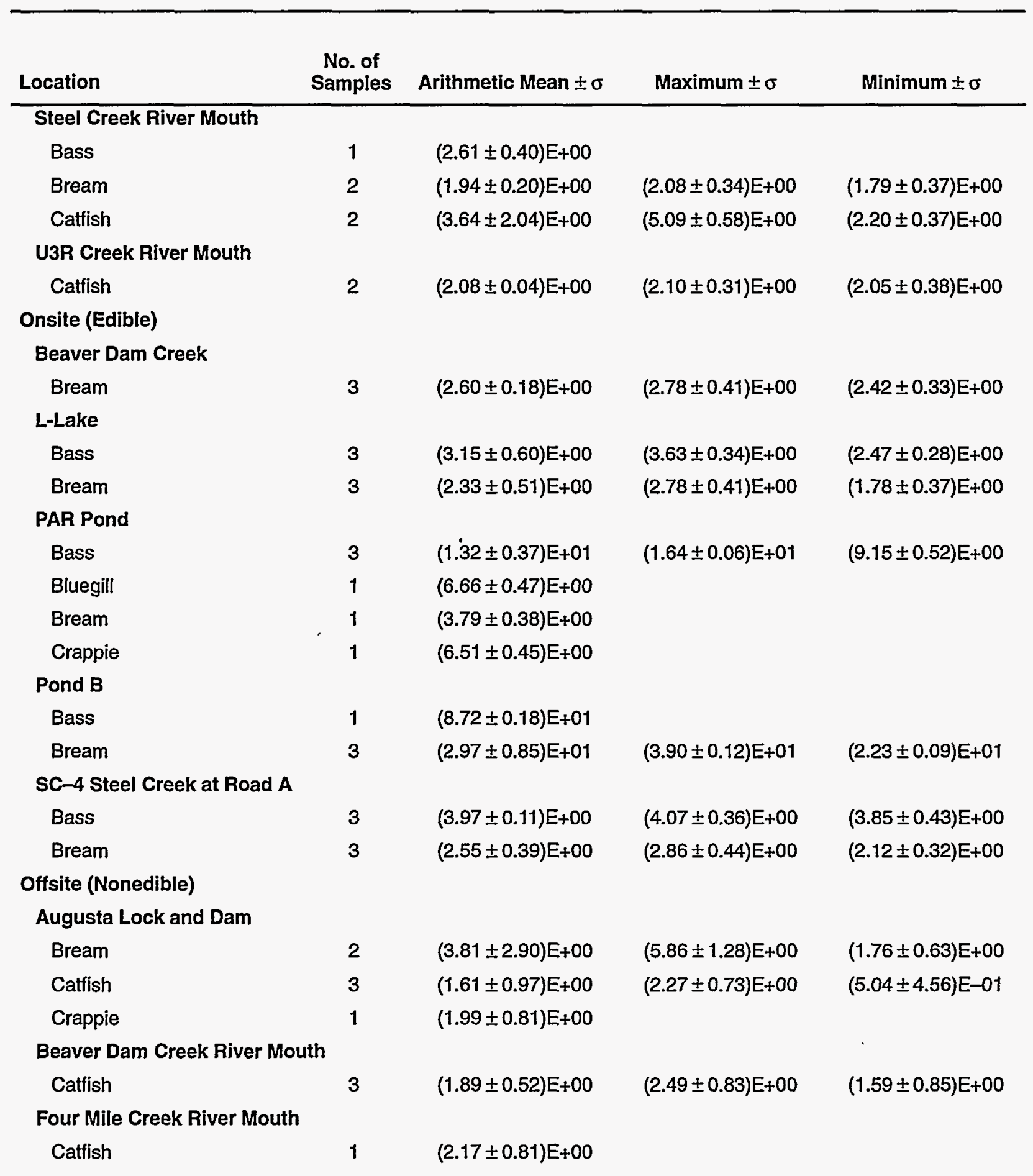




\section{Table 31}

Radioactivity in Aquatic Food Products - Freshwater Fish

Page 8 of 10

\begin{tabular}{|c|c|c|c|c|}
\hline Location & $\begin{array}{l}\text { No. of } \\
\text { Samples }\end{array}$ & Arithmetic Mean $\pm \sigma$ & Maximum $\pm \sigma$ & Minimum $\pm \sigma$ \\
\hline \multicolumn{5}{|c|}{ Hwy-301 Bridge Area } \\
\hline Bream & 2 & $(2.84 \pm 0.04) E+00$ & $(2.86 \pm 0.96) E+00$ & $(2.81 \pm 0.96) \mathrm{E}+00$ \\
\hline Catfish & 2 & $(1.75 \pm 0.37) \mathrm{E}+00$ & $(2.01 \pm 0.89) E+00$ & $(1.48 \pm 0.85) E+00$ \\
\hline \multicolumn{5}{|c|}{ L3R Creek River Mouth } \\
\hline Bream & 1 & $(1.48 \pm 0.84) E+00$ & & \\
\hline Cattish & 2 & $(1.91 \pm 1.05) E+00$ & $(2.65 \pm 0.92) E+00$ & $(1.17 \pm 0.82) E+00$ \\
\hline \multicolumn{5}{|c|}{ Steel Creek River Mouth } \\
\hline Bass & 1 & $(2.96 \pm 0.86) \mathrm{E}+00$ & & \\
\hline Bream & 2 & $(2.73 \pm 0.39) \mathrm{E}+00$ & $(3.00 \pm 0.62) E+00$ & $(2.45 \pm 0.78) E+00$ \\
\hline Cattish & 2 & $(1.61 \pm 0.70) E+00$ & $(2.10 \pm 0.73) E+00$ & $(1.12 \pm 0.66) E+00$ \\
\hline \multicolumn{5}{|c|}{ U3R Creek River Mouth } \\
\hline Cattish & 2 & $(1.83 \pm 0.16) E+00$ & $(1.94 \pm 0.81) E+00$ & $(1.71 \pm 0.74) E+00$ \\
\hline \multicolumn{5}{|c|}{ Gross Alpha, pCi/g } \\
\hline \multicolumn{5}{|c|}{ Offsite (Edible) } \\
\hline \multicolumn{5}{|c|}{ Augusta Lock and Dam } \\
\hline Bream & 2 & $(-6.34 \pm 5.59) E-02$ & $(-0.24 \pm 1.76) E-01$ & $(-1.03 \pm 0.82) E-01$ \\
\hline Cattish & 3 & $(3.35 \pm 4.54) E-02$ & $(0.66 \pm 1.48) E-01$ & $(-0.18 \pm 1.43) E-01$ \\
\hline Crappie & 1 & $(1.10 \pm 1.60) E-01$ & & \\
\hline \multicolumn{5}{|c|}{ Beaver Dam Creek River Mouth } \\
\hline Catfish & 3 & $(7.09 \pm 6.99) E-02$ & $(1.51 \pm 1.51) \mathrm{E}-01$ & $(0.26 \pm 1.23) E-01$ \\
\hline \multicolumn{5}{|c|}{ Four Mile Creek River Mouth } \\
\hline Catfish & 1 & $(-3.34 \pm 9.42) E--02$ & & \\
\hline \multicolumn{5}{|c|}{ Hwy-301 Bridge Area } \\
\hline Bream & 3 & $(-1.73 \pm 8.48) E-02$ & $(0.37 \pm 1.48) E-01$ & $(-1.15 \pm 0.89) E-01$ \\
\hline Catfișh & 2 & $(-1.02 \pm 0.01) E-01$ & $(-1.01 \pm 0.80) E-01$ & $(-1.03 \pm 0.82) E-01$ \\
\hline \multicolumn{5}{|c|}{ L3R Creek River Mouth } \\
\hline Bream & 1 & $(-0.38 \pm 1.02) E-01$ & & \\
\hline Catfish & 2 & $(-2.22 \pm 7.86) E-02$ & $(0.33 \pm 1.41) E-01$ & $(-7.77 \pm 6.34) E-02$ \\
\hline \multicolumn{5}{|c|}{ Steel Creek River Mouth } \\
\hline Bass & 1 & $(0.35 \pm 1.40) E-01$ & & \\
\hline Bream & 2 & $(1.08 \pm 1.10) E-01$ & $(1.87 \pm 1.80) E-01$ & $(0.30 \pm 1.21) E-01$ \\
\hline Cattish & 2 & $(1.86 \pm 5.92) E-02$ & $(0.60 \pm 1.37) \mathrm{E}-01$ & $(-0.23 \pm 1.73) E-01$ \\
\hline \multicolumn{5}{|c|}{ U3R Creek River Mouth } \\
\hline Catfish & 2 & $(-3.16 \pm 9.75) E-02$ & $(0.37 \pm 1.40) \mathrm{E}-01$ & $(-1.01 \pm 0.79) E-01$ \\
\hline
\end{tabular}




\section{Table 31}

\section{Radioactivity in Aquatic Food Products - Freshwater Fish}

Page 9 of 10

\begin{tabular}{|c|c|c|c|c|}
\hline Location & $\begin{array}{c}\text { No. of } \\
\text { Samples }\end{array}$ & Arithmetic Mean $\pm \sigma$ & Maximum $\pm \sigma$ & Minimum $\pm \sigma$ \\
\hline \multicolumn{5}{|c|}{ Onsite (Edible) } \\
\hline \multicolumn{5}{|c|}{ Beaver Dam Creek } \\
\hline Bream & 3 & $(-6.57 \pm 4.44) E-02$ & $(-0.39 \pm 1.06) E-01$ & $(-1.17 \pm 0.89) E-01$ \\
\hline \multicolumn{5}{|l|}{ L-Lake } \\
\hline Bass & 3 & $(1.08 \pm 1.90) E-01$ & $(2.76 \pm 1.66) E-01$ & $(-9.88 \pm 7.41) E-02$ \\
\hline Bream & 3 & $(0.61 \pm 1.72) E-01$ & $(2.60 \pm 1.86) E-01$ & $(-0.41 \pm 1.16) E-01$ \\
\hline \multicolumn{5}{|l|}{ PAR Pond } \\
\hline Bass & 3 & $(-1.25 \pm 0.29) E-01$ & $(-1.03 \pm 0.82) E-01$ & $(-1.59 \pm 0.64) E-01$ \\
\hline Bluegill & 1 & $(-9.99 \pm 8.01) E-02$ & & \\
\hline Bream & 1 & $(-0.39 \pm 1.06) E-01$ & & \\
\hline Crappie & 1 & $(-4.54 \pm 9.58) E-02$ & & \\
\hline \multicolumn{5}{|l|}{ Pond $B$} \\
\hline Bass & 1 & $(-0.61 \pm 1.76) E-01$ & & \\
\hline Bream & 3 & $(-0.44 \pm 1.09) E-01$ & $(0.82 \pm 1.81) E-01$ & $(-1.13 \pm 1.14) E-01$ \\
\hline \multicolumn{5}{|c|}{ SC-4 Steel Creek at Road A } \\
\hline Bass & 3 & $(-2.60 \pm 8.12) E-02$ & $(0.60 \pm 1.37) E-01$ & $(-1.01 \pm 0.52) E-01$ \\
\hline Bream & 3 & $(-0.16 \pm 1.28) E-01$ & $(1.22 \pm 1.74) \mathrm{E}-01$ & $(-1.31 \pm 1.00) E-01$ \\
\hline \multicolumn{5}{|c|}{ Offsite (Nonedible) } \\
\hline \multicolumn{5}{|c|}{ Augusta Lock and Dam } \\
\hline Bream & 2 & $(-5.69 \pm 5.05) E-01$ & $(-2.12 \pm 2.44) E-01$ & $(-9.26 \pm 7.30) E-01$ \\
\hline Cattish & 3 & $(2.85 \pm 2.19) E-01$ & $(5.16 \pm 3.44) \mathrm{E}-01$ & $(0.82 \pm 3.93) E-01$ \\
\hline Crappie & 1 & $(8.32 \pm 6.02) E-01$ & & \\
\hline \multicolumn{5}{|c|}{ Beaver Dam Creek River Mouth } \\
\hline Cattish & 3 & $(-1.01 \pm 2.07) E-01$ & $(1.06 \pm 3.75) \mathrm{E}-01$ & $(-3.07 \pm 2.49) E-01$ \\
\hline \multicolumn{5}{|c|}{ Four Mile Creek River Mouth } \\
\hline Cattish & 1 & $(1.20 \pm 4.16) E-01$ & & \\
\hline \multicolumn{5}{|c|}{ Hwy-301 Bridge Area } \\
\hline Bream & 2 & $(-1.27 \pm 0.00) E-01$ & $(-1.27 \pm 4.24) E-01$ & $(-1.27 \pm 4.07) E-01$ \\
\hline Cattish & 2 & $(1.22 \pm 3.15) E-01$ & $(3.44 \pm 4.68) \mathrm{E}-01$ & $(-1.01 \pm 3.43) E-01$ \\
\hline \multicolumn{5}{|c|}{ L3R Creek River Mouth } \\
\hline Bream & 1 & $(-0.95 \pm 3.18) E-01$ & & \\
\hline Cattish & 2 & $(-2.12 \pm 1.65) E-01$ & $(-0.95 \pm 3.18) E-01$ & $(-3.29 \pm 2.65) E-01$ \\
\hline
\end{tabular}


Table 31

Radioactivity in Aquatic Food Products - Freshwater Fish

Page 10 of 10

\begin{tabular}{|c|c|c|c|c|}
\hline Location & $\begin{array}{l}\text { No. of } \\
\text { Samples }\end{array}$ & Arithmetic Mean $\pm \sigma$ & Maximum $\pm \sigma$ & Minimum $\pm \sigma$ \\
\hline \multicolumn{5}{|c|}{ Steel Creek River Mouth } \\
\hline Bass & 1 & $(1.25 \pm 4.41) E-01$ & & \\
\hline Bream & 2 & $(-0.17 \pm 1.16) E-01$ & $(0.65 \pm 3.25) E-01$ & $(-0.99 \pm 3.28) E-01$ \\
\hline Catfish & 2 & $(1.61 \pm 3.79) E-01$ & $(4.29 \pm 4.50) E-01$ & $(-1.07 \pm 2.91) E-01$ \\
\hline \multicolumn{5}{|c|}{ U3R Creek River Mouth } \\
\hline Catfish & 2 & $(-0.10 \pm 1.49) E-01$ & $(0.95 \pm 3.31) E-01$ & $(-1.16 \pm 3.87) E-01$ \\
\hline
\end{tabular}


Table 32

Radioactivity in Aquatic Food Products - Marine (Saltwater) Fish

Page 1 of 1

\begin{tabular}{lcc}
\hline & \multicolumn{2}{c}{ pCi/g } \\
\cline { 2 - 3 } Location & Gross Beta & Gross Alpha \\
\hline Highway-17A Bridge Area & & \\
$\begin{array}{l}\text { Offsite (Edible) } \\
\text { Mullet }\end{array}$ & $(2.60 \pm 0.40) E+00$ & $(0.33 \pm 1.36) E-01$
\end{tabular}


Table 33

Radioactivity in Aquatic Food Products - Marine Invertebrates (Shellfish)

Page 1 of 1

No. of

Location

Samples

Arithmetic Mean $\pm \sigma$

Maximum $\pm \sigma$

Minimum $\pm \sigma$

\begin{tabular}{lcccc}
\hline Sr-89,90, pCi/g & & & & \\
\hline RM-8 Savannah River mouth & 2 & $(-2.65 \pm 2.94) E-03$ & $(-0.57 \pm 3.58) E-03$ & $(-4.73 \pm 4.18) E-03$ \\
\hline Gross Beta, pCi/g & & & & \\
\hline RM-8 Savannah River mouth & 2 & $(1.79 \pm 0.59) E+00$ & $(2.21 \pm 0.35) E+00$ & $(1.37 \pm 0.22) E+00$ \\
\hline Gross Alpha, pCi/g & & & & \\
\hline RM-8 Savannah River mouth & 2 & $(7.63 \pm 3.19) E-01$ & $(9.89 \pm 2.50) E-01$ & $(5.37 \pm 2.60) E-01$
\end{tabular}




\section{Table 34}

Comparison of Field and Laboratory Cesium-137 Measurements in Deer and Hog Muscle

Page 1 of 4

\begin{tabular}{|c|c|c|c|c|}
\hline \multirow[b]{2}{*}{ Date } & \multirow[b]{2}{*}{ Sample Number } & \multirow[b]{2}{*}{ Animal Type } & \multicolumn{2}{|c|}{$\mathrm{pCi} / \mathrm{g}$} \\
\hline & & & Field Cs-137 & Lab Cs-137 \\
\hline 01-NOV-95 & 1 & Deer & 5.1 & $5.19 \pm 0.12$ \\
\hline 01-NOV-95 & 2 & Deer & 1.8 & $2.05 \pm 0.08$ \\
\hline 01-NOV-95 & 3 & Deer & 4.8 & $4.61 \pm 0.06$ \\
\hline 01-NOV-95 & 4 & Deer & 2.7 & $3.08 \pm 0.09$ \\
\hline 01-NOV-95 & 5 (DUP)a & Deer & 10.9 & $11.5 \pm 0.17$ \\
\hline 01-NOV-95 & 5 (DUP)a & Deer & 10.9 & $11.8 \pm 0.18$ \\
\hline 01-NOV-95 & 6 & Deer & 24.2 & $20.7 \pm 0.13$ \\
\hline 01-NOV-95 & 7 & Deer & 7.4 & $9.15 \pm 0.1$ \\
\hline 01-NOV-95 & 8 & Deer & 18.4 & $10.4 \pm 0.1$ \\
\hline 01-NOV-95 & 9 & Deer & 14.3 & $11.3 \pm 0.11$ \\
\hline 01-NOV-95 & 10 & Deer & 4.1 & $3.37 \pm 0.1$ \\
\hline 01-NOV-95 & 11 & Deer & 3.5 & $3.25 \pm 0.09$ \\
\hline 01-NOV-95 & 12 & Deer & 3.9 & $3.77 \pm 0.1$ \\
\hline 01-NOV-95 & 13 & Hog & 2.4 & $3.62 \pm 0.1$ \\
\hline 04-NOV-95 & 1 & Deer & 1.7 & $1.26 \pm 0.06$ \\
\hline 04-NOV-95 & 2 & Deer & 2.7 & $3.1 \pm 0.06$ \\
\hline 04-NOV-95 & 3 & Deer & 4.4 & $5.44 \pm 0.14$ \\
\hline 04-NOV-95 & 4 & Deer & 2.7 & $2.26 \pm 0.08$ \\
\hline 04-NOV-95 & 5 (DUP) $^{a}$ & Deer & 4.6 & $3.74 \pm 0.1$ \\
\hline 04-NOV-95 & 5 (DUP)a & Deer & 4.6 & $3.29 \pm 0.09$ \\
\hline 04-NOV-95 & 6 & Deer & 1.9 & $1.83 \pm 0.08$ \\
\hline 04-NOV-95 & 7 & Deer & 1.3 & $1.64 \pm 0.07$ \\
\hline 04-NOV-95 & 8 & Deer & 3.4 & $4.52 \pm 0.11$ \\
\hline 04-NOV-95 & 9 & Deer & 3.2 & $2.69 \pm 0.09$ \\
\hline 04-NOV-95 & 10 & Deer & 4.6 & $4.97 \pm 0.13$ \\
\hline 08-NOV-95 & 1 & Deer & 4.9 & $5.01 \pm 0.13$ \\
\hline 08-NOV-95 & 2 & Deer & 3.5 & $4.07 \pm 0.1$ \\
\hline 08-NOV-95 & 3 & Deer & 6.5 & $6.82 \pm 0.15$ \\
\hline 08-NOV-95 & 4 & Deer & 9.1 & $8.35 \pm 0.17$ \\
\hline 08-NOV-95 & 5 (DUP) $^{a}$ & Deer & 6.1 & $6.04 \pm 0.09$ \\
\hline 08-NOV-95 & 5 (DUP) $^{a}$ & Deer & 6.1 & $6.24 \pm 0.13$ \\
\hline 08-NOV-95 & 6 & Deer & 6 & $8.27 \pm 0.15$ \\
\hline
\end{tabular}

a Duplicate laboratory analysis on single field sample 
Table 34

Comparison of Field and Laboratory Cesium-137 Measurements in Deer and Hog Muscle

Page 2 of 4

\begin{tabular}{|c|c|c|c|c|}
\hline \multirow[b]{2}{*}{ Date } & \multirow[b]{2}{*}{ Sample Number } & \multirow[b]{2}{*}{ Animal Type } & \multicolumn{2}{|c|}{$\mathrm{pCi} / \mathrm{g}$} \\
\hline & & & Field Cs-137 & Lab Cs-137 \\
\hline 08-NOV-95 & 7 & Deer & 5.1 & $5.95 \pm 0.13$ \\
\hline 08-NOV-95 & 8 & Deer & 9.5 & $7.78 \pm 0.16$ \\
\hline 08-NOV-95 & 9 & Deer & 2.5 & $2.58 \pm 0.08$ \\
\hline 08-NOV-95 & 10 & Deer & 2.9 & $2.11 \pm 0.08$ \\
\hline 08-NOV-95 & 11 & Deer & 3.4 & $3.06 \pm 0.06$ \\
\hline 11-NOV-95 & 1 & Deer & 3.2 & $3.49 \pm 0.1$ \\
\hline 11-NOV-95 & 2 & Deer & 2.9 & $3.87 \pm 0.1$ \\
\hline 11-NOV-95 & 3 & Deer & 2.4 & $4.13 \pm 0.11$ \\
\hline 11-NOV-95 & 4 & Deer & 4.5 & $5.24 \pm 0.12$ \\
\hline 11-NOV-95 & 5 (DUP) $^{a}$ & Deer & 6.4 & $6.78 \pm 0.14$ \\
\hline 11-NOV-95 & $5(\mathrm{DUP})^{\mathrm{a}}$ & Deer & 6.4 & $6.66 \pm 0.13$ \\
\hline 11-NOV-95 & 6 & Deer & 8.1 & $9.39 \pm 0.11$ \\
\hline 11-NOV-95 & 7 & Deer & 1 & $5 \pm 0.11$ \\
\hline $11-N O V-95$ & 8 & Deer & 3.7 & $4.67 \pm 0.11$ \\
\hline 11-NOV-95 & 9 & Deer & 3.5 & $3.91 \pm 0.1$ \\
\hline 15-NOV-95 & 1 & Deer & 3.9 & $3.72 \pm 0.11$ \\
\hline 15-NOV-95 & 2 & Deer & 15.7 & $17.7 \pm 0.13$ \\
\hline 15-NOV-95 & 3 & Deer & 2.3 & $2.81 \pm 0.1$ \\
\hline 15-NOV-95 & 4 & Deer & 4.4 & $5.22 \pm 0.13$ \\
\hline 15-NOV-95 & $5(\text { DUP })^{a}$ & Deer & 2.9 & $3.37 \pm 0.11$ \\
\hline 15-NOV-95 & $5(\text { DUP })^{a}$ & Deer & 2.9 & $3.4 \pm 0.1$ \\
\hline 15-NOV-95 & 6 & Deer & 3.9 & $4.45 \pm 0.12$ \\
\hline 15-NOV-95 & 8 & Deer & 3.1 & $4.11 \pm 0.12$ \\
\hline 15-NOV-95 & 9 & Deer & 2.8 & $3.46 \pm 0.1$ \\
\hline 15-NOV-95 & 10 & Deer & 2 & $3.37 \pm 0.11$ \\
\hline 15-NOV-95 & 11 & Deer & 3.6 & $4.87 \pm 0.13$ \\
\hline 15-NOV-95 & 12 & Deer & 2.4 & $4.14 \pm 0$ \\
\hline 15-NOV-95 & 13 & Deer & 5.7 & $6.67 \pm 0.15$ \\
\hline 15-NOV-95 & 14 & Deer & 1.8 & $2.42 \pm 0.1$ \\
\hline 15-NOV-95 & $15(D U P)^{a}$ & Deer & 6.2 & $6.93 \pm 0.13$ \\
\hline 15-NOV-95 & 15 (DUP)a $^{a}$ & Deer & 6.2 & $6.89 \pm 0.13$ \\
\hline 15-NOV-95 & 16 & Deer & 4.3 & $6.24 \pm 0.14$ \\
\hline
\end{tabular}

a Duplicate laboratory analysis on single field sample 


\section{Table 34}

Comparison of Field and Laboratory Cesium-137 Measurements in Deer and Hog Muscle

Page 3 of 4

\begin{tabular}{|c|c|c|c|c|}
\hline \multirow[b]{2}{*}{ Date } & \multirow[b]{2}{*}{ Sample Number } & \multirow[b]{2}{*}{ Animal Type } & \multicolumn{2}{|c|}{$\overline{\mathrm{pCi} / \mathrm{g}}$} \\
\hline & & & Field Cs-137 & Lab Cs-137 \\
\hline $15-$ NOV-95 & 17 & Deer & 2.3 & $2.71 \pm 0.1$ \\
\hline 18-NOV-95 & 1 & Deer & 2.6 & $2.95 \pm 0.1$ \\
\hline 18-NOV-95 & 2 & Deer & 3.2 & $3.99 \pm 0.11$ \\
\hline 18-NOV-95 & 3 & Deer & 4.5 & $6.48 \pm 0.14$ \\
\hline 18-NOV-95 & 4 & Deer & 5.1 & $5.95 \pm 0.14$ \\
\hline 18-NOV-95 & 5 (DUP) $^{a}$ & Deer & 1.7 & $1.99 \pm 0.08$ \\
\hline 18-NOV-95 & 5 (DUP) $^{a}$ & Deer & 1.7 & \\
\hline 18-NOV-95 & 6 & Deer & 7.6 & $7.41 \pm 0.16$ \\
\hline 18-NOV-95 & 7 & Deer & 5.6 & $9.01 \pm 0.09$ \\
\hline 18-NOV-95 & 8 & Deer & 2.1 & $3.77 \pm 0.11$ \\
\hline 18-NOV-95 & 9 & Deer & 2.4 & $3.26 \pm 0.1$ \\
\hline 22-NOV-95 & 1 & Deer & 3.1 & $3.92 \pm 0.1$ \\
\hline 22-NOV-95 & 2 & Deer & 2.5 & $3.39 \pm 0.1$ \\
\hline 22-NOV-95 & 3 & Deer & 2 & $2.56 \pm 0.09$ \\
\hline 22-NOV-95 & 4 & Deer & 1 & $3.87 \pm 0.11$ \\
\hline 22-NOV-95 & 5 & Deer & 2.5 & $3.22 \pm 0.09$ \\
\hline 22-NOV-95 & 6 & Deer & 2.8 & $5.38 \pm 0.1$ \\
\hline 22-NOV-95 & 7 & Deer & 3.4 & $4.47 \pm 0.11$ \\
\hline 22-NOV-95 & 8 & Deer & 2.4 & $3.37 \pm 0.09$ \\
\hline 25-NOV-95 & 1 & Deer & 1 & $6.14 \pm 0.13$ \\
\hline 25-NOV-95 & 2 & Deer & 4 & $4.67 \pm 0.11$ \\
\hline 25-NOV-95 & 3 & Deer & 1.9 & $2.15 \pm 0.08$ \\
\hline 25-NOV-95 & 4 & Deer & 1.8 & $2.41 \pm 0.08$ \\
\hline 25-NOV-95 & 5 (DUP)a & Deer & 2.4 & $2.73 \pm 0.09$ \\
\hline 25-NOV-95 & $5(D U P)^{a}$ & Deer & 2.4 & $2.61 \pm 0.08$ \\
\hline 25-NOV-95 & 6 & Deer & 2.5 & $3.9 \pm 0.1$ \\
\hline 25-NOV-95 & 7 & Deer & 2 & $3.56 \pm 0.09$ \\
\hline 29-NOV-95 & 1 & Deer & 1.4 & $2.32 \pm 0.08$ \\
\hline 29-NOV-95 & 2 & Deer & 3.9 & $5.36 \pm 0.12$ \\
\hline 29-NOV-95 & 3 & Deer & 6.8 & $8.87 \pm 0.15$ \\
\hline 02-DEC-95 & 1 & Deer & 4.3 & $4.45 \pm 0.1$ \\
\hline 02-DEC-95 & 2 & Deer & 3.6 & $5.51 \pm 0.12$ \\
\hline
\end{tabular}

a Duplicate laboratory analysis on single field sample 
Table 34

Comparison of Field and Laboratory Cesium-137 Measurements in Deer and Hog Muscle

Page 4 of 4

\begin{tabular}{|c|c|c|c|c|}
\hline \multirow[b]{2}{*}{ Date } & \multirow[b]{2}{*}{ Sample Number } & \multirow[b]{2}{*}{ Animal Type } & \multicolumn{2}{|c|}{$\mathrm{pCi} / \mathrm{g}$} \\
\hline & & & Field Cs-137 & Lab Cs-137 \\
\hline 02-DEC-95 & 3 & Deer & 3.9 & $8.06 \pm 0.14$ \\
\hline 02-DEC-95 & 4 & Deer & 5.8 & $6.22 \pm 0.13$ \\
\hline 02-DEC-95 & $5(D U P)^{a}$ & Deer & 2.3 & $4.18 \pm 0.1$ \\
\hline 02-DEC-95 & $5(\mathrm{DUP})^{\mathrm{a}}$ & Deer & 2.3 & $4.59 \pm 0.11$ \\
\hline 02-DEC-95 & 6 & Deer & 39.9 & $45.3 \pm 0.25$ \\
\hline 02-DEC-95 & 7 & Deer & 31 & $36.2 \pm 0.21$ \\
\hline 02-DEC-95 & 8 & Deer & 8 & $8.32 \pm 0.14$ \\
\hline 02-DEC-95 & 9 & Deer & 8.2 & $9.29 \pm 0.11$ \\
\hline 02-DEC-95 & 10 & Deer & 3.9 & $4.49 \pm 0.1$ \\
\hline 06-DEC-95 & 1 & Deer & 4.9 & $3.97 \pm 0.1$ \\
\hline 06-DEC-95 & 2 & Deer & 7.3 & $7.66 \pm 0.14$ \\
\hline 06-DEC-95 & 3 & Deer & 5.7 & $7.94 \pm 0.14$ \\
\hline 06-DEC-95 & 4 & Deer & 7.8 & $7.91 \pm 0.14$ \\
\hline 06-DEC-95 & 5 (DUP)a & Deer & 4.4 & $5.33 \pm 0.12$ \\
\hline 06-DEC-95 & $5(\mathrm{DUP})^{\mathrm{a}}$ & Deer & 4.4 & $5.12 \pm 0.11$ \\
\hline 06-DEC-95 & 6 & Deer & 9.7 & $9.11 \pm 0.09$ \\
\hline 06-DEC-95 & 7 & Deer & 7 & $6.57 \pm 0.08$ \\
\hline 06-DEC-95 & 8 & Deer & 4.5 & $5.9 \pm 0.12$ \\
\hline 06-DEC-95 & 9 & Deer & 8.4 & $8.41 \pm 0.14$ \\
\hline 06-DEC-95 & 10 & Hog & 1 & $1.64 \pm 0.07$ \\
\hline 06-DEC-95 & 11 & Deer & 3.5 & $3.72 \pm 0.09$ \\
\hline 09-DEC-95 & 1 & Deer & 4.6 & $5.31 \pm 0.12$ \\
\hline 09-DEC-95 & 2 & Deer & 6.1 & $9.41 \pm 0.15$ \\
\hline 09-DEC-95 & 3 & Deer & 3.9 & $4.95 \pm 0.11$ \\
\hline 09-DEC-95 & 4 & Deer & 7.4 & $9.05 \pm 0.15$ \\
\hline 09-DEC-95 & $5(D U P)^{a}$ & Deer & 2 & $3.75 \pm 0.1$ \\
\hline 09-DEC-95 & 5 (DUP) $^{a}$ & Deer & 2 & $3.8 \pm 0.1$ \\
\hline $09-D E C-95$ & 6 & Deer & 20.4 & $16 \pm 0.14$ \\
\hline 09-DEC-95 & 7 & Deer & 11.7 & $12.8 \pm 0.12$ \\
\hline 09-DEC-95 & 8 & Deer & 3.8 & $4.43 \pm 0.11$ \\
\hline 09-DEC-95 & 9 & Deer & 6.3 & $7.32 \pm 0.14$ \\
\hline
\end{tabular}

a Duplicate laboratory analysis on single field sample 


\section{Table 35}

Strontium-89,90 in Deer Bone

Page 1 of 1

\begin{tabular}{llc}
\hline Date & Sample Number & Sr-89,90 (pCi/g) \\
\hline $01-N O V-95$ & 1 & $2.97 \pm 0.34$ \\
04-NOV-95 & 1 & $1.71 \pm 0.22$ \\
08-NOV-95 & 1 & $1.03 \pm 0.13$ \\
$11-$ NOV-95 & 1 & $2.37 \pm 0.18$ \\
$15-N O V-95$ & 1 & $1.88 \pm 0.19$ \\
& 2 & $1.41 \pm 0.11$ \\
$18-N O V-95$ & 1 & $2.4 \pm 0.27$ \\
$25-N O V-95$ & 1 & $4.66 \pm 0.23$ \\
$02-D E C-95$ & 1 & $1.77 \pm 0.29$ \\
& 2 & $8.98 \pm 1.16$ \\
$09-D E C-95$ & 3 & $1.81 \pm 0.25$ \\
& 1 & $1.34 \pm 0.11$
\end{tabular}


Table 36

Strontium-89,90 in Deer Muscle

Page 1 of 1

\begin{tabular}{|c|c|c|}
\hline Date & Sample Number & $\mathrm{Sr}-89,90$ (pCi/g) \\
\hline Note: Blan & s indicate activity $w$ & $295 \mathrm{pCi} / \mathrm{g})$. \\
\hline \multirow[t]{2}{*}{$01-N O V-95$} & 1 & \\
\hline & $1(\text { DUP })^{a}$ & \\
\hline 01-NOV-95 & 2 & \\
\hline 01-NOV-95 & 3 & \\
\hline 01-NOV-95 & 4 & \\
\hline \multirow[t]{2}{*}{ 04-NOV-95 } & 1 & \\
\hline & 1 (DUP) $^{\mathbf{a}}$ & \\
\hline \multirow[t]{2}{*}{ 08-NOV-95 } & 1 & \\
\hline & 1 (DUP) $^{a}$ & \\
\hline \multirow[t]{2}{*}{ 11-NOV-95 } & 1 & \\
\hline & 1 (DUP) $^{a}$ & \\
\hline \multirow[t]{2}{*}{ 15-NOV-95 } & 1 & \\
\hline & 1 (DUP)a $^{a}$ & \\
\hline \multirow[t]{2}{*}{ 15-NOV-95 } & 2 & \\
\hline & 2 (DUP)a & \\
\hline \multirow[t]{2}{*}{ 18-NOV-95 } & 1 & \\
\hline & $1(\text { DUP })^{\mathrm{a}}$ & \\
\hline \multirow[t]{2}{*}{ 25-NOV-95 } & 1 & \\
\hline & 1 (DUP)a & \\
\hline \multirow[t]{2}{*}{ 02-DEC-95 } & 1 & \\
\hline & $1(\text { DUP })^{a}$ & \\
\hline 02-DEC-95 & 2 & \\
\hline 02-DEC-95 & 3 & \\
\hline \multirow[t]{2}{*}{ 06-DEC-95 } & 1 & $0.017 \pm 0.003$ \\
\hline & 1 (DUP)a & \\
\hline \multirow[t]{2}{*}{ 09-DEC-95 } & 1 & \\
\hline & 1 (DUP) ${ }^{a}$ & \\
\hline 09-DEC-95 & 2 & \\
\hline
\end{tabular}

a Duplicate laboratory analysis on single field sample 
Table 37

Radioactivity in Soil

Page 1 of 1

\begin{tabular}{|c|c|c|c|c|}
\hline \multirow[b]{2}{*}{ Location } & \multicolumn{4}{|c|}{$\mathrm{pCi} / \mathrm{g} \pm \sigma$; dry weight; $0-8 \mathrm{~cm}$ depth } \\
\hline & Sr-89,90 & Cs-137 & Pu-238 & Pu-239 \\
\hline \multicolumn{5}{|c|}{ Blank spaces indicate activity was below lower limit of detection. } \\
\hline \multicolumn{5}{|l|}{ F-Area } \\
\hline 2000 feet east & $(3.43 \pm 1.78) E-02$ & $(9.74 \pm 0.48) E-01$ & $(6.76 \pm 0.26) E-02$ & $(6.16 \pm 0.16) E-01$ \\
\hline 2000 feet north & $(0.26 \pm 1.14) E-02$ & $(1.14 \pm 0.27) E-01$ & $(4.23 \pm 0.22) E-02$ & $(1.04 \pm 0.04) E-01$ \\
\hline 2000 feet south & $(0.67 \pm 1.26) E-02$ & $(1.56 \pm 0.32) E-01$ & $(8.81 \pm 0.90) E-03$ & $(1.26 \pm 0.11) E-02$ \\
\hline 2000 feet west & $(0.68 \pm 1.60) E-02$ & $(6.83 \pm 0.42) E-01$ & $(6.64 \pm 0.27) E-02$ & $(8.83 \pm 0.33) E-02$ \\
\hline H-Area & & . & & \\
\hline 2000 feet east & $(1.31 \pm 1.60) E-02$ & $(4.60 \pm 0.36) E-01$ & $(6.31 \pm 0.66) E-03$ & $(1.77 \pm 0.12) E-02$ \\
\hline 2000 feet north & $(0.70 \pm 1.63) E-02$ & $(1.01 \pm 0.03) E+00$ & $(2.86 \pm 0.16) E-02$ & $(9.23 \pm 0.33) E-02$ \\
\hline 2000 feet south & $(2.35 \pm 1.69) E-02$ & $(7.80 \pm 0.51) E-01$ & $(8.14 \pm 0.68) E-03$ & $(4.33 \pm 0.18) E-02$ \\
\hline 2000 feet west & $(2.90 \pm 1.72) E-02$ & $(2.96 \pm 0.37) E-01$ & $6.80 \pm 0.69) E-03$ & $(3.31 \pm 0.17) E-02$ \\
\hline \multicolumn{5}{|l|}{ S-Area } \\
\hline$\# 1$ & $(-0.24 \pm 1.20) E-02$ & & $(7.08 \pm 3.22) E-04$ & $(2.82 \pm 0.48) E-03$ \\
\hline$\# 2$ & $(0.68 \pm 1.54) E-02$ & & $(6.73 \pm 2.27) \mathrm{E}-04$ & $(3.60 \pm 1.55) E-04$ \\
\hline \#3 & $(1.03 \pm 1.16) E-02$ & $(1.52 \pm 0.27) E-01$ & $(5.33 \pm 0.37) E-02$ & $(1.66 \pm 0.10) E-01$ \\
\hline$\# 4$ & $(3.00 \pm 1.59) E-02$ & & $(6.59 \pm 0.66) E-03$ & $(1.54 \pm 0.10) E-02$ \\
\hline \multicolumn{5}{|l|}{ Z-Area } \\
\hline$\# 1$ & $(0.21 \pm 1.44) E-02$ & & $(4.83 \pm 2.84) E-04$ & $(2.35 \pm 0.42) E-03$ \\
\hline$\# 3$ & $(-0.42 \pm 1.38) E-02$ & $(1.10 \pm 0.22) E-01$ & $(6.36 \pm 2.77) E-04$ & $(3.51 \pm 0.48) E-03$ \\
\hline$\# 5$ & $(-1.18 \pm 1.43) E-02$ & & $(1.78 \pm 1.78) E-04$ & $(0.44 \pm 1.72) E-04$ \\
\hline$\# 7$ & $(1.52 \pm 1.56) E-02$ & $(8.47 \pm 1.66) E-02$ & $(1.44 \pm 0.32) E-03$ & $(3.60 \pm 0.51) E-03$ \\
\hline \multicolumn{5}{|l|}{ Burial Ground } \\
\hline $643-26 \mathrm{E}-1$ & $(0.83 \pm 1.14) E-02$ & & & \\
\hline $643-26 E-2$ & $(0.57 \pm 1.13) E-02$ & & & \\
\hline \multicolumn{5}{|l|}{ Site Perimeter } \\
\hline NE quadrant & $(-0.26 \pm 1.55) E-02$ & $(2.52 \pm 0.29) E-01$ & $(2.78 \pm 3.08) E-04$ & $(5.92 \pm 0.76) E-03$ \\
\hline NW quadrant & $(1.50 \pm 1.58) E-02$ & $(3.78 \pm 0.38) E-01$ & $(1.65 \pm 0.42) \mathrm{E}-03$ & $(1.49 \pm 0.12) \mathrm{E}-02$ \\
\hline SE quadrant & $(1.85 \pm 1.62) E-02$ & $(4.24 \pm 0.35) E-01$ & $(1.70 \pm 0.45) E-03$ & $(8.24 \pm 0.91) E-03$ \\
\hline SW quadrant & $(1.58 \pm 1.64) E-02$ & $(3.75 \pm 0.34) E-01$ & $(1.90 \pm 0.72) E-03$ & $(1.14 \pm 0.19) \mathrm{E}-02$ \\
\hline \multicolumn{5}{|l|}{ 100-Mile Radius } \\
\hline Clinton, S.C. & $(7.41 \pm 1.12) E-02$ & $(2.92 \pm 0.40) E-01$ & $(5.78 \pm 1.60) E-04$ & $(6.81 \pm 0.44) E-03$ \\
\hline Savannah, Ga. & $(1.94 \pm 1.31) E-02$ & $(3.55 \pm 0.33) E-01$ & $(4.18 \pm 1.75) E-04$ & $(6.29 \pm 0.56) E-03$ \\
\hline
\end{tabular}


Table 38

Radioactivity in River and Stream Sediment

Page 1 of 3

\begin{tabular}{lllll}
\hline Cs-137, pCi/g (dry weight) & & & & \\
\hline Location & River Mile & 1993 & 1994 & 1995 \\
\hline
\end{tabular}

Note: Blank spaces indicate activity was below the lower limit of detection.

\begin{tabular}{|c|c|c|c|c|}
\hline \multicolumn{5}{|l|}{ Savannah River } \\
\hline Below Four Mile Creek & 150.2 & \multicolumn{2}{|l|}{$(1.66 \pm 0.35) \mathrm{E}-01$} & $(7.88 \pm 0.53) E-01$ \\
\hline Above Little Hell Landing & 136.5 & \multicolumn{2}{|l|}{$(2.89 \pm 0.24) E-01$} & $(4.33 \pm 0.41) E-01$ \\
\hline Below Little Hell Landing & 134.0 & $(1.17 \pm 0.26) E-01$ & $(2.71 \pm 0.42) \mathrm{E}-01$ & $(1.49 \pm 0.07) E+00$ \\
\hline Above Lower Three Runs & 129.5 & $(3.49 \pm 0.31) E-01$ & $(5.61 \pm 0.59) \mathrm{E}-01$ & $(1.03 \pm 0.05) E+00$ \\
\hline Highway 301 & 118.7 & $(1.51 \pm 0.29) E-01$ & $(2.40 \pm 0.38) E-01$ & $(2.03 \pm 0.36) E-01$ \\
\hline Demiere's Landing (control) & 160.5 & $(1.44 \pm 0.34) E-01$ & $(1.01 \pm 0.29) E-01$ & $(2.62 \pm 0.36) E-01$ \\
\hline \multicolumn{5}{|l|}{ SRS Streams } \\
\hline \multicolumn{2}{|l|}{ Four Mile at Road A-7 } & $(6.46 \pm 0.06) E+00$ & $(1.66 \pm 0.01) E+01$ & $(9.54 \pm 0.75) E-01$ \\
\hline \multicolumn{2}{|c|}{ Four Mile A-7A (in a beaver pond) } & $(3.15 \pm 0.07) E+00$ & $(8.88 \pm 0.05) E+01$ & $(7.73 \pm 0.57) \mathrm{E}-01$ \\
\hline \multicolumn{2}{|l|}{ Four Mile discharge at swamp } & $(6.61 \pm 0.32) E-01$ & $(8.98 \pm 0.39) E+01$ & $(4.23 \pm 0.17) \mathrm{E}-01$ \\
\hline \multicolumn{2}{|c|}{ Pen Branch discharge at swamp } & $(3.04 \pm 0.10) E+00$ & $(2.82 \pm 0.22) E+00$ & $(1.39 \pm 0.09) E+00$ \\
\hline \multicolumn{2}{|l|}{ Steel Creek at Road B } & $(1.07 \pm 0.01) E+01$ & $(2.91 \pm 0.31) E-01$ & $(3.56 \pm 0.20) E-01$ \\
\hline \multicolumn{2}{|c|}{ Steel Creek discharge at swamp } & $(1.37 \pm 0.04) E+00$ & $(1.03 \pm 0.02) E+01$ & $(4.71 \pm 0.06) E+00$ \\
\hline \multicolumn{2}{|l|}{ Steel Creek-Pen Branch mouth } & $(3.68 \pm 0.03) E+01$ & $(2.37 \pm 0.43) E-01$ & $(5.36 \pm 0.11) E+00$ \\
\hline \multicolumn{2}{|l|}{ Lower Three Runs mouth } & $(1.17 \pm 0.06) E+00$ & $(3.80 \pm 0.10) E-00$ & $(1.43 \pm 0.07) E+00$ \\
\hline \multicolumn{2}{|l|}{ Upper Three Runs mouth } & $(6.45 \pm 0.44) E-01$ & $(1.55 \pm 0.35) E-01$ & $(2.87 \pm 0.53) E-01$ \\
\hline \multicolumn{5}{|l|}{ Co-60, pCi/g (dry weight) } \\
\hline \multicolumn{2}{|r|}{ River Mile } & 1993 & 1994 & 1995 \\
\hline \multicolumn{5}{|l|}{ Savannah River } \\
\hline Below Four Mile Creek & \multicolumn{2}{|l|}{150.2} & & \\
\hline Above Little Hell Landing & \multicolumn{2}{|l|}{136.5} & & \\
\hline Below Little Hell Landing & \multicolumn{2}{|l|}{134.0} & & \\
\hline Above Lower Three Runs & \multicolumn{2}{|l|}{129.5} & & \\
\hline Highway 301 & \multicolumn{2}{|l|}{118.7} & & \\
\hline Demiere's Landing (control) & \multicolumn{2}{|l|}{160.5} & & \\
\hline \multicolumn{5}{|l|}{ SRS Streams } \\
\hline \multicolumn{2}{|l|}{ Four Mile at Road A-7 } & $(8.74 \pm 1.32) E-02$ & $(2.49 \pm 0.15) E-01$ & \\
\hline \multicolumn{2}{|c|}{ Four Mile A-7A (in a beaver pond) } & & $(1.17 \pm 0.06) E+00$ & \\
\hline \multicolumn{2}{|l|}{ Four Mile discharge at swamp } & & $(6.27 \pm 1.13) E-02$ & \\
\hline \multicolumn{2}{|c|}{ Pen Branch discharge at swamp } & $(2.61 \pm 0.47) E-01$ & & \\
\hline \multicolumn{2}{|l|}{ Steel Creek at Road B } & $(1.45 \pm 0.19) E-01$ & & \\
\hline \multicolumn{2}{|c|}{ Steel Creek discharge at swamp } & & $(3.17 \pm 0.26) E-01$ & $(2.10 \pm 0.12) E-01$ \\
\hline Steel C̣reek-Pen Branch mo & uth & $(1.02 \pm 0.05) E+00$ & & \\
\hline Lower Three Runs mouth & & & & \\
\hline Upper Three Runs mouth & & & & \\
\hline
\end{tabular}




\section{Table 38}

\section{Radioactivity in River and Stream Sediment}

Page 2 of 3

\begin{tabular}{|c|c|c|c|c|}
\hline \multicolumn{5}{|l|}{ Sr-89,90, pCi/g (dry weight) } \\
\hline Location & River Mile & 1993 & 1994 & 1995 \\
\hline \multicolumn{5}{|l|}{ Savannah River } \\
\hline Below Four Mile Creek & 150.2 & $(5.40 \pm 3.17) E-02$ & $(1.51 \pm 1.40) E-02$ & $(6.70 \pm 1.56) E-02$ \\
\hline Above Little Hell Landing & 136.5 & $(-2.49 \pm 1.41) E-02$ & $(1.23 \pm 1.11) E-02$ & $(-0.39 \pm 1.30) E-02$ \\
\hline Below Little Hell Landing & 134.0 & $(-0.18 \pm 1.91) E-02$ & $(0.74 \pm 1.47) E-02$ & $(0.94 \pm 1.39) E-02$ \\
\hline Above Lower Three Runs & 129.5 & $(-2.43 \pm 1.38) E-02$ & $(0.15 \pm 1.51) E-02$ & $(0.90 \pm 1.24) E-02$ \\
\hline Highway 301 & 118.7 & $(-1.45 \pm 1.47) \mathrm{E}-02$ & $(0.62 \pm 1.62) \mathrm{E}-02$ & $(-0.23 \pm 1.27) E-02$ \\
\hline Demiere's Landing (control) & 160.5 & $(6.68 \pm 3.23) E-02$ & $(1.28 \pm 1.46) E-02$ & $(0.83 \pm 1.14) E-02$ \\
\hline \multicolumn{5}{|l|}{ SRS Streams } \\
\hline \multicolumn{2}{|l|}{ Four Mile at Road A-7 } & $(2.47 \pm 0.26) E-01$ & $(3.42 \pm 0.20) E-01$ & $(4.17 \pm 0.27) E-01$ \\
\hline \multicolumn{2}{|c|}{ Four Mile A-7A (in a beaver pond) } & $(4.35 \pm 1.71) E-02$ & $(1.56 \pm 0.04) E+00$ & $(8.77 \pm 0.31) E-01$ \\
\hline \multicolumn{2}{|c|}{ Four Mile discharge at swamp } & $(1.15 \pm 1.56) E-02$ & $(3.03 \pm 1.82) E-02$ & $(4.36 \pm 1.69) E-02$ \\
\hline \multicolumn{2}{|c|}{ Pen Branch discharge at swamp } & $(3.48 \pm 2.38) E-02$ & $(1.25 \pm 0.23) \mathrm{E}-01$ & $(0.63 \pm 1.01) E-02$ \\
\hline \multicolumn{2}{|l|}{ Steel Creek at Road B } & $(-0.16 \pm 2.23) E-02$ & $(0.00 \pm 1.62) E-02$ & $(0.77 \pm 1.48) E-02$ \\
\hline \multicolumn{2}{|c|}{ Steel Creek discharge at swamp } & $(2.71 \pm 1.62) \mathrm{E}-02$ & $(-0.82 \pm 1.45) E-02$ & $(1.55 \pm 1.64) \mathrm{E}-02$ \\
\hline \multicolumn{2}{|c|}{ Steel Creek-Pen Branch mouth } & $(8.22 \pm 2.57) E-02$ & $(1.89 \pm 1.48) E-02$ & $(-0.09 \pm 1.53) E-02$ \\
\hline \multicolumn{2}{|l|}{ Lower Three Runs mouth } & $(-0.96 \pm 2.20) E-02$ & $(2.91 \pm 1.46) E-02$ & $(0.68 \pm 1.28) E-02$ \\
\hline \multicolumn{2}{|l|}{ Upper Three Runs mouth } & $(3.49 \pm 3.10) E-02$ & $(0.21 \pm 1.31) E-02$ & $(-1.17 \pm 1.45) E-02$ \\
\hline \multicolumn{5}{|l|}{ Pu-238, pCi/g (dry weight) } \\
\hline \multicolumn{2}{|l|}{ Location } & 1993 & 1994 & 1995 \\
\hline \multicolumn{5}{|l|}{ Savannah River } \\
\hline \multicolumn{2}{|l|}{ Below Four Mile Creek } & $(-2.15 \pm 2.15) E-04$ & $(3.06 \pm 1.31) \mathrm{E}-04$ & $(6.12 \pm 4.33) \mathrm{E}-04$ \\
\hline Above Little Hell Landing & 136.5 & $(2.02 \pm 1.78) E-04$ & $(1.59 \pm 0.92) E-04$ & $(2.93 \pm 2.32) E-04$ \\
\hline Below Little Hell Landing & 134.0 & $(3.78 \pm 1.89) E-04$ & $(2.15 \pm 1.14) E-04$ & $(1.09 \pm 0.27) E-03$ \\
\hline Above Lower Three Runs & 129.5 & $(-4.97 \pm 4.97) E-04$ & $(1.96 \pm 2.36) E-04$ & $(3.44 \pm 2.69) E-04$ \\
\hline Highway 301 & 118.7 & $(00.00 \pm 5.65) E-04$ & $(1.11 \pm 0.26) E-03$ & $(1.30 \pm 0.52) E-03$ \\
\hline Demiere's Landing (control) & 160.5 & $(2.00 \pm 2.00) E-04$ & $(1.51 \pm 1.52) E-04$ & $(1.21 \pm 0.85) E-04$ \\
\hline \multicolumn{5}{|l|}{ SRS Streams } \\
\hline \multicolumn{2}{|l|}{ Four Mile at Road A-7 } & $(7.50 \pm 0.41) E-02$ & $(2.06 \pm 0.006) E-01$ & $(5.58 \pm 7.65) E-04$ \\
\hline \multicolumn{2}{|c|}{ Four Mile A-7A (in a beaver pond) } & $(3.08 \pm 0.26) E-02$ & $(1.23 \pm 0.03) E+00$ & $(3.08 \pm 0.83) E-03$ \\
\hline \multicolumn{2}{|c|}{ Four Mile discharge at swamp } & $(4.85 \pm 0.86(E-03$ & $(4.44 \pm 0.51) E-03$ & $(2.40 \pm 0.43) E-03$ \\
\hline \multicolumn{2}{|c|}{ Pen Branch discharge at swamp } & $(4.76 \pm 1.12) E-03$ & $(5.94 \pm 0.55) E-03$ & $(1.45 \pm 0.45) E-03$ \\
\hline \multicolumn{2}{|l|}{ Steel Creek at Road B } & $(5.09 \pm 1.05) \mathrm{E}-03$ & $(6.61 \pm 3.09) E-04$ & $(1.36 \pm 0.49) E-03$ \\
\hline \multicolumn{2}{|c|}{ Steel Creek discharge at swamp } & $2.63 \pm 0.44) E-03$ & $(3.79 \pm 0.18) E-02$ & $(1.37 \pm 0.14) E-02$ \\
\hline Steel Creek-Pen Branch mc & outh & $(6.08 \pm 0.30) E-02$ & $(3.09 \pm 1.89) E-04$ & $(2.63 \pm 0.54) E-03$ \\
\hline Lower Three Runs mouth & & $(00.00 \pm 4.13) E-04$ & $(8.86 \pm 3.80) E-04$ & $(-0.58 \pm 1.75) E-04$ \\
\hline Upper Three Runs mouth & & $(3.24 \pm 0.65) E-03$ & $(1.97 \pm 0.29) E-03$ & $(2.82 \pm 0.47) E-03$ \\
\hline
\end{tabular}




\section{\&}

Table 38

Radioactivity in River and Stream Sediment

Page 3 of 3

\begin{tabular}{|c|c|c|c|c|}
\hline \multicolumn{5}{|l|}{ Pu-239, pCi/g (dry weight) } \\
\hline Location & River Mile & 1993 & 1994 & 1995 \\
\hline \multicolumn{5}{|l|}{ Savannah River } \\
\hline Below Four Mile Creek & 150.2 & $(4.29 \pm 3.03) E-04$ & $(1.25 \pm 0.36) E-03$ & $(2.89 \pm 0.77) E-03$ \\
\hline Above Little Hell Landing & 136.5 & $(1.21 \pm 0.32) E-03$ & $(5.28 \pm 1.84) E-04$ & $(2.34 \pm 0.44) \mathrm{E}-03$ \\
\hline Below Little Hell Landing & 134.0 & $(7.54 \pm 2.98) \mathrm{E}-04$ & $(1.00 \pm 0.20) E-03$ & $(5.86 \pm 0.55) E-03$ \\
\hline Above Lower Three Runs & 129.5 & $(2.48 \pm 5.55) E-04$ & $(2.48 \pm 0.43) E-03$ & $(2.17 \pm 0.41) E-03$ \\
\hline Highway 301 & 118.7 & $(00.00 \pm 5.64) E-04$ & $(3.88 \pm 1.67) E-04$ & $(8.23 \pm 1.09) E-03$ \\
\hline Demiere's Landing & 160.5 & $(9.97 \pm 3.46) \mathrm{E}-04$ & $(2.03 \pm 0.44) E-03$ & $(1.26 \pm 0.29) E-03$ \\
\hline \multicolumn{5}{|l|}{ SRS Streams } \\
\hline \multicolumn{2}{|l|}{ Four Mile at Road $A-7$} & $(3.06 \pm 0.25) E-02$ & $(8.72 \pm 0.31) \mathrm{E}-02$ & $(-7.78 \pm 6.19) E-04$ \\
\hline \multicolumn{2}{|c|}{ Four Mile A-7A (in a beaver pond) } & $(1.55 \pm 0.18) E-02$ & $(5.25 \pm 0.12) E-01$ & $(1.30 \pm 0.59) E-03$ \\
\hline \multicolumn{2}{|c|}{ Four Mile discharge at swamp } & $(3.76 \pm 0.76) E-03$ & $(4.11 \pm 0.46) E-03$ & $(1.81 \pm 0.37) E-03$ \\
\hline \multicolumn{2}{|c|}{ Pen Branch discharge at swamp } & $(2.15 \pm 0.22) E-02$ & $(1.43 \pm 0.09) E-02$ & $(1.41 \pm 0.11) E-02$ \\
\hline \multicolumn{2}{|l|}{ Steel Creek at Road B } & $(1.24 \pm 0.16) E-02$ & $(6.63 \pm 0.75) E-03$ & $(9.49 \pm 0.99) \mathrm{E}-03$ \\
\hline \multicolumn{2}{|c|}{ Steel Creek discharge at swamp } & $(2.62 \pm 0.45) E-03$ & $(2.90 \pm 0.15) \mathrm{E}-02$ & $(1.21 \pm 0.12) E-02$ \\
\hline \multicolumn{2}{|c|}{ Steel Creek-Pen Branch mouth } & $(8.15 \pm 0.36) E-02$ & $(4.10 \pm 1.79) E-04$ & $(5.24 \pm 0.76) E-03$ \\
\hline \multicolumn{2}{|l|}{ Lower Three Runs mouth } & $(1.75 \pm 0.82) E-03$ & $(4.04 \pm 0.78) E-03$ & $(1.92 \pm 0.34) E-03$ \\
\hline \multicolumn{2}{|l|}{ Upper Three Runs mouth } & $(1.52 \pm 0.14) \mathrm{E}-02$ & $(2.69 \pm 0.31) E-03$ & $(5.05 \pm 0.62) E-03$ \\
\hline
\end{tabular}


Table 39

Radioactivity in Terrestrial Vegetation (Quarterly Samples)

Page 1 of 6

\begin{tabular}{|c|c|c|c|c|}
\hline Location & $\begin{array}{l}\text { No. of } \\
\text { Samples }\end{array}$ & Arithmetic Mean $\pm \sigma$ & Maximum $\pm \sigma$ & Minimum $\pm \sigma$ \\
\hline \multicolumn{5}{|l|}{$\mathrm{H}-3, \mathrm{pCl} / \mathrm{g}$} \\
\hline \multicolumn{5}{|l|}{ On Site } \\
\hline $200-F \# 13$ & 4 & $(9.67 \pm 7.58) E+00$ & $(1.59 \pm 0.01) E+01$ & $(3.77 \pm 0.21) E-01$ \\
\hline $200-F \# 21$ & 4 & $(3.09 \pm 5.25) E+00$ & $(1.09 \pm 0.01) E+01$ & $(2.03 \pm 0.82) E-02$ \\
\hline $200-\mathrm{H} \# 10$ & 4 & $(4.27 \pm 2.85) E+00$ & $(7.30 \pm 0.06) E+00$ & $(4.37 \pm 0.21) E-01$ \\
\hline $200-H$ \#22 & 4 & $(5.91 \pm 3.67) E-01$ & $(9.40 \pm 0.21) E-01$ & $(1.97 \pm 0.06) E-01$ \\
\hline $643-26 E-1$ & 4 & $(7.10 \pm 8.31) E+00$ & $(1.87 \pm 0.02) E+01$ & $(9.28 \pm 0.24) E-01$ \\
\hline $643-26 E-2$ & 4 & $(2.54 \pm 2.61) E+00$ & $(6.41 \pm 0.04) E+00$ & $(6.92 \pm 0.23) E-01$ \\
\hline S-Area \#1 & 4 & $(9.98 \pm 3.15) E-01$ & $(1.39 \pm 0.03) E+00$ & $(6.22 \pm 0.36) E-01$ \\
\hline S-Area \#2 & 4 & $(1.43 \pm 0.50) E+00$ & $(2.04 \pm 0.02) E+00$ & $(8.33 \pm 0.55) E-01$ \\
\hline S-Area \#3 & 4 & $(3.12 \pm 2.67) E+00$ & $(5.98 \pm 0.05) E+00$ & $(5.80 \pm 0.19) E-01$ \\
\hline S-Area \#4 & 4 & $(1.02 \pm 0.67) E+00$ & $(1.66 \pm 0.03) E+00$ & $(3.47 \pm 0.23) E-01$ \\
\hline Z-Area \#1 & 4 & $(1.20 \pm 0.76) E+00$ & $(1.88 \pm 0.07) E+00$ & $(4.79 \pm 0.24) E-01$ \\
\hline Z-Area \#2 & 4 & $(7.53 \pm 6.28) E-01$ & $(1.65 \pm 0.06) E+00$ & $(1.81 \pm 0.21) E-01$ \\
\hline Z-Area \#3 & 4 & $(1.05 \pm 0.82) E+00$ & $(1.93 \pm 0.07) E+00$ & $(2.32 \pm 0.23) E-01$ \\
\hline Z-Area \#4 & 4 & $(1.08 \pm 0.70) E+00$ & $(1.64 \pm 0.05) E+00$ & $(1.03 \pm 0.19) E-01$ \\
\hline Z-Area \#5 & 4 & $(8.76 \pm 5.94) E-01$ & $(1.54 \pm 0.03) E+00$ & $(2.44 \pm 0.24) E-01$ \\
\hline Z-Area \#6 & 4 & $(0.93 \pm 1.20) E+00$ & $(2.70 \pm 0.07) \mathrm{E}+00$ & $(8.88 \pm 2.02) E-02$ \\
\hline Z-Area \#7 & 4 & $(1.14 \pm 0.97) E+00$ & $(2.49 \pm 0.07) E+00$ & $(4.16 \pm 0.45) E-01$ \\
\hline Z-Area \#8 & 4 & $(7.91 \pm 3.21) E-01$ & $(1.06 \pm 0.02) E+00$ & $(4.03 \pm 0.46) E-01$ \\
\hline \multicolumn{5}{|l|}{ Site Perimeter } \\
\hline Allendale Gate & 2 & $(0.87 \pm 1.27) E-01$ & $(1.77 \pm 0.44) E-01$ & $(-2.94 \pm 8.53) E-03$ \\
\hline Barnwell Gate & 2 & $(4.05 \pm 0.69) E-02$ & $(4.54 \pm 3.52) E-02$ & $(3.56 \pm 1.35) E-02$ \\
\hline D-Area & 3 & $(7.64 \pm 5.35) E-01$ & $(1.13 \pm 0.03) E+00$ & $(1.51 \pm 0.27) \mathrm{E}-01$ \\
\hline $\begin{array}{l}\text { Darkhorse @ Williston } \\
\text { Gate }\end{array}$ & 2 & $(1.39 \pm 1.87) E-01$ & $(2.72 \pm 0.26) E-01$ & $(6.94 \pm 8.85) E-03$ \\
\hline East Talatha & 2 & $(1.32 \pm 0.39) E-01$ & $(1.60 \pm 0.49) E-01$ & $(1.05 \pm 0.38) E-01$ \\
\hline Green Pond & 3 & $(1.36 \pm 1.13) E-01$ & $(2.36 \pm 0.39) E-01$ & $(1.40 \pm 0.81) E-02$ \\
\hline $\begin{array}{l}\text { Highway } 125 @ \\
\text { Road A-14 }\end{array}$ & 2 & $(2.42 \pm 2.42) E-01$ & $(4.13 \pm 0.29) E-01$ & $(7.09 \pm 4.35) E-02$ \\
\hline Highway 21/167 & 2 & $(4.10 \pm 1.57) E-02$ & $(5.21 \pm 3.43) E-02$ & $(2.98 \pm 1.53) E-02$ \\
\hline $\begin{array}{l}\text { Highway } 39 @ \text { Williston } \\
\text { Gate }\end{array}$ & 2 & $(1.89 \pm 2.52) E-01$ & $(3.67 \pm 0.29) E-01$ & $(1.08 \pm 0.75) E-02$ \\
\hline Jackson & 3 & $(9.44 \pm 9.07) \mathrm{E}-02$ & $(1.93 \pm 0.35) E-01$ & $(1.38 \pm 2.97) E-02$ \\
\hline Patterson Mill Road & 2 & $(8.09 \pm 4.63) E-02$ & $(1.14 \pm 0.35) E-01$ & $(4.81 \pm 3.48) E-02$ \\
\hline
\end{tabular}


Table 39

Radioactivity in Terrestrial Vegetation (Quarterly Samples)

Page 2 of 6

\begin{tabular}{|c|c|c|c|c|}
\hline Location & $\begin{array}{l}\text { No. of } \\
\text { Samples }\end{array}$ & Arithmetic Mean $\pm \sigma$ & Maximum $\pm \sigma$ & Minimum $\pm \sigma$ \\
\hline Talatha Gate & 3 & $(2.22 \pm 0.78) E-01$ & $(3.00 \pm 0.48) E-01$ & $(1.45 \pm 0.26) E-01$ \\
\hline West Jackson & 3 & $(8.11 \pm 4.46) E-02$ & $(1.32 \pm 0.18) E-01$ & $(5.02 \pm 2.09) \mathrm{E}-02$ \\
\hline Windsor Road & 2 & $(3.19 \pm 3.06) E-02$ & $(5.36 \pm 2.14) E-02$ & $(1.03 \pm 1.64) \mathrm{E}-02$ \\
\hline \multicolumn{5}{|l|}{ 25-Mile Radius } \\
\hline Allendale, S.C. & 1 & $(8.58 \pm 3.96) E-02$ & $(8.58 \pm 3.96) E-02$ & $(8.58 \pm 3.96) E-02$ \\
\hline Langley, S.C. & 2 & $(2.47 \pm 0.04) E-02$ & $(2.50 \pm 1.72) E-02$ & $(2.44 \pm 1.66) E-02$ \\
\hline Springfield, S.C. & 2 & $(4.98 \pm 5.49) E-02$ & $(8.86 \pm 3.74) E-02$ & $(1.10 \pm 3.22) E-02$ \\
\hline Waynesboro, Ga. & 2 & $(7.68 \pm 5.18) E-02$ & $(1.13 \pm 0.45) E-01$ & $(4.02 \pm 2.96) E-02$ \\
\hline \multicolumn{5}{|l|}{ 100-Mile Radius } \\
\hline Columbia, S.C. & 3 & $(3.61 \pm 1.97) \mathrm{E}-02$ & $(5.43 \pm 3.70) \mathrm{E}-02$ & $(1.51 \pm 0.91) \mathrm{E}-02$ \\
\hline Greenville, S.C. & 2 & $(2.37 \pm 3.12) E-02$ & $(4.58 \pm 1.74) \mathrm{E}-02$ & $(0.16 \pm 1.02) E-02$ \\
\hline Macon, Ga. & 2 & $(-1.13 \pm 4.29) E-02$ & $(1.90 \pm 0.70) E-02$ & $(-4.16 \pm 3.21) \mathrm{E}-02$ \\
\hline Savannah, Ga. & 4 & $(4.55 \pm 3.83) E-02$ & $(8.63 \pm 2.51) \mathrm{E}-02$ & $(-0.58 \pm 2.72) E-02$ \\
\hline \multicolumn{5}{|l|}{ Cs-137, pCi/g } \\
\hline \multicolumn{5}{|l|}{ On Site } \\
\hline $643-26 E-1$ & 2 & $(1.29 \pm 0.11) E-01$ & $(1.37 \pm 0.33) E-01$ & $(1.21 \pm 0.23) E-01$ \\
\hline F-Area & 4 & $(3.60 \pm 0.74) E-01$ & $(4.23 \pm 0.38) E-01$ & $(2.56 \pm 0.25) E-01$ \\
\hline H-Area & 4 & $(2.47 \pm 0.91) E-01$ & $(3.55 \pm 0.45) E-01$ & $(1.47 \pm 0.34) E-01$ \\
\hline S-Area \#1 & 1 & $(1.20 \pm 0.44) E-01$ & $(1.20 \pm 0.44) E-01$ & $(1.20 \pm 0.44) E-01$ \\
\hline Z-Area \#1 & 2 & $(6.70 \pm 3.57) E-02$ & $(9.22 \pm 3.27) E-02$ & $(4.18 \pm 1.56) \mathrm{E}-02$ \\
\hline Z-Area \#3 & 2 & $(1.26 \pm 0.02) E-01$ & $(1.27 \pm 0.42) \mathrm{E}-01$ & $(1.24 \pm 0.27) E-01$ \\
\hline Z-Area \#6 & 1 & $(8.48 \pm 2.68) E-02$ & $(8.48 \pm 2.68) E-02$ & $(8.48 \pm 2.68) E-02$ \\
\hline Z-Area \#8 & 3 & $(2.30 \pm 0.77) E-01$ & $(3.18 \pm 0.44) E-01$ & $(1.77 \pm 0.48) E-01$ \\
\hline \multicolumn{5}{|l|}{ Site Perimeter } \\
\hline Allendale Gate & 2 & $(3.49 \pm 1.23) E-01$ & $(4.37 \pm 0.57) \mathrm{E}-01$ & $(2.62 \pm 0.48) E-01$ \\
\hline Barnwell Gate & 2 & $(6.16 \pm 4.49) E-01$ & $(9.33 \pm 0.73) \mathrm{E}-01$ & $(2.99 \pm 0.52) \mathrm{E}-01$ \\
\hline D-Area & 1 & $(2.87 \pm 0.85) E-01$ & $(2.87 \pm 0.85) \mathrm{E}-01$ & $(2.87 \pm 0.85) E-01$ \\
\hline $\begin{array}{l}\text { Darkhorse @ Williston } \\
\text { Gate }\end{array}$ & 2 & $(2.76 \pm 0.79) E-01$ & $(3.32 \pm 0.60) \mathrm{E}-01$ & $(2.20 \pm 0.51) E-01$ \\
\hline East Talatha & 2 & $(5.39 \pm 2.55) E-01$ & $(7.19 \pm 0.76) E-01$ & $(3.59 \pm 0.56) E-01$ \\
\hline Green Pond & 1 & $(1.97 \pm 0.55) E-01$ & $(1.97 \pm 0.55) E-01$ & $(1.97 \pm 0.55) E-01$ \\
\hline $\begin{array}{l}\text { Highway } 125 @ \\
\text { Road A-14 }\end{array}$ & 1 & $(1.94 \pm 0.39) E-01$ & $(1.94 \pm 0.39) E-01$ & $(1.94 \pm 0.39) E-01$ \\
\hline Highway $21 / 167$ & 1 & $(1.57 \pm 0.38) E-01$ & $(1.57 \pm 0.38) E-01$ & $(1.57 \pm 0.38) \mathrm{E}-01$ \\
\hline
\end{tabular}




\section{Table 39}

\section{Radioactivity in Terrestrial Vegetation (Quarterly Samples)}

Page 3 of 6

\begin{tabular}{|c|c|c|c|c|}
\hline Location & $\begin{array}{l}\text { No. of } \\
\text { Samples }\end{array}$ & Arithmetic Mean $\pm \sigma$ & Maximum $\pm \sigma$ & Minimum $\pm \sigma$ \\
\hline $\begin{array}{l}\text { Highway } 39 @ \text { Williston } \\
\text { Gate }\end{array}$ & 1 & $(2.98 \pm 0.47) E-01$ & $(2.98 \pm 0.47) E-01$ & $(2.98 \pm 0.47) \mathrm{E}-01$ \\
\hline Patterson Mill Road & 1 & $(2.31 \pm 0.33) E-01$ & $(2.31 \pm 0.33) \mathrm{E}-01$ & $(2.31 \pm 0.33) E-01$ \\
\hline Site perimeter & 3 & $(1.54 \pm 0.41) E-01$ & $(1.93 \pm 0.33) \mathrm{E}-01$ & $(1.12 \pm 0.30) E-01$ \\
\hline Talatha Gate & 2 & $(2.13 \pm 0.83) E-01$ & $(2.71 \pm 0.46) E-01$ & $(1.54 \pm 0.44) E-01$ \\
\hline West Jackson & 1 & $(2.21 \pm 0.53) E-01$ & $(2.21 \pm 0.53) E-01$ & $(2.21 \pm 0.53) E-01$ \\
\hline Windsor Road & 1 & $(1.76 \pm 0.45) E-01$ & $(1.76 \pm 0.45) E-01$ & $(1.76 \pm 0.45) E-01$ \\
\hline \multicolumn{5}{|l|}{ 25-Mile Radius } \\
\hline 25 -mile radius & 1 & $(1.53 \pm 0.17) E-01$ & $(1.53 \pm 0.17) E-01$ & $(1.53 \pm 0.17) E-01$ \\
\hline Langley, S.C. & 1 & $(9.18 \pm 0.63) E-01$ & $(9.18 \pm 0.63) E-01$ & $(9.18 \pm 0.63) E-01$ \\
\hline \multicolumn{5}{|l|}{ 100-Mile Radius } \\
\hline 100 -mile radius & 2 & $(9.99 \pm 0.63) E-02$ & $(1.04 \pm 0.13) E-01$ & $(9.55 \pm 2.40) \mathrm{E}-02$ \\
\hline \multicolumn{5}{|l|}{$\mathrm{Sr}-89,90, \mathrm{pCl} / \mathrm{g}$} \\
\hline \multicolumn{5}{|l|}{ On Site } \\
\hline $643-26 E-1$ & 4 & $(2.90 \pm 1.96) E-01$ & $(5.19 \pm 0.78) E-01$ & $(5.53 \pm 6.21) E-02$ \\
\hline $643-26 E-2$ & 4 & $(0.96 \pm 1.23) E-01$ & $(2.48 \pm 0.47) E-01$ & $(-2.16 \pm 6.55) E-02$ \\
\hline F-Area & 3 & $(5.33 \pm 2.23) E-01$ & $(7.91 \pm 0.87) \mathrm{E}-01$ & $(3.98 \pm 0.63) E-01$ \\
\hline H-Area & 4 & $(4.80 \pm 2.07) E-01$ & $(7.19 \pm 0.76) \mathrm{E}-01$ & $(2.43 \pm 0.68) E-01$ \\
\hline S-Area \#1 & 4 & $(2.54 \pm 1.13) \mathrm{E}-01$ & $(3.95 \pm 0.44) \mathrm{E}-01$ & $(1.22 \pm 0.59) \mathrm{E}-01$ \\
\hline Z-Area \#1 & 4 & $(2.88 \pm 1.48) E-01$ & $(4.75 \pm 0.50) E-01$ & $(1.15 \pm 0.62) E-01$ \\
\hline \multicolumn{5}{|l|}{ Site Perimeter } \\
\hline Site perimeter & 3 & $(6.15 \pm 2.00) E-01$ & $(8.39 \pm 0.49) E-01$ & $(4.52 \pm 1.02) E-01$ \\
\hline \multicolumn{5}{|l|}{ 25-Mile Radius } \\
\hline 25-mile radjus & 2 & $(2.79 \pm 1.18) \mathrm{E}-01$ & $(3.63 \pm 0.79) \mathrm{E}-01$ & $(1.95 \pm 0.69) E-01$ \\
\hline \multicolumn{5}{|l|}{ 100-Mile Radius } \\
\hline 100 -mile radius & 3 & $(1.64 \pm 0.39) E-01$ & $(1.97 \pm 0.44) E-01$ & $(1.20 \pm 0.64) E-01$ \\
\hline \multicolumn{5}{|l|}{ Gross Beta, pCi/g } \\
\hline \multicolumn{5}{|l|}{ On Site } \\
\hline $200-F \# 13$ & 4 & $(9.65 \pm 2.83) E+00$ & $(1.19 \pm 0.13) E+01$ & $(5.51 \pm 1.07) \mathrm{E}+00$ \\
\hline 200-F \#21 & 4 & $(1.14 \pm 0.67) E+01$ & $(2.13 \pm 0.17) E+01$ & $(7.18 \pm 0.61) E+00$ \\
\hline $200 \cdot \mathrm{H} \# 10$ & 4 & $(1.14 \pm 0.52) E+01$ & $(1.73 \pm 0.11) E+01$ & $(6.89 \pm 1.09) E+00$ \\
\hline $200 \cdot H \# 22$ & 4 & $(8.09 \pm 3.87) E+00$ & $(1.32 \pm 0.14) E+01$ & $(4.62 \pm 0.73) E+00$ \\
\hline $643-26 E-1$ & 4 & $(9.25 \pm 4.82) E+00$ & $(1.61 \pm 0.16) E+01$ & $(4.98 \pm 0.75) E+00$ \\
\hline $643-26 E-2$ & 4 & $(1.61 \pm 1.08) E+01$ & $(2.83 \pm 0.20) E+01$ & $(4.98 \pm 0.75) E+00$ \\
\hline
\end{tabular}


Table 39

Radioactivity in Terrestrial Vegetation (Quarterly Samples)

Page 4 of 6

\begin{tabular}{|c|c|c|c|c|}
\hline Location & $\begin{array}{l}\text { No. of } \\
\text { Samples }\end{array}$ & Arithmetic Mean $\pm \sigma$ & Maximum $\pm \sigma$ & Minimum $\pm \sigma$ \\
\hline S-Area \#1 & 4 & $(1.26 \pm 0.68) E+01$ & $(2.20 \pm 0.17) E+01$ & $(6.58 \pm 0.58) E+00$ \\
\hline S-Area \#2 & 4 & $(1.70 \pm 0.78) E+01$ & $(2.65 \pm 0.18) E+01$ & $(9.96 \pm 0.95) E+00$ \\
\hline S-Area \#3 & 4 & $(1.34 \pm 0.81) E+01$ & $(2.25 \pm 0.17) E+01$ & $(3.91 \pm 0.68) E+00$ \\
\hline S-Area \#4 & 4 & $(9.29 \pm 2.59) E+00$ & $(1.18 \pm 0.12) E+01$ & $(5.69 \pm 0.77) E+00$ \\
\hline Z-Area \#1 & 4 & $(1.03 \pm 0.54) E+01$ & $(1.80 \pm 0.16) E+01$ & $(6.05 \pm 0.80) E+00$ \\
\hline Z-Area \#2 & 4 & $(1.22 \pm 0.33) E+01$ & $(1.63 \pm 0.14) E+01$ & $(8.53 \pm 0.91) E+00$ \\
\hline Z-Area \#3 & 4 & $(5.28 \pm 1.08) E+00$ & $(6.74 \pm 0.96) E+00$ & $(4.15 \pm 0.96) E+00$ \\
\hline Z-Area \#4 & 4 & $(1.47 \pm 0.87) E+01$ & $(2.65 \pm 0.18) E+01$ & $(6.05 \pm 0.80) E+00$ \\
\hline Z-Area \#5 & 4 & $(9.86 \pm 4.48) E+00$ & $(1.60 \pm 0.15) E+01$ & $(5.56 \pm 0.87) E+00$ \\
\hline Z-Area \#6 & 4 & $(8.91 \pm 4.10) E+00$ & $(1.35 \pm 0.13) E+01$ & $(3.56 \pm 0.67) E+00$ \\
\hline Z-Area \#7 & 4 & $(1.31 \pm 0.75) E+01$ & $(2.42 \pm 0.17) E+01$ & $(8.43 \pm 1.04) E+00$ \\
\hline Z-Area \#8 & 4 & $(1.22 \pm 0.40) E+01$ & $(1.52 \pm 0.14) E+01$ & $(6.40 \pm 0.81) E+00$ \\
\hline \multicolumn{5}{|l|}{ Site Perimeter } \\
\hline Allendale Gate & 3 & $(1.20 \pm 0.76) E+01$ & $(2.07 \pm 0.20) E+01$ & $(7.14 \pm 1.13) E+00$ \\
\hline Barnwell Gate & 3 & $(1.30 \pm 0.91) E+01$ & $(2.33 \pm 0.17) E+01$ & $(6.06 \pm 1.31) E+00$ \\
\hline D-Area & 3 & $(8.80 \pm 3.77) E+00$ & $(1.30 \pm 0.14) E+01$ & $(5.74 \pm 1.05) E+00$ \\
\hline $\begin{array}{l}\text { Darkhorse @ Williston } \\
\text { Gate }\end{array}$ & 3 & $(9.05 \pm 3.16) E+00$ & $(1.19 \pm 0.08) E+01$ & $(5.62 \pm 1.30) E+00$ \\
\hline East Talatha & 3 & $(1.68 \pm 0.68) E+01$ & $(2.24 \pm 0.16) E+01$ & $(9.18 \pm 1.24) E+00$ \\
\hline Green Pond & 3 & $(1.63 \pm 0.63) E+01$ & $(2.10 \pm 0.16) E+01$ & $(9.18 \pm 1.27) E+00$ \\
\hline $\begin{array}{l}\text { Highway } 125 \text { @ } \\
\text { Road A-14 }\end{array}$ & 3 & $(2.51 \pm 2.42) E+01$ & $(5.31 \pm 0.38) E+01$ & $(1.00 \pm 0.14) E+01$ \\
\hline Highway $21 / 167$ & 3 & $(1.67 \pm 0.55) E+01$ & $(2.29 \pm 0.16) E+01$ & $(1.33 \pm 0.14) E+01$ \\
\hline $\begin{array}{l}\text { Highway } 39 \text { @ Williston } \\
\text { Gate }\end{array}$ & 3 & $(1.57 \pm 0.60) E+01$ & $(2.25 \pm 0.16) E+01$ & $(1.12 \pm 0.15) E+01$ \\
\hline Jackson & 3 & $(1.55 \pm 1.02) E+01$ & $(2.70 \pm 0.16) E+01$ & $(7.67 \pm 0.98) E+00$ \\
\hline Patterson Mill Road & 3 & $(1.14 \pm 0.58) E+01$ & $(1.79 \pm 0.20) E+01$ & $(7.07 \pm 1.24) E+00$ \\
\hline Talatha Gate & 3 & $(7.73 \pm 3.71) E+00$ & $(1.20 \pm 0.14) E+01$ & $(5.10 \pm 1.07) E+00$ \\
\hline West Jackson & 3 & $(1.86 \pm 1.50) E+01$ & $(3.52 \pm 0.25) E+01$ & $(6.12 \pm 1.11) E+00$ \\
\hline Windsor Road & 2 & $(2.63 \pm 0.98) E+01$ & $(3.32 \pm 0.19) E+01$ & $(1.94 \pm 0.19) E+01$ \\
\hline \multicolumn{5}{|l|}{ 25-Mile Radius } \\
\hline Allendale, S.C. & 1 & $(1.06 \pm 0.15) E+01$ & $(1.06 \pm 0.15) E+01$ & $(1.06 \pm 0.15) E+01$ \\
\hline Langley, S.C. & 2 & $(1.43 \pm 0.46) E+01$ & $(1.75 \pm 0.18) E+01$ & $(1.10 \pm 0.13) E+01$ \\
\hline Springfield, S.C. & 2 & $(1.52 \pm 0.06) E+01$ & $(1.56 \pm 0.17) E+01$ & $(1.48 \pm 0.14) E+01$ \\
\hline Waynesboro, Ga. & 2 & $(1.55 \pm 0.25) E+01$ & $(1.73 \pm 0.15) E+01$ & $(1.37 \pm 0.17) E+01$ \\
\hline
\end{tabular}


Table 39

Radioactivity in Terrestrial Vegetation (Quarterly Samples)

Page 5 of 6

\begin{tabular}{|c|c|c|c|c|}
\hline Location & $\begin{array}{l}\text { No. of } \\
\text { Samples }\end{array}$ & Arithmetic Mean $\pm \sigma$ & Maximum $\pm \sigma$ & Minimum $\pm \sigma$ \\
\hline \multicolumn{5}{|l|}{ 100-Mile Radius } \\
\hline Columbia, S.C. & 3 & $(1.73 \pm 0.94) \mathrm{E}+01$ & $(2.30 \pm 0.20) E+01$ & $(6.45 \pm 1.09) E+00$ \\
\hline Greenville, S.C. & 2 & $(0.77 \pm 1.02) E+01$ & $(1.49 \pm 0.15) E+01$ & $(5.60 \pm 9.69) E-01$ \\
\hline Macon, Ga. & 2 & $(1.39 \pm 0.30) E+01$ & $(1.61 \pm 0.15) E+01$ & $(1.18 \pm 0.15) E+01$ \\
\hline Savannah, Ga. & 4 & $(1.40 \pm 0.25) E+01$ & $(1.66 \pm 0.16) E+01$ & $(1.12 \pm 0.15) E+01$ \\
\hline \multicolumn{5}{|l|}{ Gross Alpha, pCi/g } \\
\hline \multicolumn{5}{|l|}{ On Site } \\
\hline $200-F \# 13$ & 4 & $(3.64 \pm 4.38) E-01$ & $(9.01 \pm 5.30) E-01$ & $(-1.65 \pm 4.53) E-01$ \\
\hline $200-F$ \#21 & 4 & $(5.92 \pm 5.27) \mathrm{E}-01$ & $(1.33 \pm 0.34) E+00$ & $(0.92 \pm 2.16) E-01$ \\
\hline $200-\mathrm{H} \# 10$ & 4 & $(2.19 \pm 3.99) \mathrm{E}-01$ & $(6.89 \pm 4.80) \mathrm{E}-01$ & $(-1.55 \pm 1.90) E-01$ \\
\hline $200-\mathrm{H} \# 22$ & 4 & $(2.14 \pm 2.37) E-01$ & $(4.07 \pm 4.22) E-01$ & $(-1.30 \pm 3.91) E-01$ \\
\hline $643-26 E-1$ & 4 & $(0.52 \pm 1.27) E+00$ & $(2.41 \pm 1.24) E+00$ & $(-3.41 \pm 3.24) E-01$ \\
\hline $643-26 E-2$ & 4 & $(8.60 \pm 9.47) \mathrm{E}-01$ & $(2.24 \pm 1.18) E+00$ & $(1.09 \pm 4.63) E-01$ \\
\hline S-Area \#1 & 4 & $(2.78 \pm 5.09) \mathrm{E}-01$ & $(8.45 \pm 8.17) E-01$ & $(-3.90 \pm 2.88) E-01$ \\
\hline S-Area \#2 & 4 & $(6.71 \pm 8.86) E-01$ & $(1.85 \pm 0.81) E+00$ & $(-0.96 \pm 1.18) E-01$ \\
\hline S-Area \#3 & 4 & $(2.99 \pm 8.41) \mathrm{E}-01$ & $(1.45 \pm 0.78) E+00$ & $(-5.52 \pm 3.91) E-01$ \\
\hline S-Area \#4 & 4 & $(0.57 \pm 1.12) E+00$ & $(2.25 \pm 0.89) E+00$ & $(-1.47 \pm 4.06) E-01$ \\
\hline Z-Area \#1 & 4 & $(1.60 \pm 2.15) E+00$ & $(4.66 \pm 1.86) E+00$ & $(1.00 \pm 2.25) E-01$ \\
\hline Z-Area \#2 & 4 & $(4.98 \pm 5.84) E-01$ & $(1.24 \pm 0.75) E+00$ & $(-1.63 \pm 4.18) E-01$ \\
\hline Z-Area \#3 & 4 & $(1.06 \pm 3.11) E-01$ & $(5.51 \pm 5.01) E-01$ & $(-1.18 \pm 3.64) E-01$ \\
\hline Z-Area \#4 & 4 & $(3.46 \pm 7.75) E-01$ & $(1.29 \pm 0.79) E+00$ & $(-5.47 \pm 3.74) E-01$ \\
\hline Z-Area \#5 & 4 & $(1.58 \pm 2.44) E-01$ & $(3.66 \pm 5.17) E-01$ & $(-1.60 \pm 3.78) E-01$ \\
\hline Z-Area \#6 & 4 & $(4.07 \pm 4.60) E-01$ & $(1.07 \pm 0.64) E+00$ & $(0.85 \pm 1.85) E-01$ \\
\hline Z-Area \#7 & 4 & $(1.10 \pm 3.98) E-01$ & $(6.18 \pm 5.62) \mathrm{E}-01$ & $(-3.55 \pm 2.65) E-01$ \\
\hline Z-Area \#8 & 4 & $(6.77 \pm 8.43) E-01$ & $(1.74 \pm 0.92) E+00$ & $(-0.85 \pm 1.12) E-01$ \\
\hline \multicolumn{5}{|l|}{ Site Perimeter } \\
\hline Allendale Gate & 3 & $(-3.69 \pm 4.62) E-01$ & $(0.00 \pm 4.42) E-01$ & $(-8.87 \pm 7.71) E-01$ \\
\hline Barnwell Gate & 3 & $(1.41 \pm 7.10) \mathrm{E}-01$ & $(8.58 \pm 9.64) E-01$ & $(-5.61 \pm 3.88) E-01$ \\
\hline D-Area & 3 & $(0.78 \pm 5.48) E-01$ & $(5.74 \pm 5.86) E-01$ & $(-5.10 \pm 3.64) E-01$ \\
\hline $\begin{array}{l}\text { Darkhorse @ Williston } \\
\text { Gate }\end{array}$ & 3 & $(0.72 \pm 7.59) E-01$ & $(9.31 \pm 3.84) E-01$ & $(-5.10 \pm 3.61) E-01$ \\
\hline East Talatha & 3 & $(5.66 \pm 7.18) E-01$ & $(1.08 \pm 1.00) E+00$ & $(-2.55 \pm 4.45) E-01$ \\
\hline Green Pond & 3 & $(4.42 \pm 2.27) E-01$ & $(6.12 \pm 7.56) \mathrm{E}-01$ & $(1.84 \pm 5.29) E-01$ \\
\hline Highway $125 @$ & 3 & $(5.50 \pm 6.42) E-01$ & $(1.26 \pm 2.14) E+00$ & $(0.00 \pm 5.10) E-01$ \\
\hline
\end{tabular}




\section{Table 39}

\section{Radioactivity in Terrestrial Vegetation (Quarterly Samples)}

Page 6 of 6

\begin{tabular}{|c|c|c|c|c|}
\hline Location & $\begin{array}{l}\text { No. of } \\
\text { Samples }\end{array}$ & Arithmetic Mean $\pm \sigma$ & Maximum $\pm \sigma$ & Minimum $\pm \sigma$ \\
\hline Highway 21/167 & 3 & $(-0.67 \pm 1.05) E-01$ & $(0.00 \pm 4.94) E-01$ & $(-1.87 \pm 5.04) E-01$ \\
\hline $\begin{array}{l}\text { Highway } 39 \text { @ Williston } \\
\text { Gate }\end{array}$ & 3 & $(0.66 \pm 1.11) \mathrm{E}+00$ & $(1.44 \pm 0.91) E+00$ & $(-6.12 \pm 4.17) E-01$ \\
\hline Jackson & 3 & $(9.03 \pm 6.94) E-01$ & $(1.50 \pm 1.05) E+00$ & $(1.43 \pm 4.56) E-01$ \\
\hline Patterson Mill Road & 3 & $(5.46 \pm 8.46) E-01$ & $(1.52 \pm 1.44) E+00$ & $(0.00 \pm 4.84) E-01$ \\
\hline Talatha Gate & 3 & $(7.41 \pm 4.59) E-01$ & $(1.19 \pm 0.88) E+00$ & $(2.70 \pm 6.03) E-01$ \\
\hline West Jackson & 3 & $(1.79 \pm 3.67) E-01$ & $(5.06 \pm 7.65) E-01$ & $(-0.22 \pm 1.02) E+00$ \\
\hline Windsor Road & 2 & $(7.22 \pm 4.82) \mathrm{E}-01$ & $(1.06 \pm 1.03) E+00$ & $(3.81 \pm 8.33) E-01$ \\
\hline \multicolumn{5}{|l|}{ 25-Mile-Radius } \\
\hline Allendale, S.C. & 1 & $(8.12 \pm 7.65) E-01$ & $(8.12 \pm 7.65) E-01$ & $(8.12 \pm 7.65) E-01$ \\
\hline Langley, S.C. & 2 & $(-3.56 \pm 2.39) E-01$ & $(-1.87 \pm 4.00) \mathrm{E}-01$ & $(-5.25 \pm 4.15) E-01$ \\
\hline Springfield, S.C. & 2 & $(-1.94 \pm 4.77) E-01$ & $(1.44 \pm 6.85) E-01$ & $(-5.31 \pm 3.88) E-01$ \\
\hline Waynesboro, Ga. & 2 & $(-2.13 \pm 4.96) E-01$ & $(1.37 \pm 6.55) E-01$ & $(-5.64 \pm 4.06) E-01$ \\
\hline \multicolumn{5}{|l|}{ 100-Mile-Radius } \\
\hline Columbia, S.C. & 3 & $(4.41 \pm 5.36) E-01$ & $(9.43 \pm 9.86) E-01$ & $(-1.24 \pm 3.71) E-01$ \\
\hline Greenville, S.C. & 2 & $(0.49 \pm 9.86) E-01$ & $(7.46 \pm 5.53) \mathrm{E}-01$ & $(-6.48 \pm 5.40) E-01$ \\
\hline Macon, Ga. & 2 & $(1.06 \pm 0.86) E+00$ & $(1.66 \pm 0.77) E+00$ & $(4.54 \pm 6.72) E-01$ \\
\hline Savannah, Ga. & 4 & $(-2.04 \pm 2.85) E-01$ & $(1.66 \pm 5.30) E-01$ & $(-5.19 \pm 3.91) E-01$ \\
\hline
\end{tabular}


Table 40

Radioactivity in Terrestrial Vegetation

(Outside Solid Waste Disposal Facility Fences)

Page 1 of 1

\begin{tabular}{|c|c|c|c|c|}
\hline Location & $\begin{array}{l}\text { No. of } \\
\text { Samples }\end{array}$ & Arithmetic Mean $\pm \sigma$ & Maximum $\pm \sigma$ & Minimum $\pm \sigma$ \\
\hline \multicolumn{5}{|l|}{$\mathrm{Cs}-137, \mathrm{pCi} / \mathrm{g}$} \\
\hline OBG-10 outside Burial Ground & 3 & $(8.06 \pm 6.04) \mathrm{E}-01$ & $(1.32 \pm 0.09) \mathrm{E}+00$ & $(1.39 \pm 0.47) \mathrm{E}-01$ \\
\hline OBG-11 outside Burial Ground & 1 & $(3.36 \pm 0.49) E-01$ & $(3.36 \pm 0.49) E-01$ & $(3.36 \pm 0.49) E-01$ \\
\hline OBG-7 outside Burial Ground & 1 & $(2.14 \pm 0.35) E-01$ & $(2.14 \pm 0.35) E-01$ & $(2.14 \pm 0.35) E-01$ \\
\hline OBG-8 outside Burial Ground & 2 & $(1.61 \pm 0.17) E-01$ & $(1.73 \pm 0.45) E-01$ & $(1.50 \pm 0.47) E-01$ \\
\hline OBG-9 outside Burial Ground & 2 & $(1.27 \pm 0.37) E-01$ & $(1.53 \pm 0.44) E-01$ & $(1.01 \pm 0.37) E-01$ \\
\hline \multicolumn{5}{|l|}{ Gross Beta, pCi/g } \\
\hline OBG-1 outside Burial Ground & 4 & $(1.72 \pm 0.97) \mathrm{E}+01$ & $(3.15 \pm 0.20) E+01$ & $(9.71 \pm 1.06) \mathrm{E}+00$ \\
\hline OBG-10 outside Burial Ground & 4 & $(1.47 \pm 0.90) E+01$ & $(2.80 \pm 0.19) E+01$ & $(8.48 \pm 1.16) E+00$ \\
\hline OBG-11 outside Burial Ground & 4 & $(2.28 \pm 2.38) E+01$ & $(5.82 \pm 0.28) E+01$ & $(7.47 \pm 0.86) E+00$ \\
\hline OBG-2 outside Burial Ground & 4 & $(1.61 \pm 0.47) E+01$ & $(2.10 \pm 0.16) E+01$ & $(1.06 \pm 0.13) E+01$ \\
\hline OBG-3 outside Burial Ground & 4 & $(1.71 \pm 0.94) E+01$ & $(2.97 \pm 0.19) E+01$ & $(7.42 \pm 1.14) E+00$ \\
\hline OBG- 5 outside Burial Ground & 3 & $(1.42 \pm 0.62) E+01$ & $(2.04 \pm 0.16) E+01$ & $(7.95 \pm 1.14) E+00$ \\
\hline OBG- 6 outside Burial Ground & 4 & $(1.54 \pm 1.02) E+01$ & $(3.02 \pm 0.14) E+01$ & $(6.89 \pm 1.09) E+00$ \\
\hline OBG-7 outside Burial Ground & 3 & $(1.57 \pm 0.66) E+01$ & $(2.33 \pm 0.17) E+01$ & $(1.16 \pm 0.11) E+01$ \\
\hline OBG-8 outside Burial Ground & 3 & $(1.31 \pm 0.31) E+01$ & $(1.62 \pm 0.13) E+01$ & $(9.90 \pm 1.18) E+00$ \\
\hline OBG- 9 outside Burial Ground & 3 & $(1.22 \pm 0.45) \mathrm{E}+01$ & $(1.75 \pm 0.15) E+01$ & $(9.54 \pm 1.21) E+00$ \\
\hline \multicolumn{5}{|l|}{ Gross Alpha, pCi/g } \\
\hline OBG-1 outside Burial Ground & 4 & $(3.57 \pm 3.59) \mathrm{E}-01$ & $(8.74 \pm 8.83) E-01$ & $(0.94 \pm 4.70) E-01$ \\
\hline OBG-10 outside Burial Ground & 4 & $(2.70 \pm 2.07) E-01$ & $(5.59 \pm 9.21) E-01$ & $(1.02 \pm 4.41) E-01$ \\
\hline OBG-11 outside Burial Ground & 4 & $(1.92 \pm 6.46) \mathrm{E}-01$ & $(0.95 \pm 1.08) E+00$ & $(-5.08 \pm 3.67) E-01$ \\
\hline OBG-2 outside Burial Ground & 4 & $(2.00 \pm 6.33) \mathrm{E}-01$ & $(1.06 \pm 0.65) E+00$ & $(-4.66 \pm 3.40) E-01$ \\
\hline OBG-3 outside Burial Ground & 4 & $(5.71 \pm 1.91) E-01$ & $(7.95 \pm 5.81) \mathrm{E}-01$ & $(3.51 \pm 5.47) E-01$ \\
\hline OBG-5 outside Burial Ground & 3 & $(7.73 \pm 5.90) E-01$ & $(1.16 \pm 0.86) E+00$ & $(0.94 \pm 4.78) E-01$ \\
\hline OBG-6 outside Burial Ground & 4 & $(5.50 \pm 5.32) E-01$ & $(1.32 \pm 0.73) E+00$ & $(1.04 \pm 4.86) E-01$ \\
\hline OBG-7 outside Burial Ground & 3 & $(2.96 \pm 6.09) E-01$ & $(9.70 \pm 6.02) \mathrm{E}-01$ & $(-2.16 \pm 4.87) \mathrm{E}-01$ \\
\hline OBG-8 outside Burial Ground & 3 & $(0.43 \pm 1.01) E+00$ & $(1.59 \pm 0.80) E+00$ & $(-1.62 \pm 3.89) E-01$ \\
\hline OBG-9 outside Burial Ground & 3 & $(-1.41 \pm 0.27) \mathrm{E}-01$ & $(-1.16 \pm 3.27) E-01$ & $(-1.69 \pm 4.28) E-01$ \\
\hline
\end{tabular}




\section{Table 41}

\section{Radioactivity in Terrestrial Vegetation (Inside Solid Waste Disposal Facility Fences)}

Page 1 of 2

\begin{tabular}{|c|c|c|c|c|}
\hline \multirow{2}{*}{\multicolumn{2}{|c|}{ Locationa }} & \multicolumn{3}{|c|}{ pCi/g (Dry Weight) } \\
\hline & & Cs-137 & Gross Beta & Gross Alpha \\
\hline \multicolumn{5}{|c|}{$\begin{array}{l}\text { Blank spaces indicate activity was less than the lower limit of detection. The typical lower limit of detection for } \\
\text { Cs-137 in vegetation was } 1.00 \mathrm{E}-01 \mathrm{pCi} / \mathrm{g} \text {. }\end{array}$} \\
\hline \multirow{2}{*}{\multicolumn{2}{|c|}{$\begin{array}{l}\text { IBG-1 inside Burial Ground } \\
\text { IBG-1A inside Burial Ground }\end{array}$}} & $(4.96 \pm 0.83) E-01$ & $(4.27 \pm 0.71) E+00$ & $(2.60 \pm 2.61) E-01$ \\
\hline & & & $(5.33 \pm 0.75) E+00$ & $(2.67 \pm 2.68) E-01$ \\
\hline \multicolumn{2}{|c|}{ IBG-2 inside Burial Ground } & $(2.20 \pm 0.49) E-01$ & $(5.69 \pm 0.79) E+00$ & $(0.92 \pm 2.07) E-01$ \\
\hline \multicolumn{2}{|c|}{ IBG-3 inside Burial Ground } & & $(4.88 \pm 0.77) E+00$ & $(0.80 \pm 3.12) E-01$ \\
\hline \multicolumn{2}{|c|}{ IBG-3A inside Burial Ground } & & $(9.07 \pm 0.95) \mathrm{E}+00$ & $(-1.08 \pm 2.75) E-01$ \\
\hline \multicolumn{2}{|c|}{ IBG-4 inside Burial Ground } & $(2.62 \pm 0.29) E-01$ & $(7.84 \pm 0.65) E+00$ & $(-1.13 \pm 2.09) E-01$ \\
\hline \multicolumn{2}{|c|}{ IBG-4A inside Burial Ground } & $(1.94 \pm 0.09) E+00$ & $(5.23 \pm 0.20) E+01$ & $(-2.89 \pm 3.14) E-01$ \\
\hline \multicolumn{2}{|c|}{ IBG-5 inside Burial Ground } & & $(8.02 \pm 0.90) E+00$ & $(-2.55 \pm 1.88) \mathrm{E}-01$ \\
\hline \multicolumn{2}{|c|}{ IBG-6 inside Burial Ground } & & $(7.67 \pm 0.91) E+00$ & $(2.72 \pm 3.98) E-01$ \\
\hline \multicolumn{2}{|c|}{ [BG-7 inside Burial Ground } & $(1.52 \pm 0.44) E-01$ & $(5.93 \pm 0.83) E+00$ & $(-2.86 \pm 2.18) E-01$ \\
\hline \multicolumn{2}{|c|}{ IBG-8 inside Burial Ground } & $(4.04 \pm 0.64) E-01$ & $(9.07 \pm 0.96) \mathrm{E}+00$ & $(-1.15 \pm 2.90) \mathrm{E}-01$ \\
\hline \multicolumn{2}{|c|}{ IBG-8A inside Burial Ground } & $(1.38 \pm 0.05) E+00$ & $(9.07 \pm 0.96) E+00$ & $(-2.93 \pm 2.15) E-01$ \\
\hline \multicolumn{2}{|c|}{ IBG-9 inside Burial Ground } & $(2.38 \pm 0.54) E-01$ & $(1.60 \pm 0.12) \mathrm{E}+01$ & $(0.42 \pm 3.16) E-01$ \\
\hline \multicolumn{2}{|c|}{ IBG-9A inside Burial Ground } & $(5.75 \pm 0.48) E-01$ & $(1.85 \pm 0.13) E+01$ & $(-1.53 \pm 3.00) E-01$ \\
\hline \multicolumn{2}{|c|}{ IBG-10 inside Burial Ground } & $(2.53 \pm 0.50) E-01$ & $(3.84 \pm 0.18) E+01$ & $(-4.88 \pm 2.61) E-01$ \\
\hline \multicolumn{2}{|c|}{$\mid B G-11$ inside Burial Ground } & $(4.38 \pm 0.53) E-01$ & $(1.50 \pm 0.12) E+01$ & $(5.93 \pm 4.55) E-01$ \\
\hline \multicolumn{2}{|c|}{ IBG-12 inside Burial Ground } & $(5.34 \pm 0.51) E-01$ & $(2.96 \pm 0.15) E+01$ & $(3.49 \pm 4.13) E-01$ \\
\hline \multicolumn{2}{|c|}{ IBG-13 inside Burial Ground } & $(1.37 \pm 0.32) E-01$ & $(1.08 \pm 0.12) E+01$ & $(6.11 \pm 5.76) E-01$ \\
\hline \multicolumn{2}{|c|}{ IBG-14 inside Burial Ground } & & $(4.70 \pm 0.96) E+00$ & $(-3.48 \pm 2.74) E-01$ \\
\hline \multicolumn{2}{|c|}{ IBG-14A inside Burial Ground } & $(2.30 \pm 0.31) E-01$ & $(9.87 \pm 1.21) \mathrm{E}+00$ & $(1.03 \pm 4.56) E-01$ \\
\hline \multicolumn{2}{|c|}{ IBG-15 inside Burial Ground } & $(1.84 \pm 0.39) E-01$ & $(1.03 \pm 0.12) E+01$ & $(-1.41 \pm 3.67) E-01$ \\
\hline \multicolumn{2}{|c|}{ IBG-16 inside Burial Ground } & $(2.65 \pm 0.51) E-01$ & $(1.83 \pm 0.15) E+01$ & $(0.71 \pm 4.33) \mathrm{E}-01$ \\
\hline \multicolumn{2}{|c|}{ IBG-17 inside Burial Ground } & & $(6.34 \pm 0.18) E+01$ & $(0.96 \pm 3.35) E-01$ \\
\hline \multicolumn{2}{|c|}{ IBG-18 inside Burial Ground } & & $(6.58 \pm 1.06) E+00$ & $(-1.36 \pm 3.93) E-01$ \\
\hline \multicolumn{2}{|c|}{ IBG-19 inside Burial Ground } & & $(8.46 \pm 1.14) \mathrm{E}+00$ & $(-1.46 \pm 4.04) E-01$ \\
\hline \multicolumn{2}{|c|}{ IBG-19A inside Burial Ground } & & $(7.05 \pm 1.09) E+00$ & $(-1.36 \pm 3.85) E-01$ \\
\hline \multicolumn{2}{|c|}{ IBG-20 inside Burial Ground } & & & \\
\hline
\end{tabular}

a Samples were not obtained from locations IBG-20, IBG-20A, IBG-27, IBG-28, IBG-36, IBG-37, IBG-38, IBG-39, IBG-40, $I B G-41$, and IBG-42 because of lack of vegetation. 


\section{Table 41}

\section{Radioactivity in Terrestrial Vegetation (Inside Solid Waste Disposal Facility Fences)}

Page 2 of 2

\begin{tabular}{|c|c|c|c|}
\hline \multirow[b]{2}{*}{ Location ${ }^{a}$} & \multicolumn{3}{|c|}{ pCi/g (Dry Weight) } \\
\hline & Cs-137 & Gross Beta & Gross Alpha \\
\hline IBG-21 inside Burial Ground & $(2.91 \pm 0.35) \mathrm{E}-01$ & $(3.48 \pm 0.19) \mathrm{E}+01$ & $(0.13 \pm 4.58) E-01$ \\
\hline IBG-22 inside Burial Ground & & $(1.32 \pm 0.13) E+01$ & $(1.46 \pm 0.77) E+00$ \\
\hline IBG-23 inside Burial Ground & & $(7.05 \pm 1.07) E+00$ & $(3.38 \pm 4.93) \mathrm{E}-01$ \\
\hline IBG-23A inside Burial Ground & $(1.34 \pm 0.37) E-01$ & $(7.52 \pm 1.10) E+00$ & $(-3.90 \pm 2.97) E-01$ \\
\hline IBG-24 inside Burial Ground & & $(7.05 \pm 1.08) E+00$ & $(-3.81 \pm 2.90) E-01$ \\
\hline \multicolumn{4}{|l|}{ IBG-25 inside Burial Ground } \\
\hline IBG-26 inside Burial Ground & & $(7.05 \pm 1.09) E+00$ & $(1.18 \pm 4.70) E-01$ \\
\hline \multicolumn{4}{|l|}{ IBG-27 inside Burial Ground } \\
\hline \multicolumn{4}{|l|}{ IBG-28 inside Burial Ground } \\
\hline IBG-29 inside Burial Ground & & $(7.52 \pm 1.09) E+00$ & $(-1.32 \pm 3.70) E-01$ \\
\hline IBG-30 inside Burial Ground & & $(8.93 \pm 1.17) E+00$ & $(3.81 \pm 5.56) \mathrm{E}-01$ \\
\hline IBG-31 inside Burial Ground & & $(5.17 \pm 0.99) E+00$ & $(1.13 \pm 4.33) \mathrm{E}-01$ \\
\hline IBG-32 inside Burial Ground & & $(1.13 \pm 0.12) E+01$ & $(0.94 \pm 4.33) E-01$ \\
\hline IBG-33 inside Burial Ground & & $(7.95 \pm 1.14) E+00$ & $(1.43 \pm 3.18) E-01$ \\
\hline IBG-34 inside Burial Ground & $(1.81 \pm 0.66) E-01$ & $(9.54 \pm 1.22) E+00$ & $(4.19 \pm 4.31) \mathrm{E}-01$ \\
\hline IBG-35 inside Burial Ground & & $(9.24 \pm 0.85) E+00$ & $(4.32 \pm 3.11) \mathrm{E}-01$ \\
\hline \multicolumn{4}{|l|}{ IBG-36 inside Burial Ground } \\
\hline \multicolumn{4}{|l|}{ IBG-37 inside Burial Ground } \\
\hline \multicolumn{4}{|l|}{ IBG-38 inside Burial Ground } \\
\hline \multicolumn{4}{|l|}{ IBG-39 inside Burial Ground } \\
\hline \multicolumn{4}{|l|}{ IBG-40 inside Burial Ground } \\
\hline IBG-41 inside Burial Ground & & & \\
\hline IBG-42 inside Burial Ground & & & \\
\hline
\end{tabular}

a Samples were not obtained from locations IBG-20, IBG-20A, IBG-27, IBG-28, IBG-36, IBG-37, IBG-38, IBG-39, IBG-40, IBG-41, and IBG-42 because of lack of vegetation. 


\section{Table 42}

\section{Radioactivity in Terrestrial Vegetation} (Composites from Seepage and Retention Basins)

Page 1 of 1

\begin{tabular}{|c|c|c|c|c|c|}
\hline \multirow{2}{*}{\multicolumn{2}{|c|}{ Location }} & \multicolumn{4}{|c|}{ pCi/g (Dry Weight) } \\
\hline & & Cs-137 & Sr-89,90 & Gross Beta & Gross Alpha \\
\hline \multicolumn{2}{|l|}{ Note: } & $\begin{array}{l}\text { sactivity was } b \\
\text { was } 1.00 E-01\end{array}$ & he lower limit of & on. & \\
\hline \multicolumn{2}{|c|}{ A-Area seepage basin ${ }^{a}$} & $(2.67 \pm 0.93) \mathrm{E}-01$ & $(3.79 \pm 0.15) E+00$ & $(1.91 \pm 0.11) \mathrm{E}+01$ & $(4.67 \pm 4.12) E-01$ \\
\hline \multicolumn{2}{|c|}{ C-Area seepage basin ${ }^{b}$} & $(3.68 \pm 0.31) E-01$ & $(5.81 \pm 0.19) E+00$ & $(3.78 \pm 0.24) E+01$ & $(-2.91 \pm 5.22) E-01$ \\
\hline \multicolumn{2}{|c|}{ F-Area retention basinc } & $(3.82 \pm 0.72) E-01$ & $(3.52 \pm 0.70) E-01$ & $(1.49 \pm 0.17) E+01$ & $(-2.05 \pm 5.26) E-01$ \\
\hline \multicolumn{2}{|c|}{ F-Area seepage basin ${ }^{b}$} & & $(3.29 \pm 0.70) E-01$ & $(1.05 \pm 0.15) E+01$ & $(1.43 \pm 5.98) E-01$ \\
\hline \multicolumn{2}{|c|}{ H-Area retention basin ${ }^{b}$} & $(5.02 \pm 0.16) E+00$ & $(8.46 \pm 0.22) E+00$ & $(4.40 \pm 0.26) E+01$ & $(3.66 \pm 7.07) E-01$ \\
\hline \multicolumn{2}{|c|}{ H-Area seepage basin ${ }^{b}$} & -01 & $(2.02 \pm 0.12) E+00$ & $(1.74 \pm 0.17) E+01$ & $(4.34 \pm 6.50) E-01$ \\
\hline \multicolumn{2}{|c|}{ K-Area retention basin ${ }^{a}$} & & $(1.62 \pm 0.58) E-01$ & $(9.30 \pm 1.41) \mathrm{E}+00$ & $(4.46 \pm 6.50) E-01$ \\
\hline \multicolumn{2}{|c|}{ K-Area seepage basin ${ }^{\mathrm{a}}$} & $(9.33 \pm 1.96) E-02$ & $(1.47 \pm 0.50) E-01$ & $(1.30 \pm 0.16) E+01$ & $(-2.23 \pm 6.01) E-01$ \\
\hline \multicolumn{2}{|c|}{ L-Area chemical basin ${ }^{a}$} & $(2.08 \pm 0.71) E-01$ & $(1.15 \pm 0.52) E-01$ & $(1.67 \pm 0.18) E+01$ & $(5.58 \pm 8.32) E-01$ \\
\hline \multicolumn{2}{|c|}{ L-Area seepage basin ${ }^{a}$} & $(4.59 \pm 0.37) E-01$ & $(3.45 \pm 0.59) \mathrm{E}-01$ & $(1.92 \pm 0.18) E+01$ & $(-2.36 \pm 5.65) E-01$ \\
\hline \multicolumn{2}{|c|}{ P-Area seepage basin ${ }^{a}$} & $(1.65 \pm 0.09) E+00$ & $(3.81 \pm 0.65) E-01$ & $(2.11 \pm 0.19) E+01$ & $(8.68 \pm 8.98) E-01$ \\
\hline \multicolumn{2}{|c|}{ R-Area seepage basin ${ }^{b}$} & $(1.10 \pm 0.08) E+00$ & $(4.54 \pm 0.71) E-01$ & $(1.61 \pm 0.17) E+01$ & $(-2.17 \pm 5.47) E-01$ \\
\hline
\end{tabular}


Table 43

Meteorological Data

Page 1 of 7

$\begin{array}{lll}\text { USNRC Computer Code-XOQDOQ, } & \text { Version 2.0 } & \text { Run Date: 93.071 } \\ \text { 43824 Wind Stats H-Area 60 minute } & 62 \mathrm{M} \mathrm{87-91} & \text { Stability from Sigma A }\end{array}$

Joint Frequency Distribution of Wind Speed and Direction:

Atmospheric Stability Class A Extremely Unstable Conditions

\begin{tabular}{lllllrrrr}
\hline $\begin{array}{c}\text { (M/S) } \\
\text { UMAX }\end{array}$ & N & NNE & NE & ENE & E & ESE & SE & \multicolumn{1}{c}{ SSE } \\
\hline 2.00 & 0.374 & 0.406 & 0.372 & 0.422 & 0.402 & 0.374 & 0.404 & 0.356 \\
4.00 & 0.872 & 0.735 & 0.876 & 0.995 & 0.942 & 0.942 & 0.648 & 0.618 \\
6.00 & 0.573 & 0.260 & 0.157 & 0.187 & 0.148 & 0.073 & 0.066 & 0.094 \\
8.00 & 0.089 & 0.052 & 0.007 & 0.011 & 0.007 & 0.002 & 0.005 & 0.011 \\
12.00 & 0.005 & 0.000 & 0.002 & 0.002 & 0.000 & 0.000 & 0.000 & 0.000 \\
14.10 & 0.000 & 0.000 & 0.000 & 0.000 & 0.000 & 0.000 & 0.000 & 0.000 \\
TOTAL & 1.91 & 1.45 & 1.41 & 1.62 & 1.50 & 1.39 & 1.12 & 1.08
\end{tabular}

Joint Frequency Distribution of Wind Speed and Direction:

Atmospheric Stability Class A Extremely Unstable Conditions

\begin{tabular}{lllllllllr}
\hline $\begin{array}{c}\text { (M/S) } \\
\text { UMAX }\end{array}$ & S & \multicolumn{1}{c}{ SSW } & SW & WSW & W & WNW & NW & NNW & TOTAL \\
\hline 2.00 & 0.358 & 0.347 & 0.450 & 0.392 & 0.454 & 0.434 & 0.370 & 0.413 & 6.328 \\
4.00 & 0.739 & 0.721 & 1.002 & 1.282 & 1.294 & 0.942 & 0.534 & 0.602 & 13.746 \\
6.00 & 0.139 & 0.139 & 0.208 & 0.242 & 0.274 & 0.235 & 0.141 & 0.240 & 3.176 \\
8.00 & 0.021 & 0.018 & 0.016 & 0.037 & 0.032 & 0.021 & 0.014 & 0.055 & 0.397 \\
12.00 & 0.000 & 0.007 & 0.009 & 0.009 & 0.002 & 0.007 & 0.002 & 0.009 & 0.055 \\
14.10 & 0.000 & 0.000 & 0.000 & 0.000 & 0.000 & 0.000 & 0.000 & 0.000 & 0.000 \\
TOTAL & 1.26 & 1.23 & 1.68 & 1.96 & 2.06 & 1.64 & 1.06 & 1.32 & 23.70
\end{tabular}


Table 43

Meteorological Data

Page 2 of 7

USNRC Computer Code-XOQDOQ, 43824 Wind Stats H-Area 60 minute

Version 2.0 62M 87-91
Run Date: 93.071

Stability from Sigma A
(SRL 6/29/83 VERSION)

Atmospheric Stability Class B

Joint Frequency Distribution of Wind Speed and Direction: Moderately Unstable Conditions

\begin{tabular}{lllllllll}
\hline $\begin{array}{c}\text { (M/S) } \\
\text { UMAX }\end{array}$ & N & NNE & NE & ENE & E & ESE & SE & SSE \\
\hline 2.00 & 0.075 & 0.078 & 0.087 & 0.098 & 0.052 & 0.055 & 0.057 & 0.048 \\
4.00 & 0.267 & 0.411 & 0.577 & 0.621 & 0.429 & 0.342 & 0.244 & 0.217 \\
6.00 & 0.139 & 0.388 & 0.377 & 0.313 & 0.164 & 0.105 & 0.071 & 0.084 \\
8.00 & 0.007 & 0.082 & 0.027 & 0.007 & 0.005 & 0.011 & 0.002 & 0.009 \\
12.00 & 0.000 & 0.007 & 0.002 & 0.000 & 0.000 & 0.000 & 0.000 & 0.002 \\
14.10 & 0.000 & 0.000 & 0.000 & 0.000 & 0.000 & 0.000 & 0.000 & 0.000 \\
TOTAL & 0.49 & 0.97 & 1.07 & 1.04 & 0.65 & 0.51 & 0.37 & 0.36
\end{tabular}

Joint Frequency Distribution of Wind Speed and Direction:

Atmospheric Stability Class B Moderately Unstable Conditions

\begin{tabular}{lrllllllll}
\hline $\begin{array}{c}\text { (M/S) } \\
\text { UMAX }\end{array}$ & S & SSW & SW & WSW & $W$ & WNW & NW & NNW & TOTAL \\
\hline 2.00 & 0.078 & 0.071 & 0.046 & 0.050 & 0.046 & 0.075 & 0.052 & 0.071 & 1.038 \\
4.00 & 0.324 & 0.329 & 0.477 & 0.671 & 0.559 & 0.372 & 0.251 & 0.210 & 6.300 \\
6.00 & 0.187 & 0.212 & 0.317 & 0.507 & 0.511 & 0.356 & 0.126 & 0.089 & 3.945 \\
8.00 & 0.050 & 0.037 & 0.052 & 0.103 & 0.171 & 0.214 & 0.059 & 0.014 & 0.851 \\
12.00 & 0.002 & 0.002 & 0.007 & 0.007 & 0.064 & 0.064 & 0.007 & 0.002 & 0.167 \\
14.10 & 0.000 & 0.000 & 0.000 & 0.000 & 0.000 & 0.000 & 0.000 & 0.000 & 0.000 \\
TOTAL & 0.64 & 0.65 & 0.90 & 1.34 & 1.35 & 1.08 & 0.50 & 0.39 & 12.30
\end{tabular}


Table 43

Meteorological Data

Page 3 of 7

USNRC Computer Code-XOQDOQ, Version 2.0 43824 Wind Stats H-Area 60 minute

Run Date: 93.071

Stability from Sigma A

(SRL 6/29/83 VERSION)

Joint Frequency Distribution of Wind Speed and Direction:

Atmospheric Stability Class C Slightly Unstable Conditions

\begin{tabular}{lrlllllll}
\hline $\begin{array}{c}\text { (M/S) } \\
\text { UMAX }\end{array}$ & N & \multicolumn{1}{c}{ NNE } & \multicolumn{1}{c}{ NE } & \multicolumn{1}{c}{ ENE } & E & ESE & SE & \multicolumn{1}{c}{ SSE } \\
\hline 2.00 & 0.032 & 0.057 & 0.091 & 0.071 & 0.059 & 0.046 & 0.055 & 0.048 \\
4.00 & 0.173 & 0.573 & 1.125 & 1.031 & 0.600 & 0.408 & 0.411 & 0.370 \\
6.00 & 0.116 & 0.539 & 1.303 & 0.742 & 0.354 & 0.192 & 0.217 & 0.253 \\
8.00 & 0.014 & 0.098 & 0.196 & 0.082 & 0.018 & 0.032 & 0.032 & 0.059 \\
12.00 & 0.002 & 0.011 & 0.000 & 0.002 & 0.002 & 0.005 & 0.000 & 0.027 \\
14.10 & 0.000 & 0.000 & 0.000 & 0.000 & 0.000 & 0.000 & 0.000 & 0.000 \\
TOTAL & 0.34 & 1.28 & 2.72 & 1.93 & 1.03 & 0.68 & 0.71 & 0.76
\end{tabular}

Joint Frequency Distribution of Wind Speed and Direction:

Atmospheric Stability Class C Slightly Unstable Conditions

\begin{tabular}{lrllllllll}
\hline $\begin{array}{c}\text { (M/S) } \\
\text { UMAX }\end{array}$ & S & \multicolumn{1}{c}{ SSW } & SW & \multicolumn{1}{c}{ WSW } & W & \multicolumn{1}{c}{ WNW } & NW & NNW & TOTAL \\
\hline 2.00 & 0.068 & 0.048 & 0.055 & 0.050 & 0.075 & 0.046 & 0.050 & 0.052 & 0.904 \\
4.00 & 0.479 & 0.516 & 0.589 & 0.794 & 0.527 & 0.445 & 0.301 & 0.237 & 8.580 \\
6.00 & 0.470 & 0.461 & 0.561 & 0.687 & 0.643 & 0.564 & 0.212 & 0.119 & 7.432 \\
8.00 & 0.155 & 0.164 & 0.208 & 0.258 & 0.445 & 0.429 & 0.096 & 0.018 & 2.305 \\
12.00 & 0.043 & 0.041 & 0.046 & 0.059 & 0.155 & 0.167 & 0.018 & 0.007 & 0.586 \\
14.10 & 0.000 & 0.000 & 0.000 & 0.002 & 0.000 & 0.002 & 0.000 & 0.000 & 0.005 \\
TOTAL & 1.22 & 1.23 & 1.46 & 1.85 & 1.85 & 1.65 & 0.68 & 0.43 & 19.81
\end{tabular}


Table 43

Meteorological Data

Page 4 of 7

USNRC Computer Code-XOQDOQ,

Version 2.0

62M 87-91

Run Date: 93.071

Stability from Sigma A

(SRL 6/29/83 VERSION)

43824 Wind Stats H-Area 60 minute

Joint Frequency Distribution of Wind Speed and Direction: Neutral Conditions

\begin{tabular}{lllllllll}
\hline $\begin{array}{c}\text { (M/S) } \\
\text { UMAX }\end{array}$ & N & \multicolumn{1}{c}{ NNE } & NE & ENE & E & ESE & SE & \multicolumn{1}{c}{ SSE } \\
\hline 2.00 & 0.018 & 0.048 & 0.055 & 0.037 & 0.055 & 0.030 & 0.055 & 0.068 \\
4.00 & 0.103 & 0.436 & 1.072 & 0.888 & 0.552 & 0.504 & 0.705 & 0.691 \\
6.00 & 0.123 & 0.434 & 0.853 & 0.584 & 0.395 & 0.440 & 0.653 & 1.164 \\
8.00 & 0.011 & 0.052 & 0.098 & 0.023 & 0.005 & 0.011 & 0.046 & 0.180 \\
12.00 & 0.000 & 0.023 & 0.023 & 0.000 & 0.002 & 0.000 & 0.000 & 0.007 \\
14.10 & 0.000 & 0.000 & 0.000 & 0.000 & 0.000 & 0.000 & 0.000 & 0.000 \\
TOTAL & 0.26 & 0.99 & 2.10 & 1.53 & 1.01 & 0.99 & 1.46 & 2.11
\end{tabular}

Joint Frequency Distribution of Wind Speed and Direction:

Atmospheric Stability Class D Neutral Conditions

\begin{tabular}{lllllllllr}
\hline $\begin{array}{c}\text { (M/S) } \\
\text { UMAX }\end{array}$ & $\cdot$ S & SSW & SW & WSW & W & WNW & NW & NNW & TOTAL \\
\hline 2.00 & 0.055 & 0.032 & 0.071 & 0.048 & 0.037 & 0.034 & 0.050 & 0.041 & 0.732 \\
4.00 & 0.924 & 0.910 & 0.803 & 0.808 & 0.723 & 0.570 & 0.427 & 0.272 & 10.389 \\
6.00 & 1.451 & 0.778 & 0.901 & 0.769 & 0.776 & 0.648 & 0.317 & 0.089 & 10.376 \\
8.00 & 0.219 & 0.146 & 0.098 & 0.087 & 0.030 & 0.052 & 0.030 & 0.002 & 1.091 \\
12.00 & 0.016 & 0.037 & 0.002 & 0.002 & 0.007 & 0.002 & 0.000 & 0.000 & 0.121 \\
12.10 & 0.000 & 0.000 & 0.000 & 0.000 & 0.000 & 0.000 & 0.000 & 0.000 & 0.000 \\
TOTAL & 2.67 & 1.90 & 1.88 & 1.71 & 1.57 & 1.31 & $\mathbf{0 . 8 2}$ & $\mathbf{0 . 4 0}$ & 22.71
\end{tabular}


Table 43

Meteorological Data

Page 5 of 7

USNRC Computer Code-XOQDOQ, Version 2.0

43824 Wind Stats H-Area 60 minute

$62 \mathrm{M} \mathrm{87-91}$

Run Date: $93.071 \quad$ (SRL 6/29/83 VERSION)

Stability from Sigma A

Joint Frequency Distribution of Wind Speed and Direction:

Atmospheric Stability Class E Slightly Stable Conditions

\begin{tabular}{|c|c|c|c|c|c|c|c|c|}
\hline $\begin{array}{l}\text { (M/S) } \\
\text { UMAX }\end{array}$ & $\mathbf{N}$ & NNE & NE & ENE & $E$ & ESE & SE & SSE \\
\hline 2.00 & 0.011 & 0.021 & 0.039 & 0.007 & 0.009 & 0.025 & 0.030 & 0.030 \\
\hline 4.00 & 0.062 & 0.274 & 0.687 & 0.477 & 0.297 & 0.333 & 0.456 & 0.701 \\
\hline 6.00 & 0.071 & 0.527 & 0.689 & 0.705 & 0.600 & 0.454 & 0.650 & 1.006 \\
\hline 8.00 & 0.000 & 0.050 & 0.032 & 0.037 & 0.011 & 0.011 & 0.002 & 0.027 \\
\hline 12.00 & 0.000 & 0.002 & 0.002 & 0.000 & 0.000 & 0.000 & 0.000 & 0.000 \\
\hline 14.10 & 0.000 & 0.000 & 0.000 & 0.000 & 0.000 & 0.000 & 0.000 & 0.000 \\
\hline TOTAL & 0.14 & 0.87 & 1.45 & 1.23 & 0.92 & 0.82 & 1.14 & 1.76 \\
\hline
\end{tabular}

Joint Frequency Distribution of Wind Speed and Direction:

Atmospheric Stability Class E Slightly Stable Conditions

\begin{tabular}{lrllllllll}
\hline $\begin{array}{c}\text { (M/S) } \\
\text { UMAX }\end{array}$ & S & SSW & SW & WSW & W & \multicolumn{1}{c}{ WNW } & NW & NNW & TOTAL \\
\hline 2.00 & 0.016 & 0.016 & 0.011 & 0.011 & 0.021 & 0.014 & 0.023 & 0.021 & 0.303 \\
4.00 & 0.673 & 0.573 & 0.543 & 0.472 & 0.431 & 0.427 & 0.333 & 0.301 & 7.040 \\
6.00 & 1.184 & 0.940 & 0.910 & 0.894 & 0.484 & 0.402 & 0.187 & 0.141 & 9.846 \\
8.00 & 0.043 & 0.018 & 0.043 & 0.009 & 0.005 & 0.002 & 0.002 & 0.000 & 0.294 \\
12.00 & 0.000 & 0.000 & 0.000 & 0.000 & 0.000 & 0.000 & 0.000 & 0.000 & 0.005 \\
12.10 & 0.000 & 0.000 & 0.000 & 0.000 & 0.000 & 0.000 & 0.000 & 0.000 & 0.000 \\
TOTAL & 1.92 & 1.55 & 1.51 & 1.39 & 0.94 & 0.84 & 0.55 & 0.46 & 17.49
\end{tabular}


Table 43

Meteorological Data

Page 6 of 7

USNRC Computer Code-XOQDOQ, Version 2.0 43824 Wind Stats H-Area 60 minute

62M 87-91

Run Date: 93.071 (SRL 6/29/83 VERS!ON)

Stability from Sigma A

Stabilly from sigma $A$

Joint Frequency Distribution of Wind Speed and Direction:

Atmospheric Stability Class F

\begin{tabular}{|c|c|c|c|c|c|c|c|c|}
\hline $\begin{array}{l}\text { (M/S) } \\
\text { UMAX }\end{array}$ & $\mathbf{N}$ & NNE & NE & ENE & E & ESE & SE & SSE \\
\hline 2.00 & 0.000 & 0.005 & 0.007 & 0.011 & 0.007 & 0.005 & 0.002 & 0.005 \\
\hline 4.00 & 0.018 & 0.052 & 0.087 & 0.039 & 0.023 & 0.075 & 0.089 & 0.091 \\
\hline 6.00 & 0.014 & 0.256 & 0.212 & 0.144 & 0.144 & 0.187 & 0.132 & 0.162 \\
\hline 8.00 & 0.000 & 0.027 & 0.021 & 0.021 & 0.000 & 0.011 & 0.000 & 0.007 \\
\hline 12.00 & 0.000 & 0.000 & 0.000 & 0.000 & 0.000 & 0.000 & 0.000 & 0.000 \\
\hline 14.10 & 0.000 & 0.000 & 0.000 & 0.000 & 0.000 & 0.000 & 0.000 & 0.000 \\
\hline TOTAL & 0.03 & 0.34 & 0.33 & 0.21 & 0.17 & 0.28 & 0.22 & 0.26 \\
\hline
\end{tabular}

Joint Frequency Distribution of Wind Speed and Direction:

Atmospheric Stability Class F Moderately Stable Conditions

\begin{tabular}{lrllllllll}
\hline $\begin{array}{c}\text { (M/S) } \\
\text { UMAX }\end{array}$ & S & SSW & \multicolumn{1}{c}{ SW } & WSW & W & WNW & NW & NNW & TOTAL \\
\hline 2.00 & 0.007 & 0.014 & 0.018 & 0.007 & 0.007 & 0.005 & 0.011 & 0.005 & 0.114 \\
4.00 & 0.107 & 0.080 & 0.119 & 0.091 & 0.030 & 0.050 & 0.050 & 0.073 & 1.075 \\
6.00 & 0.221 & 0.214 & 0.235 & 0.228 & 0.068 & 0.039 & 0.023 & 0.041 & 2.321 \\
8.00 & 0.018 & 0.014 & 0.018 & 0.007 & 0.000 & 0.000 & 0.000 & 0.000 & 0.144 \\
12.00 & 0.000 & 0.000 & 0.000 & 0.000 & 0.000 & 0.000 & 0.000 & 0.000 & 0.000 \\
12.10 & 0.000 & 0.000 & 0.000 & 0.000 & 0.000 & 0.000 & 0.000 & 0.000 & 0.000 \\
TOTAL & $\mathbf{0 . 3 5}$ & $\mathbf{0 . 3 2}$ & $\mathbf{0 . 3 9}$ & $\mathbf{0 . 3 3}$ & $\mathbf{0 . 1 0}$ & $\mathbf{0 . 0 9}$ & $\mathbf{0 . 0 8}$ & $\mathbf{0 . 1 2}$ & $\mathbf{3 . 6 5}$
\end{tabular}




\section{Table 43}

Meteorological Data

Page 7 of 7

USNRC Computer Code-XOQDOQ,

Version 2.0

Run Date: 93.071

Stability from Sigma $A$

(SRL 6/29/83 VERSION)

43824 Wind Stats H-Area 60 minute

$62 \mathrm{M} \mathrm{87-91}$

Atmospheric Stability Class G

Joint Frequency Distribution of Wind Speed and Direction: Extremely Stable Conditions

\begin{tabular}{lllllllll}
\hline $\begin{array}{c}\text { (M/S) } \\
\text { UMAX }\end{array}$ & N & NNE & \multicolumn{1}{c}{ NE } & \multicolumn{1}{c}{ ENE } & E & \multicolumn{1}{c}{ ESE } & SE & \multicolumn{1}{c}{ SSE } \\
\hline 2.00 & 0.000 & 0.000 & 0.002 & 0.000 & 0.000 & 0.002 & 0.000 & 0.005 \\
4.00 & 0.002 & 0.000 & 0.000 & 0.000 & 0.000 & 0.002 & 0.009 & 0.002 \\
6.00 & 0.005 & 0.023 & 0.009 & 0.002 & 0.007 & 0.037 & 0.025 & 0.016 \\
8.00 & 0.000 & 0.002 & 0.000 & 0.000 & 0.000 & 0.002 & 0.002 & 0.000 \\
12.00 & 0.000 & 0.000 & 0.000 & 0.000 & 0.000 & 0.000 & 0.000 & 0.000 \\
14.10 & 0.000 & 0.000 & 0.000 & 0.000 & 0.000 & 0.000 & 0.000 & 0.000 \\
TOTAL & $\mathbf{0 . 0 1}$ & $\mathbf{0 . 0 3}$ & $\mathbf{0 . 0 1}$ & $\mathbf{0 . 0 0}$ & 0.01 & 0.04 & $\mathbf{0 . 0 4}$ & $\mathbf{0 . 0 2}$
\end{tabular}

Joint Frequency Distribution of Wind Speed and Direction:

Atmospheric Stability Class G

\begin{tabular}{lrllllllll}
\hline $\begin{array}{c}\text { (M/S) } \\
\text { UMAX }\end{array}$ & S & \multicolumn{1}{c}{ SSW } & SW & WSW & W & WNW & NW & NNW & TOTAL \\
\hline 2.00 & 0.007 & 0.009 & 0.007 & 0.002 & 0.002 & 0.000 & 0.000 & 0.002 & 0.039 \\
4.00 & 0.007 & 0.007 & 0.027 & 0.009 & 0.005 & 0.002 & 0.002 & 0.000 & 0.075 \\
6.00 & 0.021 & 0.014 & 0.011 & 0.027 & 0.002 & 0.007 & 0.002 & 0.005 & 0.212 \\
8.00 & 0.000 & 0.002 & 0.000 & 0.000 & 0.000 & 0.000 & 0.000 & 0.000 & 0.009 \\
12.00 & 0.000 & 0.000 & 0.000 & 0.000 & 0.000 & 0.000 & 0.000 & 0.000 & 0.000 \\
12.10 & 0.000 & 0.000 & 0.000 & 0.000 & 0.000 & 0.000 & 0.000 & 0.000 & 0.000 \\
TOTAL & 0.03 & 0.03 & 0.05 & 0.04 & 0.01 & 0.01 & 0.00 & 0.01 & 0.34
\end{tabular}


Table 44

80-km-Radius (50-Mile) Population Distribution Around SRS (1990 Census)

Page 1 of 1

\begin{tabular}{|c|c|c|c|c|c|c|}
\hline Dir (Miles) & $0.0-1$ & 1.-2. & 2.-3. & 3.-4. & 4. -5 . & \\
\hline $\mathbf{N}$ & $0.000 \mathrm{E}+00$ & $0.000 \mathrm{E}+00$ & $0.000 E+00$ & $0.000 E+00$ & $0.000 \mathrm{E}+00$ & \\
\hline NNE & $0.000 E+00$ & $0.000 E+00$ & $0.000 E+00$ & $0.000 E+00$ & $0.000 \mathrm{E}+00$ & \\
\hline NE & $0.000 E+00$ & $0.000 E+00$ & $0.000 E+00$ & $0.000 E+00$ & $0.000 E+00$ & \\
\hline ENE & $0.000 E+00$ & $0.000 E+00$ & $0.000 E+00$ & $0.000 E+00$ & $0.000 E+00$ & \\
\hline $\mathbf{E}$ & $0.000 E+00$ & $0.000 E+00$ & $0.000 \mathrm{E}+00$ & $0.000 E+00$ & $0.000 E+00$ & \\
\hline ESE & $0.000 E+00$ & $0.000 \mathrm{E}+00$ & $0.000 E+00$ & $0.000 E+00$ & $0.000 E+00$ & \\
\hline SE & $0.000 E+00$ & $0.000 \mathrm{E}+00$ & $0.000 E+00$ & $0.000 E+00$ & $0.000 E+00$ & \\
\hline SSE & $0.000 \mathrm{E}+00$ & $0.000 E+00$ & $0.000 E+00$ & $0.000 E+00$ & $0.000 E+00$ & \\
\hline $\mathbf{s}$ & $0.000 E+00$ & $0.000 E+00$ & $0.000 E+00$ & $0.000 E+00$ & $0.000 E+00$ & \\
\hline Ssw & $0.000 E+00$ & $0.000 E+00$ & $0.000 E+00$ & $0.000 E+00$ & $0.000 E+00$ & \\
\hline sw & $0.000 E+00$ & $0.000 \mathrm{E}+00$ & $0.000 E+00$ & $0.000 E+00$ & $0.000 E+00$ & \\
\hline wsw & $0.000 \mathrm{E}+00$ & $0.000 \mathrm{E}+00$ & $0.000 E+00$ & $0.000 E+00$ & $0.000 \mathrm{E}+00$ & \\
\hline$w$ & $0.000 E+00$ & $\dot{0} .000 E+00$ & $0.000 E+00$ & $0.000 \mathrm{E}+00$ & $0.000 E+00$ & \\
\hline WNW & $0.000 E+00$ & $0.000 \mathrm{E}+00$ & $0.000 E+00$ & $0.000 E+00$ & $0.000 E+00$ & \\
\hline NW & $0.000 E+00$ & $0.000 \mathrm{E}+00$ & $0.000 E+00$ & $0.000 E+00$ & $0.000 E+00$ & \\
\hline NNW & $0.000 E+00$ & $0.000 E+00$ & $0.000 E+00$ & $0.000 E+00$ & $0.000 E+00$ & \\
\hline Total & $0.000 E+00$ & $0.000 E+00$ & $0.000 E+00$ & $0.000 E+00$ & $0.000 \mathrm{E}+00$ & \\
\hline Dir (Miles) & 5. -10 . & 10.-20. & 20.-30. & 30. -40 . & 40. -50. & Total \\
\hline $\mathbf{N}$ & $2.600 E+01$ & $5.321 \mathrm{E}+03$ & $1.002 E+04$ & $5.067 \mathrm{E}+03$ & $1.221 E+04$ & $3.264 E+04$ \\
\hline NNE & $6.000 E+00$ & $1.320 E+03$ & $2.066 \mathrm{E}+03$ & $4.445 E+03$ & $1.437 E+04$ & $2.220 E+04$ \\
\hline NE & $1.000 E+00$ & $2.945 E+03$ & $2.928 E+03$ & $5.269 E+03$ & $1.020 E+04$ & $2.134 E+04$ \\
\hline ENE & $2.700 \mathrm{E}+\mathrm{C1}$ & $3.126 E+03$ & $4.483 E+03$ & $5.337 E+03$ & $4.077 E+04$ & $5.375 E+04$ \\
\hline $\mathbf{E}$ & $1.550 \mathrm{E}+02$ & $6.743 E+03$ & $5.305 E+03$ & $8.812 E+03$ & $4.334 E+03$ & $2.535 E+04$ \\
\hline ESE & $3.600 \mathrm{E}+01$ & $1.556 \mathrm{E}+03$ & $1.931 E+03$ & $2.711 E+03$ & $3.253 E+03$ & $9.487 E+03$ \\
\hline SE & $2.600 \mathrm{E}+01$ & $5.470 E+02$ & $6.511 E+03$ & $6.685 E+03$ & $8.577 E+03$ & $2.235 E+04$ \\
\hline SSE & $4.000 E+01$ & $3.910 E+02$ & $7.690 \mathrm{E}+02$ & $1.356 \mathrm{E}+03$ & $2.539 E+03$ & $5.095 E+03$ \\
\hline$s$ & $1.000 \mathrm{E}+00$ & $5.580 E+02$ & $1.332 \mathrm{E}+03$ & $7.251 E+03$ & $3.335 E+03$ & $1.248 \mathrm{E}+04$ \\
\hline SSW & $2.000 E+00$ & $8.970 E+02$ & $2.008 E+03$ & $4.181 E+03$ & $2.944 \mathrm{E}+03$ & $1.003 E+04$ \\
\hline sw & $1.700 \mathrm{E}+01$ & $9.440 \mathrm{E}+02$ & $2.240 \mathrm{E}+03$ & $2.606 \mathrm{E}+03$ & $2.660 \mathrm{E}+03$ & $8.467 E+03$ \\
\hline wsw & $6.000 E+01$ & $1.103 E+03$ & $7.112 E+03$ & $2.285 E+03$ & $5.818 E+03$ & $1.638 E+04$ \\
\hline w & $5.500 E+01$ & $3.314 \mathrm{E}+03$ & $7.941 \mathrm{E}+03$ & $7.994 E+03$ & $6.780 E+03$ & $2.608 E+04$ \\
\hline WNW & $4.490 E+02$ & $3.342 \mathrm{E}+03$ & $1.069 E+05$ & $5.031 E+04$ & $1.155 E+04$ & $1.725 \mathrm{E}+05$ \\
\hline NW & $2.710 E+02$ & $5.899 \mathrm{E}+03$ & $8.793 E+04$ & $2.657 E+04$ & $3.025 E+03$ & $1.237 E+05$ \\
\hline NNW & $3.63 E+02$ & $1.803 E+04$ & $2.716 \mathrm{E}+04$ & $6.665 E+03$ & $6.079 E+03$ & $5.830 \mathrm{E}+04$ \\
\hline Total & $1.535 E+03$ & $5.603 E+04$ & $2.766 \mathrm{E} \div 05$ & $1.475 E+05$ & $1.384 E+05$ & $6.201 E+05$ \\
\hline
\end{tabular}


Table 45

80-km-Radius (50-Mile) Milk, Meat, and Vegetation Production Around SRS, as of 1991

Page 1 of 2

Site Annual Milk Production (L)

\begin{tabular}{lccccccc} 
Dir (miles) & $0-5$ & $5-10$ & $10-20$ & $20-30$ & $30-40$ & $40-50$ & Total \\
\hline N & 0.0 & 0.0 & $4.200 E+04$ & $6.900 E+04$ & $1.000 E+06$ & $5.300 E+06$ & $6.411 E+06$ \\
NNE & 0.0 & 0.0 & $4.200 E+04$ & $6.900 E+04$ & $2.100 E+05$ & $5.000 E+05$ & $8.210 E+05$ \\
NE & 0.0 & 0.0 & $3.200 E+04$ & $1.000 E+06$ & $2.700 E+06$ & $2.000 E+06$ & $5.732 E+06$ \\
ENE & 0.0 & 0.0 & $2.500 E+04$ & $1.200 E+06$ & $4.400 E+06$ & $5.200 E+06$ & $1.083 E+07$ \\
E & 0.0 & 0.0 & $2.500 E+04$ & $1.400 E+06$ & $3.900 E+06$ & $4.900 E+06$ & $1.023 E+07$ \\
ESE & 0.0 & 0.0 & $2.500 E+04$ & $5.600 E+05$ & $3.000 E+04$ & $4.900 E+05$ & $1.105 E+06$ \\
SE & 0.0 & 0.0 & $2.500 E+04$ & $0.000 E+00$ & $0.000 E+00$ & $0.000 E+00$ & $2.500 E+03$ \\
SSE & 0.0 & 0.0 & $4.800 E+05$ & $8.600 E+05$ & $1.200 E+06$ & $1.200 E+06$ & $3.740 E+06$ \\
S & 0.0 & 0.0 & $1.000 E+06$ & $2.100 E+06$ & $3.000 E+06$ & $3.500 E+06$ & $9.600 E+06$ \\
SSW & 0.0 & 0.0 & $9.900 E+05$ & $3.800 E+06$ & $7.400 E+06$ & $7.600 E+06$ & $1.979 E+07$ \\
SW & 0.0 & 0.0 & $9.900 E+05$ & $2.200 E+06$ & $5.800 E+06$ & $4.800 E+06$ & $1.379 E+07$ \\
WSW & 0.0 & 0.0 & $9.900 E+05$ & $1.700 E+06$ & $2.400 E+06$ & $3.500 E+06$ & $8.590 E+06$ \\
W & 0.0 & 0.0 & $6.700 E+05$ & $1.300 E+06$ & $2.200 E+06$ & $3.600 E+06$ & $7.770 E+06$ \\
WNW & 0.0 & 0.0 & $2.300 E+05$ & $1.100 E+06$ & $1.200 E+06$ & $2.000 E+06$ & $4.530 E+06$ \\
NW & 0.0 & 0.0 & $4.200 E+04$ & $3.800 E+05$ & $1.400 E+06$ & $1.000 E+06$ & $2.822 E+06$ \\
NNW & 0.0 & 0.0 & $4.200 E+04$ & $6.000 E+04$ & $1.700 E+06$ & $3.400 E+06$ & $5.211 E+06$ \\
Total & 0.0 & 0.0 & $5.62 E+06$ & $1.781 E+07$ & $3.954 E+07$ & $4.899 E+07$ & $1.110 E+08$
\end{tabular}

Site Annual Meat Production (kg)

\begin{tabular}{lccccccc} 
Dir (miles) & $0-5$ & $\mathbf{5 - 1 0}$ & $\mathbf{1 0 - 2 0}$ & $\mathbf{2 0 - 3 0}$ & $30-40$ & $40-50$ & Total \\
\hline N & 0.0 & 0.0 & $5.300 \mathrm{E}+04$ & $8.800 \mathrm{E}+04$ & $2.500 \mathrm{E}+05$ & $9.800 \mathrm{E}+05$ & $1.371 \mathrm{E}+06$ \\
NNE & 0.0 & 0.0 & $5.300 \mathrm{E}+04$ & $8.800 \mathrm{E}+04$ & $2.000 \mathrm{E}+05$ & $4.100 \mathrm{E}+05$ & $7.510 \mathrm{E}+05$ \\
NE & 0.0 & 0.0 & $7.100 \mathrm{E}+04$ & $1.700 \mathrm{E}+05$ & $3.500 \mathrm{E}+05$ & $4.500 \mathrm{E}+05$ & $1.041 \mathrm{E}+06$ \\
ENE & 0.0 & 0.0 & $8.300 \mathrm{E}+04$ & $2.000 \mathrm{E}+05$ & $4.600 \mathrm{E}+05$ & $5.700 \mathrm{E}+05$ & $1.313 \mathrm{E}+06$ \\
E & 0.0 & 0.0 & $8.300 \mathrm{E}+04$ & $1.900 \mathrm{E}+05$ & $3.400 \mathrm{E}+05$ & $5.100 \mathrm{E}+05$ & $1.123 \mathrm{E}+06$ \\
ESE & 0.0 & 0.0 & $8.300 \mathrm{E}+04$ & $1.900 \mathrm{E}+05$ & $2.200 \mathrm{E}+05$ & $2.500 \mathrm{E}+05$ & $7.430 \mathrm{E}+05$ \\
SE & 0.0 & 0.0 & $1.200 \mathrm{E}+05$ & $2.100 \mathrm{E}+05$ & $2.600 \mathrm{E}+05$ & $3.000 \mathrm{E}+05$ & $8.900 \mathrm{E}+05$ \\
SSE & 0.0 & 0.0 & $1.100 \mathrm{E}+05$ & $1.900 \mathrm{E}+05$ & $2.600 \mathrm{E}+05$ & $2.900 \mathrm{E}+05$ & $8.500 \mathrm{E}+05$ \\
S & 0.0 & 0.0 & $9.400 \mathrm{E}+04$ & $1.500 \mathrm{E}+05$ & $2.000 \mathrm{E}+05$ & $2.700 \mathrm{E}+05$ & $7.140 \mathrm{E}+05$ \\
SSW & 0.0 & 0.0 & $9.500 \mathrm{E}+04$ & $1.800 \mathrm{E}+05$ & $2.900 \mathrm{E}+05$ & $3.900 \mathrm{E}+05$ & $9.550 \mathrm{E}+05$ \\
SW & 0.0 & 0.0 & $9.500 \mathrm{E}+04$ & $1.700 \mathrm{E}+05$ & $2.700 \mathrm{E}+05$ & $3.200 \mathrm{E}+05$ & $8.550 \mathrm{E}+05$
\end{tabular}


Table 45

80-km-Radius (50-Mile) Milk, Meat, and Vegetation Production Around SRS, as of 1991

Page 2 of 2

Site Annual Meat Production (kg), Cont'd.

\begin{tabular}{lccccccc} 
Dir (miles) & $0-5$ & $5-10$ & $10-20$ & $20-30$ & $30-40$ & $40-50$ & Total \\
\hline WSW & 0.0 & 0.0 & $9.500 \mathrm{E}+04$ & $1.600 \mathrm{E}+05$ & $2.300 \mathrm{E}+05$ & $4.000 \mathrm{E}+05$ & $8.850 \mathrm{E}+05$ \\
W & 0.0 & 0.0 & $5.800 \mathrm{E}+04$ & $1.000 \mathrm{E}+05$ & $2.100 \mathrm{E}+05$ & $4.100 \mathrm{E}+05$ & $7.780 \mathrm{E}+05$ \\
WNW & 0.0 & 0.0 & $4.800 \mathrm{E}+04$ & $6.200 \mathrm{E}+04$ & $1.300 \mathrm{E}+05$ & $2.900 \mathrm{E}+05$ & $5.300 \mathrm{E}+05$ \\
NW & 0.0 & 0.0 & $5.800 \mathrm{E}+04$ & $8.000 \mathrm{E}+04$ & $2.800 \mathrm{E}+05$ & $2.700 \mathrm{E}+05$ & $6.830 \mathrm{E}+05$ \\
NNW & 0.0 & 0.0 & $5.300 \mathrm{E}+04$ & $8.800 \mathrm{E}+04$ & $3.300 \mathrm{E}+05$ & $6.200 \mathrm{E}+05$ & $1.091 \mathrm{E}+06$ \\
Total & 0.0 & 0.0 & $1.247 \mathrm{E}+06$ & $2.316 \mathrm{E}+06$ & $4.280 \mathrm{E}+06$ & $6.730 \mathrm{E}+06$ & $1.457 \mathrm{E}+07$
\end{tabular}

Site Annual Vegetation Production (kg)

\begin{tabular}{lccccccc} 
Dir (miles) & $\mathbf{0 - 5}$ & $\mathbf{5 - 1 0}$ & $\mathbf{1 0 - 2 0}$ & $\mathbf{2 0 - 3 0}$ & $\mathbf{3 0 - 4 0}$ & $\mathbf{4 0 - 5 0}$ & Total \\
\hline N & 0.0 & 0.0 & $3.600 \mathrm{E}+05$ & $6.000 \mathrm{E}+05$ & $8.400 \mathrm{E}+05$ & $8.700 \mathrm{E}+05$ & $2.670 \mathrm{E}+06$ \\
NNE & 0.0 & 0.0 & $3.600 \mathrm{E}+05$ & $6.000 \mathrm{E}+05$ & $5.100 \mathrm{E}+05$ & $6.300 \mathrm{E}+03$ & $1.476 \mathrm{E}+06$ \\
NE & 0.0 & 0.0 & $3.600 \mathrm{E}+05$ & $6.900 \mathrm{E}+05$ & $1.000 \mathrm{E}+06$ & $5.000 \mathrm{E}+05$ & $2.550 \mathrm{E}+06$ \\
ENE & 0.0 & 0.0 & $3.600 \mathrm{E}+05$ & $6.600 \mathrm{E}+05$ & $1.200 \mathrm{E}+06$ & $1.500 \mathrm{E}+06$ & $3.270 \mathrm{E}+06$ \\
E & 0.0 & 0.0 & $3.600 \mathrm{E}+05$ & $5.900 \mathrm{E}+05$ & $8.500 \mathrm{E}+05$ & $1.400 \mathrm{E}+06$ & $3.200 \mathrm{E}+06$ \\
ESE & 0.0 & 0.0 & $3.600 \mathrm{E}+05$ & $2.200 \mathrm{E}+06$ & $1.900 \mathrm{E}+06$ & $1.100 \mathrm{E}+06$ & $5.560 \mathrm{E}+06$ \\
SE & 0.0 & 0.0 & $2.500 \mathrm{E}+06$ & $4.500 \mathrm{E}+06$ & $3.000 \mathrm{E}+06$ & $1.100 \mathrm{E}+06$ & $1.110 \mathrm{E}+07$ \\
SSE & 0.0 & 0.0 & $1.700 \mathrm{E}+06$ & $2.900 \mathrm{E}+06$ & $3.600 \mathrm{E}+06$ & $1.100 \mathrm{E}+06$ & $9.300 \mathrm{E}+06$ \\
S & 0.0 & 0.0 & $7.200 \mathrm{E}+04$ & $5.400 \mathrm{E}+05$ & $8.400 \mathrm{E}+05$ & $9.700 \mathrm{E}+05$ & $2.422 \mathrm{E}+06$ \\
SSW & 0.0 & 0.0 & $3.500 \mathrm{E}+02$ & $1.200 \mathrm{E}+05$ & $2.500 \mathrm{E}+05$ & $1.100 \mathrm{E}+05$ & $4.804 \mathrm{E}+05$ \\
SW & 0.0 & 0.0 & $3.500 \mathrm{E}+02$ & $7.800 \mathrm{E}+02$ & $2.200 \mathrm{E}+03$ & $3.100 \mathrm{E}+05$ & $3.133 \mathrm{E}+05$ \\
WSW & 0.0 & 0.0 & $3.500 \mathrm{E}+02$ & $5.800 \mathrm{E}+02$ & $7.900 \mathrm{E}+03$ & $2.900 \mathrm{E}+03$ & $4.820 \mathrm{E}+03$ \\
W & 0.0 & 0.0 & $4.500 \mathrm{E}+04$ & $2.300 \mathrm{E}+04$ & $1.700 \mathrm{E}+04$ & $5.200 \mathrm{E}+04$ & $1.370 \mathrm{E}+05$ \\
WNW & 0.0 & 0.0 & $2.600 \mathrm{E}+05$ & $3.800 \mathrm{E}+04$ & $6.200 \mathrm{E}+04$ & $1.100 \mathrm{E}+06$ & $1.848 \mathrm{E}+06$ \\
NW & 0.0 & 0.0 & $3.600 \mathrm{E}+05$ & $4.300 \mathrm{E}+05$ & $8.400 \mathrm{E}+05$ & $1.100 \mathrm{E}+06$ & $2.730 \mathrm{0}+06$ \\
NNW & 0.0 & 0.0 & $3.600 \mathrm{E}+05$ & $6.000 \mathrm{E}+05$ & $8.400 \mathrm{E}+05$ & $1.100 \mathrm{E}+06$ & $2.900 \mathrm{E}+06$ \\
Total & 0.0 & 0.0 & $7.458 \mathrm{E}+06$ & $1.449 \mathrm{E}+07$ & $1.614 \mathrm{E}+07$ & $1.232 \mathrm{E}+07$ & $5.041 \mathrm{E}+07$
\end{tabular}


Table 46

Release Locations for Maximally Exposed Individual Dose

Page 1 of 1

\begin{tabular}{lccccc}
\hline & \multicolumn{5}{c}{ Release Source Area } \\
\cline { 2 - 6 } & F,H,P,K,C & M & D & $\begin{array}{c}\text { Savannah River } \\
\text { Technology Center }\end{array}$ & Diffuse \& Fugitive \\
\cline { 2 - 6 } & 61 & 0 & 16 & 31 & 0 \\
Release height, $m$ \\
$\begin{array}{l}\text { Release location } \\
\text { Site coordinate }\end{array}$ & & & & & \\
East & 58000 & 50041 & 20938 & 51863 & 58000 \\
$\quad$ North & 62000 & 104828 & 65284 & 106670 & 62000
\end{tabular}


Table 47

Parameters Used for Adult Consumption Rates and for Atmospheric Dose Calculations ${ }^{a}$

Page 1 of 1

Pathway

Maximally Exposed Individual

Population

Fruits, vegetables, and grains (kg/yr)

276

163

Leafy vegetables $(\mathrm{kg} / \mathrm{yr})$

43

21

Milk (L/yr)

230

120

Meat (beef) $(\mathrm{kg} / \mathrm{yr})$

81

43

Inhalation ( $\left.\mathrm{m}^{3} / \mathrm{yr}\right)$

8,000

8,000

Fraction of external dose received after taking structural shielding into account

a Values developed by SRTC for SRS 


\section{Table 48}

Site-Specific Parameters Used with CAP88 Code Used for NESHAPS

Calculations

Page 1 of 1

Particle size, AMADa

$\mathrm{H}-3, \mathrm{C}-14, \mathrm{Kr}-85$

0

All other

Environmental dose commitment integrating period, yrs

Maximum individual

40

Population

100

Meteorological data

Plume rise

1987-91; H-Area

Number of stacks

None

Stack heights, $m$

2

Height of lid, $m$

Rainfall, $\mathrm{cm} / \mathrm{yr}$

0 and 61

1000

122.4

Average air temperature, $\mathrm{C}$

17.8

Surface roughness length, $m$

0.4

Height of wind measurements, $m$

61

Average wind speed, $\mathrm{m} / \mathrm{s}$

3.82

Population size

620,100

Food supply fractions (fraction from local sources)

0.700

Vegetable

0.442

Meat

0.399

a Activity Median Aerodynamic Diameter 
Table 49

Parameters Used for Adult Consumption Rates and for Liquid Dose Calculations ${ }^{a}$

Page 1 of 1

\begin{tabular}{lcc} 
Pathway & Maximally Exposed Individual & Population \\
\hline Water consumption $(\mathrm{L} / \mathrm{yr})$ & 730 & 370 \\
Fish consumption $(\mathrm{kg} / \mathrm{yr})$ & 19 & 9 \\
Marine invertebrates $(\mathrm{kg} / \mathrm{yr})_{\text {Boating }^{b}}$ & 8 & 2 \\
Swimming $^{\mathrm{b}}$ & 21 & $1,100,000$ \\
Shoreline recreation $^{\mathrm{b}}$ & 8.9 & 160,000
\end{tabular}

Water treatment plants ${ }^{c}$

Water consumption (L/yr)

730

370

Values developed by SRTC for SRS

Maximum individual values are in units of hours/year; population values are in units of person-hours.

Dose is calculated for the maximally exposed individual and population through the drinking water pathway from the downriver

Beaufort-Jasper Water Treatment Plant near Beaufort, S.C., and the City of Savannah Industrial and Domestic Water Supply

Plant at Port Wentworth, Ga. 


\section{Table 50}

Site-Specific Parameters Used in Liquid Dose Calculations

Page 1 of 1

River flow rate at Highway 301 for 1995 (cu ft/sec) ${ }^{a}$

9,973

River dilution in estuary

Transit time from process areas to river (hr)

24

Transit time from SRS to water treatment plants (hr)

72

Water treatment time $(\mathrm{hr})$

24

Edible aquatic food harvest $(\mathrm{kg} / \mathrm{yr})$

Fish-sport

$35,000^{\mathrm{b}}$

Fish - commercial

$2,700^{\mathrm{b}}$

Invertebrates - salt water

$390,000^{\mathrm{b}}$

Irrigation

None $e^{b, c}$

Shore width factor

0.2

Fish bioaccumulation factor for cesium

3,000

The effective river flow rate was based on tritium measurements. The measured river flow rate was 12,781 cfs.

Irrigation is considered a "special case" scenario. 
Table 51

Committed Dose to the Maximally Exposed Individual from Atmospheric Releases (MAXIGASP Code - Using Consumption of Cow Milk Pathway))

Page 1 of 1

By Pathway

\begin{tabular}{lcc} 
Pathway & Maximally Exposed Individual Dose (mrem) & Percent of Total Dose \\
\hline Plume & $7.06 \mathrm{E}-09$ & 0.00001 \\
Ground & $2.01 \mathrm{E}-03$ & 3.6 \\
Inhalation & $2.45 \mathrm{E}-02$ & 44.1 \\
Vegetation & $2.04 \mathrm{E}-02$ & 36.7 \\
Cow milk & $6.24 \mathrm{E}-03$ & 11.2 \\
Meat & $2.50 \mathrm{E}-03$ & 4.5 \\
Total & $5.56 \mathrm{E}-02$ &
\end{tabular}

By Radionuclide

\begin{tabular}{lcc} 
Radionuclide & Maximally Exposed Individual Dose (mrem) & Percent of Total Dose $^{\mathrm{b}}$ \\
\hline Gases and Vapors & & 77.5 \\
H-3 & $4.31 \mathrm{E}-02$ & 4.8 \\
I-129 & $2.65 \mathrm{E}-03$ & 0.4 \\
l-131 & $2.05 \mathrm{E}-04$ & \\
Particulates & & 0.9 \\
Sr-90 & & 4.4 \\
Cs-137 & $5.20 \mathrm{E}-04$ & 0.1 \\
U-234 & $2.42 \mathrm{E}-03$ & 0.7 \\
U-235 & $5.93 \mathrm{E}-05$ & 0.4 \\
U-238 & $3.99 \mathrm{E}-04$ & 2.8 \\
Pu-238 & $2.46 \mathrm{E}-04$ & 7.9 \\
Pu-239 & $1.53 \mathrm{E}-03$ & 0.2 \\
Am-241 & $4.41 \mathrm{E}-03$ & \\
Total & $9.07 \mathrm{E}-05$ & \\
& $5.56 \mathrm{E}-02$ & \\
\end{tabular}

a Committed effective dose equivalent

b Radionuclides contributing $\mathbf{0 . 1 \%}$ or more of the total dose 


\section{Table 52}

Committed Dose to the Maximally Exposed Individual from Atmospheric Releases (MAXIGASP Code - Using Consumption of Goat Milk Pathway)

Page 1 of 1

\begin{tabular}{lcc}
$\begin{array}{l}\text { By Pathway } \\
\text { Pathway }\end{array}$ & Maximally Exposed Individual Dose (mrem) & \\
\hline Plume & $7.06 \mathrm{E}-09$ & Percent of Total Dose \\
Ground & $2.01 \mathrm{E}-03$ & 0.00001 \\
Inhalation & $2.45 \mathrm{E}-02$ & 3.2 \\
Vegetation & $2.04 \mathrm{E}-02$ & 38.6 \\
Goat Milk & $1.41 \mathrm{E}-02$ & 32.1 \\
Meat & $2.50 \mathrm{E}-03$ & 22.2 \\
Total & $6.35 \mathrm{E}-02$ & 3.9
\end{tabular}

By Radionuclide

Radionuclide

Gases and Vapors

$\mathrm{H}-3$

$5.06 \mathrm{E}-02$

79.7

1-129

2.72E-03

4.3

|-131

2.31E-04

0.4

Particulates

Sr-90

5.31E-04

0.8

Cs-137

2.65E-03

4.2

U-234

5.92E-05

0.1

U-235

3.99E-04

0.6

U-238

2.46E-04

0.4

Pu-238

$1.53 \mathrm{E}-03$

2.4

Pu-239

4.41E-03

6.9

Am-241

$9.06 \mathrm{E}-05$

0.1

Total

$6.35 \mathrm{E}-02$

a Committed effective dose equivalent

b Radionuclides contributing $0.1 \%$ or more of the total dose 
Table 53

80-km (50-Mile) Collective Dose from Atmospheric Releases (POPGASP Code)

Page 1 of 1

By Pathway

Pathway

Population Dose (person-rem) ${ }^{\mathrm{a}}$

Percent of Total Dose

\begin{tabular}{lcc}
\hline Plume & $7.76 \mathrm{E}-07$ & 0.00002 \\
Ground & $1.65 \mathrm{E}-01$ & 4.7 \\
Inhalation & $2.47 \mathrm{E}+00$ & 71.0 \\
Vegetation & $5.35 \mathrm{E}-01$ & 15.4 \\
Cow Milk & $2.55 \mathrm{E}-01$ & 7.3 \\
Meat & $6.14 \mathrm{E}-02$ & 1.8 \\
Total & $3.48 \mathrm{E}+00$ &
\end{tabular}

By Radionuclide

Radionuclide

Population Dose (person-rem) ${ }^{\mathrm{a}}$

Percent of Total Dose

Gases and Vapors

$\mathrm{H}-3$

$2.87 \mathrm{E}+00$

82.5

I-129

8.78E-02

2.5

$\mid-131$

$7.00 \mathrm{E}-03$

0.2

Particulates

Sr-90

1.03E-02

0.3

Cs-137

1.64E-01

4.7

U-234

5.93E-03

0.2

U-235

4.31E-02

1.2

U-238

2.45E-02

0.7

Pu-238

1.11E-01

3.2

Pu-239

1.52E-01

4.4

Am-241

$6.55 \mathrm{E}-03$

0.2

Total

$3.48 E+00$

a Committed effective dose equivalent

b Radionuclides contributing $0.1 \%$ or more of the total dose 


\section{Table 54}

\section{Total Site Releases and Maximally Exposed Individual}

Effective Dose Equivalent by Radionuclide

(CAP88 Dose Calculations for 1995 NESHAP Report to EPA)

Page 1 of 2

\begin{tabular}{|c|c|c|c|}
\hline Radionuclide & Releases (curies) & Maximally Exposed Individual EDE (mrem) & Percent of Dose \\
\hline $\mathrm{H}-3$ (oxide) & $5.50 \mathrm{E}+04$ & $7.42 E-02$ & 95.9 \\
\hline Pu-239a & $7.26 \mathrm{E}-04$ & $7.99 E-04$ & 1.0 \\
\hline Cs-137 & $1.50 \mathrm{E}-02$ & $7.67 E-04$ & 1.0 \\
\hline Pu-238 & $5.92 E-04$ & $5.96 \mathrm{E}-04$ & 0.8 \\
\hline$U-235$ & $8.59 E-04$ & $3.33 E-04$ & 0.4 \\
\hline $\mid-129$ & $4.70 E-03$ & $3.11 E-04$ & 0.4 \\
\hline$U-238$ & $5.79 E-04$ & $2.07 E-04$ & 0.3 \\
\hline Sr-89,90b & $5.52 E-03$ & $6.90 E-05$ & 0.1 \\
\hline $\mathrm{U}-234$ & $1.29 \mathrm{E}-04$ & $5.18 E-05$ & 0.1 \\
\hline Am-241 & $3.04 \mathrm{E}-05$ & $5.02 E-05$ & 0.1 \\
\hline $1-135$ & $7.19 E-02$ & $2.48 E-05$ & 0.03 \\
\hline H-3 (elemental) & 4.17E+04 & $2.25 E-05$ & 0.03 \\
\hline $\mid-131$ & $2.06 E-02$ & $1.23 \mathrm{E}-05$ & 0.02 \\
\hline $\mathrm{Xe}-135$ & $3.36 \mathrm{E}-02$ & $1.13 E-05$ & 0.01 \\
\hline$R u-106$ & $1.81 E-04$ & $3.50 \mathrm{E}-06$ & 0.005 \\
\hline $\mathrm{Cm}-244$ & $3.40 E-06$ & $2.96 \mathrm{E}-06$ & 0.004 \\
\hline Co-60 & $5.76 \mathrm{E}-05$ & $2.63 E-06$ & 0.003 \\
\hline $\mathrm{Ce}-144$ & 2.32E-04 & $1.21 \mathrm{E}-06$ & 0.002 \\
\hline$S b-125$ & $1.20 E-04$ & $6.08 E-07$ & 0.001 \\
\hline Cs-134 & $3.01 E-05$ & $4.88 E-07$ & 0.001 \\
\hline $\mathrm{Zn}-65$ & $6.24 E-05$ & $1.58 E-07$ & 0.0002 \\
\hline Zr-95 & $4.51 E-05$ & $6.48 E-08$ & 0.0001 \\
\hline $1-133$ & $1.72 E-03$ & $2.63 E-08$ & 0.00003 \\
\hline Co-58 & $2.60 E-05$ & $2.52 E-08$ & 0.00003 \\
\hline$R u-103$ & $3.72 E-05$ & $1.23 E-08$ & 0.00002 \\
\hline $\mathrm{Nb}-95$ & 2.67E-05 & $1.21 E-08$ & 0.00002 \\
\hline Eu-154 & $3.02 E-07$ & $1.07 E-08$ & 0.00001 \\
\hline Ce-141 & $5.30 E-05$ & $6.45 E-09$ & 0.00001 \\
\hline Eu-155 & $7.50 \mathrm{E}-07$ & $1.05 E-09$ & 0.000001 \\
\hline Sb-124 & $1.81 \mathrm{E}-07$ & $2.72 E-10$ & 0.0000004 \\
\hline Pm-147 & $7.92 E-07$ & $1.35 \mathrm{E}-10$ & 0.0000002 \\
\hline
\end{tabular}

a Includes unidentified alpha (assigned to $\mathrm{Pu}$-239)

b Includes unidentified beta (assigned to Sr-90) 
Table 54

Total Site Releases and Maximally Exposed Individual

Effective Dose Equivalent by Radionuclide

(CAP88 Dose Calculations for 1995 NESHAP Report to EPA)

Page 2 of 2

\begin{tabular}{lccl}
\hline Radionuclide & Releases (curies) & Maximally Exposed Individual EDE (mrem) & Percent of Dose \\
\hline $\mathrm{Cm}-243$ & $4.90 \mathrm{E}-14$ & $6.63 \mathrm{E}-14$ & 0.0000000001 \\
$\mathrm{Hg}-203$ & $1.00 \mathrm{E}-12$ & $2.62 \mathrm{E}-16$ & 0.0000000000003 \\
$\mathrm{Cm}-242$ & $2.03 \mathrm{E}-16$ & $2.61 \mathrm{E}-16$ & 0.0000000000003 \\
$\mathrm{~S}-35$ & $5.26 \mathrm{E}-12$ & $1.16 \mathrm{E}-16$ & 0.0000000000001 \\
$\mathrm{Cm}-248$ & $9.20 \mathrm{E}-18$ & $6.97 \mathrm{E}-17$ & 0.0000000000001 \\
$\mathrm{Am}-243$ & $2.30 \mathrm{E}-17$ & $4.64 \mathrm{E}-17$ & 0.0000000000001 \\
$\mathrm{Co}-57$ & $2.50 \mathrm{E}-14$ & $1.28 \mathrm{E}-17$ & 0.00000000000002 \\
$\mathrm{Ni}-63$ & $2.00 \mathrm{E}-13$ & $7.31 \mathrm{E}-18$ & 0.00000000000001 \\
$\mathrm{C}-14$ & $9.80 \mathrm{E}-15$ & $8.55 \mathrm{E}-19$ & 0.000000000000001 \\
$\mathrm{Rb}-86$ & $2.00 \mathrm{E}-15$ & $4.52 \mathrm{E}-19$ & 0.000000000000001 \\
$\mathrm{Sn}-113$ & $3.80 \mathrm{E}-16$ & $6.81 \mathrm{E}-20$ & 0.0000000000000001 \\
$\mathrm{Cr}-51$ & $1.00 \mathrm{E}-16$ & $1.50 \mathrm{E}-21$ & 0.000000000000000002 \\
Total & & $7.74 \mathrm{E}-02$ &
\end{tabular}




\section{Table 55}

\section{NESHAP Report Data: CAP88 Compared With MAXIGASP}

Page 1 of 1 .

Maximally Exposed Individual Dose Commitment at Site Boundary from Atmospheric Releases

\begin{tabular}{cc}
\multicolumn{2}{c}{ CAP88 Code } \\
\cline { 2 - 3 } mrem $^{2}$ & Percent of Dose \\
\hline
\end{tabular}

\begin{tabular}{ll}
\multicolumn{2}{c}{ MAXIGASP Code } \\
\hline mrem $^{\mathrm{a}}$ & Percent of Dose \\
\hline
\end{tabular}

\section{By Pathway}

Plume

Ground

Inhalation

Food ${ }^{b}$

Total

1.25E-07

$7.83 E-04$

$2.27 \mathrm{E}-02$

$5.39 \mathrm{E}-02$

7.74E-02

By Radionuclide

\section{Gases and Vapors}

$\mathrm{H}-3^{\mathrm{C}}$

I-129

$\mid-131$

$$
\begin{aligned}
& 7.42 E-02 \\
& 3.11 E-04 \\
& 1.23 E-05
\end{aligned}
$$

\section{Particulates}

Sr-90

Cs-137

U-234

U-235

U-238

Pu-238

Pu-239

Am-241

Total
$6.90 E-05$

7.67E-04

5.18E-05

3.33E-04

2.07E-04

$5.96 \mathrm{E}-04$

$7.99 \mathrm{E}-04$

5.02E-05

7.74E-02

0.0002
1.0
29.3
69.6

95.9

0.4

0.02

0.09

1.0

0.1

0.4

0.3

0.8

1.0

0.06
4.31E-02

77.5

2.65E-03

4.8

2.05E-04

0.4

7.06E-09

2.45E-02

\subsection{E-02}

0.00001

3.6

44.1

52.3

52.3

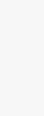


Table 56

NESHAP Report Data: CAP88 Compared With POPGASP

Page 1 of 1

\begin{tabular}{|c|c|c|c|c|}
\hline & \multicolumn{4}{|c|}{ Collective Committed Dose from Atmospheric Releases } \\
\hline & \multicolumn{2}{|c|}{ CAP88 Code } & \multicolumn{2}{|c|}{ POPGASP Code } \\
\hline & person-rem $^{\mathbf{a}}$ & Percent of Dose & person-rem ${ }^{2}$ & Percent of Dose \\
\hline \multicolumn{5}{|c|}{ By Pathway } \\
\hline Plume & $1.06 \mathrm{E}-05$ & 0.0001 & $7.76 \mathrm{E}-07$ & 0.00002 \\
\hline Ground & $1.03 E-01$ & 1.2 & $1.65 E-01$ & 4.7 \\
\hline Inhalation & $2.91 E+00$ & 34.8 & $2.47 \mathrm{E}+00$ & 71.0 \\
\hline Food $^{b}$ & $5.35 \mathrm{E}+00$ & 64.0 & $8.52 E-01$ & 24.5 \\
\hline Total & $8.36 \mathrm{E}+00$ & & $3.48 E+00$ & \\
\hline \multicolumn{5}{|c|}{ By Radionuclide } \\
\hline \multicolumn{5}{|c|}{ Gases and Vapors } \\
\hline $\mathrm{H}-3^{\mathrm{c}}$ & $8.01 E+00$ & 95.9 & $2.87 E+00$ & 82.5 \\
\hline $1-129$ & $1.41 E-02$ & 0.2 & $8.78 E-02$ & 2.5 \\
\hline $\mid-131$ & $6.29 E-04$ & 0.01 & $7.00 \mathrm{E}-03$ & 0.2 \\
\hline \multicolumn{5}{|c|}{ Particulates } \\
\hline Sr-90 & $6.49 E-03$ & 0.08 & $1.03 \mathrm{E}-02$ & 0.3 \\
\hline Cs-137 & $1.02 E-01$ & 1.2 & $1.64 \mathrm{E}-01$ & 4.7 \\
\hline U-234 & $5.68 E-03$ & 0.1 & $5.93 E-03$ & 0.2 \\
\hline U-235 & $3.68 E-02$ & 0.4 & $4.31 \mathrm{E}-02$ & 1.2 \\
\hline U-238 & 2.27E-02 & 0.3 & $2.45 E-02$ & 0.7 \\
\hline Pu-238 & $6.48 E-02$ & 0.8 & $1.11 \mathrm{E}-01$ & 3.2 \\
\hline Pu-239 & 8.65E-02 & 1.0 & $1.52 E-01$ & 4.4 \\
\hline Am-241 & $5.51 E-03$ & 0.07 & $6.55 E-03$ & 0.2 \\
\hline Total & 8.36E+00 & & $3.48 E+00$ & \\
\hline
\end{tabular}

a Committed effective dose equivalent

b Meat, milk, and vegetables

c Dose from tritium in foods calculated with absolute humidity of $11.4 \mathrm{~g}$ water/cubic meter of air 
Table 57

Committed Dose to Maximally Exposed Individual from Liquid Releases

Page 1 of 1

By Pathway

Pathway

Individual Dose (mrem) ${ }^{a}$

Percent of Total Dose

Fish

6.95E-02

49.9

Water

$6.96 \mathrm{E}-02$

50.0

Shoreline

2.21E-04

0.2

Swimming

$1.50 \mathrm{E}-07$

0.0001

Boating

1.77E-07

0.0001

Total

1.39E-01

By Radionuclide

Radionuclide

Individual Dose (mrem) ${ }^{a}$

Percent of Total Dose

H-3 (oxide)

6.02E-02

43.2

Co-60

$1.61 E-05$

0.0

Sr-90b

$7.55 \mathrm{E}-03$

5.4

1-129

3.03E-04

0.2

Cs-137

$6.53 \mathrm{E}-02$

46.8

Pm-147

3.37E-07

0.0002

U-234

3.32E-06

0.002

U-235

2.59E-07

0.0

U-238

3.35E-06

0.0

Pu-238

1.37E-05

0.0

Pu-239c

5.99E-03

4.3

Am-241

1.22E-06

0.0

Cm-244

1.13E-06

0.0

Total

1.39E-01

\footnotetext{
a Committed effective dose equivalent

b Includes unidentified beta (assigned to Sr-90)

c Includes unidentified alpha (assigned to Pu-239)
} 
Table 58

Committed Dose to Maximally Exposed Individual from Public Water Supplies at Beaufort-Jasper Water Treatment Plant

Page 1 of 1

\begin{tabular}{lcc}
\hline Radionuclide & Individual Dose, mrem & Percent of Total Dose \\
\hline$H-3$ (oxide) & $3.79 E-02$ & 84.5 \\
Co-60 & $3.12 E-06$ & 0.01 \\
Sr-90 & $2.73 E-03$ & 6.1 \\
H-129 & $1.40 E-04$ & 0.3 \\
Cs-137 & $5.29 E-04$ & 1.2 \\
Pm-147 & $1.31 E-07$ & 0.0003 \\
U-234 & $2.03 E-06$ & 0.005 \\
U-235 & $1.55 E-07$ & 0.0003 \\
U-238 & $2.05 E-06$ & 0.005 \\
Pu-238 & $8.11 E-06$ & 0.02 \\
Pu-239c & $3.54 E-03$ & 7.9 \\
Am-241 & $4.75 E-07$ & 0.001 \\
Cm-244 & $4.40 E-07$ & 0.001 \\
Total & $4.48 E-02$ &
\end{tabular}




\section{Table 59}

Committed Dose to Maximally Exposed Individual from Public Water Supplies at Port Wentworth Water Treatment Plant

Page 1 of 1

\begin{tabular}{lcc}
\hline Radionuclide & Individual Dose, mrem & \\
\hline H-3 (oxide) & $4.51 \mathrm{E}-02$ & Percent of Total Dose \\
Co-60 & $3.72 \mathrm{E}-06$ & 84.5 \\
Sr-90b & $3.25 \mathrm{E}-03$ & 0.01 \\
l-129 & $1.67 \mathrm{E}-04$ & 6.1 \\
Cs-137 & $6.30 \mathrm{E}-04$ & 0.3 \\
Pm-147 & $1.56 \mathrm{E}-07$ & 1.2 \\
U-234 & $2.42 \mathrm{E}-06$ & 0.0003 \\
U-235 & $1.85 \mathrm{E}-07$ & 0.005 \\
U-238 & $2.45 \mathrm{E}-06$ & 0.0003 \\
Pu-238 & $9.67 \mathrm{E}-06$ & 0.005 \\
Pu-239c & $4.22 \mathrm{E}-03$ & 0.02 \\
Am-241 & $5.66 \mathrm{E}-07$ & 7.9 \\
Cm-244 & $5.24 \mathrm{E}-07$ & 0.001 \\
Total & $5.34 \mathrm{E}-02$ & 0.001
\end{tabular}


Table 60

Collective Dose from Liquid Releases

Page 1 of 1

By Pathway

Pathway

Sport fish

Commercial fish

Beaufort-Jasper

Port Wentworth

Salt water invertebrates

Recreation-river

Total

By Radionuclide

Radionuclide

$\mathrm{H}-3$

Co-60

Sr-90 b

I-129

Cs-137

Pm-147

U-234

U-235

U-238

Pu-238

Pu-239c

Am-241

Cm-244

Total

\section{Collective Dose (person-rem) ${ }^{\mathrm{a}}$}

$1.26 \mathrm{E}-01$

$1.23 \mathrm{E}-03$

$1.14 \mathrm{E}+00$

4.06E-01

$3.69 \mathrm{E}-04$

$9.24 \mathrm{E}-03$

$1.68 E+00$
Percent of Total Dose

7.5

0.1

67.7

24.1

0.02

0.5
Collective Dose (person-rem) ${ }^{a}$

$1.31 \mathrm{E}+00$

3.27E-04

$1.00 \mathrm{E}-01$

$5.02 E-03$

$1.45 \mathrm{E}-01$

4.83E-06

$7.04 \mathrm{E}-05$

$5.57 \mathrm{E}-06$

7.11E-05

2.83E-04

1.23E-01

1.75E-05

1.62E-05

$1.68 \mathrm{E}+00$
Percent of Total Dose

77.8

0.02

6.0

0.3

8.6

0.0003

0.004

0.0003

0.004

0.02

7.3

0.001

0.001

Committed effective dose equivalent

b Includes unidentified beta (assigned to Sr-90)

c Includes unidentified alpha (assigned to Pu-239) 


\section{Table 61}

Potential Doses from Irrigation Pathways

Page 1 of 1

Effective Dose Equivalent

Food Type ${ }^{a}$

Maximally Exposed Individual (mrem) ${ }^{\mathrm{a}}$

Population (person-rem) ${ }^{\mathrm{a}}$

Vegetation
Leafy vegetable
Milk

$5.05 \mathrm{E}-02$

3.15E+00

$7.87 \mathrm{E}-03$

2.03E-01

Meat

2.02E-02

$2.75 \mathrm{E}+00$

Total

$6.72 \mathrm{E}-03$

3.60E-01

8.53E-02

$6.46 E+00$

a Irrigated acreage for each food type assumed to be 1,000 acres 
Table 62

Dose from Consumption of Fish from SRS Creek Mouths and River Mile

Page 1 of 2

Number of Composites with Quantifiable Activitya

\begin{tabular}{llccccc} 
Location & Species & H-3 & Sr-90 & Cs-137 & Pu-238 & Pu-239 \\
\hline BDC & Catfish & 3 & 2 & 2 & 3 & 3 \\
FMC & Catfish & 1 & 1 & 0 & 1 & 1 \\
L3R & Catfish & 2 & 2 & 2 & 1 & 1 \\
& Bream & 1 & 1 & 1 & 1 & 1 \\
SC & Bass & 1 & 1 & 1 & 1 & 1 \\
& Catfish & 2 & 2 & 2 & 2 & 2 \\
& Bream & 2 & 2 & 2 & 2 & 2 \\
U3R & Catfish & 2 & 2 & 2 & 2 & 2 \\
RM 120 & Catfish & 2 & 2 & 2 & 3 & 3
\end{tabular}

Average Concentration in Composites, $\mathrm{pCi} / \mathrm{g}$

\begin{tabular}{llccccc} 
Location & Species & H-3 & Sr-90 & Cs-137 & Pu-238 & Pu-239 \\
\hline BDC & Catfish & $9.36 E-02$ & $1.23 E-03$ & $9.00 E-02$ & $4.46 E-04$ & $-1.14 E-06$ \\
FMC & Catfish & $7.19 E-01$ & $2.98 E-03$ & NA & $1.12 E-04$ & $6.07 E-06$ \\
L3R & Catfish & $1.71 E-01$ & $1.02 E-03$ & $2.54 E-01$ & $4.23 E-05$ & $-2.11 E-05$ \\
& Bream & $2.66 E-01$ & $-3.00 E-05$ & $1.55 E-01$ & $-1.23 E-05$ & $1.63 E-05$ \\
SC & Bass & $1.97 E+00$ & $5.62 E-03$ & $1.21 E+00$ & $7.34 E-06$ & $4.89 E-06$ \\
& Catfish & $1.59 E+00$ & $1.40 E-02$ & $8.23 E-02$ & $2.42 E-05$ & $2.74 E-06$ \\
& Bream & $2.08 E+00$ & $8.25 E-03$ & $1.71 E-01$ & $3.10 E-06$ & $2.69 E-06$ \\
U3R & Catfish & $1.89 E-01$ & $2.24 E-03$ & $9.10 E-02$ & $7.30 E-06$ & $2.09 E-06$ \\
RM 120 & Catfish & $3.06 E-01$ & $3.40 E-03$ & $5.69 E-02$ & $-3.56 E-05$ & $-4.29 E-05$ \\
& Bream & $2.79 E-01$ & $5.56 E-03$ & $7.50 E-02$ & $1.08 E-05$ & $3.60 E-06$
\end{tabular}

a All composites were 5 fish.

b Not available (no quantifiable activity) 


\section{Table 62}

Dose from Consumption of Fish from SRS Creek Mouths and River Mile

Page 2 of 2

\begin{tabular}{|c|c|c|c|c|c|c|c|}
\hline \multicolumn{8}{|c|}{ Dose from Consumption of $19 \mathrm{~kg} / \mathrm{year}$ (42 lbs), mrem } \\
\hline Location & Species & H-3 & Sr-90 & Cs-137 & Pu-238 & Pu-239 & Total \\
\hline BDC & Catfish & $1.12 \mathrm{E}-04$ & $3.04 E-03$ & $8.55 E-02$ & $3.22 E-02$ & b & $1.21 \mathrm{E}-01$ \\
\hline FMC & Catfish & $8.61 E-04$ & $7.36 \mathrm{E}-03$ & $N A^{c}$ & $8.09 E-03$ & $4.96 \mathrm{E}-04$ & $1.68 \mathrm{E}-02$ \\
\hline \multirow[t]{2}{*}{ L3R } & Catfish & $2.05 E-04$ & $2.52 E-03$ & $2.41 E-01$ & $3.05 E-03$ & & $2.47 \mathrm{E}-01$ \\
\hline & Bream & $3.18 E-04$ & & 1.47E-01 & & $1.33 \mathrm{E}-03$ & $1.49 \mathrm{E}-01$ \\
\hline \multirow[t]{3}{*}{ SC } & Bass & $2.36 E-03$ & $1.39 \mathrm{E}-02$ & $1.15 \mathrm{E}+00$ & $5.30 E-04$ & $4.00 E-04$ & $1.17 \mathrm{E}+00$ \\
\hline & Cattish & $1.90 \mathrm{E}-03$ & $3.46 \mathrm{E}-02$ & $7.82 E-02$ & $1.75 E-03$ & $2.24 E-04$ & $1.17 \mathrm{E}-01$ \\
\hline & Bream & $2.49 E-03$ & $2.04 \mathrm{E}-02$ & $1.62 E-01$ & $2.24 \mathrm{E}-04$ & $2.20 E-04$ & $1.86 \mathrm{E}-01$ \\
\hline U3R & Cattish & $2.26 \mathrm{E}-04$ & $5.53 E-03$ & $8.65 E-02$ & $5.27 E-04$ & $1.71 \mathrm{E}-04$ & $9.29 E-02$ \\
\hline \multirow[t]{2}{*}{ RM 120} & Cattish & $3.66 \mathrm{E}-04$ & $8.40 \mathrm{E}-03$ & $5.41 E-02$ & & & $6.28 \mathrm{E}-02$ \\
\hline & Bream & $3.34 \mathrm{E}-04$ & $1.37 \mathrm{E}-02$ & $7.13 E-02$ & $7.80 \mathrm{E}-04$ & $2.94 \mathrm{E}-04$ & $8.64 \mathrm{E}-02$ \\
\hline
\end{tabular}

a Dose is based on maximum consumption of $19 \mathrm{~kg} / \mathrm{year}$. To obtain dose from average consumption of fish ( $9 \mathrm{~kg} / \mathrm{year}$ ), multiply doses in this table by 0.474 .

b Dose based on negative concentration of individual radionuclides in fish was not calculated (shaded areas) and was not used in the total dose column.

c Not available (no quantifiable activity) 
Table 63

Calculated Doses to Aquatic Biota from SRS Releases

Page 1 of 1

\begin{tabular}{|c|c|c|c|c|c|}
\hline \multirow[b]{2}{*}{ Stream } & \multirow[b]{2}{*}{ Location } & \multirow[b]{2}{*}{ Flow, cfs } & \multicolumn{3}{|c|}{ Dose to Biota, rad/da,b } \\
\hline & & & Fish & Invertebrate & Algae \\
\hline U3R & $\begin{array}{l}\text { Tim's Branch } 5 \\
\text { (Road C) }\end{array}$ & 7 & $3.78 E-04$ & $4.32 E-04$ & $1.19 \mathrm{E}-04$ \\
\hline $\mathrm{BDC}$ & Below 400-D & 72 & $6.43 E-06$ & $1.08 \mathrm{E}-05$ & 7.86E-06 \\
\hline FMC & $\begin{array}{l}\text { FM3 above } \\
\text { Road E }\end{array}$ & 10 & $6.67 \mathrm{E}-04$ & $8.26 \mathrm{E}-04$ & $9.53 \mathrm{E}-04$ \\
\hline PB & $K-018$ & 37 & $2.36 \mathrm{E}-05$ & $3.46 E-05$ & $9.21 E-05$ \\
\hline SC & $\begin{array}{l}\text { Steel Creek 2A } \\
\text { above Road B }\end{array}$ & 5 & $6.43 E-05$ & $1.62 E-04$ & $6.75 \mathrm{E}-04$ \\
\hline \multirow[t]{2}{*}{ L3R } & $\begin{array}{l}\text { L3R1A above } \\
\text { Road B }\end{array}$ & 19 & $3.83 E-06$ & $8.94 E-06$ & $3.56 \mathrm{E}-05$ \\
\hline & & & \multicolumn{2}{|c|}{ Dose to Biota, $\mathrm{rad} / \mathrm{da}, \mathrm{b}$} & \\
\hline Stream & Location & Flow, cfs & Raccoon & Duck & \\
\hline U3R & $\begin{array}{l}\text { Tim's Branch } 5 \\
\text { (Road C) }\end{array}$ & 7 & $1.53 \mathrm{E}-04$ & $1.54 \mathrm{E}-03$ & \\
\hline $\mathrm{BDC}$ & Below 400-D & 72 & $4.94 \mathrm{E}-06$ & $8.45 E-05$ & \\
\hline FMC & $\begin{array}{l}\text { FM3 above } \\
\text { Road E }\end{array}$ & 10 & $5.29 E-04$ & $1.14 \mathrm{E}-02$ & \\
\hline PB & $K-018$ & 37 & $4.05 E-05$ & $1.12 E-03$ & \\
\hline SC & $\begin{array}{l}\text { Steel Creek 2A } \\
\text { above Road B }\end{array}$ & 5 & $3.40 E-04$ & $9.86 E-03$ & \\
\hline L3R & $\begin{array}{l}\text { L3R1A above } \\
\text { Road B }\end{array}$ & 19 & $1.84 \mathrm{E}-05$ & $5.35 E-04$ & \\
\hline
\end{tabular}

a Calculated with CRITR methodology incorporated in the LADTAPIl computer program

b The DOE limit is $1 \mathrm{rad} / \mathrm{d}$. 


\section{Table 64}

\section{Toxic/Hazardous Air Pollutant Emissions (1994)}

Page 1 of 4

Pollutant

Actual Tons/Year

\begin{tabular}{|c|c|c|}
\hline Note: & $\begin{array}{l}\text { Emissions are calculated eac } \\
\text { compiled and emissions calc } \\
\text { process, which begins in Jan } \\
\text { emissions only. Actual emissi } \\
\text { Environmental Report for } 199\end{array}$ & $\begin{array}{l}\text { tory. In 1995, operating data were } \\
\text { ission sources. Because this } \\
\text { his report provides } 1994 \\
\text { in depth in the SRS }\end{array}$ \\
\hline$\overline{1,1,1-}$ & RICHLOROETHANE & $1.92 E-01$ \\
\hline $1,1,2,2$ & -TETRACHLOROETHANE & $1.15 E-04$ \\
\hline $1,1,2-$ & RICHLOROETHANE & $2.01 E-06$ \\
\hline $1,1-D$ & HLORETHYLENE & $1.78 E-04$ \\
\hline $1,1-D$ & HLOROETHANE & $4.56 E-07$ \\
\hline $1,2,4-$ & RICHLOROBENZENE & $1.17 E-04$ \\
\hline $1,3-B$ & TADIENE & $9.06 E-02$ \\
\hline $2,2,4-$ & RIMETHYLPENTANE & $1.28 \mathrm{E}-02$ \\
\hline $2,4-D$ & IITROPHENOL & 4.43E-07 \\
\hline 2-BIP & ENYLOL & $5.00 E-06$ \\
\hline ACET & LDEHYDE & $3.27 E-01$ \\
\hline ACET & NITRILE & $4.66 \mathrm{E}-01$ \\
\hline ACRO & EIN & $1.41 \mathrm{E}-02$ \\
\hline ALIPH & TIC POLYISOCYANATE & $00 E+00$ \\
\hline AMMC & VIUM CHLORIDE & $4.89 E-03$ \\
\hline ANILII & & $1.92 E-04$ \\
\hline ANTIN & DNY & $1.02 E-01$ \\
\hline ARSE & & $4.46 E-01$ \\
\hline BENZ & & $2.72 E+00$ \\
\hline BERY & LIUM & $2.42 E-03$ \\
\hline BIS- $(2$ & -ETHYLHEXYL)PHTHALATE & $7.00 \mathrm{E}-03$ \\
\hline BRON & DFORM & $3.00 E+00$ \\
\hline CADM & & $2.09 E-02$ \\
\hline CARB & N DISULFIDE & $1.39 E-02$ \\
\hline CARB & N TETRACHLORIDE & $4.78 E-03$ \\
\hline CHLO & DANE & $6.77 E-03$ \\
\hline CHLO & INE & $1.01 E-02$ \\
\hline $\mathrm{CHLO}$ & OBENZENE & $8.35 E-05$ \\
\hline CHLO & OFORM & $3.94 \mathrm{E}+00$ \\
\hline $\mathrm{CHRO}$ & MIC OXIDE & $8.46 E-09$ \\
\hline $\mathrm{CHRO}$ & AIUM & $6.60 \mathrm{E}-02$ \\
\hline
\end{tabular}


Table 64

Toxic/Hazardous Air Pollutant Emissions (1994)

Page 2 of 4

Pollutant

CHROMIUM (+6) COMPOUNDS

CHROMIUM (PM10)

COBALT

CRESOL

CUMENE

CYANIDE

DIBUTYL PHTHALATE

DIOXANE

DIPHENYL

DIPHENYLMERCURY

ETHYL BENZENE

ETHYLENE DIBROMIDE

ETHYLENE DICHLORIDE

ETHYLENE GLYCOL

FORMALDEHYDE

FORMIC ACID

FURFURYL ALCOHOL

GLYCOL ETHERS

HEPTACHLOR

HEXACHLOROBENZENE

HEXACHLOROCYLOPENTADIENE

HEXANE

HYDRAZINE

HYDROCHLORIC ACID (HYDROGEN CHLORIDE)

HYDROGEN FLUORIDE

HYDROGEN SULFIDE

HYDROQUINONE

ISOPHORONE

LEAD

LEAD OXIDE

LEAD SULFATE

M-XYLENE

MANGANESE

MANGANESE (PM10)

MANGANESE DIOXIDE
Actual Tons/Year

8.34E-05

1.62E-07

$1.78 \mathrm{E}-03$

1.12E-04

$1.56 \mathrm{E}-02$

$1.95 \mathrm{E}-03$

2.00E-03

$1.50 \mathrm{E}-05$

2.71E-03

$5.44 \mathrm{E}-05$

7.32E-01

7.81E-06

3.61E-05

2.61E-01

2.61E+00

9.08E-01

$8.20 E-03$

$5.70 \mathrm{E}-03$

3.90E-05

1.16E-04

9.76E-04

$2.46 \mathrm{E}+00$

1.76E-06

$1.85 \mathrm{E}+00$

$6.74 \mathrm{E}-02$

1.44E+01

5.45E-03

6.95E-03

1.46E-01

9.56E-02

$4.00 \mathrm{E}-13$

9.68E-02

5.28E-02

7.30E-09

9.81E-08 


\section{Table 64}

Toxic/Hazardous Air Pollutant Emissions (1994)

Page 3 of 4

\begin{tabular}{|c|c|}
\hline Pollutant & Actual Tons/Year \\
\hline MANGANESE FORMATE & $1.14 \mathrm{E}-07$ \\
\hline MANGANESE TETROXIDE & $1.00 E-11$ \\
\hline MERCURIC NITRATE & 5.77E-04 \\
\hline MERCURY & $1.39 E-01$ \\
\hline MERCURY OXIDE & $00 E+00$ \\
\hline METHOXYCHLOR & $1.24 \mathrm{E}-01$ \\
\hline METHYL ALCOHOL & $9.39 E-01$ \\
\hline METHYL CYANOACRYLATE & $5.94 \mathrm{E}-04$ \\
\hline METHYL ETHYL KETONE (2-BUTONE) & $3.01 E-01$ \\
\hline METHYL ISOBUTYL KETONE & $7.57 E-01$ \\
\hline METHYL METHACRYLATE & 2.10E-06 \\
\hline METHYL TERT-BUTYL ETHER & $7.48 E-02$ \\
\hline METHYL-2-CYANOACETATE & $5.00 E-06$ \\
\hline METHYLENE CHLORIDE & $2.30 E-01$ \\
\hline MINERAL OIL & $8.79 E-03$ \\
\hline NAPHTHALENE & 1.07E-02 \\
\hline NICKEL & $5.00 \mathrm{E}-02$ \\
\hline NICKEL (PM10) & $8.40 E-08$ \\
\hline NICKEL FORMATE & $2.02 E-09$ \\
\hline NICKEL MONOXIDE & $5.00 E-08$ \\
\hline NITRIC ACID & $3.68 \mathrm{E}+00$ \\
\hline NITRIC ACID (64\%) & $3.84 \mathrm{E}-03$ \\
\hline NITROBENZENE & 3.35E-06 \\
\hline O-TERPHENYL & 4.97E-06 \\
\hline O-XYLENE & 4.85E-01 \\
\hline OXALIC ACID & $2.31 \mathrm{E}-02$ \\
\hline P-NITROSOPHENOL & 2.17E-04 \\
\hline P-XYLENE & $1.27 E-01$ \\
\hline PHENOL & $8.44 E-03$ \\
\hline PHOSPHORIC ACID & $1.07 E-01$ \\
\hline PHOSPHORUS (YELLOW OR WHITE) & $2.64 E-06$ \\
\hline POLYCHLORINATED BIPHENYLS (PCB) & $6.28 \mathrm{E}-03$ \\
\hline POLYCYCLIC ORGANIC MATTER & $2.50 E-02$ \\
\hline POTASSIUM PERMANGANATE4 & $67 E-10$ \\
\hline RADIONUCLIDES & $4.91 E-01$ \\
\hline
\end{tabular}


Table 64

Toxic/Hazardous Air Pollutant Emissions (1994)

Page 4 of 4

Pollutant

SELENIUM

SODIUM HYDROXIDE

STYRENE

SULFURIC ACID

TETRACHLOROETHYLENE $(C L 2 C=C C L)$

TOLUENE

TOXAPHENE

TRICHLOROETHYLENE (TCE)

VINYL ACETATE

VINYL CHLORIDE

XYLENE (MIXED ISOMERS)

ZINC POTASSIUM CHROMATE

ZINC POTASSIUM CHROMATE (PM10)
Actual Tons/Year

2.55E-03

$43 E-01$

$6.51 E-02$

$7.41 E+00$

$3.55 E+00$

$1.59 E+00$

3.73E-01

$5.68 \mathrm{E}+00$

$7.50 E-06$

1.97E-04

$5.30 E+00$

$2.09 \mathrm{E}-03$

$9.66 \mathrm{E}-04$ 
Table 65

National Pollutant Discharge Elimination System Monitoring Data

Page 1 of 22

\begin{tabular}{lllllll}
\hline Measurement & Units & $\begin{array}{c}\text { Freq/ } \\
\text { Year }\end{array}$ & Maximum & Minimum & $\begin{array}{c}\text { No. in } \\
\text { Average }\end{array}$ & Average \\
\hline
\end{tabular}

Permit SC0000175

Note: Permit levels can be found in WSRC NPDES Environmental Monitoring Program (ESH-EMS-910110) or in the permit itself. The permit is available from the South Carolina Department of Health and Environmental Control.

\section{Outfall A-001}

Effluent consists of the following types of wastewater: cooling water, steam condensate, and laboratory drain water.

$\begin{array}{lllllll}\text { BOD } & \mathrm{mg} / \mathrm{L} & 15 & 1.81 \mathrm{E}+02 & <1.00 \mathrm{E}+00 & 9 & 2.22 \mathrm{E}+01 \\ \text { FLO } & \mathrm{MGD} & 15 & 2.88 \mathrm{E}-01 & 8.64 \mathrm{E}-02 & 15 & 2.11 \mathrm{E}-01 \\ \text { O\&G } & \mathrm{mg} / \mathrm{L} & 14 & 3.10 \mathrm{E}+00 & <1.00 \mathrm{E}+00 & 2 & 2.05 \mathrm{E}+00 \\ \mathrm{PH} & \mathrm{pH} & 15 & 7.40 & 6.20 & & \\ \text { TMP } & \mathrm{Deg} \mathrm{C} & 15 & 25.00 & 17.00 & 15 & 22.47 \\ \text { TSS } & \mathrm{mg} / \mathrm{L} & 14 & 8.00 \mathrm{E}+00 & <1.00 \mathrm{E}+00 & 6 & 3.48 \mathrm{E}+00\end{array}$

Outfall A-003

Effluent consists of the following types of wastewater: cooling water and steam condensate.

$\begin{array}{lllllll}\text { CR } & \mathrm{mg} / \mathrm{L} & 28 & 2.10 \mathrm{E}-02 & <5.00 \mathrm{E}-03 & 5 & 1.19 \mathrm{E}-02 \\ \mathrm{FLO} & \mathrm{MGD} & 65 & 2.59 \mathrm{E}-01 & 5.76 \mathrm{E}-02 & 65 & 8.30 \mathrm{E}-02 \\ \mathrm{O} \& \mathrm{G} & \mathrm{mg} / \mathrm{L} & 17 & 4.40 \mathrm{E}+00 & <1.00 \mathrm{E}+00 & 2 & 2.80 \mathrm{E}+00 \\ \mathrm{PH} & \mathrm{pH} & 17 & 7.70 & 6.30 & & \\ \mathrm{TMP} & \mathrm{Deg} \mathrm{C} & 17 & 27.00 & 10.00 & 17 & 20.65 \\ \text { TSS } & \mathrm{mg} / \mathrm{L} & 17 & 2.00 \mathrm{E}+00 & <1.00 \mathrm{E}+00 & 4 & 1.60 \mathrm{E}+00\end{array}$

Outfall A-005

Effluent consists of the following types of wastewater: wastewater from animal holding area, steam condensate, steam cleaning wastewater, and cooling water.

$\begin{array}{lllllll}\text { BOD } & \mathrm{mg} / \mathrm{L} & 14 & 5.60 \mathrm{E}+00 & <1.00 \mathrm{E}+00 & 8 & 2.97 \mathrm{E}+00 \\ \text { FEC } & \# / 100 \mathrm{~mL} & 17 & 16000 . & <2 . & 44475 . & \\ \text { FLO } & \mathrm{MGD} & 14 & 4.32 \mathrm{E}-01 & 1.44 \mathrm{E}-01 & 14 & 2.67 \mathrm{E}-01 \\ \text { O\&G } & \mathrm{mg} / \mathrm{L} & 14 & 3.20 \mathrm{E}+00 & <1.00 \mathrm{E}+00 & 1 & 3.20 \mathrm{E}+00 \\ \text { PERCL } & \mathrm{ug} / \mathrm{L} & 14 & <2.00 \mathrm{E}+00 & <2.00 \mathrm{E}+00 & & \\ \text { PH } & \mathrm{pH} & 14 & 7.40 & 6.50 & & \\ \text { TMP } & \mathrm{Deg} C & 14 & 25.00 & 12.00 & 14 & 19.86 \\ \text { TRICL } & \mathrm{ug} / \mathrm{L} & 14 & 3.40 \mathrm{E}+00 & <2.00 \mathrm{E}+00 & 2 & 3.30 \mathrm{E}+00 \\ \text { TSS } & \mathrm{mg} / \mathrm{L} & 14 & 2.60 \mathrm{E}+01 & <1.00 \mathrm{E}+00 & 10 & 5.07 \mathrm{E}+00\end{array}$




\section{Table 65}

National Pollutant Discharge Elimination System Monitoring Data

Page 2 of 22

\begin{tabular}{lllllll}
\hline Measurement & Units & $\begin{array}{l}\text { Freql } \\
\text { Year }\end{array}$ & Maximum & Minimum & $\begin{array}{c}\text { No. in } \\
\text { Average }\end{array}$ & Average \\
\hline
\end{tabular}

Permit SC0000175

Outfall A-011

Effluent consists of the following types of wastewater: cooling water.

$\begin{array}{lllllll}\text { BOD } & \mathrm{mg} / \mathrm{L} & 15 & 4.20 \mathrm{E}+00 & <1.00 \mathrm{E}+00 & 3 & 2.50 \mathrm{E}+00 \\ \mathrm{FLO} & \mathrm{MGD} & 67 & 1.44 \mathrm{E}-01 & 1.44 \mathrm{E}-03 & 67 & 1.75 \mathrm{E}-02 \\ \text { O\&G } & \mathrm{mg} / \mathrm{L} & 15 & <1.00 \mathrm{E}+00 & <1.00 \mathrm{E}+00 & & \\ \mathrm{PH} & \mathrm{pH} & 16 & 7.50 & 6.00 & & \\ \text { TMP } & \mathrm{Deg} \mathrm{C} & 16 & 31.00 & 10.00 & 16 & 20.88 \\ \text { TSS } & \mathrm{mg} / \mathrm{L} & 16 & 1.78 \mathrm{E}+01 & <1.00 \mathrm{E}+00 & 7 & 6.71 \mathrm{E}+00\end{array}$

Outfall A-014

Effluent consists of the following types of wastewater: air stripper wastewater and noncontact cooling water.

$\begin{array}{lllllll}\text { BOD } & \mathrm{mg} / \mathrm{L} & 14 & 2.38 \mathrm{E}+01 & <1.00 \mathrm{E}+00 & 12 & 4.39 \mathrm{E}+00 \\ \text { FLO } & \mathrm{MGD} & 17 & 1.12 \mathrm{E}+01 & 3.53 \mathrm{E}-01 & 17 & 2.53 \mathrm{E}+00 \\ \text { O\&G } & \mathrm{mg} / \mathrm{L} & 14 & 3.40 \mathrm{E}+00 & <1.00 \mathrm{E}+00 & 2 & 2.90 \mathrm{E}+00 \\ \text { PERCL } & \mathrm{ug} / \mathrm{L} & 15 & 4.80 \mathrm{E}+00 & <2.00 \mathrm{E}+00 & 2 & 4.50 \mathrm{E}+00 \\ \mathrm{PH} & \mathrm{pH} & 24 & 7.20 & 6.20 & \\ \text { TCE } & \mathrm{ug} / \mathrm{L} & 14 & <5.00 \mathrm{E}+00 & <2.00 \mathrm{E}+00 & & \\ \text { TMP } & \mathrm{Deg} \mathrm{C} & 23 & 27.00 & 10.00 & 23 & 20 . \\ \text { TRICL } & \mathrm{ug} / \mathrm{L} & 15 & <5.00 \mathrm{E}+00 & <2.00 \mathrm{E}+00 & & \\ \text { TSS } & \mathrm{mg} / \mathrm{L} & 14 & 5.69 \mathrm{E}+01 & <1.00 \mathrm{E}+00 & 13 & 1.91 \mathrm{E}+01\end{array}$

Outfall A-015

Effluent consists of the following types of wastewater: sanitary wastewater.

$\begin{array}{lllllll}\text { BOD } & \mathrm{mg} / \mathrm{L} & 5 & 5.30 \mathrm{E}+00 & 2.00 \mathrm{E}+00 & 5 & 3.36 \mathrm{E}+00 \\ \mathrm{FEC} & \# / 100 \mathrm{~mL} & 4 & 26 . & <2 . & 1 & 26 . \\ \mathrm{FLO} & \mathrm{MGD} & 151 & 2.15 \mathrm{E}-01 & 0.00 \mathrm{E}+00 & 151 & 9.16 \mathrm{E}-02 \\ \mathrm{PH} & \mathrm{pH} & 5 & 7.20 & 7.01 & & \\ \text { TSS } & \mathrm{mg} / \mathrm{L} & 5 & 7.60 \mathrm{E}+00 & <1.00 \mathrm{E}+00 & 3 & 4.57 \mathrm{E}+00\end{array}$

Outfall C-001

Effluent consists of the following types of wastewater: cooling water.

$\begin{array}{llllllr}\text { FLO } & \text { MGD } & 365 & 1.55 \mathrm{E}-01 & 1.29 \mathrm{E}-02 & 365 & 2.68 \mathrm{E}-02 \\ \mathrm{O} \& \mathrm{G} & \mathrm{mg} / \mathrm{L} & 15 & 1.10 \mathrm{E}+00 & <1.00 \mathrm{E}+00 & 1 & 1.10 \mathrm{E}+00 \\ \mathrm{PH} & \mathrm{pH} & 15 & 7.20 & 5.60 & & \end{array}$


Table 65

National Pollutant Discharge Elimination System Monitoring Data

Page 3 of 22

\begin{tabular}{lllllll}
\hline Measurement & Units & $\begin{array}{c}\text { Freq/ } \\
\text { Year }\end{array}$ & Maximum & Minimum & $\begin{array}{c}\text { No. in } \\
\text { Average }\end{array}$ & \multicolumn{1}{c}{ Average } \\
\hline & & & & & & \\
Permit SC0000175 & & & & & & \\
\hline TMP & Deg C & 15 & 27.00 & 10.50 & 15 & 19.03 \\
TSS & $\mathrm{mg} / \mathrm{L}$ & 16 & $2.23 \mathrm{E}+01$ & $<1.00 \mathrm{E}+00$ & 12 & $4.80 \mathrm{E}+00$
\end{tabular}

Outfall C-003

Effluent consists of the following types of wastewater: cooling water.

$\begin{array}{lllllll}\text { FLO } & \text { MGD } & 365 & 1.29 \mathrm{E}+00 & 6.46 \mathrm{E}-02 & 365 & 2.81 \mathrm{E}-01 \\ \text { O\&G } & \mathrm{mg} / \mathrm{L} & 15 & <1.00 \mathrm{E}+00 & <1.00 \mathrm{E}+00 & & \\ \mathrm{PH} & \mathrm{pH} & 15 & 7.20 & 6.30 & & \\ \text { TMP } & \text { Deg C } & 15 & 24.00 & 12.00 & 15 & 20.83 \\ \text { TSS } & \mathrm{mg} / \mathrm{L} & 15 & 1.20 \mathrm{E}+01 & <1.00 \mathrm{E}+00 & 8 & 4.78 \mathrm{E}+00\end{array}$

Outfall C-004

Effluent consists of the following types of wastewater: neutralization water, senvice wastewater, and C-Reactor cooling water.

$\begin{array}{lllllll}\text { FLO } & \text { MGD } & 365 & 4.91 \mathrm{E}+01 & 2.91 \mathrm{E}-01 & 365 & 4.05 \mathrm{E}+00 \\ \text { O\&G } & \mathrm{mg} / \mathrm{L} & 13 & <1.00 \mathrm{E}+00 & <1.00 \mathrm{E}+00 & & \\ \mathrm{PH} & \mathrm{pH} & 13 & 7.00 & 6.10 & & \\ \text { TMP } & \text { Deg C } & 365 & 27.80 & 5.90 & 365 & 18.79 \\ \text { TSS } & \mathrm{mg} / \mathrm{L} & 17 & 1.05 \mathrm{E}+02 & <1.00 \mathrm{E}+00 & 14 & 1.98 \mathrm{E}+01\end{array}$

Outfall C-004A

Effluent consists of the following types of wastewater: sanitary wastewater.

$\begin{array}{lllllll}\text { BOD } & \mathrm{mg} / \mathrm{L} & 5 & 3.70 \mathrm{E}+00 & <1.00 \mathrm{E}+00 & 3 & 2.77 \mathrm{E}+00 \\ \text { FEC } & \# / 100 \mathrm{~mL} & 4 & 6 . & <2 . & 1 & 6 . \\ \text { FLO } & \text { MGD } & 151 & 3.04 \mathrm{E}-02 & 0.00 \mathrm{E}+00 & 151 & 1.17 \mathrm{E}-02 \\ \mathrm{PH} & \mathrm{pH} & 5 & 8.01 & 7.01 & & \\ \text { TSS } & \mathrm{mg} / \mathrm{L} & 5 & 3.50 \mathrm{E}+00 & 1.20 \mathrm{E}+00 & 5 & 2.28 \mathrm{E}+00\end{array}$

Outfall D-001

Effluent consists of the following types of wastewater: cooling water, neutralization water, and heavy water.

$\begin{array}{lllllll}\text { FLO } & \text { MGD } & 272 & 6.27 E+01 & 4.07 \mathrm{E}+01 & 272 & 4.95 \mathrm{E}+01 \\ \text { O\&G } & \mathrm{mg} / \mathrm{L} & 11 & 1.10 \mathrm{E}+00 & <1.00 \mathrm{E}+00 & 1 & 1.10 \mathrm{E}+00 \\ \mathrm{PH} & \mathrm{pH} & 18 & 6.90 & 5.30 & & \\ \text { TMP } & \text { Deg C } & 43 & 27.40 & 0.00 & 43 & 20.95 \\ \text { TSS } & \mathrm{mg} / \mathrm{L} & 11 & 2.20 \mathrm{E}+01 & 7.40 \mathrm{E}+00 & 11 & 1.36 \mathrm{E}+01\end{array}$




\section{Table 65}

National Pollutant Discharge Elimination System Monitoring Data

Page 4 of 22

\begin{tabular}{lllllll}
\hline Measurement & Units & $\begin{array}{l}\text { Freq } \\
\text { Year }\end{array}$ & Maximum & Minimum & $\begin{array}{c}\text { No. in } \\
\text { Average }\end{array}$ & Average \\
\hline
\end{tabular}

Permit SC0000175

Outfall D-001A

Effluent consists of the following types of wastewater: sanitary wastewater.

$\begin{array}{lllllll}\text { BOD } & \mathrm{mg} / \mathrm{L} & 13 & 1.40 \mathrm{E}+01 & <1.00 \mathrm{E}+00 & 9 & 4.33 \mathrm{E}+00 \\ \text { FEC } & \# / 100 \mathrm{~mL} & 16 & 68 . & <2 . & 6 & 16 . \\ \text { FLO } & \text { MGD } & 365 & 6.54 \mathrm{E}-02 & 8.52 \mathrm{E}-04 & 365 & 1.19 \mathrm{E}-02 \\ \text { PH } & \mathrm{pH} & 13 & 8.01 & 7.02 & & \\ \text { TSS } & \mathrm{mg} / \mathrm{L} & 13 & 2.00 \mathrm{E}+01 & 1.50 \mathrm{E}+00 & 13 & 6.29 \mathrm{E}+00\end{array}$

Outfall D-001B

Effluent consists of the following types of wastewater: coal pile runoff.

$\begin{array}{lclllll}\text { AL } & \mathrm{mg} / \mathrm{L} & 20 & 8.27 \mathrm{E}+01 & 5.48 \mathrm{E}+01 & 20 & 6.83 \mathrm{E}+01 \\ \mathrm{FE} & \mathrm{mg} / \mathrm{L} & 20 & 4.72 \mathrm{E}+02 & 2.94 \mathrm{E}+02 & 20 & 4.10 \mathrm{E}+02 \\ \mathrm{FLO} & \mathrm{MGD} & 191 & 1.73 \mathrm{E}-01 & 0.00 \mathrm{E}+00 & 191 & 6.42 \mathrm{E}-02 \\ \mathrm{MG} & \mathrm{mg} / \mathrm{L} & 19 & 3.74 \mathrm{E}+01 & 2.61 \mathrm{E}+01 & 19 & 3.15 \mathrm{E}+01 \\ \mathrm{MN} & \mathrm{mg} / \mathrm{L} & 20 & 4.23 \mathrm{E}+00 & 2.98 \mathrm{E}+00 & 20 & 3.56 \mathrm{E}+00 \\ \mathrm{PH} & \mathrm{pH} & 8 & 6.90 & 6.30 & & \\ \mathrm{SO} 4 & \mathrm{mg} / \mathrm{L} & 21 & 5.09 \mathrm{E}+03 & 1.32 \mathrm{E}+03 & 21 & 1.90 \mathrm{E}+03 \\ \mathrm{TSS} & \mathrm{mg} / \mathrm{L} & 18 & 1.76 \mathrm{E}+01 & <1.00 \mathrm{E}+00 & 17 & 5.04 \mathrm{E}+00 \\ \mathrm{ZN} & \mathrm{mg} / \mathrm{L} & 20 & 9.76 \mathrm{E}+00 & 1.40 \mathrm{E}+00 & 20 & 2.10 \mathrm{E}+00\end{array}$

Outfall D-001C

Effluent consists of the following types of wastewater: ash basin discharge.

$\begin{array}{lllllll}\text { FLO } & \text { MGD } & 10 & 6.24 E+00 & 3.99 E+00 & 10 & 4.78 E+00 \\ \text { O\&G } & \mathrm{mg} / \mathrm{L} & 10 & <1.00 \mathrm{E}+00 & <1.00 \mathrm{E}+00 & & \\ \mathrm{PH} & \mathrm{pH} & 9 & 6.80 & 6.20 & & \\ \text { TSS } & \mathrm{mg} / \mathrm{L} & 10 & 2.10 \mathrm{E}+01 & 2.80 \mathrm{E}+00 & 10 & 7.71 \mathrm{E}+00\end{array}$

Outfall D-003

Effluent consists of the following types of wastewater: powerhouse washdown water and water treatment plant wastewater.

FLO

$O \& G$

$\mathrm{PH}$

TSS

$\begin{array}{clllll}\text { MGD } & 273 & 8.40 E-01 & 1.23 E-01 & 273 & 2.16 E-01 \\ \mathrm{mg} / \mathrm{L} & 11 & 1.20 \mathrm{E}+00 & <1.00 \mathrm{E}+00 & 1 & 1.20 \mathrm{E}+00 \\ \mathrm{pH} & 11 & 7.10 & 6.10 & & \\ \mathrm{mg} / \mathrm{L} & 15 & 1.04 \mathrm{E}+02 & <1.00 \mathrm{E}+00 & 14 & 1.08 \mathrm{E}+01\end{array}$


Table 65

National Pollutant Discharge Elimination System Monitoring Data

Page 5 of 22

\begin{tabular}{|c|c|c|c|c|c|c|}
\hline Measurement & Units & $\begin{array}{l}\text { Freq/ } \\
\text { Year }\end{array}$ & Maximum & Minimum & $\begin{array}{c}\text { No. in } \\
\text { Average }\end{array}$ & Average \\
\hline
\end{tabular}

Permit SC0000175

Outfall D-005 (facility in service but did not discharge)

Effluent consists of the following types of wastewater: water filter backwash overflow.
FLO
MGD
8
$0.00 E+00$
8
$0.00 \mathrm{E}+00$

Outfall D-006

Effluent consists of the following types of wastewater: cooling water, powerhouse washdown water, and heavy water process water.

$\begin{array}{lllllll}\text { FEC } & \# / 100 \mathrm{~mL} & 11 & 3000 . & 17 . & 11 & 344 . \\ \text { FLO } & \text { MGD } & 273 & 7.76 \mathrm{E}+00 & 7.76 \mathrm{E}-01 & 273 & 1.88 \mathrm{E}+00 \\ \text { O\&G } & \mathrm{mg} / \mathrm{L} & 11 & 2.00 \mathrm{E}+00 & <1.00 \mathrm{E}+00 & 1 & 2.00 \mathrm{E}+00 \\ \mathrm{PH} & \mathrm{pH} & 11 & 7.00 & 6.00 & & 20.91 \\ \text { TMP } & \mathrm{Deg} \mathrm{C} & 263 & 27.30 & 9.20 & 263 & 200 \\ \text { TSS } & \mathrm{mg} / \mathrm{L} & 15 & 1.55 \mathrm{E}+02 & 2.90 \mathrm{E}+00 & 15 & 1.69 \mathrm{E}+01\end{array}$

Outfall DW-001 (facility not in service)

Effluent consists of the following types of wastewater. washdown water from concrete transit mixer trucks.
FLO
MGD $\quad 60$
$0.00 \mathrm{E}+00$
60
$0.00 \mathrm{E}+00$

Outfall DW-002

Effluent consists of the following types of wastewater: steam cleaning wastewater, stormwater runoff, and equipment wash water.

$\begin{array}{lllllll}\text { FLO } & \text { MGD } & 31 & 7.20 E-03 & 0.00 E+00 & 31 & 1.70 E-03 \\ \text { O\&G } & \mathrm{mg} / \mathrm{L} & 18 & <1.00 E+00 & <1.00 \mathrm{E}+00 & & \\ \mathrm{PH} & \mathrm{pH} & 13 & 7.60 & 6.40 & \end{array}$

\section{Outfall DW-003}

Effluent consists of the following types of wastewater: sanitary wastewater from DWPF (S-Area).

$\begin{array}{llllllr}\text { BOD } & \mathrm{mg} / \mathrm{L} & 5 & 1.61 \mathrm{E}+01 & <1.00 \mathrm{E}+00 & 2 & 9.00 \mathrm{E}+00 \\ \text { FEC } & \# / 100 \mathrm{~mL} & 4 & <2 . & <2 . & 1.29 \mathrm{E}-02 \\ \text { FLO } & \mathrm{MGD} & 151 & 2.88 \mathrm{E}-02 & 0.00 \mathrm{E}+00 & 151 & \\ \mathrm{PH} & \mathrm{pH} & 5 & 8.60 & 7.02 & & 1.72 \mathrm{E}+00 \\ \text { TSS } & \mathrm{mg} / \mathrm{L} & 5 & 2.40 \mathrm{E}+00 & 1.00 \mathrm{E}+00 & 5 & \end{array}$


Table 65

National Pollutant Discharge Elimination System Monitoring Data

Page 6 of 22

\begin{tabular}{|c|c|c|c|c|c|c|}
\hline Measurement & Units & $\begin{array}{l}\text { Freql } \\
\text { Year }\end{array}$ & Maximum & Minimum & $\begin{array}{c}\text { No. in } \\
\text { Average }\end{array}$ & Average \\
\hline
\end{tabular}

\section{Permit SC0000175}

Outfall DW-004

Effluent consists of the following types of wastewater: neutralization, cooling water, and steam condensate.

$\begin{array}{lllllll}\text { BOD } & \mathrm{mg} / \mathrm{L} & 29 & 6.65 \mathrm{E}+01 & <1.00 \mathrm{E}+00 & 23 & 8.88 \mathrm{E}+00 \\ \mathrm{CHL} & \mathrm{mg} / \mathrm{L} & 25 & 2.00 \mathrm{E}+00 & <1.00 \mathrm{E}-01 & 17 & 4.49 \mathrm{E}-01 \\ \text { FLO } & \mathrm{MGD} & 53 & 1.73 \mathrm{E}-01 & 0.00 \mathrm{E}+00 & 53 & 6.23 \mathrm{E}-02 \\ \mathrm{O \& G} & \mathrm{mg} / \mathrm{L} & 31 & 5.70 \mathrm{E}+00 & <1.00 \mathrm{E}+00 & 5 & 3.88 \mathrm{E}+00 \\ \mathrm{PH} & \mathrm{pH} & 29 & 7.90 & 6.70 & & \\ \text { TMP } & \mathrm{Deg} \mathrm{C} & 29 & 28.00 & 15.00 & 29 & 21.31 \\ \text { TSS } & \mathrm{mg} / \mathrm{L} & 31 & 1.20 \mathrm{E}+01 & <1.00 \mathrm{E}+00 & 26 & 4.80 \mathrm{E}+00\end{array}$

Outfall F-001

Effluent consists of the following types of wastewater: nonprocess cooling water.

$\begin{array}{lllllll}\text { FLO } & \text { MGD } & 14 & 1.44 \mathrm{E}-01 & 2.88 \mathrm{E}-02 & 14 & 6.02 \mathrm{E}-02 \\ \text { O\&G } & \mathrm{mg} / \mathrm{L} & 14 & 1.00 \mathrm{E}+00 & <1.00 \mathrm{E}+00 & 1 & 1.00 \mathrm{E}+00 \\ \mathrm{PH} & \mathrm{pH} & 14 & 8.00 & 6.80 & & \\ \text { TMP } & \text { Deg C } & 14 & 29.00 & 15.00 & 14 & 23.29 \\ \text { TSS } & \mathrm{mg} / \mathrm{L} & 14 & 8.00 \mathrm{E}+00 & <1.00 \mathrm{E}+00 & 5 & 3.16 \mathrm{E}+00\end{array}$

Outfall F-002

Effluent consists of the following types of wastewater: nonprocess cooling water.

$\begin{array}{lllllll}\text { FLO } & \text { MGD } & 14 & 1.01 \mathrm{E}-01 & 1.44 \mathrm{E}-02 & 14 & 4.73 \mathrm{E}-02 \\ \text { O\&G } & \mathrm{mg} / \mathrm{L} & 14 & 1.30 \mathrm{E}+00 & <1.00 \mathrm{E}+00 & 1 & 1.30 \mathrm{E}+00 \\ \mathrm{PH} & \mathrm{pH} & 14 & 7.40 & 6.50 & & \\ \text { TMP } & \text { Deg C } & 14 & 28.00 & 10.00 & 14 & 19.21 \\ \text { TSS } & \mathrm{mg} / \mathrm{L} & 14 & 1.00 \mathrm{E}+01 & <1.00 \mathrm{E}+00 & 5 & 2.90 \mathrm{E}+00\end{array}$

Outfall F-003

Effluent consists of the following types of wastewater: cooling water and steam condensate.

$\begin{array}{lllllll}\text { BOD } & \mathrm{mg} / \mathrm{L} & 14 & 2.80 \mathrm{E}+00 & <1.00 \mathrm{E}+00 & 8 & 2.00 \mathrm{E}+00 \\ \text { FLO } & \mathrm{MGD} & 14 & 1.15 \mathrm{E}-01 & 4.32 \mathrm{E}-03 & 14 & 3.39 \mathrm{E}-02 \\ \text { O\&G } & \mathrm{mg} / \mathrm{L} & 14 & 3.50 \mathrm{E}+00 & <1.00 \mathrm{E}+00 & 2 & 2.45 \mathrm{E}+00 \\ \mathrm{PH} & \mathrm{pH} & 14 & 7.60 & 5.80 & & \\ \text { TMP } & \mathrm{Deg} \mathrm{C} & 14 & 29.00 & 10.00 & 14 & 20.25 \\ \text { TSS } & \mathrm{mg} / \mathrm{L} & 16 & 2.46 \mathrm{E}+01 & <1.00 \mathrm{E}+00 & 13 & 6.91 \mathrm{E}+00\end{array}$




\section{Table 65}

\section{National Pollutant Discharge Elimination System Monitoring Data}

Page 7 of 22

\begin{tabular}{llllll}
\hline Measurement & Units & $\begin{array}{c}\text { Freq/ } \\
\text { Year }\end{array}$ & Maximum & Minimum & No. in \\
Average & Average \\
\hline
\end{tabular}

Permit SC0000175

Outfall F-003A

Effluent consists of the following types of wastewater: sanitary wastewater.

$\begin{array}{lllllll}\text { BOD } & \mathrm{mg} / \mathrm{L} & 5 & 3.20 \mathrm{E}+00 & 1.40 \mathrm{E}+00 & 5 & 2.26 \mathrm{E}+00 \\ \text { FEC } & \# / 100 \mathrm{~mL} & 4 & <2 . & <2 . & & \\ \text { FLO } & \mathrm{MGD} & 151 & 9.58 \mathrm{E}-02 & 0.00 \mathrm{E}+00 & 151 & 3.03 \mathrm{E}-02 \\ \mathrm{PH} & \mathrm{pH} & 5 & 8.70 & 7.00 & & \\ \text { TSS } & \mathrm{mg} / \mathrm{L} & 5 & 9.80 \mathrm{E}+00 & 3.20 \mathrm{E}+00 & 5 & 5.26 \mathrm{E}+00\end{array}$

Outfall F-005

Effluent consists of the following types of wastewater: cooling water and steam condensate.

$\begin{array}{lllllll}\text { FLO } & \text { MGD } & 14 & 2.16 \mathrm{E}-01 & 1.44 \mathrm{E}-02 & 14 & 6.02 \mathrm{E}-02 \\ \text { O\&G } & \mathrm{mg} / \mathrm{L} & 14 & 4.80 \mathrm{E}+00 & <1.00 \mathrm{E}+00 & 2 & 3.35 \mathrm{E}+00 \\ \mathrm{PH} & \mathrm{pH} & 14 & 7.60 & 4.90 & & \\ \text { TMP } & \text { Deg C } & 14 & 28.00 & 16.00 & 14 & 23.07 \\ \text { TSS } & \mathrm{mg} / \mathrm{L} & 16 & 2.24 \mathrm{E}+01 & <1.00 \mathrm{E}+00 & 9 & 4.88 \mathrm{E}+00\end{array}$

Outfall F -007 (facility in service but did not discharge)

Effluent consists of the following types of wastewater: ash basin discharge.
FLO
MGD
13
$0.00 E+00$
13
$0.00 E+00$

Outfall F -008

Effluent consists of the following types of wastewater: neutralization, cooling water, steam condensate, process wastewater, and Outfall F-012 and Outfall F-013.

$\begin{array}{lllllll}\text { AL } & \mathrm{mg} / \mathrm{L} & 7 & 2.89 \mathrm{E}-01 & <5.00 \mathrm{E}-02 & 5 & 2.48 \mathrm{E}-01 \\ \mathrm{AN} & \mathrm{mg} / \mathrm{L} & 7 & 5.76 \mathrm{E}-01 & <5.00 \mathrm{E}-02 & 2 & 4.68 \mathrm{E}-01 \\ \mathrm{CR} & \mathrm{mg} / \mathrm{L} & 7 & <1.00 \mathrm{E}-02 & <5.00 \mathrm{E}-03 & & \\ \mathrm{CU} & \mathrm{mg} / \mathrm{L} & 7 & 1.24 \mathrm{E}-02 & <5.00 \mathrm{E}-03 & 3 & 8.80 \mathrm{E}-03 \\ \mathrm{FLO} & \mathrm{MGD} & 365 & 9.69 \mathrm{E}+00 & 7.76 \mathrm{E}-01 & 365 & 1.70 \mathrm{E}+00 \\ \mathrm{HG} & \mathrm{mg} / \mathrm{L} & 7 & <1.00 \mathrm{E}-04 & <1.00 \mathrm{E}-04 & & \\ \mathrm{MN} & \mathrm{mg} / \mathrm{L} & 7 & 5.50 \mathrm{E}-02 & 9.00 \mathrm{E}-03 & 5 & 1.91 \mathrm{E}-02 \\ \mathrm{NI} & \mathrm{mg} / \mathrm{L} & 7 & 5.40 \mathrm{E}-03 & <5.00 \mathrm{E}-03 & 1 & 5.40 \mathrm{E}-03 \\ \mathrm{NO3} & \mathrm{mg} / \mathrm{L} & 7 & 1.71 \mathrm{E}+00 & <2.00 \mathrm{E}-02 & 4 & 7.28 \mathrm{E}-01 \\ \text { O\&G } & \mathrm{mg} / \mathrm{L} & 14 & 2.40 \mathrm{E}+00 & <1.00 \mathrm{E}+00 & 1 & 2.40 \mathrm{E}+00 \\ \mathrm{~PB} & \mathrm{mg} / \mathrm{L} & 7 & <2.00 \mathrm{E}-02 & <3.00 \mathrm{E}-03 & & \\ \mathrm{PH} & \mathrm{pH} & 14 & 7.40 & 4.70 & & \end{array}$


Table 65

National Pollutant Discharge Elimination System Monitoring Data

Page 8 of 22

\begin{tabular}{lllllll}
\hline Measurement & Units & $\begin{array}{c}\text { Freq/ } \\
\text { Year }\end{array}$ & Maximum & Minimum & $\begin{array}{c}\text { No. in } \\
\text { Average }\end{array}$ & Average \\
\hline & & & & & & \\
Permit SC0000175 & & & & & & \\
\hline TMP & Deg C & 14 & 27.00 & 13.00 & 14 & 22.00 \\
TSS & $\mathrm{mg} / \mathrm{L}$ & 14 & $1.00 \mathrm{E}+01$ & $<1.00 \mathrm{E}+00$ & 12 & $3.35 \mathrm{E}+00$ \\
U308 & $\mathrm{mg} / \mathrm{L}$ & 7 & $<5.00 \mathrm{E}-01$ & $<2.00 \mathrm{E}-02$ & & \\
ZN & $\mathrm{mg} / \mathrm{L}$ & 7 & $6.40 \mathrm{E}-02$ & $1.20 \mathrm{E}-02$ & 7 & $3.05 \mathrm{E}-02$
\end{tabular}

Outfall F-008A

Effluent consists of the following types of wastewater: sanitary wastewater.

$\begin{array}{lllllll}\text { BOD } & \mathrm{mg} / \mathrm{L} & 5 & 2.44 \mathrm{E}+01 & 1.30 \mathrm{E}+00 & 5 & 6.40 \mathrm{E}+00 \\ \text { FEC } & \# / 100 \mathrm{~mL} & 4 & <2 . & <2 . & & \\ \text { FLO } & \mathrm{MGD} & 151 & 9.35 \mathrm{E}-02 & 0.00 \mathrm{E}+00 & 151 & 3.72 \mathrm{E}-02 \\ \mathrm{PH} & \mathrm{pH} & 5 & 7.90 & 7.01 & & \\ \text { TSS } & \mathrm{mg} / \mathrm{L} & 5 & 9.50 \mathrm{E}+00 & <1.00 \mathrm{E}+00 & 4 & 4.95 \mathrm{E}+00\end{array}$

Outfall F-012

Effluent consists of the following types of wastewater: stormwater runoff retention basin effluent.

$\begin{array}{lllllll}\text { AL } & \mathrm{mg} / \mathrm{L} & 19 & 7.35 \mathrm{E}-01 & <5.00 \mathrm{E}-02 & 15 & 2.49 \mathrm{E}-01 \\ \mathrm{AN} & \mathrm{mg} / \mathrm{L} & 19 & 1.46 \mathrm{E}+00 & <5.00 \mathrm{E}-02 & 10 & 3.01 \mathrm{E}-01 \\ \mathrm{CR} & \mathrm{mg} / \mathrm{L} & 19 & <1.00 \mathrm{E}-02 & <5.00 \mathrm{E}-03 & & \\ \mathrm{CU} & \mathrm{mg} / \mathrm{L} & 19 & 2.66 \mathrm{E}-02 & <5.00 \mathrm{E}-03 & 11 & 1.26 \mathrm{E}-02 \\ \mathrm{FLO} & \mathrm{MGD} & 19 & 1.62 \mathrm{E}+00 & 4.23 \mathrm{E}-01 & 19 & 9.67 \mathrm{E}-01 \\ \mathrm{HG} & \mathrm{mg} / \mathrm{L} & 19 & 4.00 \mathrm{E}-04 & <1.00 \mathrm{E}-04 & 2 & 2.00 \mathrm{E}-04 \\ \mathrm{MN} & \mathrm{mg} / \mathrm{L} & 19 & 7.40 \mathrm{E}-02 & 8.00 \mathrm{E}-03 & 15 & 2.54 \mathrm{E}-02 \\ \mathrm{NI} & \mathrm{mg} / \mathrm{L} & 19 & 5.90 \mathrm{E}-03 & <5.00 \mathrm{E}-03 & 1 & 5.90 \mathrm{E}-03 \\ \mathrm{NO3} & \mathrm{mg} / \mathrm{L} & 18 & 3.88 \mathrm{E}+00 & <2.00 \mathrm{E}-02 & 10 & 1.02 \mathrm{E}+00 \\ \mathrm{~PB} & \mathrm{mg} / \mathrm{L} & 19 & 3.60 \mathrm{E}-03 & <3.00 \mathrm{E}-03 & 2 & 3.50 \mathrm{E}-03 \\ \mathrm{PH} & \mathrm{pH} & 19 & 9.10 & 6.20 & & \\ \mathrm{TSS} & \mathrm{mg} / \mathrm{L} & 19 & 2.20 \mathrm{E}+01 & 1.00 \mathrm{E}+00 & 19 & 9.68 \mathrm{E}+00 \\ \mathrm{U} 308 & \mathrm{mg} / \mathrm{L} & 19 & 5.00 \mathrm{E}-01 & <2.00 \mathrm{E}-02 & 1 & 5.00 \mathrm{E}-01 \\ \mathrm{ZN} & \mathrm{mg} / \mathrm{L} & 19 & 1.74 \mathrm{E}-01 & 1.60 \mathrm{E}-02 & 19 & 9.39 \mathrm{E}-02\end{array}$

Outfall F-013

Effluent consists of the following types of wastewater: cooling water retention basin effluent.

$\begin{array}{lllllll}\mathrm{AL} & \mathrm{mg} / \mathrm{L} & 6 & 9.22 \mathrm{E}-01 & <5.00 \mathrm{E}-02 & 4 & 3.54 \mathrm{E}-01 \\ \mathrm{AN} & \mathrm{mg} / \mathrm{L} & 6 & 1.86 \mathrm{E}-01 & <5.00 \mathrm{E}-02 & 3 & 1.05 \mathrm{E}-01 \\ \mathrm{CR} & \mathrm{mg} / \mathrm{L} & 6 & <1.00 \mathrm{E}-02 & <5.00 \mathrm{E}-03 & & \end{array}$


Table 65

National Pollutant Discharge Elimination System Monitoring Data

Page 9 of 22

\begin{tabular}{lcclllc}
\hline Measurement & Units & $\begin{array}{l}\text { Freql } \\
\text { Year }\end{array}$ & Maximum & Minimum & $\begin{array}{c}\text { No. in } \\
\text { Average }\end{array}$ & Average \\
\hline Permit SC0000175 & & & & & & \\
\hline CU & $\mathrm{mg} / \mathrm{L}$ & 6 & $1.83 \mathrm{E}-02$ & $<5.00 \mathrm{E}-03$ & 3 & $1.36 \mathrm{E}-02$ \\
$\mathrm{FLO}$ & $\mathrm{MGD}$ & 6 & $1.62 \mathrm{E}+00$ & $3.73 \mathrm{E}-01$ & 6 & $1.32 \mathrm{E}+00$ \\
$\mathrm{HG}$ & $\mathrm{mg} / \mathrm{L}$ & 6 & $<1.00 \mathrm{E}-04$ & $<1.00 \mathrm{E}-04$ & & \\
$\mathrm{MN}$ & $\mathrm{mg} / \mathrm{L}$ & 6 & $9.75 \mathrm{E}-02$ & $5.50 \mathrm{E}-03$ & 5 & $3.31 \mathrm{E}-02$ \\
$\mathrm{NI}$ & $\mathrm{mg} / \mathrm{L}$ & 6 & $<1.00 \mathrm{E}-02$ & $<5.00 \mathrm{E}-03$ & & \\
$\mathrm{NO} 3$ & $\mathrm{mg} / \mathrm{L}$ & 6 & $2.70 \mathrm{E}-01$ & $<2.00 \mathrm{E}-02$ & 3 & $1.03 \mathrm{E}-01$ \\
$\mathrm{~PB}$ & $\mathrm{mg} / \mathrm{L}$ & 6 & $6.20 \mathrm{E}-03$ & $<3.00 \mathrm{E}-03$ & 2 & $4.70 \mathrm{E}-03$ \\
$\mathrm{PH}$ & $\mathrm{pH}$ & 6 & 8.10 & 6.50 & & \\
TSS & $\mathrm{mg} / \mathrm{L}$ & 6 & $2.09 \mathrm{E}+01$ & $3.80 \mathrm{E}+00$ & 6 & $8.85 \mathrm{E}+00$ \\
U308 & $\mathrm{mg} / \mathrm{L}$ & 6 & $<5.00 \mathrm{E}-01$ & $<2.00 \mathrm{E}-02$ & & \\
ZN & $\mathrm{mg} / \mathrm{L}$ & 6 & $7.90 \mathrm{E}-01$ & $2.40 \mathrm{E}-02$ & 6 & $1.83 \mathrm{E}-01$
\end{tabular}

Outfall FS-001

Effluent consists of the following types of wastewater: cooling water and greenhouse wastewater.

$\begin{array}{lllllll}\text { FEC } & \# / 100 \mathrm{~mL} & 9 & 230 . & 8 . & 9 & 123 . \\ \text { FLO } & \text { MGD } & 14 & 4.32 E-02 & 0.00 E+00 & 14 & 1.44 \mathrm{E}-02\end{array}$

Outfall FS-002 (facility in service but did not discharge)

Effluent consists of the following types of wastewater: cooling water and greenhouse wastewater.

$\begin{array}{llllll}\text { FLO } & \text { MGD } & 13 & 0.00 E+00 & 13 & 0.00 E+00\end{array}$

Outfall $\mathrm{H}-002$

Effluent consists of the following types of wastewater: nonprocess cooling water and stormwater.

$\begin{array}{llllrlr}\mathrm{C} & \mathrm{mg} / \mathrm{L} & 5 & 2.14 \mathrm{E}+00 & <1.00 \mathrm{E}+00 & 2 & 1.63 \mathrm{E}+00 \\ \mathrm{COD} & \mathrm{mg} / \mathrm{L} & 6 & 1.54 \mathrm{E}+01 & <1.00 \mathrm{E}+01 & 1 & 1.54 \mathrm{E}+01 \\ \mathrm{FLO} & \mathrm{MGD} & 15 & 7.20 \mathrm{E}-02 & 2.88 \mathrm{E}-02 & 15 & 4.17 \mathrm{E}-02 \\ \text { O\&G } & \mathrm{mg} / \mathrm{L} & 15 & 2.00 \mathrm{E}+00 & <1.00 \mathrm{E}+00 & 2 & 1.60 \mathrm{E}+00 \\ \mathrm{PH} & \mathrm{pH} & 15 & 7.00 & 5.80 & & \\ \text { TMP } & \mathrm{Deg} \mathrm{C} & 15 & 26.00 & 14.00 & 15 & 19.20 \\ \text { TSS } & \mathrm{mg} / \mathrm{L} & 15 & 8.60 \mathrm{E}+00 & <1.00 \mathrm{E}+00 & 9 & 4.27 \mathrm{E}+00\end{array}$

Outfall H-004

Effluent consists of the following types of wastewater: nonprocess cooling water.

$\begin{array}{lllllll}\text { FLO } & \text { MGD } & 15 & 2.88 E-02 & 0.00 E+00 & 15 & 9.00 E-03 \\ \text { O\&G } & \mathrm{mg} / \mathrm{L} & 13 & <1.00 \mathrm{E}+00 & <1.00 \mathrm{E}+00 & & \end{array}$


Table 65

National Pollutant Discharge Elimination System Monitoring Data

Page 10 of 22

\begin{tabular}{lllllll}
\hline Measurement & Units & $\begin{array}{l}\text { Freq/ } \\
\text { Year }\end{array}$ & Maximum & Minimum & $\begin{array}{c}\text { No. in } \\
\text { Average }\end{array}$ & Average \\
\hline
\end{tabular}

Permit SC0000175

\begin{tabular}{lllllll}
\hline PH & $\mathrm{pH}$ & 13 & 7.10 & 6.30 & & \\
TMP & Deg C & 13 & 25.00 & 12.00 & 13 & 18.77 \\
TSS & $\mathrm{mg} / \mathrm{L}$ & 13 & $9.00 \mathrm{E}+00$ & $<1.00 \mathrm{E}+00$ & 8 & $3.85 \mathrm{E}+00$
\end{tabular}

Outfall $\mathrm{H}-006$

Effluent consists of the following types of wastewater: stormwater.

$\begin{array}{lllllll}\mathrm{C} & \mathrm{mg} / \mathrm{L} & 1 & <1.00 \mathrm{E}+00 & <1.00 \mathrm{E}+00 & & \\ \text { COD } & \mathrm{mg} / \mathrm{L} & 1 & <1.00 \mathrm{E}+01 & <1.00 \mathrm{E}+01 & & \\ \text { FLO } & \mathrm{MGD} & 2 & 1.44 \mathrm{E}-03 & 0.00 \mathrm{E}+00 & 2 & 7.00 \mathrm{E}-04 \\ \text { O\&G } & \mathrm{mg} / \mathrm{L} & 1 & <1.00 \mathrm{E}+00 & <1.00 \mathrm{E}+00 & & \\ \mathrm{PH} & \mathrm{pH} & 1 & 7.30 & 7.30 & \end{array}$

Outfall $\mathrm{H}-007$

Effluent consists of the following types of wastewater: cooling tower blowdown, air compressor cooling water, and freeze protection purge from a water tank filling line.

$\begin{array}{lllllll}\text { CHL } & \mathrm{mg} / \mathrm{L} & 10 & 1.10 \mathrm{E}-01 & <1.00 \mathrm{E}-01 & 1 & 1.10 \mathrm{E}-01 \\ \mathrm{FLO} & \mathrm{MGD} & 13 & 3.60 \mathrm{E}-01 & 0.00 \mathrm{E}+00 & 13 & 8.14 \mathrm{E}-02 \\ \mathrm{O \& G} & \mathrm{mg} / \mathrm{L} & 11 & 3.90 \mathrm{E}+00 & <1.00 \mathrm{E}+00 & 1 & 3.90 \mathrm{E}+00 \\ \mathrm{PH} & \mathrm{pH} & 13 & 6.70 & 3.80 & & \\ \text { TMP } & \mathrm{Deg} \mathrm{C} & 11 & 26.00 & 14.00 & 11 & 18.18 \\ \text { TSS } & \mathrm{mg} / \mathrm{L} & 11 & 1.80 \mathrm{E}+01 & <1.00 \mathrm{E}+00 & 10 & 7.29 \mathrm{E}+00\end{array}$

Outfall $\mathrm{H}-008$

Effluent consists of the following types of wastewater: cooling water, steam condensate, neutralization wastewater, and powerhouse wastewater.

FLO

$O \& G$

$\mathrm{PH}$

TMP

TSS

MGD 365

$\mathrm{mg} / \mathrm{L} \quad 15$

$\mathrm{pH} \quad 15$

Deg C 15

$\mathrm{mg} / \mathrm{L} \quad 15$

$5.69 \mathrm{E}+00$
$5.30 \mathrm{E}+00$
7.20

28.00

$6.70 \mathrm{E}+00$

$\begin{array}{ll}3.17 \mathrm{E}-01 & 365 \\ <1.00 \mathrm{E}+00 & 2\end{array}$

5.60

11.00

$<1.00 \mathrm{E}+00$
9.06E-01

$3.75 \mathrm{E}+00$

22.07

$3.06 \mathrm{E}+00$

\section{Outfall $\mathrm{H}-008 \mathrm{~A}$}

Effluent consists of the following types of wastewater: ash basin discharge.

$\begin{array}{lllllll}\text { FLO } & \text { MGD } & 14 & 6.26 \mathrm{E}-01 & 0.00 \mathrm{E}+00 & 14 & 8.66 \mathrm{E}-02 \\ \text { O\&G } & \mathrm{mg} / \mathrm{L} & 6 & <1.00 \mathrm{E}+00 & <1.00 \mathrm{E}+00 & & \end{array}$


Table 65

National Pollutant Discharge Elimination System Monitoring Data

Page 11 of 22

\begin{tabular}{lllllll}
\hline Measurement & Units & $\begin{array}{c}\text { Freq' } \\
\text { Year }\end{array}$ & Maximum & Minimum & $\begin{array}{c}\text { No. in } \\
\text { Average }\end{array}$ & Average \\
\hline & & & & & & \\
Permit SC0000175 & & & & & & \\
\hline PH & $\mathrm{pH}$ & 6 & 7.20 & 6.50 & & \\
TSS & $\mathrm{mg} / \mathrm{L}$ & 6 & $1.03 \mathrm{E}+01$ & $1.20 \mathrm{E}+00$ & 6 & $4.32 \mathrm{E}+00$
\end{tabular}

Outfall $\mathrm{H}-012$

Effluent consists of the following types of wastewater: cooling and steam condensate, Replacement Tritium Facility's decontamination showers, floor drains, Outfall $\mathrm{H}-017$, and Outfall H-018.

\begin{tabular}{|c|c|c|c|c|c|c|}
\hline$A L$ & $\mathrm{mg} / \mathrm{L}$ & 9 & $2.58 \mathrm{E}-01$ & $<5.00 E-02$ & 7 & $1.70 E-01$ \\
\hline AN & $\mathrm{mg} / \mathrm{L}$ & 5 & $1.67 E+00$ & $<5.00 E-02$ & 3 & $8.91 E-01$ \\
\hline CR & $\mathrm{mg} / \mathrm{L}$ & 9 & $<1.00 E-02$ & $<5.00 E-03$ & & \\
\hline $\mathrm{CU}$ & $\mathrm{mg} / \mathrm{L}$ & 9 & $1.81 E-02$ & $<5.00 E-03$ & 5 & $8.80 E-03$ \\
\hline FLO & MGD & 15 & $7.20 E-01$ & $7.20 \mathrm{E}-02$ & 15 & $3.10 E-01$ \\
\hline$H G$ & $\mathrm{mg} / \mathrm{L}$ & 9 & $<1.00 \mathrm{E}-04$ & $<1.00 \mathrm{E}-04$ & & \\
\hline MN & $\mathrm{mg} / \mathrm{L}$ & 8 & $2.46 \mathrm{E}-02$ & $<1.00 E-02$ & 6 & $1.87 E-02$ \\
\hline $\mathrm{NI}$ & $\mathrm{mg} / \mathrm{L}$ & 9 & $<1.00 E-02$ & $<5.00 E-03$ & & \\
\hline NO3 & $\mathrm{mg} / \mathrm{L}$ & 5 & $1.48 E+00$ & $<2.00 E-02$ & 4 & $7.15 E-01$ \\
\hline O\&G & $\mathrm{mg} / \mathrm{L}$ & 15 & $2.70 E+00$ & $<1.00 E+00$ & 2 & $2.35 E+00$ \\
\hline PB & $\mathrm{mg} / \mathrm{L}$ & 9 & $<2.00 E-02$ & $<3.00 E-03$ & & \\
\hline $\mathrm{PH}$ & $\mathrm{pH}$ & 15 & 7.00 & 6.00 & & \\
\hline SO4 & $\mathrm{mg} / \mathrm{L}$ & 8 & $1.29 E+01$ & $6.40 E+00$ & 8 & $1.08 E+01$ \\
\hline TMP & $\operatorname{Deg} C$ & 15 & 28.00 & 15.00 & 15 & 21.47 \\
\hline TSS & $\mathrm{mg} / \mathrm{L}$ & 15 & $3.13 E+01$ & $<1.00 \mathrm{E}+00$ & 10 & $5.72 E+00$ \\
\hline U308 & $\mathrm{mg} / \mathrm{L}$ & 9 & $<5.00 E-01$ & $<2.00 \mathrm{E}-02$ & & \\
\hline $\mathrm{ZN}$ & $\mathrm{mg} / \mathrm{L}$ & 9 & $7.16 \mathrm{E}-02$ & $9.00 \mathrm{E}-03$ & 9 & $2.76 E-02$ \\
\hline
\end{tabular}

\section{Outfall $\mathrm{H}-013$}

Effluent consists of the following types of wastewater: sanitany wastewater.

$\begin{array}{lllllll}\text { BOD } & \mathrm{mg} / \mathrm{L} & 6 & 3.24 \mathrm{E}+01 & <1.00 \mathrm{E}+00 & 5 & 9.52 \mathrm{E}+00 \\ \text { FEC } & \# / 100 \mathrm{~mL} & 4 & <2 . & <2 . & \\ \text { FLO } & \mathrm{MGD} & 151 & 1.46 \mathrm{E}-01 & 0.00 \mathrm{E}+00 & 151 & 4.25 \mathrm{E}-02 \\ \mathrm{PH} & \mathrm{pH} & 5 & 7.80 & 7.00 & \\ \text { TSS } & \mathrm{mg} / \mathrm{L} & 5 & 7.30 \mathrm{E}+00 & 1.90 \mathrm{E}+00 & 5 & 3.88 \mathrm{E}+00\end{array}$




\section{Table 65}

National Pollutant Discharge Elimination System Monitoring Data

Page 12 of 22

\begin{tabular}{lllllll}
\hline Measurement & Units & Freq/ & Year & Maximum & Minimum & No. in \\
Average & Average \\
\hline
\end{tabular}

Permit SC0000175

Table 65

National Pollutant Discharge Elimination System Monitoring Data

Page 14 of 22

\begin{tabular}{lllllll}
\hline Measurement & Units & $\begin{array}{c}\text { Freq/ } \\
\text { Year }\end{array}$ & Maximum & Minimum & $\begin{array}{c}\text { No. in } \\
\text { Average }\end{array}$ & Average \\
\hline & & & & & & \\
Permit SC0000175 & Deg C & 14 & 26.00 & 10.00 & 14 & 19.14 \\
\hline TMP & $\mathrm{mg} / \mathrm{L}$ & 14 & $7.30 \mathrm{E}+00$ & $<1.00 \mathrm{E}+00$ & 8 & $4.18 \mathrm{E}+00$
\end{tabular}

Outfall K-008

Effluent consists of the following types of wastewater: nonprocess cooling water.

$\begin{array}{lllllll}\text { FLO } & \text { MGD } & 14 & 8.64 \mathrm{E}-02 & 0.00 \mathrm{E}+00 & 14 & 4.32 \mathrm{E}-02 \\ \text { O\&G } & \mathrm{mg} / \mathrm{L} & 12 & <1.00 \mathrm{E}+00 & <1.00 \mathrm{E}+00 & & \\ \mathrm{PH} & \mathrm{pH} & 12 & 7.20 & 6.40 & & \\ \mathrm{TMP} & \mathrm{Deg} \mathrm{C} & 12 & 31.00 & 12.00 & 12 & 23.17 \\ \text { TSS } & \mathrm{mg} / \mathrm{L} & -12 & 8.30 \mathrm{E}+00 & <1.00 \mathrm{E}+00 & 11 & 3.32 \mathrm{E}+00\end{array}$

Outfall $\mathrm{K}-010$

Effluent consists of the following types of wastewater: nonprocess cooling water.

$\begin{array}{lllllll}\text { FLO } & \text { MGD } & 13 & 5.76 \mathrm{E}-02 & 0.00 \mathrm{E}+00 & 13 & 1.96 \mathrm{E}-02 \\ \text { O\&G } & \mathrm{mg} / \mathrm{L} & 10 & 1.10 \mathrm{E}+00 & <1.00 \mathrm{E}+00 & 1 & 1.10 \mathrm{E}+00 \\ \mathrm{PH} & \mathrm{pH} & 10 & 7.20 & 6.50 & & \\ \mathrm{TMP} & \mathrm{Deg} \mathrm{C} & 10 & 26.00 & 14.00 & 10 & 20.20 \\ \text { TSS } & \mathrm{mg} / \mathrm{L} & 10 & 1.10 \mathrm{E}+01 & <1.00 \mathrm{E}+00 & 9 & 4.63 \mathrm{E}+00\end{array}$

Outfall K-012

Effluent consists of the following types of wastewater: sanitary wastewater.

\begin{tabular}{|c|c|c|c|c|c|c|}
\hline BOD & $\mathrm{mg} / \mathrm{L}$ & 15 & $4.09 E+01$ & $<1.00 E+00$ & 14 & $1.02 \mathrm{E}+01$ \\
\hline FEC & $\# / 100 \mathrm{~mL}$ & 14 & 35. & $<2$ & 5 & 18. \\
\hline FLO & MGD & 365 & $3.43 E-02$ & $0.00 E+00$ & 365 & $6.70 E-03$ \\
\hline $\mathrm{PH}$ & $\mathrm{pH}$ & 12 & 8.90 & 6.70 & & \\
\hline TSS & $\mathrm{ma} / \mathrm{L}$ & 14 & 4 गחF & 1 nncinn & +1 & nes \\
\hline
\end{tabular}


Table 65

National Pollutant Discharge Elimination System Monitoring Data

Page 11 of 22

\begin{tabular}{lllllll}
\hline Measurement & Units & $\begin{array}{l}\text { Freq/ } \\
\text { Year }\end{array}$ & Maximum & Minimum & $\begin{array}{c}\text { No. in } \\
\text { Average }\end{array}$ & Average \\
\hline Permit SC0000175 & & & & & & \\
\hline $\mathrm{PH}$ & $\mathrm{pH}$ & 6 & 7.20 & 6.50 & & \\
TSS & $\mathrm{mg} / \mathrm{L}$ & 6 & $1.03 \mathrm{E}+01$ & $1.20 \mathrm{E}+00$ & 6 & $4.32 \mathrm{E}+00$
\end{tabular}

Outfall $\mathrm{H}-012$

Effluent consists of the following types of wastewater: cooling and steam condensate, Replacement Tritium Facility's decontamination showers, floor drains, Outfall H-017, and Outfall H-018.

AL

AN

CR

$\mathrm{CU}$

FLO

HG

MN

NI

NO3

O\&G

PB

$\mathrm{PH}$

$\mathrm{SO} 4$

TMP

TSS

U308

$\mathrm{ZN}$

\begin{abstract}
$\mathrm{mg} / \mathrm{L} \quad 9$
\end{abstract}
$\mathrm{mg} / \mathrm{L} \quad 5$

$\mathrm{mg} / \mathrm{L} \quad 9$

$\mathrm{mg} / \mathrm{L} \quad 9$

MGD $\quad 15$

$\mathrm{mg} / \mathrm{L} \quad 9$

$\mathrm{mg} / \mathrm{L} \quad 8$

$\mathrm{mg} / \mathrm{L} \quad 9$

$\mathrm{mg} / \mathrm{L} \quad 5$

$\mathrm{mg} / \mathrm{L} \quad 15$

$\mathrm{mg} / \mathrm{L} \quad 9$

$\mathrm{pH}$

$\mathrm{mg} / \mathrm{L} \quad 8$

Deg C 15

$\mathrm{mg} / \mathrm{L} \quad 15$

$\mathrm{mg} / \mathrm{L} \quad 9$ $\mathrm{mg} / \mathrm{L} \quad 9$
2.58E-01

$1.67 \mathrm{E}+00$

$<1.00 \mathrm{E}-02$

1.81E-02

$7.20 \mathrm{E}-01$

$<1.00 \mathrm{E}-04$

2.46E-02

$<1.00 \mathrm{E}-02$

$1.48 \mathrm{E}+00$

$2.70 \mathrm{E}+00$

$<2.00 E-02$

7.00

$1.29 \mathrm{E}+01$

28.00

3.13E+01

$<5.00 \mathrm{E}-01$

7.16E-02
$<5.00 \mathrm{E}-02 \quad 7$

$<5.00 \mathrm{E}-02 \quad 3$

$<5.00 \mathrm{E}-03$

$<5.00 \mathrm{E}-03$

$7.20 \mathrm{E}-02$

5

15

$<1.00 \mathrm{E}-04$

$<1.00 E-02$

6

$<5.00 \mathrm{E}-03$

$<2.00 \mathrm{E}-02$

$<1.00 \mathrm{E}+00$

4

7.15E-01

$<3.00 \mathrm{E}-03$

6.00

$6.40 E+00$

15.00

$<1.00 \mathrm{E}+00$

$<2.00 \mathrm{E}-02$

$9.00 E-03$

9

8

$1.08 \mathrm{E}+01$

15

21.47

10

$5.72 E+00$

Outfall H-013

Effluent consists of the following types of wastewater: sanitary wastewater.

$\begin{array}{lllllll}\text { BOD } & \mathrm{mg} / \mathrm{L} & 6 & 3.24 \mathrm{E}+01 & <1.00 \mathrm{E}+00 & 5 & 9.52 \mathrm{E}+00 \\ \text { FEC } & \# / 100 \mathrm{~mL} & 4 & <2 . & <2 . & & \\ \text { FLO } & \mathrm{MGD} & 151 & 1.46 \mathrm{E}-01 & 0.00 \mathrm{E}+00 & 151 & 4.25 \mathrm{E}-02 \\ \mathrm{PH} & \mathrm{pH} & 5 & 7.80 & 7.00 & & \\ \text { TSS } & \mathrm{mg} / \mathrm{L} & 5 & 7.30 \mathrm{E}+00 & 1.90 \mathrm{E}+00 & 5 & 3.88 \mathrm{E}+00\end{array}$




\section{Table 65}

\section{National Pollutant Discharge Elimination System Monitoring Data}

Page 12 of 22

\begin{tabular}{lllllll}
\hline Measurement & Units & $\begin{array}{l}\text { Freq/ } \\
\text { Year }\end{array}$ & Maximum & Minimum & $\begin{array}{c}\text { No. in } \\
\text { Average }\end{array}$ & Average \\
\hline
\end{tabular}

Permit SC0000175

Outfall $\mathrm{H}-015$ (facility not in service but point still on permit)

Effluent consists of the following types of wastewater: noncontact cooling water, steam condensate, and cooling tower blowdown.

Outfall H-016

Effluent consists of the following types of wastewater: process water, cooling water, and stormwater.

\begin{tabular}{|c|c|c|c|c|c|c|}
\hline$A L$ & $\mathrm{mg} / \mathrm{L}$ & 13 & $5.00 E-02$ & $<2.00 E-02$ & 1 & $5.00 \mathrm{E}-02$ \\
\hline AN & $\mathrm{mg} / \mathrm{L}$ & 52 & $1.08 \mathrm{E}+00$ & $<5.00 \mathrm{E}-02$ & 23 & $2.94 \mathrm{E}-01$ \\
\hline BOD & $\mathrm{mg} / \mathrm{L}$ & 52 & $1.97 \mathrm{E}+01$ & $<1.00 E+00$ & 16 & $4.09 E+00$ \\
\hline $\mathrm{CHL}$ & $\mathrm{mg} / \mathrm{L}$ & 12 & $1.20 \mathrm{E}-01$ & $<1.00 \mathrm{E}-01$ & 1 & $1.20 E-01$ \\
\hline CR & $\mathrm{mg} / \mathrm{L}$ & 52 & $1.60 E-02$ & $<5.00 E-03$ & 1 & $1.60 \mathrm{E}-02$ \\
\hline $\mathrm{CU}$ & $\mathrm{mg} / \mathrm{L}$ & 52 & $1.50 E-02$ & $<5.00 E-03$ & 4 & $1.25 E-02$ \\
\hline FLO & MGD & 366 & $3.06 \mathrm{E}-01$ & $0.00 E+00$ & 366 & $4.51 E-02$ \\
\hline$H G$ & $\mathrm{mg} / \mathrm{L}$ & 52 & $3.00 \mathrm{E}-04$ & $<1.00 E-04$ & 3 & $2.00 E-04$ \\
\hline MN & $\mathrm{mg} / \mathrm{L}$. & 52 & $5.30 \mathrm{E}-02$ & $<5.00 \mathrm{E}-03$ & 4 & $3.56 E-02$ \\
\hline $\mathrm{NI}$ & $\mathrm{mg} / \mathrm{L}$ & 13 & $<1.00 \mathrm{E}-02$ & $<5.00 E-03$ & & \\
\hline NO3 & $\mathrm{mg} / \mathrm{L}$ & 52 & $3.77 \mathrm{E}+02$ & $1.98 \mathrm{E}+00$ & 52 & $1.79 E+01$ \\
\hline$O \& G$ & $\mathrm{mg} / \mathrm{L}$ & 51 & $1.00 E+01$ & $<1.00 \mathrm{E}+00$ & 5 & $4.78 E+00$ \\
\hline PB & $\mathrm{mg} / \mathrm{L}$ & 52 & $<2.00 \mathrm{E}-02$ & $<3.00 \mathrm{E}-03$ & & \\
\hline $\mathrm{PH}$ & $\mathrm{pH}$ & 24 & 8.60 & 6.20 & & \\
\hline TMP & $\operatorname{Deg} C$ & 82 & 184570.00 & 0.00 & 82 & 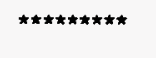 \\
\hline TSS & $\mathrm{mg} / \mathrm{L}$ & 52 & $1.00 E+01$ & $<1.00 \mathrm{E}+00$ & 16 & $2.95 \mathrm{E}+00$ \\
\hline U308 & $\mathrm{mg} / \mathrm{L}$ & 13 & $<5.00 \mathrm{E}-01$ & $<2.00 \mathrm{E}-02$ & & \\
\hline $\mathrm{ZN}$ & $\mathrm{mg} / \mathrm{L}$ & 52 & $1.41 E-01$ & $<5.00 \mathrm{E}-03$ & 37 & $1.75 \mathrm{E}-02$ \\
\hline
\end{tabular}

Outfall $\mathrm{H}-017$

Effluent consists of the following types of wastewater: stormwater runoff retention basin effluent.

$\begin{array}{lllllll}\text { AL } & \mathrm{mg} / \mathrm{L} & 21 & 9.58 \mathrm{E}-01 & <5.00 \mathrm{E}-02 & 17 & 3.66 \mathrm{E}-01 \\ \mathrm{AN} & \mathrm{mg} / \mathrm{L} & 21 & 9.14 \mathrm{E}-01 & <5.00 \mathrm{E}-02 & 9 & 2.45 \mathrm{E}-01 \\ \mathrm{CR} & \mathrm{mg} / \mathrm{L} & 21 & <1.00 \mathrm{E}-02 & <5.00 \mathrm{E}-03 & & \\ \mathrm{CU} & \mathrm{mg} / \mathrm{L} & 21 & 1.70 \mathrm{E}-02 & <5.00 \mathrm{E}-03 & 14 & 1.03 \mathrm{E}-02 \\ \mathrm{FLO} & \mathrm{MGD} & 21 & 1.10 \mathrm{E}+00 & 5.53 \mathrm{E}-01 & 21 & 9.62 \mathrm{E}-01 \\ \mathrm{HG} & \mathrm{mg} / \mathrm{L} & 21 & <1.00 \mathrm{E}-04 & <1.00 \mathrm{E}-04 & & \\ \mathrm{MN} & \mathrm{mg} / \mathrm{L} & 21 & 7.70 \mathrm{E}-02 & 6.60 \mathrm{E}-03 & 16 & 2.82 \mathrm{E}-02\end{array}$


Table 65

National Pollutant Discharge Elimination System Monitoring Data

Page 13 of 22

\begin{tabular}{lccllll}
\hline Measurement & Units & $\begin{array}{c}\text { Freq/ } \\
\text { Year }\end{array}$ & Maximum & Minimum & $\begin{array}{c}\text { No. in } \\
\text { Average }\end{array}$ & Average \\
\hline Permit SC0000175 & & & & & & \\
\hline $\mathrm{NI}$ & $\mathrm{mg} / \mathrm{L}$ & 21 & $<1.00 \mathrm{E}-02$ & $<5.00 \mathrm{E}-03$ & & \\
$\mathrm{NO3}$ & $\mathrm{mg} / \mathrm{L}$ & 21 & $3.58 \mathrm{E}+02$ & $<2.00 \mathrm{E}-02$ & 18 & $2.04 \mathrm{E}+01$ \\
$\mathrm{~PB}$ & $\mathrm{mg} / \mathrm{L}$ & 21 & $4.30 \mathrm{E}-03$ & $<3.00 \mathrm{E}-03$ & 2 & $4.00 \mathrm{E}-03$ \\
$\mathrm{PH}$ & $\mathrm{pH}$ & 21 & 8.30 & 6.30 & & \\
TSS & $\mathrm{mg} / \mathrm{L}$ & 21 & $3.70 \mathrm{E}+01$ & $4.90 \mathrm{E}+00$ & 21 & $1.19 \mathrm{E}+01$ \\
$\mathrm{U} 308$ & $\mathrm{mg} / \mathrm{L}$ & 21 & $<5.00 \mathrm{E}-01$ & $<2.00 \mathrm{E}-02$ & & \\
$\mathrm{ZN}$ & $\mathrm{mg} / \mathrm{L}$ & 21 & $3.36 \mathrm{E}-01$ & $5.10 \mathrm{E}-02$ & 21 & $1.56 \mathrm{E}-01$
\end{tabular}

Outfall $\mathrm{H}-018$

Effluent consists of the following types of wastewater cooling water retention basin effluent.

$\begin{array}{lclllll}\text { AL } & \mathrm{mg} / \mathrm{L} & 4 & 4.66 \mathrm{E}-01 & <5.00 \mathrm{E}-02 & 2 & 3.10 \mathrm{E}-01 \\ \mathrm{AN} & \mathrm{mg} / \mathrm{L} & 4 & 2.44 \mathrm{E}-01 & <1.00 \mathrm{E}-01 & 3 & 1.54 \mathrm{E}-01 \\ \mathrm{CR} & \mathrm{mg} / \mathrm{L} & 4 & <1.00 \mathrm{E}-02 & <5.00 \mathrm{E}-03 & & \\ \mathrm{CU} & \mathrm{mg} / \mathrm{L} & 4 & 2.20 \mathrm{E}-02 & 8.80 \mathrm{E}-03 & 3 & 1.49 \mathrm{E}-02 \\ \mathrm{FLO} & \mathrm{MGD} & 4 & 1.22 \mathrm{E}+00 & 6.84 \mathrm{E}-01 & 4 & 9.25 \mathrm{E}-01 \\ \mathrm{HG} & \mathrm{mg} / \mathrm{L} & 4 & <1.00 \mathrm{E}-04 & <1.00 \mathrm{E}-04 & & \\ \mathrm{MN} & \mathrm{mg} / \mathrm{L} & 4 & 7.90 \mathrm{E}-02 & 7.60 \mathrm{E}-03 & 2 & 4.33 \mathrm{E}-02 \\ \mathrm{NI} & \mathrm{mg} / \mathrm{L} & 4 & <1.00 \mathrm{E}-02 & <5.00 \mathrm{E}-03 & & \\ \mathrm{NO3} & \mathrm{mg} / \mathrm{L} & 4 & 1.35 \mathrm{E}-01 & <2.00 \mathrm{E}-02 & 2 & 8.25 \mathrm{E}-02 \\ \mathrm{~PB} & \mathrm{mg} / \mathrm{L} & 4 & 4.20 \mathrm{E}-03 & 4.20 \mathrm{E}-03 & 1 & 4.20 \mathrm{E}-03 \\ \mathrm{PH} & \mathrm{pH} & 4 & 8.60 & 7.00 & & \\ \text { TSS } & \mathrm{mg} / \mathrm{L} & 4 & 1.20 \mathrm{E}+01 & 2.50 \mathrm{E}+00 & 4 & 6.38 \mathrm{E}+00 \\ \mathrm{U} 308 & \mathrm{mg} / \mathrm{L} & 4 & <5.00 \mathrm{E}-01 & <2.00 \mathrm{E}-02 & & \\ \text { ZN } & \mathrm{mg} / \mathrm{L} & 4 & 1.34 \mathrm{E}-01 & 1.50 \mathrm{E}-02 & 4 & 5.75 \mathrm{E}-02\end{array}$

Outfall K-001 (facility in service but did not discharge)

Effluent consists of the following types of wastewater. cooling water.
FLO
MGD 13
$0.00 E+00$
13
$0.00 E+00$

Outfall K-006

Effluent consists of the following types of wastewater: nonprocess cooling water, ash sluice leakage, and powerhouse wastewater.

$\begin{array}{lllllll}\text { FLO } & \text { MGD } & 15 & 5.04 \mathrm{E}-02 & 0.00 \mathrm{E}+00 & 15 & 3.09 \mathrm{E}-02 \\ \text { O\&G } & \mathrm{mg} / \mathrm{L} & 14 & <1.00 \mathrm{E}+00 & <1.00 \mathrm{E}+00 & & \\ \mathrm{PH} & \mathrm{pH} & 14 & 7.00 & 6.60 & \end{array}$


Table 65

National Pollutant Discharge Elimination System Monitoring Data

Page 14 of 22

\begin{tabular}{lllllll}
\hline Measurement & Units & $\begin{array}{c}\text { Freq/ } \\
\text { Year }\end{array}$ & Maximum & Minimum & $\begin{array}{c}\text { No. in } \\
\text { Average }\end{array}$ & Average \\
\hline Permit SC0000175 & & & & & & \\
\hline TMP & Deg C & 14 & 26.00 & 10.00 & 14 & 19.14 \\
TSS & $\mathrm{mg} / \mathrm{L}$ & 14 & $7.30 \mathrm{E}+00$ & $<1.00 \mathrm{E}+00$ & 8 & $4.18 \mathrm{E}+00$
\end{tabular}

Outfall K-008

Effluent consists of the following types of wastewater: nonprocess cooling water.

$\begin{array}{lllllll}\text { FLO } & \text { MGD } & 14 & 8.64 \mathrm{E}-02 & 0.00 \mathrm{E}+00 & 14 & 4.32 \mathrm{E}-02 \\ \mathrm{O \& G} & \mathrm{mg} / \mathrm{L} & 12 & <1.00 \mathrm{E}+00 & <1.00 \mathrm{E}+00 & & \\ \mathrm{PH} & \mathrm{pH} & 12 & 7.20 & 6.40 & & \\ \mathrm{TMP} & \mathrm{Deg} \mathrm{C} & 12 & 31.00 & 12.00 & 12 & 23.17 \\ \text { TSS } & \mathrm{mg} / \mathrm{L} & 12 & 8.30 \mathrm{E}+00 & <1.00 \mathrm{E}+00 & 11 & 3.32 \mathrm{E}+00\end{array}$

Outfall K-010

Effluent consists of the following types of wastewater: nonprocess cooling water.

$\begin{array}{lllllll}\text { FLO } & \text { MGD } & 13 & 5.76 \mathrm{E}-02 & 0.00 \mathrm{E}+00 & 13 & 1.96 \mathrm{E}-02 \\ \mathrm{O \& G} & \mathrm{mg} / \mathrm{L} & 10 & 1.10 \mathrm{E}+00 & <1.00 \mathrm{E}+00 & 1 & 1.10 \mathrm{E}+00 \\ \mathrm{PH} & \mathrm{pH} & 10 & 7.20 & 6.50 & \\ \mathrm{TMP} & \mathrm{Deg} \mathrm{C} & 10 & 26.00 & 14.00 & 10 & 20.20 \\ \text { TSS } & \mathrm{mg} / \mathrm{L} & 10 & 1.10 \mathrm{E}+01 & <1.00 \mathrm{E}+00 & 9 & 4.63 \mathrm{E}+00\end{array}$

Outfall $\mathrm{K}-012$

Effluent consists of the following types of wastewater: sanitary wastewater.

$\begin{array}{lllllll}\text { BOD } & \mathrm{mg} / \mathrm{L} & 15 & 4.09 \mathrm{E}+01 & <1.00 \mathrm{E}+00 & 14 & 1.02 \mathrm{E}+01 \\ \mathrm{FEC} & \# / 100 \mathrm{~mL} & 14 & 35 . & <2 . & 5 & 18 . \\ \mathrm{FLO} & \mathrm{MGD} & 365 & 3.43 \mathrm{E}-02 & 0.00 \mathrm{E}+00 & 365 & 6.70 \mathrm{E}-03^{*} \\ \mathrm{PH} & \mathrm{pH} & 12 & 8.90 & 6.70 & & \\ \text { TSS } & \mathrm{mg} / \mathrm{L} & 14 & 4.20 \mathrm{E}+00 & 1.00 \mathrm{E}+00 & 14 & 2.11 \mathrm{E}+00\end{array}$

Outfall L-007

Effluent consists of the following types of wastewater: treated low-activity wastewater, treated sanitary and neutralization tank waste, reactor cooling water, water treatment plant backflush, and reservoir overflow.

$\begin{array}{lllllll}\text { FLO } & \text { MGD } & 359 & 8.27 \mathrm{E}+01 & 1.68 \mathrm{E}+01 & 359 & 3.91 \mathrm{E}+01 \\ \text { O\&G } & \mathrm{mgl} & 15 & 2.00 \mathrm{E}+00 & <1.00 \mathrm{E}+00 & 1 & 2.00 \mathrm{E}+00 \\ \mathrm{PH} & \mathrm{pH} & 15 & 7.10 & 6.60 & & \\ \text { TMP } & \mathrm{Deg} \mathrm{C} & 367 & 25.80 & 7.30 & 367 & 17.88 \\ \text { TMP1 } & \text { Deg C } & 0 & & & \end{array}$


Table 65

National Pollutant Discharge Elimination System Monitoring Data

Page 15 of 22

\begin{tabular}{|c|c|c|c|c|c|c|}
\hline Measurement & Units & $\begin{array}{l}\text { Freq' } \\
\text { Year }\end{array}$ & Maximum & Minimum & $\begin{array}{l}\text { No. in } \\
\text { Average }\end{array}$ & Average \\
\hline
\end{tabular}

Permit SC0000175

\begin{tabular}{lllllll}
\hline TMP2 & Deg C & 0 & & & & \\
TSS & $\mathrm{mg} / \mathrm{L}$ & 15 & $1.57 \mathrm{E}+01$ & $<1.00 \mathrm{E}+00$ & 14 & $6.46 \mathrm{E}+00$
\end{tabular}

Outfall L-007A

Effluent consists of the following types of wastewater. sanitary wastewater.

$\begin{array}{lllllll}\text { BOD } & \mathrm{mg} / \mathrm{L} & 13 & 1.20 \mathrm{E}+01 & <1.00 \mathrm{E}+00 & 9 & 4.00 \mathrm{E}+00 \\ \text { FEC } & \# / 100 \mathrm{~mL} & 14 & 20 . & <2 . & 5 & 6 . \\ \text { FLO } & \text { MGD } & 365 & 2.64 \mathrm{E}-02 & 0.00 \mathrm{E}+00 & 365 & 3.60 \mathrm{E}-03 \\ \mathrm{PH} & \mathrm{pH} & 12 & 7.50 & 6.90 & & \\ \text { TSS } & \mathrm{mg} / \mathrm{L} & 13 & 2.64 \mathrm{E}+01 & <1.00 \mathrm{E}+00 & 11 & 9.26 \mathrm{E}+00\end{array}$

Outfall $L-008$

Effluent consists of the following types of wastewater: nonprocess cooling water and water reservoir sump wastewater.

FLO

O\&G

MGD $\quad 16$

7.78E-02

2.88E-02

16

5.30E-02

$\mathrm{PH}$

$\mathrm{mg} / \mathrm{L} \quad 15$

$2.60 \mathrm{E}+00$

$<1.00 \mathrm{E}+00$

$2.10 \mathrm{E}+00$

TMP

$\mathrm{pH} \quad 16$

7.30

6.70

TSS

Deg C 13

27.00

11.00

2

20.77

$\mathrm{mg} / \mathrm{L} \quad 15$

$8.00 \mathrm{E}+00$

$<1.00 \mathrm{E}+00 \quad 9$

$2.14 E+00$

Outfall L-010 (facility in service but did not discharge)

Effluent consists of the following types of wastewater: stormwater.

FLO

MGD 3

$0.00 E+00 \quad 3$

$0.00 E+00$

Outfall M-004

Effluent consists of the following types of wastewater: Liquid Effluent Treatment Facility wastewater.

$A G$

AL

$C D$

$\mathrm{CN}$

$C R$

CU

FLO

$\mathrm{NI}$

NO3

O\&G

$\begin{array}{cll}\mathrm{mg} / \mathrm{L} & 5 & <5.00 \mathrm{E}-03 \\ \mathrm{mg} / \mathrm{L} & 18 & 6.42 \mathrm{E}-01 \\ \mathrm{mg} / \mathrm{L} & 5 & <5.00 \mathrm{E}-03 \\ \mathrm{mg} / \mathrm{L} & 3 & <2.00 \mathrm{E}-02 \\ \mathrm{mg} / \mathrm{L} & 5 & <1.00 \mathrm{E}-02 \\ \mathrm{mg} / \mathrm{L} & 18 & 2.00 \mathrm{E}-02 \\ \mathrm{MGD} & 48 & 1.70 \mathrm{E}-01 \\ \mathrm{mg} / \mathrm{L} & 18 & 9.00 \mathrm{E}-02 \\ \mathrm{mg} / \mathrm{L} & 12 & 8.46 \mathrm{E}+01 \\ \mathrm{mg} / \mathrm{L} & 17 & 1.60 \mathrm{E}+01\end{array}$

$<5.00 \mathrm{E}-04$

$<5.00 \mathrm{E}-02$

$<5.00 \mathrm{E}-03$

$<1.00 \mathrm{E}-02$

$<5.00 \mathrm{E}-03$

$<5.00 \mathrm{E}-03$

$0.00 \mathrm{E}+00$

7

1.14E-02

$<5.00 \mathrm{E}-03$

48

$8.00 E-03$

$<2.00 \mathrm{E}-02$

5

3.21E-02

$<1.00 E+00$

10

3.13E +01

$5.62 \mathrm{E}+00$ 
Table 65

National Pollutant Discharge Elimination System Monitoring Data

Page 16 of 22

\begin{tabular}{lllllll}
\hline Measurement & Units & Freql & Year & Maximum & Minimum & No. in \\
Average & Average \\
\hline
\end{tabular}

Permit SC0000175

\begin{tabular}{lllllll}
\hline PB & $\mathrm{mg} / \mathrm{L}$ & 18 & $<2.00 \mathrm{E}-02$ & $<3.00 \mathrm{E}-03$ & & \\
$\mathrm{PH}$ & $\mathrm{pH}$ & 17 & 7.10 & 6.20 & & \\
PHOS & $\mathrm{mg} / \mathrm{L}$ & 11 & $3.70 \mathrm{E}-01$ & $1.30 \mathrm{E}-02$ & 11 & $1.79 \mathrm{E}-01$ \\
TSS & $\mathrm{mg} / \mathrm{L}$ & 18 & $1.00 \mathrm{E}+01$ & $<1.00 \mathrm{E}+00$ & 15 & $3.86 \mathrm{E}+00$ \\
U308 & $\mathrm{mg} / \mathrm{L}$ & 18 & $<5.00 \mathrm{E}-01$ & $<2.00 \mathrm{E}-02$ & & \\
ZN & $\mathrm{mg} / \mathrm{L}$ & 6 & $3.75 \mathrm{E}-01$ & $9.81 \mathrm{E}-02$ & 6 & $2.02 \mathrm{E}-01$
\end{tabular}

Outfall $\mathrm{M}-005$

Effluent consists of the following types of wastewater: air stripper.

$\begin{array}{lllllll}\text { FLO } & \text { MGD } & 54 & 7.49 E-01 & 0.00 E+00 & 54 & 5.03 E-01 \\ \text { PERCL } & u g / L & 46 & 1.18 E+01 & <2.00 E+00 & 2 & 7.75 E+00 \\ \text { PH } & \mathrm{pH} & 26 & 7.10 & 5.20 & & \\ \text { TRICL } & \mathrm{ug} / \mathrm{L} & 46 & 3.10 \mathrm{E}+00 & <2.00 \mathrm{E}+00 & 2 & 3.10 \mathrm{E}+00\end{array}$

Outfall P-005 (facility in service but did not discharge)

Effluent consists of the following types of wastewater: ash basin discharge.

$\begin{array}{lllllll}\text { FLO } & \text { MGD } & 12 & 0.00 E+00 & 12 & 0.00 E+00\end{array}$

Outfall $P-007$

Effluent consists of the following types of wastewater: Powerhouse wastewater and nonprocess cooling water.

$\begin{array}{lllllll}\text { FLO } & \text { MGD } & 367 & 4.14 \mathrm{E}-01 & 0.00 E+00 & 367 & 8.90 \mathrm{E}-03\end{array}$

Outfall P-013

Effluent consists of the following types of wastewater: neutralization, reactor process, and cooling water.

$\begin{array}{lllllll}\text { FLO } & \text { MGD } & 365 & 6.40 E+00 & 1.36 E-01 & 365 & 2.34 E+00 \\ \text { O\&G } & \mathrm{mg} / \mathrm{L} & 13 & 2.30 \mathrm{E}+00 & <1.00 \mathrm{E}+00 & 1 & 2.30 \mathrm{E}+00 \\ \mathrm{PH} & \mathrm{pH} & 13 & 7.30 & 6.10 & & \\ \text { TMP } & \mathrm{Deg} \mathrm{C} & 367 & 27.00 & 6.30 & 367 & 18.42 \\ \text { TSS } & \mathrm{mg} / \mathrm{L} & 13 & 1.80 \mathrm{E}+01 & 1.20 \mathrm{E}+00 & 13 & 5.09 \mathrm{E}+00\end{array}$

Outfall P-014

Effluent consists of the following types of wastewater: sanitary wastewater.

$\begin{array}{lllllll}\text { BOD } & \mathrm{mg} / \mathrm{L} & 14 & 2.50 \mathrm{E}+01 & <1.00 \mathrm{E}+00 & 9 & 3.87 \mathrm{E}+00 \\ \text { FEC } & \# / 100 \mathrm{~mL} & 17 & 7500 . & <2 . & 5 & 1721 . \\ \text { FLO } & \mathrm{MGD} & 365 & 2.84 \mathrm{E}-02 & 0.00 \mathrm{E}+00 & 365 & 6.00 \mathrm{E}-03\end{array}$




\section{Table 65}

National Pollutant Discharge Elimination System Monitoring Data

Page 17 of 22

\begin{tabular}{lccllll}
\hline Measurement & Units & $\begin{array}{c}\text { Freq/ } \\
\text { Year }\end{array}$ & Maximum & Minimum & $\begin{array}{c}\text { No. in } \\
\text { Average }\end{array}$ & Average \\
\hline & & & & & & \\
Permit SC0000175 & & & & & & \\
\hline PH & $\mathrm{pH}$ & 12 & 7.60 & 6.90 & & \\
TSS & $\mathrm{mg} / \mathrm{L}$ & 14 & $2.60 \mathrm{E}+01$ & $1.50 \mathrm{E}+00$ & 14 & $7.31 \mathrm{E}+00$
\end{tabular}

Outfall P-019

Effluent consists of the following types of wastewater: P-Reactor cooling water.

$\begin{array}{lllllll}\text { FLO } & \text { MGD } & 365 & 6.98 \mathrm{E}+01 & 1.29 \mathrm{E}+00 & 365 & 5.16 \mathrm{E}+00 \\ \text { O\&G } & \mathrm{mg} / \mathrm{L} & 14 & 1.20 \mathrm{E}+00 & <1.00 \mathrm{E}+00 & 1 & 1.20 \mathrm{E}+00 \\ \mathrm{PH} & \mathrm{pH} & 14 & 8.60 & 6.00 & & \\ \text { TMP } & \mathrm{Deg} C & 367 & 26.60 & 5.90 & 367 & 17.92 \\ \text { TSS } & \mathrm{mg} / \mathrm{L} & 16 & 2.48 \mathrm{E}+01 & <1.00 \mathrm{E}+00 & 14 & 5.54 \mathrm{E}+00\end{array}$

Outfall PP-001

Effluent consists of the following types of wastewater: stormwater.

$\begin{array}{lllllll}\text { FLO } & \text { MGD } & 5 & 2.88 \mathrm{E}-02 & 7.20 \mathrm{E}-03 & 5 & 1.87 \mathrm{E}-02 \\ \mathrm{O} \& \mathrm{G} & \mathrm{mg} / \mathrm{L} & 5 & 2.00 \mathrm{E}+00 & <1.00 \mathrm{E}+00 & 1 & 2.00 \mathrm{E}+00 \\ \mathrm{PH} & \mathrm{pH} & 5 & 6.80 & 6.10 & & \end{array}$

Outfall S-002 (CS-002)

Effluent consists of the following types of wastewater: automotive shop and cooling wastewater.

$\begin{array}{lllllll}\text { AL } & \mathrm{mg} / \mathrm{L} & 1 & 7.65 \mathrm{E}+00 & 7.65 \mathrm{E}+00 & 1 & 7.65 \mathrm{E}+00 \\ \mathrm{BOD} & \mathrm{mg} / \mathrm{L} & 1 & 1.15 \mathrm{E}+01 & 1.15 \mathrm{E}+01 & 1 & 1.15 \mathrm{E}+01 \\ \mathrm{FE} & \mathrm{mg} / \mathrm{L} & 1 & 8.58 \mathrm{E}+00 & 8.58 \mathrm{E}+00 & 1 & 8.58 \mathrm{E}+00 \\ \mathrm{FLO} & \mathrm{MGD} & 52 & 1.44 \mathrm{E}-01 & 0.00 \mathrm{E}+00 & 52 & 2.80 \mathrm{E}-03 \\ \mathrm{O} \& \mathrm{G} & \mathrm{mg} / \mathrm{L} & 1 & <1.00 \mathrm{E}+00 & <1.00 \mathrm{E}+00 & & \\ \mathrm{PH} & \mathrm{pH} & 1 & 6.40 & 6.40 & & \\ \mathrm{TMP} & \mathrm{Deg} \mathrm{C} & 1 & 8.00 & 8.00 & 1 & 8.00 \\ \text { TSS } & \mathrm{mg} / \mathrm{L} & 1 & 1.95 \mathrm{E}+02 & 1.95 \mathrm{E}+02 & 1 & 1.95 \mathrm{E}+02\end{array}$

Outfall S-008 (facility in service but did not discharge)

Effluent consists of the following types of wastewater: stormwater runoff and cooling wastewater.

$\begin{array}{llllll}\text { FLO } & \text { MGD } & 12 & 0.00 \mathrm{E}+00 & 12 & 0.00 \mathrm{E}+00\end{array}$ 


\section{Table 65}

\section{National Pollutant Discharge Elimination System Monitoring Data}

Page 18 of 22

\begin{tabular}{lllllll}
\hline Measurement & Units & $\begin{array}{l}\text { Freq/ } \\
\text { Year }\end{array}$ & Maximum & Minimum & $\begin{array}{c}\text { No. in } \\
\text { Average }\end{array}$ & Average \\
\hline
\end{tabular}

\section{Permit SC0000175}

Outfall S-011

Effluent consists of the following types of wastewater: sanitary wastewater from Central Shops wastewater treatment plant.

$\begin{array}{lllllll}\text { BOD } & \mathrm{mg} / \mathrm{L} & 5 & 2.40 \mathrm{E}+00 & <1.00 \mathrm{E}+00 & 4 & 1.77 \mathrm{E}+00 \\ \text { FEC } & \# / 100 \mathrm{~mL} & 4 & <2 . & <2 . & & \\ \text { FLO } & \text { MGD } & 151 & 6.32 \mathrm{E}-02 & 0.00 \mathrm{E}+00 & 151 & 2.45 \mathrm{E}-02 \\ \text { PH } & \mathrm{pH} & 5 & 7.50 & 7.02 & & \\ \text { TSS } & \mathrm{mg} / \mathrm{L} & 5 & 5.20 \mathrm{E}+00 & 1.20 \mathrm{E}+00 & 5 & 2.62 \mathrm{E}+00\end{array}$

Outfall S-014 (facility in service but did not discharge)

Effluent consists of the following types of wastewater: stormwater.
FLO
MGD
4
$0.00 E+00$
$0.00 E+00$

\section{Outfall SC-004}

Effluent consists of the following types of wastewater: Steel Creek water monitoring below the dam at Road A.

$\begin{array}{lllllll}\text { AG } & \mathrm{mg} / \mathrm{L} & 12 & 3.30 \mathrm{E}-01 & <5.00 \mathrm{E}-04 & 1 & 3.30 \mathrm{E}-01 \\ \mathrm{AS} & \mathrm{mg} / \mathrm{L} & 12 & <2.00 \mathrm{E}-02 & <5.00 \mathrm{E}-03 & & \\ \mathrm{BA} & \mathrm{mg} / \mathrm{L} & 12 & 2.10 \mathrm{E}-02 & <5.00 \mathrm{E}-03 & 11 & 1.34 \mathrm{E}-02 \\ \mathrm{CD} & \mathrm{mg} / \mathrm{L} & 12 & <5.00 \mathrm{E}-03 & <5.00 \mathrm{E}-03 & & \\ \mathrm{CR} & \mathrm{mg} / \mathrm{L} & 12 & <1.00 \mathrm{E}-02 & <5.00 \mathrm{E}-03 & & \\ \text { DO } & \mathrm{mg} / \mathrm{L} & 359 & 1.30 \mathrm{E}+01 & 2.00 \mathrm{E}+00 & 359 & 8.39 \mathrm{E}+00 \\ \text { FLO } & \mathrm{MGD} & 365 & 1.41 \mathrm{E}+02 & 2.39 \mathrm{E}+01 & 365 & 4.84 \mathrm{E}+01 \\ \mathrm{HG} & \mathrm{mg} / \mathrm{L} & 12 & 3.00 \mathrm{E}-04 & <1.00 \mathrm{E}-04 & 1 & 3.00 \mathrm{E}-04 \\ \mathrm{NO3} & \mathrm{mg} / \mathrm{L} & 13 & 3.30 \mathrm{E}-01 & <2.00 \mathrm{E}-02 & 12 & 9.61 \mathrm{E}-02 \\ \text { PB } & \mathrm{mg} / \mathrm{L} & 12 & <2.00 \mathrm{E}-02 & <3.00 \mathrm{E}-03 & & \\ \text { PH } & \mathrm{pH} & 12 & 7.60 & 6.00 & & \\ \text { PHOS } & \mathrm{mg} / \mathrm{L} & 13 & 7.21 \mathrm{E}-01 & <1.00 \mathrm{E}-02 & 11 & 1.46 \mathrm{E}-01 \\ \text { SE } & \mathrm{mg} / \mathrm{L} & 12 & <2.00 \mathrm{E}-02 & <5.00 \mathrm{E}-03 & & \\ \text { TMP } & \mathrm{Deg} \mathrm{C} & 359 & 29.50 & 8.00 & 359 & 19.53\end{array}$




\section{Table 65}

\section{National Pollutant Discharge Elimination System Monitoring Data}

Page 19 of 22

\begin{tabular}{lllllll}
\hline Measurement & Units & Freq/ & Year & Maximum & Minimum & No. in \\
Average & Average \\
\hline
\end{tabular}

Permit SC0000175

Outfall T-001 (facility in service but did not discharge)

Effluent consists of the following types of wastewater: artificial stream water.

$\begin{array}{llllll}\text { FLO } & \text { MGD } & 13 & 0.00 E+00 & 13 & 0.00 E+00\end{array}$

Outfall T-005 (facility in service but did not discharge)

Effluent consists of the following types of wastewater: cooling water, boiler blowdown, and neutralized acid solutions.

FLO

MGD $\quad 14$

$0.00 \mathrm{E}+00$

14

$0.00 E+00$

Outfall T-007

Effluent consists of the following types of wastewater: sanitary wastewater from B-Area.

$\begin{array}{lllllll}\text { BOD } & \mathrm{mg} / \mathrm{L} & 8 & 3.20 \mathrm{E}+00 & <1.00 \mathrm{E}+00 & 7 & 2.43 \mathrm{E}+00 \\ \mathrm{CHL} & \mathrm{mg} / \mathrm{L} & 8 & 3.95 \mathrm{E}+01 & 3.00 \mathrm{E}-02 & 7 & 1.59 \mathrm{E}+01 \\ \text { FEC } & \# / 100 \mathrm{~mL} & 6 & & 0 . & 1 & \\ \text { FLO } & \mathrm{MGD} & 151 & 2.88 \mathrm{E}-02 & 0.00 \mathrm{E}+00 & 151 & 7.90 \mathrm{E}-03 \\ \mathrm{PH} & \mathrm{pH} & 8 & 8.00 & 7.04 & & \\ \text { TSS } & \mathrm{mg} / \mathrm{L} & 8 & 5.20 \mathrm{E}+00 & <1.00 \mathrm{E}+00 & 7 & 2.16 \mathrm{E}+00\end{array}$

Outfall X-004

Effluent consists of the following types of wastewater: cooling water.

$\begin{array}{lllllll}\text { FLO } & \text { MGD } & 366 & 7.76 E-02 & 0.00 E+00 & 366 & 2.70 E-02 \\ \text { O\&G } & \mathrm{mg} / \mathrm{L} & 15 & 3.50 \mathrm{E}+00 & <1.00 \mathrm{E}+00 & 1 & 3.50 \mathrm{E}+00 \\ \mathrm{PH} & \mathrm{pH} & 15 & 7.00 & 6.40 & & \\ \text { TMP } & \mathrm{Deg} \mathrm{C} & 15 & 31.50 & 19.00 & 15 & 23.63 \\ \text { TSS } & \mathrm{mg} / \mathrm{L} & 15 & 6.00 \mathrm{E}+00 & <1.00 \mathrm{E}+00 & 13 & 2.99 \mathrm{E}+00\end{array}$

Outfall X-008

Effluent consists of the following types of wastewater. cooling water, cooling tower blowdown, and domestic water overflow.

$\mathrm{AL}$

$\mathrm{mg} / \mathrm{L} \quad 15$

$\mathrm{mg} / \mathrm{L} \quad 15$

$1.55 \mathrm{E}-01$

$<2.00 E-02 \quad 3$

1.11E-01

FE

1.79E+00

9.06E-01 15

1.15E+00

FLO

MGD $\quad 15$

3.60E-01

1.93E-01

2.72E-01

O\&G

$\mathrm{mg} / \mathrm{L} \quad 15$

$4.40 \mathrm{E}+00$

$<1.00 \mathrm{E}+00$

$4.40 \mathrm{E}+00$

$\mathrm{PH}$

$\mathrm{pH}$

7.00

6.00

TMP

Deg C 15

28.00

19.00

23.67

TSS

$\mathrm{mg} / \mathrm{L} \quad 15$

$1.00 E+01$

$1.10 \mathrm{E}+00$

15

$3.91 E+00$ 
Table 65

National Pollutant Discharge Elimination System Monitoring Data

Page 20 of 22

\begin{tabular}{llllll}
\hline Measurement & Units & $\begin{array}{l}\text { Freq/ } \\
\text { Year }\end{array}$ & Maximum & Minimum & $\begin{array}{c}\text { No. in } \\
\text { Average }\end{array}$ \\
\hline
\end{tabular}

Permit SC0000175

Outfall X-011

Effluent consists of the following types of wastewater: cooling water, cooling tower blowdown, and domestic water overflow.

FLO

7.20E-03

$0.00 \mathrm{E}+00$

12

$1.40 \mathrm{E}-03$

Outfall X-013 (replaced by Outfall X-008A, permitted by SC0044903)

Effluent consists of the following types of wastewater: sanitary wastewater.

Outfall $\mathrm{X}-014$

Effluent consists of the following types of wastewater: treated wastewater from TNX-ETP.

$\begin{array}{lllllll}\text { BEN } & \mathrm{ug} / \mathrm{L} & 46 & 5.49 \mathrm{E}+00 & <5.00 \mathrm{E}-01 & 1 & 5.49 \mathrm{E}+00 \\ \mathrm{BOD} & \mathrm{mg} / \mathrm{L} & 46 & 3.50 \mathrm{E}+01 & <1.00 \mathrm{E}+00 & 18 & 3.69 \mathrm{E}+00 \\ \mathrm{C} & \mathrm{mg} / \mathrm{L} & 42 & 8.70 \mathrm{E}+00 & <1.00 \mathrm{E}+00 & 41 & 4.10 \mathrm{E}+00 \\ \mathrm{FLO} & \mathrm{MGD} & 365 & 4.60 \mathrm{E}-02 & 0.00 \mathrm{E}+00 & 365 & 4.10 \mathrm{E}-03 \\ \mathrm{HG} & \mathrm{ug} / \mathrm{L} & 46 & 3.80 \mathrm{E}-01 & <1.00 \mathrm{E}-04 & 23 & 1.46 \mathrm{E}-01 \\ \mathrm{O \& G} & \mathrm{mg} / \mathrm{L} & 46 & 4.50 \mathrm{E}+00 & <1.00 \mathrm{E}+00 & 7 & 2.74 \mathrm{E}+00 \\ \mathrm{PH} & \mathrm{pH} & 100 & 8.03 & 6.80 & & \\ \mathrm{PHE} & \mathrm{mg} / \mathrm{L} & 46 & 7.70 \mathrm{E}-02 & <2.00 \mathrm{E}-03 & 10 & 2.62 \mathrm{E}-02 \\ \text { TSS } & \mathrm{mg} / \mathrm{L} & 46 & 3.90 \mathrm{E}+01 & 3.00 \mathrm{E}+00 & 46 & 9.65 \mathrm{E}+00\end{array}$

Outfall Y-001 (facility in service but did not discharge)

Effluent consists of the following types of wastewater: cooling water and locomotive wash water.

FLO

MGD $\quad 12$

$0.00 \mathrm{E}+00$

12

$0.00 E+00$ 


\section{Table 65}

\section{National Pollutant Discharge Elimination System Monitoring Data}

Page 21 of 22

\begin{tabular}{|c|c|c|c|c|c|c|c|}
\hline \multicolumn{2}{|c|}{ Measurement } & Units & $\begin{array}{l}\text { Freq } \\
\text { Year }\end{array}$ & Maximum & Minimum & $\begin{array}{c}\text { No. in } \\
\text { Average }\end{array}$ & Average \\
\hline \multicolumn{8}{|c|}{ Permit SC0044903 } \\
\hline Note: & \multicolumn{7}{|c|}{$\begin{array}{l}\text { Permit levels can be found in WSRC NPDES Environmental Monitoring Program (ESH-EMS-910110) o } \\
\text { in the permit itself. The permit is available from the South Carolina Department of Health and } \\
\text { Environmental Control. }\end{array}$} \\
\hline
\end{tabular}

Outfall A-001A

Effluent consists of the following types of wastewater: air stripper effluent.

$\begin{array}{lllllll}\text { FLO } & \text { MGD } & 366 & 9.07 \mathrm{E}-02 & 0.00 \mathrm{E}+00 & 366 & 5.97 \mathrm{E}-02 \\ \text { PERCL } & \mathrm{ug} / \mathrm{L} & 44 & 1.33 \mathrm{E}+01 & <2.00 \mathrm{E}+00 & 3 & 9.60 \mathrm{E}+00 \\ \text { PH } & \mathrm{pH} & 27 & 7.00 & 4.70 & & \\ \text { TRICL } & \mathrm{ug} / \mathrm{L} & 45 & 8.50 \mathrm{E}+00 & <2.00 \mathrm{E}+00 & 3 & 5.90 \mathrm{E}+00\end{array}$

Outfall A-028 (facility not yet in service) Effluent consists of the following types of wastewater: Powerhouse wastewater, water from floor drains, cooling water, and well flush water.

Outfall A-029 (facility not yet in service)

Effluent consists of the following types of wastewater: well flush water and tank overflow.

Outfall G-010 (new central sanitary treatment plant)

Effluent consists of the following types of wastewater: treated sanitary wastewater.

$\begin{array}{lllllll}\text { AN-S } & \mathrm{mg} / \mathrm{L} & 13 & 1.35 \mathrm{E}+00 & <5.00 \mathrm{E}-02 & 10 & 4.77 \mathrm{E}-01 \\ \text { AN-W } & \mathrm{mg} / \mathrm{L} & 4 & 5.32 \mathrm{E}-01 & <1.00 \mathrm{E}-01 & 3 & 4.77 \mathrm{E}-01 \\ \text { ATOX } & \text { PASS/FA } & 7 & & 0.00 \mathrm{E}+00 & 7 & 0.00 \mathrm{E}+00 \\ \text { BOD } & \mathrm{mg} / \mathrm{L} & 20 & 1.40 \mathrm{E}+01 & <1.00 \mathrm{E}+00 & 19 & 5.23 \mathrm{E}+00 \\ \text { CTOX } & \text { PASS/FA } & 7 & & 0.00 \mathrm{E}+00 & 7 & 0.00 \mathrm{E}+00 \\ \text { DO } & \mathrm{mg} / \mathrm{L} & 17 & 1.04 \mathrm{E}+01 & 5.80 \mathrm{E}+00 & 17 & 7.39 \mathrm{E}+00 \\ \text { FEC } & \# / 100 \mathrm{~mL} & 20 & 270 . & <2 . & 10 & 47 . \\ \text { FLO } & \mathrm{MGD} & 245 & 6.56 \mathrm{E}-01 & 0.00 \mathrm{E}+00 & 245 & 1.86 \mathrm{E}-01 \\ \text { PH } & \mathrm{pH} & 18 & 7.53 & 6.60 & & \\ \text { TSS } & \mathrm{mg} / \mathrm{L} & 23 & 5.50 \mathrm{E}+01 & 4.00 \mathrm{E}+00 & 23 & 1.44 \mathrm{E}+01\end{array}$

Outfall K-018 (formerly Outfall K-011)

Effluent consists of the following types of wastewater: neutralization, K-Reactor cooling tower blowdown, and reservoir wastewater.

$\begin{array}{lllllll}\mathrm{AL} & \mathrm{mg} / \mathrm{L} & 25 & 1.20 \mathrm{E}+00 & <5.00 \mathrm{E}-02 & 19 & 2.57 \mathrm{E}-01 \\ \mathrm{BOD} & \mathrm{mg} / \mathrm{L} & 25 & 1.40 \mathrm{E}+01 & <1.00 \mathrm{E}+00 & 19 & 2.71 \mathrm{E}+00 \\ \mathrm{CHL} & \mathrm{mg} / \mathrm{L} & 104 & <1.00 \mathrm{E}-01 & <1.00 \mathrm{E}-01 & & \end{array}$




\section{Table 65}

National Pollutant Discharge Elimination System Monitoring Data

Page 22 of 22

\begin{tabular}{lllllll}
\hline Measurement & Units & $\begin{array}{l}\text { Freql } \\
\text { Year }\end{array}$ & Maximum & Minimum & $\begin{array}{c}\text { No. in } \\
\text { Average }\end{array}$ & Average \\
\hline & & & & & & \\
Permit SC0044903 & & & & & & \\
\hline FEC & $\# / 100 \mathrm{~mL}$ & 25 & 110. & 2. & 24 & 26. \\
FLO & MGD & 365 & $3.68 \mathrm{E}+01$ & $1.81 \mathrm{E}+01$ & 365 & $2.25 \mathrm{E}+01$ \\
O\&G & $\mathrm{mg} / \mathrm{L}$ & 25 & $1.80 \mathrm{E}+00$ & $<1.00 \mathrm{E}+00$ & 1 & $1.80 \mathrm{E}+00$ \\
PH & $\mathrm{pH}$ & 365 & 7.10 & 5.80 & & \\
TMP & Deg C & 355 & 26.90 & 7.20 & 355 & 18.56 \\
TMPD & Deg C & 0 & & & & \\
TSS & $\mathrm{mg} / \mathrm{L}$ & 26 & $1.60 \mathrm{E}+01$ & $1.60 \mathrm{E}+00$ & 25 & $5.11 \mathrm{E}+00$
\end{tabular}

Outfall PP-004

Effluent consists of the following filter backwash wastewater.

$\begin{array}{lccllll}\text { FE } & \mathrm{mg} / \mathrm{L} & 12 & 6.16 \mathrm{E}-01 & <2.00 \mathrm{E}-02 & 8 & 3.77 \mathrm{E}-01 \\ \mathrm{FLO} & \mathrm{MGD} & 12 & 3.83 \mathrm{E}-02 & 8.70 \mathrm{E}-04 & 12 & 1.21 \mathrm{E}-02 \\ \mathrm{MN} & \mathrm{mg} / \mathrm{L} & 12 & 3.18 \mathrm{E}+02 & 3.44 \mathrm{E}+00 & 12 & 3.68 \mathrm{E}+01 \\ \mathrm{PH} & \mathrm{pH} & 12 & 6.70 & 6.00 & & \\ \text { TSS } & \mathrm{mg} / \mathrm{L} & 12 & 3.50 \mathrm{E}+00 & <1.00 \mathrm{E}+00 & 9 & 2.03 \mathrm{E}+00\end{array}$

Outfall $X-008 A$

Effluent consists of the following types of wastewater: sanitary wastewater.

$\begin{array}{lllllll}\text { BOD } & \mathrm{mg} / \mathrm{L} & 24 & 1.34 \mathrm{E}+01 & <1.00 \mathrm{E}+00 & 20 & 2.64 \mathrm{E}+00 \\ \text { FEC } & \# / 100 \mathrm{~mL} & 24 & 8 . & 0 . & 10 & 4 . \\ \text { FLO } & \text { MGD } & 365 & 2.14 \mathrm{E}-02 & 0.00 \mathrm{E}+00 & 365 & 6.50 \mathrm{E}-03 \\ \mathrm{PH} & \mathrm{pH} & 24 & 8.20 & 6.80 & & \\ \text { TSS } & \mathrm{mg} / \mathrm{L} & 24 & 1.10 \mathrm{E}+01 & <1.00 \mathrm{E}+00 & 23 & 4.22 \mathrm{E}+00\end{array}$


Table 66

National Pollutant Discharge Elimination System Stormwater Monitoring Data

Page 1 of 14

Measurement

Units

Type Sample/Concentration

Permit SCR000000

OIL \& GREASE

TSS

BOD

$\mathrm{NO} 2, \mathrm{NO} 3$

PO4-P

COD

TOC

TKN

$\mathrm{pH}$

AIR TEMP

WATER TEMP

RAIN GAUGE

TOTAL RAIN

RAIN DATE

LAST .01 RAIN

$\begin{array}{lccc}\begin{array}{c}\text { Background } \\ \text { Grab }\end{array} & \text { Composite } & \text { Grab } \\ \text { No Flow } & <1 & 37.8 \\ \text { No Flow } & 4.6 & 5.3 \\ \text { No Flow } & 3.2 & 0.22 \\ \text { No Flow } & 0.17 & 0.097 \\ \text { No Flow } & 0.082 & <10 \\ \text { No Flow } & <10 & 7.77 \\ \text { No Flow } & 6.08 & 0.4 \\ \text { No Flow } & 0.53 & 6.8 \\ \text { No Flow } & 7.1 & 8 \\ \text { No Flow } & & 6 \\ \text { No Flow } & & 0.24 \\ & & 1.23 \\ & 1.25 & 2 / 10 / 95 \\ & 1 / 14 / 95 & 2 / 7 / 95\end{array}$




\begin{tabular}{|c|c|c|c|c|c|c|c|}
\hline \multicolumn{8}{|c|}{$\begin{array}{l}\text { Table } 66 \\
\text { National Pollutant Discharge Elimination System Stormwater Monitoring Data } \\
\text { Page } 2 \text { of } 14\end{array}$} \\
\hline Measurement & Units & & & Type Sam & le/Concentra & & \\
\hline \multicolumn{8}{|l|}{ Permit SCR000000 } \\
\hline Outfall CS-006 & & $\begin{array}{c}\text { Backgrour } \\
\text { Grab }\end{array}$ & & Composite & Composite & Grab & \\
\hline OIL \& GREASE & $\mathrm{mg} / \mathrm{L}$ & $<1$ & & & $<1$ & & \\
\hline TSS & $\mathrm{mg} / \mathrm{L}$ & 4 & 4.2 & 6.7 & 48.5 & & \\
\hline BOD & $\mathrm{mg} / \mathrm{L}$ & $<1$ & $<1$ & 3.1 & 4.6 & & \\
\hline $\mathrm{NO} 2, \mathrm{NO} 3$ & $\mathrm{mg} / \mathrm{L}$ & 0.02 & 0.05 & 0.03 & 0.16 & & \\
\hline PO4-P & $\mathrm{mg} / \mathrm{L}$ & 0.043 & 0.142 & 0.042 & 0.02 & & \\
\hline COD & $\mathrm{mg} / \mathrm{L}$ & $<10$ & $<10$ & 11.4 & 19.5 & & \\
\hline TOC & $\mathrm{mg} / \mathrm{L}$ & 4.26 & 3.78 & 5 & 7.33 & & \\
\hline TKN & $\mathrm{mg} / \mathrm{L}$ & 0.36 & $<0.2$ & $<0.2$ & 0.48 & & \\
\hline PHENOL & $\mathrm{mg} / \mathrm{L}$ & $<0.002$ & & & 0.007 & & \\
\hline TETRACHLOROETHYLENE & $\mu \mathrm{g} / \mathrm{L}$ & $<2$ & & & $<2$ & & \\
\hline TRICHLOROETHYLENE & $\mu g / L$ & $<2$ & & & $<2$ & & \\
\hline 1,1,1-TRICHLOROETHYLENE & $\mu \mathrm{g} / \mathrm{L}$ & $<2$ & & & $<2$ & & \\
\hline BENZENE & $\mathrm{mg} / \mathrm{L}$ & $<0.8$ & & & $<0.8$ & & \\
\hline AL & $\mathrm{mg} / \mathrm{L}$ & 0.459 & 0.6 & 0.285 & 2.89 & & \\
\hline CR & $\mathrm{mg} / \mathrm{L}$ & $<0.005$ & $<0.005$ & $<0.005$ & $<0.005$ & & \\
\hline $\mathrm{CU}$ & $\mathrm{mg} / \mathrm{L}$ & $<0.005$ & $<0.005$ & $<0.005$ & 0.0094 & & \\
\hline FE & $\mathrm{mg} / \mathrm{L}$ & 1.06 & 0.98 & 1.17 & 4.32 & & \\
\hline PB & $\mathrm{mg} / \mathrm{L}$ & $<0.003$ & $<0.003$ & $<0.003$ & 0.0068 & & \\
\hline
\end{tabular}




\section{Table 66}

National Pollutant Discharge Elimination System Stormwater Monitoring Data

$\begin{array}{lll}\text { Measurement } \quad \text { Units } & \text { Type Sample/Concentration }\end{array}$

Permit SCR000000

MG

NI

$A G$

ZN

$\mathrm{pH}$

AIR TEMP

WATER TEMP

RAIN GAUGE

TOTAL RAIN

RAIN DATE

LAST .01 RAIN

Outfall CS-12A

OIL \& GREASE

TSS

BOD

NO2,NO3

PO4-P

COD

TOC $\mathrm{mg} / \mathrm{L}$

$\mathrm{mg} / \mathrm{L}$

$\mathrm{mg} / \mathrm{L}$

$\mathrm{mg} / \mathrm{L}$

$\mathrm{pH}$

${ }^{\circ} \mathrm{C}$

${ }^{\circ} \mathrm{C}$

in.

in.

$1 / 12 / 95$
$1 / 6 / 95$

0.4

2/15/95

$2 / 11 / 95$

Background

$$
\text { Grab }
$$

$\mathrm{mg} / \mathrm{L}$

$\mathrm{mg} / \mathrm{L}$

No Flow

$\mathrm{mg} / \mathrm{L} \quad$ No Flow

$\mathrm{mg} / \mathrm{L} \quad$ No Flow

$\mathrm{mg} / \mathrm{L} \quad$ No Flow

$\mathrm{mg} / \mathrm{L} \quad$ No Flow

$\mathrm{mg} / \mathrm{L} \quad$ No Flow

Type Sample/Concentration

$\begin{array}{cccc} & \begin{array}{c}\text { Composite } \\ <1\end{array} & \text { Grab } & \text { Composite } \\ 43.8 & 768 & 52.5 & \\ 5.8 & 3.9 & 3.7 & \\ 0.96 & 0.24 & 0.5 & \\ 0.106 & 3.14 & 0.119 & \\ 35.1 & 167 & <10 & \\ 5.04 & 6.16 & & \end{array}$


Table 66

National Pollutant Discharge Elimination System Stormwater Monitoring Data

Page 4 of 14

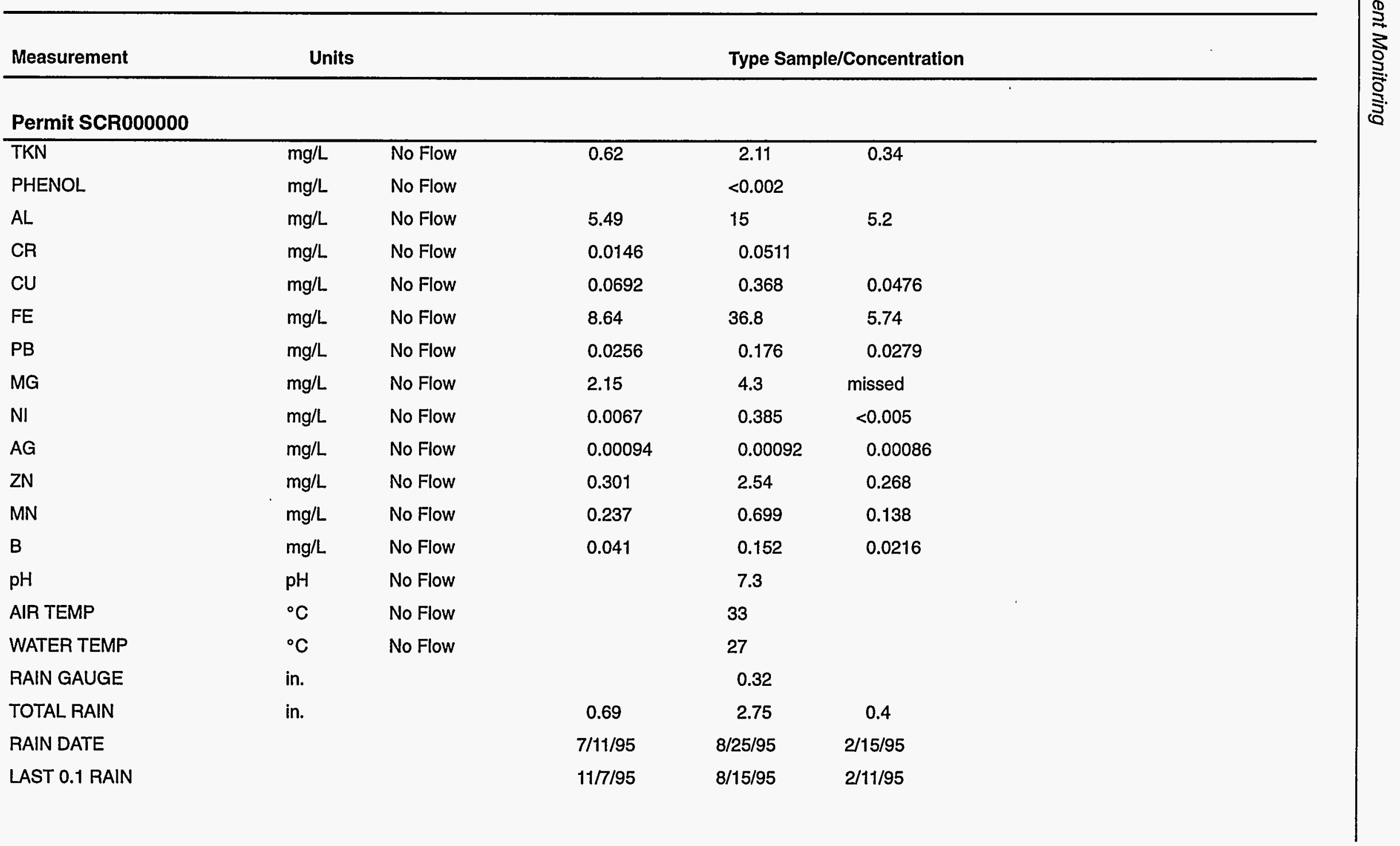


Table 66

National Pollutant Discharge Elimination System Stormwater Monitoring Data

Page 5 of 14

\begin{tabular}{lll} 
Measurement & Units & Type Sample/Concentration \\
\hline
\end{tabular}

\section{Permit SCR000000}

\section{Outfall E-001}

OIL \& GREASE

TSS

BOD

TDS

NH3-N

$\mathrm{NO} 2, \mathrm{NO} 3$

PO4-P

COD

TOC

TKN

' PHENOL

SO4

BROMIDE

$\mathrm{CN}$

$A G$

AL

AS

$B$

BA

$\begin{array}{lcc} & \text { Grab } & \text { Grab } \\ \mathrm{mg} / \mathrm{L} & <1 & <1 \\ \mathrm{mg} / \mathrm{L} & 8.3 & 20 \\ \mathrm{mg} / \mathrm{L} & 2.1 & 2.1 \\ \mathrm{mg} / \mathrm{L} & 39 & <10 \\ \mathrm{mg} / \mathrm{L} & <0.05 & 0.127 \\ \mathrm{mg} / \mathrm{L} & 0.07 & <0.02 \\ \mathrm{mg} / \mathrm{L} & 0.061 & 0.313 \\ \mathrm{mg} / \mathrm{L} & <10 & 199 \\ \mathrm{mg} / \mathrm{L} & 2.92 & 3.3 \\ \mathrm{mg} / \mathrm{L} & 0.45 & 0.426 \\ \mathrm{mg} / \mathrm{L} & <0.002 & <0.006 \\ \mathrm{mg} / \mathrm{L} & 8.1 & 5.33 \\ \mathrm{mg} / \mathrm{L} & <2 & <2 \\ \mathrm{mg} / \mathrm{L} & <0.01 & <0.01 \\ \mathrm{mg} / \mathrm{L} & <0.0005 & <0.005 \\ \mathrm{mg} / \mathrm{L} & 0.677 & 1.31 \\ \mathrm{mg} / \mathrm{L} & <0.005 & <0.02 \\ \mathrm{mg} / \mathrm{L} & 0.014 & 0.057 \\ \mathrm{mg} / \mathrm{L} & 0.0146 & 0.022 \\ & & \\ & & \end{array}$




\begin{tabular}{|c|c|c|c|c|}
\hline \multicolumn{5}{|c|}{$\begin{array}{l}\text { Table } 66 \\
\text { National Pollutant Discharge Elimination System Stormwater Monitoring Data }\end{array}$} \\
\hline Measurement & Units & & Type Sample/Concentration & \\
\hline \multicolumn{5}{|l|}{ Permit SCR000000 } \\
\hline $\mathrm{CD}$ & $\mathrm{mg} / \mathrm{L}$ & $<0.005$ & $<0.005$ & \\
\hline CR & $\mathrm{mg} / \mathrm{L}$ & $<0.005$ & $<0.01$ & \\
\hline $\mathrm{CU}$ & $\mathrm{mg} / \mathrm{L}$ & $<0.005$ & $<0.005$ & \\
\hline $\mathrm{FE}$ & $\mathrm{mg} / \mathrm{L}$ & 0.75 & 1.93 & \\
\hline$H G$ & $\mathrm{mg} / \mathrm{L}$ & $<0.0001$ & 0.0004 & \\
\hline MG & $\mathrm{mg} / \mathrm{L}$ & 1.01 & 0.931 & \\
\hline MN & $\mathrm{mg} / \mathrm{L}$ & 0.047 & 0.117 & \\
\hline NI & $\mathrm{mg} / \mathrm{L}$ & $<0.005$ & $<0.01$ & \\
\hline PB & $\mathrm{mg} / \mathrm{L}$ & $<0.003$ & $<0.02$ & \\
\hline SB & $\mathrm{mg} / \mathrm{L}$ & $<0.005$ & $<0.02$ & \\
\hline SE & $\mathrm{mg} / \mathrm{L}$ & $<0.005$ & $<0.2$ & \\
\hline SN & $\mathrm{mg} / \mathrm{L}$ & $<0.005$ & $<0.5$ & \\
\hline$u$ & $\mathrm{mg} / \mathrm{L}$ & $<0.02$ & $<0.5$ & \\
\hline $\mathrm{ZN}$ & $\mathrm{mg} / \mathrm{L}$ & 0.0101 & 0.01 & \\
\hline MG (DISSOLVED) & $\mathrm{mg} / \mathrm{L}$ & & 0.794 & \\
\hline TETRACHLOROETHYLENE & $\mu \mathrm{g} / \mathrm{L}$ & $<2$ & $<2$ & \\
\hline TRICHLOROETHYLENE & $\mu \mathrm{g} / \mathrm{L}$ & $<2$ & $<2$ & \\
\hline 1,1,1-TRICHLOROETHYLENE & $\mu \mathrm{g} / \mathrm{L}$ & $<2$ & $<2$ & \\
\hline $\mathrm{pH}$ & $\mathrm{pH}$ & 6.2 & 7 & \\
\hline AIR TEMP & ${ }^{\circ} \mathrm{C}$ & 27 & 23 & \\
\hline WATER TEMP & ${ }^{\circ} \mathrm{C}$ & 31 & 24 & \\
\hline
\end{tabular}


Table 66

National Pollutant Discharge Elimination System Stormwater Monitoring Data

Page 7 of 14

\begin{tabular}{|c|c|c|c|c|c|c|c|c|}
\hline Measurement & Units & & & Type Sam & Concentra & & & \\
\hline \multicolumn{9}{|c|}{ Permit SCR000000 } \\
\hline RAIN GAUGE & in. & 0.21 & 0.43 & & {[} & & & \\
\hline TOTAL RAIN & in. & 2 & 0.22 & & & & & \\
\hline RAIN DATE & & $7 / 26 / 95$ & $9 / 14 / 95$ & & & & . & - \\
\hline LAST 0.1 RAIN & & $7 / 23 / 95$ & $9 / 7 / 95$ & & & & & \\
\hline Outfall GS-002 & & $\begin{array}{c}\text { Background } \\
\text { Grab }\end{array}$ & & Composite & Grab & Grab & Composite & \\
\hline O\&G & $\mathrm{mg} / \mathrm{L}$ & No Flow & & $<1$ & $<1$ & & & \\
\hline BOD & $\mathrm{mg} / \mathrm{L}$ & & 1.5 & $<1$ & $<1$ & 1.6 & & \\
\hline TSS & $\mathrm{mg} / \mathrm{L}$ & & 5.5 & 6.1 & 7.6 & 25.2 & & \\
\hline SO4 & $\mathrm{mg} / \mathrm{L}$ & & 3.1 & 1.4 & 7.1 & 4.9 & & \\
\hline NH3-N & $\mathrm{mg} / \mathrm{L}$ & & 0.06 & $<0.05$ & 0.06 & $<0.05$ & & \\
\hline $\mathrm{NO} 2, \mathrm{NO} 3$ & $\mathrm{mg} / \mathrm{L}$ & & 0.39 & 0.06 & 0.71 & 0.24 & & \\
\hline PO4-P & $\mathrm{mg} / \mathrm{L}$ & & 0.054 & 0.028 & 0.447 & 0.131 & & \\
\hline COD & $\mathrm{mg} / \mathrm{L}$ & & $<10$ & $<10$ & 16.6 & & & \\
\hline TOC & $\mathrm{mg} / \mathrm{L}$ & & 1.75 & $<1$ & 1.49 & & & \\
\hline TKN & $\mathrm{mg} / \mathrm{L}$ & & 0.23 & $<0.2$ & 0.87 & 0.29 & & \\
\hline SB & $\mathrm{mg} / \mathrm{L}$ & & $<0.01$ & $<0.005$ & $<0.005$ & 0.0089 & & \\
\hline $\mathrm{CR}$ & $\mathrm{mg} / \mathrm{L}$ & & $<0.005$ & $<0.005$ & 0.0105 & 0.0144 & & \\
\hline $\mathrm{CU}$ & $\mathrm{mg} / \mathrm{L}$ & & 0.0099 & $<0.005$ & 0.0452 & 0.049 & & \\
\hline$H G$ & $\mathrm{mg} / \mathrm{L}$ & & $<0.0001$ & $<0.0001$ & $<0.0001$ & $<0.0001$ & & \\
\hline
\end{tabular}


Table 66

National Pollutant Discharge Elimination System Stormwater Monitoring Data

Page 8 of 14

\begin{tabular}{|c|c|c|c|c|c|c|c|}
\hline Permit SCR0000 & \multicolumn{2}{|l|}{ Units } & \multicolumn{4}{|c|}{ Type Sample/Concentration } & \\
\hline $\mathrm{NI}$ & $\mathrm{mg} / \mathrm{L}$ & & $<0.005$ & $<0.005$ & $<0.005$ & $<0.005$ & \\
\hline PB & $\mathrm{mg} / \mathrm{L}$ & & 0.147 & 0.0636 & 1.2 & 0.612 & \\
\hline SN & $\mathrm{mg} / \mathrm{L}$ & & $<0.005$ & $<0.005$ & $<0.005$ & $<0.005$ & \\
\hline $\mathrm{pH}$ & $\mathrm{pH}$ & & 7 & 4.4 & 7 & & \\
\hline AIR TEMP & ${ }^{\circ} \mathrm{C}$ & & & 11 & 10 & & \\
\hline WATER TEMP & ${ }^{\circ} \mathrm{C}$ & & & 9 & 8 & & \\
\hline RAIN GAUGE & in. & & & 0.26 & 0.12 & & \\
\hline TOTAL RAIN & in. & & 0.15 & 1.23 & 0.7 & 0.7 & \\
\hline RAIN DATE & & & $1 / 19 / 95$ & $2 / 10 / 95$ & 2/15/95 & $2 / 15 / 95$ & \\
\hline LAST 0.1 RAIN & & & $1 / 14 / 95$ & $2 / 7 / 95$ & $2 / 11 / 95$ & $2 / 11 / 95$ & \\
\hline Outfall H-007A & & $\begin{array}{l}\text { Background } \\
\text { Grab }\end{array}$ & & Composite & Grab & Composite & Grab \\
\hline O\&G & $\mathrm{mg} / \mathrm{L}$ & No Flow & & $<1$ & & 1.1 & \\
\hline BOD & $\mathrm{mg} / \mathrm{L}$ & & 2.8 & 1.3 & 2.8 & 3.4 & \\
\hline TSS & $\mathrm{mg} / \mathrm{L}$ & & 18.7 & 13.5 & 71.5 & 15.9 & \\
\hline $\mathrm{NO} 2, \mathrm{NO} 3$ & $\mathrm{mg} / \mathrm{h}$ & & 0.57 & 0.81 & 0.32 & 1.11 & \\
\hline PO4-P & $\mathrm{mg} / \mathrm{L}$ & & 0.172 & 0.089 & 0.153 & 0.111 & \\
\hline COD & $\mathrm{mg} / \mathrm{L}$ & & 13.8 & $<10$ & $<10$ & 17.3 & \\
\hline TOC & $\mathrm{mg} / \mathrm{L}$ & & 3.4 & 4.57 & 2.11 & 7.2 & \\
\hline TKN & $\mathrm{mg} / \mathrm{L}$ & & $<0.2$ & 0.44 & 0.26 & 0.4 & \\
\hline
\end{tabular}


Table 66

National Pollutant Discharge Elimination System Stormwater Monitoring Data

Page 9 of 14

\begin{tabular}{|c|c|c|c|c|c|c|c|c|}
\hline Measurement & Units & & & Type Sa & le/Concentra & & & \\
\hline \multicolumn{9}{|c|}{ Permit SCR000000 } \\
\hline SO4 & $\mathrm{mg} / \mathrm{L}$ & & 8.1 & 13.4 & 11.4 & 17.4 & & \\
\hline$A L$ & $\mathrm{mg} / \mathrm{L}$ & & 1.73 & 1.99 & 5.62 & 2.32 & & \\
\hline FE & $\mathrm{mg} / \mathrm{L}$ & & 2.19 & 1.71 & 7.58 & 2.89 & & \\
\hline $\mathrm{pH}$ & $\mathrm{pH}$ & & & 6.9 & & 6.4 & & \\
\hline AIR TEMP & ${ }^{\circ} \mathrm{C}$ & & & 10 & & 29 & & \\
\hline WATER TEMP & ${ }^{\circ} \mathrm{C}$ & & & 11 & & 26 & & \\
\hline RAIN GAUGE & in. & & & 0.11 & & & & \\
\hline TOTAL RAIN & in. & & 1 & 0.5 & 0.5 & 0.27 & & \\
\hline RAIN DATE & & & $1 / 28 / 95$ & 2/15/95 & $2 / 15 / 95$ & $8 / 2 / 95$ & & \\
\hline LAST 0.1 RAIN & & & $1 / 23 / 95$ & $2 / 11 / 945$ & $2 / 11 / 95$ & $7 / 28 / 95$ & & \\
\hline Outfall G-020 & & Background & Composite & Grab & Composite & Grab & Composite & Composite \\
\hline O\&G & $\mathrm{mg} / \mathrm{L}$ & No Flow & & & & & & \\
\hline $\mathrm{BOD}$ & $\mathrm{mg} / \mathrm{L}$ & & & & & & 3.4 & 7.4 \\
\hline TSS & $\mathrm{mg} / \mathrm{L}$ & & 28.2 & & 63.5 & & & \\
\hline $\mathrm{NO} 2, \mathrm{NO} 3$ & $\mathrm{mg} / \mathrm{L}$ & & 0.03 & & 0.11 & & & \\
\hline PO4-P & $\mathrm{mg} / \mathrm{L}$ & & 0.137 & & 0.063 & & & \\
\hline TKN & $\mathrm{mg} / \mathrm{L}$ & & 0.8 & & 0.85 & & & \\
\hline COD & $\mathrm{mg} / \mathrm{L}$ & & 84.3 & & 93 & & & \\
\hline TOC & $\mathrm{mg} / \mathrm{L}$ & & 24.5 & & 39.7 & & & \\
\hline PHENOL & $\mathrm{mg} / \mathrm{L}$ & & & & & & & \\
\hline
\end{tabular}




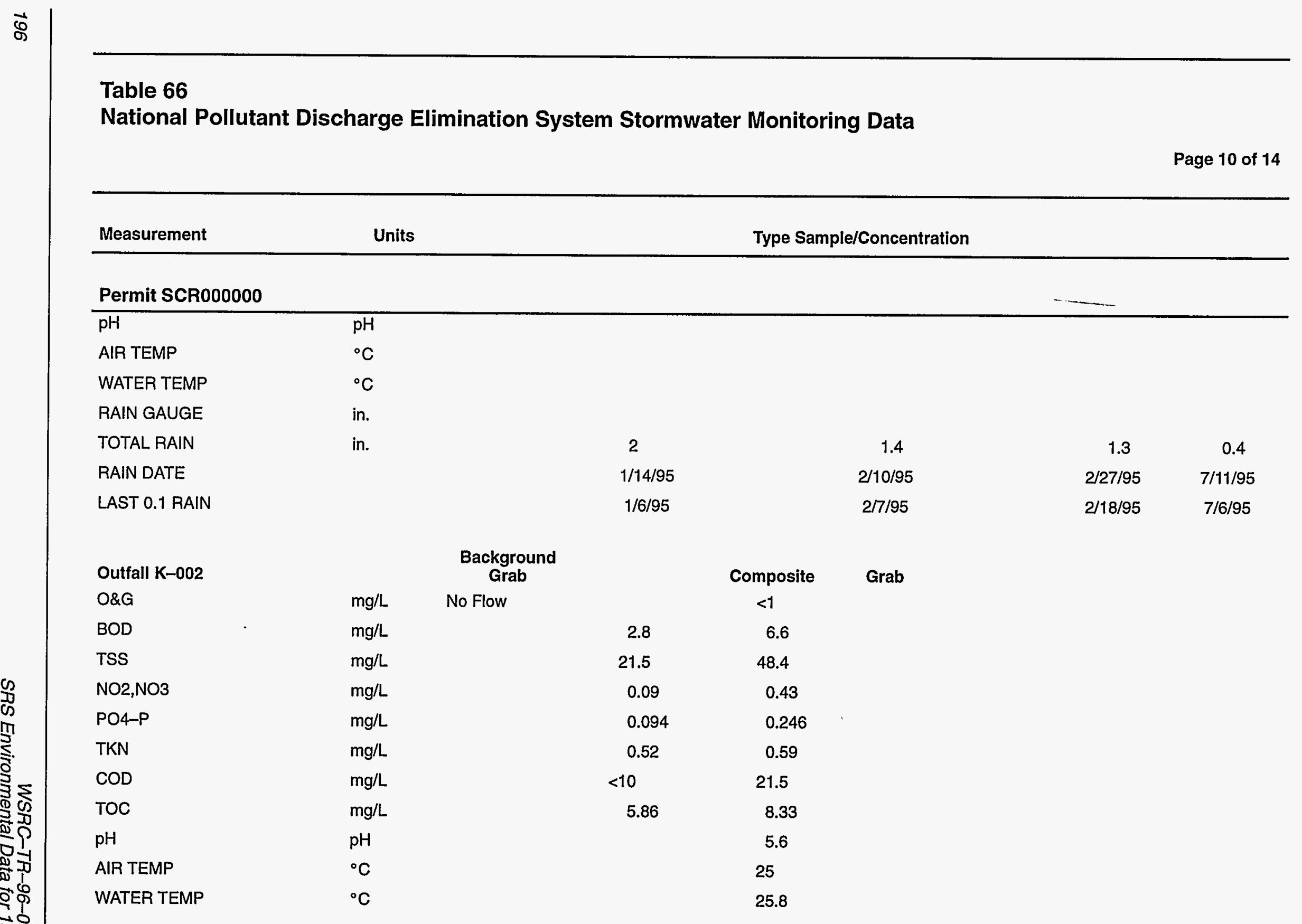


Table 66

National Pollutant Discharge Elimination System Stormwater Monitoring Data

Page 11 of 14

\begin{tabular}{|c|c|c|c|c|c|c|}
\hline \multirow[t]{2}{*}{ Measurement } & \multicolumn{2}{|l|}{ Units } & \multicolumn{4}{|c|}{ Type Sample/Concentration } \\
\hline & & & & & & \\
\hline RAIN GAUGE & in. & & & 0.68 & & \\
\hline TOTAL RAIN & in. & & 1.73 & 1.5 & & \\
\hline RAIN DATE & & & 1/14/95 & $7 / 26 / 95$ & & \\
\hline LAST 0.1 RAIN & & & $1 / 6 / 95$ & $7 / 23 / 95$ & & \\
\hline Outfall K-004 & & $\begin{array}{l}\text { Background } \\
\text { Grab }\end{array}$ & & Composite & Composite & Grab \\
\hline$O \& G$ & $\mathrm{mg} / \mathrm{h}$ & No Flow. & & & $<1$ & \\
\hline BOD & $\mathrm{mg} / \mathrm{L}$ & & & 2.1 & 4.9 & \\
\hline TSS & $\mathrm{mg} / \mathrm{h}$ & & & 7.5 & 55.2 & \\
\hline $\mathrm{NO2,NO3}$ & $\mathrm{mg} / \mathrm{L}$ & & 0.14 & & 0.41 & \\
\hline PO4-P & $\mathrm{mg} / \mathrm{L}$ & & 1 & & 0.251 & \\
\hline TKN & $\mathrm{mg} / \mathrm{L}$ & & 1.6 & & 0.34 & \\
\hline COD & $\mathrm{mg} / \mathrm{L}$ & & 51 & & 14.9 & \\
\hline TOC & $\mathrm{mg} / \mathrm{L}$ & & 6.98 & & 9.2 & \\
\hline $\mathrm{pH}$ & $\mathrm{pH}$ & & & & 6.3 & \\
\hline AIR TEMP & ${ }^{\circ} \mathrm{C}$ & & & & 25 & \\
\hline WATER TEMP & ${ }^{\circ} \mathrm{C}$ & & & & 26 & \\
\hline RAIN GAUGE & in. & & & & 0.6 & \\
\hline TOTAL RAIN & in. & & 1.71 & 0.7 & 1.5 & \\
\hline RAIN DATE & & & 1/14/95 & $1 / 28 / 95$ & $7 / 26 / 95$ & \\
\hline
\end{tabular}




\begin{tabular}{|c|c|c|c|c|c|c|}
\hline \multicolumn{7}{|c|}{$\begin{array}{l}\text { Table } 66 \\
\text { National Pollutant Discharge Elimination System Stormwater Monitoring Data }\end{array}$} \\
\hline Measurement & Units & & & Type Sam & /Concentration & \\
\hline \multicolumn{7}{|c|}{ Permit SCR000000 } \\
\hline LAST 0.1 RAIN & & & $1 / 6 / 95$ & $1 / 23 / 95$ & $7 / 23 / 95$ & \\
\hline Outfall S-005 & & $\begin{array}{l}\text { Backgrouno } \\
\text { Grab }\end{array}$ & & Grab & Grab & \\
\hline O\&G & $\mathrm{mg} / \mathrm{L}$ & $<1$ & & $<1$ & & \\
\hline BOD & $\mathrm{mg} / \mathrm{L}$ & 2.1 & 3.8 & 1.9 & & \\
\hline TSS & $\mathrm{mg} / \mathrm{L}$ & 58 & 144 & 16.2 & & \\
\hline $\mathrm{NO2,NO3}$ & $\mathrm{mg} / \mathrm{L}$ & 0.83 & 0.18 & 0.04 & & \\
\hline PO4-P & $\mathrm{mg} / \mathrm{L}$ & 0.288 & 0.328 & 0.1 & & \\
\hline COD & $\mathrm{mg} / \mathrm{L}$ & $<10$ & 14.4 & $<10$ & & \\
\hline TOC & $\mathrm{mg} / \mathrm{L}$ & 2.82 & 8.33 & & & \\
\hline TKN & $\mathrm{mg} / \mathrm{L}$ & 0.65 & 1.08 & 0.27 & & \\
\hline SO4 & $\mathrm{mg} / \mathrm{L}$ & 6.7 & 8.82 & 9.4 & & \\
\hline $\mathrm{NH} 3-\mathrm{N}$ & $\mathrm{mg} / \mathrm{L}$ & $<0.05$ & 0.43 & $<0.05$ & & \\
\hline PHENOL & $\mathrm{mg} / \mathrm{L}$ & $<0.002$ & $<0.002$ & 0.002 & & \\
\hline BENZENE & $\mathrm{mg} / \mathrm{L}$ & $<0.8$ & $<0.8$ & $<0.8$ & & \\
\hline B & $\mathrm{mg} / \mathrm{L}$ & $<0.03$ & 0.0404 & 0.0122 & & \\
\hline $\mathrm{CR}$ & $\mathrm{mg} / \mathrm{L}$. & 0.0099 & $<0.005$ & $<0.005$ & & \\
\hline $\mathrm{CU}$ & $\mathrm{mg} / \mathrm{L}$ & 0.009 & 0.019 & 0.0076 & & \\
\hline$H G$ & $\mathrm{mg} / \mathrm{L}$ & $<0.0001$ & $<0.0001$ & $<0.0001$ & & \\
\hline PB & $\mathrm{mg} / \mathrm{L}$ & 0.0128 & 0.0614 & 0.0193 & & \\
\hline
\end{tabular}


Table 66

National Pollutant Discharge Elimination System Stormwater Monitoring Data

Page 13 of 14

\begin{tabular}{|c|c|c|c|c|c|c|c|}
\hline Measurement & \multicolumn{2}{|c|}{ Units } & \multicolumn{4}{|c|}{ Type Sample/Concentration } & \\
\hline$\overline{Z N}$ & $\mathrm{mg} / \mathrm{L}$ & 0.136 & 0.149 & 0.0572 & & & \\
\hline $\mathrm{pH}$ & $\mathrm{pH}$ & 6.8 & 5.7 & 6.6 & & & \\
\hline AIR TEMP & ${ }^{\circ} \mathrm{C}$ & 16 & 25 & 29 & & & \\
\hline WATER TEMP & ${ }^{\circ} \mathrm{C}$ & 11 & 26.5 & 24 & & & \\
\hline RAIN GAUGE & in. & & 0.45 & & & & \\
\hline TOTAL RAIN & in. & & 1.4 & 0.3 & & & \\
\hline RAIN DATE & & & $7 / 26 / 95$ & $8 / 2 / 95$ & & & \\
\hline LAST 0.1 RAIN & & $1 / 6 / 95$ & 7/23/95 & $7 / 28 / 95$ & & & \\
\hline Outfall X-001 & & $\begin{array}{l}\text { Background } \\
\text { Grab }\end{array}$ & & Composite & Grab & Composite & Grab \\
\hline O\&G & $\mathrm{mg} / \mathrm{L}$ & No Flow & & $<1$ & & & \\
\hline BOD & $\mathrm{mg} / \mathrm{L}$ & & 4.8 & 3.9 & 6.8 & & \\
\hline TSS & $\mathrm{mg} / \mathrm{L}$ & & 74 & 162 & 19.4 & & \\
\hline NO2,NO3 & $\mathrm{mg} / \mathrm{L}$ & & 0.36 & $\widehat{0.41}$ & 1.49 & & \\
\hline PO4-P & $\mathrm{mg} / \mathrm{L}$ & & 0.106 & 0.052 & 0.291 & & \\
\hline TKN & $\mathrm{mg} / \mathrm{L}$ & & 0.46 & 0.47 & 0.4 & & \\
\hline COD & mg/L & & 14.3 & 17.8 & 13 & & \\
\hline TOC & $\mathrm{mg} / \mathrm{L}$ & & & 4.22 & 7.5 & & \\
\hline PHENOL & $\mathrm{mg} / \mathrm{L}$ & & & $<0.002$ & & & \\
\hline BENZENE & $\mathrm{mg} / \mathrm{L}$ & & & $<0.8$ & & & \\
\hline
\end{tabular}




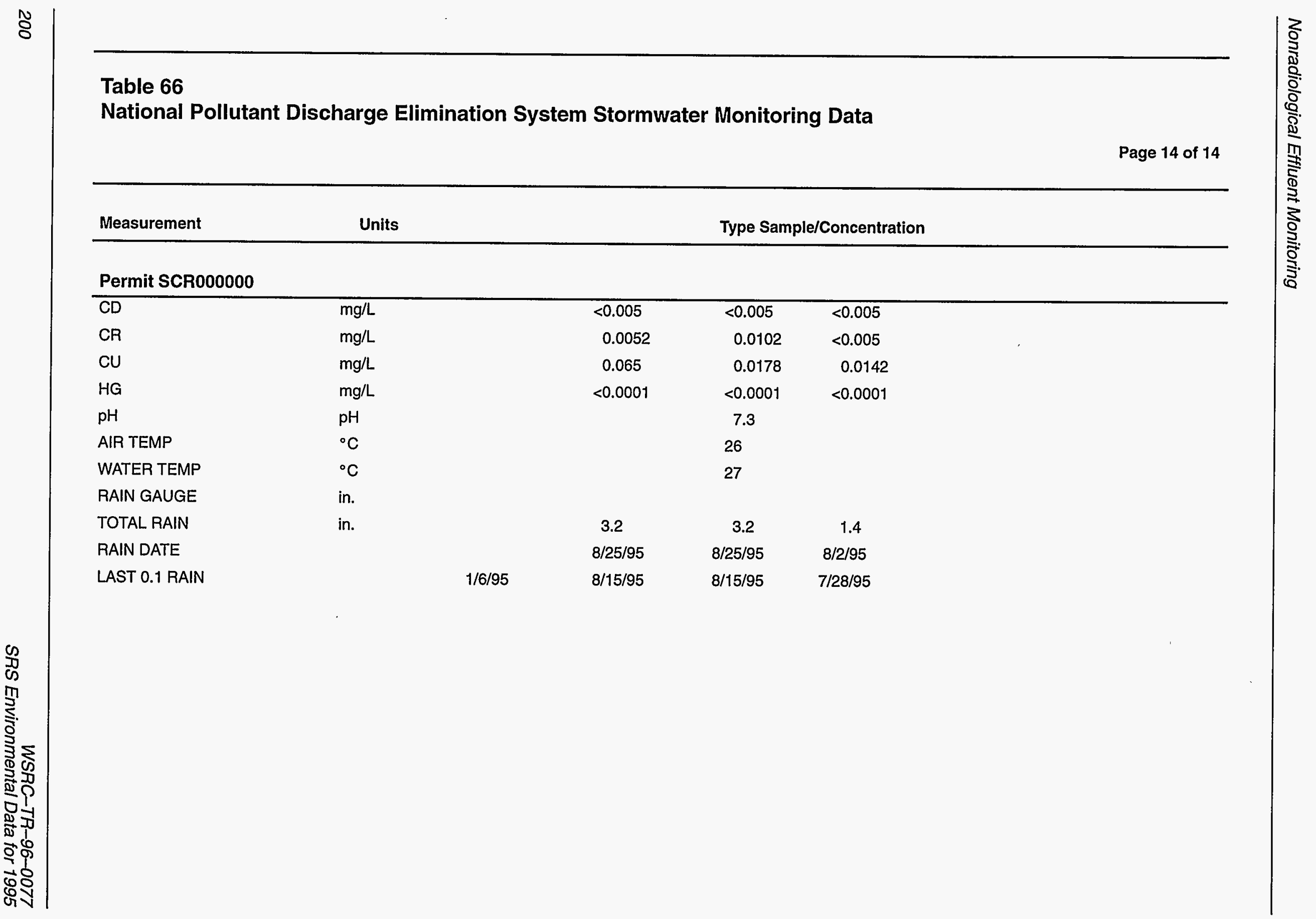




\section{Table 67}

SRS Stream Water Quality: Inorganic Contaminants

Page 1 of 20

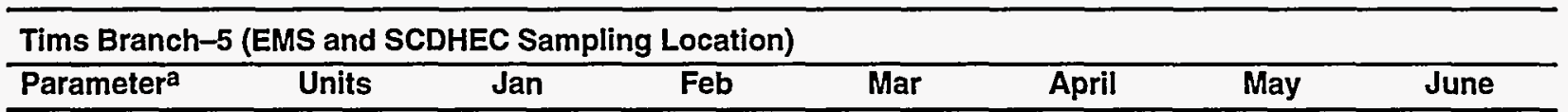

Notes: 1. "ND" denotes "not detected"

2. Fecal coliform results can be found in table 72 on page 232 .

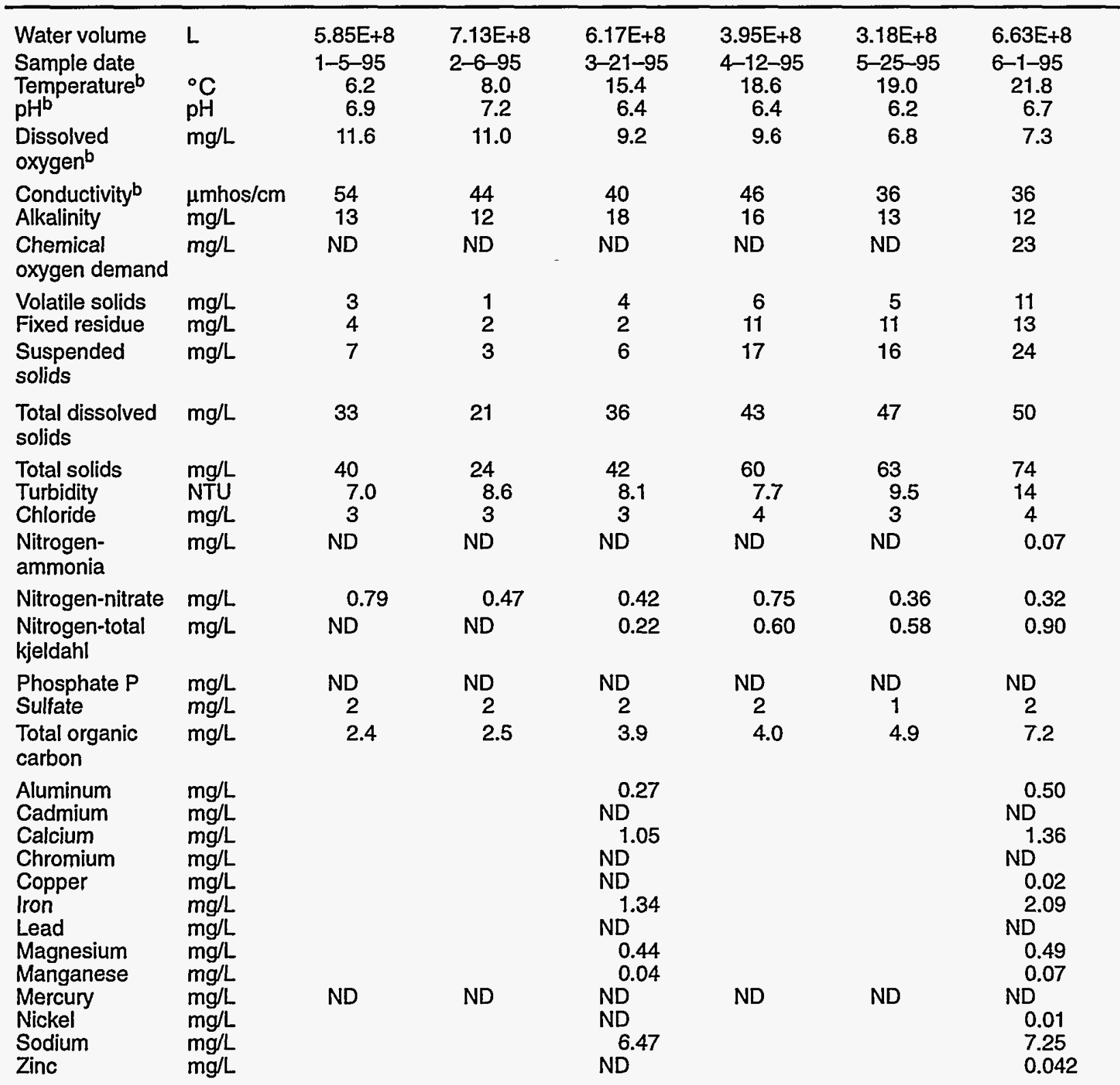

Metals (except mercury) are analyzed quarterly from monthly grab composites. Mercury is analyzed from a composite of
monthly grab samples.

b Field measurement 
Table 67

SRS Stream Water Quality: Inorganic Contaminants

Page 2 of 20

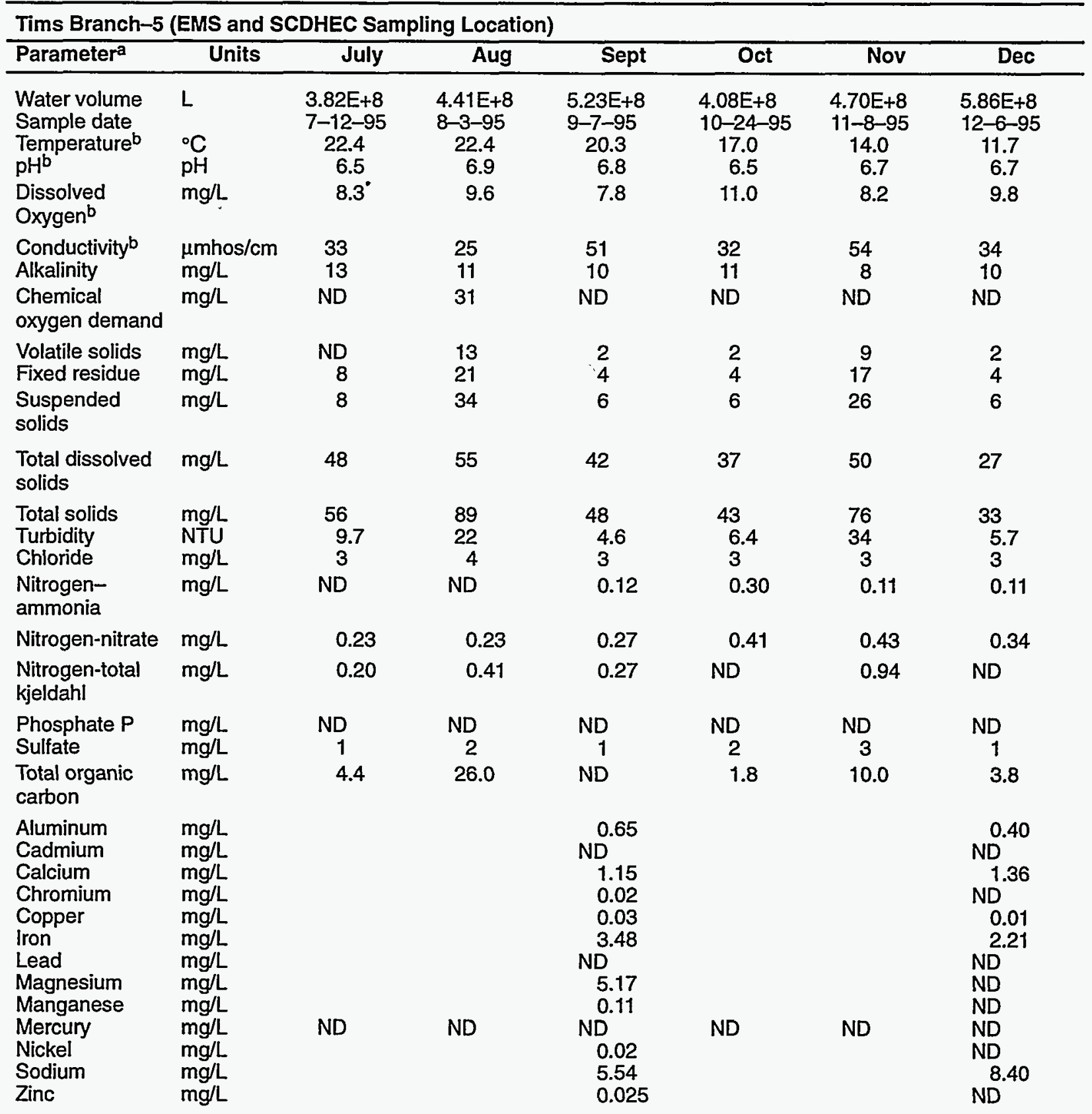

\footnotetext{
a Metals (except mercury) are analyzed quarterly from monthly grab composite. Mercury is analyzed from a composite of monthly grab samples.
}

b Field measurement 
Table 67

SRS Stream Water Quality: Inorganic Contaminants

Page 3 of 20

\begin{tabular}{|c|c|c|c|c|c|c|c|}
\hline \multicolumn{8}{|c|}{ Steel Creek-4 at Road A (EMS and SCDHEC Sampling Location) } \\
\hline Parameter ${ }^{a}$ & Units & Jan & Feb & Mar & April & May & June \\
\hline Water volume & $L$ & $7.48 E+9$ & $4.61 E+9$ & $5.42 E+9$ & $5.45 E+9$ & $5.44 E+9$ & $6.44 E+9$ \\
\hline Sample date & & $1-5-95$ & $2-7-95$ & $3-21-95$ & $4-11-95$ & $5-25-95$ & $6-1-95$ \\
\hline Temperature ${ }^{b}$ & ${ }^{\circ} \mathrm{C}$ & 9.9 & 10.2 & 14.5 & 19.0 & 23.0 & 23.0 \\
\hline $\mathrm{pH}^{\mathrm{b}}$ & $\mathrm{pH}$ & 7.2 & 7.1 & 6.6 & 7.3 & 6.7 & 6.7 \\
\hline $\begin{array}{l}\text { Dissolved } \\
\text { oxygen b }^{b}\end{array}$ & $\mathrm{mg} / \mathrm{L}$ & 10.5 & 12.2 & 10.4 & 8.8 & 7.5 & 7.5 \\
\hline Conductivityb & $\mu \mathrm{mhos} / \mathrm{cm}$ & 70 & 68 & 59 & 52 & 66 & 65 \\
\hline Alkalinity & $\mathrm{mg} / \mathrm{L}$ & 14 & 13 & 17 & 13 & 14 & 13 \\
\hline $\begin{array}{l}\text { Chemical } \\
\text { oxygen demand }\end{array}$ & $\mathrm{mg} / \mathrm{L}$ & ND & ND & ND & ND & ND & ND \\
\hline Volatile solids & $\mathrm{mg} / \mathrm{L}$ & 1 & 1 & 2 & 3 & ND & 2 \\
\hline Fixed residue & $\mathrm{mg} / \mathrm{L}$ & 1 & 1 & ND & 4 & 6 & 5 \\
\hline $\begin{array}{l}\text { Suspended } \\
\text { solids }\end{array}$ & $\mathrm{mg} / \mathrm{L}$ & 2 & 2 & 2 & 7 & 6 & 7 \\
\hline $\begin{array}{l}\text { Total dissolved } \\
\text { solids }\end{array}$ & $\mathrm{mg} / \mathrm{L}$ & 39 & 38 & 42 & 41 & 53 & 49 \\
\hline Total solids & $\mathrm{mg} / \mathrm{L}$ & 41 & 40 & 44 & 48 & 59 & 56 \\
\hline Turbidity & NTU & 1.6 & 1.8 & 2.5 & 1.8 & 2.8 & 4.4 \\
\hline Chloride & $\mathrm{mg} / \mathrm{L}$ & 7 & 5 & 6 & 6 & 7 & 7 \\
\hline $\begin{array}{l}\text { Nitrogen- } \\
\text { ammonia }\end{array}$ & $\mathrm{mg} / \mathrm{L}$ & 0.06 & ND & ND & ND & ND & ND \\
\hline Nitrogen-nitrate & $\mathrm{mg} / \mathrm{L}$ & 0.25 & 0.19 & 0.21 & 0.31 & 0.19 & 0.15 \\
\hline $\begin{array}{l}\text { Nitrogen-total } \\
\text { kjeldahl }\end{array}$ & $\mathrm{mg} / \mathrm{L}$ & 0.31 & ND & ND & 0.37 & ND & ND \\
\hline Phosphate P & $\mathrm{mg} / \mathrm{L}$ & ND & ND & ND & ND & ND & ND \\
\hline Sulfate & $\mathrm{mg} / \mathrm{L}$ & 6 & 5 & 6 & 6 & 7 & 6 \\
\hline $\begin{array}{l}\text { Total organic } \\
\text { carbon }\end{array}$ & $\mathrm{mg} / \mathrm{L}$ & 1.2 & 2.8 & 3.3 & 3.9 & 3.5 & 3.0 \\
\hline Aluminum & $\mathrm{mg} / \mathrm{L}$ & & & 0.13 & & & 0.14 \\
\hline Cadmium & $\mathrm{mg} / \mathrm{L}$ & & & ND & & & ND \\
\hline Calcium & $\mathrm{mg} / \mathrm{L}$ & & & 2.73 & & & 2.32 \\
\hline Chromium & $\mathrm{mg} / \mathrm{L}$ & & & ND & & & ND \\
\hline Copper & $\mathrm{mg} / \mathrm{L}$ & & & 0.01 & & & 0.01 \\
\hline Iron & $\mathrm{mg} / \mathrm{L}$ & & & 0.36 & & & 0.24 \\
\hline Lead & $\mathrm{mg} / \mathrm{L}$ & & & ND & & & ND \\
\hline Magnesium & $\mathrm{mg} / \mathrm{L}$ & & & 1.05 & & & 1.09 \\
\hline Manganese & $\mathrm{mg} / \mathrm{L}$ & & & 0.04 & & & 0.03 \\
\hline Mercury & $\mathrm{mg} / \mathrm{L}$ & ND & ND & ND & ND & ND & ND \\
\hline Nickel & $\mathrm{mg} / \mathrm{L}$ & & & ND & & & ND \\
\hline Sodium & $\mathrm{mg} / \mathrm{L}$ & & & 6.66 & & & 7.37 \\
\hline Zinc & $\mathrm{mg} / \mathrm{L}$ & & & ND & & & ND \\
\hline
\end{tabular}

a Metals (except mercury) are analyzed quarterly from monthly grab composite. Mercury is analyzed from a composite of monthly grab samples.

b Field measurement 
Table 67

SRS Stream Water Quality: Inorganic Contaminants

Page 4 of 20

\begin{tabular}{|c|c|c|c|c|c|c|c|}
\hline \multicolumn{8}{|c|}{ Steel Creek -4 at Road A (EMS and SCDHEC Sampling Location) } \\
\hline Parameter ${ }^{\mathrm{a}}$ & Units & July & Aug & Sept & $\overline{\text { Oct }}$ & Nov & $\overline{\text { Dec }}$ \\
\hline Water volume & $\mathbf{L}$ & $6.05 E+9$ & $6.59 E+9$ & $6.27 E+9$ & $6.02 E+9$ & $4.66 E+9$ & $4.89 E+9$ \\
\hline Sample date & & $7-12-95$ & $8-3-95$ & $9-7-95$ & $10-24-95$ & $11-8-95$ & $12-6-95$ \\
\hline Temperature $^{\mathrm{b}}$ & ${ }^{\circ} \mathrm{C}$ & 28.9 & 26.0 & 24.5 & 20.4 & 17.4 & 13.2 \\
\hline $\mathrm{pH}^{\mathrm{b}}$ & $\mathrm{pH}$ & 6.8 & 6.9 & 7.0 & 6.2 & 6.7 & 6.7 \\
\hline $\begin{array}{l}\text { Dissolved } \\
\text { oxygen }^{\mathrm{b}}\end{array}$ & $\mathrm{mg} / \mathrm{L}$ & 5.9 & 6.0 & 7.0 & 9.0 & 7.9 & 7.3 \\
\hline Conductivityb & $\mu \mathrm{mhos} / \mathrm{cm}$ & 69 & 55 & 78 & 70 & 78 & 77 \\
\hline Alkalinity & $\mathrm{mg} / \mathrm{L}$ & 15 & 17 & 18 & 18 & 15 & 17 \\
\hline $\begin{array}{l}\text { Chemical } \\
\text { oxygen demand }\end{array}$ & $\mathrm{mg} / \mathrm{L}$ & ND & 20 & ND & ND & ND & ND \\
\hline Volatile solids & $\mathrm{mg} / \mathrm{L}$ & ND & 8 & 2 & 1 & 3 & 1 \\
\hline Fixed residue & $\mathrm{mg} / \mathrm{L}$ & 3 & 11 & 3 & 2 & 3 & 1 \\
\hline $\begin{array}{l}\text { Suspended } \\
\text { solids }\end{array}$ & $\mathrm{mg} / \mathrm{L}$ & 3 & 19 & 5 & 3 & 6 & 1 \\
\hline $\begin{array}{l}\text { Total dissolved } \\
\text { solids }\end{array}$ & $\mathrm{mg} / \mathrm{L}$ & 64 & 66 & 60 & 53 & 54 & 45 \\
\hline Total solids & $\mathrm{mg} / \mathrm{L}$ & 67 & 85 & 65 & 56 & 60 & 46 \\
\hline Turbidity & NTU & 3.0 & 10 & 3.0 & 2.9 & 1.5 & 1.9 \\
\hline Chloride & $\mathrm{mg} / \mathrm{L}$ & 7 & 7 & 8 & 7 & 7 & 7 \\
\hline $\begin{array}{l}\text { Nitrogen- } \\
\text { ammonia }\end{array}$ & $\mathrm{mg} / \mathrm{L}$ & ND & ND & ND & 0.18 & ND & 0.12 \\
\hline Nitrogen-nitrate & $\mathrm{mg} / \mathrm{L}$ & 0.15 & 0.21 & 0.23 & 0.20 & 0.17 & 0.18 \\
\hline $\begin{array}{l}\text { Nitrogen-total } \\
\text { kjeldahl }\end{array}$ & $\mathrm{mg} / \mathrm{L}$ & ND & 0.92 & 0.64 & 0.12 & 0.15 & 0.17 \\
\hline Phosphate P & $\mathrm{mg} / \mathrm{L}$ & ND & ND & ND & ND & ND & ND \\
\hline Sulfate & $\mathrm{mg} / \mathrm{L}$ & 7 & 6 & 7 & 6 & 6 & 6 \\
\hline $\begin{array}{l}\text { Total organic } \\
\text { carbon }\end{array}$ & $\mathrm{mg} / \mathrm{L}$ & 4.8 & 7.4 & 4.3 & 2.5 & 6.0 & 4.3 \\
\hline Aluminum & $\mathrm{mg} / \mathrm{L}$ & & & ND & & & ND \\
\hline Cadmium & $\mathrm{mg} / \mathrm{L}$ & & & ND & & & ND \\
\hline Calcium & $\mathrm{mg} / \mathrm{L}$ & & & 2.53 & & & 3.06 \\
\hline Chromium & $\mathrm{mg} / \mathrm{L}$ & & & ND & & & ND \\
\hline Copper & $\mathrm{mg} / \mathrm{L}$ & & & 0.04 & & & 0.02 \\
\hline Iron & $\mathrm{mg} / \mathrm{L}$ & & & 0.24 & & & 0.25 \\
\hline Lead & $\mathrm{mg} / \mathrm{L}$ & & & ND & & & ND \\
\hline Magnesium & $\mathrm{mg} / \mathrm{L}$ & & & 1.41 & & & 0.85 \\
\hline Manganese & $\mathrm{mg} / \mathrm{L}$ & & & ND & & & ND \\
\hline Mercury & $\mathrm{mg} / \mathrm{L}$ & ND & ND & ND & ND & ND & ND \\
\hline Nickel & $\mathrm{mg} / \mathrm{L}$ & & & ND & & & ND \\
\hline Sodium & $\mathrm{mg} / \mathrm{L}$ & & & 8.74 & & & 11.70 \\
\hline Zinc & $\mathrm{mg} / \mathrm{L}$ & & & 0.045 & & & ND \\
\hline
\end{tabular}

a Metals (except mercury) are analyzed quarterly from monthly grab composite. Mercury is analyzed from a composite of monthly grab samples.

b Field measurement 


\section{Table 67}

\section{SRS Stream Water Quality: Inorganic Contaminants}

Page 5 of 20

\begin{tabular}{|c|c|c|c|c|c|c|c|}
\hline \multicolumn{8}{|c|}{ Upper Three Runs-4 at Road A (EMS and SCDHEC Sampling Location) } \\
\hline Parameter $^{\mathbf{a}}$ & Units & Jan & Feb & Mar & April & May & June \\
\hline Water volume & L & $2.78 E+10$ & $2.69 E+10$ & $2.45 E+10$ & $1.75 E+10$ & $1.49 E+10$ & $2.27 E+10$ \\
\hline Sample date & & $1-5-95$ & $2-7-95$ & $3-21-95$ & $4-11-95$ & $5-25-95$ & $6-1-95$ \\
\hline Temperature $^{b}$ & ${ }^{\circ} \mathrm{C}$ & 7.1 & 8.4 & 15.8 & 20.0 & 20.3 & 22.0 \\
\hline $\mathrm{pH}^{\mathrm{b}}$ & $\mathrm{pH}$ & 7.1 & 6.9 & 6.2 & 7.2 & 6.2 & 6.6 \\
\hline $\begin{array}{l}\text { Dissolved } \\
\text { oxygen }^{b}\end{array}$ & $\mathrm{mg} / \mathrm{L}$ & 11.7 & 11.0 & 10.8 & 9.3 & 7.8 & 7.3 \\
\hline Conductivityb & $\mu \mathrm{mhos} / \mathrm{cm}$ & 30 & 29 & 19 & 21 & 22 & 20 \\
\hline Alkalinity & $\mathrm{mg} / \mathrm{L}$ & 4 & 4 & 6 & 5 & 6 & 4 \\
\hline $\begin{array}{l}\text { Chemical } \\
\text { oxygen demand }\end{array}$ & $\mathrm{mg} / \mathrm{L}$ & ND & ND & ND & ND & ND & ND \\
\hline Volatile solids & $\mathrm{mg} / \mathrm{L}$ & 2 & 1 & 3 & 7 & 4 & 5 \\
\hline Fixed residue & $\mathrm{mg} / \mathrm{L}$ & 1 & 2 & ND & 12 & 10 & 8 \\
\hline $\begin{array}{l}\text { Suspended } \\
\text { solids }\end{array}$ & $\mathrm{mg} / \mathrm{L}$ & 3 & 3 & 3 & 19 & 14 & 12 \\
\hline $\begin{array}{l}\text { Total dissolved } \\
\text { solids }\end{array}$ & $\mathrm{mg} / \mathrm{L}$ & 23 & 21 & 23 & 12 & 30 & 29 \\
\hline Total solid's & $\mathrm{mg} / \mathrm{L}$ & 26 & 24 & 26 & 31 & 44 & 41 \\
\hline Turbidity & NTU & 2.4 & 2.3 & 4.3 & 3.8 & 5.2 & 6.6 \\
\hline Chloride & $\mathrm{mg} / \mathrm{L}$ & 2 & 2 & 2 & 3 & 2 & 2 \\
\hline $\begin{array}{l}\text { Nitrogen- } \\
\text { ammonia }\end{array}$ & $\mathrm{mg} / \mathrm{L}$ & ND & ND & ND & ND & ND & ND \\
\hline Nitrogen-nitrate & $\mathrm{mg} / \mathrm{L}$ & 0.29 & 0.22 & 0.22 & 0.34 & 0.27 & 0.23 \\
\hline $\begin{array}{l}\text { Nitrogen-total } \\
\text { kjeldahl }\end{array}$ & $\mathrm{mg} / \mathrm{L}$ & ND & ND & ND & 0.37 & ND & 0.26 \\
\hline $\begin{array}{l}\text { Phosphate P } \\
\text { Sulfate }\end{array}$ & $\begin{array}{l}\mathrm{mg} / \mathrm{L} \\
\mathrm{mg} / \mathrm{L}\end{array}$ & $\begin{array}{r}\text { ND } \\
2\end{array}$ & $\begin{array}{r}\text { ND } \\
2\end{array}$ & $\begin{array}{r}\text { ND } \\
1\end{array}$ & $\begin{array}{r}\text { ND } \\
2\end{array}$ & $\begin{array}{r}\text { ND } \\
2\end{array}$ & $\begin{array}{r}\text { ND } \\
2\end{array}$ \\
\hline $\begin{array}{l}\text { Total organic } \\
\text { carbon }\end{array}$ & $\mathrm{mg} / \mathrm{L}$ & 1.0 & 2.5 & 4.0 & 3.8 & 2.7 & 3.9 \\
\hline Aluminum & $\mathrm{mg} / \mathrm{L}$ & & & 0.24 & & & 0.26 \\
\hline Cadmium & $\mathrm{mg} / \mathrm{L}$ & & & ND & & & ND \\
\hline Calcium & $\mathrm{mg} / \mathrm{L}$ & & & 1.48 & & & 1.60 \\
\hline Chromium & $\mathrm{mg} / \mathrm{L}$ & & & ND & & & ND \\
\hline Copper & $\mathrm{mg} / \mathrm{L}$ & & & ND & & & 0.01 \\
\hline Iron & $\mathrm{mg} / \mathrm{L}$ & & & 0.56 & & & 0.67 \\
\hline Lead & $\mathrm{mg} / \mathrm{L}$ & & & ND & & & ND \\
\hline Magnesium & $\mathrm{mg} / \mathrm{L}$ & & & 0.34 & & & 0.34 \\
\hline Manganese & $\mathrm{mg} / \mathrm{L}$ & & & 0.02 & & & 0.02 \\
\hline Mercury & $\mathrm{mg} / \mathrm{L}$ & ND & ND & ND & ND & ND & ND \\
\hline Nickel & $\mathrm{mg} / \mathrm{L}$ & & & ND & & & ND \\
\hline Sodium & $\mathrm{mg} / \mathrm{L}$ & & & 1.57 & & & 1.52 \\
\hline Zinc & $\mathrm{mg} / \mathrm{L}$ & & & 0.005 & & & ND \\
\hline
\end{tabular}

a Metals (except mercury) are analyzed quarterly from monthly grab composite. Mercury is analyzed from a composite of monthly grab samples.

b Field measurement 
Table 67

\section{SRS Stream Water Quality: Inorganic Contaminants}

Page 6 of 20

\begin{tabular}{|c|c|c|c|c|c|c|c|}
\hline \multicolumn{8}{|c|}{ Upper Three Runs-4 at Road A (EMS and SCDHEC Sampling Location) } \\
\hline Parameter ${ }^{\mathrm{a}}$ & Units & July & Aug & Sept & Oct & Nov & Dec \\
\hline Water volume & L & $1.68 E+10$ & $2.03 \dot{E}+10$ & $1.95 E+10$ & $1.81 E+10$ & $1.91 \mathrm{E}+10$ & $2.12 E+10$ \\
\hline Sample date & & $7-12-95$ & $8-3-95$ & $9-7-95$ & $10-24-95$ & $11-8-95$ & $12-6-95$ \\
\hline Temperature & ${ }^{\circ} \mathrm{C}$ & 23.4 & 24.0 & 20.6 & 21.5 & 14.7 & 12.6 \\
\hline $\mathrm{pH}^{\mathrm{b}}$ & $\mathrm{pH}$ & 6.3 & 6.7 & 6.6 & 5.7 & 6.7 & 6.3 \\
\hline $\begin{array}{l}\text { Dissolved } \\
\text { oxygen }^{b}\end{array}$ & $\mathrm{mg} / \mathrm{L}$ & 7.1 & 6.3 & 7.2 & 6.0 & 7.8 & 7.9 \\
\hline Conductivityb & $\mu \mathrm{mhos} / \mathrm{cm}$ & 21 & 20 & 32 & 17 & 36 & 23 \\
\hline Alkalinity & $\mathrm{mg} / \mathrm{L}$ & 5 & 6 & 5 & 6 & 5 & 5 \\
\hline $\begin{array}{l}\text { Chemical } \\
\text { oxygen demand }\end{array}$ & $\mathrm{mg} / \mathrm{L}$ & ND & ND & ND & ND & ND & ND \\
\hline Volatile solids & $\mathrm{mg} / \mathrm{L}$ & 4 & 6 & 2 & 2 & 5 & 1 \\
\hline Fixed residue & $\mathrm{mg} / \mathrm{L}$ & 9 & 12 & 3 & 2 & 8 & 2 \\
\hline $\begin{array}{l}\text { Suspended } \\
\text { solids }\end{array}$ & $\mathrm{mg} / \mathrm{L}$ & 12 & 18 & 5 & 4 & 13 & 3 \\
\hline $\begin{array}{l}\text { Total dissolved } \\
\text { solids }\end{array}$ & $\mathrm{mg} / \mathrm{L}$ & 35 & 42 & 30 & 28 & 46 & 26 \\
\hline Total solids & $\mathrm{mg} / \mathrm{L}$ & 47 & 60 & 35 & 32 & 59 & 29 \\
\hline Turbidity & NTU & 9.2 & 13 & 3.2 & 3.1 & 14 & 2.8 \\
\hline Chloride & $\mathrm{mg} / \mathrm{L}$ & 2 & 3 & 3 & 3 & 3 & 3 \\
\hline $\begin{array}{l}\text { Nitrogen- } \\
\text { ammonia }\end{array}$ & $\mathrm{mg} / \mathrm{L}$ & ND & ND & 0.11 & 0.66 & ND & 0.10 \\
\hline Nitrogen-nitrate & $\mathrm{mg} / \mathrm{L}$ & 0.22 & 0.28 & 0.27 & 0.30 & 0.19 & 0.25 \\
\hline $\begin{array}{l}\text { Nitrogen-total } \\
\text { kjeldahl }\end{array}$ & $\mathrm{mg} / \mathrm{L}$ & ND & 0.36 & 0.18 & 0.19 & 0.29 & 0.34 \\
\hline Phosphate P & $\mathrm{mg} / \mathrm{L}$ & ND & ND & ND & ND & ND & ND \\
\hline Sulfate & $\mathrm{mg} / \mathrm{L}$ & 2 & 2 & 2 & 2 & 2 & 2 \\
\hline $\begin{array}{l}\text { Total organic } \\
\text { carbon }\end{array}$ & $\mathrm{mg} / \mathrm{L}$ & 2.9 & 4.9 & 1.1 & 1.9 & 12.4 & 4.0 \\
\hline Aluminum & $\mathrm{mg} / \mathrm{L}$ & & & ND & & & ND \\
\hline Cadmium & $\mathrm{mg} / \mathrm{L}$ & & & ND & & & ND \\
\hline Calcium & $\mathrm{mg} / \mathrm{L}$ & & & 1.32 & & & 1.83 \\
\hline Chromium & $\mathrm{mg} / \mathrm{L}$ & & & ND & & & ND \\
\hline Copper & $\mathrm{mg} / \mathrm{L}$ & & & 0.03 & & & 0.02 \\
\hline Iron & $\mathrm{mg} / \mathrm{L}$ & & & 0.90 & & & 0.54 \\
\hline Lead & $\mathrm{mg} / \mathrm{L}$ & & & ND & & & ND \\
\hline Magnesium & $\mathrm{mg} / \mathrm{L}$ & & & 0.44 & & & ND \\
\hline Manganese & $\mathrm{mg} / \mathrm{L}$ & & & ND & & & ND \\
\hline Mercury & $\mathrm{mg} / \mathrm{L}$ & ND & 0.0001 & ND & ND & ND & ND \\
\hline Nickel & $\mathrm{mg} / \mathrm{L}$ & & & ND & & & ND \\
\hline Sodium & $\mathrm{mg} / \mathrm{L}$ & & & 0.29 & & & 5.57 \\
\hline Zinc & $\mathrm{mg} / \mathrm{L}$ & & & 0.03 & & & ND \\
\hline
\end{tabular}

a Metals (except mercury) are analyzed quarterly from monthly grab composite. Mercury is analyzed from a composite of monthly grab samples.

b Field measurement 
Table 67

SRS Stream Water Quality: Inorganic Contaminants

Page 7 of 20

Four Mile Creek at Road A7 (EMS and SCDHEC Sampling Location)

\begin{tabular}{|c|c|c|c|c|c|c|c|}
\hline Parameter ${ }^{a}$ & Units & Jan & Feb & Mar & April & May & June \\
\hline Water volume & $\mathbf{L}$ & $2.69 E+9$ & $2.83 E+9$ & $1.69 E+9$ & $8.37 E+8$ & $6.20 E+8$ & $1.89 E+9$ \\
\hline Sample date & & $1-5-95$ & $2-6-95$ & $3-21-95$ & $4-12-95$ & $5-25-95$ & $6-1-95$ \\
\hline Temperatureb & ${ }^{\circ} \mathrm{C}$ & 4.5 & 6.0 & 16.0 & 20.5 & 21.0 & 23.0 \\
\hline $\mathrm{pH}^{\mathrm{b}}$ & $\mathrm{pH}$ & 7.1 & 6.8 & 6.3 & 6.9 & 6.7 & 6.6 \\
\hline $\begin{array}{l}\text { Dissolved } \\
\text { oxygen }^{b}\end{array}$ & $\mathrm{mg} / \mathrm{L}$ & 13.1 & 11.1 & 9.1 & 9.0 & 7.3 & 6.9 \\
\hline Conductivityb & $\mu \mathrm{mhos} / \mathrm{cm}$ & 77 & 35 & 51 & 59 & 89 & 85 \\
\hline Alkalinity & $\mathrm{mg} / \mathrm{L}$ & 8 & 7 & 12 & 12 & 18 & 18 \\
\hline $\begin{array}{l}\text { Chemical } \\
\text { oxygen demand }\end{array}$ & $\mathrm{mg} / \mathrm{L}$ & ND & ND & ND & ND & ND & ND \\
\hline Volatile solids & $\mathrm{mg} / \mathrm{L}$ & 1 & 2 & 1 & 5 & ND & 1 \\
\hline Fixed residue & $\mathrm{mg} / \mathrm{L}$ & 3 & 4 & ND & 8 & 4 & 3 \\
\hline $\begin{array}{l}\text { Suspended } \\
\text { solids }\end{array}$ & $\mathrm{mg} / \mathrm{L}$ & 4 & 6 & 1 & 13 & 4 & 4 \\
\hline $\begin{array}{l}\text { Total dissolved } \\
\text { solids }\end{array}$ & $\mathrm{mg} / \mathrm{L}$ & 43 & 37 & 40 & 40 & 81 & 73 \\
\hline Total solids & $\mathrm{mg} / \mathrm{L}$ & 47 & 43 & 41 & 53 & 85 & 77 \\
\hline Turbidity & NTU & 2.5 & 4.4 & 3.6 & 4.5 & 3.3 & 3.3 \\
\hline Chloride & $\mathrm{mg} / \mathrm{L}$ & 3 & 4 & 3 & 3 & 4 & 4 \\
\hline $\begin{array}{l}\text { Nitrogen- } \\
\text { ammonia }\end{array}$ & $\mathrm{mg} / \mathrm{L}$ & ND & ND & ND & ND & ND & ND \\
\hline Nitrogen-nitrate & $\mathrm{mg} / \mathrm{L}$ & 2.25 & 1.98 & 1.77 & 1.42 & 3.00 & 3.00 \\
\hline $\begin{array}{l}\text { Nitrogen-total } \\
\text { kjeldahl }\end{array}$ & $\mathrm{mg} / \mathrm{L}$ & ND & ND & ND & 0.42 & 0.22 & 0.28 \\
\hline Phosphate P & $\mathrm{mg} / \mathrm{L}$ & ND & ND & ND & ND & ND & ND \\
\hline Sulfate & $\mathrm{mg} / \mathrm{L}$ & 11 & 8 & 4 & 4 & 7 & 5 \\
\hline $\begin{array}{l}\text { Total organic } \\
\text { carbon }\end{array}$ & $\mathrm{mg} / \mathrm{L}$ & ND & 1.7 & 2.6 & 3.8 & 2.8 & 2.3 \\
\hline Aluminum & $\mathrm{mg} / \mathrm{L}$ & & & 0.22 & & & 0.16 \\
\hline Cadmium & $\mathrm{mg} / \mathrm{L}$ & & & ND & & & ND \\
\hline Calcium & $\mathrm{mg} / \mathrm{L}$ & & & 2.59 & & & 2.62 \\
\hline Chromium & $\mathrm{mg} / \mathrm{L}$ & & & ND & & & ND \\
\hline Copper & $\mathrm{mg} / \mathrm{L}$ & & & ND & & & 0.01 \\
\hline Iron & $\mathrm{mg} / \mathrm{L}$ & & & 0.61 & & & 0.72 \\
\hline Lead & $\mathrm{mg} / \mathrm{L}$ & & & ND & & & ND \\
\hline Magnesium & $\mathrm{mg} / \mathrm{L}$ & & & 0.58 & & & 0.48 \\
\hline Manganese & $\mathrm{mg} / \mathrm{L}$ & & & 0.08 & & & 0.05 \\
\hline Mercury & $\mathrm{mg} / \mathrm{L}$ & ND & ND & ND & ND & ND & ND \\
\hline Nickel & $\mathrm{mg} / \mathrm{L}$ & & & ND & & & ND \\
\hline Sodium & $\mathrm{mg} / \mathrm{L}$ & & & 6.81 & & & 9.27 \\
\hline Zinc & $\mathrm{mg} / \mathrm{L}$ & & & 0.011 & & & ND \\
\hline
\end{tabular}

a Metals (except mercury) are analyzed quarterly from monthly grab composite. Mercury is analyzed from a composite of monthly grab samples.

b Field measurement 
Table 67

SRS Stream Water Quality: Inorganic Contaminants

Page 8 of 20

\begin{tabular}{|c|c|c|c|c|c|c|c|}
\hline \multicolumn{8}{|c|}{ Four Mile Creek at Road A7 (EMS and SCDHEC Sampling Location) } \\
\hline Parameter $^{\mathbf{a}}$ & Units & July & Aug & Sept & Oct & Nov & Dec \\
\hline Water volume & $\mathrm{L}$ & $1.05 E+9$ & $1.42 E+9$ & $1.31 \mathrm{E}+9$ & $9.78 \mathrm{E}+8$ & $1.11 E+9$ & $1.60 E+9$ \\
\hline Sample date & & $7-12-95$ & $8-3-95$ & $9-7-95$ & $10-24-95$ & $11-8-95$ & $12-6-95$ \\
\hline Temperature $^{\mathrm{b}}$ & ${ }^{\circ} \mathrm{C}$ & 25.3 & 23.7 & 21.5 & 21.0 & 14.5 & 12.1 \\
\hline $\mathrm{pH}^{\mathrm{b}}$ & $\mathrm{pH}$ & 6.6 & 6.5 & 7.0 & 6.0 & 6.6 & 6.1 \\
\hline $\begin{array}{l}\text { Dissolved } \\
\text { oxygen }^{b}\end{array}$ & $\mathrm{mg} / \mathrm{L}$ & 5.9 & 5.1 & 6.7 & 15.9 & 7.0 & 7.8 \\
\hline Conductivityb & $\mu \mathrm{mhos} / \mathrm{cm}$ & 84 & 55 & 93 & 94 & 70 & 92 \\
\hline Alkalinity & $\mathrm{mg} / \mathrm{L}$ & 24 & 15 & 17 & 17 & 10 & 13 \\
\hline $\begin{array}{l}\text { Chemical } \\
\text { oxygen demand }\end{array}$ & $\mathrm{mg} / \mathrm{L}$ & ND & 20 & ND & ND & ND & ND \\
\hline Volatile solids & $\mathrm{mg} / \mathrm{L}$ & ND & 3 & 2 & 1 & 4 & 1 \\
\hline Fixed residue & $\mathrm{mg} / \mathrm{L}$ & 5 & 5 & 3 & 2 & 5 & 2 \\
\hline $\begin{array}{l}\text { Suspended } \\
\text { solids }\end{array}$ & $\mathrm{mg} / \mathrm{L}$ & 5 & 7 & 4 & 3 & 8 & 3 \\
\hline $\begin{array}{l}\text { Total dissolved } \\
\text { solids }\end{array}$ & $\mathrm{mg} / \mathrm{L}$ & 90 & 59 & 80 & 79 & 63 & 58 \\
\hline Total solids & $\mathrm{mg} / \mathrm{L}$ & 95 & 66 & 84 & 82 & 71 & 61 \\
\hline Turbidity & NTU & 7.8 & 8.3 & 2.7 & 2.0 & 19 & 2.8 \\
\hline Chloride & $\mathrm{mg} / \mathrm{L}$ & 4 & 3 & 6 & 7 & 4 & 5 \\
\hline $\begin{array}{l}\text { Nitrogen- } \\
\text { ammonia }\end{array}$ & $\mathrm{mg} / \mathrm{L}$ & ND & ND & 0.14 & 0.33 & ND & 0.18 \\
\hline Nitrogen-nitrate & $\mathrm{mg} / \mathrm{L}$ & 2.49 & 2.31 & 3.88 & 4.59 & 1.96 & 3.07 \\
\hline $\begin{array}{l}\text { Nitrogen-total } \\
\text { kjeldahl }\end{array}$ & $\mathrm{mg} / \mathrm{L}$ & 0.31 & 0.89 & 0.59 & 0.63 & 2.30 & ND \\
\hline $\begin{array}{l}\text { Phosphate P } \\
\text { Sulfate }\end{array}$ & $\begin{array}{l}\mathrm{mg} / \mathrm{L} \\
\mathrm{mg} / \mathrm{L}\end{array}$ & $\begin{array}{r}\text { ND } \\
5\end{array}$ & $\begin{array}{r}\text { ND } \\
3\end{array}$ & $\begin{array}{r}\text { ND } \\
6\end{array}$ & $\begin{array}{r}\text { ND } \\
6\end{array}$ & $\begin{array}{r}\text { ND } \\
5\end{array}$ & $\begin{array}{r}\text { ND } \\
7\end{array}$ \\
\hline $\begin{array}{l}\text { Total organic } \\
\text { carbon }\end{array}$ & $\mathrm{mg} / \mathrm{L}$ & 5.8 & 6.5 & ND & 1.9 & 8.4 & 3.6 \\
\hline Aluminum & $\mathrm{mg} / \mathrm{L}$ & & & ND & & & ND \\
\hline Cadmium & $\mathrm{mg} / \mathrm{L}$ & & & ND & & & ND \\
\hline Calcium & $\mathrm{mg} / \mathrm{L}$ & & & 2.91 & & & 3.56 \\
\hline Chromium & $\mathrm{mg} / \mathrm{L}$ & & & ND & & & ND \\
\hline Copper & $\mathrm{mg} / \mathrm{L}$ & & & 0.03 & & & 0.01 \\
\hline Iron & $\mathrm{mg} / \mathrm{L}$ & & & 1.06 & & & 0.81 \\
\hline Lead & $\mathrm{mg} / \mathrm{L}$ & & & ND & & & ND \\
\hline Magnesium & $\mathrm{mg} / \mathrm{L}$ & & & 1.59 & & & 0.82 \\
\hline Manganese & $\mathrm{mg} / \mathrm{L}$ & & & 0.05 & & & ND \\
\hline Mercury & $\mathrm{mg} / \mathrm{L}$ & 0.0002 & ND & ND & ND & ND & ND \\
\hline Nickel & $\mathrm{mg} / \mathrm{L}$ & & & ND & & & ND \\
\hline Sodium & $\mathrm{mg} / \mathrm{L}$ & & & 9.24 & & & 14.30 \\
\hline Zinc & $\mathrm{mg} / \mathrm{L}$ & & & 0.073 & & & 0.037 \\
\hline
\end{tabular}

a Metals (except mercury) are analyzed quarterly from monthly grab composite. Mercury is analyzed from a composite of monthly grab samples.

b Field measurement 


\section{Table 67}

SRS Stream Water Quality: Inorganic Contaminants

Page 9 of 20

\begin{tabular}{|c|c|c|c|c|c|c|c|}
\hline \multicolumn{8}{|c|}{ Crouch Branch } \\
\hline Parameter $^{2}$ & Units & Jan & Feb & Mar & April & May & June \\
\hline Water volume & $L$ & $2.73 E+7$ & $3.22 E+7$ & $1.21 E+7$ & $2.64 \mathrm{E}+6$ & $3.94 \mathrm{E}+6$ & $3.23 E+7$ \\
\hline Sample date & & $1-5-95$ & $2-6-95$ & $3-15-95$ & $4-11-95$ & $5-25-95$ & $6-1-95$ \\
\hline Temperature $^{\mathrm{b}}$ & ${ }^{\circ} \mathrm{C}$ & 6.0 & 4.0 & 12.7 & 17.3 & 19.0 & 25.9 \\
\hline $\mathrm{pH}^{\mathrm{b}}$ & $\mathrm{pH}$ & 6.6 & 6.4 & 6.2 & 6.0 & 6.1 & 5.4 \\
\hline $\begin{array}{l}\text { Dissolved } \\
\text { oxygenb }\end{array}$ & $\mathrm{mg} / \mathrm{L}$ & 8.2 & 8.9 & 8.6 & 5.8 & 7.5 & 5.2 \\
\hline Conductivityb & $\mu \mathrm{mhos} / \mathrm{cm}$ & 66 & 76 & 27 & 65 & 60 & 63 \\
\hline Turbidity & NTU & 8.3 & 18 & 9.7 & 5.8 & 28 & 15 \\
\hline $\begin{array}{l}\text { Suspended } \\
\text { solids }\end{array}$ & $\mathrm{mg} / \mathrm{L}$ & 2 & 7 & 5 & 13 & 47 & 14 \\
\hline
\end{tabular}

a Metals (except mercury) are analyzed quarterly from monthly grab composite. Mercury is analyzed from a composite of monthly grab samples.

b Field measurement

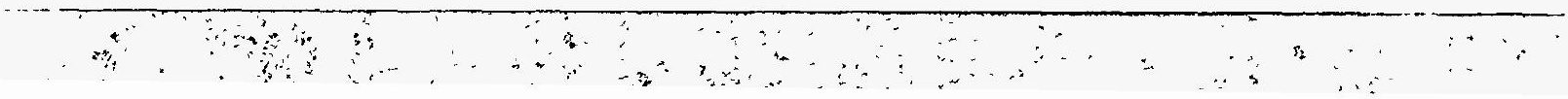


Table 67

SRS Stream Water Quality: Inorganic Contaminants

Page 10 of 20

\begin{tabular}{|c|c|c|c|c|c|c|c|}
\hline \multicolumn{8}{|c|}{ Crouch Branch } \\
\hline Parameter ${ }^{\mathbf{a}}$ & Units & July & Aug & Sept & Oct & Nov & Dec \\
\hline Water volume & L & $8.34 E+6$ & $2.20 E+7$ & $1.25 E+7$ & $4.85 E+6$ & $7.34 \mathrm{E}+6$ & $1.06 E+7$ \\
\hline Sample date & & $7-12-95$ & $8-3-95$ & $9-7-95$ & $10-24-95$ & $11-8-95$ & $12-6-95$ \\
\hline Temperature $^{b}$ & ${ }^{\circ} \mathrm{C}$ & $24.0^{\circ}$ & 24.5 & 21.0 & 16.8 & 14.5 & 15.0 \\
\hline $\mathrm{pH}^{\mathrm{b}}$ & $\mathrm{pH}$ & 5.7 & 6.2 & 6.3 & 5.3 & 5.5 & 5.3 \\
\hline $\begin{array}{l}\text { Dissolved } \\
\text { oxygen b }^{b}\end{array}$ & $\mathrm{mg} / \mathrm{L}$ & 4.8 & 6.7 & 6.9 & 5.2 & 7.5 & 7.2 \\
\hline Conductivity $^{b}$ & $\mu \mathrm{mhos} / \mathrm{cm}$ & 54 & 49 & 51 & 36 & 56 & 59 \\
\hline Turbidity & NTU & 56 & 55 & 13 & 7.6 & 60 & 2.4 \\
\hline Suspended & $\mathrm{mg} / \mathrm{L}$ & 89 & 24 & 8 & 5 & 14 & 5 \\
\hline
\end{tabular}

a Metals (except mercury) are analyzed quarterly from monthly grab composite. Mercury is analyzed from a composite of monthly grab samples.

b Field measurement 


\section{Table 67}

\section{SRS Stream Water Quality: Inorganic Contaminants}

Page 11 of 20

\begin{tabular}{|c|c|c|c|c|c|c|c|}
\hline Parameter ${ }^{\mathrm{a}}$ & Units & Jan & Feb & Mar & April & May & June \\
\hline Water volume & $L$ & $5.73 \mathrm{E}+9$ & $4.73 E \div 9$ & $4.44 E+9$ & $3.29 E+9$ & $2.34 \mathrm{E}+9$ & $5.02 E+9$ \\
\hline Sample date & & $1-5-95$ & $2-6-95$ & $3-15-95$ & $4-11-95$ & $5-25-95$ & $6-1-95$ \\
\hline Temperature $^{b}$ & ${ }^{\circ} \mathrm{C}$ & 7.1 & 8.1 & 14.1 & 18.8 & 20.5 & 21.6 \\
\hline $\mathrm{pH}^{\mathrm{b}}$ & $\mathrm{pH}$ & 7.0 & 6.8 & 6.6 & 6.7 & 6.2 & 6.6 \\
\hline $\begin{array}{l}\text { Dissolved } \\
\text { oxygen }^{b}\end{array}$ & $\mathrm{mg} / \mathrm{L}$ & 9.8 & 10.9 & 9.8 & 7.8 & 8.5 & 8.9 \\
\hline Conductivityb & $\mu \mathrm{mhos} / \mathrm{cm}$ & 92 & 86 & 81 & 96 & 80 & 95 \\
\hline Alkalinity & $\mathrm{mg} / \mathrm{L}$ & 40 & 36 & 40 & 35 & 37 & 40 \\
\hline $\begin{array}{l}\text { Chemical } \\
\text { oxygen demand }\end{array}$ & $\mathrm{mg} / \mathrm{L}$ & ND & ND & ND & ND & ND & 20 \\
\hline Volatile solids & $\mathrm{mg} / \mathrm{L}$ & 1 & ND & 2 & 6 & 18 & 8 \\
\hline Fixed residue & $\mathrm{mg} / \mathrm{L}$ & 1 & ND & 2 & 14 & 36 & 8 \\
\hline $\begin{array}{l}\text { Suspended } \\
\text { solids }\end{array}$ & $\mathrm{mg} / \mathrm{L}$ & 2 & ND & 3 & 20 & 54 & 16 \\
\hline $\begin{array}{l}\text { Total dissolved } \\
\text { solids }\end{array}$ & $\mathrm{mg} / \mathrm{L}$ & 61 & 49 & 54 & 46 & 69 & 70 \\
\hline Total solids & $\mathrm{mg} / \mathrm{L}$ & 63 & 49 & 57 & 66 & 123 & 86 \\
\hline Turbidity & NTU & 1.5 & 2.1 & 2.0 & 2.2 & 16 & 6.5 \\
\hline Chloride & $\mathrm{mg} / \mathrm{L}$ & 4 & 3 & 3 & 4 & 3 & 3 \\
\hline $\begin{array}{l}\text { Nitrogen- } \\
\text { ammonia }\end{array}$ & $\mathrm{mg} / \mathrm{L}$ & ND & ND & ND & ND & ND & ND \\
\hline Nitrogen-nitrate & $\mathrm{mg} / \mathrm{L}$ & 0.25 & 0.21 & 0.20 & 0.35 & 0.27 & 0.27 \\
\hline Phosphate P & $\mathrm{mg} / \mathrm{L}$ & ND & ND & ND & ND & ND & ND \\
\hline Sulfate & $\mathrm{mg} / \mathrm{L}$ & 2 & 2 & 2 & 2 & 3 & 2 \\
\hline Aluminum & $\mathrm{mg} / \mathrm{L}$ & & & 0.08 & & & 0.73 \\
\hline Cadmium & $\mathrm{mg} / \mathrm{L}$ & & & ND & & & ND \\
\hline Calcium & $\mathrm{mg} / \mathrm{L}$ & & & 12.90 & & & 13.40 \\
\hline Chromium & $\mathrm{mg} / \mathrm{L}$ & & & ND & & & ND \\
\hline Copper & $\mathrm{mg} / \mathrm{L}$ & & & ND & & & 0.01 \\
\hline Iron & $\mathrm{mg} / \mathrm{L}$ & & & 0.24 & & & 1.60 \\
\hline Lead & $\mathrm{mg} / \mathrm{L}$ & & & ND & & & ND \\
\hline Magnesium & $\mathrm{mg} / \mathrm{L}$ & $i$ & & 0.61 & & & 0.73 \\
\hline Manganese & $\mathrm{mg} / \mathrm{L}$ & & & 0.02 & & & 0.45 \\
\hline Mercury & $\mathrm{mg} / \mathrm{L}$ & ND & ND & ND & ND & ND & ND \\
\hline Nickel & $\mathrm{mg} / \mathrm{L}$ & & & ND & & & ND \\
\hline Sodium & $\mathrm{mg} / \mathrm{L}$ & & & 2.34 & & & 2.82 \\
\hline Zinc & $\mathrm{mg} / \mathrm{L}$ & & & ND & & & 0.006 \\
\hline
\end{tabular}

\footnotetext{
a Metals (except mercury) are analyzed quarterly from monthly grab composite. Mercury is analyzed from a composite of monthly grab samples.

b Field measurement
} 
Table 67

SRS Stream Water Quality: Inorganic Contaminants

Page 12 of 20

\begin{tabular}{|c|c|c|c|c|c|c|c|}
\hline \multicolumn{8}{|c|}{ Lower Three Runs-2 } \\
\hline Parameter ${ }^{\mathbf{a}}$ & Units & July & Aug & Sept & Oct & Nov & Dec \\
\hline Water volume & L & $3.57 E+9$ & $5.68 E+9$ & $4.79 E+9$ & $3.44 E+9$ & $4.00 E+9$ & $5.08 E+9$ \\
\hline Sample date & & $7-12-95$ & $8-3-95$ & $9-7-95$ & $10-24-95$ & $11-8-95$ & $12-6-95$ \\
\hline Temperature $^{\mathrm{b}}$ & ${ }^{\circ} \mathrm{C}$ & 25.6 & 23.0 & 23.0 & 18.9 & 14.5 & 14.6 \\
\hline $\mathrm{pH}^{\mathrm{b}}$ & $\mathrm{pH}$ & 6.5 & 6.5 & 6.8 & 6.7 & 6.2 & 6.3 \\
\hline $\begin{array}{l}\text { Dissolved } \\
\text { oxygenb }\end{array}$ & $\mathrm{mg} / \mathrm{L}$ & 4.8 & 6.3 & 7.7 & 6.3 & 7.5 & 8.6 \\
\hline Conductivityb & $\mu \mathrm{mhos} / \mathrm{cm}$ & 95 & 78 & 75 & 104 & 67 & 83 \\
\hline Alkalinity & $\mathrm{mg} / \mathrm{L}$ & 32 & 32 & 29 & 39 & 22 & 31 \\
\hline $\begin{array}{l}\text { Chemical } \\
\text { oxygen demand }\end{array}$ & $\mathrm{mg} / \mathrm{L}$ & ND & 20 & ND & ND & 32 & ND \\
\hline Volatile solids & $\mathrm{mg} / \mathrm{L}$ & 3 & 12 & 4 & 1 & 3 & 1 \\
\hline Fixed residue & $\mathrm{mg} / \mathrm{L}$ & 3 & 13 & 7 & 2 & 1 & 1 \\
\hline $\begin{array}{l}\text { Suspended } \\
\text { solids }\end{array}$ & $\mathrm{mg} / \mathrm{L}$ & 6 & 24 & 11 & 3 & 4 & 2 \\
\hline $\begin{array}{l}\text { Total dissolved } \\
\text { solids }\end{array}$ & $\mathrm{mg} / \mathrm{L}$ & 67 & 66 & 59 & 68 & 80 & 58 \\
\hline Total solids & $\mathrm{mg} / \mathrm{L}$ & 73 & 90 & 70 & 71 & 84 & 60 \\
\hline Turbidity & NTU & 5.4 & 8.1 & 2.1 & 2.1 & 4.1 & 1.8 \\
\hline Chloride & $\mathrm{mg} / \mathrm{L}$ & 4 & 3 & 4 & 3 & 4 & 4 \\
\hline $\begin{array}{l}\text { Nitrogen- } \\
\text { ammonia }\end{array}$ & $\mathrm{mg} / \mathrm{L}$ & ND & ND & 0.13 & 0.17 & 0.10 & 0.14 \\
\hline Nitrogen-nitrate & $\mathrm{mg} / \mathrm{L}$ & 0.23 & 0.22 & 0.23 & 0.26 & 0.16 & 0.25 \\
\hline Phosphate P & $\mathrm{mg} / \mathrm{L}$ & ND & ND & ND & ND & ND & ND \\
\hline Sulfate & $\mathrm{mg} / \mathrm{L}$ & 2 & 2 & 2 & 2 & 2 & 2 \\
\hline Aluminum & $\mathrm{mg} / \mathrm{L}$ & & & 0.53 & & & ND \\
\hline Cadmium & $\mathrm{mg} / \mathrm{L}$ & & & ND & & & ND \\
\hline Calcium & $\mathrm{mg} / \mathrm{L}$ & & & 9.29 & & & 10.20 \\
\hline Chromium & $\mathrm{mg} / \mathrm{L}$ & & & ND & & & ND \\
\hline Copper & $\mathrm{mg} / \mathrm{L}$ & & & 0.03 & & & 0.02 \\
\hline Iron & $\mathrm{mg} / \mathrm{L}$ & & & 1.26 & & & 1.04 \\
\hline Lead & $\mathrm{mg} / \mathrm{L}$ & & & ND & & & ND \\
\hline Magnesium & $\mathrm{mg} / \mathrm{L}$ & & & 1.19 & & & 0.65 \\
\hline Manganese & $\mathrm{mg} / \mathrm{L}$ & & & 0.32 & & & 0.20 \\
\hline Mercury & $\mathrm{mg} / \mathrm{L}$ & ND & ND & ND & ND & ND & ND \\
\hline Nickel & $\mathrm{mg} / \mathrm{L}$ & & & ND & & & ND \\
\hline Sodium & $\mathrm{mg} / \mathrm{L}$ & & & 1.47 & & & 7.03 \\
\hline Zinc & $\mathrm{mg} / \mathrm{L}$ & & & 0.009 & & & ND \\
\hline
\end{tabular}

a Metals (except mercury) are analyzed quarterly from monthly grab composite. Mercury is analyzed from a composite of monthly
grab samples.

b Field measurement 


\section{Table 67}

SRS Stream Water Quality: Inorganic Contaminants

Page 13 of 20

\begin{tabular}{|c|c|c|c|c|c|c|c|}
\hline \multicolumn{8}{|c|}{ McQueens Branch } \\
\hline Parametera & Units & Jan & Feb & Mar & April & May & June \\
\hline Water volume & $\mathbf{L}$ & $2.34 E+8$ & $2.80 E+8$ & $1.43 E+8$ & $7.78 E+7$ & $3.94 E+7$ & $1.24 \mathrm{E}+8$ \\
\hline Sample date & & $1-5-95$ & $2-6-95$ & $3-15-95$ & $4-11-95$ & $5-25-95$ & $6-1-95$ \\
\hline Temperature $^{b}$ & ${ }^{\circ} \mathrm{C}$ & 6.5 & 6.0 & 12.8 & 17.4 & 19.0 & 24.6 \\
\hline $\mathrm{pH}^{\mathrm{b}}$ & $\mathrm{pH}$ & 6.7 & 6.9 & 6.2 & 6.0 & 6.1 & 5.9 \\
\hline $\begin{array}{l}\text { Dissolved } \\
\text { oxygen }^{b}\end{array}$ & $\mathrm{mg} / \mathrm{L}$ & 11.8 & 10.8 & 7.8 & 7.9 & 8.9 & 7.8 \\
\hline Conductivity ${ }^{b}$ & $\mu \dot{m h o s} / \mathrm{cm}$ & 39 & 62 & 50 & 41 & 50 & 60 \\
\hline Turbidity & NTU & 5.5 & 6.4 & 4.5 & 3.5 & 8.4 & 10 \\
\hline $\begin{array}{l}\text { Suspended } \\
\text { solids }\end{array}$ & $\mathrm{mg} / \mathrm{L}$ & 8 & 3 & 3 & 14 & 13 & 12 \\
\hline
\end{tabular}

a Metals (except mercury) are analyzed quarterly from monthly grab composite. Mercury is analyzed from a composite of monthly grab samples.

b Field measurement 


\section{Table 67}

\section{SRS Stream Water Quality: Inorganic Contaminants}

Page 14 of 20

\begin{tabular}{|c|c|c|c|c|c|c|c|}
\hline \multicolumn{8}{|c|}{ McQueens Branch } \\
\hline Parameter ${ }^{a}$ & Units & July & Aug & Sept & Oct & Nov & Dec \\
\hline Water volume & $L$ & $7.21 \mathrm{E}+7$ & $9.10 \mathrm{E}+7$ & $6.90 \mathrm{E}+7$ & $5.99 E+7$ & $6.46 E+7$ & $8.27 E+7$ \\
\hline Sample date & & $7-12-95$ & $8-3-95$ & $9-7-95$ & $10-24-95$ & $11-8-95$ & $12-6-95$ \\
\hline Temperatureb & ${ }^{\circ} \mathrm{C}$ & 22.2 & 23.0 & 20.0 & 16.9 & 14.5 & 14.9 \\
\hline $\mathrm{pH}^{\mathrm{b}}$ & $\mathrm{pH}$ & 5.2 & 6.2 & 6.4 & 6.6 & 5.9 & 5.8 \\
\hline $\begin{array}{l}\text { Dissolved } \\
\text { oxygen b }^{\text {by }}\end{array}$ & $\mathrm{mg} / \mathrm{L}$ & 5.6 & 6.8 & 8.9 & 6.4 & 9.0 & 8.7 \\
\hline Conductivity $^{b}$ & $\mu \mathrm{mhos} / \mathrm{cm}$ & 18 & 56 & 55 & 25 & 42 & 47 \\
\hline Turbidity & NTU & 35 & 60 & 4.8 & 4.5 & 29 & 2.1 \\
\hline $\begin{array}{l}\text { Suspended } \\
\text { solids }\end{array}$ & $\mathrm{mg} / \mathrm{L}$ & 16 & 37 & 3 & 4 & 10 & 5 \\
\hline
\end{tabular}

a Metals (except mercury) are analyzed quarterly from monthly grab composite. Mercury is analyzed from a composite of monthly grab samples.

b Field measurement 
Table 67

SRS Stream Water Quality: Inorganic Contaminants

Page 15 of 20

\begin{tabular}{|c|c|c|c|c|c|c|c|}
\hline \multicolumn{8}{|l|}{ Pen Branch-3 } \\
\hline Parameter $^{\mathrm{a}}$ & Units & Jan & Feb & Mar & April & May & June \\
\hline Water volume & $L$ & $6.57 E+9$ & $5.10 E+9$ & $4.21 E+9$ & $2.77 E+9$ & $2.67 E+9$ & $3.48 E+9$ \\
\hline Sample date & & $1-5-95$ & $2-6-95$ & $3-15-95$ & $4-11-95$ & $5-25-95$ & $6-1-95$ \\
\hline Temperature $^{b}$ & ${ }^{\circ} \mathrm{C}$ & 6.7 & 4.9 & 13.2 & 18.8 & 22.0 & 23.9 \\
\hline $\mathrm{pH}^{\mathrm{b}}$ & $\mathrm{pH}$ & 6.6 & 6.0 & 6.4 & 5.8 & 6.2 & 6.5 \\
\hline $\begin{array}{l}\text { Dissolved } \\
\text { oxygen }^{b}\end{array}$ & $\mathrm{mg} / \mathrm{L}$ & 12.4 & 12.1 & 11.0 & 8.9 & 9.0 & 9.6 \\
\hline Conductivityb & $\mu \mathrm{mhos} / \mathrm{cm}$ & 69 & 67 & 51 & 118 & 80 & 78 \\
\hline Alkalinity & $\mathrm{mg} / \mathrm{L}$ & 16 & 14 & 17 & 19 & 20 & 18 \\
\hline $\begin{array}{l}\text { Chemical } \\
\text { oxygen demand }\end{array}$ & $\mathrm{mg} / \mathrm{L}$ & ND & ND & ND & ND & ND & ND \\
\hline Volatile solids & $\mathrm{mg} / \mathrm{L}$ & 1 & 1 & 3 & 5 & ND & 6 \\
\hline Fixed residue & $\mathrm{mg} / \mathrm{L}$ & 2 & 2 & 7 & 11 & 6 & 7 \\
\hline $\begin{array}{l}\text { Suspended } \\
\text { solids }\end{array}$ & $\mathrm{mg} / \mathrm{L}$ & 3 & 3 & 9 & 16 & 6 & 12 \\
\hline $\begin{array}{l}\text { Total dissolved } \\
\text { solids }\end{array}$ & $\mathrm{mg} / \mathrm{L}$ & 48 & 47 & 46 & 43 & 70 & 66 \\
\hline Total solids & $\mathrm{mg} / \mathrm{L}$ & 51 & 50 & 55 & 59 & 76 & 78 \\
\hline Turbidity & NTU & 4.6 & 6.7 & 6.5 & 7.2 & 5.9 & 7.9 \\
\hline Chloride & $\mathrm{mg} / \mathrm{L}$ & 6 & 6 & 4 & 6 & 8 & 7 \\
\hline $\begin{array}{l}\text { Nitrogen- } \\
\text { ammonia }\end{array}$ & $\mathrm{mg} / \mathrm{L}$ & ND & 0.09 & ND & ND & ND & ND \\
\hline Nitrogen-nitrate & $\mathrm{mg} / \mathrm{L}$ & 0.37 & 0.34 & 0.18 & 0.44 & 0.40 & 0.34 \\
\hline Phosphate $P$ & $\mathrm{mg} / \mathrm{L}$ & ND & ND & ND & ND & ND & ND \\
\hline Sulfate & $\mathrm{mg} / \mathrm{L}$ & 6 & 7 & 4 & 6 & 8 & 7 \\
\hline Aluminum & $\mathrm{mg} / \mathrm{L}$ & & & 0.50 & & & 0.12 \\
\hline Cadmium & $\mathrm{mg} / \mathrm{L}$ & & & ND & & & ND \\
\hline Calcium & $\mathrm{mg} / \mathrm{L}$ & & & 4.27 & & & 4.87 \\
\hline Chromium & $\mathrm{mg} / \mathrm{L}$ & & & ND & & & ND \\
\hline Copper & $\mathrm{mg} / \mathrm{L}$ & & & 0.04 & & & 0.01 \\
\hline Iron & $\mathrm{mg} / \mathrm{L}$ & & & 0.99 & & & 0.48 \\
\hline Lead & $\mathrm{mg} / \mathrm{L}$ & & & ND & & & ND \\
\hline Magnesium & $\mathrm{mg} / \mathrm{L}$ & & & 0.86 & & & 1.05 \\
\hline Manganese & $\mathrm{mg} / \mathrm{L}$ & & & 0.07 & & & 0.02 \\
\hline Mercury & $\mathrm{mg} / \mathrm{L}$ & ND & ND & ND & ND & ND & ND \\
\hline Nickel & $\mathrm{mg} / \mathrm{L}$ & & & ND & & & ND \\
\hline Sodium & $\mathrm{mg} / \mathrm{L}$ & & & 4.49 & & & 7.98 \\
\hline Zinc & $\mathrm{mg} / \mathrm{L}$ & & & 0.007 & & & ND \\
\hline
\end{tabular}

\footnotetext{
a Metals (except mercury) are analyzed quarterly from monthly grab composite. Mercury is analyzed from a composite of monthly grab samples.

b Field measurement
} 
Table 67

SRS Stream Water Quality: Inorganic Contaminants

Page 16 of 20

\begin{tabular}{|c|c|c|c|c|c|c|c|}
\hline \multicolumn{8}{|l|}{ Pen Branch-3 } \\
\hline Parameter $^{\mathrm{a}}$ & Units & July & Aug & Sept & Oct & Nov & Dec \\
\hline Water volume & L & $2.58 E+9$ & $3.48 E+9$ & $2.94 \mathrm{E}+9$ & $1.37 E+9$ & $1.50 \mathrm{E}+9$ & $2.20 \mathrm{E}+9$ \\
\hline Sample date & & $7-12-95$ & 8-3-95 & $9-7-95$ & $10-24-95$ & $11-8-95$ & $12-6-95$ \\
\hline Temperature $^{\mathrm{b}}$ & ${ }^{\circ} \mathrm{C}$ & 21.4 & 24.0 & 23.0 & 20.0 & 14.0 & 16.0 \\
\hline $\mathrm{pH}^{\mathrm{b}}$ & $\mathrm{pH}$ & 8.6 & 6.4 & 6.8 & 6.8 & 6.2 & 6.4 \\
\hline $\begin{array}{l}\text { Dissolved } \\
\text { oxygen }^{\mathrm{b}}\end{array}$ & $\mathrm{mg} / \mathrm{L}$ & 7.8 & 6.9 & 8.9 & 7.2 & 8.4 & 8.4 \\
\hline Conductivityb & $\mu \mathrm{mhos} / \mathrm{cm}$ & 118 & 65 & 71 & 70 & 60 & 73 \\
\hline Alkalinity & $\mathrm{mg} / \mathrm{L}$ & 19 & 16 & 18 & 18 & 15 & 19 \\
\hline $\begin{array}{l}\text { Chemical } \\
\text { oxygen demand }\end{array}$ & $\mathrm{mg} / \mathrm{L}$ & ND & 20 & ND & ND & ND & ND \\
\hline Volatile solids & $\mathrm{mg} / \mathrm{L}$ & ND & 8 & 2 & 1 & 6 & 1 \\
\hline Fixed residue & $\mathrm{mg} / \mathrm{L}$ & 3 & 21 & 4 & 4 & 12 & 3 \\
\hline $\begin{array}{l}\text { Suspended } \\
\text { solids }\end{array}$ & $\mathrm{mg} / \mathrm{L}$ & 3 & 28 & 5 & 5 & 18 & 4 \\
\hline $\begin{array}{l}\text { Total dissolved } \\
\text { solids }\end{array}$ & $\mathrm{mg} / \mathrm{L}$ & 69 & 58 & 54 & 55 & 61 & 47 \\
\hline Total solids & $\mathrm{mg} / \mathrm{L}$ & 72 & 86 & 59 & 60 & 79 & 51 \\
\hline Turbidity & NTU & 4.3 & 14 & 3.7 & 4.8 & 14 & 5.9 \\
\hline Chloride & $\mathrm{mg} / \mathrm{L}$ & 8 & 5 & 5 & 5 & 5 & 6 \\
\hline $\begin{array}{l}\text { Nitrogen- } \\
\text { ammonia }\end{array}$ & $\mathrm{mg} / \mathrm{L}$ & ND & ND & ND & 0.15 & ND & 0.12 \\
\hline Nitrogen-nitrate & $\mathrm{mg} / \mathrm{L}$ & 0.29 & 0.32 & 0.35 & 0.31 & 0.30 & 0.30 \\
\hline Phosphate P & $\mathrm{mg} / \mathrm{L}$ & ND & ND & ND & ND & ND & ND \\
\hline Sulfate & $\mathrm{mg} / \mathrm{L}$ & 7 & 5 & 5 & 5 & 4 & 5 \\
\hline Aluminum & $\mathrm{mg} / \mathrm{L}$ & & & ND & & & 0.56 \\
\hline Cadmium & $\mathrm{mg} / \mathrm{L}$ & & & ND & & & ND \\
\hline Calcium & $\mathrm{mg} / \mathrm{L}$ & & & 4.36 & & & 4.86 \\
\hline Chromium & $\mathrm{mg} / \mathrm{L}$ & & & ND & & & ND \\
\hline Copper & $\mathrm{mg} / \mathrm{L}$ & & & 0.03 & & & 0.02 \\
\hline Iron & $\mathrm{mg} / \mathrm{L}$ & & & 0.45 & & & 1.07 \\
\hline Lead & $\mathrm{mg} / \mathrm{L}$ & & & ND & & & ND \\
\hline Magnesium & $\mathrm{mg} / \mathrm{L}$ & & & 1.11 & & & 0.95 \\
\hline Manganese & $\mathrm{mg} / \mathrm{L}$ & & & 0.06 & & & 0.10 \\
\hline Mercury & $\mathrm{mg} / \mathrm{L}$ & ND & ND & 0.0005 & ND & ND & ND \\
\hline Nickel & $\mathrm{mg} / \mathrm{L}$ & & & ND & & & ND \\
\hline Sodium & $\mathrm{mg} / \mathrm{L}$ & & & 7.04 & & & 9.99 \\
\hline Zinc & $\mathrm{mg} / \mathrm{L}$ & & & 0.018 & & & 0.013 \\
\hline
\end{tabular}

\footnotetext{
a Metals (except mercury) are analyzed quarterly from monthly grab composite. Mercury is analyzed from a composite of monthly grab samples.
}

b Field measurement 
Table 67

SRS Stream Water Quality: Inorganic Contaminants

Page 17 of 20

\begin{tabular}{|c|c|c|c|c|c|c|c|}
\hline Parameter $^{\mathrm{a}}$ & Units & Jan & Feb & Mar & April & May & June \\
\hline Water volume & $L$ & $4.32 E+9$ & 4.47E+9 & $3.38 E+9$ & $2.08 E+9$ & $1.63 E+9$ & $3.44 \mathrm{E}+9$ \\
\hline Sample date & & $1-5-95$ & $2-6-95$ & $3-15-95$ & $4-11-95$ & $5-25-95$ & $6-1-95$ \\
\hline Temperature $^{b}$ & ${ }^{\circ} \mathrm{C}$ & 5.1 & 4.2 & 14.7 & 19.6 & 21.0 & 24.5 \\
\hline $\mathrm{pH}^{\mathrm{b}}$ & $\mathrm{pH}$ & 7.3 & 6.8 & 6.7 & 5.2 & 6.5 & 6.2 \\
\hline $\begin{array}{l}\text { Dissolved } \\
\text { oxygen }^{b}\end{array}$ & $\mathrm{mg} / \mathrm{L}$ & 12.7 & 11.6 & 8.9 & 9.2 & 8.4 & 8.7 \\
\hline $\begin{array}{l}\text { Conductivityb } \\
\text { Alkalinity }\end{array}$ & $\mu \mathrm{mhos} / \mathrm{cm}$ & 54 & 52 & 44 & 135 & 70 & 67 \\
\hline Alkalinity & $\mathrm{mg} / \mathrm{L}$ & 9 & 8 & 11 & 13 & 17 & 15 \\
\hline $\begin{array}{l}\text { Chemical } \\
\text { oxygen demand }\end{array}$ & $\mathrm{mg} / \mathrm{L}$ & ND & ND & ND & ND & ND & ND \\
\hline Volatile solids & $\mathrm{mg} / \mathrm{L}$ & 1 & ND & 2 & 5 & ND & 3 \\
\hline Fixed residue & $\mathrm{mg} / \mathrm{L}$ & 1 & ND & 3 & 6 & 6 & 5 \\
\hline $\begin{array}{l}\text { Suspended } \\
\text { solids }\end{array}$ & $\mathrm{mg} / \mathrm{L}$ & 2 & ND & 5 & 11 & 6 & 7 \\
\hline $\begin{array}{l}\text { Total dissolved } \\
\text { solids }\end{array}$ & $\mathrm{mg} / \mathrm{L}$ & 36 & 33 & 45 & 37 & 57 & 57 \\
\hline Total solids & $\mathrm{mg} / \mathrm{L}$ & 38 & 33 & 50 & 48 & 63 & 64 \\
\hline Turbidity & NTU & 2.4 & 4.8 & 3.2 & 3.6 & 3.6 & 5.0 \\
\hline Chloride & $\mathrm{mg} / \mathrm{L}$ & 3 & 3 & 3 & 4 & 5 & 5 \\
\hline $\begin{array}{l}\text { Nitrogen- } \\
\text { ammonia }\end{array}$ & $\mathrm{mg} / \mathrm{L}$ & ND & ND & ND & ND & ND & ND \\
\hline Nitrogen-nitrate & $\mathrm{mg} / \mathrm{L}$ & 1.36 & 1.05 & 0.92 & 1.00 & 0.68 & 1.07 \\
\hline Phosphate P & $\mathrm{mg} / \mathrm{L}$ & ND & ND & ND & ND & ND & ND \\
\hline Sulfate & $\mathrm{mg} / \mathrm{L}$ & 6 & 5 & 4 & 4 & 5 & 4 \\
\hline Aluminum & $\mathrm{mg} / \mathrm{L}$ & & & 0.29 & & & 0.10 \\
\hline Cadmium & $\mathrm{mg} / \mathrm{L}$ & & & ND & & & ND \\
\hline Calcium & $\mathrm{mg} / \mathrm{L}$ & & & 2.81 & & & 2.93 \\
\hline Chromium & $\mathrm{mg} / \mathrm{L}$ & & & ND & & & ND \\
\hline Copper & $\mathrm{mg} / \mathrm{L}$ & & & ND & & & 0.01 \\
\hline Iron & $\mathrm{mg} / \mathrm{L}$ & & & 0.68 & & & 0.57 \\
\hline Lead & $\mathrm{mg} / \mathrm{L}$ & & & ND & & & ND \\
\hline Magnesium & $\mathrm{mg} / \mathrm{L}$ & & & 0.63 & & & 0.63 \\
\hline Manganese & $\mathrm{mg} / \mathrm{L}$ & & & 0.04 & & & 0.03 \\
\hline Mercury & $\mathrm{mg} / \mathrm{L}$ & ND & ND & ND & ND & ND & ND \\
\hline Nickel & $\mathrm{mg} / \mathrm{L}$ & & & ND & & & ND \\
\hline Sodium & $\mathrm{mg} / \mathrm{L}$ & & & 4.67 & & & 6.01 \\
\hline Zinc & $\mathrm{mg} / \mathrm{L}$ & & & 0.006 & & & ND \\
\hline
\end{tabular}

\footnotetext{
a Metals (except mercury) are analyzed quarterly from monthly grab composite. Mercury is analyzed from a composite of monthly grab samples.

b Field measurement
} 
Table 67

SRS Stream Water Quality: Inorganic Contaminants

Page 18 of 20

\begin{tabular}{|c|c|c|c|c|c|c|c|}
\hline \multicolumn{8}{|c|}{ Four Mile Creek-6 } \\
\hline Parameter $^{\mathbf{a}}$ & Units & July & Aug & Sept & Oct & Nov & Dec \\
\hline Water volume & L & $1.93 E+9$ & $2.99 E+9$ & $2.66 \mathrm{E}+9$ & $2.05 E+9$ & $2.13 E+9$ & $2.65 E+9$ \\
\hline Sample date & & $7-12-95$ & $8-3-95$ & $9-7-95$ & $10-24-95$ & $11-8-95$ & $12-6-95$ \\
\hline Temperature $^{\mathrm{b}}$ & ${ }^{\circ} \mathrm{C}$ & 24.5 & 24.0 & 22.0 & 18.0 & 14.0 & 16.1 \\
\hline $\mathrm{pH}^{\mathrm{b}}$ & $\mathrm{pH}$ & 6.3 & 6.3 & 6.6 & 6.5 & 6.2 & 6.1 \\
\hline $\begin{array}{l}\text { Dissolved } \\
\text { oxygen }^{b}\end{array}$ & $\mathrm{mg} / \mathrm{L}$ & 7.8 & 6.4 & 8.4 & 7.3 & 8.5 & 8.0 \\
\hline Conductivity ${ }^{b}$ & $\mu \mathrm{mhos} / \mathrm{cm}$ & 67 & 58 & 67 & 62 & 65 & 65 \\
\hline Alkalinity & $\mathrm{mg} / \mathrm{L}$ & 17 & 17 & 17 & 15 & 12 & 14 \\
\hline $\begin{array}{l}\text { Chemical } \\
\text { oxygen demand }\end{array}$ & $\mathrm{mg} / \mathrm{L}$ & ND & ND & ND & ND & ND & ND \\
\hline Volatile solids & $\mathrm{mg} / \mathrm{L}$ & ND & 3 & 1 & 1 & 3 & 1 \\
\hline Fixed residue & $\mathrm{mg} / \mathrm{L}$ & 1 & 4 & 2 & 3 & 3 & 3 \\
\hline $\begin{array}{l}\text { Suspended } \\
\text { solids }\end{array}$ & $\mathrm{mg} / \mathrm{L}$ & 1 & 7 & 3 & 3 & 6 & 4 \\
\hline $\begin{array}{l}\text { Total dissolved } \\
\text { solids }\end{array}$ & $\mathrm{mg} / \mathrm{L}$ & 61 & 50 & 54 & 51 & 62 & 47 \\
\hline Total solids & $\mathrm{mg} / \mathrm{L}$ & 62 & 57 & 57 & 54 & 68 & 51 \\
\hline Turbidity & NTU & 3.9 & 5.2 & 2.0 & 2.2 & 7.6 & 3.5 \\
\hline Chloride & $\mathrm{mg} / \mathrm{L}$ & 4 & 4 & 5 & 5 & 5 & 4 \\
\hline $\begin{array}{l}\text { Nitrogen- } \\
\text { ammonia }\end{array}$ & $\mathrm{mg} / \mathrm{L}$ & ND & ND & ND & 0.48 & ND & 0.14 \\
\hline Nitrogen-nitrate & $\mathrm{mg} / \mathrm{L}$ & 0.78 & 0.60 & 1.06 & 1.29 & 1.30 & 1.46 \\
\hline Phosphate P & $\mathrm{mg} / \mathrm{L}$ & ND & ND & ND & ND & ND & ND \\
\hline Sulfate & $\mathrm{mg} / \mathrm{L}$ & 4 & 3 & 4 & 4 & 4 & 5 \\
\hline Aluminum & $\mathrm{mg} / \mathrm{L}$ & & & ND & & & ND \\
\hline Cadmium & $\mathrm{mg} / \mathrm{L}$ & & & ND & & & ND \\
\hline Calcium & $\mathrm{mg} / \mathrm{L}$ & & & 2.99 & & & 4.15 \\
\hline Chromium & $\mathrm{mg} / \mathrm{L}$ & & & ND & & & ND \\
\hline Copper & $\mathrm{mg} / \mathrm{L}$ & & & 0.04 & & & 0.03 \\
\hline Iron & $\mathrm{mg} / \mathrm{L}$ & & & 1.23 & & & 1.00 \\
\hline Lead & $\mathrm{mg} / \mathrm{L}$ & & & ND & & & ND \\
\hline Magnesium & $\mathrm{mg} / \mathrm{L}$ & & & 6.08 & & & 0.75 \\
\hline Manganese & $\mathrm{mg} / \mathrm{L}$ & & & 0.08 & & & ND \\
\hline Mercury & $\mathrm{mg} / \mathrm{L}$ & ND & ND & ND & ND & ND & ND \\
\hline Nickel & $\mathrm{mg} / \mathrm{L}$ & & & ND & & & ND \\
\hline Sodium & $\mathrm{mg} / \mathrm{L}$ & & & 6.34 & & & 11.00 \\
\hline Zinc & $\mathrm{mg} / \mathrm{L}$ & & & 0.006 & & & 0.017 \\
\hline
\end{tabular}

a Metals (except mercury) are analyzed quarterly from monthly grab composite. Mercury is analyzed from a composite of monthly

b Field measurement 
Table 67

SRS Stream Water Quality: Inorganic Contaminants

Page 19 of 20

\begin{tabular}{|c|c|c|c|c|c|c|c|}
\hline \multicolumn{8}{|c|}{ Upper Three Runs-1A } \\
\hline Parameter ${ }^{\mathrm{a}}$ & Units & Jan & Feb & Mar & April & May & June \\
\hline Water volume & $L$ & $1.06 E+10$ & $9.87 E+9$ & $9.94 E+9$ & $8.52 E+9$ & $8.19 E+9$ & $9.76 E+9$ \\
\hline Sample date & & $1-5-95$ & $2-6-95$ & $3-15-95$ & $4-11-95$ & $5-25-95$ & $6-1-95$ \\
\hline Temperature $^{\mathrm{b}}$ & ${ }^{\circ} \mathrm{C}$ & 9.5 & 8.0 & 14.8 & 18.0 & 21.5 & 19.9 \\
\hline $\mathrm{pH}^{\mathrm{b}}$ & $\mathrm{pH}$ & 6.6 & 6.9 & 6.0 & 5.6 & 6.7 & 5.2 \\
\hline $\begin{array}{l}\text { Dissolved } \\
\text { oxygen }^{b}\end{array}$ & $\mathrm{mg} / \mathrm{L}$ & 10.3 & 10.0 & 9.4 & 7.4 & 9.1 & 8.5 \\
\hline Conductivityb & $\mu \mathrm{mhos} / \mathrm{cm}$ & 15 & 61 & 23 & 55 & 30 & 15 \\
\hline Alkalinity & $\mathrm{mg} / \mathrm{L}$ & 2 & 2 & 3 & 3 & 2 & 2 \\
\hline $\begin{array}{l}\text { Chemical } \\
\text { oxygen demand }\end{array}$ & $\mathrm{mg} / \mathrm{L}$ & ND & ND & ND & ND & ND & ND \\
\hline Volatile solids & $\mathrm{mg} / \mathrm{L}$ & 2 & 1 & 2 & 4 & ND & 2 \\
\hline Fixed residue & $\mathrm{mg} / \mathrm{L}$ & ND & ND & 2 & 9 & 6 & 2 \\
\hline $\begin{array}{l}\text { Suspended } \\
\text { solids }\end{array}$ & $\mathrm{mg} / \mathrm{L}$ & 2 & 1 & 4 & 13 & 7 & 4 \\
\hline $\begin{array}{l}\text { Total dissolved } \\
\text { solids }\end{array}$ & $\mathrm{mg} / \mathrm{L}$ & 22 & 12 & 13 & 6 & 25 & 31 \\
\hline Total solids & $\mathrm{mg} / \mathrm{L}$ & 24 & 13 & 17 & 19 & 32 & 35 \\
\hline Turbidity & NTU & 1.4 & 1.9 & 2.0 & 2.0 & 2.6 & 3.6 \\
\hline Chloride & $\mathrm{mg} / \mathrm{L}$ & 3 & 2 & 2 & 2 & 3 & 2 \\
\hline $\begin{array}{l}\text { Nitrogen- } \\
\text { ammonia }\end{array}$ & mg/L & ND & ND & ND & ND & ND & ND \\
\hline Nitrogen-nitrate & $\mathrm{mg} / \mathrm{L}$ & 0.36 & 0.31 & 0.31 & 0.38 & 0.33 & 0.28 \\
\hline Phosphate P & $\mathrm{mg} / \mathrm{L}$ & ND & ND & ND & ND & ND & ND \\
\hline Sulfate & $\mathrm{mg} / \mathrm{L}$ & 1 & 1 & 1 & 1 & 1 & 1 \\
\hline Aluminum & $\mathrm{mg} / \mathrm{L}$ & & & 0.16 & & & 0.10 \\
\hline Cadmium & $\mathrm{mg} / \mathrm{L}$ & & & ND & & & ND \\
\hline Calcium & $\mathrm{mg} / \mathrm{L}$ & & & 0.52 & & & 0.45 \\
\hline Chromium & $\mathrm{mg} / \mathrm{L}$ & & & ND & & & ND \\
\hline Copper & $\mathrm{mg} / \mathrm{L}$ & & & ND & & & ND \\
\hline Iron & $\mathrm{mg} / \mathrm{L}$ & & & 0.48 & & & 0.35 \\
\hline Lead & mg/L & & & ND & & & ND \\
\hline Magnesium & $\mathrm{mg} / \mathrm{L}$ & & & 0.38 & & & 0.32 \\
\hline Manganese & $\mathrm{mg} / \mathrm{L}$ & & & 0.14 & & & 0.01 \\
\hline Mercury & $\mathrm{mg} / \mathrm{L}$ & ND & ND & ND & ND & ND & ND \\
\hline Nickel & $\mathrm{mg} / \mathrm{L}$ & & & ND & & & ND \\
\hline Sodium & $\mathrm{mg} / \mathrm{L}$ & & & 1.40 & & & 1.27 \\
\hline Zinc & mg/L & & & 0.007 & & & ND \\
\hline
\end{tabular}

\footnotetext{
a Metals (except mercury) are analyzed quarterly from monthly grab composite. Mercury is analyzed from a composite of monthly grab samples.

b Field measurement
} 
Table 67

SRS Stream Water Quality: Inorganic Contaminants

Page 20 of 20

\begin{tabular}{|c|c|c|c|c|c|c|c|}
\hline \multicolumn{8}{|c|}{ Upper Three Runs-1A } \\
\hline Parameter $^{a}$ & Units & July & $\overline{\text { Aug }}$ & $\overline{\text { Sept }}$ & Oct & Nov & Dec \\
\hline Water volume & L & $8.65 E+9$ & $1.02 E+10$ & $9.40 E+9$ & $9.25 E+9$ & $9.40 E+9$ & $9.86 E+9$ \\
\hline Sample date & & $7-12-95$ & $8-3-95$ & $9-7-95$ & $10-24-95$ & $11-8-95$ & $12-6-95$ \\
\hline Temperature ${ }^{b}$ & ${ }^{\circ} \mathrm{C}$ & 21.0 & 22.0 & 20.0 & 17.0 & 15.0 & 15.2 \\
\hline $\mathrm{pH}^{\mathrm{b}}$ & $\mathrm{pH}$ & 4.3 & 5.5 & 6.2 & 6.6 & 4.5 & 4.7 \\
\hline $\begin{array}{l}\text { Dissolved } \\
\text { oxygen }^{\mathbf{b}}\end{array}$ & $\mathrm{mg} / \mathrm{L}$ & 7.0 & 6.8 & 7.8 & 9.1 & 8.0 & 7.4 \\
\hline Conductivityb & $\mu \mathrm{mhos} / \mathrm{cm}$ & 24 & 16 & 16 & 22 & 20 & 23 \\
\hline Alkalinity & $\mathrm{mg} / \mathrm{L}$ & 3 & 2 & 3 & 4 & 2 & 3 \\
\hline $\begin{array}{l}\text { Chemical } \\
\text { oxygen demand }\end{array}$ & $\mathrm{mg} / \mathrm{L}$ & ND & ND & ND & ND & ND & ND \\
\hline Volatile solids & $\mathrm{mg} / \mathrm{L}$ & 1 & 4 & 2 & 1 & 3 & 2 \\
\hline Fixed residue & $\mathrm{mg} / \mathrm{L}$ & 4 & 5 & 2 & 3 & 2 & 2 \\
\hline $\begin{array}{l}\text { Suspended } \\
\text { solids }\end{array}$ & $\mathrm{mg} / \mathrm{L}$ & 5 & 8 & 4 & 4 & 5 & 3 \\
\hline $\begin{array}{l}\text { Total dissolved } \\
\text { solids }\end{array}$ & $\mathrm{mg} / \mathrm{L}$ & 30 & 29 & 25 & 23 & 43 & 24 \\
\hline Total solids & $\mathrm{mg} / \mathrm{L}$ & 35 & 37 & 29 & 27 & 48 & 27 \\
\hline Turbidity & NTU & 4.3 & 5.4 & 2.0 & 2.4 & 9.0 & 1.9 \\
\hline Chloride & $\mathrm{mg} / \mathrm{L}$ & 3 & 2 & 2 & 2 & 2 & 4 \\
\hline $\begin{array}{l}\text { Nitrogen- } \\
\text { ammonia }\end{array}$ & $\mathrm{mg} / \mathrm{L}$ & ND & ND & ND & 0.79 & 0.12 & 0.25 \\
\hline Nitrogen-nitrate & $\mathrm{mg} / \mathrm{L}$ & 0.28 & 0.28 & 0.34 & 0.34 & 0.24 & 0.34 \\
\hline Phosphate P & $\mathrm{mg} / \mathrm{L}$ & ND & ND & ND & ND & ND & ND \\
\hline Sulfate & $\mathrm{mg} / \mathrm{L}$ & 1 & 1 & 1 & 1 & 1 & 1 \\
\hline Aluminum & $\mathrm{mg} / \mathrm{L}$ & & & 0.25 & & & ND \\
\hline Cadmium & $\mathrm{mg} / \mathrm{L}$ & & & ND & & & ND \\
\hline Calcium & $\mathrm{mg} / \mathrm{L}$ & & & 0.22 & & & 0.83 \\
\hline Chromium & $\mathrm{mg} / \mathrm{L}$ & & & ND & & & ND \\
\hline Copper & $\mathrm{mg} / \mathrm{L}$ & & & 0.07 & & & 0.02 \\
\hline Iron & $\mathrm{mg} / \mathrm{L}$ & & & 0.41 & & & 0.38 \\
\hline Lead & $\mathrm{mg} / \mathrm{L}$ & & & ND & & & ND \\
\hline Magnesium & $\mathrm{mg} / \mathrm{L}$ & & & ND & & & 0.42 \\
\hline Manganese & $\mathrm{mg} / \mathrm{L}$ & & & ND & & & ND \\
\hline Mercury & $\mathrm{mg} / \mathrm{L}$ & ND & ND & ND & ND & ND & ND \\
\hline Nickel & $\mathrm{mg} / \mathrm{L}$ & & & ND & & & ND \\
\hline Sodium & $\mathrm{mg} / \mathrm{L}$ & & & 1.65 & & & 4.92 \\
\hline Zinc & $\mathrm{mg} / \mathrm{L}$ & & & 0.061 & & & ND \\
\hline
\end{tabular}

a Metals (except mercury) are analyzed quarterly from monthly grab composite. Mercury is analyzed from a composite of monthly
grab samples.

b Field measurement 


\section{Table 68}

SRS Stream Water Quality: Pesticides, Herbicides, and Volatile Organic Compounds

Page 1 of 1

Note: $\quad$ EMS samples from the following SCDHEC locations were analyzed monthly for the constituents listed below. Practical quantification limits (PQLS) listed are achieved for pure water in ideal laboratory conditions. Actual PQLs reported to Westinghouse Savannah River Company by the laboratory may vary because of impurities in the water, instrument variations, sample volume size, and/or sample dilution factors.

\begin{tabular}{|c|c|c|c|c|c|}
\hline & \multirow{2}{*}{$\begin{array}{c}\text { Practical } \\
\text { Quantification } \\
\text { Limit } \\
(\mu \mathrm{g} / \mathrm{L})\end{array}$} & \multicolumn{4}{|c|}{$\mu g / L$} \\
\hline & & Tims Branch-5 & $\begin{array}{l}\text { Four Mile } \\
\text { Creek-A7 }\end{array}$ & Steel Creek-4 & $\begin{array}{c}\text { Upper Three } \\
\text { Runs Creek-4 } \\
\text { at Road A }\end{array}$ \\
\hline \multicolumn{6}{|l|}{ Pesticides } \\
\hline Aldrin & 0.05 & $<0.05$ & $<0.05$ & $<0.05$ & $<0.05$ \\
\hline Alpha-BHC & 0.05 & $<0.05$ & $<0.05$ & $<0.05$ & $<0.05$ \\
\hline Beta-BHC & 0.05 & $<0.05$ & $<0.05$ & $<0.05$ & $<0.05$ \\
\hline Delta-BHC & 0.05 & $<0.05$ & $<0.05$ & $<0.05$ & $<0.05$ \\
\hline Lindane & 0.05 & $<0.05$ & $<0.05$ & $<0.05$ & $<0.05$ \\
\hline Chlordane & 0.50 & $<0.50$ & $<0.50$ & $<0.50$ & $<0.50$ \\
\hline 4,4-DDD & 0.05 & $<0.05$ & $<0.05$ & $<0.05$ & $<0.05$ \\
\hline 4,4-DDE & 0.05 & $<0.05$ & $<0.05$ & $<0.05$ & $<0.05$ \\
\hline 4,4-DDT & 0.05 & $<0.05$ & $<0.05$ & $<0.05$ & $<0.05$ \\
\hline Dieldrin & 0.05 & $<0.05$ & $<0.05$ & $<0.05$ & $<0.05$ \\
\hline Endosulfan I & 0.05 & $<0.05$ & $<0.05$ & $<0.05$ & $<0.05$ \\
\hline Endosulfan II & 0.05 & $<0.05$ & $<0.05$ & $<0.05$ & $<0.05$ \\
\hline Endosulfan sulfate & 0.05 & $<0.05$ & $<0.05$ & $<0.05$ & $<0.05$ \\
\hline Endrin & 0.05 & $<0.05$ & $<0.05$ & $<0.05$ & $<0.05$ \\
\hline Endrin aldehyde & 0.05 & $<0.05$ & $<0.05$ & $<0.05$ & $<0.05$ \\
\hline Heptachlor & 0.05 & 0.052 & $<0.05$ & $<0.05$ & $<0.05$ \\
\hline Heptachlor epoxide & 0.05 & 0.052 & $<0.05$ & $<0.05$ & $<0.05$ \\
\hline Methoxychlor & 0.20 & 0.52 & $<0.20$ & $<0.20$ & $<0.20$ \\
\hline Toxaphene & 5.00 & 5.2 & $<5.00$ & 4.8 & $<5.00$ \\
\hline \multicolumn{6}{|l|}{ Herbicides } \\
\hline $2,4-D$ & 0.10 & $<0.10$ & $<0.10$ & $<0.10$ & $<0.10$ \\
\hline 2,4, 5-TP (Silvex) & 0.10 & $<0.10$ & $<0.10$ & $<0.10$ & 0.28 \\
\hline \multicolumn{6}{|c|}{ Volatile Organic Compounds } \\
\hline Tetrachloroethene & 2.0 & 3.4 & $<2.0$ & $<2.0$ & 3.7 \\
\hline 111-Trichloroethane & 2.0 & $<2.0$ & $<2.0$ & $<2.0$ & $<2.0$ \\
\hline Trichloroethene & 2.0 & $<2.0$ & $<2.0$ & $<2.0$ & $<2.0$ \\
\hline
\end{tabular}


Table 69

SRS Stream and Savannah River Water Quality: Pesticides and Herbicides

Page 1 of 2

$\mu \mathrm{g} / \mathrm{L}$

\begin{tabular}{llllll} 
& Steel Creek & $\begin{array}{c}\text { PAR Pond } \\
\text { Pump House }\end{array}$ & $\begin{array}{c}\text { Lower Three } \\
\text { Runs-3 }\end{array}$ & $\begin{array}{c}\text { Upper Three } \\
\text { Runs-4 }\end{array}$ & $\begin{array}{c}\text { Upper Three } \\
\text { Runs-Road F }\end{array}$ \\
\hline Pesticides & & & & & \\
\hline Aldrin & $<0.050$ & $<0.056$ & $<0.11$ & $<0.055$ & Not collected \\
Alpha-BHC & $<0.050$ & $<0.056$ & $<0.11$ & $<0.055$ & Not collected \\
Beta-BHC & $<0.050$ & $<0.056$ & $<0.11$ & $<0.055$ & Not collected \\
Delta-BHC & $<0.050$ & $<0.056$ & $<0.11$ & $<0.055$ & Not collected \\
Lindane & $<0.050$ & $<0.056$ & $<0.11$ & $<0.055$ & Not collected \\
Chlordane & $<0.50$ & $<0.56$ & $<1.1$ & $<0.55$ & Not collected \\
4,4-DDD & $<0.10$ & $<0.11$ & $<0.22$ & $<0.11$ & Not collected \\
4,4-DDE & $<0.10$ & $<0.11$ & $<0.22$ & $<0.11$ & Not collected \\
4,4-DDT & $<0.10$ & $<0.11$ & $<0.22$ & $<0.11$ & Not collected \\
Dieldrin & $<0.10$ & $<0.11$ & $<0.22$ & $<0.11$ & Not collected \\
Endosulfan I & $<0.050$ & $<0.056$ & $<0.11$ & $<0.055$ & Not collected \\
Endosulfan II & $<0.10$ & $<0.11$ & $<0.22$ & $<0.11$ & Not collected \\
Endosulfan sulfate & $<0.10$ & $<0.11$ & $<0.22$ & $<0.11$ & Not collected \\
Endrin & $<0.10$ & $<0.11$ & $<0.22$ & $<0.11$ & Not collected \\
Endrin aldehyde & $<0.10$ & $<0.11$ & $<0.22$ & $<0.11$ & Not collected \\
Heptachlor & $<0.050$ & $<0.056$ & $<0.11$ & $<0.055$ & Not collected \\
Heptachlor epoxide & $<0.050$ & $<0.056$ & $<0.11$ & $<0.055$ & Not collected \\
Methoxychlor & $<0.50$ & $<0.56$ & $<1.1$ & $<0.55$ & Not collected \\
Toxaphene & $<5.0$ & $<5.6$ & $<11$ & $<5.5$ & Not collected \\
\hline Herbicides & $<1.0$ & $<1.1$ & $<2.2$ & $<1.1$ & Not collected \\
\hline 2,4-D & $<0.21$ & $<0.22$ & $<0.44$ & $<0.21$ & Not collected \\
2,4,5-TP (Silvex) & & & & &
\end{tabular}




\section{Table 69}

SRS Stream and Savannah River Water Quality: Pesticides and Herbicides

Page 2 of 2

$\mu \mathrm{g} / \mathrm{L}$

\begin{tabular}{|c|c|c|c|c|}
\hline & \multicolumn{2}{|l|}{ - } & \multicolumn{2}{|l|}{$\mu \mathrm{g} / \mathrm{L}$} \\
\hline & River 2 & River 10 & Pen Branch 3 & Four Mile 6 \\
\hline \multicolumn{5}{|l|}{ Pesticides } \\
\hline Aldrin & $<0.052$ & $<0.052$ & $<0.10$ & $<0.054$ \\
\hline Alpha-BHC & $<0.052$ & $<0.052$ & $<0.10$ & $<0.054$ \\
\hline Beta-BHC & $<0.052$ & $<0.052$ & $<0.10$ & $<0.054$ \\
\hline Delta-BHC & $<0.052$ & $<0.052$ & $<0.10$ & $<0.054$ \\
\hline Lindane & $<0.052$ & $<0.052$ & $<0.10$ & $<0.054$ \\
\hline Chlordane & $<0.52$ & $<0.52$ & $<1.0$ & $<0.54$ \\
\hline 4,4-DDD & $<0.10$ & $<0.10$ & $<0.21$ & $<0.11$ \\
\hline $4,4-\mathrm{DDE}$ & $<0.10$ & $<0.10$ & $<0.21$ & $<0.11$ \\
\hline 4,4-DDT & $<0.10$ & $<0.10$ & $<0.21$ & $<0.11$ \\
\hline Dieldrin & $<0.10$ & $<0.10$ & $<0.21$ & $<0.11$ \\
\hline Endosulfan I & $<0.052$ & $<0.052$ & $<0.10$ & $<0.054$ \\
\hline Endosulfan II & $<0.10$ & $<0.10$ & $<0.21$ & $<0.11$ \\
\hline Endosulfan sulfate & $<0.10$ & $<0.10$ & $<0.21$ & $<0.11$ \\
\hline Endrin & $<0.10$ & $<0.10$ & $<0.21$ & $<0.11$ \\
\hline Endrin aldehyde & $<0.10$ & $<0.10$ & $<0.21$ & $<0.11$ \\
\hline Heptachlor & $<0.052$ & $<0.052$ & $<0.10$ & $<0.054$ \\
\hline Heptachlor epoxide & $<0.052$ & $<0.052$ & $<0.10$ & $<0.054$ \\
\hline Methoxychlor & $<0.52$ & $<0.52$ & $<1.0$ & $<0.54$ \\
\hline Toxaphene & $<5.2$ & $<5.2$ & $<10$ & $<5.4$ \\
\hline \multicolumn{5}{|l|}{ Herbicides } \\
\hline $2,4-D$ & $<1.0$ & $<1.2$ & $<2.1$ & $<1.1$ \\
\hline 2,4,5-TP (Silvex) & $<0.20$ & 0.24 & $<0.42$ & $<0.21$ \\
\hline
\end{tabular}


Table 70

Savannah River Water Quality: Inorganic Contaminants

Page 1 of 6

\begin{tabular}{llllllll}
\hline RM-160 (Above SRS) & & & & & & \\
\hline Parameter & Units & Jan & Feb & Mar & April & May & June \\
\hline
\end{tabular}

Notes: "ND" denotes "not detected."

Fecal coliform results can be found in table 72 on page 232.

\begin{tabular}{|c|c|c|c|c|c|c|c|}
\hline Water volume & $L$ & $1.15 \mathrm{E}+12$ & b & b & $6.44 E+11$ & $5.24 \mathrm{E}+11$ & $5.39 E+11$ \\
\hline Sample date & & $1-3-95$ & $2-7-95$ & $3-7-95$ & $4-11-95$ & $5-23-95$ & $6-13-95$ \\
\hline Temperature ${ }^{b}$ & ${ }^{\circ} \mathrm{C}$ & 11.2 & 8.0 & 11.3 & 16.1 & 21.2 & 23.3 \\
\hline $\mathrm{pH}^{\mathrm{b}}$ & $\mathrm{pH}$ & 6.8 & 6.7 & 6.8 & 6.9 & 6.6 & 6.4 \\
\hline $\begin{array}{l}\text { Dissolved } \\
\text { oxygen }\end{array}$ & $\mathrm{mg} / \mathrm{L}$ & 9.8 & 9.2 & 10.8 & 6.7 & 7.7 & 7.0 \\
\hline Conductivityb & $\mu \mathrm{mhos} / \mathrm{cm}$ & 79 & 82 & 63 & 80 & 107 & 103 \\
\hline Alkalinity & $\mathrm{mg} / \mathrm{L}$ & 18 & 19 & 18 & 20 & 22 & 23 \\
\hline $\begin{array}{l}\text { Chemical } \\
\text { oxygen demand }\end{array}$ & $\mathrm{mg} / \mathrm{L}$ & ND & ND & ND & ND & ND & ND \\
\hline Volatile solids & $\mathrm{mg} / \mathrm{L}$ & 1 & 1 & ND & 4 & 4 & 1 \\
\hline Fixed residue & $\mathrm{mg} / \mathrm{L}$ & 4 & 4 & 9 & 12 & 3 & 8 \\
\hline $\begin{array}{l}\text { Suspended } \\
\text { solids }\end{array}$ & $\mathrm{mg} / \mathrm{L}$ & 5 & 5 & 9 & 16 & 7 & 9 \\
\hline $\begin{array}{l}\text { Total dissolved } \\
\text { solids }\end{array}$ & $\mathrm{mg} / \mathrm{L}$ & 51 & 55 & 51 & 48 & 91 & 86 \\
\hline Total solids & $\mathrm{mg} / \mathrm{L}$ & 56 & 60 & 60 & 64 & 98 & 95 \\
\hline Turbidity & NTU & 6.3 & 10 & 9.6 & 9.7 & 8.5 & 8.9 \\
\hline Chloride & $\mathrm{mg} / \mathrm{L}$ & 7 & 7 & 5 & 8 & 11 & 11 \\
\hline $\begin{array}{l}\text { Nitrogen- } \\
\text { ammonia }\end{array}$ & $\mathrm{mg} / \mathrm{L}$ & 0.10 & 0.08 & 0.05 & 0.08 & 0.08 & 0.09 \\
\hline Nitrogen-nitrate & $\mathrm{mg} / \mathrm{L}$ & 0.34 & 0.30 & 0.27 & 0.45 & 0.39 & 0.45 \\
\hline Phosphate P & $\mathrm{mg} / \mathrm{L}$ & ND & ND & ND & ND & ND & ND \\
\hline Sulfate & $\mathrm{mg} / \mathrm{L}$ & 6 & 7 & 6 & 7 & 11 & 10 \\
\hline Aluminum & $\mathrm{mg} / \mathrm{L}$ & & & 0.31 & & & 0.33 \\
\hline Cadmium & $\mathrm{mg} / \mathrm{L}$ & & & ND & & & ND \\
\hline Calcium & $\mathrm{mg} / \mathrm{L}$ & & & 3.52 & & & 4.00 \\
\hline Chromium & $\mathrm{mg} / \mathrm{L}$ & & & ND & & & ND \\
\hline Copper & $\mathrm{mg} / \mathrm{L}$ & & & ND & & & 0.01 \\
\hline Iron & $\mathrm{mg} / \mathrm{L}$ & & & 0.59 & & & 0.68 \\
\hline Lead & $\mathrm{mg} / \mathrm{L}$ & & & ND & & & ND \\
\hline Magnesium & $\mathrm{mg} / \mathrm{L}$ & & & 1.39 & & & 1.33 \\
\hline Manganese & $\mathrm{mg} / \mathrm{L}$ & & & 0.06 & & & 0.07 \\
\hline Mercury & $\mathrm{mg} / \mathrm{L}$ & ND & ND & ND & ND & ND & ND \\
\hline Nickel & $\mathrm{mg} / \mathrm{L}$ & & & ND & & & ND \\
\hline Sodium & $\mathrm{mg} / \mathrm{L}$ & & & 7.58 & & & 11.3 \\
\hline Zinc & $\mathrm{mg} / \mathrm{L}$ & & & ND & & & 0.005 \\
\hline
\end{tabular}

\footnotetext{
a Metals (except mercury) are analyzed quarterly from monthly grab composites. Mercury is analyzed from a composite of monthly grab samples.

b Water volume was too high for flow meter to register.

c Field measurement
} 
Table 70

Savannah River Water Quality: Inorganic Contaminants

Page 2 of 6

\begin{tabular}{|c|c|c|c|c|c|c|c|}
\hline \multicolumn{8}{|c|}{ RM-160 (Above SRS) } \\
\hline Parametera $^{a}$ & Units & July & Aug & Sept & Oct & Nov & Dec \\
\hline Water volume & $L$ & $5.80 E+11$ & $6.96 \mathrm{E}+11$ & $7.85 E+11$ & $8.58 E+11$ & 0 & $1.10 \mathrm{E}+12$ \\
\hline Sample date & & $7-11-95$ & $8-1-95$ & $9-6-95$ & $10-24-95$ & $11-7-95$ & $12-5-95$ \\
\hline Temperature ${ }^{b}$ & ${ }^{\circ} \mathrm{C}$ & 24.4 & 24.5 & 21.0 & 20.7 & 17.7 & 13.9 \\
\hline $\mathrm{pH}^{\mathrm{b}}$ & $\mathrm{pH}$ & 6.0 & 6.6 & 6.4 & 6.0 & 7.0 & 6.4 \\
\hline $\begin{array}{l}\text { Dissolved } \\
\text { oxygen }^{6}\end{array}$ & $\mathrm{mg} / \mathrm{L}$ & 6.9 & 8.7 & 7.9 & 7.9 & 8.3 & 9.9 \\
\hline Conductivityb & $\mu \mathrm{mhos} / \mathrm{cm}$ & 99 & 92 & 61 & 78 & 83 & 62 \\
\hline Alkalinity & $\mathrm{mg} / \mathrm{L}$ & 23 & 21 & 17 & 17 & 19 & 18 \\
\hline $\begin{array}{l}\text { Chemical } \\
\text { oxygen demand }\end{array}$ & $\mathrm{mg} / \mathrm{L}$ & ND & ND & ND & ND & ND & ND \\
\hline Volatile solids & $\mathrm{mg} / \mathrm{L}$ & ND & 2 & 2 & 1 & 4 & 2 \\
\hline Fixed residue & $\mathrm{mg} / \mathrm{L}$ & 3 & 8 & 11 & 9 & 6 & 13 \\
\hline $\begin{array}{l}\text { Suspended } \\
\text { solids }\end{array}$ & $\mathrm{mg} / \mathrm{L}$ & 3 & 9 & 13 & 10 & 9 & 15 \\
\hline $\begin{array}{l}\text { Total dissolved } \\
\text { solids }\end{array}$ & $\mathrm{mg} / \mathrm{L}$ & 83 & 68 & 55 & 53 & 69 & 54 \\
\hline Total solids & $\mathrm{mg} / \mathrm{L}$ & 86 & 77 & 68 & 63 & 78 & 69 \\
\hline Turbidity & NTU & 6.0 & 8.9 & 7.4 & 6.8 & 4.8 & 15 \\
\hline Chloride & $\mathrm{mg} / \mathrm{L}$ & 10 & 8 & 5 & 5 & 8 & 5 \\
\hline $\begin{array}{l}\text { Nitrogen- } \\
\text { ammonia }\end{array}$ & $\mathrm{mg} / \mathrm{L}$ & ND & ND & ND & 0.16 & ND & 0.15 \\
\hline Nitrogen-nitrate & $\mathrm{mg} / \mathrm{L}$ & 0.37 & 0.34 & 0.31 & 0.29 & 0.32 & 0.31 \\
\hline Phosphate P & $\mathrm{mg} / \mathrm{L}$ & ND & ND & ND & ND & ND & ND \\
\hline Sulfate & $\mathrm{mg} / \mathrm{L}$ & 9 & 8 & 4 & 5 & 7 & 5 \\
\hline Aluminum & $\mathrm{mg} / \mathrm{L}$ & & & 0.65 & & & 0.48 \\
\hline Cadmium & $\mathrm{mg} / \mathrm{L}$ & & & ND & & & ND \\
\hline Calcium & $\mathrm{mg} / \mathrm{L}$ & & & 3.41 & & & 3.20 \\
\hline Chromium & $\mathrm{mg} / \mathrm{L}$ & & & ND & & & ND \\
\hline Copper & $\mathrm{mg} / \mathrm{L}$ & & & 0.02 & & & 0.01 \\
\hline Iron & $\mathrm{mg} / \mathrm{L}$ & & & 0.64 & & & 0.61 \\
\hline Lead & $\mathrm{mg} / \mathrm{L}$ & & & ND & & & ND \\
\hline Magnesium & $\mathrm{mg} / \mathrm{L}$ & & & 1.37 & & & 1.22 \\
\hline Manganese & $\mathrm{mg} / \mathrm{L}$ & & & 0.11 & & & 0.07 \\
\hline Mercury & $\mathrm{mg} / \mathrm{L}$ & ND & ND & 0.0006 & ND & ND & ND \\
\hline Nickel & $\mathrm{mg} / \mathrm{L}$ & & & ND & & & ND \\
\hline Sodium & $\mathrm{mg} / \mathrm{L}$ & & & 9.24 & & & 10.70 \\
\hline Zinc & $\mathrm{mg} / \mathrm{L}$ & & & 0.059 & & & 0.020 \\
\hline
\end{tabular}


Table 70

Savannah River Water Quality: Inorganic Contaminants

Page 3 of 6

\begin{tabular}{|c|c|c|c|c|c|c|c|}
\hline \\
\hline \multicolumn{7}{|c|}{$\begin{array}{l}\text { Plant Vogtle Discharge } \\
\text { Parametera Units }\end{array}$} & June \\
\hline Water volume & L & $1.20 E+12$ & b & b & $6.30 \mathrm{E}+11$ & $4.98 \mathrm{E}+11$ & $5.26 E+11$ \\
\hline Sample date & & $1-3-95$ & $2-7-95$ & $3-7-95$ & $4-11-95$ & $5-23-95$ & $6-13-95$ \\
\hline Temperature $^{b}$ & ${ }^{\circ} \mathrm{C}$ & 11.0 & 8.1 & 11.3 & 16.8 & 21.9 & 24.1 \\
\hline $\mathrm{pH}^{\mathrm{b}}$ & $\mathrm{pH}$ & 6.8 & 6.7 & 6.8 & 6.9 & 6.7 & 6.4 \\
\hline $\begin{array}{l}\text { Dissolved } \\
\text { oxygen }^{b}\end{array}$ & $\mathrm{mg} / \mathrm{L}$ & 9.7 & 11.2 & 10.1 & 6.1 & 7.3 & 5.3 \\
\hline Conductivityb & $\mu \mathrm{mhos} / \mathrm{cm}$ & 77 & 85 & 68 & 81 & 107 & 102 \\
\hline Alkalinity & $\mathrm{mg} / \mathrm{L}$ & 18 & 18 & 17 & 21 & 23 & 25 \\
\hline $\begin{array}{l}\text { Chemical } \\
\text { oxygen demand }\end{array}$ & $\mathrm{mg} / \mathrm{L}$ & ND & ND & ND & ND & ND & ND \\
\hline Volatile solids & $\mathrm{mg} / \mathrm{L}$ & 1 & 5 & ND & 4 & 3 & 2 \\
\hline Fixed residue & $\mathrm{mg} / \mathrm{L}$ & 6 & 5 & 7 & 13 & 6 & 10 \\
\hline $\begin{array}{l}\text { Suspended } \\
\text { solids }\end{array}$ & $\mathrm{mg} / \mathrm{L}$ & 7 & 10 & 7 & 16 & 9 & 12 \\
\hline $\begin{array}{l}\text { Total dissolved } \\
\text { solids }\end{array}$ & $\mathrm{mg} / \mathrm{L}$ & 54 & 58 & 51 & 53 & 90 & 88 \\
\hline Total solids & $\mathrm{mg} / \mathrm{L}$ & 61 & 68 & 58 & 69 & 99 & 100 \\
\hline Turbidity & NTU & 6.0 & 10 & 9.3 & 10 & 9.2 & 11 \\
\hline Chloride & $\mathrm{mg} / \mathrm{L}$ & 7 & 6 & 5 & 8 & 11 & 11 \\
\hline $\begin{array}{l}\text { Nitrogen- } \\
\text { ammonia }\end{array}$ & $\mathrm{mg} / \mathrm{L}$ & 0.08 & 0.10 & ND & ND & 0.08 & 0.11 \\
\hline Nitrogen-nitrate & $\mathrm{mg} / \mathrm{L}$ & 0.35 & 0.32 & 0.26 & 0.42 & 0.42 & 0.44 \\
\hline Phosphate P & $\mathrm{mg} / \mathrm{L}$ & ND & ND & ND & ND & ND & ND \\
\hline Sulfate & $\mathrm{mg} / \mathrm{L}$ & 6 & 7 & 6 & 7 & 11 & 10 \\
\hline Aluminum & $\mathrm{mg} / \mathrm{L}$ & & & 0.30 & & & 0.44 \\
\hline Cadmium & $\mathrm{mg} / \mathrm{L}$ & & & ND & & & ND \\
\hline Calcium & $\mathrm{mg} / \mathrm{L}$ & & & 3.83 & & & 4.45 \\
\hline Chromium & $\mathrm{mg} / \mathrm{L}$ & & & ND & & & ND \\
\hline Copper & $\mathrm{mg} / \mathrm{L}$ & & & ND & & & 0.01 \\
\hline Iron & $\mathrm{mg} / \mathrm{L}$ & & & 0.58 & & & 0.79 \\
\hline Lead & $\mathrm{mg} / \mathrm{L}$ & & & ND & & & ND \\
\hline Magnesium & $\mathrm{mg} / \mathrm{L}$ & & & 1.51 & & & 1.43 \\
\hline Manganese & $\mathrm{mg} / \mathrm{L}$ & & & 0.07 & & & 0.07 \\
\hline Mercury & $\mathrm{mg} / \mathrm{L}$ & ND & ND & ND & ND & ND & ND \\
\hline Nickel & $\mathrm{mg} / \mathrm{L}$ & & & ND & & & ND \\
\hline Sodium & $\mathrm{mg} / \mathrm{L}$ & & & 7.67 & & & 11.20 \\
\hline Zinc & $\mathrm{mg} / \mathrm{L}$ & & & 0.009 & & & 0.010 \\
\hline
\end{tabular}

a Metals (except mercury) are analyzed quarterly from monthly grab composites. Mercury is analyzed from a composite of monthly grab samples.

b Water volume was too high for flow meter to register

c Field measurement 
Table 70

Savannah River Water Quality: Inorganic Contaminants

Page 4 of 6

\begin{tabular}{|c|c|c|c|c|c|c|c|}
\hline \multicolumn{8}{|c|}{ Plant Vogtle Discharge } \\
\hline Parameter $^{\mathbf{a}}$ & Units & July & Aug & Sept & Oct & Nov & Dec \\
\hline Water volume & $\mathbf{L}$ & $5.52 E+11$ & $6.83 E+11$ & $7.70 E+11$ & $8.41 E+11$ & b & 1.17E+12 \\
\hline Sample date & & $7-11-95$ & $8-1-95$ & $9-6-95$ & $10-24-95$ & $11-7-95$ & $12-5-95$ \\
\hline Temperature $^{b}$ & ${ }^{\circ} \mathrm{C}$ & 25.2 & 25.0 & 21.2 & 20.5 & 17.5 & 14.1 \\
\hline $\mathrm{pH}^{\mathrm{b}}$ & $\mathrm{pH}$ & 6.1 & 6.5 & 5.9 & 5.9 & 6.5 & 6.9 \\
\hline $\begin{array}{l}\text { Dissolved } \\
\text { oxygen }^{b}\end{array}$ & $\mathrm{mg} / \mathrm{L}$ & 6.1 & 6.9 & 7.4 & 7.0 & 7.1 & 9.3 \\
\hline Conductivityb & $\mu \mathrm{mhos} / \mathrm{cm}$ & 109 & 92 & 63 & 70 & 80 & 70 \\
\hline Alkalinity & $\mathrm{mg} / \mathrm{L}$ & 23 & 21 & 18 & 17 & 20 & 20 \\
\hline $\begin{array}{l}\text { Chemical } \\
\text { oxygen demand }\end{array}$ & $\mathrm{mg} / \mathrm{L}$ & ND & ND & ND & ND & ND & ND \\
\hline Volatile solids & $\mathrm{mg} / \mathrm{L}$ & ND & 3 & 3 & 1 & 4 & 3 \\
\hline Fixed residue & $\mathrm{mg} / \mathrm{L}$ & 6 & 7 & 17 & 9 & 7 & 16 \\
\hline $\begin{array}{l}\text { Suspended } \\
\text { solids }\end{array}$ & $\mathrm{mg} / \mathrm{L}$ & 6 & 9 & 20 & 10 & 11 & 18 \\
\hline $\begin{array}{l}\text { Total dissolved } \\
\text { solids }\end{array}$ & $\mathrm{mg} / \mathrm{L}$ & 90 & 66 & 52 & 55 & 70 & 62 \\
\hline Total solids & $\mathrm{mg} / \mathrm{L}$ & 96 & 75 & 72 & 65 & 81 & 80 \\
\hline Turbidity & NTU & 7.2 & 8.8 & 11 & 6.9 & 7.6 & 16 \\
\hline Chloride & $\mathrm{mg} / \mathrm{L}$ & 12 & 8 & 5 & 5 & 8 & 7 \\
\hline $\begin{array}{l}\text { Nitrogen- } \\
\text { ammonia }\end{array}$ & $\mathrm{mg} / \mathrm{L}$ & ND & ND & 0.15 & 0.12 & 0.14 & 0.14 \\
\hline Nitrogen-nitrate & $\mathrm{mg} / \mathrm{L}$ & 0.42 & 0.35 & 0.34 & 0.29 & 0.33 & 0.35 \\
\hline Phosphate P & $\mathrm{mg} / \mathrm{L}$ & ND & ND & ND & ND & ND & ND \\
\hline Sulfate & $\mathrm{mg} / \mathrm{L}$ & 10 & 8 & 5 & 5 & 7 & 6 \\
\hline Aluminum & $\mathrm{mg} / \mathrm{L}$ & & & 0.29 & & & ND \\
\hline Cadmium & $\mathrm{mg} / \mathrm{L}$ & & & ND & & & ND \\
\hline Calcium & $\mathrm{mg} / \mathrm{L}$ & & & 2.97 & & & 3.32 \\
\hline Chromium & $\mathrm{mg} / \mathrm{L}$ & & & ND & & & ND \\
\hline Copper & $\mathrm{mg} / \mathrm{L}$ & & & 0.02 & & & 0.01 \\
\hline Iron & $\mathrm{mg} / \mathrm{L}$ & & & 0.39 & & & 0.73 \\
\hline Lead & $\mathrm{mg} / \mathrm{L}$ & & & ND & & & ND \\
\hline Magnesium & $\mathrm{mg} / \mathrm{L}$ & & & 1.24 & & & 1.41 \\
\hline Manganese & $\mathrm{mg} / \mathrm{L}$ & & & 0.11 & & & 0.07 \\
\hline Mercury & $\mathrm{mg} / \mathrm{L}$ & ND & ND & 0.0004 & ND & ND & ND \\
\hline Nickel & $\mathrm{mg} / \mathrm{L}$ & & & ND & & & ND \\
\hline Sodium & $\mathrm{mg} / \mathrm{L}$ & & & 7.04 & & & 11.90 \\
\hline Zinc & $\mathrm{mg} / \mathrm{L}$ & & & 0.015 & & & 0.009 \\
\hline
\end{tabular}

a Metals (except mercury) are analyzed quarterly from monthly grab composites. Mercury is analyzed from a composite of monthly grab samples.

b Water volume was too high for flow meter to register.

c Field measurement 


\section{Table 70}

Savannah River Water Quality: Inorganic Contaminants

Page 5 of 6

\begin{tabular}{|c|c|c|c|c|c|c|c|}
\hline \multicolumn{8}{|c|}{ RM-120 (Below SRS) } \\
\hline Parameter ${ }^{\mathbf{a}}$ & Units & Jan & Feb & Mar & April & May & June \\
\hline Water volume & L & $1.26 \mathrm{E}+12$ & $1.31 E+12$ & $1.87 E+12$ & $6.15 E+11$ & $4.72 E+11$ & $5.13 E+11$ \\
\hline Sample date & & $1-3-95$ & $2-7-95$ & $3-7-95$ & $4-11-95$ & $5-23-95$ & $6-13-95$ \\
\hline Temperature $^{b}$ & ${ }^{\circ} \mathrm{C}$ & 10.8 & 8.4 & 12.3 & 17.3 & 21.9 & 24.2 \\
\hline $\mathrm{pH}^{\mathrm{b}}$ & $\mathrm{pH}$ & 6.9 & 7.1 & 6.9 & 6.7 & 6.6 & 6.3 \\
\hline $\begin{array}{l}\text { Dissolved } \\
\text { oxygen }^{b}\end{array}$ & $\mathrm{mg} / \mathrm{L}$ & 9.3 & 7.2 & 9.4 & 6.6 & 7.5 & 6.0 \\
\hline Conductivityb & $\mu \mathrm{mhos} / \mathrm{cm}$ & 83 & 94 & 54 & 87 & 102 & 98 \\
\hline Alkalinity & $\mathrm{mg} / \mathrm{L}$ & 19 & 19 & 18 & 22 & 22 & 22 \\
\hline $\begin{array}{l}\text { Chemical } \\
\text { oxygen demand }\end{array}$ & $\mathrm{mg} / \mathrm{L}$ & ND & ND & ND & ND & ND & ND \\
\hline Volatile solids & $\mathrm{mg} / \mathrm{L}$ & 1 & 2 & ND & 4 & 4 & 3 \\
\hline Fixed residue & $\mathrm{mg} / \mathrm{L}$ & 7 & 6 & 5 & 14 & 8 & 16 \\
\hline $\begin{array}{l}\text { Suspended } \\
\text { solids }\end{array}$ & $\mathrm{mg} / \mathrm{L}$ & 8 & 8 & 5 & 17 & 11 & 18 \\
\hline $\begin{array}{l}\text { Total dissolved } \\
\text { solids }\end{array}$ & $\mathrm{mg} / \mathrm{L}$ & 59 & 57 & 54 & 58 & 89 & 82 \\
\hline Total solids & $\mathrm{mg} / \mathrm{L}$ & 67 & 65 & 59 & 75 & 100 & 100 \\
\hline Turbidity & NTU & 6.7 & 9.1 & 10 & 9.9 & 9.1 & 11 \\
\hline Chloride & $\mathrm{mg} / \mathrm{L}$ & 8 & 7 & 5 & 8 & 10 & 9 \\
\hline $\begin{array}{l}\text { Nitrogen- } \\
\text { ammonia }\end{array}$ & $\mathrm{mg} / \mathrm{L}$ & 0.09 & 0.09 & ND & ND & ND & 0.08 \\
\hline Nitrogen-nitrate & $\mathrm{mg} / \mathrm{L}$ & 0.38 & 0.34 & 0.27 & 0.47 & 0.46 & 0.46 \\
\hline Phosphate P & $\mathrm{mg} / \mathrm{L}$ & ND & ND & ND & ND & ND & ND \\
\hline Sulfate & $\mathrm{mg} / \mathrm{L}$ & 7 & 9 & 6 & 8 & 11 & 10 \\
\hline Aluminum & $\mathrm{mg} / \mathrm{L}$ & & & 0.47 & & & 0.43 \\
\hline Cadmium & $\mathrm{mg} / \mathrm{L}$ & & & ND & & & ND \\
\hline Calcium & $\mathrm{mg} / \mathrm{L}$ & & & 4.22 & & & 4.79 \\
\hline Chromium & $\mathrm{mg} / \mathrm{L}$ & & & ND & & & ND \\
\hline Copper & $\mathrm{mg} / \mathrm{L}$ & & & ND & & & 0.01 \\
\hline Iron & $\mathrm{mg} / \mathrm{L}$ & & & 0.96 & & & 0.91 \\
\hline Lead & $\mathrm{mg} / \mathrm{L}$ & & & ND & & & ND \\
\hline Magnesium & $\mathrm{mg} / \mathrm{L}$ & & & 1.51 & & & 1.48 \\
\hline Manganese & $\mathrm{mg} / \mathrm{L}$ & & & 0.06 & & & 0.07 \\
\hline Mercury & $\mathrm{mg} / \mathrm{L}$ & ND & ND & ND & ND & ND & ND \\
\hline Nickel & $\mathrm{mg} / \mathrm{L}$ & & & ND & & & ND \\
\hline Sodium & $\mathrm{mg} / \mathrm{L}$ & & & 8.32 & & & 10.6 \\
\hline Zinc & $\mathrm{mg} / \mathrm{L}$ & & & ND & & & 0.036 \\
\hline
\end{tabular}

\footnotetext{
Metals (except mercury) are analyzed quarterly from monthly grab composites. Mercury is analyzed from a composite of monthly grab samples.
}

b Field measurement 
Table 70

Savannah River Water Quality: Inorganic Contaminants

Page 6 of 6

\begin{tabular}{|c|c|c|c|c|c|c|c|}
\hline \multicolumn{8}{|c|}{ RM-120 (Below SRS) } \\
\hline Parameter ${ }^{\mathbf{a}}$ & Units & July & Aug & Sept & Oct & Nov & Dec \\
\hline Water volume & L & $5.23 E+11$ & $6.70 E+11$ & $7.55 E+11$ & $8.24 \mathrm{E}+12$ & $1.35 E+12$ & $1.23 E+12$ \\
\hline Sample date & & $7-11-95$ & $8-1-95$ & $9-6-95$ & $10-24-95$ & $11-7-95$ & $12-5-95$ \\
\hline Temperature $^{\mathrm{b}}$ & ${ }^{\circ} \mathrm{C}$ & 26.2 & 25.7 & 22.0 & 19.9 & 17.2 & 13.1 \\
\hline $\mathrm{pH}^{\mathrm{b}}$ & $\mathrm{pH}$ & 6.7 & 6.6 & 6.7 & 5.9 & 6.8 & 4.9 \\
\hline $\begin{array}{l}\text { Dissolved } \\
\text { oxygen }^{6}\end{array}$ & $\mathrm{mg} / \mathrm{L}$ & 6.5 & 6.6 & 7.3 & 7.6 & 7.7 & 7.3 \\
\hline Conductivityb & $\mu \mathrm{mhos} / \mathrm{cm}$ & 104 & 102 & 65 & 88 & 102 & 84 \\
\hline Alkalinity & $\mathrm{mg} / \mathrm{L}$ & 23 & 20 & 18 & 17 & 22 & 21 \\
\hline $\begin{array}{l}\text { Chemical } \\
\text { oxygen demand }\end{array}$ & $\mathrm{mg} / \mathrm{L}$ & ND & ND & ND & ND & ND & ND \\
\hline Volatile solids & $\mathrm{mg} / \mathrm{L}$ & 1 & 2 & 4 & 3 & 4 & 2 \\
\hline Fixed residue & $\mathrm{mg} / \mathrm{L}$ & 6 & 10 & 23 & 14 & 7 & 6 \\
\hline $\begin{array}{l}\text { Suspended } \\
\text { solids }\end{array}$ & $\mathrm{mg} / \mathrm{L}$ & 7 & 12 & 27 & 17 & 11 & 7 \\
\hline $\begin{array}{l}\text { Total dissolved } \\
\text { solids }\end{array}$ & $\mathrm{mg} / \mathrm{L}$ & 82 & 71 & 55 & 52 & 80 & 63 \\
\hline Total solids & $\mathrm{mg} / \mathrm{L}$ & 89 & 83 & 82 & 69 & 91 & 70 \\
\hline Turbidity & NTU & 6.8 & 10 & 12 & 9.1 & 5.4 & 14 \\
\hline Chloride & $\mathrm{mg} / \mathrm{L}$ & 11 & 9 & 5 & 5 & 9 & 7 \\
\hline $\begin{array}{l}\text { Nitrogen- } \\
\text { ammonia }\end{array}$ & $\mathrm{mg} / \mathrm{L}$ & ND & ND & 0.13 & 0.55 & 0.24 & 0.14 \\
\hline Nitrogen-nitrate & $\mathrm{mg} / \mathrm{L}$ & 0.41 & 0.35 & 0.35 & 0.28 & 0.33 & 0.29 \\
\hline Phosphate $P$ & $\mathrm{mg} / \mathrm{L}$ & ND & ND & ND & ND & ND & ND \\
\hline Sulfate & $\mathrm{mg} / \mathrm{L}$ & 10 & 9 & 5 & 5 & 8 & 7 \\
\hline Aluminum & $\mathrm{mg} / \mathrm{L}$ & & & ND & & & ND \\
\hline Cadmium & $\mathrm{mg} / \mathrm{L}$ & & & ND & & & ND \\
\hline Calcium & $\mathrm{mg} / \mathrm{L}$ & & & 3.86 & & & 3.77 \\
\hline Chromium & $\mathrm{mg} / \mathrm{L}$ & & & ND & & & ND \\
\hline Copper & $\mathrm{mg} / \mathrm{L}$ & & & 0.03 & & & 0.02 \\
\hline Iron & $\mathrm{mg} / \mathrm{L}$ & & & 0.93 & & & 0.84 \\
\hline Lead & $\mathrm{mg} / \mathrm{L}$ & & & ND & & & ND \\
\hline Magnesium & $\mathrm{mg} / \mathrm{L}$ & & & 1.44 & & & 1.18 \\
\hline Manganese & $\mathrm{mg} / \mathrm{L}$ & & & 0.11 & & & ND \\
\hline Mercury & $\mathrm{mg} / \mathrm{L}$ & ND & ND & 0.0015 & ND & ND & ND \\
\hline Nickel & $\mathrm{mg} / \mathrm{L}$ & & & ND & & & ND \\
\hline Sodium & $\mathrm{mg} / \mathrm{L}$ & & & 12.80 & & & 12.60 \\
\hline Zinc & $\mathrm{mg} / \mathrm{L}$ & & & 0.043 & & & 0.008 \\
\hline
\end{tabular}

a Metals (except mercury) are analyzed quarterly from monthly grab composites. Mercury is analyzed from a composite of monthly grab samples.

b Field measurement 


\section{Table 71}

Savannah River Water Quality:

Georgia Department of Natural Resources and EMS Sampling Location

Page 1 of 2

\begin{tabular}{|c|c|c|c|c|c|}
\hline Parameter & Units & June & Duplicate & December & Duplicate \\
\hline \multicolumn{6}{|c|}{$\begin{array}{l}\text { Note: "ND" denotes "none detected." } \\
\text { All analyses were analyzed from grab samples. }\end{array}$} \\
\hline \multicolumn{6}{|l|}{$681-5 G^{a}$} \\
\hline \multicolumn{6}{|l|}{ Field Analyses } \\
\hline Sample Date & & $6-13-95$ & $6-13-95$ & $12-5-95$ & $12-5-95$ \\
\hline \multicolumn{6}{|c|}{ Laboratory Analyses } \\
\hline $\mathrm{pH}$ & $\mathrm{pH}$ & 6.95 & 6.95 & 7.32 & 7.26 \\
\hline Conductivity & $\mu \mathrm{mhos} / \mathrm{cm}$ & 102 & 102 & 69 & 71 \\
\hline Alkalinity & $\mathrm{mg} / \mathrm{L}$ & 23 & 23 & 19 & 18 \\
\hline $\begin{array}{l}\text { Chemical oxygen de- } \\
\text { mand }\end{array}$ & $\mathrm{mg} / \mathrm{L}$ & ND & ND & ND & ND \\
\hline Volatile solids & $\mathrm{mg} / \mathrm{L}$ & 1 & 1 & 2 & 2 \\
\hline Fixed residue & $\mathrm{mg} / \mathrm{L}$ & 6 & 6 & 15 & 15 \\
\hline Suspended solids & $\mathrm{mg} / \mathrm{L}$ & 7 & 7 & 17 & 16 \\
\hline Total dissolved solids & $\mathrm{mg} / \mathrm{L}$ & 83 & 83 & 55 & 54 \\
\hline Total solids & $\mathrm{mg} / \mathrm{L}$ & 90 & 90 & 72 & 70 \\
\hline Turbidity & NTU & 9.7 & 9.6 & 15 & 15 \\
\hline Chloride & $\mathrm{mg} / \mathrm{L}$ & 10 & 10 & 6 & 6 \\
\hline Nitrogen- ammonia & $\mathrm{mg} / \mathrm{L}$ & 0.08 & 0.08 & 0.16 & 0.14 \\
\hline Nitrogen- nitrate & $\mathrm{mg} / \mathrm{L}$ & 0.42 & 0.43 & 0.33 & 0.32 \\
\hline Phosphate P & $\mathrm{mg} / \mathrm{L}$ & ND & ND & ND & ND \\
\hline Sulfate & $\mathrm{mg} / \mathrm{L}$ & 9 & 9 & 5 & 5 \\
\hline \multicolumn{6}{|l|}{ Offsite Analyses } \\
\hline Aluminum & $\mathrm{mg} / \mathrm{L}$ & 0.33 & 0.42 & ND & 0.69 \\
\hline Cadmium & $\mathrm{mg} / \mathrm{L}$ & ND & ND & ND & ND \\
\hline Calcium & $\mathrm{mg} / \mathrm{L}$ & 4.23 & 4.25 & 3.36 & 3.64 \\
\hline Chromium & $\mathrm{mg} / \mathrm{L}$ & ND & ND & ND & ND \\
\hline Copper & $\mathrm{mg} / \mathrm{L}$ & ND & ND & 0.01 & 0.01 \\
\hline Iron & $\mathrm{mg} / \mathrm{L}$ & 0.75 & 0.78 & 1.20 & 1.19 \\
\hline Lead & $\mathrm{mg} / \mathrm{L}$ & ND & ND & ND & ND \\
\hline Magnesium & $\mathrm{mg} / \mathrm{L}$ & 1.35 & 1.37 & 1.13 & 1.75 \\
\hline Manganese & $\mathrm{mg} / \mathrm{L}$ & 0.07 & 0.073 & ND & 0.12 \\
\hline
\end{tabular}

a This location is sampled monthly by the Georgia Department of Natural Resources and twice yearly by the Environmental Monitoring Section. 


\section{Table 71}

Savannah River Water Quality:

Georgia Department of Natural Resources and EMS Sampling Location

Page 2 of 2

\begin{tabular}{llcccc}
\hline Parameter & \multicolumn{1}{c}{ Units } & June & Duplicate & December & Duplicate \\
\hline Mercury & $\mathrm{mg} / \mathrm{L}$ & $\mathrm{ND}$ & $\mathrm{ND}$ & $\mathrm{ND}$ & $\mathrm{ND}$ \\
Nickel & $\mathrm{mg} / \mathrm{L}$ & $\mathrm{ND}$ & $\mathrm{ND}$ & $\mathrm{ND}$ & $\mathrm{ND}$ \\
Sodium & $\mathrm{mg} / \mathrm{L}$ & 11.2 & 11.2 & 10.3 & 11.7 \\
Zinc & $\mathrm{mg} / \mathrm{L}$ & $\mathrm{ND}$ & $\mathrm{ND}$ & 0.009 & $\mathrm{ND}$
\end{tabular}


Table 72

SRS Streams and Savannah River Water Quality:

Fecal Coliform Bacteria Exceedances

Page 1 of 2

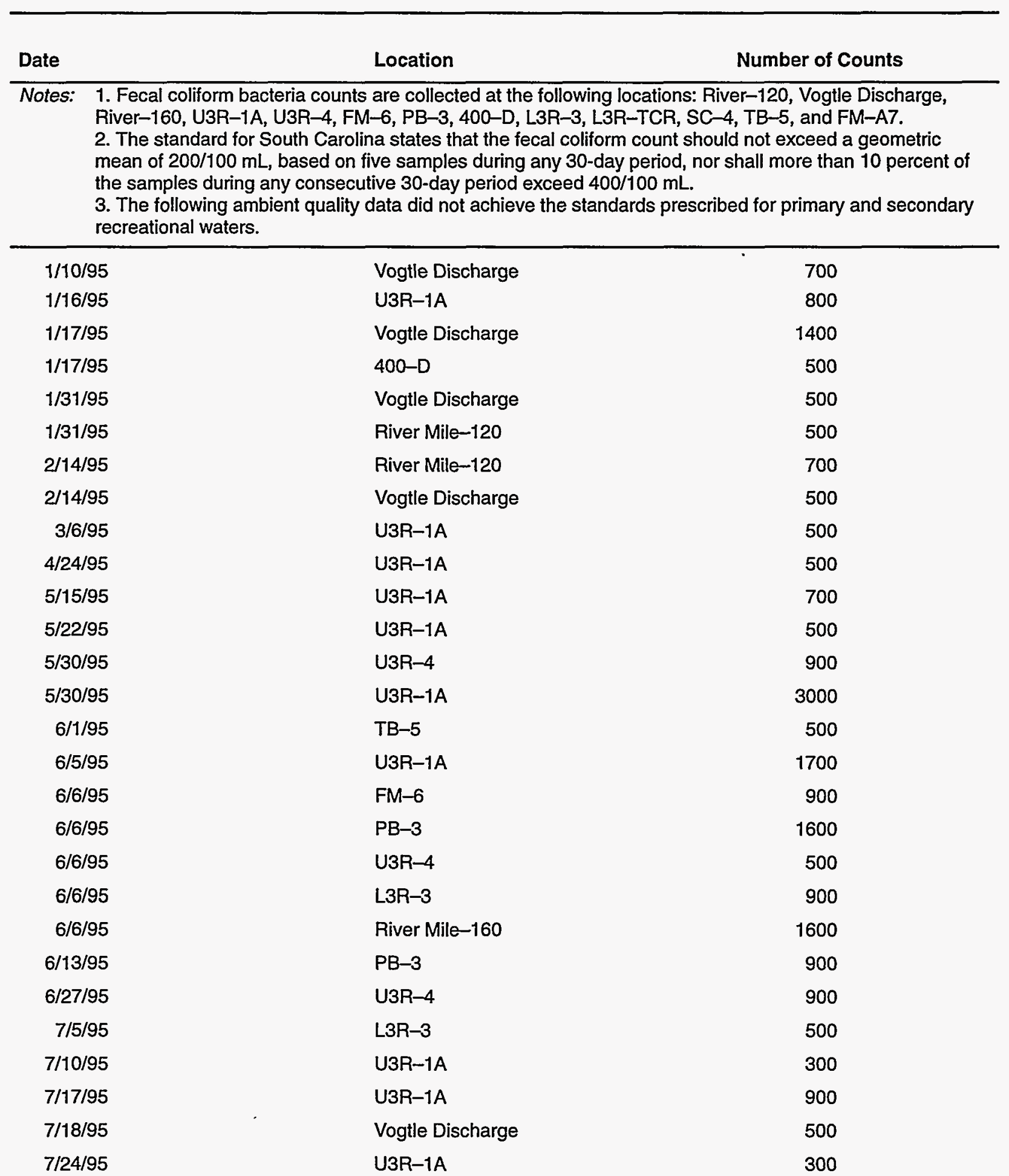




\section{Table 72}

SRS Streams and Savannah River Water Quality:

Fecal Coliform Bacteria Exceedances

Page 2 of 2

\begin{tabular}{rlr}
\hline \multicolumn{1}{l}{ Date } & Location & Number of Counts \\
\hline $7 / 25 / 95$ & L3R-3 & 500 \\
$7 / 25 / 95$ & U3R-4 & $>1600$ \\
$8 / 3 / 95$ & FM-7A & 1600 \\
$8 / 7 / 95$ & U3R-1 & 500 \\
$8 / 8 / 95$ & L3R-3 & 1600 \\
$8 / 8 / 95$ & U3R-4 & 500 \\
$8 / 22 / 95$ & L3R-3 & 1600 \\
$9 / 12 / 95$ & U3R-4 & 2200 \\
$9 / 12 / 95$ & U3R-1 & 2600 \\
$9 / 19 / 95$ & River Mile-120 & $>600$ \\
$9 / 26 / 95$ & L3R-3 & 480 \\
$9 / 26 / 95$ & PB-3 & 500 \\
$9 / 26 / 95$ & $400-D$ & 400 \\
$9 / 26 / 95$ & Vogtle Discharge & 2600 \\
$9 / 26 / 95$ & River Mile-160 & 3200 \\
$10 / 4 / 95$ & L3R-3 & 230 \\
$10 / 10 / 95$ & L3R-3 & 220 \\
$12 / 19 / 95$ & Vogtle Discharge & 2300 \\
$12 / 19 / 95$ & River Mile-160 & 1900 \\
$12 / 19 / 95$ & L3R-3 & 700 \\
$12 / 19 / 95$ & FM-6 & 800 \\
\hline & &
\end{tabular}


Table 73

Sediment Surveillance: Pesticides and Herbicides

Page 1 of 2

\begin{tabular}{|c|c|c|c|c|c|}
\hline & \multicolumn{5}{|c|}{$\mu g / 1$} \\
\hline & Steel Creek-4 & $\begin{array}{l}\text { PAR Pond } \\
\text { Pump House }\end{array}$ & $\begin{array}{c}\text { Lower Three } \\
\text { Runs-3 }\end{array}$ & $\begin{array}{l}\text { Upper Three } \\
\text { Runs-4 }\end{array}$ & $\begin{array}{l}\text { Upper Three } \\
\text { Runs-Road F }\end{array}$ \\
\hline \multicolumn{6}{|l|}{ Pesticides } \\
\hline Aldrin & $<8.7$ & $<2.4$ & $<5.5$ & $<3.5$ & Not collected \\
\hline Alpha-BHC & $<8.7$ & $<2.4$ & $<5.5$ & $<3.5$ & Not collected \\
\hline Beta-BHC & $<8.7$ & $<2.4$ & $<5.5$ & $<3.5$ & Not collected \\
\hline Delta-BHC & $<8.7$ & $<2.4$ & $<5.5$ & $<3.5$ & Not cọllected \\
\hline Lindane & $<8.7$ & $<2.4$ & $<5.5$ & $<3.5$ & Not collected \\
\hline Chlordane & $<87$ & $<24$ & $<55$ & $<35$ & Not collected \\
\hline 4,4-DDD & $<17$ & $<4.8$ & $<11$ & 17 & Not collected \\
\hline 4,4 - DDE & $<17$ & $<4.8$ & 24 & 10 & Not collected \\
\hline 4,4-DDT & $<17$ & $<4.8$ & $<11$ & $<6.7$ & Not collected \\
\hline Dieldrin & $<17$ & $<4.8$ & $<11$ & $<6.7$ & Not collected \\
\hline Endosulfan I & $<8.7$ & $<2.4$ & $<5.5$ & $<3.5$ & Not collected \\
\hline Endosulfan II & $<17$ & $<4.8$ & $<11$ & $<6.7$ & Not collected \\
\hline Endosulfan sulfate & $<17$ & $<4.8$ & $<11$ & $<6.7$ & Not collected \\
\hline Endrin & $<17$ & $<4.8$ & $<11$ & $<6.7$ & Not collected \\
\hline Endrin aldehyde & $<17$ & $<4.8$ & 15 & $<6.7$ & Not collected \\
\hline Heptachlor & $<8.7$ & $<2.4$ & $<5.5$ & $<3.5$ & Not collected \\
\hline Heptachlor epoxide & $<8.7$ & $<2.4$ & $<5.5$ & $<3.5$ & Not collected \\
\hline Methoxychlor & $<87$ & $<244$ & $<55$ & $<35$ & Not collected \\
\hline Toxaphene & $<870$ & $<240$ & $<550$ & $<350$ & Not collected \\
\hline \multicolumn{6}{|l|}{ Herbicides } \\
\hline $2,4-D$ & $<1300$ & $<360$ & $<820$ & $<520$ & Not collected \\
\hline 2,4,5-TP (Silvex) & $<210$ & $<58$ & $<130$ & $<83$ & Not collected \\
\hline
\end{tabular}




\section{Table 73}

Sediment Surveillance: Pesticides and Herbicides

Page 2 of 2

$\mu g /$

\begin{tabular}{|c|c|c|c|c|}
\hline & \multicolumn{4}{|c|}{$\mu g /$} \\
\hline & River-2 & River-10 & Pen Branch-3 & Four Mile-6 \\
\hline \multicolumn{5}{|l|}{ Pesticides } \\
\hline Aldrin & $<2.7$ & $<2.9$ & $<2.2$ & $<2.7$ \\
\hline Alpha-BHC & $<2.7$ & $<2.9$ & $<2.2$ & $<2.7$ \\
\hline Beta-BHC & $<2.7$ & $<2.9$ & $<2.2$ & $<2.7$ \\
\hline Delta-BHC & $<2.7$ & $<2.9$ & $<2.2$ & $<2.7$ \\
\hline Lindane & $<2.7$ & $<2.9$ & $<2.2$ & $<2.7$ \\
\hline Chlordane & $<27$ & $<29$ & $<22$ & $<27$ \\
\hline 4,4-DDD & $<5.2$ & $<5.6$ & $<4.4$ & $<5.3$ \\
\hline $4,4-D D E$ & $<5.2$ & $<5.6$ & $<4.4$ & $<5.3$ \\
\hline 4,4-DDT & $<5.2$ & $<5.6$ & $<4.4$ & $<5.3$ \\
\hline Dieldrin & $<5.2$ & $<5.6$ & $<4.4$ & $<5.3$ \\
\hline Endosulfan I & $<2.7$ & $<2.9$ & $<2.2$ & $<2.7$ \\
\hline Endosulfan II & $<5.2$ & $<5.6$ & $<4.4$ & $<5.3$ \\
\hline Endosulfan sulfate & $<5.2$ & $<5.6$ & $<4.4$ & $<5.3$ \\
\hline Endrin & $<5.2$ & $<5.6$ & $<4.4$ & $<5.3$ \\
\hline Endrin aldehyde & $<5.2$ & $<5.6$ & $<4.4$ & $<5.3$ \\
\hline Heptachlor & $<2.7$ & $<2.9$ & $<2.2$ & $<2.7$ \\
\hline Heptachlor epoxide & $<2.7$ & $<2.9$ & $<2.2$ & $<2.7$ \\
\hline Methoxychlor & $<27$ & $<29$ & $<22$ & $<27$ \\
\hline Toxaphene & $<270$ & $<290$ & $<220$ & $<270$ \\
\hline \multicolumn{5}{|l|}{ Herbicides } \\
\hline $2,4-D$ & $<400$ & $<440$ & $<340$ & $<400$ \\
\hline 2,4,5-TP (Silvex) & $<64$ & $<70$ & $<54$ & $<65$ \\
\hline
\end{tabular}


Table 74

Fish Surveillance: Mercury

Page 1 of 2

\begin{tabular}{|c|c|c|c|c|c|c|c|c|c|}
\hline \multirow{2}{*}{\multicolumn{2}{|c|}{ Location/Species }} & \multicolumn{8}{|c|}{ Mercury ( $\mu \mathrm{g} \mathrm{Hg} / \mathrm{g}$ - Parts Per Million) } \\
\hline & & \multicolumn{5}{|c|}{ Analytical Results } & & \multirow[t]{2}{*}{ Min } & \multirow[t]{2}{*}{$\operatorname{Max}$} \\
\hline Note: & \multicolumn{7}{|c|}{ ND denotes "not detected; the result is less than $0.17 \mu \mathrm{g} / \mathrm{g} . "$} & & \\
\hline \multicolumn{10}{|c|}{ Augusta Lock and Dam } \\
\hline & fish & ND & 0.17 & 0.20 & ND & 0.37 & & ND & 0.37 \\
\hline & fish & ND & ND & ND & ND & ND & & ND & ND \\
\hline \multicolumn{10}{|c|}{ Beaver Dam Creek } \\
\hline & & 0.17 & ND & ND & ND & 0.17 & & ND & 0.17 \\
\hline \multicolumn{10}{|c|}{$\begin{array}{l}\text { Beaver Dam Creek @ } \\
\text { Creek Mouth }\end{array}$} \\
\hline $\mathrm{Ba}$ & & & 0.63 & & & & & 0.63 & 0.83 \\
\hline $\mathrm{Br}$ & & ND & 0.17 & ND & ND & & & ND & 0.17 \\
\hline & ish & 0.17 & ND & ND & ND & ND & 0.27 & ND & 0.27 \\
\hline
\end{tabular}

Four Mile Creek

@ Creek Mouth

Cattish

0.20

Highway 301

Bass
Bream
Catfish

L-Lake

Bass

Bream

Lower Three Runs-2

Panfish [Crappie]

Lower Three Runs @ Creek Mouth

Catfish

Panfish

ND

ND

ND

0.20

PAR Pond

Bass
Bluegill
Panfish

1.13

ND

0.43

0.70

0.70

0.70

0.23

0.47

0.77

Pond B

Bass
Bluegill
Bream

0.33

ND

0.23
0.70
0.27

0.57
0.50

0.43

1.40

ND
0.43

1.13

$\begin{array}{cc}0.37 & 0.53 \\ 0.20 & 0.23 \\ \text { ND } & 0.63 \\ & \\ 1.00 & 1.70 \\ \text { ND } & \text { ND }\end{array}$

ND

ND

ND ND

$0.20 \quad 0.20$

ND $\quad 0.17$

$0.23 \quad 0.77$

$0.33 \quad 1.40$

ND $\quad 0.27$

ND $\quad 0.57$ 


\section{Table 74}

Fish Surveillance: Mercury

Page 2 of 2

\begin{tabular}{|c|c|c|c|c|c|c|c|}
\hline \multirow[b]{2}{*}{ Location/Species } & \multicolumn{7}{|c|}{ Mercury ( $\mu \mathrm{g} \mathrm{Hg} / \mathrm{g}$ - Parts Per Million) } \\
\hline & \multicolumn{5}{|c|}{ Analytical Results } & Min & Max \\
\hline \multicolumn{8}{|l|}{ Steel Creek-4 } \\
\hline Bass & 1.47 & 0.33 & 1.03 & 0.50 & 1.10 & 0.33 & 1.47 \\
\hline Panfish [Crappie] & 0.17 & ND & 0.17 & ND & ND & ND & 0.17 \\
\hline \multicolumn{8}{|c|}{ Steel Creek @ Creek Mouth } \\
\hline Bass & 0.43 & 0.67 & 0.70 & 0.17 & 0.23 & 0.17 & 0.70 \\
\hline Cattish & 0.23 & 0.27 & 0.23 & 0.27 & 0.23 & 0.23 & 0.27 \\
\hline Crappie & 0.77 & & & & & & \\
\hline \multicolumn{8}{|l|}{ Upper Three Runs-4 } \\
\hline Bass & 0.57 & & & & & 0.57 & 0.57 \\
\hline Cattish & 0.17 & & & & & 0.17 & 0.17 \\
\hline Panfish [Crappie] & 0.87 & & & & & 0.87 & 0.87 \\
\hline \multicolumn{8}{|c|}{$\begin{array}{l}\text { Upper Three Runs @ Creek } \\
\text { Mouth }\end{array}$} \\
\hline Bass & 0.47 & & & & & 0.47 & 0.47 \\
\hline Cattish & 0.23 & 0.17 & ND & 0.43 & 0.70 & ND & 0.43 \\
\hline Crappie & ND & 0.40 & ND & & & ND & 0.47 \\
\hline Pan fish & ND & & & & & ND & ND \\
\hline
\end{tabular}


Table 75

Blind Sample Results for pH Field Measurements

Page 1 of 1

\begin{tabular}{|c|c|c|c|}
\hline \multirow[b]{2}{*}{ Sample Identification } & \multicolumn{3}{|c|}{ pH Units } \\
\hline & Measured Value & Actual Value & Difference \\
\hline $\mathrm{BpH}-01-1$ & 4.00 & 3.93 & 0.07 units \\
\hline $\mathrm{BpH}-01-2$ & 6.82 & 6.84 & 0.02 units \\
\hline $\mathrm{BpH}-01-3$ & 7.40 & 7.44 & 0.04 units \\
\hline $\mathrm{BpH}-02-1$ & 4.00 & 3.89 & 0.11 units \\
\hline $\mathrm{BpH}-02-2$ & 7.10 & 6.83 & 0.27 units \\
\hline $\mathrm{BpH}-02-3$ & 7.40 & 7.42 & 0.02 units \\
\hline $\mathrm{BpH}-03-1$ & 3.85 & 3.91 & 0.06 units \\
\hline $\mathrm{BpH}-03-2$ & 6.80 & 6.86 & 0.06 units \\
\hline $\mathrm{BpH}-03-3$ & 7.50 & 7.47 & 0.03 units \\
\hline $\mathrm{BpH}-04-1$ & 7.40 & 7.41 & 0.01 units \\
\hline $\mathrm{BpH}-04-2$ & 4.00 & 4.01 & 0.01 units \\
\hline $\mathrm{BpH}-04-3$ & 9.9 & 10.21 & 0.31 units \\
\hline $\mathrm{BpH}-05-1$ & 7.40 & 4.39 & 0.01 units \\
\hline $\mathrm{BpH}-05-2$ & 4.10 & 4.04 & 0.06 units \\
\hline $\mathrm{BpH}-05-3$ & 9.80 & 9.94 & 0.14 units \\
\hline $\mathrm{BpH}-06-1$ & 6.80 & 6.82 & 0.02 units \\
\hline $\mathrm{BpH}-06-2$ & 4.02 & 4.01 & 0.01 units \\
\hline $\mathrm{BpH}-06-3$ & 7.38 & 7.47 & 0.09 units \\
\hline $\mathrm{BpH}-07-1$ & 4.00 & 4.06 & 0.06 units \\
\hline $\mathrm{BpH}-07-2$ & 6.85 & 6.82 & 0.03 units \\
\hline $\mathrm{BpH}-07-3$ & 9.84 & 10.24 & 0.40 units \\
\hline $\mathrm{BpH}-08-1$ & 4.07 & 4.01 & 0.06 units \\
\hline $\mathrm{BpH}-08-2$ & 6.86 & 6.85 & 0.01 units \\
\hline $\mathrm{BpH}-08-3$ & 10.30 & 10.30 & 0.00 units \\
\hline BpH-09-1 & 3.90 & 4.01 & 0.11 units \\
\hline $\mathrm{BpH}-09-2$ & 6.90 & 6.79 & 0.11 units \\
\hline $\mathrm{BpH}-09-3$ & 9.04 & 9.08 & 0.04 units \\
\hline $\mathrm{BpH}-10-1$ & 3.90 & 4.05 & 0.15 units \\
\hline $\mathrm{BpH}-10-2$ & 6.90 & 6.86 & 0.04 units \\
\hline $\mathrm{BpH}-10-3$ & 8.80 & 9.14 & 0.34 units \\
\hline $\mathrm{BpH}-11-1$ & 4.00 & 4.09 & 0.09 units \\
\hline $\mathrm{BpH}-11-2$ & 6.80 & 6.87 & 0.07 units \\
\hline $\mathrm{BpH}-11-3$ & 8.85 & 9.16 & 0.31 units \\
\hline $\mathrm{BpH}-12-1$ & 6.82 & 6.84 & 0.02 units \\
\hline $\mathrm{BpH}-12-2$ & 9.20 & 9.08 & 0.12 units \\
\hline $\mathrm{BpH}-12-3$ & 7.30 & 7.42 & 0.12 units \\
\hline
\end{tabular}


Table 76

Blind Sample Results for Conductivity Field Measurements

Page 1 of 1

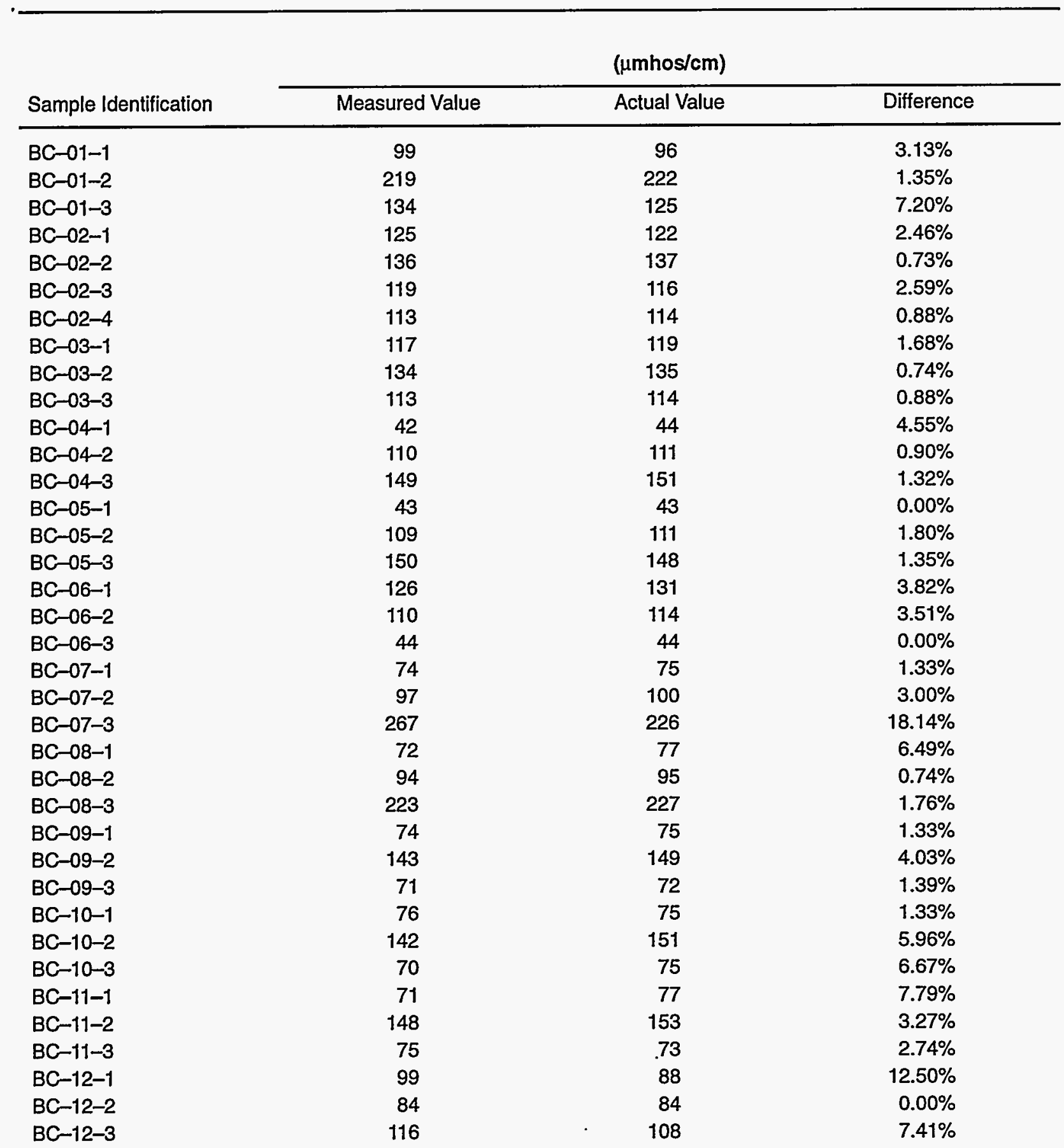


Table 77

EMS Blind Sample Results for Tritium

Page 1 of 1

\begin{tabular}{|c|c|c|c|c|c|}
\hline \multirow[b]{2}{*}{ Sample Date } & \multicolumn{2}{|c|}{$\mathrm{pCi} / \mathrm{L}$} & \multirow[b]{2}{*}{ Ratio } & \multicolumn{2}{|c|}{ Range } \\
\hline & Measured Value & Known Value & & $\begin{array}{c}\text { Lower } \\
\text { Control Limita }\end{array}$ & $\begin{array}{c}\text { Upper } \\
\text { Control Limita }\end{array}$ \\
\hline $02-17-95$ & $0.84 \pm 0.31$ & $0.67 \pm 0.60$ & 1.25 & 0.43 & 1.24 \\
\hline $03-16-95$ & $4.75 \pm 0.37$ & $5.88 \pm 0.60$ & 0.81 & 3.76 & 10.87 \\
\hline $04-11-95$ & $7.41 \pm 0.41$ & $7.71 \pm 0.60$ & 0.96 & 4.93 & 14.26 \\
\hline $05-10-95$ & $7.26 \pm 0.3$ & $8.77 \pm 0.60$ & 0.83 & 5.61 & 16.22 \\
\hline $07-17-95$ & $1.31 \pm 0.3$ & $1.16 \pm 0.60$ & 1.13 & 0.74 & 2.14 \\
\hline $07-17-95$ & $5.36 \pm 0.38$ & $5.82 \pm 0.60$ & 0.92 & 3.72 & 10.77 \\
\hline $08-21-95$ & $5.31 \pm 0.37$ & $5.78 \pm 0.60$ & 0.92 & 3.70 & 10.70 \\
\hline $10-17-95$ & $0.75 \pm 0.35$ & $0.95 \pm 0.6$ & 0.79 & 0.61 & 1.76 \\
\hline $11-16-95$ & $13.1 \pm 0.55$ & $14.10 \pm 0.60$ & 0.93 & 9.02 & 26.09 \\
\hline $12-16-95$ & $4.36 \pm 0.43$ & $4.39 \pm 0.60$ & 0.99 & 2.81 & 8.12 \\
\hline
\end{tabular}

a Tritium control limits are according to DOE/EML limits. 
Table 78

EMS Blind Sample Results for Gross Alpha and Beta and Gamma-Emitting Radionuclides

Page 1 of 1

\begin{tabular}{|c|c|c|c|c|c|c|}
\hline \multirow[b]{2}{*}{$\begin{array}{l}\text { Sample } \\
\text { Number }\end{array}$} & \multirow[b]{2}{*}{ Nuclide } & \multicolumn{2}{|c|}{$\mathrm{pCi} / \mathrm{L}$} & \multirow[b]{2}{*}{$\begin{array}{c}\text { Ratio } \\
\text { Found/Actual }\end{array}$} & \multicolumn{2}{|c|}{ Range } \\
\hline & & Found Value & Actual Value & & $\begin{array}{c}\text { Lower } \\
\text { Control Limita }\end{array}$ & $\begin{array}{c}\text { Upper } \\
\text { Control Limita }\end{array}$ \\
\hline \multicolumn{7}{|c|}{ Gross Alpha and Beta } \\
\hline \multirow[t]{2}{*}{139} & Alpha & $44.3 \pm 6.45$ & $57 \pm 14$ & 0.78 & 33 & 81 \\
\hline & Beta & $24.1 \pm 5.13$ & $23 \pm 5$ & 1.05 & 14 & 32 \\
\hline \multirow[t]{2}{*}{142} & Alpha & $2.08 \pm 1.06$ & $5 \pm 5$ & 0.42 & -4 & 14 \\
\hline & Beta & $7.62 \pm 1.93$ & $5 \pm 5$ & 1.52 & -4 & 14 \\
\hline \multicolumn{7}{|l|}{ Gamma } \\
\hline \multirow[t]{5}{*}{138} & $\mathrm{Co}-60$ & $62 \pm 3$ & $59 \pm 5$ & 1.05 & 50 & 68 \\
\hline & $Z n-65$ & $88.6 \pm 3$ & $100 \pm 10$ & 0.89 & 83 & 117 \\
\hline & Cs-134 & $19.4 \pm 3$ & $24 \pm 5$ & 0.81 & 15 & 33 \\
\hline & Cs-137 & $64.8 \pm 3$ & $49 \pm 5$ & 1.32 & 40 & 58 \\
\hline & $\mathrm{Ba}-133$ & $66.7 \pm 3$ & $73 \pm 7$ & 0.91 & 61 & 85 \\
\hline \multirow[t]{5}{*}{147} & Co-60 & $40.9 \pm 2$ & $40.0 \pm 5$ & 1.02 & 31 & 49 \\
\hline & $Z n-65$ & $90.3 \pm 7$ & $76 \pm 8$ & 1.19 & 62 & 90 \\
\hline & Cs-134 & $45.9 \pm 2$ & $50 \pm 5$ & 0.92 & 41 & 59 \\
\hline & Cs-137 & $36.3 \pm 3$ & $35 \pm 5$ & 1.04 & 26 & 44 \\
\hline & $\mathrm{Ba}-133$ & $80.3 \pm 4$ & $79 \pm 8$ & 1.02 & 65 & 93 \\
\hline
\end{tabular}

a Alpha, beta, and gamma control limits are according to QAD/EPA limits. 
Table 79

NPDES Blind Sample Results

Page 1 of 3

\begin{tabular}{|c|c|c|c|c|c|}
\hline NPDES S & Parameter Sampled & Units & Result 1 & Result 2 & Dlfference \\
\hline Note: & $\begin{array}{l}\text { The laboratories performing the an } \\
\text { Services, Inc. This table compares }\end{array}$ & $\begin{array}{l}\text { vere Thermo } \\
\text { lytical result }\end{array}$ & $\begin{array}{l}\text { alytical, Inc. } \\
\text { m each labo }\end{array}$ & $\begin{array}{l}\text { 1A) and She } \\
\text { ory. }\end{array}$ & Environmental \\
\hline$A-001$ & 1,1,1-Trichloroethylene & $\mu g / L$ & $<2$ & $<2$ & $<2$ \\
\hline$M-005$ & 1,1,1-Trichloroethylene & $\mu g / L$ & $<2$ & $<2$ & $<2$ \\
\hline$A-001 A$ & 1,1,1-Trichloroethylene & $\mu g / L$ & $<2$ & $<2$ & $<2$ \\
\hline$A-014$ & 1,1,1-Trichloroethylene & $\mu g / L$ & $<2$ & $<2$ & $<2$ \\
\hline$A-005$ & 1,1,1-Trichloroethylene & $\mu g / L$ & $<2$ & $<2$ & $<2$ \\
\hline $\mathrm{H}-017$ & Aluminum & $\mathrm{mg} / \mathrm{L}$ & 0.101 & 0.956 & -0.855 \\
\hline$M-004$ & Aluminum & $\mathrm{mg} / \mathrm{L}$ & $<0.05$ & $<0.05$ & $<0.05$ \\
\hline$F-008$ & Aluminum & $\mathrm{mg} / \mathrm{L}$ & $<0.005$ & 0.522 & $<0.522$ \\
\hline $\mathrm{F}-012$ & Ammonia & $\mathrm{mgSO} 4 / \mathrm{L}$ & $<0.05$ & $<0.05$ & $<0.05$ \\
\hline$x-014$ & Benzene & $\mathrm{mg} / \mathrm{L}$ & $<0.8$ & $<0.8$ & $<0.8$ \\
\hline$x-014$ & Benzene & $\mathrm{mg} / \mathrm{L}$ & $<0.5$ & $<0.5$ & $<0.5$ \\
\hline$x-014$ & Biological Oxygen Demand & $\mathrm{mg} / \mathrm{L}$ & $<1$ & $<1$ & $<1$ \\
\hline DW-004 & Biological Oxygen Demand & $\mathrm{mg} / \mathrm{L}$ & 2.1 & 1.7 & 0.4 \\
\hline$x-014$ & Biological Oxygen Demand & $\mathrm{mg} / \mathrm{L}$ & 1.2 & 1.2 & 0 \\
\hline$K-018$ & Biological Oxygen Demand & $\mathrm{mg} / \mathrm{L}$ & $<1$ & $<1$ & $<1$ \\
\hline$A-001$ & Biological Oxygen Demand & $\mathrm{mg} / \mathrm{L}$ & $<1$ & $<1$ & $<1$ \\
\hline$F-003$ & Biological Oxygen Demand & $\mathrm{mg} / \mathrm{L}$ & $<1$ & $<1$ & $<1$ \\
\hline $\mathrm{H}-017$ & Chromium & $\mathrm{mg} / \mathrm{L}$ & $<0.005$ & $<0.005$ & $<0.005$ \\
\hline$M-004$ & Chromium & $\mathrm{mg} / \mathrm{L}$ & $<0.01$ & $<0.01$ & $<0.01$ \\
\hline$A-003$ & Chromium & $\mathrm{mg} / \mathrm{L}$ & $<0.01$ & $<0.01$ & $<0.01$ \\
\hline$F-008$ & Chromium & $\mathrm{mg} / \mathrm{L}$ & $<0.01$ & $<0.01$ & $<0.01$ \\
\hline $\mathrm{H}-017$ & Copper & $\mathrm{mg} / \mathrm{L}$ & $<0.005$ & $<0.005$ & $<0.005$ \\
\hline$M-004$ & Copper & $\mathrm{mg} / \mathrm{L}$ & $<0.005$ & $<0.005$ & $<0.005$ \\
\hline$F-008$ & Copper & $\mathrm{mg} / \mathrm{L}$ & $<0.01$ & $<0.01$ & $<0.01$ \\
\hline$M-004$ & Cyanide & $\mathrm{mg} / \mathrm{L}$ & $<0.01$ & $<0.01$ & $<0.01$ \\
\hline$x-008$ & Iron & $\mathrm{mg} / \mathrm{L}$ & 0.924 & 1.01 & -0.086 \\
\hline $\mathrm{H}-017$ & Lead & $\mathrm{mg} / \mathrm{L}$ & 0.0043 & 0.0042 & 0.0001 \\
\hline$M-004$ & Lead & $\mathrm{mg} / \mathrm{L}$ & $<0.02$ & $<0.02$ & $<0.02$ \\
\hline$F-008$ & Lead & $\mathrm{mg} / \mathrm{L}$ & $<0.02$ & $<0.02$ & $<0.02$ \\
\hline $\mathrm{H}-017$ & Manganese & $\mathrm{mg} / \mathrm{L}$ & 0.0296 & 0.0302 & -0.0006 \\
\hline$F-008$ & Manganese & $\mathrm{mg} / \mathrm{L}$ & $<0.01$ & $<0.01$ & $<0.01$ \\
\hline $\mathrm{H}-017$ & Mercury & $\mathrm{mg} / \mathrm{L}$ & $<0.005$ & $<0.005$ & $<0.005$ \\
\hline$M-004$ & Nickel & $\mathrm{mg} / \mathrm{L}$ & $<0.01$ & $<0.01$ & $<0.01$ \\
\hline
\end{tabular}




\section{Table 79}

\section{NPDES Blind Sample Results}

Page 2 of 3

\begin{tabular}{|c|c|c|c|c|c|}
\hline NPDES Site & Parameter Sampled & Units & Result 1 & Result 2 & Difference \\
\hline$F-008$ & Nickel & $\mathrm{mg} / \mathrm{L}$ & $<0.01$ & $<0.01$ & $<0.01$ \\
\hline$F-012$ & Nitrate & $\mathrm{mgN} / \mathrm{L}$ & 1.84 & 1.86 & -0.02 \\
\hline$F-008$ & Nitrate & $\mathrm{mgN} / \mathrm{L}$ & $<0.02$ & $<0.02$ & $<0.02$ \\
\hline$A-001$ & Oil \& Grease & $\mathrm{mg} / \mathrm{L}$ & $<1$ & $<1$ & $<1$ \\
\hline$F-001$ & Oil \& Grease & $\mathrm{mg} / \mathrm{L}$ & $<1$ & $<1$ & $<1$ \\
\hline $\mathrm{C}-001$ & Oil \& Grease & $\mathrm{mg} / \mathrm{L}$ & $<1$ & $<1$ & $<1$ \\
\hline $\mathrm{C}-004$ & Oil \& Grease & $\mathrm{mg} / \mathrm{L}$ & $<1$ & $<1$ & $<1$ \\
\hline$A-011$ & Oil \& Grease & $\mathrm{mg} / \mathrm{L}$ & $<1$ & $<1$ & $<1$ \\
\hline$A-005$ & Oil \& Grease & $\mathrm{mg} / \mathrm{L}$ & 2.1 & $<1$ & $<2.1$ \\
\hline$x-014$ & Phenol & $\mathrm{mg} / \mathrm{L}$ & $<0.002$ & $<0.002$ & $<0.002$ \\
\hline$x-014$ & Phenol & $\mathrm{mg} / \mathrm{L}$ & $<0.006$ & $<0.006$ & $<0.006$ \\
\hline$M-004$ & Phosphate & $\mathrm{mgP} / \mathrm{L}$ & 0.109 & 0.122 & -0.013 \\
\hline $\mathrm{H}-017$ & Silver & $\mathrm{mg} / \mathrm{L}$ & $<0.0005$ & $<0.0005$ & $<0.0005$ \\
\hline$A-001$ & Tetrachloroethylene & $\mu \mathrm{g} / \mathrm{L}$ & $<2$ & $<2$ & $<2$ \\
\hline$M-005$ & Tetrachloroethylene & $\mu g / L$ & $<2$ & $<2$ & $<2$ \\
\hline$A-001 A$ & Tetrachloroethylene & $\mu g / L$ & $<2$ & $<2$ & $<2$ \\
\hline$A-014$ & Tetrachloroethylene & $\mu \mathrm{g} / \mathrm{L}$ & $<2$ & $<2$ & $<2$ \\
\hline$A-005$ & Tetrachloroethylene & $\mu g / L$ & $<2.0$ & $<2.0$ & $<2.0$ \\
\hline$x-014$ & Total Organic Carbon & $\mathrm{mg} / \mathrm{L}$ & 4.3 & 4.6 & -0.3 \\
\hline$x-014$ & Total Organic Carbon & $\mathrm{mg} / \mathrm{L}$ & 1.8 & 2.2 & -0.4 \\
\hline$x-014$ & Total Susp'ended Solids & $\mathrm{mg} / \mathrm{L}$ & 6.2 & 2.8 & 3.4 \\
\hline$x-014$ & Total Suspended Solids & $\mathrm{mg} / \mathrm{L}$ & 10 & 56 & 46 \\
\hline$K-018$ & Total Suspended Solids & $\mathrm{mg} / \mathrm{L}$ & 12 & 10 & 2 \\
\hline$A-001$ & Total Suspended Solids & $\mathrm{mg} / \mathrm{L}$ & $<1$ & $<1$ & $<1$ \\
\hline $\mathrm{H}-007$ & Total Suspended Solids & $\mathrm{mg} / \mathrm{L}$ & 8 & 2 & 6 \\
\hline $\mathrm{H}-008$ & Total Suspended Solids & $\mathrm{mg} / \mathrm{L}$ & 2.2 & 1 & 1.2 \\
\hline $\mathrm{F}-003$ & Total Suspended Solids & $\mathrm{mg} / \mathrm{L}$ & $<1$ & $<1$ & $<1$ \\
\hline $\mathrm{C}-004$ & Total Suspended Solids & $\mathrm{mg} / \mathrm{L}$ & 3 & 6 & -3 \\
\hline$F-008$ & Total Suspended Solids & $\mathrm{mg} / \mathrm{L}$ & 2 & 2 & 0 \\
\hline$C-001$ & Total Suspended Solids & $\mathrm{mg} / \mathrm{L}$ & 10 & 10 & 0 \\
\hline$F-001$ & Total Suspended Solids & $\mathrm{mg} / \mathrm{L}$ & $<1$ & $<1$ & $<1$ \\
\hline$A-001$ & Trichloroethylene & $\mu \mathrm{g} / \mathrm{L}$ & $<2$ & $<2$ & $<2$ \\
\hline$M-005$ & Trichloroethylene & $\mu \mathrm{g} / \mathrm{L}$ & $<2$ & $<2$ & $<2$ \\
\hline$A-001 A$ & Trichloroethylene & $\mu \mathrm{g} / \mathrm{L}$ & $<2$ & $<2$ & $<2$ \\
\hline$A-014$ & Trichloroethylene & $\mu g / L$ & $<2$ & $<2$ & $<2$ \\
\hline$A-005$ & Trichloroethylene & $\mu \mathrm{g} / \mathrm{L}$ & $<2.0$ & $<2.0$ & $<2.0$ \\
\hline
\end{tabular}


Table 79

NPDES Blind Sample Results

Page 3 of 3

\begin{tabular}{llcccc}
\hline NPDES Site & Parameter Sampled & Units & Result 1 & Result 2 & Difference \\
\hline H-017 & Uranium & $\mathrm{mg} / \mathrm{L}$ & $<0.02$ & $<0.02$ & $<0.02$ \\
$\mathrm{~F}-008$ & Uranium & $\mathrm{mg} / \mathrm{L}$ & $<0.5$ & $<0.5$ & $<0.5$ \\
$\mathrm{H}-017$ & Zinc & $\mathrm{mg} / \mathrm{L}$ & 0.126 & 0.17 & -0.044 \\
$\mathrm{~F}-008$ & Zinc & $\mathrm{mg} / \mathrm{L}$ & 0.012 & 0.02 & -0.008
\end{tabular}




\section{Table 80}

\section{NPDES Duplicate Sample Results}

Page 1 of 6

\begin{tabular}{|c|c|c|c|c|c|}
\hline Sample & Analyte & Units & Result 1 & Result 2 & Difference \\
\hline Note: & \multicolumn{5}{|c|}{$\begin{array}{l}\text { Result } 1 \text { and Result } 2 \text { were duplicate samples analyzed by the same laboratory, ThermoAnalytical, Inc. } \\
\text { (TMA). }\end{array}$} \\
\hline $\mathrm{X}-008$ & Total Suspended Solids & $m g / L$ & 2.8 & 1.9 & 0.9 \\
\hline$A-001$ & 1,1,1-Trichloroethylene & $\mu g / L$ & $<2$ & $<2$ & $<2$ \\
\hline$A-005$ & 1,1,1-Trichloroethylene & $\mu \mathrm{g} / \mathrm{L}$ & $<2$ & $<2$ & $<2$ \\
\hline$A-001 A$ & 1,1,1-Trichloroethylene & $\mu g / L$ & $<2$ & $<2$ & $<2$ \\
\hline$A-001 A$ & 1,1,1-Trichloroethylene & $\mu \mathrm{g} / \mathrm{L}$ & $<2$ & $<2$ & $<2$ \\
\hline$A-001$ & 1,1,1-Trichloroethylene & $\mu g / L$ & $<2$ & $<2$ & $<2$ \\
\hline$A-005$ & 1,1,1-Trichloroethylene & $\mu g / L$ & $<2$ & $<2$ & $<2$ \\
\hline$A-014$ & 1,1,1-Trichloroethylene & $\mu \mathrm{g} / \mathrm{L}$ & $<2$ & $<2$ & $<2$ \\
\hline$M-005$ & $1,1,1-$ Trichloroethylene & $\mu g / L$ & $<2$ & $<2$ & $<2$ \\
\hline$A-003$ & 1,1,1-Trichloroethylene & $\mu \mathrm{g} / \mathrm{L}$ & $<2$ & $<2$ & $<2$ \\
\hline$A-003$ & $1,1,1-$ Trichloroethylene & $\mu \mathrm{g} / \mathrm{L}$ & $<2$ & $<2$ & $<2$ \\
\hline$A-001$ & 1,1,1-Trichloroethylene & $\mu \mathrm{g} / \mathrm{L}$ & $<2$ & $<2$ & $<2$ \\
\hline$A-001 A$ & $1,1,1-$ Trichloroethylene & $\mu \mathrm{g} / \mathrm{L}$ & $<2$ & $<2$ & $<2$ \\
\hline$A-001$ & $1,1,1$-Trichloroethylene & $\mu \mathrm{g} / \mathrm{L}$ & $<2$ & $<2$ & $<2$ \\
\hline$F-012$ & Aluminum & $\mathrm{mg} / \mathrm{L}$ & 0.312 & 0.357 & -0.045 \\
\hline $\mathrm{K}-018$ & Aluminum & $\mathrm{mg} / \mathrm{L}$ & 0.256 & 0.257 & -0.001 \\
\hline$M-004$ & Aluminum & $\mathrm{mg} / \mathrm{L}$ & 0.13 & 0.113 & 0.017 \\
\hline$F-012$ & Aluminum & $\mathrm{mg} / \mathrm{L}$ & 0.194 & 0.166 & 0.028 \\
\hline$K-018$ & Aluminum & $\mathrm{mg} / \mathrm{L}$ & 0.282 & 0.14 & 0.142 \\
\hline$K-018$ & Aluminum & $\mathrm{mg} / \mathrm{L}$ & 0.19 & 0.204 & -0.014 \\
\hline$M-004$ & Aluminum & $\mathrm{mg} / \mathrm{L}$ & 0.194 & 0.187 & 0.007 \\
\hline $\mathrm{K}-018$ & Aluminum & $\mathrm{mg} / \mathrm{L}$ & 0.109 & 0.121 & -0.012 \\
\hline$F-008$ & Aluminum & $\mathrm{mg} / \mathrm{L}$ & 0.2 & 0.198 & 0.002 \\
\hline $\mathrm{H}-012$ & Aluminum & $\mathrm{mg} / \mathrm{L}$ & 0.116 & 0.116 & 0 \\
\hline$F-012$ & Aluminum & $\mathrm{mg} / \mathrm{L}$ & 0.142 & 0.149 & -0.007 \\
\hline$F-012$ & Aluminum & $\mathrm{mg} / \mathrm{L}$ & 0.185 & 0.155 & 0.03 \\
\hline $\mathrm{K}-018$ & Aluminum & $\mathrm{mg} / \mathrm{L}$ & 0.284 & 0.455 & -0.171 \\
\hline$F-008$ & Aluminum & $\mathrm{mg} / \mathrm{L}$ & 0.274 & $<0.05$ & $<0.274$ \\
\hline $\mathrm{H}-012$ & Aluminum & $\mathrm{mg} / \mathrm{L}$ & 0.24 & $<0.05$ & $<0.24$ \\
\hline$F-012$ & Aluminum & $\mathrm{mg} / \mathrm{L}$ & 0.336 & $<0.05$ & $<0.336$ \\
\hline$M-004$ & Aluminum & $\mathrm{mg} / \mathrm{L}$ & $<0.05$ & $<0.05$ & $<0.05$ \\
\hline$F-012$ & Ammonia & $\mathrm{mg} / \mathrm{L}$ & $<.05$ & $<.05$ & $<.05$ \\
\hline $\mathrm{H}-016$ & Ammonia & $\mathrm{mgSO} 4 / \mathrm{L}$ & $<.05$ & $<.05$ & $<.05$ \\
\hline $\mathrm{H}-017$ & Ammonia & $\mathrm{mgSO} 4 / \mathrm{L}$ & 0.18 & 0.26 & -0.08 \\
\hline$F-013$ & Ammonia & mgSO4/L & $<.05$ & $<.05$ & $<.05$ \\
\hline$F-013$ & Ammonia & $\mathrm{mgSO} 4 / \mathrm{L}$ & $<.05$ & $<.05$ & $<.05$ \\
\hline$F-012$ & Ammonia & $\mathrm{mgSO} 4 / \mathrm{L}$ & $<.05$ & $<.05$ & $<.05$ \\
\hline $\mathrm{H}-017$ & Ammonia & mgSO4/L & $<0.05$ & $<0.05$ & $<0.05$ \\
\hline $\mathrm{H}-016$ & Ammonia & $\mathrm{mgSO} 4 / \mathrm{L}$ & 0.13 & 0.16 & -0.03 \\
\hline$F-008$ & Ammonia & mgSO4/L & $<0.05$ & $<0.05$ & $<0.05$ \\
\hline $\mathrm{H}-012$ & Ammonia & mgSO $4 / \mathrm{L}$ & 0.09 & $<0.05$ & $<0.09$ \\
\hline $\mathrm{H}-017$ & Ammonia & $\mathrm{mgSO} 4 / \mathrm{L}$ & $<0.05$ & $<0.05$ & $<0.05$ \\
\hline $\mathrm{F}-012$ & Ammonia & mgSO4/L & $<0.05$ & $<0.05$ & $<0.05$ \\
\hline$F-012$ & Ammonia & $\mathrm{mgSO} 4 / \mathrm{L}$ & $<0.05$ & $<0.05$ & $<0.05$ \\
\hline $\mathrm{H}-017$ & Ammonia & mgSO4/L & $<0.05$ & $<0.05$ & $<0.05$ \\
\hline
\end{tabular}


Table 80

NPDES Duplicate Sample Results

Page 2 of 6

\begin{tabular}{|c|c|c|c|c|c|}
\hline Sample & Analyte & Units & Result 1 & Result 2 & Difference \\
\hline$F-012$ & Ammonia & $\mathrm{mgSO} 4 / \mathrm{L}$ & $\overline{0.12}$ & 0.111 & 0.009 \\
\hline $\mathrm{H}-017$ & Ammonia & mgSO4/L & 0.142 & 0.124 & 0.018 \\
\hline$F-008$ & Ammonia & $\mathrm{mgSO} 4 / \mathrm{L}$ & 0.576 & 0.452 & 0.124 \\
\hline $\mathrm{H}-012$ & Ammonia & $\mathrm{mgSO} 4 / \mathrm{L}$ & 0.913 & 0.783 & 0.13 \\
\hline$F-012$ & Ammonia & $\mathrm{mgSO} 4 / \mathrm{L}$ & 1.46 & 2.36 & -0.9 \\
\hline $\mathrm{H}-017$ & Ammonia & mgso4/L & $<0.1$ & $<0.1$ & $<0.1$ \\
\hline$F-012$ & Ammonia & $\mathrm{mgSO} 4 / \mathrm{L}$ & 0.299 & 0.115 & 0.184 \\
\hline SC-004 & Arsenic & $\mathrm{mg} / \mathrm{L}$ & $<.005$ & $<.005$ & $<.005$ \\
\hline SC-004 & Arsenic & $\mathrm{mg} / \mathrm{L}$ & $<.005$ & $<.005$ & $<.005$ \\
\hline SC-004 & Arsenic & $\mathrm{mg} / \mathrm{L}$ & $<0.02$ & $<0.02$ & $<0.02$ \\
\hline SC-004 & Arsenic & $\mathrm{mg} / \mathrm{L}$ & $<0.02$ & $<0.02$ & $<0.02$ \\
\hline $\mathrm{SC}-004$ & Barium & $\mathrm{mg} / \mathrm{L}$ & 0.0124 & 0.0124 & 0 \\
\hline SC-004 & Barium & $\mathrm{mg} / \mathrm{L}$ & 0.0129 & 0.0126 & 0.0003 \\
\hline SC-004 & Barium & $\mathrm{mg} / \mathrm{L}$ & 0.021 & 0.032 & -0.011 \\
\hline SC-004 & Barium & $\mathrm{mg} / \mathrm{L}$ & $<0.005$ & $<0.005$ & $<0.005$ \\
\hline$x-014$ & Benzene & $\mathrm{mg} / \mathrm{L}$ & $<0.8$ & $<0.8$ & $<0.8$ \\
\hline$x-014$ & Benzene & $\mathrm{mg} / \mathrm{L}$ & $<0.8$ & $<0.8$ & $<0.8$ \\
\hline$x-014$ & Benzene & $\mathrm{mg} / \mathrm{L}$ & $<0.8$ & $<0.8$ & $<0.8$ \\
\hline$x-014$ & Benzene & $\mathrm{mg} / \mathrm{L}$ & $<0.8$ & $<0.8$ & $<0.8$ \\
\hline$x-014$ & Benzene & $\mathrm{mg} / \mathrm{L}$ & $<0.5$ & $<0.5$ & $<0.5$ \\
\hline$x-014$ & Benzene & $\mathrm{mg} / \mathrm{L}$ & $<0.5$ & $<0.5$ & $<0.5$ \\
\hline$F-003$ & Biological Oxygen Demand & $\mathrm{mg} / \mathrm{L}$ & 1.6 & 1.3 & 0.3 \\
\hline DW-004 & Biological Oxygen Demand & $\mathrm{mg} / \mathrm{L}$ & $<1$ & $<1$ & $<1$ \\
\hline A-001 & Biological Oxygen Demand & $\mathrm{mg} / \mathrm{L}$ & 2.3 & 1.3 & 1 \\
\hline$A-005$ & Biological Oxygen Demand & $\mathrm{mg} / \mathrm{L}$ & $<1$ & $<1$ & $<1$ \\
\hline $\mathrm{K}-018$ & Biological Oxygen Demand & $\mathrm{mg} / \mathrm{L}$ & $<1$ & 1.5 & $<1.5$ \\
\hline$A-001$ & Biological Oxygen Demand & $\mathrm{mg} / \mathrm{L}$ & $<1$ & $<1$ & $<1$ \\
\hline$A-005$ & Biological Oxygen Demand & $\mathrm{mg} / \mathrm{L}$ & $<1$ & $<1$ & $<1$ \\
\hline$K-018$ & Biological Oxygen Demand & $\mathrm{mg} / \mathrm{L}$ & 2.1 & 1.8 & 0.3 \\
\hline $\mathrm{K}-018$ & Biological Oxygen Demand & $\mathrm{mg} / \mathrm{L}$ & 1.6 & 1 & 0.6 \\
\hline $\mathrm{K}-018$ & Biological Oxygen Demand & $\mathrm{mg} / \mathrm{L}$ & 2 & 2 & 0 \\
\hline$x-014$ & Biological Oxygen Demand & $\mathrm{mg} / \mathrm{L}$ & $<1$ & $<1$ & $<1$ \\
\hline A-001 & Biological Oxygen Demand & $\mathrm{mg} / \mathrm{L}$ & 1.2 & $<1$ & $<1.2$ \\
\hline A-011 & Biological Oxygen Demand & $\mathrm{mg} / \mathrm{L}$ & $<1$ & $<1$ & $<1$ \\
\hline $\mathrm{F}-003$ & Biological Oxygen Demand & $\mathrm{mg} / \mathrm{L}$ & $<1$ & $<1$ & $<1$ \\
\hline$A-001$ & Biological Oxygen Demand & $\mathrm{mg} / \mathrm{L}$ & $<1$ & $<1$ & $<1$ \\
\hline$A-005$ & Biological Oxygen Demand & $\mathrm{mg} / \mathrm{L}$ & $<1$ & $<1$ & $<1$ \\
\hline$A-011$ & Biological Oxygen Demand & $\mathrm{mg} / \mathrm{L}$ & $<1$ & $<1$ & $<1$ \\
\hline$x-014$ & Biological Oxygen Demand & $\mathrm{mg} / \mathrm{L}$ & $<1$ & $<1$ & $<1$ \\
\hline$A-011$ & Biological Oxygen Demand & $\mathrm{mg} / \mathrm{L}$ & 1.2 & 1.3 & -0.1 \\
\hline$x-014$ & Biological Oxygen Demand & $\mathrm{mg} / \mathrm{L}$ & 1.3 & 1.8 & -0.5 \\
\hline$A-001$ & Biological Oxygen Demand & $\mathrm{mg} / \mathrm{L}$ & 181 & $<1$ & $<181$ \\
\hline $\mathrm{K}-018$ & Biological Oxygen Demand & $\mathrm{mg} / \mathrm{L}$ & 14 & 7.6 & 6.4 \\
\hline $\mathrm{F}-003$ & Biological Oxygen Demand & $\mathrm{mg} / \mathrm{L}$ & 2.2 & 7 & -4.8 \\
\hline$A-005$ & Biological Oxygen Demand & $\mathrm{mg} / \mathrm{L}$ & $<1$ & 1.5 & $<1.5$ \\
\hline$A-011$ & Biological Oxygen Demand & $\mathrm{mg} / \mathrm{L}$ & $<1$ & 1.7 & $<1.7$ \\
\hline$A-001$ & Biological Oxygen Demand & $\mathrm{mg} / \mathrm{L}$ & $<1$ & $<1$ & $<1$ \\
\hline A-001 & Biological Oxygen Demand & $\mathrm{mg} / \mathrm{L}$ & $<1$ & $<1$ & $<1$ \\
\hline
\end{tabular}


Table 80

NPDES Duplicate Sample Results

Page 3 of 6

\begin{tabular}{|c|c|c|c|c|c|}
\hline Sample & Analyte & Units & Result 1 & Result 2 & Difference \\
\hline $\mathrm{K}-018$ & Biological Oxygen Demand & $\mathrm{mg} / \mathrm{L}$ & 1.6 & $<1$ & $<1.6$ \\
\hline$x-014$ & Biological Oxygen Demand & $\mathrm{mg} / \mathrm{L}$ & $<1$ & $<1$ & $<1$ \\
\hline$A-011$ & Biological Oxygen Demand & $\mathrm{mg} / \mathrm{L}$ & $<1$ & $<1$ & $<1$ \\
\hline DW-004 & Biological Oxygen Demand & $\mathrm{mg} / \mathrm{L}$ & 3.6 & 3.3 & 0.3 \\
\hline A-011 & Biological Oxygen Demand & $\mathrm{mg} / \mathrm{L}$ & 4 & 3.6 & 0.4 \\
\hline $\mathrm{F}-003$ & Biological Oxygen Demand & $\mathrm{mg} / \mathrm{L}$ & 2 & 2.1 & -0.1 \\
\hline$A-005$ & Biological Oxygen Demand & $\mathrm{mg} / \mathrm{L}$ & 2.1 & 1.5 & 0.6 \\
\hline$A-001$ & Biological Oxygen Demand & $\mathrm{mg} / \mathrm{L}$ & 5.8 & 6.3 & -0.5 \\
\hline $\mathrm{H}-016$ & Biological Ozygen Demand & $\mathrm{mg} / \mathrm{L}$ & $<1$ & $<1$ & $<1$ \\
\hline$x-014$ & Biological Ozygen Demand & $\mathrm{mg} / \mathrm{L}$ & $<1$ & $<1$ & $<1$ \\
\hline SC-004 & Cadmium & $\mathrm{mg} / \mathrm{L}$ & $<0.005$ & $<0.005$ & $<0.005$ \\
\hline SC-004 & Cadmium & $\mathrm{mg} / \mathrm{L}$ & $<0.005$ & $<0.005$ & $<0.005$ \\
\hline$M-004$ & Cadmium & $\mathrm{mg} / \mathrm{L}$ & $<0.005$ & $<0.005$ & $<0.005$ \\
\hline $\mathrm{SC}-004$ & Cadmium & $\mathrm{mg} / \mathrm{L}$ & $<0.005$ & $<0.005$ & $<0.005$ \\
\hline SC-004 & Cadmium & $\mathrm{mg} / \mathrm{L}$ & $<0.005$ & $<0.005$ & $<0.005$ \\
\hline$F-012$ & Chromium & $\mathrm{mg} / \mathrm{L}$ & $<0.005$ & $<0.005$ & $<0.005$ \\
\hline $\mathrm{H}-016$ & Chromium & $\mathrm{mg} / \mathrm{L}$ & $<0.005$ & $<0.005$ & $<0.005$ \\
\hline SC-004 & Chromium & $\mathrm{mg} / \mathrm{L}$ & $<0.005$ & $<0.005$ & $<0.005$ \\
\hline SC-004 & Chromium & $\mathrm{mg} / \mathrm{L}$ & $<0.005$ & $<0.005$ & $<0.005$ \\
\hline $\mathrm{F}-012$ & Chromium & $\mathrm{mg} / \mathrm{L}$ & $<0.005$ & $<0.005$ & $<0.005$ \\
\hline$M-004$ & Chromium & $\mathrm{mg} / \mathrm{L}$ & $<0.005$ & $<0.005$ & $<0.005$ \\
\hline $\mathrm{H}-016$ & Chromium & $\mathrm{mg} / \mathrm{L}$ & $<0.005$ & $<0.005$ & $<0.005$ \\
\hline$F-008$ & Chromium & $\mathrm{mg} / \mathrm{L}$ & $<0.005$ & $<0.005$ & $<0.005$ \\
\hline $\mathrm{H}-012$ & Chromium & $\mathrm{mg} / \mathrm{L}$ & $<0.005$ & $<0.005$ & $<0.005$ \\
\hline$F-012$ & Chromium & $\mathrm{mg} / \mathrm{L}$ & $<0.005$ & $<0.005$ & $<0.005$ \\
\hline$F-012$ & Chromium & $\mathrm{mg} / \mathrm{L}$ & $<0.01$ & $<0.01$ & $<0.01$ \\
\hline $\mathrm{SC}-004$ & Chromium & $\mathrm{mg} / \mathrm{L}$ & $<0.01$ & $<0.01$ & $<0.01$ \\
\hline$F-008$ & Chromium & $\mathrm{mg} / \mathrm{L}$ & $<0.01$ & $<0.01$ & $<0.01$ \\
\hline $\mathrm{H}-012$ & Chromium & $\mathrm{mg} / \mathrm{L}$ & $<0.01$ & $<0.01$ & $<0.01$ \\
\hline$F-012$ & Chromium & $\mathrm{mg} / \mathrm{L}$ & $<0.01$ & $<0.01$ & $<0.01$ \\
\hline SC-004 & Chromium & $\mathrm{mg} / \mathrm{L}$ & $<0.01$ & $<0.01$ & $<0.01$ \\
\hline$F-012$ & Copper & $\mathrm{mg} / \mathrm{L}$ & 0.0113 & 0.0076 & 0.0037 \\
\hline $\mathrm{H}-016$ & Copper & $\mathrm{mg} / \mathrm{L}$ & $<.005$ & 0.0135 & $<0.0135$ \\
\hline $\mathrm{H}-017$ & Copper & $\mathrm{mg} / \mathrm{L}$ & 0.0079 & 0.0075 & 0.0004 \\
\hline$M-004$ & Copper & $\mathrm{mg} / \mathrm{L}$ & $<.005$ & 0.0053 & $<0.0053$ \\
\hline$F-012$ & Copper & $\mathrm{mg} / \mathrm{L}$ & $<0.005$ & $<0.005$ & $<0.005$ \\
\hline$F-013$ & Copper & $\mathrm{mg} / \mathrm{L}$ & $<0.005$ & $<0.005$ & $<0.005$ \\
\hline$F-013$ & Copper & $\mathrm{mg} / \mathrm{L}$ & $<0.005$ & $<0.005$ & $<0.005$ \\
\hline $\mathrm{H}-017$ & Copper & $\mathrm{mg} / \mathrm{L}$ & $<0.005$ & $<0.005$ & $<0.005$ \\
\hline$M-004$ & Copper & $\mathrm{mg} / \mathrm{L}$ & $<0.005$ & $<0.005$ & $<0.005$ \\
\hline $\mathrm{H}-016$ & Copper & $\mathrm{mg} / \mathrm{L}$ & $<0.005$ & $<0.005$ & $<0.005$ \\
\hline$F-008$ & Copper & $\mathrm{mg} / \mathrm{L}$ & 0.0124 & $<0.005$ & $<0.0124$ \\
\hline $\mathrm{H}-012$ & Copper & $\mathrm{mg} / \mathrm{L}$ & 0.0083 & 0.0088 & -0.0005 \\
\hline$F-012$ & Copper & $\mathrm{mg} / \mathrm{L}$ & 0.0107 & 0.0119 & -0.0012 \\
\hline$F-012$ & Copper & $\mathrm{mg} / \mathrm{L}$ & $<0.01$ & $<0.01$ & $<0.01$ \\
\hline$F-008$ & Copper & $\mathrm{mg} / \mathrm{L}$ & $<0.005$ & $<0.005$ & $<0.005$ \\
\hline $\mathrm{H}-012$ & Copper & $\mathrm{mg} / \mathrm{L}$ & $<0.005$ & 0.007 & $<0.007$ \\
\hline$F-012$ & Copper & $\mathrm{mg} / \mathrm{L}$ & $<0.005$ & 0.008 & $<0.008$ \\
\hline
\end{tabular}


Table 80

\section{NPDES Duplicate Sample Results}

Page 4 of 6

\begin{tabular}{|c|c|c|c|c|c|}
\hline Sample & Analyte & Units & Result 1 & Result 2 & Difference \\
\hline$M-004$ & Copper & $\mathrm{mg} / \mathrm{L}$ & $<0.01$ & $<0.01$ & $<0.01$ \\
\hline$M-004$ & Cyanide & $\mathrm{mg} / \mathrm{L}$ & $<10$ & $<10$ & $<10$ \\
\hline$x-008$ & Iron & $\mathrm{mg} / \mathrm{L}$ & 1.79 & 1.66 & 0.13 \\
\hline D-001B & Iron & $\mathrm{mg} / \mathrm{L}$ & 295 & 308 & -13 \\
\hline D-001B & Iron & $\mathrm{mg} / \mathrm{L}$ & 344 & 342 & 2 \\
\hline$F-012$ & Lead & $\mathrm{mg} / \mathrm{L}$ & $<.003$ & $<.003$ & $<.003$ \\
\hline $\mathrm{H}-016$ & Lead & $\mathrm{mg} / \mathrm{L}$ & $<.003$ & $<.003$ & $<.003$ \\
\hline SC-004 & Lead & $\mathrm{mg} / \mathrm{L}$ & $<.003$ & $<.003$ & $<.003$ \\
\hline$M-004$ & Lead & $\mathrm{mg} / \mathrm{L}$ & $<.003$ & $<.003$ & $<.003$ \\
\hline SC-004 & Lead & $\mathrm{mg} / \mathrm{L}$ & $<.003$ & $<.003$ & $<.003$ \\
\hline$F-012$ & Lead & $\mathrm{mg} / \mathrm{L}$ & $<0.003$ & $<0.003$ & $<0.003$ \\
\hline$M-004$ & Lead & $\mathrm{mg} / \mathrm{L}$ & $<0.015$ & $<0.015$ & $<0.015$ \\
\hline $\mathrm{H}-016$ & Lead & $\mathrm{mg} / \mathrm{L}$ & $<0.003$ & $<0.003$ & $<0.003$ \\
\hline$F-008$ & Lead & $\mathrm{mg} / \mathrm{L}$ & $<0.003$ & $<0.003$ & $<0.003$ \\
\hline $\mathrm{H}-012$ & Lead & $\mathrm{mg} / \mathrm{L}$ & $<0.003$ & $<0.003$ & $<0.003$ \\
\hline$F-012$ & Lead & $\mathrm{mg} / \mathrm{L}$ & 0.0036 & $<0.003$ & $<.0036$ \\
\hline$F-012$ & Lead & $\mathrm{mg} / \mathrm{L}$ & $<0.02$ & 0.033 & $<0.033$ \\
\hline SC-004 & Lead & $\mathrm{mg} / \mathrm{L}$ & $<0.02$ & $<0.02$ & $<0.02$ \\
\hline$F-008$ & Lead & $\mathrm{mg} / \mathrm{L}$ & $<0.02$ & $<0.02$ & $<0.02$ \\
\hline $\mathrm{H}-012$ & Lead & $\mathrm{mg} / \mathrm{L}$ & $<0.02$ & $<0.02$ & $<0.02$ \\
\hline$F-012$ & Lead & $\mathrm{mg} / \mathrm{L}$ & $<0.02$ & $<0.02$ & $<0.02$ \\
\hline SC-004 & Lead & $\mathrm{mg} / \mathrm{L}$ & $<0.02$ & $<0.02$ & $<0.02$ \\
\hline M-004 & Lead & $\mathrm{mg} / \mathrm{L}$ & $<0.02$ & $<0.02$ & $<0.02$ \\
\hline $\mathrm{D}-001 \mathrm{~B}$ & Magnesium & $\mathrm{mg} / \mathrm{L}$ & 26.1 & 27.5 & -1.4 \\
\hline$D-001 B$ & Magnesium & $\mathrm{mg} / \mathrm{L}$ & 32.1 & 34.3 & -2.2 \\
\hline$F-012$ & Manganese & $\mathrm{mg} / \mathrm{L}$ & 0.0087 & 0.0088 & -0.0001 \\
\hline $\mathrm{H}-016$ & Manganese & $\mathrm{mg} / \mathrm{L}$ & $<.005$ & $<.005$ & $<.005$ \\
\hline $\mathrm{H}-017$ & Manganese & $\mathrm{mg} / \mathrm{L}$ & 0.0579 & 0.0576 & 0.0003 \\
\hline$F-012$ & Manganese & $\mathrm{mg} / \mathrm{L}$ & 0.03598 & 0.0015 & 0.03448 \\
\hline$F-013$ & Manganese & $\mathrm{mg} / \mathrm{L}$ & 0.0131 & 0.0124 & 0.0007 \\
\hline$F-013$ & Manganese & $\mathrm{mg} / \mathrm{L}$ & 0.0288 & 0.0229 & 0.0059 \\
\hline $\mathrm{F}-012$ & Manganese & $\mathrm{mg} / \mathrm{L}$ & 0.0163 & 0.0165 & -0.0002 \\
\hline $\mathrm{H}-017$ & Manganese & $\mathrm{mg} / \mathrm{L}$ & 0.0296 & 0.0295 & 0.0001 \\
\hline$H-016$ & Manganese & $\mathrm{mg} / \mathrm{L}$ & $<0.005$ & $<0.005$ & $<0.005$ \\
\hline $\mathrm{F}-008$ & Manganese & $\mathrm{mg} / \mathrm{L}$ & 0.0102 & 0.0102 & 0 \\
\hline $\mathrm{H}-012$ & Manganese & $\mathrm{mg} / \mathrm{L}$ & 0.012 & 0.0117 & 0.0003 \\
\hline$F-012$ & Manganese & $\mathrm{mg} / \mathrm{L}$ & 0.0176 & 0.0177 & 0.0001 \\
\hline$F-012$ & Manganese & $\mathrm{mg} / \mathrm{L}$ & 0.045 & 0.084 & -0.039 \\
\hline$F-008$ & Manganese & $\mathrm{mg} / \mathrm{L}$ & 0.055 & $<0.01$ & $<0.055$ \\
\hline $\mathrm{H}-012$ & Manganese & $\mathrm{mg} / \mathrm{L}$ & $<0.01$ & $<0.01$ & $<0.01$ \\
\hline$F-012$ & Manganese & $\mathrm{mg} / \mathrm{L}$ & $<0.01$ & $<0.01$ & $<0.01$ \\
\hline$F-012$ & Mercury & $\mathrm{mg} / \mathrm{L}$ & $<.0001$ & 0.00048 & $<0.00048$ \\
\hline$x-014$ & Mercury & $\mathrm{mg} / \mathrm{L}$ & 0.1 & 0.14 & -0.04 \\
\hline$x-014$ & Mercury & $\mathrm{mg} / \mathrm{L}$ & 0.2 & 0.32 & -0.12 \\
\hline $\mathrm{H}-016$ & Mercury & $\mathrm{mg} / \mathrm{L}$ & $<0.0001$ & $<0.0001$ & $<0.0001$ \\
\hline SC-004 & Mercury & $\mathrm{mg} / \mathrm{L}$ & $<0.0001$ & $<0.0001$ & $<0.0001$ \\
\hline $\mathrm{H}-017$ & Mercury & $\mathrm{mg} / \mathrm{L}$ & $<0.0001$ & $<0.0001$ & $<0.0001$ \\
\hline$F-012$ & Mercury & $\mathrm{mg} / \mathrm{L}$ & $<0.0001$ & $<0.0001$ & $<0.0001$ \\
\hline
\end{tabular}


Table 80

NPDES Duplicate Sample Results

Page 5 of 6

\begin{tabular}{|c|c|c|c|c|c|}
\hline Sample & Analyte & Units & Result 1 & Resuit 2 & Difference \\
\hline$F=013$ & Mercury & $\mathrm{mg} / \mathrm{L}$ & $<0.0001$ & $<0.0001$ & $<0,0001$ \\
\hline SC-004 & Mercury & $\mathrm{mg} / \mathrm{L}$ & $<0.0001$ & $<0.0001$ & $<0.0001$ \\
\hline$F-013$ & Mercury & $\mathrm{mg} / \mathrm{L}$ & $<0.0001$ & $<0.0001$ & $<0.0001$ \\
\hline$F-012$ & Mercury & $\mathrm{mg} / \mathrm{L}$ & $<0.0001$ & $<0.0001$ & $<0.0001$ \\
\hline $\mathrm{H}-017$ & Mercury & $\mathrm{mg} / \mathrm{L}$ & $<0.0001$ & $<0.0001$ & $<0.0001$ \\
\hline$x-014$ & Mercury & $\mathrm{mg} / \mathrm{L}$ & 0.14 & $<0.1$ & $<0.14$ \\
\hline$H-016$ & Mercury & $\mathrm{mg} / \mathrm{L}$ & $<0.0001$ & $<0.0001$ & $<0.0001$ \\
\hline$F-008$ & Mercury & $\mathrm{mg} / \mathrm{L}$ & $<0.0001$ & $<0.0001$ & $<0.0001$ \\
\hline $\mathrm{H}-012$ & Mercury & $\mathrm{mg} / \mathrm{L}$ & $<0.0001$ & $<0.0001$ & $<0.0001$ \\
\hline$F-012$ & Mercury & $\mathrm{mg} / \mathrm{L}$ & $<0.0001$ & $<0.0001$ & $<0.0001$ \\
\hline$F=012$ & Mercury & $\mathrm{mg} / \mathrm{h}$ & $<0.0001$ & 0.0004 & $<0.0004$ \\
\hline SC-004 & Mercury & $\mathrm{mg} / \mathrm{L}$ & 0.0003 & $<0.001$ & $<0.003$ \\
\hline$F-008$ & Mercury & $\mathrm{mg} / \mathrm{L}$ & $<0.0001$ & $<0.0001$ & $<0.0001$ \\
\hline $\mathrm{H}-012$ & Mercury & $\mathrm{mg} / \mathrm{L}$ & $<0.0001$ & $<0.0001$ & $<0.0001$ \\
\hline$F-012$ & Mercury & $\mathrm{mg} / \mathrm{L}$ & $<0.0001$ & $<0.0001$ & $<0.0001$ \\
\hline SC-004 & Mercury & $\mathrm{mg} / \mathrm{L}$ & $<0.0001$ & $<0.0001$ & $<0.0001$ \\
\hline$F-012$ & Nickel & $\mathrm{mg} / \mathrm{L}$ & $<.005$ & $<.005$ & $<.005$ \\
\hline$M-004$ & Nickel & $\mathrm{mg} / \mathrm{L}$ & 0.0202 & 0.0151 & 0.0051 \\
\hline$M-004$ & Nickel & $\mathrm{mg} / \mathrm{L}$ & 0.0114 & 0.0134 & -0.002 \\
\hline$F-008$ & Nickel & $\mathrm{mg} / \mathrm{L}$ & $<0.005$ & $<0.005$ & $<0.005$ \\
\hline $\mathrm{H}-012$ & Nickel & $\mathrm{mg} / \mathrm{L}$ & $<0.005$ & $<0.005$ & $<0.005$ \\
\hline$F-012$ & Nickel & $\mathrm{mg} / \mathrm{L}$ & $<0.005$ & $<0.005$ & $<0.005$ \\
\hline$F-012$ & Nickel & $\mathrm{mg} / \mathrm{L}$ & $<0.01$ & $<0.01$ & $<0.01$ \\
\hline$F-008$ & Nickel & $\mathrm{mg} / \mathrm{L}$ & $<0.01$ & $<0.01$ & $<0.01$ \\
\hline $\mathrm{H}-012$ & Nickel & $\mathrm{mg} / \mathrm{L}$ & $<0.01$ & $<0.01$ & $<0.01$ \\
\hline$F-012$ & Nickel & $\mathrm{mg} / \mathrm{L}$ & $<0.01$ & $<0.01$ & $<0.01$ \\
\hline$M-004$ & Nickel & $\mathrm{mg} / \mathrm{L}$ & $<0.01$ & $<0.01$ & $<0.01$ \\
\hline $\mathrm{H}-016$ & Nitrate & $\mathrm{mgN} / \mathrm{L}$ & 8.74 & 9.07 & -0.33 \\
\hline SC-004 & Nitrate & $\mathrm{mgN} / \mathrm{L}$ & 0.1 & 0.09 & 0.01 \\
\hline M-004 & Nitrate & $\mathrm{mgN} / \mathrm{L}$ & 35.9 & 34.7 & 1.2 \\
\hline$F-012$ & Nitrate & $\mathrm{mgN} / \mathrm{L}$ & 3.81 & 4.04 & -0.23 \\
\hline SC-004 & Nitrate & $\mathrm{mgN} / \mathrm{L}$ & 0.05 & 0.07 & -0.02 \\
\hline $\mathrm{H}-016$ & Nitrate & $\mathrm{mgN} / \mathrm{L}$ & 23 & 20.8 & 2.2 \\
\hline$M-004$ & Nitrate & $\mathrm{mgN} / \mathrm{L}$ & 84.6 & 111 & -26.4 \\
\hline$F-008$ & Nitrate & $\mathrm{mgN} / \mathrm{L}$ & 0.03 & 0.03 & 0 \\
\hline $\mathrm{H}-012$ & Nitrate & $\mathrm{mgN} / \mathrm{L}$ & $<0.02$ & 0.04 & $<0.04$ \\
\hline $\mathrm{H}-017$ & Nitrate & $\mathrm{mgN} / \mathrm{L}$ & $<0.02$ & $<0.02$ & $<0.02$ \\
\hline$F-012$ & Nitrate & $\mathrm{mgN} / \mathrm{L}$ & $<0.02$ & $<0.02$ & $<0.02$ \\
\hline$F-012$ & Nitrate & $\mathrm{mgN} / \mathrm{L}$ & 0.05 & 0.04 & 0.01 \\
\hline $\mathrm{H}-017$ & Nitrate & $\mathrm{mgN} / \mathrm{L}$ & 0.1 & 0.09 & 0.01 \\
\hline$F-012$ & Nitrate & $\mathrm{mgN} / \mathrm{L}$ & 0.117 & 0.123 & -0.006 \\
\hline $\mathrm{H}-017$ & Nitrate & $\mathrm{mgN} / \mathrm{L}$ & 0.219 & 0.207 & 0.012 \\
\hline SC-004 & Nitrate & $\mathrm{mgN} / \mathrm{L}$ & 0.026 & 0.025 & 0.001 \\
\hline$F-008$ & Nitrate & $\mathrm{mgN} / \mathrm{L}$ & $<0.02$ & $<0.02$ & $<0.02$ \\
\hline $\mathrm{H}-012$ & Nitrate & $\mathrm{mgN} / \mathrm{L}$ & 0.02 & $<0.02$ & $<0.02$ \\
\hline$F-012$ & Nitrate & $\mathrm{mgN} / \mathrm{L}$ & $<0.02$ & $<0.02$ & $<0.02$ \\
\hline SC-004 & Nitrate & $\mathrm{mgN} / \mathrm{L}$ & $<0.02$ & 0.061 & $<0.061$ \\
\hline $\mathrm{H}-017$ & Nitrate & $\mathrm{mgN} / \mathrm{L}$ & $<0.02$ & $<0.02$ & $<0.02$ \\
\hline
\end{tabular}


Table 80

NPDES Duplicate Sample Results

Page 6 of 6

\begin{tabular}{lllllc}
\hline Sample & Analyte & Units & Result 1 & Result 2 & Difference \\
\hline F-012 & Nitrate & $\mathrm{mg} / \mathrm{L}$ & $<0.02$ & $<0.02$ & $<0.02$ \\
K-010 & Oil \& Grease & $\mathrm{mg} / \mathrm{L}$ & $<1$ & $<1$ & $<1$ \\
$\mathrm{D}-006$ & Oil \& Grease & $\mathrm{mg} / \mathrm{L}$ & $<1$ & $<1$ & $<1$ \\
$\mathrm{~L}-007$ & Oil \& Grease & $\mathrm{mg} / \mathrm{L}$ & $<1$ & $<1$ & $<1$ \\
L-008 & Oil \& Grease & $\mathrm{mg} / \mathrm{L}$ & $<1$ & $<1$ & $<1$ \\
$\mathrm{H}-007 \mathrm{~A}$ & Oil \& Grease & $\mathrm{mg} / \mathrm{L}$ & $<1$ & $<1$ & $<1$ \\
$\mathrm{~F}-001$ & Oil \& Grease & $\mathrm{mg} / \mathrm{L}$ & $<1$ & $<1$ & $<1$ \\
$\mathrm{~F}-002$ & Oil \& Grease & $\mathrm{mg} / \mathrm{L}$ & $<1$ & $<1$ & $<1$ \\
$\mathrm{X}-014$ & Oil \& Grease & $\mathrm{mg} / \mathrm{L}$ & $<1$ & $<1$ & $<1$ \\
$\mathrm{~L}-007$ & Oil \& Grease & $\mathrm{mg} / \mathrm{L}$ & $<1$ & $<1$ & $<1$ \\
$\mathrm{~A}-001$ & Oil \& Grease & $\mathrm{mg} / \mathrm{L}$ & $<1$ & $<1$ & $<1$ \\
$\mathrm{~A}-005$ & Oil \& Grease & $\mathrm{mg} / \mathrm{L}$ & $<1$ & $<1$ & $<1$ \\
$\mathrm{~K}-018$ & Oil \& Grease & $\mathrm{mg} / \mathrm{L}$ & $<1$ & $<1$ & $<1$ \\
$\mathrm{H}-016$ & Oil \& Grease & $\mathrm{mg} / \mathrm{L}$ & $<1$ & $<1$ & $<1$ \\
$\mathrm{M}-004$ & Oil \& Grease & $\mathrm{mg} / \mathrm{L}$ & $<1$ & $<1$ & $<1$
\end{tabular}




\section{Table 81}

\section{QAP Interlaboratory Comparison of Analytical Results}

Page 1 of 3

\begin{tabular}{|c|c|c|c|c|c|}
\hline \multirow{2}{*}{ Nuclide } & \multirow[b]{2}{*}{ SRS Value ${ }^{a}$} & \multirow[b]{2}{*}{ QAP Value ${ }^{b}$} & \multirow[b]{2}{*}{$\begin{array}{c}\text { SRS/QAP } \\
\text { Ratio }\end{array}$} & \multicolumn{2}{|c|}{ Range } \\
\hline & & & & $\begin{array}{c}\text { Lower Control } \\
\text { Limitc }\end{array}$ & $\begin{array}{l}\text { Upper Control } \\
\text { Limit }^{c}\end{array}$ \\
\hline \multicolumn{6}{|c|}{ March-June 1995} \\
\hline \multicolumn{6}{|l|}{ Air } \\
\hline Alpha & $3.64 \pm 0.2$ & 3.22 & 1.13 & 1.61 & 4.83 \\
\hline Beta & $1.99 \pm 0.07$ & 1.85 & 1.08 & 0.925 & 2.77 \\
\hline $\mathrm{Ce}-144$ & $66.0 \pm 2.5$ & 91.2 & 0.56 & 53.8 & 124. \\
\hline Co-57 & $9.6 \pm 0.5$ & 12.7 & 0.57 & 8.13 & 18.4 \\
\hline Co-60 & $3.2 \pm 0.2$ & 3.76 & 0.69 & 2.67 & 4.85 \\
\hline Cs-134 & $5.4 \pm 0.6$ & 5.75 & 0.73 & 3.74 & 7.01 \\
\hline Cs-137 & $4.4 \pm 0.6$ & 5.28 & 0.66 & 3.64 & 6.97 \\
\hline$M n-54$ & $4.1 \pm 0.5$ & 4.71 & 0.66 & 3.48 & 6.41 \\
\hline $\mathrm{Pu}-238$ & $0.063 \pm 0.003$ & 0.122 & 0.52 & 0.059 & 0.214 \\
\hline Pu-239 & $0.033 \pm 0.002$ & 0.0623 & 0.53 & 0.036 & 0.1 \\
\hline $\mathrm{Sb}-125$ & $8.9 \pm 1$ & 9.42 & 0.73 & 4.71 & 17. \\
\hline \multicolumn{6}{|l|}{ Soil } \\
\hline Cs-137 & $299 \pm 10$ & 266. & 1.12 & 184. & 388. \\
\hline$K-40$ & $393 \pm 41$ & 384. & 1.02 & 257. & 588. \\
\hline \multicolumn{6}{|c|}{ Vegetation } \\
\hline Co-60 & $11.2 \pm 5.9$ & 10.7 & 1.05 & 5.35 & 16.1 \\
\hline Cs-137 & $133.6 \pm 9.8$ & 117. & 1.14 & 58.5 & 176. \\
\hline$K-40$ & $1161 \pm 236$ & 1030. & 1.13 & 515. & 1545. \\
\hline Pu-238 & $0.13 \pm 0.04$ & 0.0887 & 1.47 & 0.044 & 0.133 \\
\hline Pu-239 & $1.19 \pm 0.15$ & 1.12 & 1.06 & 0.56 & 1.68 \\
\hline Sr-90 & $274 \pm 28$ & 512. & 0.54 & 256. & 768. \\
\hline \multicolumn{6}{|l|}{ Water } \\
\hline Alpha & $1236 \pm 47$ & 1340 & 0.92 & 670. & 2010. \\
\hline$A m-241$ & $1.34 \pm 0.19$ & 1.33 & 1.01 & 0.758 & 2. \\
\hline Beta & $666 \pm 26$ & 653. & 1.02 & 326. & 980. \\
\hline Co-60 & $216 \pm 4$ & 196. & 1.10 & 155. & 231. \\
\hline Cs-134 & $88 \pm 2$ & 83.5 & 1.05 & 61.8 & 108. \\
\hline Cs-137 & $89 \pm 3$ & 76.8 & 1.16 & 63. & 99.1 \\
\hline $\mathrm{H}-3$ & $71 \pm 5$ & 60.3 & 1.18 & 38.6 & 112. \\
\hline $\begin{array}{ll}\text { a } & \text { Value } \\
\text { b } & \text { Qualit } \\
& \text { Divisic } \\
\text { c } & \text { Contrc }\end{array}$ & $\begin{array}{l}\text { 1, Bq/filter. } \\
\text { ogram conducte } \\
\text { emistry laborator } \\
\text { blished by EML }\end{array}$ & $\begin{array}{l}\text { A National Exp } \\
\text { om historical d }\end{array}$ & Research & atory Characterizatio & Research \\
\hline
\end{tabular}


Table 81

QAP Interlaboratory Comparison of Analytical Results

Page 2 of 3

\begin{tabular}{|c|c|c|c|c|c|}
\hline \multirow[b]{2}{*}{ Nuclide } & \multirow[b]{2}{*}{ SRS Value ${ }^{a}$} & \multirow[b]{2}{*}{ QAP Value ${ }^{b}$} & \multirow[b]{2}{*}{$\begin{array}{c}\text { SRS/QAP } \\
\text { Ratio }\end{array}$} & \multicolumn{2}{|c|}{ Range } \\
\hline & & & & $\begin{array}{c}\text { Lower Control } \\
\text { Limitc }^{c}\end{array}$ & $\begin{array}{l}\text { Upper Control } \\
\text { Limit }^{c}\end{array}$ \\
\hline $\mathrm{Mn}-54$ & $50 \pm 1$ & 43.5 & 1.15 & 35.2 & 54.4 \\
\hline$P u-239$ & $0.588 \pm 0.021$ & 0.591 & 0.99 & 0.319 & 0.792 \\
\hline Sr-90 & $2.2 \pm 0.5$ & 2.4 & 0.92 & 1.37 & 3.43 \\
\hline$U-234$ & $0.356 \pm 0.056$ & 0.373 & 0.95 & 0.283 & 0.571 \\
\hline U-235 & $0.252 \pm 0.052$ & 0.196 & 1.29 & 0.149 & 0.3 \\
\hline$U-238$ & $0.028 \pm 0.014$ & 0.0727 & 0.39 & 0.056 & 0.105 \\
\hline \multicolumn{6}{|c|}{ September-December 1995} \\
\hline \multicolumn{6}{|l|}{ Air } \\
\hline Am-241 & $0.16 \pm 0.01$ & 0.189 & 0.85 & 0.102 & 0.31 \\
\hline $\mathrm{Ce}-144$ & $35 \pm 3$ & 52.1 & 0.67 & 30.7 & 70.9 \\
\hline Co-57 & $10.1 \pm 0.9$ & 14.7 & 0.69 & 9.41 & 21.3 \\
\hline Co-60 & $26 \pm 1$ & 32.6 & 0.80 & 23.1 & 42.1 \\
\hline Cs-134 & $15 \pm 1$ & 17.9 & 0.84 & 11.6 & 21.8 \\
\hline Cs-137 & $5.5 \pm 0.9$ & 7.25 & 0.76 & 5. & 9.57 \\
\hline GA & $3 \pm 0.09$ & 3.3 & 0.91 & 1.65 & 4.95 \\
\hline GB & $1.63 \pm 0.06$ & 1.12 & 1.46 & 0.56 & 1.68 \\
\hline$M n-54$ & $4.2 \pm 0.7$ & 5.34 & 0.79 & 3.95 & 7.26 \\
\hline $\mathrm{Pu}-238$ & $0.099 \pm 0.016$ & 0.0962 & 1.03 & 0.046 & 0.168 \\
\hline $\mathrm{Pu}-239$ & $0.205 \pm 0.028$ & 0.0927 & 2.21 & 0.053 & 0.148 \\
\hline$R u-106$ & $17 \pm 8$ & 17. & 1.00 & 8.5 & 25.5 \\
\hline $\mathrm{Sb}-125$ & $10 \pm 2$ & 11.4 & 0.88 & 5.7 & 20.5 \\
\hline$U-234$ & $0.058 \pm 0.003$ & 0.0517 & 1.12 & 0.036 & 0.09 \\
\hline$U-238$ & $0.059 \pm 0.003$ & 0.0533 & 1.11 & 0.039 & 0.1 \\
\hline \multicolumn{6}{|l|}{ Soil } \\
\hline Cs-137 & $202 \pm 16$ & 207. & 0.98 & 143. & 302. \\
\hline$K-40$ & $406 \pm 33$ & 377. & 1.08 & 253. & 577. \\
\hline $\mathrm{Pu}-238$ & $19.3 \pm 2.1$ & 17.5 & 1.10 & 3.67 & 24.7 \\
\hline Pu-239 & $5.83 \pm 0.71$ & 5.17 & 1.13 & 2.84 & 10.6 \\
\hline \multicolumn{6}{|c|}{ Vegetation } \\
\hline $\mathrm{Co}-60$ & $9 \pm 2$ & 9.17 & 0.98 & 6.33 & 12.8 \\
\hline Cs-137 & $107 \pm 10$ & 97.2 & 1.10 & 67.1 & 151. \\
\hline
\end{tabular}

\footnotetext{
a Values are $\mathrm{Bq} / \mathrm{h}, \mathrm{Bq} / \mathrm{Kg}, \mathrm{Bq} / \mathrm{filter}$.

b Quality Assessment Program conducted by EPA National Exposure Research Laboratory Characterization Research Division-Las Vegas chemistry laboratories

c Control limits were established by EMUQAP from historical data.
} 
Table 81

QAP Interlaboratory Comparison of Analytical Results

Page 3 of 3

\begin{tabular}{|c|c|c|c|c|c|}
\hline \multirow[b]{2}{*}{ Nuclide } & \multirow[b]{2}{*}{ SRS Value ${ }^{a}$} & \multirow[b]{2}{*}{ QAP Valueb } & \multirow[b]{2}{*}{$\begin{array}{c}\text { SRS/QAP } \\
\text { Ratio }\end{array}$} & \multicolumn{2}{|c|}{ Range } \\
\hline & & & & $\begin{array}{c}\text { Lower Control } \\
\text { Limit }^{c}\end{array}$ & $\begin{array}{l}\text { Upper Control } \\
\text { Limit }^{c}\end{array}$ \\
\hline $\mathrm{K}-40$ & $407 \pm 22$ & 352. & 1.16 & 151. & 521. \\
\hline $\mathrm{Pu}-239$ & $0.85 \pm 0.18$ & 0.98 & 0.87 & 0.549 & 1.9 \\
\hline Sr-90 & $376 \pm 58$ & 587. & 0.64 & 258. & 816. \\
\hline \multicolumn{6}{|l|}{ Water } \\
\hline Co-60 & $214 \pm 4$ & 196. & 1.09 & 155. & 231. \\
\hline Cs-137 & $84 \pm 3$ & 75.2 & 1.12 & 61.7 & 97. \\
\hline $\mathrm{GA}$ & $1223.88 \pm 122$ & 1310. & 0.93 & 655. & 2358. \\
\hline GB & $398.77 \pm 39$ & 410. & 0.97 & 205. & 738. \\
\hline$H-3$ & $270 \pm 7$ & 168. & 1.61 & 108. & 311. \\
\hline$M n-54$ & $52 \pm 3$ & 44.9 & 1.16 & 36.4 & 56.1 \\
\hline$P u-238$ & $1.458 \pm 0.018$ & 1.41 & 1.03 & 0.55 & 2.51 \\
\hline Pu-239 & $0.29 \pm 0.006$ & 0.272 & 1.07 & 0.147 & 0.364 \\
\hline Sr-90 & $3 \pm 0.23$ & 2. & 1.50 & 1.14 & 2.86 \\
\hline$U-234$ & $0.346 \pm 0.052$ & 0.306 & 1.13 & 0.233 & 0.468 \\
\hline$U-238$ & $0.325 \pm 0.048$ & 0.311 & 1.05 & 0.239 & 0.451 \\
\hline
\end{tabular}

a Values are $\mathrm{Bq} / \mathrm{L}, \mathrm{Bq} / \mathrm{Kg}, \mathrm{Bq} /$ filter.

b Quality Assessment Program conducted by EPA National Exposure Research Laboratory Characterization Research Division-Las Vegas chemistry laboratories

c Control limits were established by EML/QAP from historical data. 


\section{Table 82}

QAD Interlaboratory Comparison of Analytical Results

Page 1 of 2

\begin{tabular}{|c|c|c|c|c|c|c|}
\hline \multirow[b]{2}{*}{$\begin{array}{l}\text { Sample } \\
\text { Date }\end{array}$} & \multirow[b]{2}{*}{ Nuclide } & \multirow[b]{2}{*}{ SRS Value } & \multirow[b]{2}{*}{ QADa Value } & \multirow[b]{2}{*}{$\begin{array}{c}\text { SRS/QAD } \\
\text { Ratio }\end{array}$} & \multicolumn{2}{|c|}{ Range } \\
\hline & & & & & $\begin{array}{l}\text { Lower Control } \\
\text { Limit }^{\mathbf{b}}\end{array}$ & $\begin{array}{l}\text { Upper Control } \\
\text { Limit }^{\mathrm{b}}\end{array}$ \\
\hline \multicolumn{7}{|c|}{ Air Filter Samples (pCi/filter) } \\
\hline $08-25-95$ & Alpha & $29.8 \pm 3.4$ & $25 \pm 6.3$ & 1.19 & 14.1 & 35.9 \\
\hline $08-25-95$ & Beta & $75.9 \pm 4.0$ & $86.6 \pm 10$ & 0.88 & 69.3 & 103.9 \\
\hline $08-25-95$ & Cs-137 & $24 \pm 5$ & $25 \pm 5$ & 0.96 & 16.3 & 33.7 \\
\hline \multicolumn{7}{|c|}{ Milk Samples (pCi/L) } \\
\hline $09-29-95$ & Cs-137 & $53 \pm 4$ & $50 \pm 5$ & 1.06 & 41.3 & 58.7 \\
\hline $09-29-95$ & $\mid-131$ & $102 \pm 8$ & $99 \pm 10$ & 1.03 & 81.7 & 116.3 \\
\hline $09-29-95$ & Sr-90 & $18 \pm 5$ & $15 \pm 5$ & 1.20 & 6.3 & 23.7 \\
\hline \multicolumn{7}{|c|}{ Water Samples (pCi/L) } \\
\hline $01-27-95$ & Alpha & $3.3 \pm 3$ & $5 \pm 5$ & 0.66 & -3.7 & 13.7 \\
\hline $04-18-95$ & & $50.4 \pm 2.2$ & $47.5 \pm 11.9$ & 1.06 & 26.9 & 68.1 \\
\hline $07-21-95$ & & $15.6 \pm 5.5$ & $27.5 \pm 6.9$ & 0.57 & 15.6 & 39.4 \\
\hline $10-27-95$ & & $26.1 \pm 3.6$ & $51.2 \pm 12.8$ & 0.51 & 29.0 & 73.4 \\
\hline $06-09-95$ & $\mathrm{Ba}-133$ & $76 \pm 4$ & $79 \pm 8$ & 0.96 & 65.2 & 92.8 \\
\hline $11-03-95$ & & $99 \pm 5$ & $99 \pm 10$ & 1.00 & 81.7 & 116.3 \\
\hline $01-27-95$ & Beta & $12 \pm 4$ & $5 \pm 5$ & 2.40 & -3.7 & 13.7 \\
\hline $04-18-95$ & & $76.2 \pm 2.0$ & $86.6 \pm 10$ & 0.88 & 69.3 & 103.9 \\
\hline $07-21-95$ & & $17.1 \pm 4.3$ & $19.4 \pm 5$ & 0.88 & 10.7 & 28.1 \\
\hline $10-27-95$ & & $19.2 \pm 2.6$ & $24.8 \pm 5.0$ & 0.77 & 16.1 & 33.5 \\
\hline $04-18-95$ & Co-60 & $29 \pm 4$ & $29 \pm 5$ & 1.00 & 20.3 & 37.7 \\
\hline $06-09-95$ & & $43 \pm 3$ & $40 \pm 5$ & 1.08 & 31.3 & 48.7 \\
\hline $10-17-95$ & & $52 \pm 5$ & $49 \pm 5$ & 1.06 & 40.3 & 57.7 \\
\hline $11-03-95$ & & $66 \pm 8$ & $60 \pm 5$ & 1.10 & 51.3 & 68.7 \\
\hline $04-18-95$ & Cs-134 & $19 \pm 4$ & $20 \pm 5$ & 0.95 & 11.3 & 28.7 \\
\hline $06-09-95$ & & $46 \pm 3$ & $50 \pm 5$ & 0.92 & 41.3 & 58.7 \\
\hline $10-17-95$ & & $37.3 \pm 4$ & $40 \pm 5$ & 0.93 & 31.3 & 48.7 \\
\hline $11-03-95$ & & $43 \pm 6$ & $40 \pm 4$ & 1.08 & 33.1 & 46.9 \\
\hline
\end{tabular}

a EPA Quality Assurance Division

b Control limits were established by EPA National Exposure Research Laboratory Characterization Research Division-Las Vegas. 
Table 82

QAD Interlaboratory Comparison of Analytical Results

Page 2 of 2

\begin{tabular}{|c|c|c|c|c|c|c|}
\hline \multirow[b]{2}{*}{$\begin{array}{l}\text { Sample } \\
\text { Date }\end{array}$} & \multirow[b]{2}{*}{ Nuclide } & \multirow[b]{2}{*}{ SRS Value } & \multirow[b]{2}{*}{ QADa Value } & \multirow[b]{2}{*}{$\begin{array}{c}\text { SRS/QAD } \\
\text { Ratio }\end{array}$} & \multicolumn{2}{|c|}{ Range } \\
\hline & & & & & $\begin{array}{c}\text { Lower Control } \\
\text { Limit }^{b}\end{array}$ & $\begin{array}{l}\text { Upper Control } \\
\text { Limit }^{\mathrm{b}}\end{array}$ \\
\hline $04-18-95$ & Cs-137 & $11 \pm 4$ & $11 \pm 5$ & 1.00 & 2.3 & 19.7 \\
\hline $06-09-95$ & & $40 \pm 4$ & $35 \pm 5$ & 1.14 & 26.3 & 43.7 \\
\hline $10-17-95$ & & $31 \pm 4$ & $30 \pm 5$ & 1.03 & 21.3 & 38.7 \\
\hline $11-03-95$ & & $54 \pm 9$ & $49 \pm 5$ & 1.10 & 40.3 & 57.7 \\
\hline $03-10-95$ & $\mathrm{H}-3$ & $7250 \pm 108$ & $7435 \pm 744$ & 0.98 & 6147 & 8723 \\
\hline 08-04-95 & & $4663 \pm 155$ & $4872 \pm 487$ & 0.96 & 4029 & 5715 \\
\hline $03-17-95$ & Pu-239 & $10.7 \pm 1.0$ & $11.1 \pm 1.1$ & 0.96 & 9.2 & 13.0 \\
\hline $01-13-95$ & Sr-89 & $7 \pm 1.1$ & $20 \pm 5$ & 0.35 & 11.3 & 28.7 \\
\hline $04-18-95$ & & $18 \pm 2.3$ & $20 \pm 5$ & 0.90 & 11.3 & 28.7 \\
\hline $07-14-95$ & & $13 \pm 1.1$ & $20 \pm 5$ & 0.65 & 11.3 & 28.7 \\
\hline $10-17-95$ & & $12 \pm 6$ & $20 \pm 5$ & 0.60 & 11.3 & 28.7 \\
\hline $01-13-95$ & Sr-90 & $9.3 \pm 1.3$ & $15 \pm 5$ & 0.62 & 6.3 & 23.7 \\
\hline $04-18-95$ & & $17 \pm 0.98$ & $15 \pm 5$ & 1.13 & 6.3 & 23.7 \\
\hline $07-14-95$ & & $7 \pm 1.1$ & $8 \pm 5$ & 0.88 & -0.7 & 16.7 \\
\hline $10-17-95$ & & $10.3 \pm 2$ & $10 \pm 5$ & 1.03 & 1.3 & 18.7 \\
\hline $06-09-95$ & $Z n-65$ & $78 \pm 8$ & $76 \pm 8$ & 1.03 & 62.2 & 89.8 \\
\hline $11-03-95$ & & $128 \pm 12$ & $125 \pm 13$ & 1.02 & 102.5 & 147.5 \\
\hline
\end{tabular}

a EPA Quality Assurance Division

b Control limits were established by EPA National Exposure Research Laboratory Characterization Research Division-Las Vegas. 
Table 83

Metals Analysis on Split Duplicate Samples

Page 1 of 2

\begin{tabular}{|c|c|c|c|c|}
\hline \multirow[b]{2}{*}{ Parameter } & \multicolumn{2}{|c|}{$R M-160$} & \multicolumn{2}{|c|}{ U3R-4 } \\
\hline & Weston & ThermoAnalytical & Weston & ThermoAnalytical \\
\hline First Quarter & \multicolumn{4}{|c|}{$(\mathrm{mg} / \mathrm{L})$} \\
\hline Aluminum & 0.153 & 0.308 & 0.182 & 0.24 \\
\hline Calcium & 3.55 & 3.52 & 1.7 & 1.48 \\
\hline Cadmium & $<0.0047$ & $<0.005$ & $<0.0047$ & $<0.005$ \\
\hline Chromium & $<0.01$ & $<0.005$ & $<0.01$ & $<0.005$ \\
\hline Copper & $<0.018$ & $<0.005$ & 0.002 & $<0.005$ \\
\hline Iron & 0.368 & 0.59 & 0.428 & 0.558 \\
\hline Magnesium & 1.4 & 1.39 & 0.478 & 0.344 \\
\hline Manganese & 0.0591 & 0.0619 & 0.0193 & 0.021 \\
\hline Sodium & 7.24 & 7.58 & 1.43 & 1.57 \\
\hline Nickel & 0.00063 & $<0.005$ & 0.0017 & $<0.005$ \\
\hline Lead & $<0.013$ & $<0.003$ & $<0.013$ & $<0.003$ \\
\hline \multirow[t]{3}{*}{ Zinc } & 0.0072 & $<0.005$ & 0.0094 & 0.0054 \\
\hline & \multicolumn{2}{|c|}{ PB-3 } & \multicolumn{2}{|c|}{ TB-5 } \\
\hline & Weston & ThermoAnalytical & Weston & ThermoAnalytical \\
\hline Second Quarter & \multicolumn{4}{|c|}{ (mg/L) } \\
\hline Aluminum & 0.106 & 0.121 & 0.261 & 0.502 \\
\hline Calcium & 3.58 & 4.87 & 1.32 & 1.36 \\
\hline Cadmium & $<0.0047$ & $<0.005$ & $<0.0047$ & $<0.005$ \\
\hline Chromium & $<0.01$ & $<0.005$ & $<0.01$ & $<0.005$ \\
\hline Copper & $<0.018$ & 0.0086 & $<0.018$ & 0.02 \\
\hline Iron & 0.292 & 0.484 & 1.59 & 2.09 \\
\hline Magnesium & 0.8 & 1.05 & 0.483 & 0.488 \\
\hline Manganese & 0.0161 & 0.0221 & 0.0589 & 0.0686 \\
\hline Sodium & 5.73 & 7.98 & 6.2 & 7.25 \\
\hline Nickel & $<0.0054$ & $<0.005$ & 0.0106 & 0.0095 \\
\hline Lead & $<0.013$ & $<0.003$ & $<0.013$ & $<0.003$ \\
\hline Zinc & $<0.0097$ & $<0.005$ & 0.0412 & 0.0421 \\
\hline
\end{tabular}


Table 83

Metals Analysis on Split Duplicate Samples

Page 2 of 2

\begin{tabular}{|c|c|c|c|c|}
\hline \multirow{3}{*}{$\begin{array}{l}\text { Parameter } \\
\text { Third Quarter }\end{array}$} & \multicolumn{2}{|c|}{ SC-004 } & \multicolumn{2}{|c|}{ U3R-1A } \\
\hline & Weston & Shealy & Weston & Shealy \\
\hline & \multicolumn{4}{|c|}{$(\mathrm{mg} / \mathrm{L})$} \\
\hline Aluminum & $<0.087$ & $<0.05$ & $<0.087$ & 0.254 \\
\hline Calcium & 3.04 & 2.53 & 1.29 & 0.7 \\
\hline Cadmium & $<0.0047$ & $<0.005$ & $<0.0047$ & $<0.005$ \\
\hline Chromium & $<0.01$ & $<0.01$ & $<0.01$ & $<0.01$ \\
\hline Copper & 0.0322 & 0.039 & 0.0391 & 0.065 \\
\hline Iron & 0.19 & 0.24 & 0.315 & 0.408 \\
\hline Magnesium & 1.31 & 1.41 & 0.779 & $<0.05$ \\
\hline Manganese & 0.0242 & $<0.01$ & $<0.0057$ & $<0.01$ \\
\hline Sodium & 7.31 & 8.74 & 1.35 & 1.65 \\
\hline Nickel & $<0.0054$ & $<0.01$ & $<0.0054$ & $<0.01$ \\
\hline Lead & $<0.013$ & $<0.02$ & $<0.013$ & $<0.02$ \\
\hline Zinc & 0.0258 & 0.045 & 0.0179 & 0.061 \\
\hline
\end{tabular}


Table 84

Metals Analysis on Split Blind Quarterly Composites

Page 1 of 2

ThermoAnalytical, Inc.

\begin{tabular}{|c|c|c|c|c|c|c|c|c|}
\hline Metals & Duplicate 1 & Duplicate 2 & Duplicate 3 & Average & Duplicate 1 & Duplicate 2 & Duplicate 3 & Average \\
\hline First Quarter & \multicolumn{8}{|c|}{ (mg/L) } \\
\hline Aluminum & 0.149 & 0.138 & 0.162 & 0.1497 & 0.225 & 0.224 & 0.249 & 0.2327 \\
\hline Cadmium & $<0.0047$ & $<0.0047$ & $<0.0047$ & $<0.0047$ & $<0.005$ & $<0.005$ & $<0.005$ & $<0.005$ \\
\hline Calcium & 3.82 & 3.79 & 3.84 & 3.8167 & 3.82 & 3.98 & 4.02 & 3.9400 \\
\hline Chromium & $<0.01$ & $<0.01$ & $<0.01$ & $<0.01$ & $<0.005$ & $<0.005$ & $<0.005$ & $<0.005$ \\
\hline Copper & 0.008 & 0.0085 & 0.0075 & 0.0080 & 0.0088 & 0.0107 & 0.0092 & 0.0096 \\
\hline Iron & 0.381 & 0.394 & 0.37 & 0.3817 & 0.541 & 0.554 & 0.788 & 0.6277 \\
\hline Lead & $<0.013$ & $<0.013$ & $<0.013$ & $<0.013$ & $<0.003$ & $<0.003$ & $<0.003$ & $<0.003$ \\
\hline Magnesium & 0.685 . & 0.652 & 0.644 & 0.6603 & 0.62 & 0.669 & 0.67 & 0.6530 \\
\hline Manganese & 0.0276 & 0.0281 & 0.0287 & 0.0281 & 0.0323 & 0.0335 & 0.0351 & 0.0336 \\
\hline Nickel & 0.00099 & 0.0012 & 0.0011 & 0.0011 & $<0.005$ & $<0.005$ & $<0.005$ & $<0.005$ \\
\hline Sodium & 3.28 & 3.3 & 3.3 & 3.2933 & 3.44 & 3.58 & 3.47 & 3.4967 \\
\hline \multirow[t]{2}{*}{ Zinc } & 0.0071 & 0.0087 & 0.0093 & 0.0084 & $<0.005$ & $<0.005$ & $<0.005$ & $<0.005$ \\
\hline & \multicolumn{4}{|c|}{ Weston } & \multicolumn{4}{|c|}{ ThermoAnalytical, Inc. } \\
\hline Second Quarter & \multicolumn{8}{|c|}{ (mg/L) } \\
\hline Aluminum & 0.142 & 0.174 & 0.14 & 0.1520 & 0.219 & 0.21 & 0.326 & 0.2517 \\
\hline Cadmium & $<0.0047$ & $<0.0047$ & $<0.0047$ & $<0.0047$ & $<0.005$ & $<0.005$ & $<0.005$ & $<0.005$ \\
\hline Calcium & 3.81 & 4.42 & 3.58 & 3.9367 & 4.34 & 4.26 & 4.32 & 4.3067 \\
\hline Chromium & $<0.01$ & $<0.01$ & $<0.01$ & $<0.01$ & $<0.005$ & $<0.005$ & $<0.005$ & $<0.005$ \\
\hline Copper & $<0.018$ & $<0.018$ & $<0.018$ & $<0.018$ & 0.0062 & 0.0084 & $<0.005$ & 0.0065 \\
\hline Iron & 0.455 & 0.517 & 0.445 & 0.4723 & 0.658 & 0.643 & 0.71 & 0.6703 \\
\hline
\end{tabular}


Table 84

Metals Analysis on Split Blind Quarterly Composites

Page 2 of 2

\begin{tabular}{|c|c|c|c|c|c|c|c|c|}
\hline \multirow[b]{2}{*}{ Metals } & \multicolumn{4}{|c|}{ Weston } & \multicolumn{4}{|c|}{ ThermoAnalytical, Inc. } \\
\hline & Duplicate 1 & Duplicate 2 & Duplicate 3 & Average & Duplicate 1 & Duplicate 2 & Duplicate 3 & Average \\
\hline Magnesium & 0.667 & 0.831 & 0.62 & 0.7060 & 0.696 & 0.689 & 0.703 & 0.6960 \\
\hline Maganese & 0.0764 & 0.0886 & 0.0718 & 0.0789 & 0.981 & 0.955 & 0.984 & 0.9733 \\
\hline Nickel & $<0.0054$ & $<0.0054$ & $<0.0054$ & $<0.0054$ & $<0.005$ & $<0.005$ & $<0.005$ & $<0.005$ \\
\hline Zinc & \multicolumn{4}{|c|}{ Weston } & \multicolumn{4}{|c|}{ Shealy } \\
\hline Third Quarter & \multicolumn{8}{|c|}{ (mg/L) } \\
\hline Aluminum & 0.115 & 0.135 & 0.0886 & 0.1129 & $<0.05$ & 0.376 & 0.21 & 0.2120 \\
\hline Cadmium & $<0.0047$ & $<0.0047$ & $<0.0047$ & $<0.0047$ & $<0.005$ & $<0.005$ & $<0.005$ & $<0.005$ \\
\hline Iron & 0.539 & 0.607 & 0.618 & 0.5880 & 0.745 & 0.724 & 0.588 & 0.6857 \\
\hline Lead & $<0.013$ & 0.0264 & 0.0184 & 0.0192 & $<0.02$ & $<0.02$ & $<0.02$ & $<0.02$ \\
\hline Magnesium & 1.49 & 1.12 & 1.29 & 1.3000 & 0.788 & 0.791 & 0.704 & 0.7610 \\
\hline Manganese & 0.0876 & 0.0953 & 0.0979 & 0.0936 & 0.103 & 0.077 & 0.071 & 0.0837 \\
\hline Nickel & $<0.0054$ & $<0.0054$ & $<0.0054$ & $<0.0054$ & $<0.01$ & $<0.01$ & $<0.01$ & $<0.01$ \\
\hline Sodium & 4 & 4.09 & 4.34 & 4.1433 & 4.84 & 3.64 & 3.24 & 3.9067 \\
\hline Zinc & 0.0185 & 0.0156 & 0.0289 & 0.0210 & 0.017 & 0.011 & 0.014 & 0.0140 \\
\hline
\end{tabular}




\section{Errata from 1994 Report}

The following information was either incorrect in, or missing from, Savannah River Site Environmental Data for 1994, WSRC-TR-95-077:

Table 23, Radioactivity in SRS Stream Water, page 81, should have included:

\begin{tabular}{lcccc}
\hline Location & $\begin{array}{c}\text { No. of } \\
\text { Samples }\end{array}$ & Arithmetic Mean $\pm \sigma$ & Maximum $\pm \sigma$ & Minimum $\pm \sigma$ \\
\hline $\begin{array}{l}\text { Gross A, } \mu \text { Ci/mL } \\
\text { Upper Three Runs }\end{array}$ & & & & \\
Crouch Branch at Road 4 & 25 & $(2.79 \pm 3.30) \mathrm{E}-10$ & $(1.29 \pm 0.54) \mathrm{E}-09$ & $(-0.78 \pm 2.48) \mathrm{E}-10$ \\
U3R-1A Treadway Bridge RD & 52 & $(1.49 \pm 0.85) \mathrm{E}-09$ & $(3.48 \pm 0.60) \mathrm{E}-09$ & $(3.82 \pm 2.50) \mathrm{E}-10$ \\
8-1 & & & & \\
U3R-3 at Road C & 25 & $(1.81 \pm 0.99) \mathrm{E}-09$ & $(4.46 \pm 0.72) \mathrm{E}-09$ & $(4.87 \pm 2.55) \mathrm{E}-10$ \\
U3R-4 at Road A & 26 & $(1.57 \pm 0.79) \mathrm{E}-09$ & $(3.09 \pm 0.65) \mathrm{E}-09$ & $(2.15 \pm 2.20) \mathrm{E}-10$ \\
Four Mile Creek & & & & \\
FM-2 at Road 4 & 25 & $(1.19 \pm 0.40) \mathrm{E}-09$ & $(1.79 \pm 0.56) \mathrm{E}-09$ & $(4.99 \pm 3.21) \mathrm{E}-10$ \\
FM-2B Above F-Area Effluent & 24 & $(3.50 \pm 2.23) \mathrm{E}-10$ & $/(8.08 \pm 5.30) \mathrm{E}-10$ & $(0.08 \pm 2.50) \mathrm{E}-10$ \\
FM-3A Below F-Area EHfluent & 26 & $(1.51 \pm 0.72) \mathrm{E}-09$ & $(3.51 \pm 0.97) \mathrm{E}-09$ & $(5.76 \pm 3.36) \mathrm{E}-10$ \\
FM-6 at Road A-12.2 & 26 & $(3.02 \pm 2.92) \mathrm{E}-10$ & $(9.10 \pm 4.15) \mathrm{E}-10$ & $(-1.56 \pm 1.27) \mathrm{E}-10$ \\
FM-A7 at Road A-7 & 25 & $(8.81 \pm 3.68) \mathrm{E}-10$ & $(1.77 \pm 0.57) \mathrm{E}-09$ & $(2.52 \pm 3.30) \mathrm{E}-10$ \\
H-008 Outfall & 51 & $(2.34 \pm 1.20) \mathrm{E}-09$ & $(7.72 \pm 1.00) \mathrm{E}-09$ & $((9.48 \pm 5.14) \mathrm{E}-10$ \\
HP-50 Tritium Facility Outfall & 25 & $(3.81 \pm 2.19) \mathrm{E}-09$ & $(9.63 \pm 1.13) \mathrm{E}-09$ & $(2.70 \pm 3.04) \mathrm{E}-10$
\end{tabular}

